\title{
C-N Cross-Couplings for Site-selective Late-Stage Diversification via Aryl Sulfonium Salts
}

Pascal S. Engl, Andreas P. Häring, Florian Berger, Georg Berger, Alberto Peréz-Bitrián and Tobias Ritter*

Max-Planck-Institute für Kohlenforschung,

Kaiser-Wilhelm Platz 1, D-45470 Mülheim an der Ruhr

*E-mail: ritter@kofo.mpg.de 


\section{TABLE OF CONTENTS}

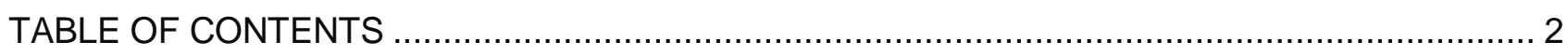

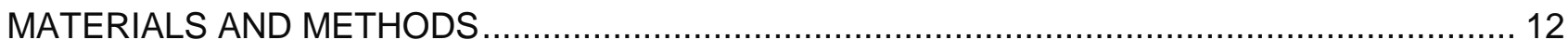

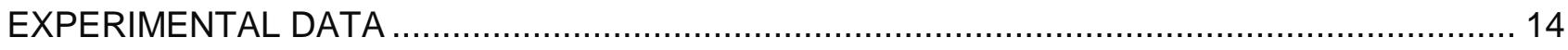

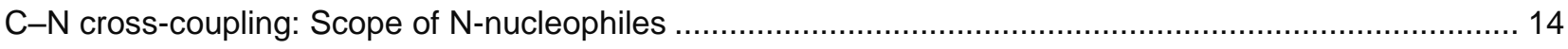

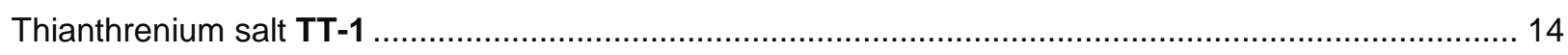

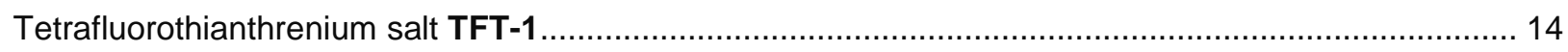

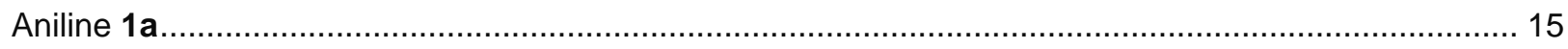

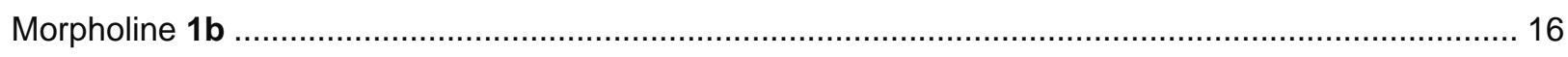

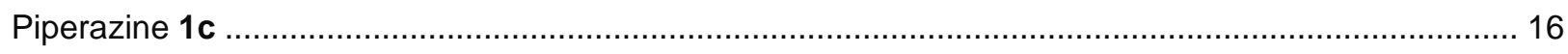

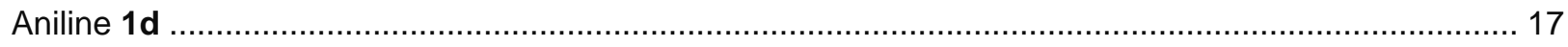

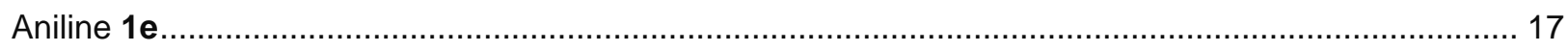

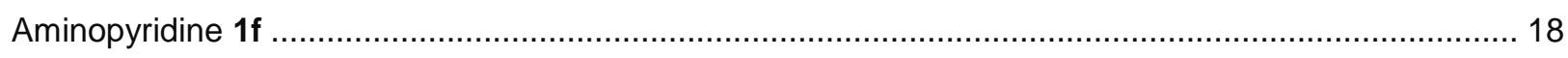

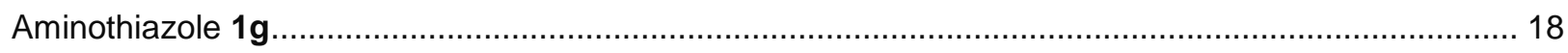

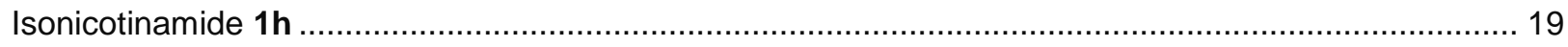

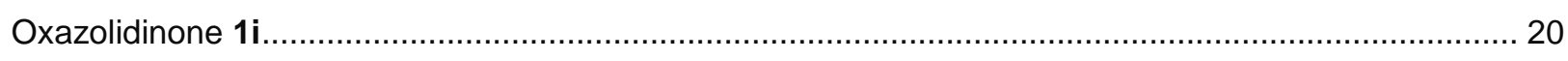

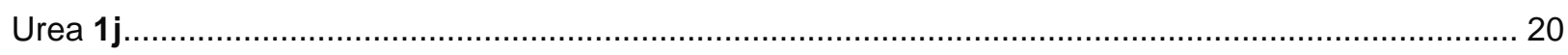

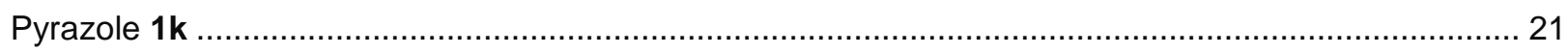

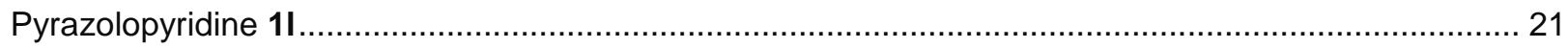

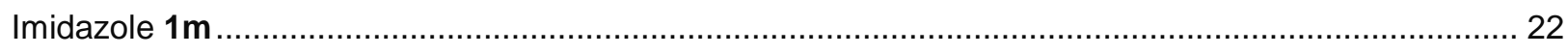

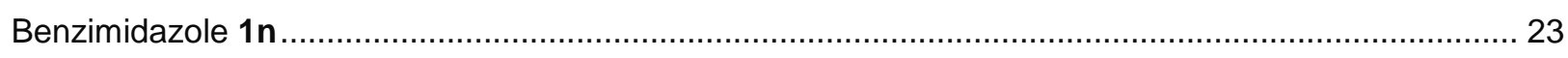

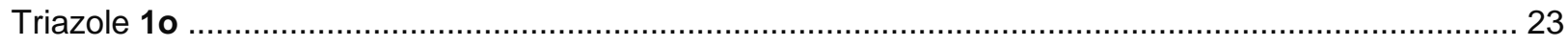

Phthalimide 1p

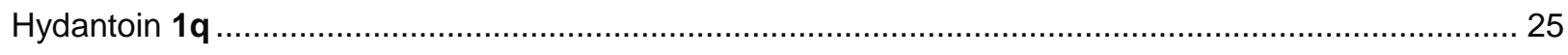

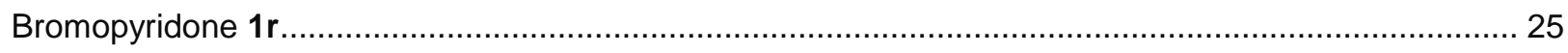

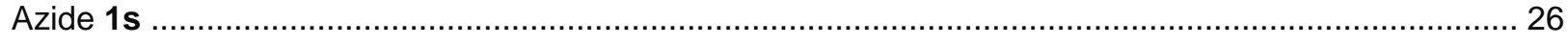

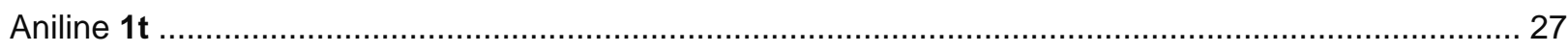




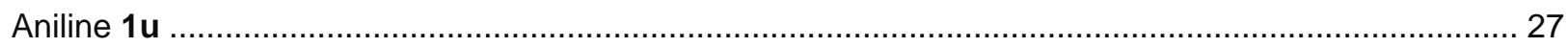

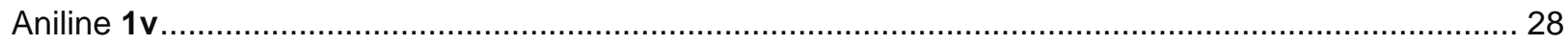

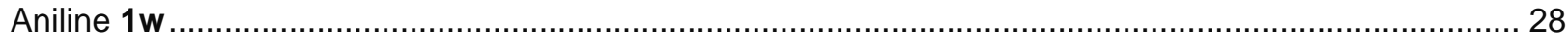

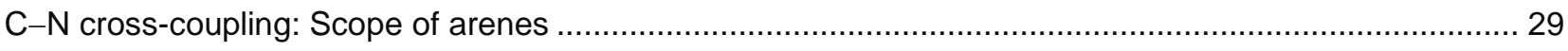

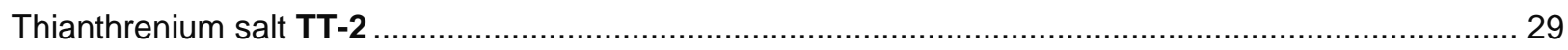

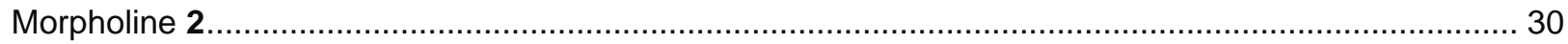

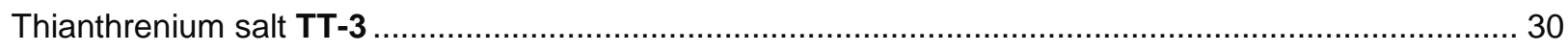

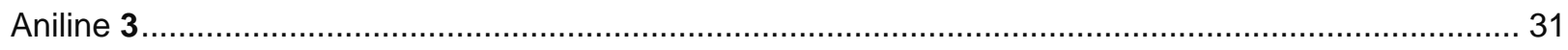

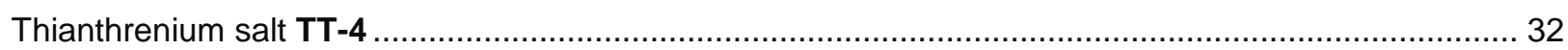

Aniline 4

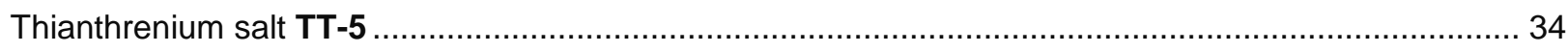

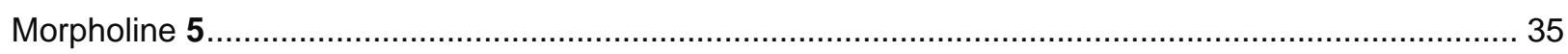

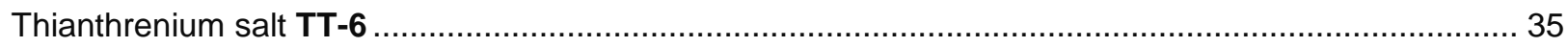

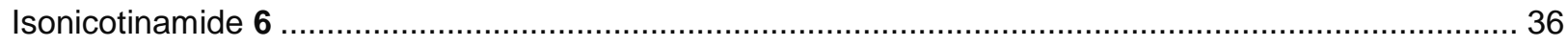

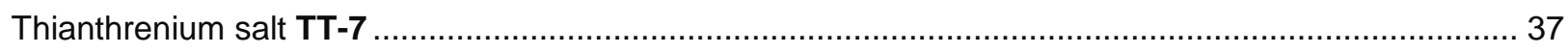

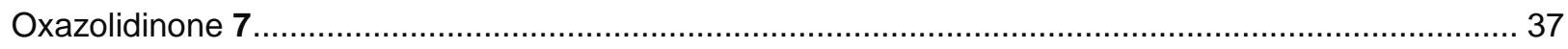

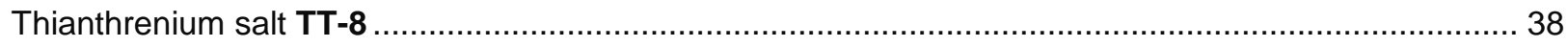

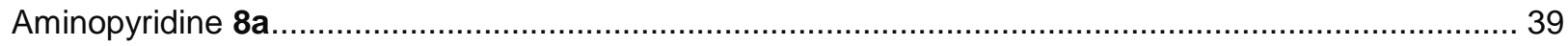

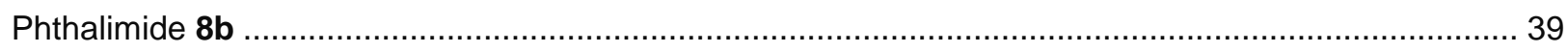

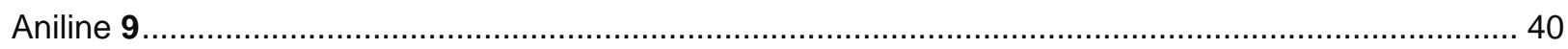

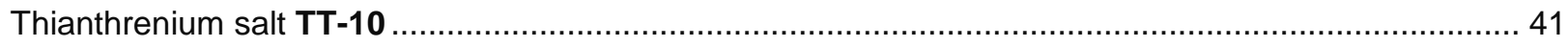

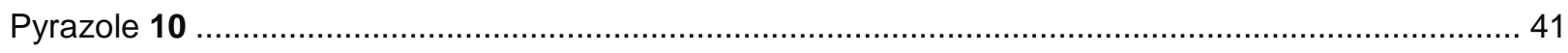

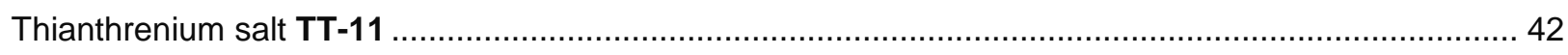

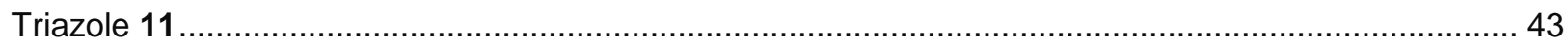

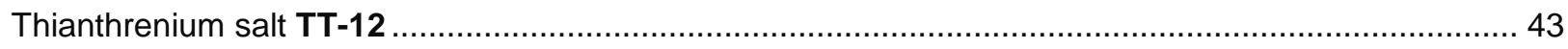

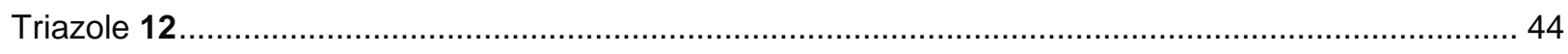

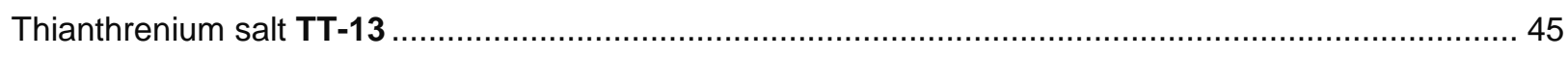

Triazole 13

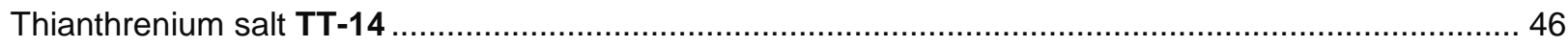




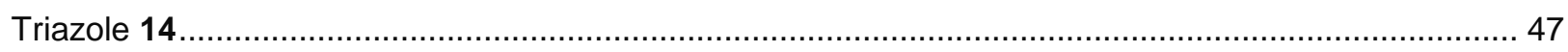

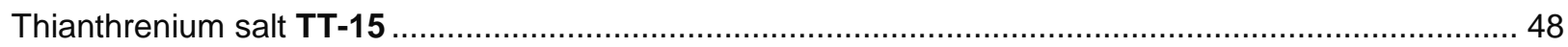

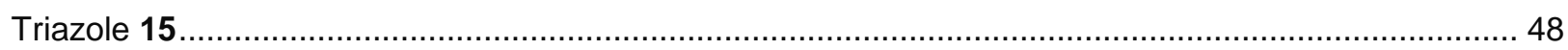

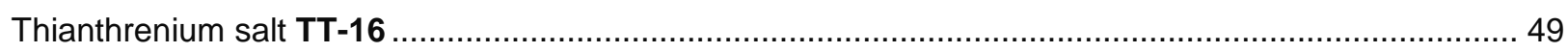

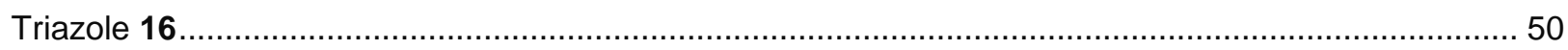

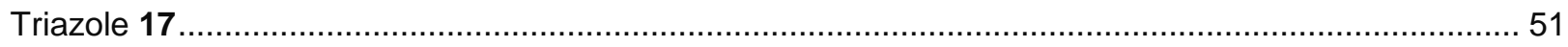

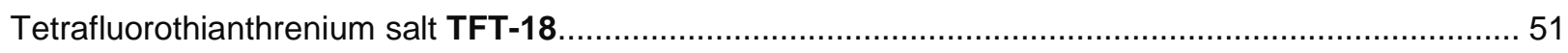

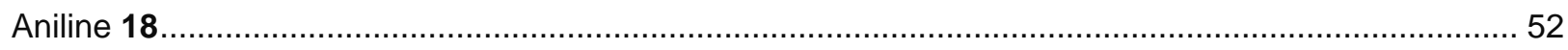

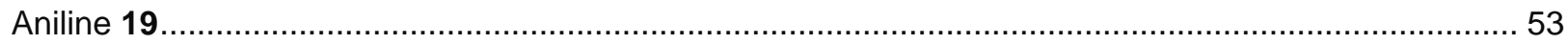

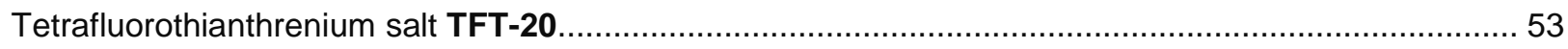

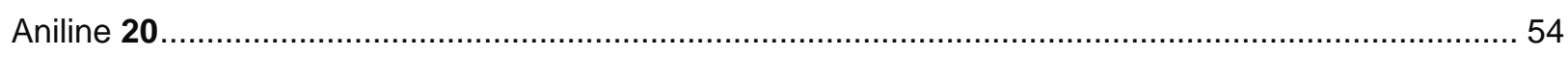

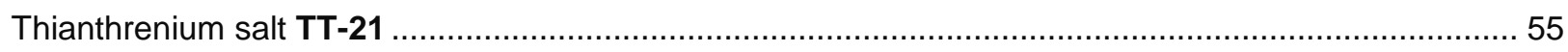

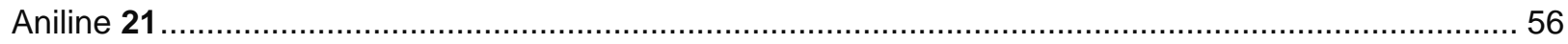

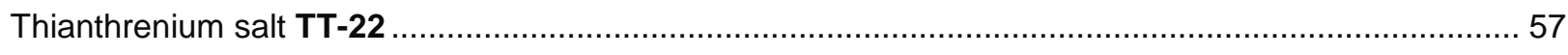

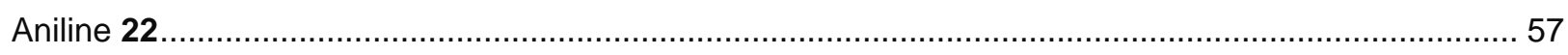

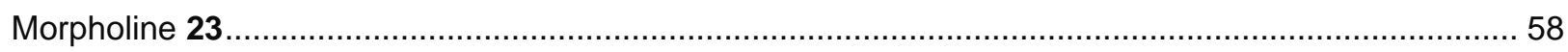

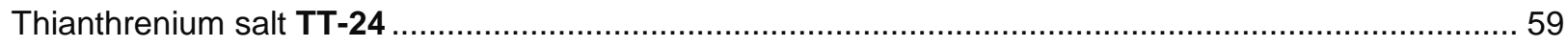

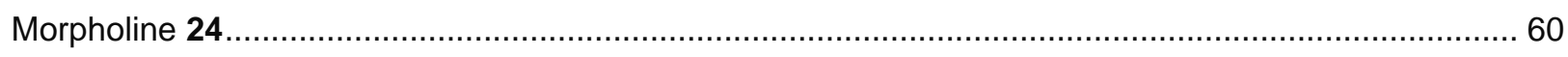

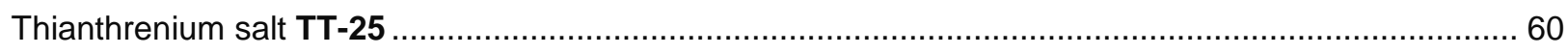

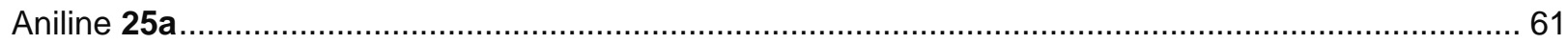

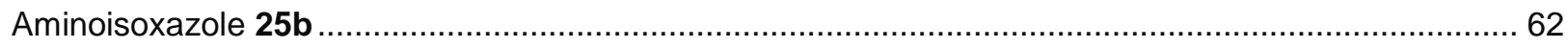

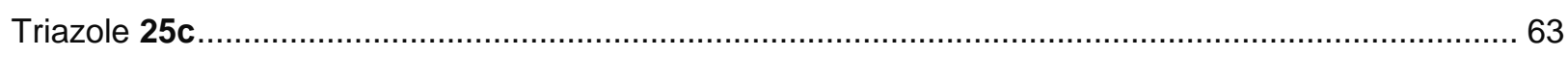

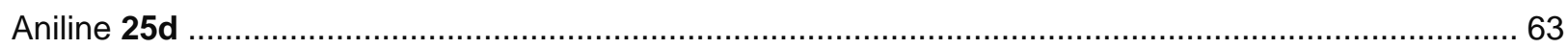

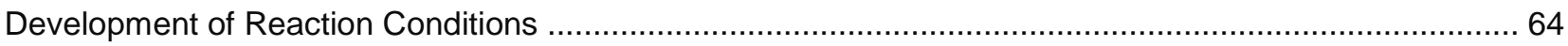

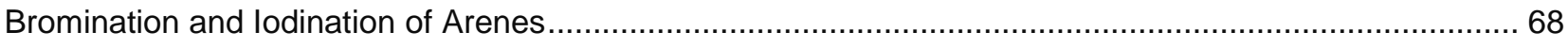

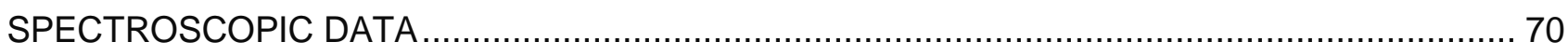

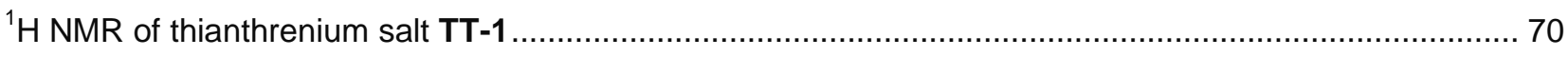

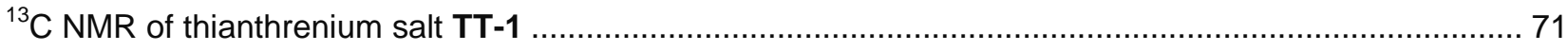

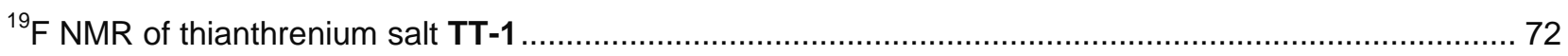




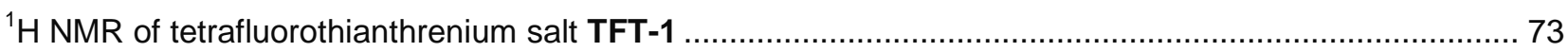

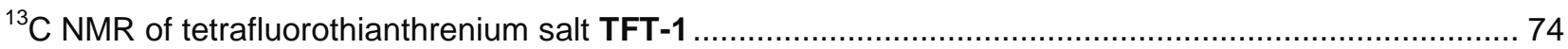

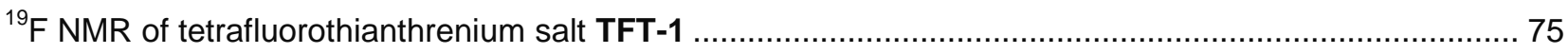

${ }^{1} \mathrm{H}$ NMR of aniline $1 \mathbf{1}$

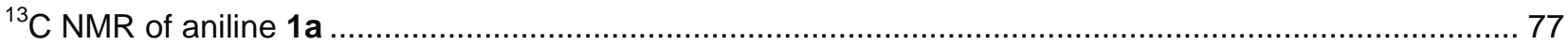

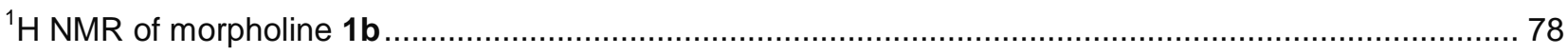

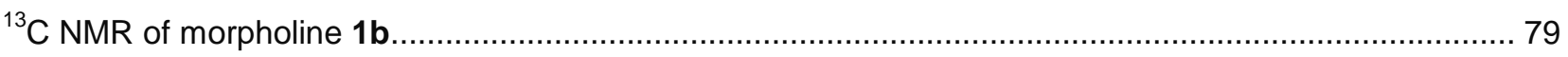

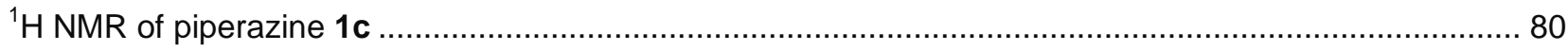

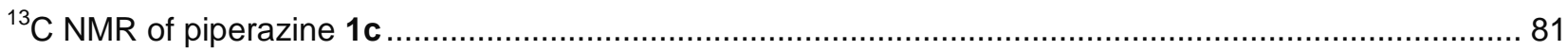

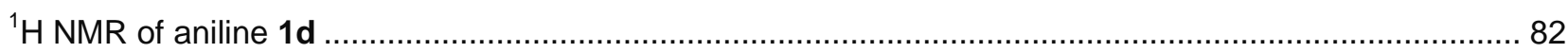

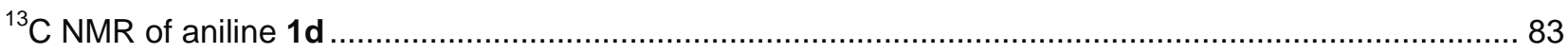

${ }^{1} \mathrm{H}$ NMR of aniline $1 \mathrm{e}$

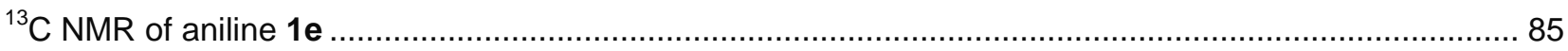

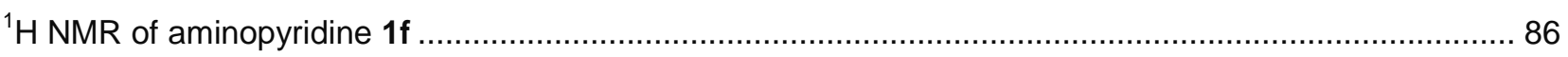

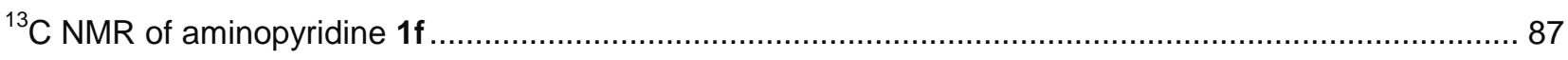

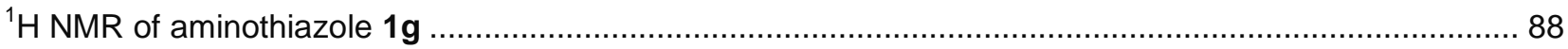

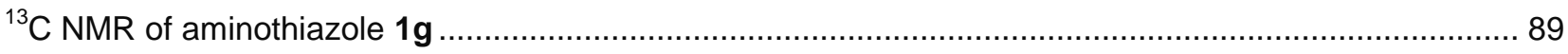

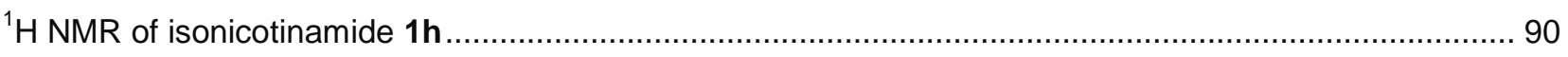

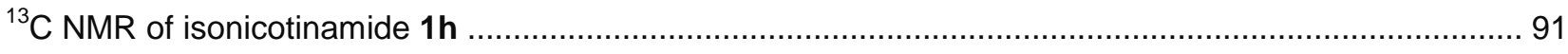

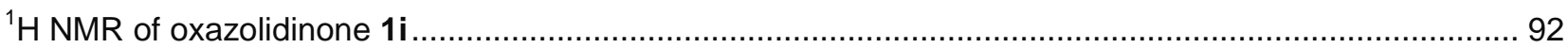

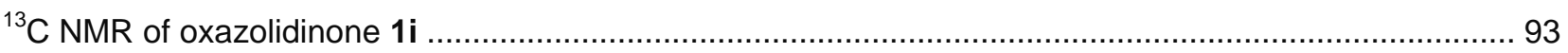

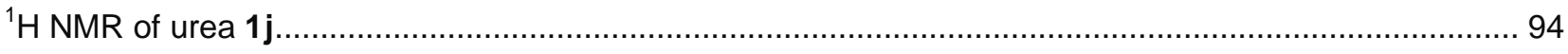

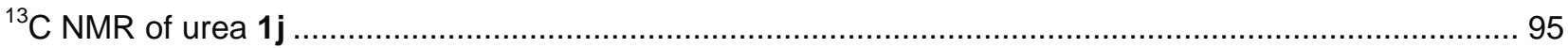

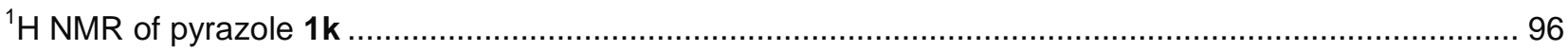

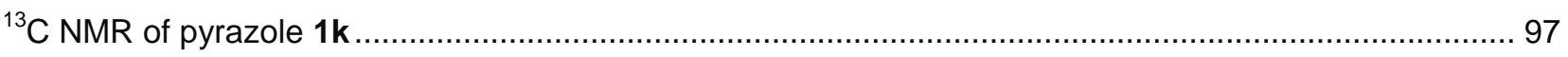

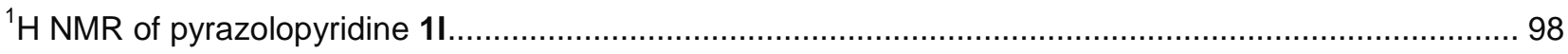

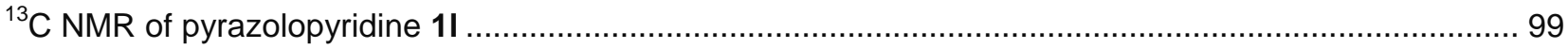

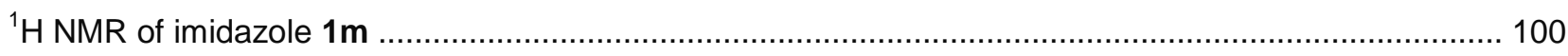

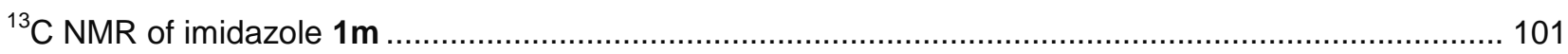




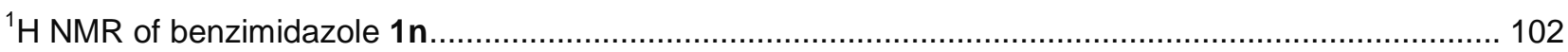

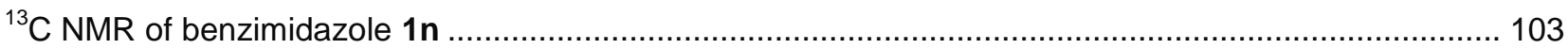

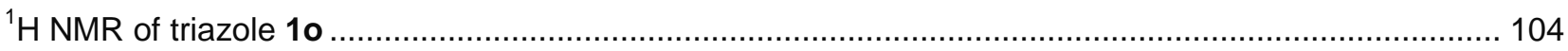

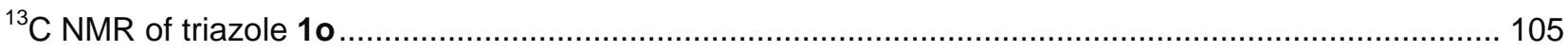

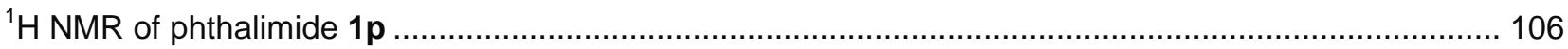

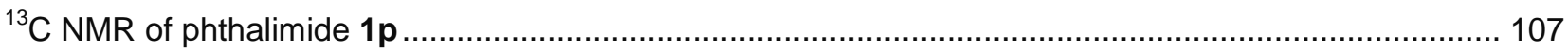

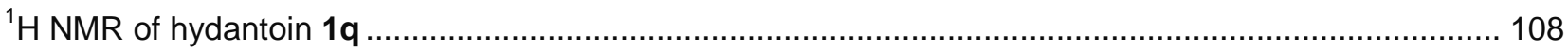

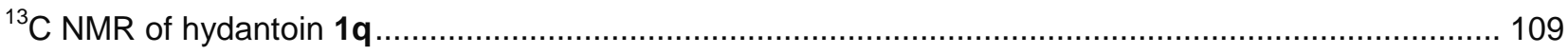

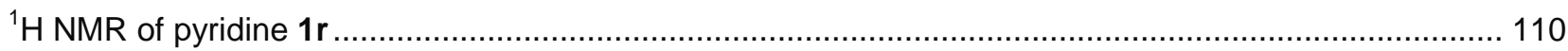

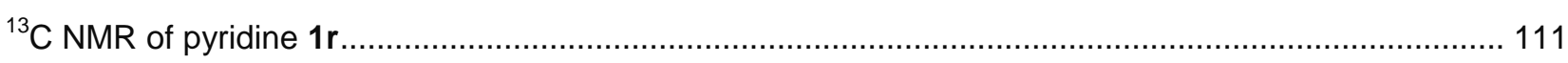

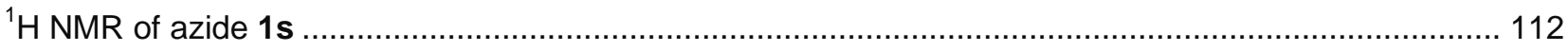

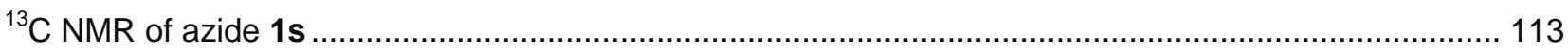

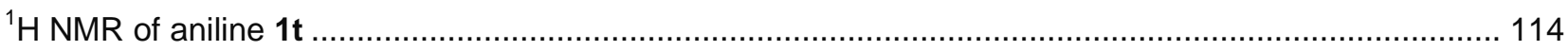

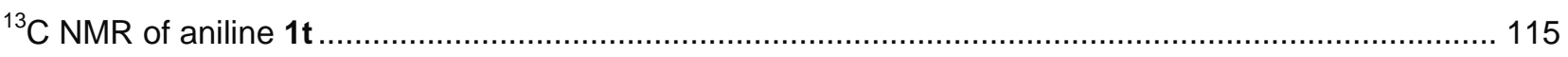

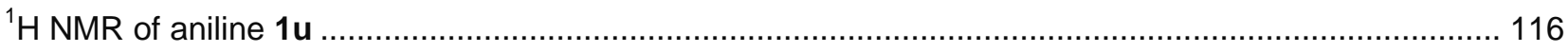

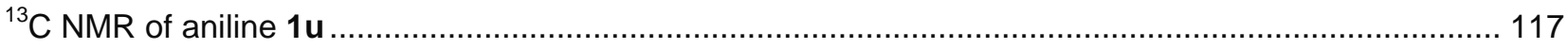

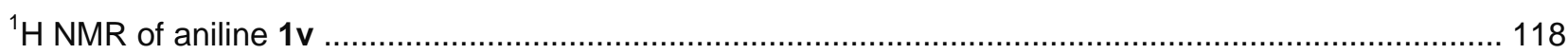

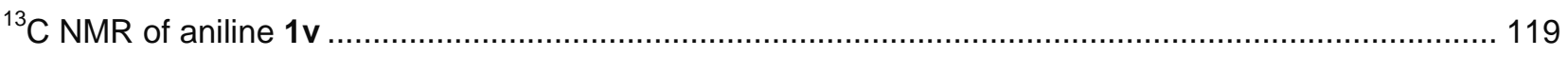

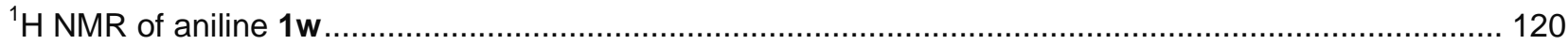

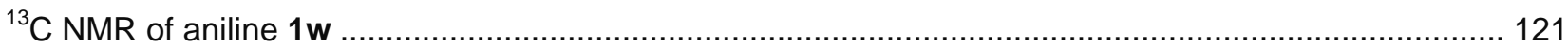

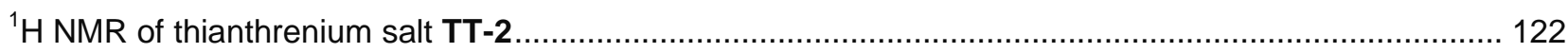

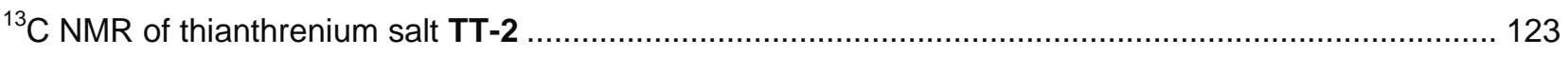

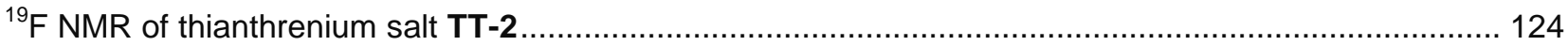

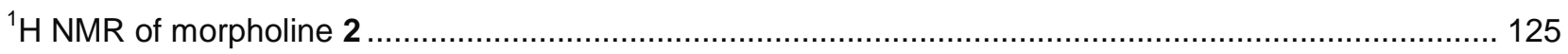

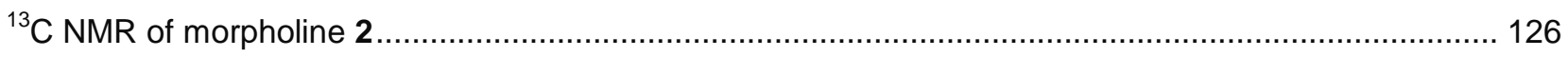

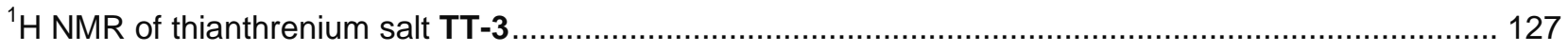

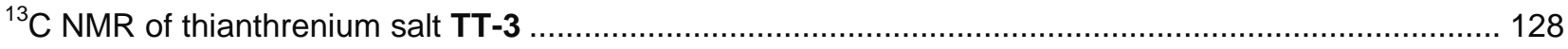

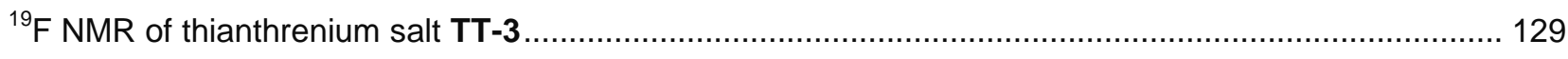

${ }^{1} \mathrm{H}$ NMR of aniline 3 
${ }^{13} \mathrm{C}$ NMR of aniline 3

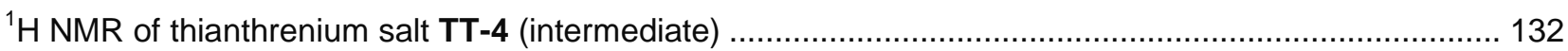

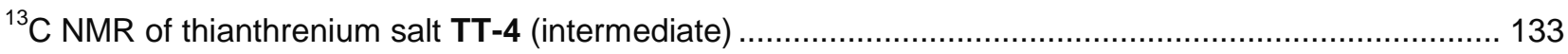

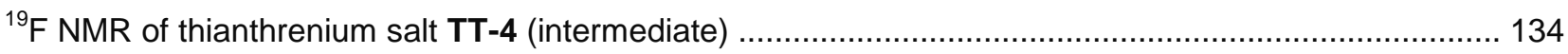

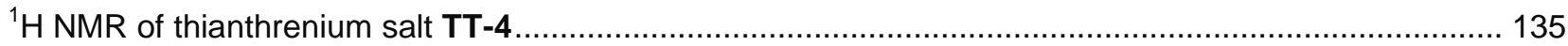

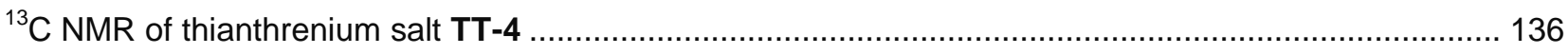

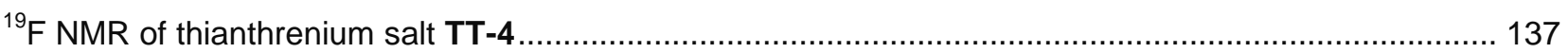

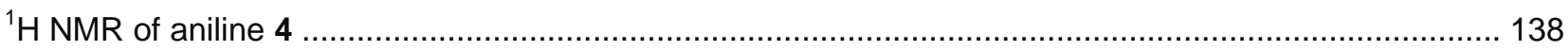

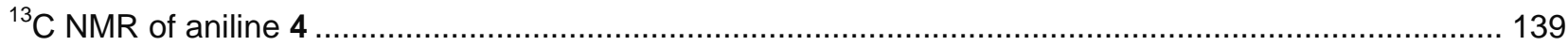

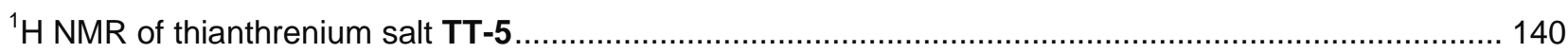

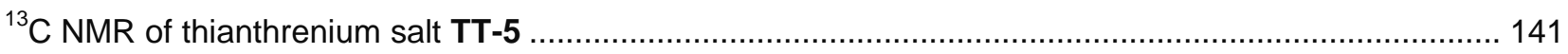

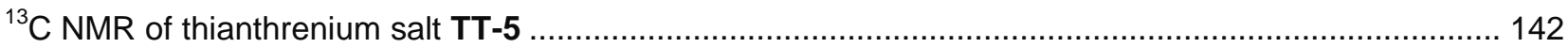

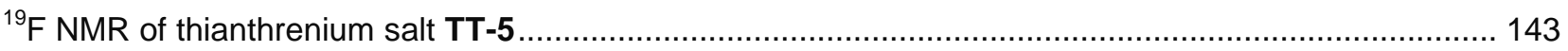

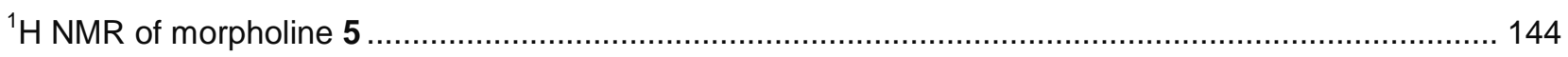

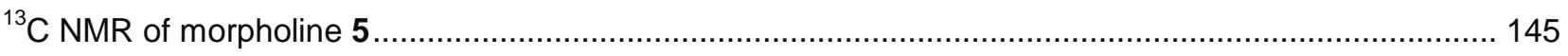

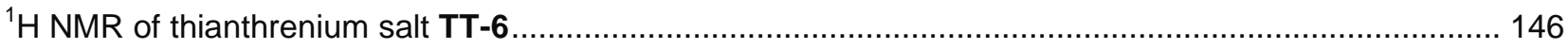

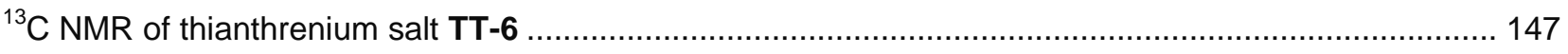

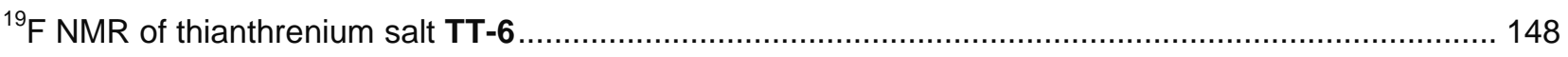

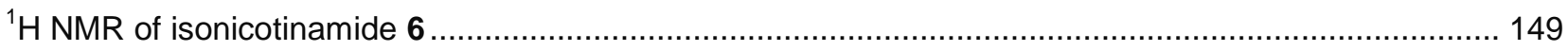

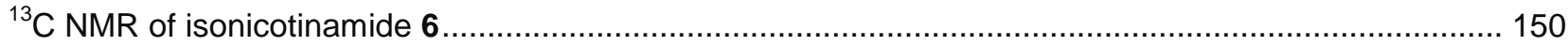

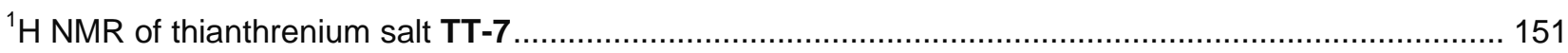

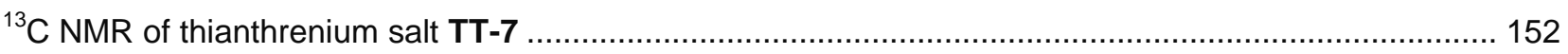

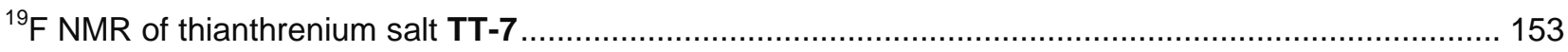

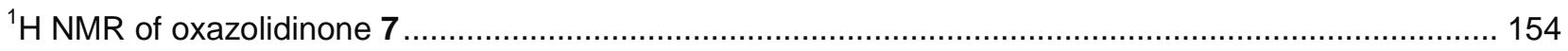

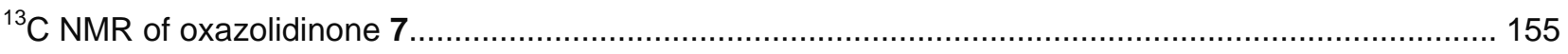

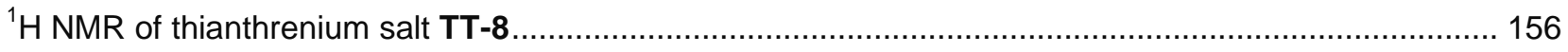

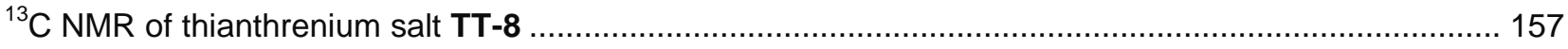

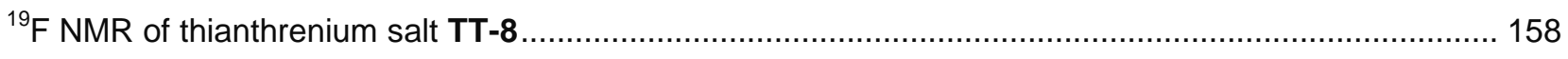

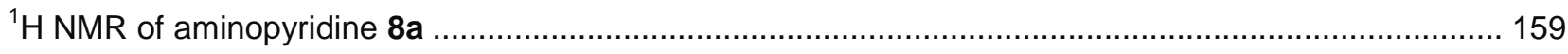


${ }^{13} \mathrm{C}$ NMR of aminopyridine $8 \mathrm{a}$ 160

${ }^{1} \mathrm{H}$ NMR of phthalimide $8 \mathrm{~b}$ 161

${ }^{13} \mathrm{C}$ NMR of phthalimide $\mathbf{8 b}$ 162

${ }^{1} \mathrm{H}$ NMR of aniline 9 163

${ }^{13} \mathrm{C}$ NMR of aniline 9 164

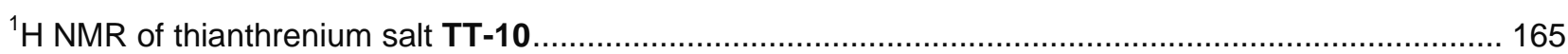

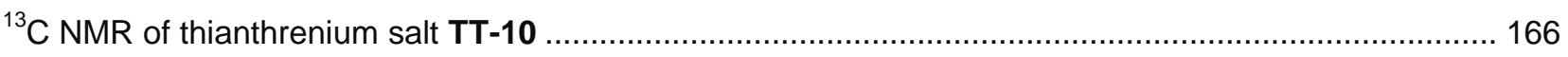

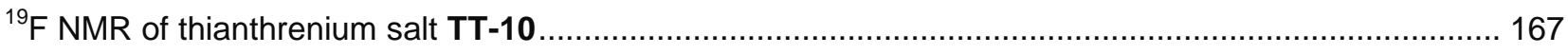

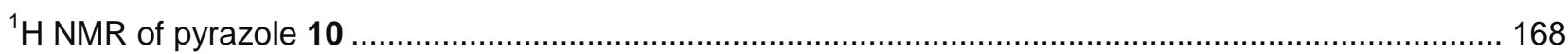

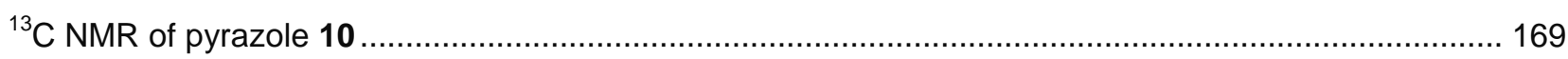

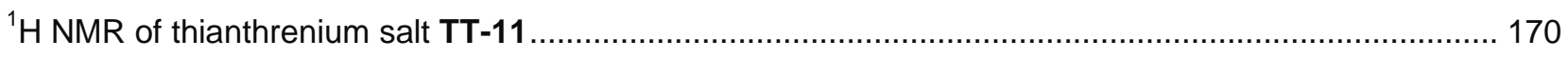

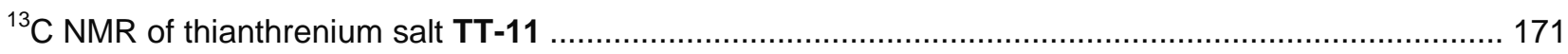

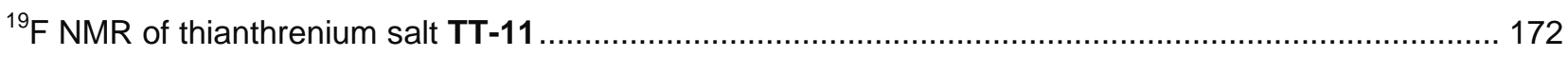

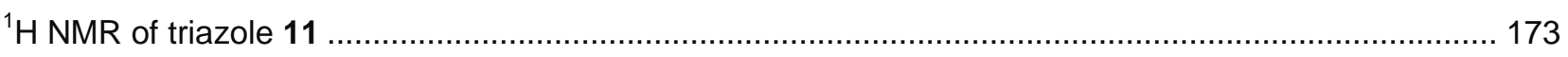

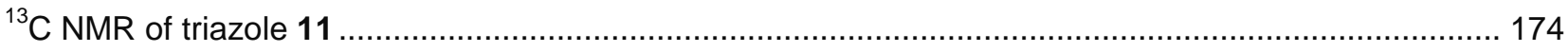

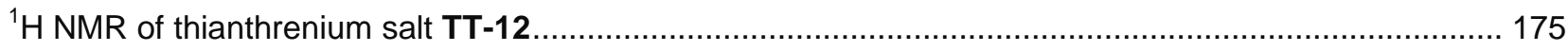

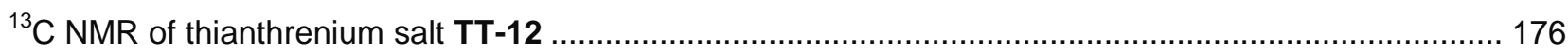

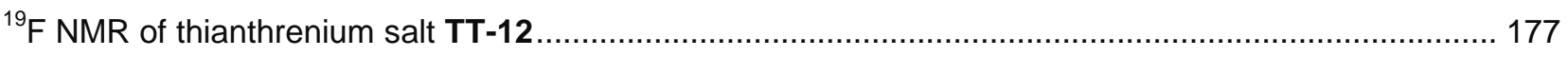

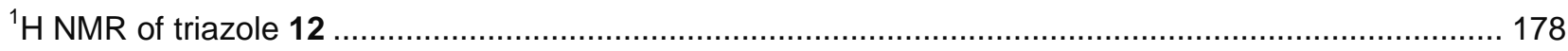

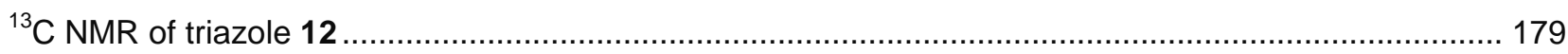

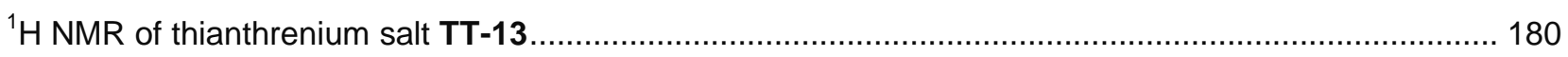

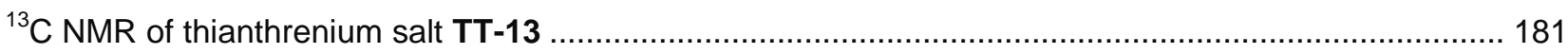

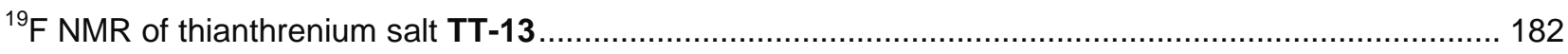

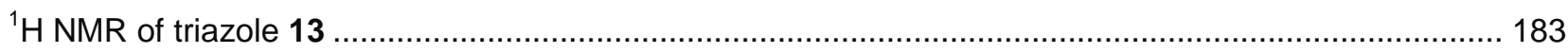

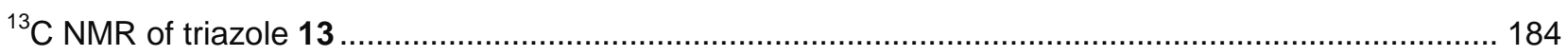

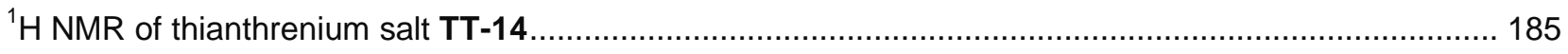

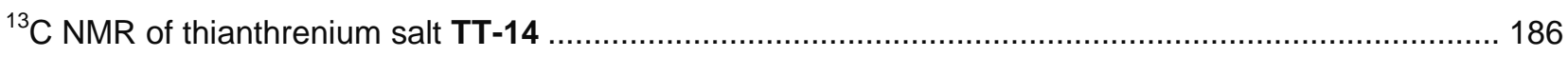

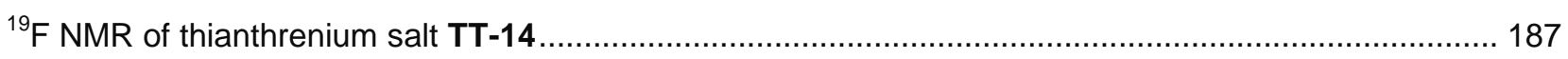

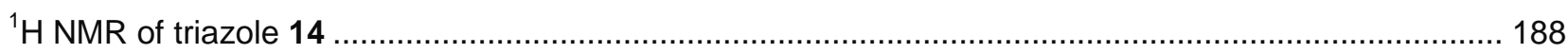


${ }^{13} \mathrm{C}$ NMR of triazole 14

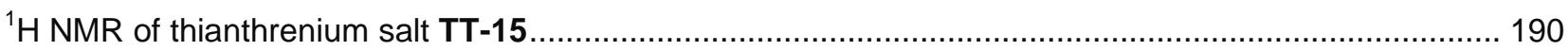

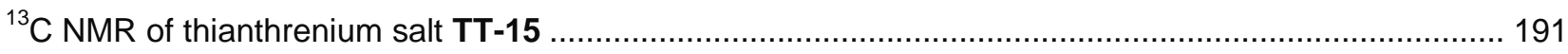

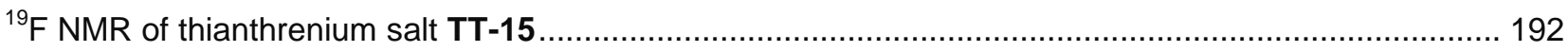

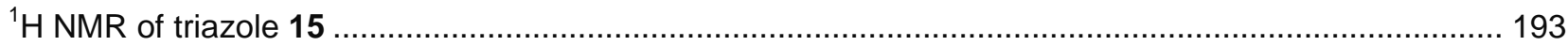

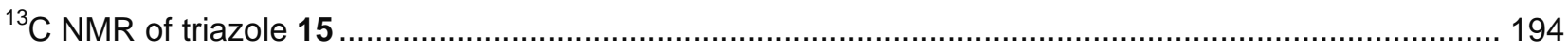

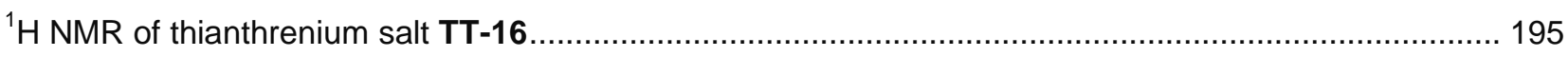

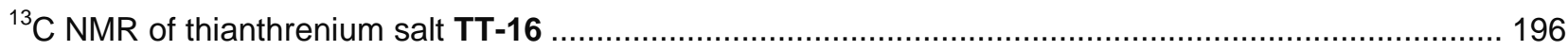

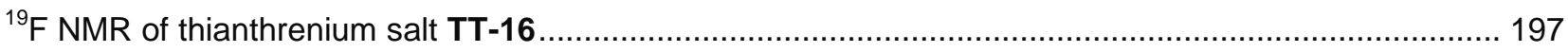

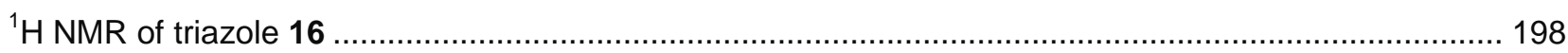

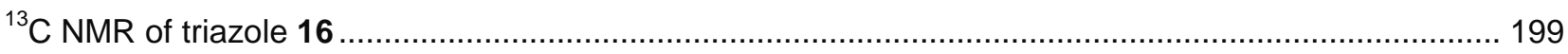

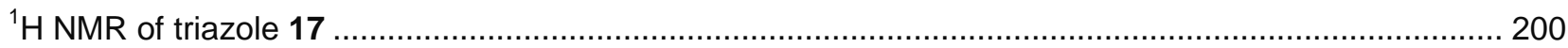

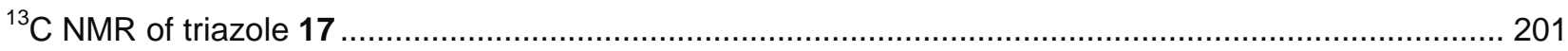

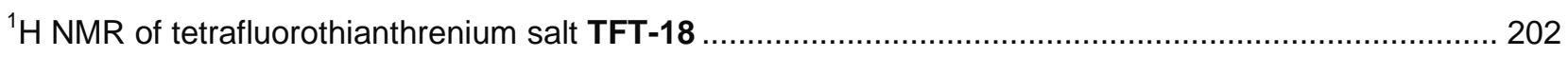

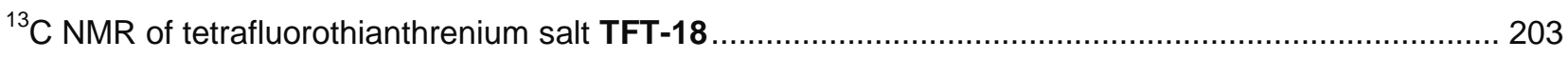

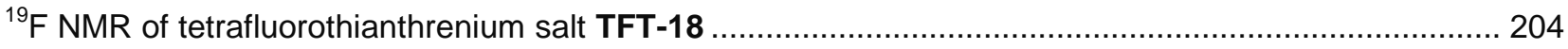

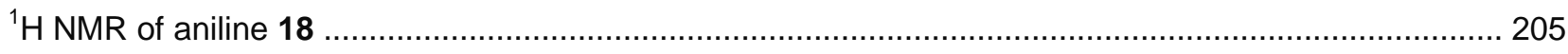

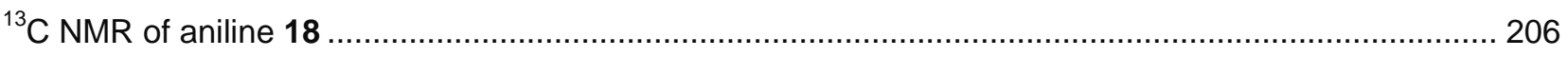

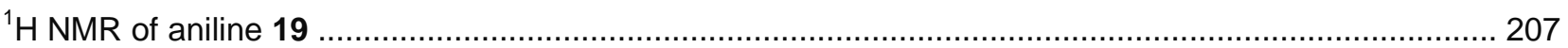

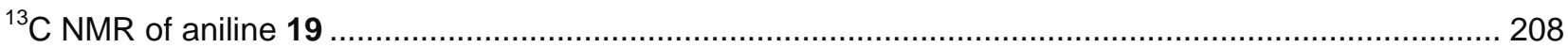

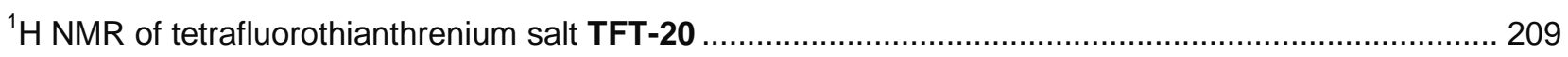

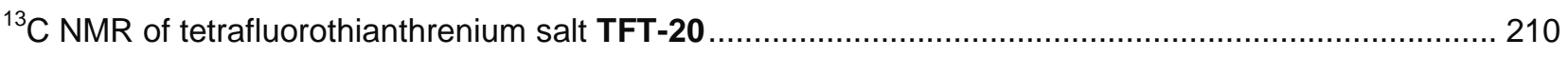

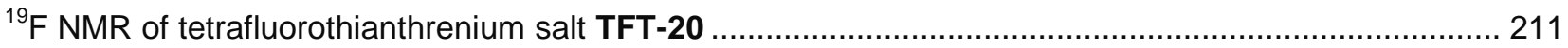

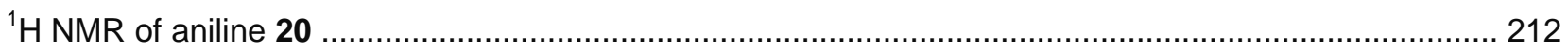

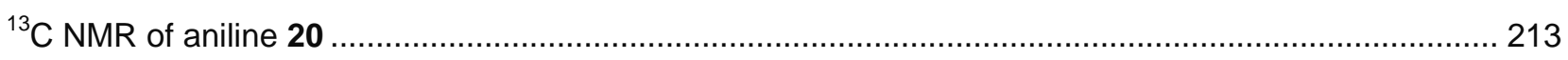

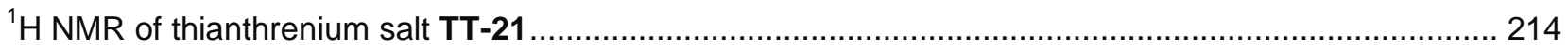

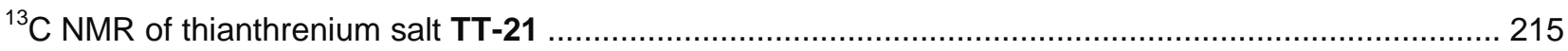

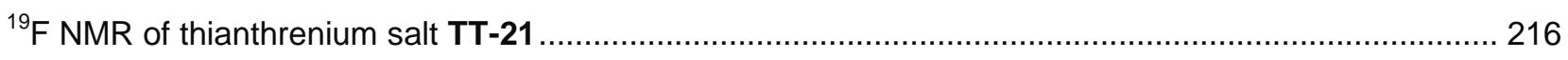

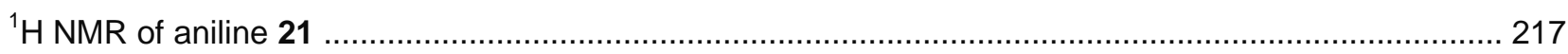


${ }^{13} \mathrm{C}$ NMR of aniline 21

${ }^{1} \mathrm{H}$ NMR of thianthrenium salt TT-22.

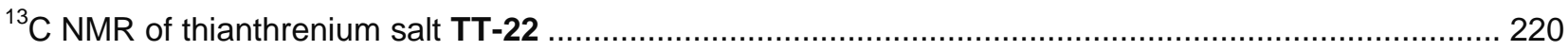

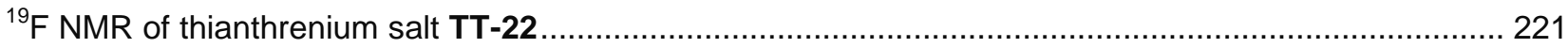

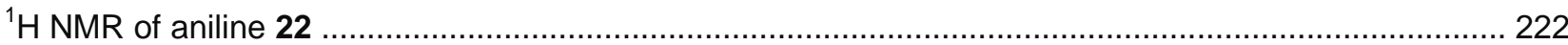

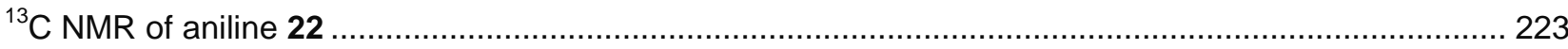

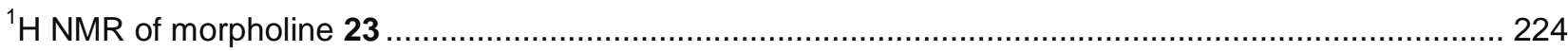

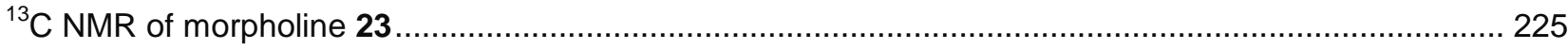

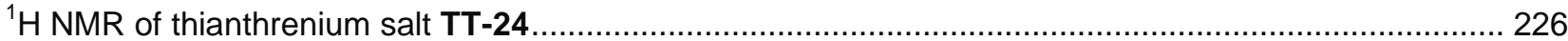

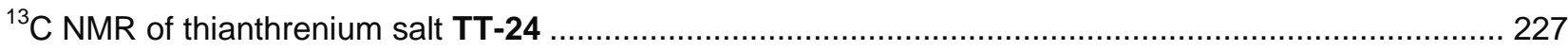

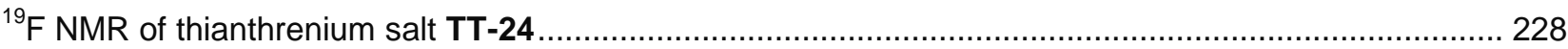

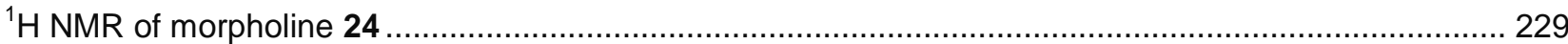

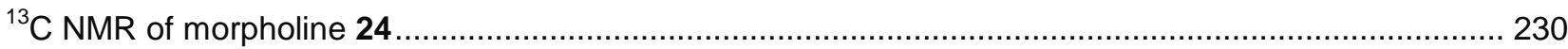

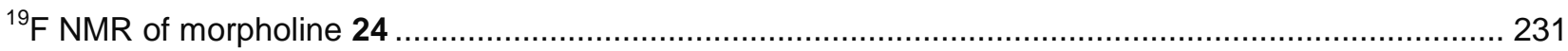

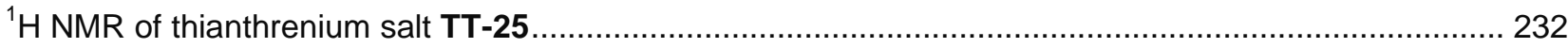

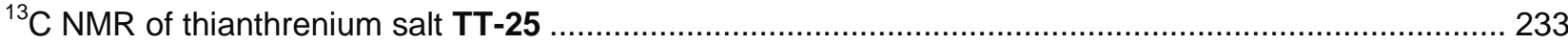

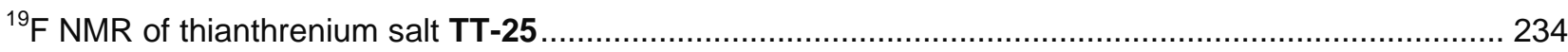

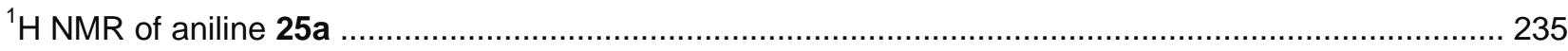

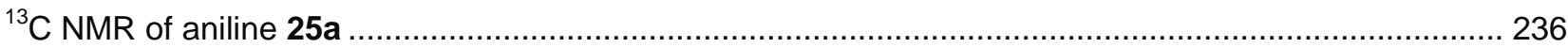

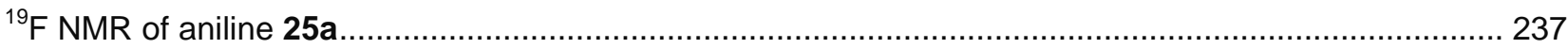

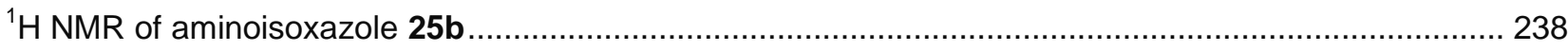

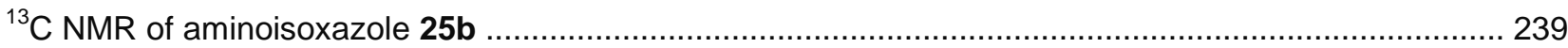

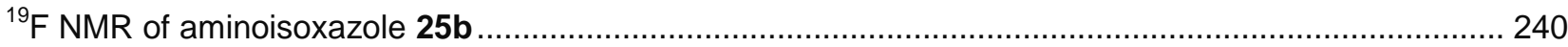

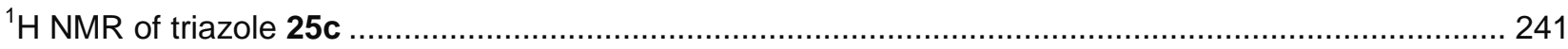

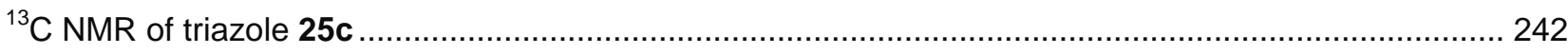

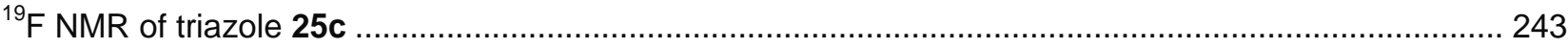

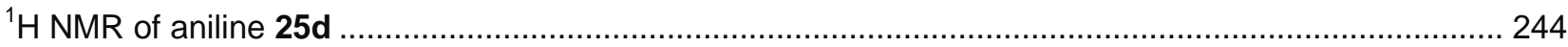

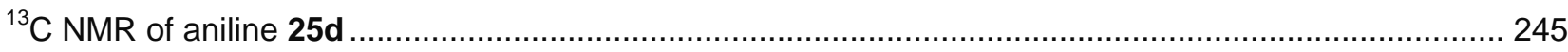

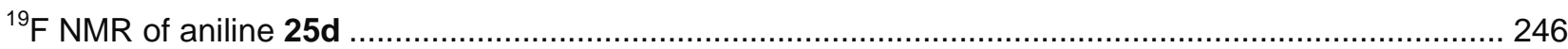




\section{MATERIALS AND METHODS}

All reactions were carried out under ambient atmosphere unless otherwise stated. High-resolution mass spectra were obtained using $Q$ Exactive Plus from Thermo. Concentration under reduced pressure was performed by rotary evaporation at $25-40^{\circ} \mathrm{C}$ at an appropriate pressure. Purified compounds were further dried under vacuum $\left(10^{-6}-10^{-3}\right.$ bar). Yields refer to purified and spectroscopically pure compounds, unless otherwise stated.

\section{Solvents}

DMSO was purchased from Chemsolute and used as received. Anhydrous DMF was purchased from SigmaAldrich, degassed via three freeze-pump-thaw cycles and stored under a nitrogen atmosphere. Anhydrous acetonitrile, tetrahydrofuran, dichloromethane, and toluene were obtained from Phoenix Solvent Drying Systems. All deuterated solvents were purchased from Euriso-Top.

\section{Chromatography}

Thin layer chromatography (TLC) was performed using EMD TLC plates pre-coated with $250 \mu$ m thickness silica gel $60 \mathrm{~F}_{254}$ plates and visualized by irradiation UV light or by dipping the TLC plate onto a dilute, alkaline, aqueous $\mathrm{KMnO}_{4}$-solution. Flash chromatography was performed using silica gel (40-63 $\mu \mathrm{m}$ particle size) purchased from Geduran.

\section{Photochemistry}

All reactions with blue light were carried out using a photoreactor equipped with a blue LED module (KTElektronik, "100W Power LED blau 450 nm Aquarium", 450 nm, $100 \mathrm{~W}$ ), consisting out of 100 LED-chips. The power of the LED was adjusted using a linear regulator. To avoid overheating of the reaction mixture, vials were cooled with two Peltier-elements (TEC1-12706) while being irradiated with blue light.

\section{Spectroscopy and Instruments}

NMR spectra were recorded on a Bruker Ascend ${ }^{T M} 500$ spectrometer operating at $500 \mathrm{MHz}, 471 \mathrm{MHz}$, and $126 \mathrm{MHz}$, for ${ }^{1} \mathrm{H},{ }^{19} \mathrm{~F}$, and ${ }^{13} \mathrm{C}$ acquisitions, respectively. Chemical shifts are reported in ppm with the solvent residual peak as the internal standard. For ${ }^{1} \mathrm{H} \mathrm{NMR:} \mathrm{CDCl}_{3}, \delta 7.260 ; \mathrm{CD}_{3} \mathrm{CN}, \delta 1.940 ; \mathrm{CD}_{2} \mathrm{Cl}_{2}, \delta 5.320$; DMSO- $\mathrm{d}_{6}, \delta$ 2.500; For ${ }^{13} \mathrm{C}$ NMR: $\mathrm{CDCl}_{3}, \delta 77.16 ; \mathrm{CD}_{3} \mathrm{CN}, \delta 1.32 ; \mathrm{CD}_{2} \mathrm{Cl}_{2}, \delta 53.84 ; \mathrm{DMSO}_{6}, \delta 39.52 .{ }^{19} \mathrm{~F}$ NMR spectra were referenced using a unified chemical shift scale based on the ${ }^{1} \mathrm{H}$ resonance of tetramethylsilane $(1 \% \mathrm{v} / \mathrm{v}$ solution in the respective solvent). Data is reported as follows: $\mathrm{s}=\operatorname{singlet}, \mathrm{d}=$ doublet, $\mathrm{t}=$ triplet, $\mathrm{q}=$ quartet, quin = quintet, sext = sextet, sept = septet, $\mathrm{m}=$ multiplet, $\mathrm{bs}=$ broad singlet; coupling constants in $\mathrm{Hz}$; integration.

\section{Starting materials}

All substrates were used as received from commercial suppliers, unless otherwise stated. Chemicals were purchased from Sigma-Aldrich, Chempur, TCl, or Alfa Aesar. Thianthrenium salts TFT-9, TT-17, TFT-19, and 
TT-23 were prepared as described before. ${ }^{1}$

${ }^{1}$ Nature 2019, 567, 223-228. 


\section{EXPERIMENTAL DATA}

\section{C-N cross-coupling: Scope of $\mathbf{N}$-nucleophiles}

Thianthrenium salt TT-1

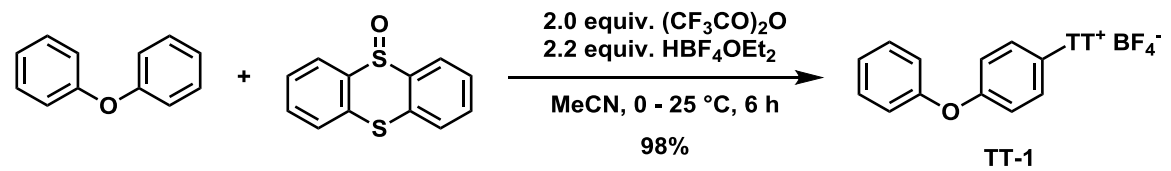

Under ambient atmosphere, a $500 \mathrm{~mL}$ round-bottom flask equipped with a magnetic stir bar was charged with diphenyl ether (7.0 mL, $7.5 \mathrm{~g}, 44 \mathrm{mmol}, 1.0$ equiv.) and $\mathrm{MeCN}(0.10 \mathrm{~L}, \mathrm{C}=0.44 \mathrm{M})$. Trifluoroacetic anhydride (12.2 mL, $18.4 \mathrm{~g}, 87.8 \mathrm{mmol}, 2.0$ equiv.) was added at ambient temperature while stirring. After cooling to $0^{\circ} \mathrm{C}$, thianthrene S-oxide (10.2 g, $43.9 \mathrm{mmol}, 0.99$ equiv.) was added in one portion, followed by the addition of $\mathrm{HBF}_{4} \cdot \mathrm{OEt}_{2}\left(13.2 \mathrm{~mL}, 15.7 \mathrm{~g}, 97.0 \mathrm{mmol}, 2.2\right.$ equiv.) in one portion. The mixture was stirred at $0{ }^{\circ} \mathrm{C}$ for $1 \mathrm{~h}$, then at ambient temperature for $5 \mathrm{~h}$. The reaction mixture was concentrated under reduced pressure, and subsequently diluted with DCM $(300 \mathrm{~mL})$. The solution was poured onto a saturated aqueous $\mathrm{NaHCO}_{3}$ solution $(300 \mathrm{~mL})$, and the layers were separated. The organic phase was washed with aqueous $\mathrm{NaBF}_{4}$ solution $(2 \times 300 \mathrm{~mL}, 10 \%)$, and with water $(2 \times 300 \mathrm{~mL})$. The organic phase was dried over $\mathrm{MgSO}_{4}$, and the solvent was removed under reduced pressure. The residue was purified by chromatography on silica gel eluting with DCM / MeOH (1:0-10:1 (v/v)) to afford $20 \mathrm{~g}(98 \%)$ of TT-1 as colorless foam.

$\mathbf{R} \boldsymbol{f}=0.46(\mathrm{DCM} / \mathrm{MeOH}, 94: 6(\mathrm{v} / \mathrm{v}))$.

\section{NMR Spectroscopy:}

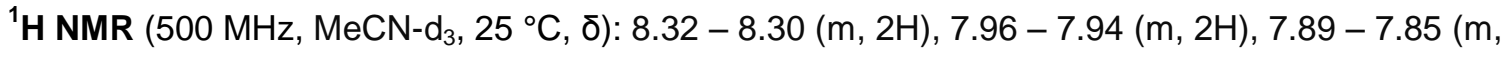
$2 \mathrm{H}), 7.81-7.78(\mathrm{~m}, 2 \mathrm{H}), 7.43-7.40(\mathrm{~m}, 2 \mathrm{H}), 7.27-7.23(\mathrm{~m}, 2 \mathrm{H}), 7.04-7.02(\mathrm{~m}, 2 \mathrm{H}), 6.99-$ $6.98(\mathrm{~m}, 2 \mathrm{H})$.

${ }^{13} \mathrm{C}$ NMR $\left\{{ }^{1} \mathrm{H}\right\}\left(126 \mathrm{MHz}, \mathrm{MeCN}-\mathrm{d}_{3}, 25^{\circ} \mathrm{C}, \delta\right): 163.1,155.6,137.2,136.0,135.6,131.6,131.6$, $131.4,130.9,126.4,121.3,120.0,119.9,116.7$.

${ }^{19} \mathrm{~F}$ NMR $\left\{{ }^{1} \mathrm{H}\right\}\left(471 \mathrm{MHz}, \mathrm{MeCN}-\mathrm{d}_{3}, 25^{\circ} \mathrm{C}, \delta\right):-151.4,-151.5$.

HRMS-ESI (m/z) calc'd. for $\mathrm{C}_{24} \mathrm{H}_{17} \mathrm{OS}_{2}^{+}\left[\mathrm{M}-\mathrm{BF}_{4}\right]^{+}$, 385.071530; found, 385.071535; deviation: +0.01 ppm.

Tetrafluorothianthrenium salt TFT-1

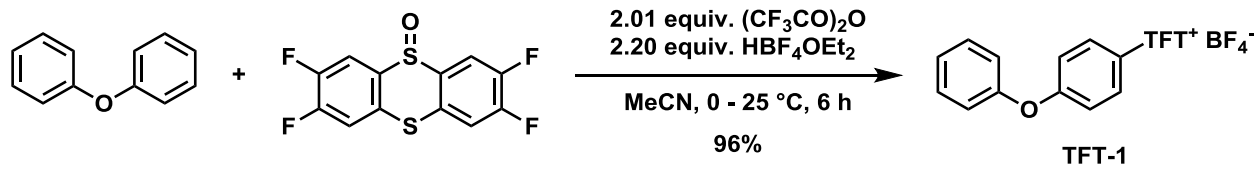

Under ambient atmosphere, a $250 \mathrm{~mL}$ round-bottom flask equipped with a magnetic stir bar was charged with diphenyl ether ( $1.00 \mathrm{~mL}, 1.07 \mathrm{~g}, 6.30 \mathrm{mmol}, 1.00$ equiv.) and $\mathrm{MeCN}(25 \mathrm{~mL}, \mathrm{C}=0.25 \mathrm{M}$ ). Trifluoroacetic anhydride (1.76 mL, $2.66 \mathrm{~g}, 12.7 \mathrm{mmol}, 2.01$ equiv.) was added at ambient temperature while stirring. After 
cooling to $0^{\circ} \mathrm{C}$, tetrafluorothianthrene S-oxide $(1.92 \mathrm{~g}, 6.31 \mathrm{mmol}, 1.00$ equiv.) was added in one portion, followed by the addition of $\mathrm{HBF}_{4} \cdot \mathrm{OEt}_{2}(1.89 \mathrm{~mL}, 2.25 \mathrm{~g}, 13.9 \mathrm{mmol}, 2.20$ equiv.) in one portion. The mixture was stirred at $0{ }^{\circ} \mathrm{C}$ for $1 \mathrm{~h}$, then at ambient temperature for $5 \mathrm{~h}$. The reaction mixture was concentrated under reduced pressure, and subsequently diluted with $\mathrm{DCM}(100 \mathrm{~mL})$. The solution was poured onto a saturated aqueous $\mathrm{NaHCO}_{3}$ solution $(100 \mathrm{~mL})$, and the layers were separated. The organic phase was washed with aqueous $\mathrm{NaBF}_{4}$ solution $(2 \times 100 \mathrm{~mL}, 10 \%)$, and with water $(2 \times 100 \mathrm{~mL})$. The organic phase was dried over $\mathrm{MgSO}_{4}$, and the solvent was removed under reduced pressure. The residue was purified by chromatography on silica gel eluting with DCM / MeOH (1:0-10:1 (v/v)) to afford $3.25 \mathrm{~g}(96 \%)$ of TFT-1 as colorless foam.

$\mathbf{R}_{\boldsymbol{f}}=0.48(\mathrm{DCM} / \mathrm{MeOH}, 94: 6(\mathrm{v} / \mathrm{v}))$.

\section{NMR Spectroscopy:}

${ }^{1}$ H NMR $\left(500 \mathrm{MHz}, \mathrm{MeCN}_{-} \mathrm{d}_{3}, 25^{\circ} \mathrm{C}, \delta\right): 8.37$ (dd, $\left.J=9.2,7.2 \mathrm{~Hz}, 2 \mathrm{H}\right), 7.95(\mathrm{dd}, J=10.0,7.0 \mathrm{~Hz}$, $2 \mathrm{H}), 7.45-7.41(\mathrm{~m}, 2 \mathrm{H}), 7.27-7.23(\mathrm{~m}, 4 \mathrm{H}), 7.06-7.00(\mathrm{~m}, 4 \mathrm{H})$.

${ }^{13} \mathrm{C}$ NMR $\left\{{ }^{1} \mathrm{H}\right\}\left(126 \mathrm{MHz}, \mathrm{MeCN}-\mathrm{d}_{3}, 25^{\circ} \mathrm{C}, \delta\right): 163.5,155.5,154.7(\mathrm{dd}, J=261.6,13.2 \mathrm{~Hz}), 151.6$ (dd, $J=255.6,13.6 \mathrm{~Hz}$ ), 134.9 (dd, $J=8.6,3.8 \mathrm{~Hz}$ ), 132.0, 131.5, 126.6, 125.2 (dd, $J=22.2,2.4$ $\mathrm{Hz}), 121.4,121.1$ (d, $J=21.7 \mathrm{~Hz}), 119.9,115.9$ (dd, $J=7.3,3.4 \mathrm{~Hz}), 115.2$.

${ }^{19} \mathrm{~F}$ NMR $\left\{{ }^{1} \mathrm{H}\right\}\left(471 \mathrm{MHz}, \mathrm{MeCN}-\mathrm{d}_{3}, 25^{\circ} \mathrm{C}, \delta\right):-125.7(\mathrm{~d}, J=20.5 \mathrm{~Hz}),-133.8(\mathrm{~d}, J=20.6 \mathrm{~Hz}),-$ 151.0 .

HRMS-ESI (m/z) calc'd. for $\mathrm{C}_{24} \mathrm{H}_{13} \mathrm{~F}_{4} \mathrm{OS}_{2}{ }^{+}\left[\mathrm{M}-\mathrm{BF}_{4}\right]^{+}, 457.033850$; found, 457.033849; deviation: 0.0 ppm.

Aniline 1a

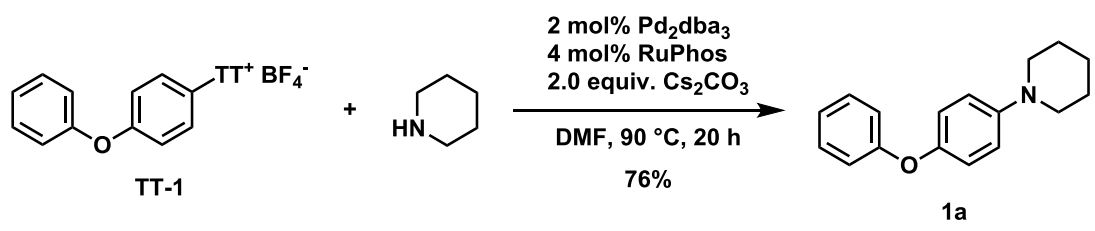

In an argon-filled glovebox, a $4 \mathrm{~mL}$ glass-vial equipped with a magnetic stir bar was charged with TT-1 (94 mg, 0.20 mmol, 1.0 equiv.), $\mathrm{Pd}_{2} \mathrm{dba}_{3}$ (4 mg, 4 mol, 2 mol\%), RuPhos (4 mg, $9 \mu \mathrm{mol}, 4 \mathrm{~mol} \%$ ), $\mathrm{Cs}_{2} \mathrm{CO}_{3}$ (130 mg, $0.40 \mathrm{mmol}, 2.0$ equiv.), and DMF ( $1 \mathrm{~mL}, \mathrm{c}=0.2 \mathrm{M}$ ). Piperidine $(59 \mu \mathrm{L}, 51 \mathrm{mg}, 0.60 \mathrm{mmol}$, 3.0 equiv.) was added, and the sealed vial was taken out from the glovebox. The suspension was stirred at $90{ }^{\circ} \mathrm{C}$ for $20 \mathrm{~h}$. The solvent was removed under reduced pressure, and the residue was purified by chromatography on silica gel eluting with hexanes / EtOAc $(1: 0-30: 1(\mathrm{v} / \mathrm{v}))$ to afford $39 \mathrm{mg}(76 \%)$ of $1 \mathrm{a}$ as yellow oil.

$\mathbf{R}_{\boldsymbol{f}}=0.22$ (hexanes / EtOAc, 95:5 (v/v)).

\section{NMR Spectroscopy:}

${ }^{1} \mathrm{H}$ NMR $\left(500 \mathrm{MHz}, \mathrm{CDCl}_{3}, 25^{\circ} \mathrm{C}, \delta\right): 7.29$ (t, $\left.J=8.0 \mathrm{~Hz}, 2 \mathrm{H}\right), 7.03$ (t, $\left.J=7.0 \mathrm{~Hz}, 1 \mathrm{H}\right), 6.98-6.90$ $(\mathrm{m}, 6 \mathrm{H}), 3.14-3.07(\mathrm{~m}, 4 \mathrm{H}), 1.78-1.69(\mathrm{~m}, 4 \mathrm{H}), 1.61-1.53(\mathrm{~m}, 2 \mathrm{H})$. 
${ }^{13} \mathrm{C}$ NMR $\left\{{ }^{1} \mathrm{H}\right\}\left(126 \mathrm{MHz}, \mathrm{CDCl}_{3}, 25{ }^{\circ} \mathrm{C}, \delta\right): 158.8,149.5,149.1,129.7,122.4,120.6,118.2,117.7$, 51.6, 26.2, 24.3.

HRMS-ESI (m/z) calc'd. for $\mathrm{C}_{17} \mathrm{H}_{20} \mathrm{NO}^{+}[\mathrm{M}+\mathrm{H}]^{+}$, 254.153939; found, 254.153990; deviation: -0.20 ppm.

Morpholine 1b

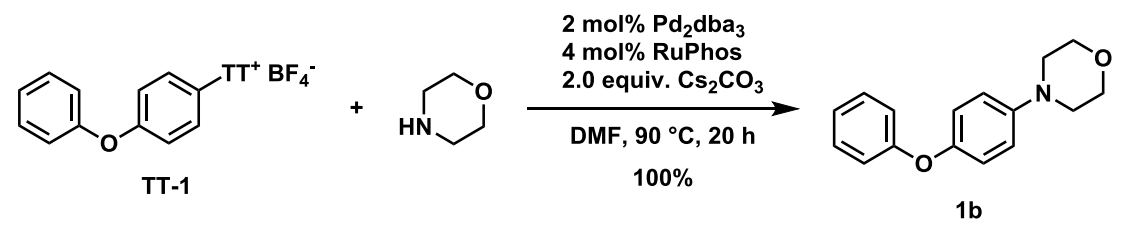

In an argon-filled glovebox, a $4 \mathrm{~mL}$ glass-vial equipped with a magnetic stir bar was charged with TT-1 (94 mg, 0.20 mmol, 1.0 equiv.), $\mathrm{Pd}_{2} \mathrm{dba}_{3}$ (4 mg, 4 mol, 2 mol\%), RuPhos (4 mg, $9 \mu \mathrm{mol}, 4 \mathrm{~mol} \%$ ), $\mathrm{Cs}_{2} \mathrm{CO}_{3}$ (130 mg, $0.40 \mathrm{mmol}, 2.0$ equiv.), and DMF ( $1 \mathrm{~mL}, \mathrm{C}=0.2 \mathrm{M}$ ). Morpholine ( $26 \mu \mathrm{L}, 26 \mathrm{mg}, 0.30 \mathrm{mmol}$, 1.5 equiv.) was added, and the sealed vial was taken out from the glovebox. The suspension was stirred at $90{ }^{\circ} \mathrm{C}$ for $20 \mathrm{~h}$. The solvent was removed under reduced pressure, and the residue was purified by chromatography on silica gel eluting with hexanes / EtOAc (1:0-19:1 (v/v)) to afford $51 \mathrm{mg}(100 \%)$ of $1 \mathrm{~b}$ as yellow oil.

$\mathbf{R}_{\boldsymbol{f}}=0.20($ hexanes / EtOAc, 9:1 (v/v)).

\section{NMR Spectroscopy:}

${ }^{1} \mathrm{H}$ NMR $\left(500 \mathrm{MHz}, \mathrm{CDCl}_{3}, 25^{\circ} \mathrm{C}, \delta\right): 7.32-7.26(\mathrm{~m}, 2 \mathrm{H}), 7.06-7.03(\mathrm{~m}, 1 \mathrm{H}), 6.99-6.90(\mathrm{~m}$, $6 \mathrm{H}), 3.88-3.87(\mathrm{~m}, 4 \mathrm{H}), 3.14-3.12(\mathrm{~m}, 4 \mathrm{H})$.

${ }^{13} \mathrm{C}$ NMR $\left\{{ }^{1} \mathrm{H}\right\}\left(126 \mathrm{MHz}, \mathrm{CDCl}_{3}, 25^{\circ} \mathrm{C}, \delta\right): 158.6,150.2,147.9,129.8,122.6,120.7,117.8,117.4$, 67.1, 50.3.

HRMS-ESI (m/z) calc'd. for $\mathrm{C}_{16} \mathrm{H}_{18} \mathrm{NO}_{2}{ }^{+}[\mathrm{M}+\mathrm{H}]^{+}$, 256.133204; found, 256.133150; deviation: +0.21 ppm.

Piperazine 1c

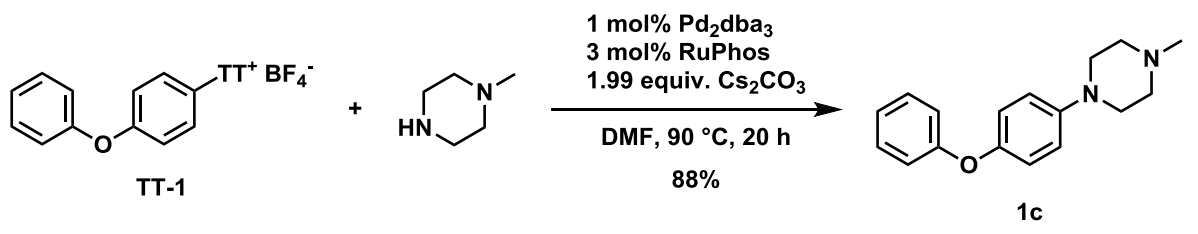

In an argon-filled glovebox, a $4 \mathrm{~mL}$ glass-vial equipped with a magnetic stir bar was charged with TT-1 (142 mg, $301 \mu \mathrm{mol}, 1.00$ equiv.), $\mathrm{Pd}_{2} \mathrm{dba}_{3}$ (4 mg, $4 \mu \mathrm{mol}, 1 \mathrm{~mol} \%$ ), RuPhos (4 mg, $9 \mu \mathrm{mol}, 3 \mathrm{~mol} \%$ ), $\mathrm{Cs}_{2} \mathrm{CO}_{3}$ (195 mg, $598 \mu \mathrm{mol}, 1.99$ equiv.), and DMF (2 mL, c = $0.2 \mathrm{M}$ ). $N$-Methylpiperazin ( $50 \mu \mathrm{L}, 45 \mathrm{mg}, 0.45 \mathrm{mmol}$, 1.5 equiv.) was added, and the sealed vial was taken out from the glovebox. The suspension was stirred at $90{ }^{\circ} \mathrm{C}$ for $20 \mathrm{~h}$. The solvent was removed under reduced pressure, and the residue was purified by chromatography on silica gel eluting with hexanes / EtOAc $(1: 0-95: 5(\mathrm{v} / \mathrm{v}))$ to afford $71 \mathrm{mg}(88 \%)$ of $1 \mathrm{c}$ as yellow oil. 
$\mathbf{R} \boldsymbol{f}=0.17(\mathrm{DCM} / \mathrm{MeOH}, 95: 5(\mathrm{v} / \mathrm{v}))$.

\section{NMR Spectroscopy:}

${ }^{1}$ H NMR $\left(500 \mathrm{MHz}, \mathrm{CDCl}_{3}, 25^{\circ} \mathrm{C}, \delta\right): 7.32-7.25(\mathrm{~m}, 2 \mathrm{H}), 7.06-7.00(\mathrm{~m}, 2 \mathrm{H}), 6.99-6.88(\mathrm{~m}$, $5 \mathrm{H}), 3.19$ (br s, 3H), 2.62 (br s, 4H), 2.39 (br s, 4H).

${ }^{13} \mathrm{C}$ NMR $\left\{{ }^{1} \mathrm{H}\right\}\left(126 \mathrm{MHz}, \mathrm{CDCl}_{3}, 25^{\circ} \mathrm{C}, \delta\right): 158.6,150.0,147.9,129.7,122.5,120.6,117.9,55.3$, $49.9,46.2$.

HRMS-EI (m/z) calc'd. for $\mathrm{C}_{17} \mathrm{H}_{20} \mathrm{~N}_{2} \mathrm{O}^{+}[\mathrm{M}]^{+}$, 268.157012; found, 268.157490; deviation: -1.78 ppm.

Aniline 1d

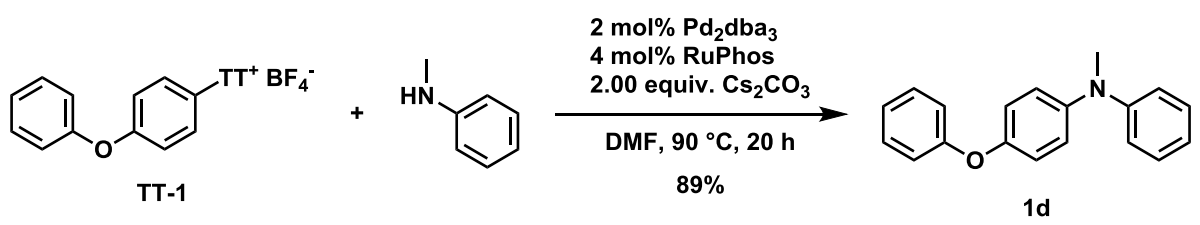

In an argon-filled glovebox, a $4 \mathrm{~mL}$ glass-vial equipped with a magnetic stir bar was charged with TT-1

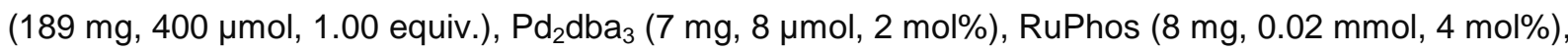
$\mathrm{Cs}_{2} \mathrm{CO}_{3}(261 \mathrm{mg}, 801 \mu \mathrm{mol}, 2.00$ equiv.), and DMF (2 mL, c = $0.2 \mathrm{M})$. $N$-Methylaniline $(65 \mu \mathrm{L}, 64 \mathrm{mg}$, $0.60 \mathrm{mmol}, 1.5$ equiv.) was added, and the sealed vial was taken out from the glovebox. The suspension was stirred at $90{ }^{\circ} \mathrm{C}$ for $20 \mathrm{~h}$. The solvent was removed under reduced pressure, and the residue was purified by chromatography on silica gel eluting with hexanes / EtOAc $(1: 0-97: 3(\mathrm{v} / \mathrm{v}))$ to afford $98 \mathrm{mg}(89 \%)$ of $\mathbf{1 d}$ as yellow oil.

$\mathbf{R} \boldsymbol{f}=0.26$ (hexanes / EtOAc, 95:5 (v/v)).

\section{NMR Spectroscopy:}

${ }^{1} \mathbf{H}$ NMR $\left(500 \mathrm{MHz}, \mathrm{CDCl}_{3}, 25^{\circ} \mathrm{C}, \delta\right): 7.35-7.31(\mathrm{~m}, 2 \mathrm{H}), 7.27-7.23(\mathrm{~m}, 2 \mathrm{H}), 7.09-7.05(\mathrm{~m}$, $3 \mathrm{H}), 7.03-7.01(\mathrm{~m}, 2 \mathrm{H}), 6.99-6.96(\mathrm{~m}, 2 \mathrm{H}), 6.94-6.93(\mathrm{~m}, 2 \mathrm{H}), 6.90-6.87(\mathrm{~m}, 1 \mathrm{H})$.

${ }^{13} \mathrm{C}$ NMR $\left\{{ }^{1} \mathrm{H}\right\}\left(126 \mathrm{MHz}, \mathrm{CDCl}_{3}, 25{ }^{\circ} \mathrm{C}, \delta\right): 158.0,152.3,149.5,144.9,129.8,129.3,124.1,123.0$, $120.3,120.1,118.4,118.3,40.6$.

HRMS-ESI (m/z) calc'd. for $\mathrm{C}_{19} \mathrm{H}_{18} \mathrm{NO}^{+}\left[\mathrm{M}+\mathrm{H}^{+}, 276.138288\right.$; found, 276.138360; deviation: $-0.26 \mathrm{ppm}$.

Aniline 1e

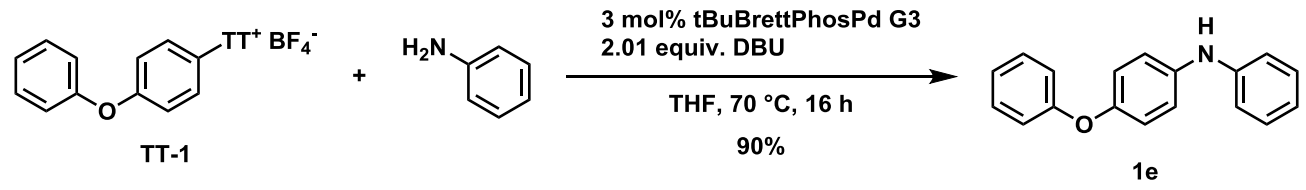

In an argon-filled glovebox, a $4 \mathrm{~mL}$ glass-vial equipped with a magnetic stir bar was charged with TT-1 (236 mg, $500 \mu \mathrm{mol}, 1.00$ equiv.), tBuBrettPhosPd G3 (13 mg, $15 \mu \mathrm{mol}, 3.0 \mathrm{~mol} \%$ ), and THF (0.5 mL, c = $1 \mathrm{M}$ ). Aniline (68 $\mu \mathrm{L}, 69 \mathrm{mg}, 0.75 \mathrm{mmol}, 1.5$ equiv.) and DBU (150 $\mu \mathrm{L}, 153 \mathrm{mg}, 1.00 \mathrm{mmol}, 2.01$ equiv.) were added, 
and the sealed vial was taken out from the glovebox. The solution was stirred at $70{ }^{\circ} \mathrm{C}$ for $16 \mathrm{~h}$. The solvent was removed under reduced pressure, and the residue was purified by chromatography on silica gel eluting with hexanes / EtOAc (1:0-19:1 (v/v)) to afford $118 \mathrm{mg}(90 \%)$ of $1 \mathrm{e}$ as white solid.

$\mathbf{R}_{\boldsymbol{f}}=0.15$ (hexanes / EtOAc, 95:5 (v/v)).

\section{NMR Spectroscopy:}

${ }^{1} \mathrm{H}$ NMR $\left(500 \mathrm{MHz}, \mathrm{CDCl}_{3}, 25^{\circ} \mathrm{C}, \delta\right): 7.26-7.32(\mathrm{~m}, 2 \mathrm{H}), 7.30-7.26(\mathrm{~m}, 2 \mathrm{H}), 7.11-7.07(\mathrm{~m}$, $3 \mathrm{H}), 7.04-6.98(\mathrm{~m}, 6 \mathrm{H}), 6.94-6.91(\mathrm{~m}, 1 \mathrm{H}), 5.62(\mathrm{~s}, 1 \mathrm{H})$.

${ }^{13} \mathrm{C}$ NMR $\left\{{ }^{1} \mathrm{H}\right\}\left(126 \mathrm{MHz}, \mathrm{CDCl}_{3}, 25{ }^{\circ} \mathrm{C}, \delta\right): 158.3,151.3,144.1,138.9,129.8,129.5,122.8,120.6$, $120.5,118.1,117.0$.

HRMS-ESI (m/z) calc'd. for $\mathrm{C}_{18} \mathrm{H}_{16} \mathrm{NO}^{+}[\mathrm{M}+\mathrm{H}]^{+}$, 262.122639; found, 262.122660; deviation: -0.08 ppm.

\section{Aminopyridine $1 f$}

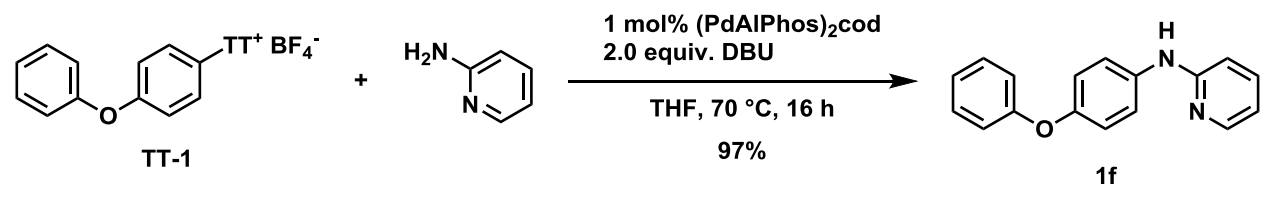

In an argon-filled glovebox, a $4 \mathrm{~mL}$ glass-vial equipped with a magnetic stir bar was charged with TT-1 (94 mg, 0.20 mmol, 1.0 equiv.), (PdAlphos) ${ }_{2} \operatorname{cod}$ ( $4 \mathrm{mg}, 2 \mu \mathrm{mol}, 1 \mathrm{~mol} \%$ ), and THF ( $1 \mathrm{~mL}, \mathrm{c}=0.2 \mathrm{M}$ ).

2-Aminopyridine (23 mg, $0.24 \mathrm{mmol}, 1.2$ equiv.) and DBU (60 $\mu \mathrm{L}, 61 \mathrm{mg}, 0.40 \mathrm{mmol}, 2.0$ equiv.) were added, and the sealed vial was taken out from the glovebox. The solution was stirred at $70{ }^{\circ} \mathrm{C}$ for $16 \mathrm{~h}$. The solvent was removed under reduced pressure, and the residue was purified by chromatography on silica gel eluting with hexanes / EtOAc (1:0-10:1 (v/v)) to afford $51 \mathrm{mg}(97 \%)$ of $1 \mathrm{f}$ as white solid.

$\mathbf{R}_{\boldsymbol{f}}=0.16$ (hexanes / EtOAc, 9:1 (v/v)).

\section{NMR Spectroscopy:}

${ }^{1} \mathrm{H}$ NMR $\left(500 \mathrm{MHz}, \mathrm{CDCl}_{3}, 25^{\circ} \mathrm{C}, \delta\right): 8.20-8.19(\mathrm{~m}, 1 \mathrm{H}), 7.49-7.46(\mathrm{~m}, 1 \mathrm{H}), 7.35-7.31(\mathrm{~m}$, $4 \mathrm{H}), 7.10-7.07(\mathrm{~m}, 1 \mathrm{H}), 7.03-7.01(\mathrm{~m}, 4 \mathrm{H}), 6.85(\mathrm{br} \mathrm{s}, 1 \mathrm{H}), 6.80-6.78(\mathrm{~m}, 1 \mathrm{H}), 6.73-6.70(\mathrm{~m}$, $1 \mathrm{H})$.

${ }^{13} \mathrm{C}$ NMR $\left\{{ }^{1} \mathrm{H}\right\}\left(126 \mathrm{MHz}, \mathrm{CDCl}_{3}, 25{ }^{\circ} \mathrm{C}, \delta\right): 157.9,156.7,152.7,148.5,137.8,136.2,129.8,123.0$, 122.8, 120.3, 118.4, 114.9, 108.0.

HRMS-ESI (m/z) calc'd. for $\mathrm{C}_{17} \mathrm{H}_{15} \mathrm{~N}_{2} \mathrm{O}^{+}[\mathrm{M}+\mathrm{H}]^{+}$, 263.117887; found, 263.118000; deviation: -0.43 ppm.

\section{Aminothiazole 1g}

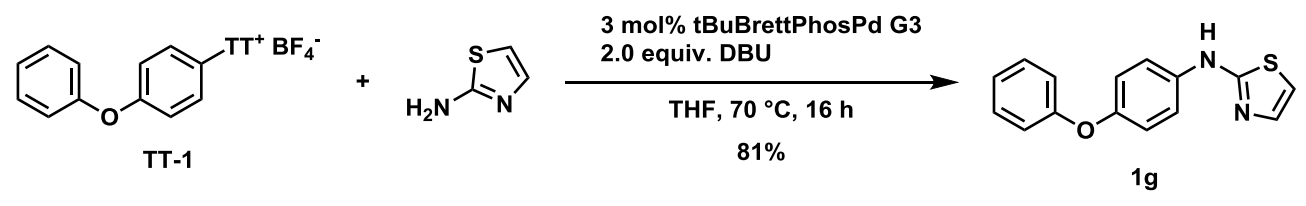


In an argon-filled glovebox, a $4 \mathrm{~mL}$ glass-vial equipped with a magnetic stir bar was charged with TT-1 (94 mg, $0.20 \mathrm{mmol}, 1.0$ equiv.), tBuBrettPhosPd G3 (5 mg, $6 \mu \mathrm{mol}, 3 \mathrm{~mol} \%$ ), and THF ( $1 \mathrm{~mL}, \mathrm{c}=0.2 \mathrm{M}$ ). 2-Aminothiazole (30 mg, $0.30 \mathrm{mmol}, 1.5$ equiv.) and DBU (60 $\mu \mathrm{L}, 61 \mathrm{mg}, 0.40 \mathrm{mmol}, 2.0$ equiv.) were added, and the sealed vial was taken out from the glovebox. The solution was stirred at $70{ }^{\circ} \mathrm{C}$ for $16 \mathrm{~h}$. The solvent was removed under reduced pressure, and the residue was purified by chromatography on silica gel eluting with hexanes / EtOAc (1:0-10:1 (v/v)) to afford $44 \mathrm{mg}(81 \%)$ of $\mathbf{1 g}$ as off-white solid.

$\mathbf{R}_{\boldsymbol{f}}=0.16($ hexanes / EtOAc, 3:7 (v/v)).

\section{NMR Spectroscopy:}

${ }^{1} \mathrm{H}$ NMR $\left(500 \mathrm{MHz}, \mathrm{CDCl}_{3}, 25^{\circ} \mathrm{C}, \delta\right): 8.34(\mathrm{~s}, 1 \mathrm{H}), 7.37-7.32(\mathrm{~m}, 4 \mathrm{H}), 7.28-7.27(\mathrm{~m}, 1 \mathrm{H}), 7.11$

$-7.08(\mathrm{~m}, 1 \mathrm{H}), 7.05-7.00(\mathrm{~m}, 4 \mathrm{H}), 6.61-6.60(\mathrm{~m}, 1 \mathrm{H})$.

${ }^{13} \mathrm{C}$ NMR $\left\{{ }^{1} \mathrm{H}\right\}\left(126 \mathrm{MHz}, \mathrm{CDCl}_{3}, 25{ }^{\circ} \mathrm{C}, \delta\right): 167.0,157.8,152.9,138.7,136.6,129.9,123.2,120.6$, 120.4, 118.5, 107.3.

HRMS-EI (m/z) calc'd. for $\mathrm{C}_{15} \mathrm{H}_{12} \mathrm{~N}_{2} \mathrm{OS}^{+}[\mathrm{M}]^{+}, 268.066485$; found, 268.066740; deviation: -0.95 ppm.

Isonicotinamide $1 \mathrm{~h}$
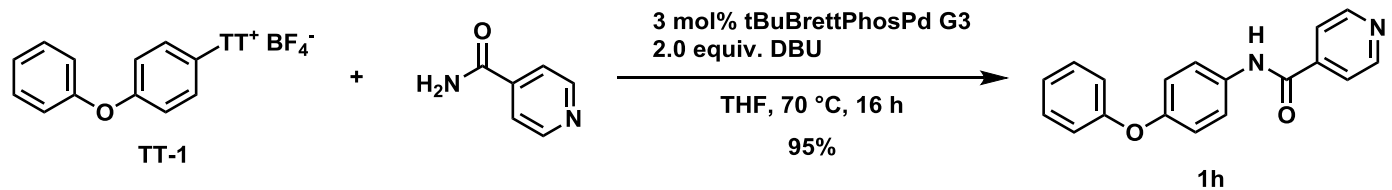

In an argon-filled glovebox, a $4 \mathrm{~mL}$ glass-vial equipped with a magnetic stir bar was charged with TT-1 (94 mg, 0.20 mmol, 1.0 equiv.), tBuBrettPhosPd G3 (5 mg, $6 \mu \mathrm{mol}, 3 \mathrm{~mol} \%$ ), and THF (1 mL, c = $0.2 \mathrm{M}$ ). Isonicotinamide (37 mg, $0.30 \mathrm{mmol}, 1.5$ equiv.) and $\mathrm{DBU}(60 \mu \mathrm{L}, 61 \mathrm{mg}, 0.40 \mathrm{mmol}, 2.0$ equiv.) were added, and the sealed vial was taken out from the glovebox. The solution was stirred at $70{ }^{\circ} \mathrm{C}$ for $16 \mathrm{~h}$. The solvent was removed under reduced pressure, and the residue was purified by chromatography on silica gel eluting with hexanes / EtOAc (1:0-2:3 (v/v)) to afford $55 \mathrm{mg}(95 \%)$ of $\mathbf{1 h}$ as off-white solid.

$\mathbf{R}_{\boldsymbol{f}}=0.19$ (hexanes / EtOAc, 4:6(v/v)).

\section{NMR Spectroscopy:}

${ }^{1} \mathrm{H}$ NMR $\left(500 \mathrm{MHz}, \mathrm{CDCl}_{3}, 25^{\circ} \mathrm{C}, \delta\right): 8.78$ (apt d, $J=5.8 \mathrm{~Hz}, 2 \mathrm{H}$ ), 7.99 (s, $1 \mathrm{H}$ ), $7.71-7.70$ (apt d, $J=5.8 \mathrm{~Hz}, 2 \mathrm{H}), 7.59($ apt $d, J=8.8 \mathrm{~Hz}, 2 \mathrm{H}), 7.36-7.33(\mathrm{~m}, 2 \mathrm{H}), 7.13-7.10(\mathrm{~m}, 1 \mathrm{H}), 7.04-7.00$ $(\mathrm{m}, 4 \mathrm{H})$.

${ }^{13} \mathrm{C}$ NMR $\left\{{ }^{1} \mathrm{H}\right\}\left(126 \mathrm{MHz}, \mathrm{CDCl}_{3}, 25{ }^{\circ} \mathrm{C}, \delta\right): 163.8,157.3,154.6,150.9,142.1,132.6,130.0,123.5$, 122.4, 121.0, 119.7, 118.9.

HRMS-ESI (m/z) calc'd. for $\mathrm{C}_{18} \mathrm{H}_{15} \mathrm{~N}_{2} \mathrm{O}_{2}{ }^{+}[\mathrm{M}+\mathrm{H}]^{+}$, 291.112803; found, 291.112700; deviation: +0.35 ppm. 


\section{Oxazolidinone 1i}

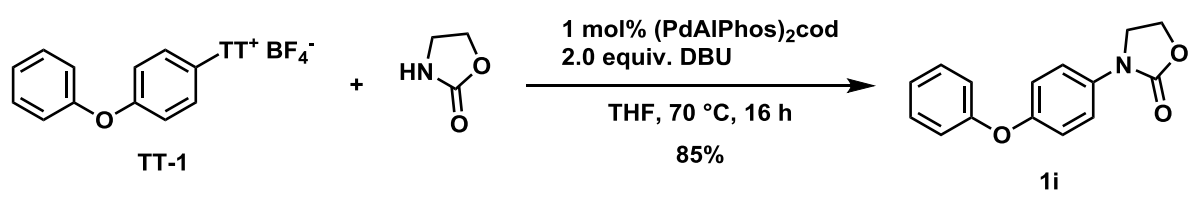

In an argon-filled glovebox, a $4 \mathrm{~mL}$ glass-vial equipped with a magnetic stir bar was charged with TT-1 (94 mg, $0.20 \mathrm{mmol}, 1.0$ equiv.), (PdAlphos) ${ }_{2} \operatorname{cod}(4 \mathrm{mg}, 2 \mu \mathrm{mol}, 1 \mathrm{~mol} \%$ ), and THF (0.5 mL, c = $0.4 \mathrm{M}$ ).

2-Oxazolidinone (17 mg, $0.20 \mathrm{mmol}, 0.98$ equiv.) and DBU (60 $\mu \mathrm{L}, 61 \mathrm{mg}, 0.40 \mathrm{mmol}, 2.0$ equiv.) were added, and the sealed vial was taken out from the glovebox. The solution was stirred at $70{ }^{\circ} \mathrm{C}$ for $16 \mathrm{~h}$. The solvent was removed under reduced pressure, and the residue was purified by chromatography on silica gel eluting with hexanes / EtOAc (1:0-5:2 (v/v)) to afford $43 \mathrm{mg}(85 \%)$ of $1 \mathbf{i}$ as colorless oil.

$\mathbf{R}_{\boldsymbol{f}}=0.23$ (hexanes / EtOAc, 7:3 (v/v)).

\section{NMR Spectroscopy:}

${ }^{1} \mathbf{H}$ NMR $\left(500 \mathrm{MHz}, \mathrm{CDCl}_{3}, 25^{\circ} \mathrm{C}, \delta\right): 7.51-7.49(\mathrm{~m}, 2 \mathrm{H}), 7.35-7.31(\mathrm{~m}, 2 \mathrm{H}), 7.11-7.05(\mathrm{~m}$, $3 \mathrm{H}), 7.00-6.98(\mathrm{~m}, 2 \mathrm{H}), 4.51-4.47(\mathrm{~m}, 2 \mathrm{H}), 4.08-4.04(\mathrm{~m}, 2 \mathrm{H})$.

${ }^{13} \mathrm{C}$ NMR $\left\{{ }^{1} \mathrm{H}\right\}\left(126 \mathrm{MHz}, \mathrm{CDCl}_{3}, 25{ }^{\circ} \mathrm{C}, \delta\right): 157.5,155.6,153.6,133.9,129.9,123.3,120.2,119.8$, 118.6, 61.4, 45.7.

HRMS-El (m/z) calc'd. for $\mathrm{C}_{15} \mathrm{H}_{13} \mathrm{NO}_{3}{ }^{+}[\mathrm{M}]^{+}$, 255.088994; found, 255.089320; deviation: -1.28 ppm.

\section{Urea $1 \mathrm{j}$}
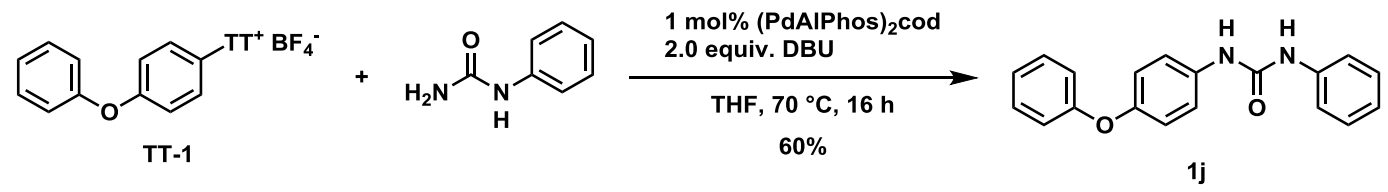

In an argon-filled glovebox, a $4 \mathrm{~mL}$ glass-vial equipped with a magnetic stir bar was charged with TT-1 (94 mg, 0.20 mmol, 1.0 equiv.), (PdAlphos) ${ }_{2} \operatorname{cod}(4 \mathrm{mg}, 2 \mu \mathrm{mol}, 1 \mathrm{~mol} \%$ ), and THF ( $1 \mathrm{~mL}, \mathrm{c}=0.2 \mathrm{M}$ ). $\mathrm{N}$-Phenylurea (33 mg, $0.24 \mathrm{mmol}, 1.2$ equiv.) and DBU (60 $\mu \mathrm{L}, 61 \mathrm{mg}, 0.40 \mathrm{mmol}, 2.0$ equiv.) were added, and the sealed vial was taken out from the glovebox. The solution was stirred at $70{ }^{\circ} \mathrm{C}$ for $16 \mathrm{~h}$. The solvent was removed under reduced pressure, and the residue was purified by chromatography on silica gel eluting with hexanes / EtOAc (1:0-2:5 (v/v)) to afford $37 \mathrm{mg}(60 \%)$ of $\mathbf{1 j}$ as white solid.

$\mathbf{R}_{\boldsymbol{f}}=0.24$ (hexanes / EtOAc, 8:2(v/v)).

\section{NMR Spectroscopy:}

${ }^{1} \mathrm{H}$ NMR $\left(500 \mathrm{MHz}, \mathrm{DMSO}-\mathrm{d}_{6}, 25^{\circ} \mathrm{C}, \delta\right): 8.66(\mathrm{~s}, 1 \mathrm{H}), 8.63(\mathrm{~s}, 1 \mathrm{H}), 7.48-7.44(\mathrm{~m}, 4 \mathrm{H}), 7.37-$ $7.34(\mathrm{~m}, 2 \mathrm{H}), 7.29-7.26(\mathrm{~m}, 2 \mathrm{H}), 7.10-7.07(\mathrm{~m}, 1 \mathrm{H}), 6.99-6.95(\mathrm{~m}, 5 \mathrm{H})$.

${ }^{13} \mathrm{C}$ NMR $\left\{{ }^{1} \mathrm{H}\right\}\left(126 \mathrm{MHz}, \mathrm{DMSO}-\mathrm{d}_{6}, 25^{\circ} \mathrm{C}, \delta\right): 157.7,152.6,150.6,139.7,135.7,129.9,128.8$, 122.7, 121.8, 119.9, 119.8, 118.2, 117.6. 
HRMS-ESI (m/z) calc'd. for $\mathrm{C}_{19} \mathrm{H}_{16} \mathrm{~N}_{2} \mathrm{O}_{2} \mathrm{Na}^{+}[\mathrm{M}+\mathrm{Na}]^{+}$, 327.110396; found, 327.110410; deviation: -0.04 ppm.

Pyrazole 1k

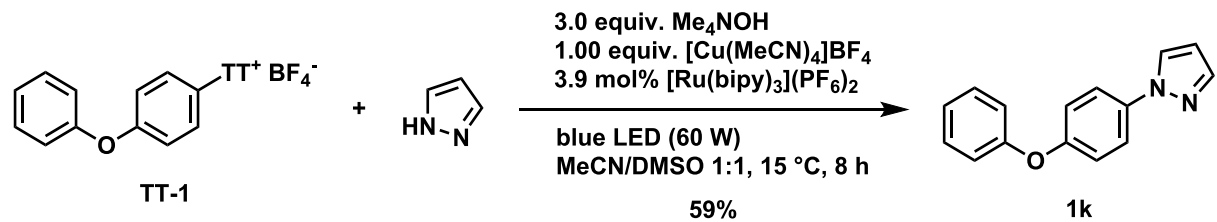

Under ambient atmosphere, a $4 \mathrm{~mL}$ glass-vial was charged with pyrazole (104 mg, $1.53 \mathrm{mmol}, 3.01$ equiv.) and tetramethylammonium hydroxide ( $25 \%$ in methanol, $0.64 \mathrm{~mL}, 0.55 \mathrm{~g}, 1.5 \mathrm{mmol}, 3.0$ equiv.), and the solvent was removed under reduced pressure. Thianthrenium salt TT-1 (240 mg, 0.508 mmol, 1.00 equiv.), $\left[\mathrm{Cu}(\mathrm{MeCN})_{4}\right] \mathrm{BF}_{4}\left(160 \mathrm{mg}, 0.509 \mathrm{mmol}, 1.00\right.$ equiv.), [Ru(bipy) $\left.{ }_{3}\right]\left(\mathrm{PF}_{6}\right)_{2}(17 \mathrm{mg}, 20 \mu \mathrm{mol}, 3.9 \mathrm{~mol} \%)$, acetonitrile $(1.5 \mathrm{~mL})$ and dimethylsulfoxide $\left(1.5 \mathrm{~mL}, \mathrm{c}_{\text {total }}=0.17 \mathrm{M}\right)$ were added, and the suspension was degassed by bubbling through argon with a cannula for $5 \mathrm{~min}$. While stirring, the suspension was irradiated for $8 \mathrm{~h}$ at $15^{\circ} \mathrm{C}$ using a blue LED (60 W). The suspension was added to water $(30 \mathrm{~mL})$, and the aqueous phase was extracted with DCM $(3 \times 20 \mathrm{~mL})$. The organic phase was dried over $\mathrm{MgSO}_{4}$, and the solvent was removed under reduced pressure. The residue was purified by chromatography on silica gel eluting with hexanes / EtOAc (1:0-3:1 (v/v)) to afford $71 \mathrm{mg}(59 \%)$ of $\mathbf{1 k}$ as white solid.

$\mathbf{R} \boldsymbol{f}=0.42($ hexanes / EtOAc, 85:15 (v/v)).

\section{NMR Spectroscopy:}

${ }^{1} \mathrm{H}$ NMR $\left(500 \mathrm{MHz}, \mathrm{CDCl}_{3}, 25^{\circ} \mathrm{C}, \delta\right): 7.87(\mathrm{~d}, \mathrm{~J}=2.4 \mathrm{~Hz}, 1 \mathrm{H}), 7.72(\mathrm{~s}, 1 \mathrm{H}), 7.67-7.64(\mathrm{~m}, 2 \mathrm{H})$, $7.38-7.35(\mathrm{~m}, 2 \mathrm{H}), 7.15-7.09(\mathrm{~m}, 3 \mathrm{H}), 7.05-7.03(\mathrm{~m}, 2 \mathrm{H}), 6.46(\mathrm{t}, J=2.1 \mathrm{~Hz}, 1 \mathrm{H})$.

${ }^{13} \mathrm{C}$ NMR $\left\{{ }^{1} \mathrm{H}\right\}\left(126 \mathrm{MHz}, \mathrm{CDCl}_{3}, 25{ }^{\circ} \mathrm{C}, \delta\right): 157.2,155.8,141.1,136.1,130.0,126.9,123.6,121.0$, 119.8, 119.0, 107.6 .

HRMS-El (m/z) calc'd. for $\mathrm{C}_{15} \mathrm{H}_{12} \mathrm{~N}_{2} \mathrm{O}^{+}[\mathrm{M}]^{+}$, 236.094412; found, 236.094490; deviation: -0.33 ppm.

\section{Pyrazolopyridine 11}

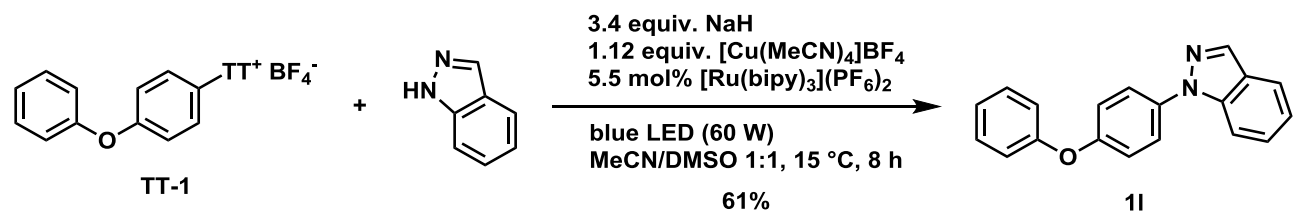

Under ambient atmosphere, a $4 \mathrm{~mL}$ glass-vial was charged with $1 \mathrm{H}$-pyrazolo[3,4-b]pyridine (143 mg,

$1.20 \mathrm{mmol}, 3.35$ equiv.), THF ( $1 \mathrm{~mL}$ ), and $\mathrm{NaH}$ (60\% dispersion in mineral oil, $48 \mathrm{mg}, 1.2 \mathrm{mmol}, 3.4$ equiv.) in three portions, and the solvent was removed under reduced pressure. Thianthrenium salt TT-1 (169 mg, $0.358 \mathrm{mmol}, 1.00$ equiv.), [Cu(MeCN$\left.)_{4}\right]_{B F}\left(126 \mathrm{mg}, 0.401 \mathrm{mmol}, 1.12\right.$ equiv.), [Ru(bipy) $\left.{ }_{3}\right]\left(\mathrm{PF}_{6}\right)_{2}(17 \mathrm{mg}$, $20 \mu \mathrm{mol}, 5.5 \mathrm{~mol} \%)$, acetonitrile $(1 \mathrm{~mL})$ and dimethylsulfoxide $\left(1 \mathrm{~mL}, \mathrm{c}_{\text {total }}=0.2 \mathrm{M}\right)$ were added, and the suspension was degassed by bubbling through argon with a cannula for $5 \mathrm{~min}$. While stirring, the suspension 
was irradiated for $8 \mathrm{~h}$ at $15^{\circ} \mathrm{C}$ using a blue LED (60 W). The suspension was added to water (30 mL), and the aqueous phase was extracted with DCM $(3 \times 20 \mathrm{~mL})$. The organic phase was dried over $\mathrm{MgSO}_{4}$, and the solvent was removed under reduced pressure. The residue was purified by chromatography on silica gel eluting with hexanes / EtOAc (1:0-3:1 (v/v)) to afford $70 \mathrm{mg}(61 \%)$ of 11 as yellow oil.

$\mathbf{R} \boldsymbol{f}=0.32$ (hexanes / EtOAc, 85:15 (v/v)).

\section{NMR Spectroscopy:}

${ }^{1} \mathrm{H}$ NMR $\left(500 \mathrm{MHz}, \mathrm{CDCl}_{3}, 25^{\circ} \mathrm{C}, \delta\right): 8.63(\mathrm{dd}, J=4.5,1.6 \mathrm{~Hz}, 1 \mathrm{H}), 8.21-8.18(\mathrm{~m}, 3 \mathrm{H}), 8.14(\mathrm{dd}$, $J=8.0,1.6 \mathrm{~Hz}, 1 \mathrm{H}), 7.38-7.34(\mathrm{~m}, 2 \mathrm{H}), 7.23-7.17(\mathrm{~m}, 3 \mathrm{H}), 7.14-7.11(\mathrm{~m}, 1 \mathrm{H}), 7.08-7.06(\mathrm{~m}$, $2 \mathrm{H})$.

${ }^{13} \mathrm{C}$ NMR $\left\{{ }^{1} \mathrm{H}\right\}\left(126 \mathrm{MHz}, \mathrm{CDCl}_{3}, 25{ }^{\circ} \mathrm{C}, \delta\right): 157.4,155.4,150.1,149.3,135.0,133.8,130.4,129.9$, 123.5, 123.2, 119.7, 118.9, 117.7, 117.2 .

HRMS-EI (m/z) calc'd. for $\mathrm{C}_{18} \mathrm{H}_{13} \mathrm{~N}_{3} \mathrm{O}^{+}[\mathrm{M}]^{+}$, 287.105311; found, 287.105800; deviation: -1.70 ppm.

Imidazole 1m

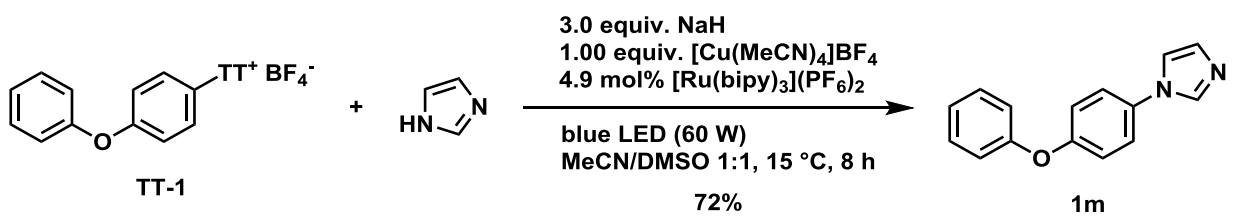

Under ambient atmosphere, a $4 \mathrm{~mL}$ glass-vial was charged with imidazole (82 mg, $1.2 \mathrm{mmol}, 3.0$ equiv.), THF (1 mL), and sodium hydride (60\% dispersion in mineral oil, $48 \mathrm{mg}, 1.2 \mathrm{mmol}, 3.0$ equiv.) in three portions, and the solvent was removed under reduced pressure. Thianthrenium salt TT-1 (189 mg, $0.400 \mathrm{mmol}$, 1.00 equiv.), [Cu(MeCN$\left.)_{4}\right]_{B F}\left(126 \mathrm{mg}, 0.401 \mathrm{mmol}, 1.00 \text { equiv.), [Ru(bipy) } 3 \text { ( } \mathrm{PF}_{6}\right)_{2}(17 \mathrm{mg}, 20 \mu \mathrm{mol}$, $4.9 \mathrm{~mol} \%)$, acetonitrile $(1 \mathrm{~mL})$ and dimethylsulfoxide $\left(1 \mathrm{~mL}, \mathrm{c}_{\text {total }}=0.2 \mathrm{M}\right)$ were added, and the suspension was degassed by bubbling through argon with a cannula for $5 \mathrm{~min}$. While stirring, the suspension was irradiated for $8 \mathrm{~h}$ at $15^{\circ} \mathrm{C}$ using a blue LED $(60 \mathrm{~W})$. The suspension was added to water $(30 \mathrm{~mL})$, and the aqueous phase was extracted with DCM $(3 \times 20 \mathrm{~mL})$. The organic phase was dried over $\mathrm{MgSO}_{4}$, and the solvent was removed under reduced pressure. The residue was purified by chromatography on silica gel eluting with hexanes / EtOAc (1:0-15:1 (v/v)) to afford $68 \mathrm{mg}(72 \%)$ of $1 \mathrm{~m}$ as yellow oil.

$\mathbf{R}_{\boldsymbol{f}}=0.33(\mathrm{DCM} / \mathrm{MeOH}, 95: 5(\mathrm{v} / \mathrm{v}))$.

NMR Spectroscopy:

${ }^{1} \mathrm{H}$ NMR $\left(500 \mathrm{MHz}, \mathrm{CDCl}_{3}, 25^{\circ} \mathrm{C}, \delta\right): 7.87(\mathrm{~d}, \mathrm{~J}=2.4 \mathrm{~Hz}, 1 \mathrm{H}), 7.72(\mathrm{~s}, 1 \mathrm{H}), 7.67-7.64(\mathrm{~m}, 2 \mathrm{H})$, $7.38-7.35(\mathrm{~m}, 2 \mathrm{H}), 7.15-7.09(\mathrm{~m}, 3 \mathrm{H}), 7.05-7.03(\mathrm{~m}, 2 \mathrm{H}), 6.46(\mathrm{t}, J=2.1 \mathrm{~Hz}, 1 \mathrm{H})$.

${ }^{13} \mathrm{C}$ NMR $\left\{{ }^{1} \mathrm{H}\right\}\left(126 \mathrm{MHz}, \mathrm{CDCl}_{3}, 25{ }^{\circ} \mathrm{C}, \delta\right): 157.2,155.8,141.1,136.1,130.0,126.9,123.6,121.0$, 119.8, 119.0, 107.6.

HRMS-EI (m/z) calc'd. for $\mathrm{C}_{15} \mathrm{H}_{12} \mathrm{~N}_{2} \mathrm{O}^{+}[\mathrm{M}]^{+}, 236.094412$; found, 236.094520; deviation: -1.46 ppm. 
Benzimidazole 1n

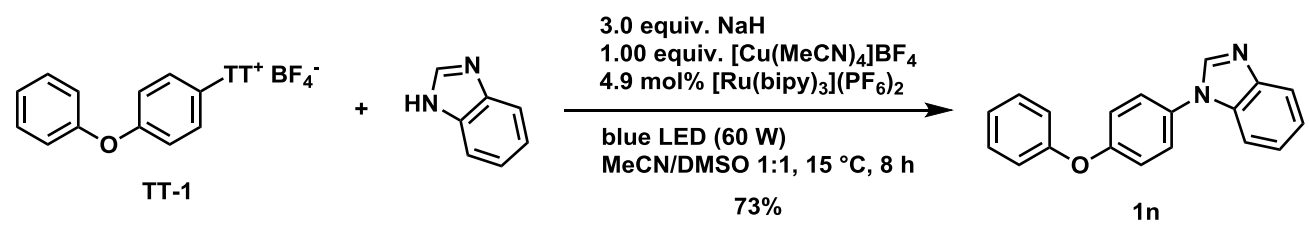

Under ambient atmosphere, a $4 \mathrm{~mL}$ glass-vial was charged with $1 H$-benzo[d]imidazole (143 mg, $1.21 \mathrm{mmol}$, 3.02 equiv.), THF ( $1 \mathrm{~mL}$ ), and sodium hydride (60\% dispersion in mineral oil, $48 \mathrm{mg}, 1.2 \mathrm{mmol}, 3.0$ equiv.) in three portions, and the solvent was removed under reduced pressure. Thianthrenium salt TT-1 (189 mg, $0.400 \mathrm{mmol}, 1.00$ equiv.), [Cu(MeCN$\left.)_{4}\right] \mathrm{BF}_{4}$ (126 mg, $0.401 \mathrm{mmol}, 1.00$ equiv.), [Ru(bipy) $\left.{ }_{3}\right]\left(\mathrm{PF}_{6}\right)_{2}(17 \mathrm{mg}$, $20 \mu \mathrm{mol}, 4.9 \mathrm{~mol} \%)$, acetonitrile $(1 \mathrm{~mL})$ and dimethylsulfoxide $\left(1 \mathrm{~mL}, \mathrm{c}_{\text {total }}=0.2 \mathrm{M}\right)$ were added, and the suspension was degassed by bubbling through argon with a cannula for $5 \mathrm{~min}$. While stirring, the suspension was irradiated for $8 \mathrm{~h}$ at $15^{\circ} \mathrm{C}$ using a blue LED (60 W). The suspension was added to water (30 mL), and the aqueous phase was extracted with $\mathrm{DCM}(3 \times 20 \mathrm{~mL})$. The organic phase was dried over $\mathrm{MgSO}_{4}$, and the solvent was removed under reduced pressure. The residue was purified by chromatography on silica gel eluting with $\mathrm{DCM} / \mathrm{MeOH}(1: 0-15: 1(\mathrm{v} / \mathrm{v}))$ to afford $84 \mathrm{mg}(73 \%)$ of $\mathbf{1 n}$ as yellow oil.

$\mathbf{R} \boldsymbol{f}=0.49(\mathrm{DCM} / \mathrm{MeOH}, 95: 5(\mathrm{v} / \mathrm{v}))$.

\section{NMR Spectroscopy:}

${ }^{1} \mathrm{H}$ NMR $\left(500 \mathrm{MHz}, \mathrm{CDCl}_{3}, 25^{\circ} \mathrm{C}, \delta\right): 8.13(\mathrm{br} \mathrm{s}, 1 \mathrm{H}), 7.91$ (br s, $\left.1 \mathrm{H}\right), 7.54$ (br s, $\left.1 \mathrm{H}\right), 7.47-7.43$ $(\mathrm{m}, 2 \mathrm{H}), 7.43-7.39(\mathrm{~m}, 2 \mathrm{H}), 7.36-7.32(\mathrm{~m}, 2 \mathrm{H}), 7.21-7.17(\mathrm{~m}, 3 \mathrm{H}), 7.12-7.09(\mathrm{~m}, 2 \mathrm{H})$.

${ }^{13} \mathrm{C}$ NMR $\left\{{ }^{1} \mathrm{H}\right\}\left(126 \mathrm{MHz}, \mathrm{CDCl}_{3}, 25^{\circ} \mathrm{C}, \delta\right): 157.5,156.5,130.2,125.9,124.3,123.9,122.5,121.1$, 119.7, 119.6.

HRMS-ESI (m/z) calc'd. for $\mathrm{C}_{19} \mathrm{H}_{15} \mathrm{~N}_{2} \mathrm{O}^{+}[\mathrm{M}+\mathrm{H}]^{+}$, 287.117887; found, 287.118170; deviation: -0.98 ppm.

Triazole 10

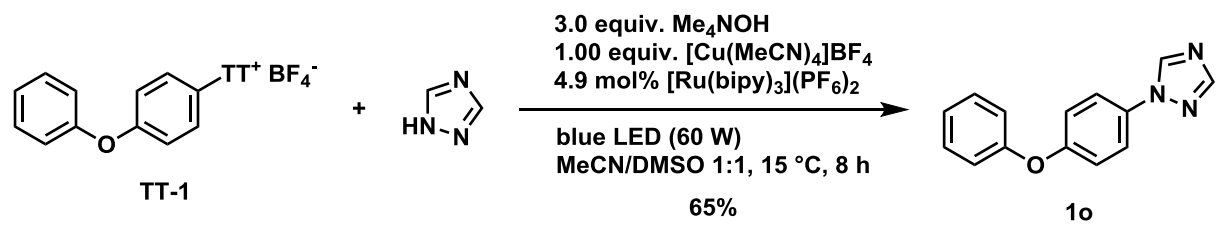

Under ambient atmosphere, a $4 \mathrm{~mL}$ glass-vial was charged with 1,2,4-triazole (83 mg, $1.2 \mathrm{mmol}, 3.0$ equiv.) and tetramethylammonium hydroxide (25\% in methanol, $438 \mathrm{mg}, 0.51 \mathrm{~mL}, 1.2 \mathrm{mmol}, 3.0$ equiv.), and the solvent was removed under reduced pressure. Thianthrenium salt TT-1 (189 mg, $0.400 \mathrm{mmol}, 1.00$ equiv.), $\left[\mathrm{Cu}(\mathrm{MeCN})_{4}\right] \mathrm{BF}_{4}$ (126 mg, $0.401 \mathrm{mmol}, 1.00$ equiv.), [Ru(bipy) $\left.{ }_{3}\right]\left(\mathrm{PF}_{6}\right)_{2}(17 \mathrm{mg}, 20 \mu \mathrm{mol}, 4.9 \mathrm{~mol} \%)$, acetonitrile $(1.0 \mathrm{~mL})$ and dimethylsulfoxide $\left(1.0 \mathrm{~mL}, \mathrm{c}_{\text {total }}=0.20 \mathrm{M}\right)$ were added, and the suspension was degassed by bubbling through argon with a cannula for $5 \mathrm{~min}$. While stirring, the suspension was irradiated for $8 \mathrm{~h}$ at $15^{\circ} \mathrm{C}$ using a blue LED (60 W). The suspension was added to water ( $\left.30 \mathrm{~mL}\right)$, and the aqueous phase was extracted with DCM $(3 \times 20 \mathrm{~mL})$. The organic phase was washed with saturated $\mathrm{NaCl}$ solution 
(30 $\mathrm{mL})$, dried over $\mathrm{MgSO}_{4}$, and the solvent was removed under reduced pressure. The residue was purified by chromatography on silica gel eluting with hexanes / EtOAc $(10: 0-2: 8(\mathrm{v} / \mathrm{v}))$ to afford $62 \mathrm{mg}(65 \%)$ of 10 as colorless oil.

$\mathbf{R}_{\boldsymbol{f}}=0.15($ hexanes / EtOAc, 7:3 (v/v)).

\section{NMR Spectroscopy:}

${ }^{1} \mathrm{H}$ NMR $\left(500 \mathrm{MHz}, \mathrm{CDCl}_{3}, 25^{\circ} \mathrm{C}, \delta\right): 8.58(\mathrm{~s}, 1 \mathrm{H}), 8.10(\mathrm{~s}, 1 \mathrm{H}), 7.64-7.60(\mathrm{~m}, 2 \mathrm{H}), 7.39-7.35$

$(\mathrm{m}, 2 \mathrm{H}), 7.18-7.14(\mathrm{~m}, 1 \mathrm{H}), 7.13-7.10(\mathrm{~m}, 2 \mathrm{H}), 7.06-7.04(\mathrm{~m}, 2 \mathrm{H})$.

${ }^{13} \mathrm{C}$ NMR $\left\{{ }^{1} \mathrm{H}\right\}\left(126 \mathrm{MHz}, \mathrm{CDCl}_{3}, 25{ }^{\circ} \mathrm{C}, \delta\right): 157.6,156.5,152.3,140.9,132.3,130.1,124.2,122.0$, 119.6, 119.4 .

HRMS-EI (m/z) calc'd. for $\mathrm{C}_{14} \mathrm{H}_{11} \mathrm{~N}_{3} \mathrm{O}^{+}[\mathrm{M}]^{+}$, 237.089661; found, 237.089850; deviation: -0.80 ppm.

Phthalimide 1p
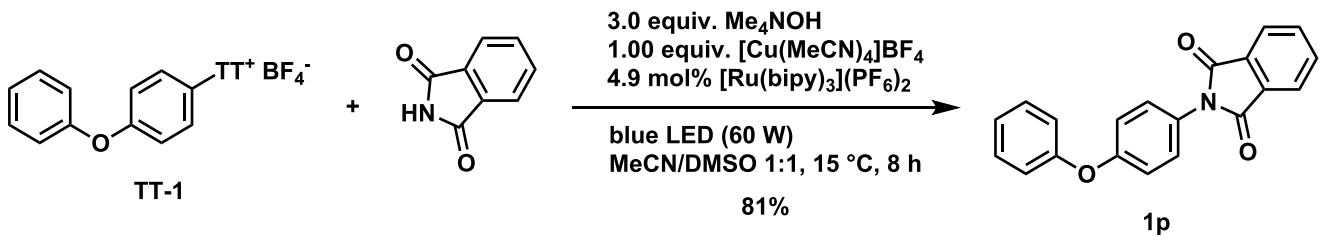

Under ambient atmosphere, a $4 \mathrm{~mL}$ glass-vial was charged with phthalimide (177 mg, $1.20 \mathrm{mmol}$,

3.01 equiv.) and tetramethylammonium hydroxide ( $25 \%$ in methanol, $438 \mathrm{mg}, 0.51 \mathrm{~mL}, 1.2 \mathrm{mmol}, 3.0$ equiv.), and the solvent was removed under reduced pressure. Thianthrenium salt TT-1 (189 mg, $0.400 \mathrm{mmol}$, 1.00 equiv.), [Cu(MeCN)$\left.)_{4}\right] \mathrm{BF}_{4}$ (126 mg, 0.401 mmol, 1.00 equiv.), [Ru(bipy) $\left.)_{3}\right]\left(\mathrm{PF}_{6}\right)_{2}(17 \mathrm{mg}, 20 \mu \mathrm{mol}$, $4.9 \mathrm{~mol} \%)$, acetonitrile $(1.0 \mathrm{~mL})$ and dimethylsulfoxide $\left(1.0 \mathrm{~mL}, \mathrm{c}_{\text {total }}=0.20 \mathrm{M}\right)$ were added, and the suspension was degassed by bubbling through argon with a cannula for $5 \mathrm{~min}$. While stirring, the suspension was irradiated for $8 \mathrm{~h}$ at $15^{\circ} \mathrm{C}$ using a blue LED (60 W). The suspension was added to water (30 mL), and the aqueous phase was extracted with $\mathrm{DCM}(3 \times 20 \mathrm{~mL})$. The organic phase was washed with saturated $\mathrm{NaCl}$ solution $(30 \mathrm{~mL})$, dried over $\mathrm{MgSO}_{4}$, and the solvent was removed under reduced pressure. The residue was purified by chromatography on silica gel eluting with hexanes / EtOAc (10:0-2:8 (v/v)) to afford $102 \mathrm{mg}(81 \%)$ of $1 \mathrm{p}$ as colorless oil.

$\mathbf{R} \boldsymbol{f}=0.22($ hexanes / EtOAc, 8:2 (v/v)).

\section{NMR Spectroscopy:}

${ }^{1} \mathrm{H}$ NMR $\left(500 \mathrm{MHz}, \mathrm{CDCl}_{3}, 25^{\circ} \mathrm{C}, \delta\right): 7.98-7.94(\mathrm{~m}, 2 \mathrm{H}), 7.81-7.78(\mathrm{~m}, 2 \mathrm{H}), 7.40-7.36(\mathrm{~m}$, $4 \mathrm{H}), 7.17-7.08(\mathrm{~m}, 5 \mathrm{H})$.

${ }^{13} \mathrm{C}$ NMR $\left\{{ }^{1} \mathrm{H}\right\}\left(126 \mathrm{MHz}, \mathrm{CDCl}_{3}, 25{ }^{\circ} \mathrm{C}, \delta\right): 167.5,157.3,156.6,134.5,131.9,130.0,128.2,126.5$, 124.0, 123.9, 119.7, 119.0.

HRMS-ESI (m/z) calc'd. for $\mathrm{C}_{20} \mathrm{H}_{14} \mathrm{NO}_{3}{ }^{+}[\mathrm{M}+\mathrm{H}]^{+}$, 316.096819; found, 316.096780; deviation: +0.12 ppm. 


\section{Hydantoin 1q}
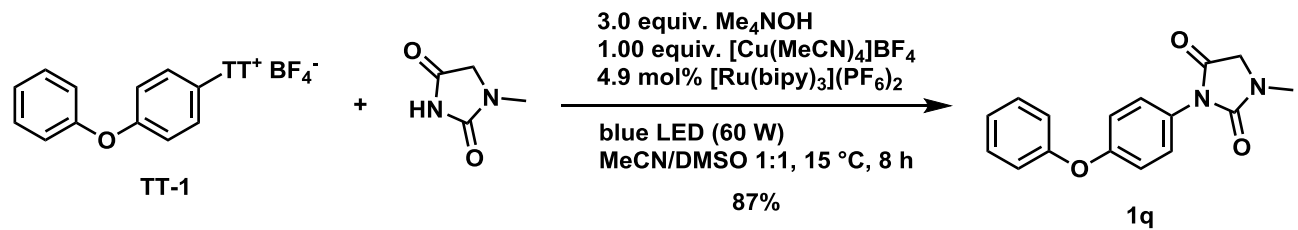

Under ambient atmosphere, a $4 \mathrm{~mL}$ glass-vial was charged with 1-methylhydantoin $(137 \mathrm{mg}, 1.20 \mathrm{mmol}$, 3.00 equiv.) and tetramethylammonium hydroxide ( $25 \%$ in methanol, $438 \mathrm{mg}, 0.51 \mathrm{~mL}, 1.2 \mathrm{mmol}, 3.0$ equiv.), and the solvent was removed under reduced pressure. Thianthrenium salt TT-1 (189 mg, $0.400 \mathrm{mmol}$, 1.00 equiv.), [Cu(MeCN)$\left.)_{4}\right] \mathrm{BF}_{4}$ (126 mg, $0.401 \mathrm{mmol}, 1.00$ equiv.), $\left[\mathrm{Ru}(\text { bipy })_{3}\right]\left(\mathrm{PF}_{6}\right)_{2}(17 \mathrm{mg}, 20 \mu \mathrm{mol}$, $4.9 \mathrm{~mol} \%)$, acetonitrile $(1.0 \mathrm{~mL})$ and dimethylsulfoxide $\left(1.0 \mathrm{~mL}, \mathrm{c}_{\text {total }}=0.20 \mathrm{M}\right)$ were added, and the suspension was degassed by bubbling through argon with a cannula for $5 \mathrm{~min}$. While stirring, the suspension was irradiated for $8 \mathrm{~h}$ at $15^{\circ} \mathrm{C}$ using a blue LED (60 W). The suspension was added to water (30 mL), and the aqueous phase was extracted with $\mathrm{DCM}(3 \times 20 \mathrm{~mL})$. The organic phase was washed with saturated $\mathrm{NaCl}$ solution $(30 \mathrm{~mL})$, dried over $\mathrm{MgSO}_{4}$, and the solvent was removed under reduced pressure. The residue was purified by chromatography on silica gel eluting with hexanes / EtOAc (10:0-2:3 (v/v)) to afford $98 \mathrm{mg}(87 \%)$ of $1 \mathrm{q}$ as brown oil.

$\mathbf{R}_{\boldsymbol{f}}=0.26($ hexanes / EtOAc, 4:6 (v/v)).

\section{NMR Spectroscopy:}

${ }^{1}$ H NMR $\left(500 \mathrm{MHz}, \mathrm{CDCl}_{3}, 25^{\circ} \mathrm{C}, \delta\right): 7.37-7.32(\mathrm{~m}, 4 \mathrm{H}), 7.16(\mathrm{~m}, 1 \mathrm{H}), 7.07-7.04(\mathrm{~m}, 4 \mathrm{H}), 4.04$ $(\mathrm{s}, 2 \mathrm{H}), 3.09(\mathrm{~s}, 3 \mathrm{H})$.

${ }^{13} \mathrm{C}$ NMR $\left\{{ }^{1} \mathrm{H}\right\}\left(126 \mathrm{MHz}, \mathrm{CDCl}_{3}, 25{ }^{\circ} \mathrm{C}, \delta\right): 168.9,157.3,156.6,156.0,130.0,127.7,126.7,124.0$, 119.6, 119.0, 51.8, 30.0.

HRMS-EI (m/z) calc'd. for $\mathrm{C}_{16} \mathrm{H}_{14} \mathrm{~N}_{2} \mathrm{O}_{3}{ }^{+}[\mathrm{M}]^{+}$, 282.099892; found, 282.100310; deviation: -1.48 ppm.

\section{Bromopyridone 1r}

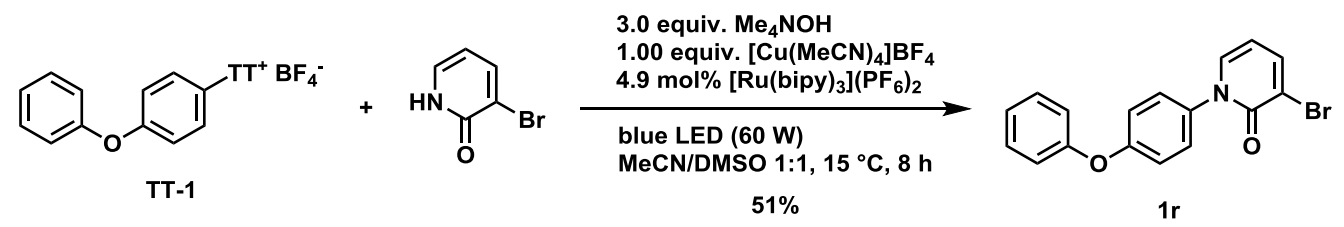

Under ambient atmosphere, a $4 \mathrm{~mL}$ glass-vial was charged with 3-bromo-2-hydroxypyridine (209 mg, $1.20 \mathrm{mmol}, 3.00$ equiv.) and tetramethylammonium hydroxide ( $25 \%$ in methanol, $438 \mathrm{mg}, 0.51 \mathrm{~mL}, 1.2 \mathrm{mmol}$, 3.0 equiv.), and the solvent was removed under reduced pressure. Thianthrenium salt TT-1 (189 mg, $0.400 \mathrm{mmol}, 1.00$ equiv.), [Cu(MeCN$\left.)_{4}\right]_{B F}\left(126 \mathrm{mg}, 0.401 \mathrm{mmol}, 1.00\right.$ equiv.), [Ru(bipy) $\left.{ }_{3}\right]\left(\mathrm{PF}_{6}\right)_{2}(17 \mathrm{mg}$, $20 \mu \mathrm{mol}, 4.9 \mathrm{~mol} \%)$, acetonitrile $(1.0 \mathrm{~mL})$ and dimethylsulfoxide $\left(1.0 \mathrm{~mL}, \mathrm{c}_{\text {total }}=0.20 \mathrm{M}\right)$ were added, and the suspension was degassed by bubbling through argon with a cannula for $5 \mathrm{~min}$. While stirring, the suspension 
was irradiated for $8 \mathrm{~h}$ at $15^{\circ} \mathrm{C}$ using a blue LED (60 W). The suspension was added to water (30 mL), and the aqueous phase was extracted with DCM $(3 \times 20 \mathrm{~mL})$. The organic phase was washed with saturated $\mathrm{NaCl}$ solution $\left(30 \mathrm{~mL}\right.$ ), dried over $\mathrm{MgSO}_{4}$, and the solvent was removed under reduced pressure. The residue was purified by chromatography on silica gel eluting with hexanes / EtOAc (10:0-4:1 (v/v)) to afford $70 \mathrm{mg}(51 \%)$ of $1 \mathrm{r}$ as white solid.

$\mathbf{R} \boldsymbol{f}=0.12($ hexanes / EtOAc, 85:15 (v/v)).

\section{NMR Spectroscopy:}

${ }^{1} \mathrm{H}$ NMR $\left(500 \mathrm{MHz}, \mathrm{CDCl}_{3}, 25^{\circ} \mathrm{C}, \delta\right): 7.80$ (apt dd, $\left.J=7.2,1.9 \mathrm{~Hz}, 1 \mathrm{H}\right), 7.39-7.35(\mathrm{~m}, 3 \mathrm{H}), 7.33$ $-7.30(\mathrm{~m}, 2 \mathrm{H}), 7.18-7.14(\mathrm{~m}, 1 \mathrm{H}), 7.08-7.06(\mathrm{~m}, 4 \mathrm{H}), 6.14($ apt $\mathrm{t}, J=7.0 \mathrm{~Hz}, 1 \mathrm{H})$.

${ }^{13} \mathrm{C}$ NMR $\left\{{ }^{1} \mathrm{H}\right\}\left(126 \mathrm{MHz}, \mathrm{CDCl}_{3}, 25{ }^{\circ} \mathrm{C}, \delta\right): 158.9,157.9,156.4,142.0,137.7,135.6,130.1,127.9$, 124.2, 119.7, 118.9, 117,6, 106.0.

HRMS-ESI (m/z) calc'd. for $\mathrm{C}_{17} \mathrm{H}_{12} \mathrm{BrNO}_{2} \mathrm{Na}^{+}[\mathrm{M}+\mathrm{Na}]^{+}$, 363.994373; found, 363.994320; deviation: $+0.15 \mathrm{ppm}$.

Azide 1s

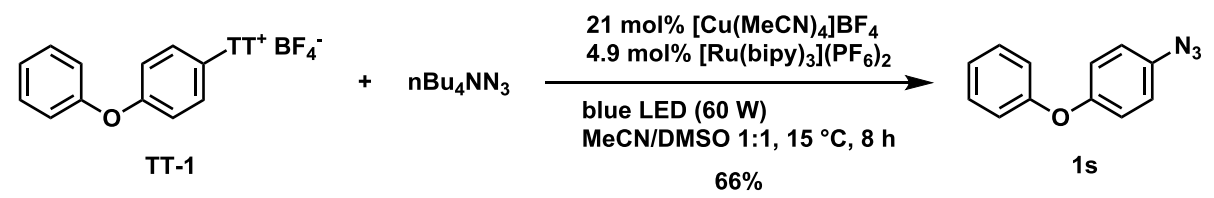

Under ambient atmosphere, a $4 \mathrm{~mL}$ glass-vial was charged with thianthrenium salt TT-1 (189 mg, $0.400 \mathrm{mmol}, 1.00$ equiv.), [Cu(MeCN$\left.)_{4}\right] \mathrm{BF}_{4}(26 \mathrm{mg}, 83 \mu \mathrm{mol}, 21 \mathrm{~mol} \%),\left[\mathrm{Ru}(\mathrm{bipy})_{3}\right]\left(\mathrm{PF}_{6}\right)_{2}(17 \mathrm{mg}, 20 \mu \mathrm{mol}$, $4.9 \mathrm{~mol} \%), \mathrm{nBuNN}_{3}$ (341 mg, $1.20 \mathrm{mmol}, 3.00$ equiv.), acetonitrile $(2.0 \mathrm{~mL}, \mathrm{c}=0.20 \mathrm{M})$, and the suspension was degassed by bubbling through argon with a cannula for $5 \mathrm{~min}$. While stirring, the suspension was irradiated for $8 \mathrm{~h}$ at $15^{\circ} \mathrm{C}$ using a blue LED $(60 \mathrm{~W})$. The solvent was removed under reduced pressure. The residue was purified by chromatography on silica gel eluting with hexanes / EtOAc (1:0-98:2 (v/v)) to afford $56 \mathrm{mg}(66 \%)$ of $1 \mathrm{~s}$ as yellow oil.

$\mathbf{R}_{\boldsymbol{f}}=0.58$ (hexanes / EtOAc, 95:5 (v/v)).

\section{NMR Spectroscopy:}

${ }^{1} \mathrm{H}$ NMR $\left(500 \mathrm{MHz}, \mathrm{CD}_{2} \mathrm{Cl}_{2}, 25^{\circ} \mathrm{C}, \delta\right): 7.36-7.33(\mathrm{~m}, 2 \mathrm{H}), 7.13-7.10(\mathrm{~m}, 1 \mathrm{H}), 7.02-6.98(\mathrm{~m}$, $6 \mathrm{H})$.

${ }^{13} \mathrm{C}$ NMR $\left\{{ }^{1} \mathrm{H}\right\}\left(126 \mathrm{MHz}, \mathrm{CD}_{2} \mathrm{Cl}_{2}, 25^{\circ} \mathrm{C}, \delta\right): 157.9,154.9,135.6,130.4,123.9,120.9,120.8,119.1$.

HRMS- $\mathrm{Cl}(\mathbf{m} / \mathbf{z})$ calc'd. for $\mathrm{C}_{12} \mathrm{H}_{10} \mathrm{~N}_{3} \mathrm{O}^{+}[\mathrm{M}+\mathrm{H}]^{+}, 212.081836$; found, 212.082080; deviation: -1.15 ppm. 
Aniline 1t

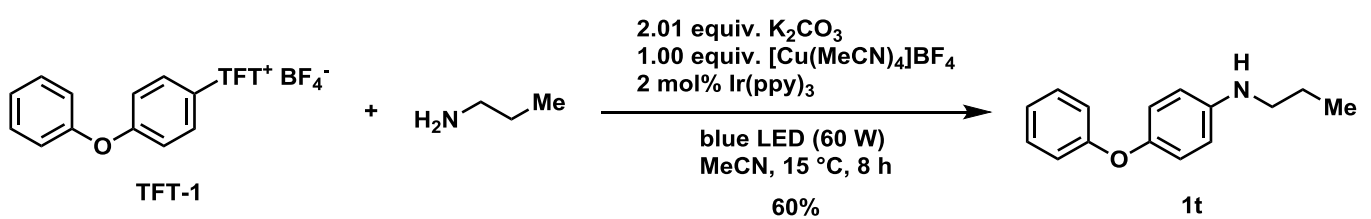

Under ambient atmosphere, a $4 \mathrm{~mL}$ glass-vial was charged with tetrafluorothianthrenium salt TFT-1 (218 mg, $0.401 \mathrm{mmol}, 1.00$ equiv.), [Cu(MeCN)$)_{4} \mathrm{BF}_{4}$ (126 mg, $0.401 \mathrm{mmol}, 1.00$ equiv.), $\operatorname{lr}(\mathrm{ppy})_{3}$ (4 mg, $6 \mu \mathrm{mol}$, $2 \mathrm{~mol} \%), \mathrm{K}_{2} \mathrm{CO}_{3}$ (111 mg, $0.803 \mathrm{mmol}, 2.01$ equiv.), and acetonitrile (2 $\left.\mathrm{mL}, \mathrm{C}=0.2 \mathrm{M}\right)$. Propylamine $(49 \mu \mathrm{L}$, $35 \mathrm{mg}, 0.60 \mathrm{mmol}, 1.5$ equiv.) was added, and the suspension was degassed by bubbling through argon with a cannula for $5 \mathrm{~min}$. While stirring, the suspension was irradiated for $8 \mathrm{~h}$ at $15^{\circ} \mathrm{C}$ using a blue LED $(60 \mathrm{~W})$. The solvent was removed under reduced pressure. The residue was purified by chromatography on silica gel eluting with hexanes / EtOAc (1:0-9:1 (v/v)) to afford $55 \mathrm{mg}(60 \%)$ of $1 \mathrm{t}$ as brown oil.

$\mathbf{R}_{\boldsymbol{f}}=0.57$ (hexanes / EtOAc, 9:1 (v/v)).

\section{NMR Spectroscopy:}

${ }^{1} \mathrm{H}$ NMR $\left(500 \mathrm{MHz}, \mathrm{CDCl}_{3}, 25^{\circ} \mathrm{C}, \delta\right): 7.30-7.26(\mathrm{~m}, 2 \mathrm{H}), 7.02-6.99(\mathrm{~m}, 1 \mathrm{H}), 6.95-6.90(\mathrm{~m}$, $4 \mathrm{H}$ ), $6.62-6.60(\mathrm{~m}, 2 \mathrm{H}), 3.55$ (br s, 1H), 3.08 (t, $J=7.1 \mathrm{~Hz}, 2 \mathrm{H}$ ), 1.66 (hept, $J=7.4 \mathrm{~Hz}, 2 \mathrm{H}$ ), 1.02 (t, $J=7.4 \mathrm{~Hz}, 3 \mathrm{H})$.

${ }^{13} \mathrm{C}$ NMR $\left\{{ }^{1} \mathrm{H}\right\}\left(126 \mathrm{MHz}, \mathrm{CDCl}_{3}, 25{ }^{\circ} \mathrm{C}, \delta\right): 159.3,147.5,145.4,129.6,122.0,121.4,117.1,113.8$, 46.5, 22.9, 11.8 .

HRMS-ESI (m/z) calc'd. for $\mathrm{C}_{15} \mathrm{H}_{18} \mathrm{NO}^{+}[\mathrm{M}+\mathrm{H}]^{+}$, 228.138289; found, 228.138420; deviation: -0.57 ppm.

Aniline 1u

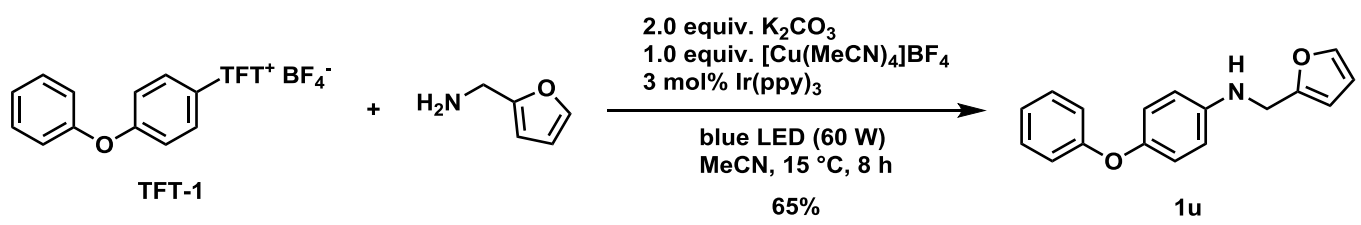

Under ambient atmosphere, a $4 \mathrm{~mL}$ glass-vial was charged with tetrafluorothianthrenium salt TFT-1 (109 mg, 0.200 mmol, 1.00 equiv.), [Cu(MeCN) $)_{4} \mathrm{BF}_{4}$ (63 mg, 0.20 mmol, 1.0 equiv.), $\operatorname{lr}(\mathrm{ppy})_{3}$ (4 mg, $6 \mu \mathrm{mol}, 3 \mathrm{~mol} \%$ ), $\mathrm{K}_{2} \mathrm{CO}_{3}$ (55 mg, $0.40 \mathrm{mmol}, 2.0$ equiv.), and acetonitrile ( $\left.1 \mathrm{~mL}, \mathrm{C}=0.2 \mathrm{M}\right)$. Furfurylamine $(27 \mu \mathrm{L}, 30 \mathrm{mg}$, $0.31 \mathrm{mmol}, 1.5$ equiv.) was added, and the suspension was degassed by bubbling through argon with a cannula for $5 \mathrm{~min}$. While stirring, the suspension was irradiated for $8 \mathrm{~h}$ at $15^{\circ} \mathrm{C}$ using a blue LED $(60 \mathrm{~W})$. The solvent was removed under reduced pressure. The residue was purified by chromatography on silica gel eluting with hexanes / EtOAc (1:0-9:1 (v/v)) to afford $35 \mathrm{mg}(65 \%)$ of $1 \mathbf{u}$ as yellow oil.

$\mathbf{R}_{\boldsymbol{f}}=0.47$ (hexanes / EtOAc, 9:1 (v/v)).

\section{NMR Spectroscopy:}


${ }^{1} \mathrm{H}$ NMR $\left(500 \mathrm{MHz}, \mathrm{CDCl}_{3}, 25^{\circ} \mathrm{C}, \delta\right): 7.39-7.38(\mathrm{~m}, 1 \mathrm{H}), 7.30-7.26(\mathrm{~m}, 2 \mathrm{H}), 7.03-7.00(\mathrm{~m}$, $1 \mathrm{H}), 6.94-6.90(\mathrm{~m}, 4 \mathrm{H}), 6.69-6.66(\mathrm{~m}, 2 \mathrm{H}), 6.35-6.34(\mathrm{~m}, 1 \mathrm{H}), 6.26-6.25(\mathrm{~m}, 1 \mathrm{H}), 4.31(\mathrm{~s}$, 2H), 3.96 (br s, 1H).

${ }^{13} \mathrm{C}$ NMR $\left\{{ }^{1} \mathrm{H}\right\}\left(126 \mathrm{MHz}, \mathrm{CDCl}_{3}, 25{ }^{\circ} \mathrm{C}, \delta\right): 159.1,152.8,148.4,144.3,142.1,129.6,122.2,121.3$, $117.4,114.4,110.5,107.2,42.1$.

HRMS-EI (m/z) calc'd. for $\mathrm{C}_{17} \mathrm{H}_{15} \mathrm{NO}_{2}{ }^{+}[\mathrm{M}]^{+}$, 265.109728; found, 265.110140; deviation: -1.55 ppm.

Aniline 1v

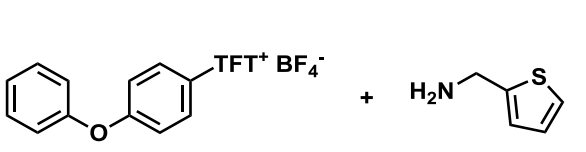

TFT-1

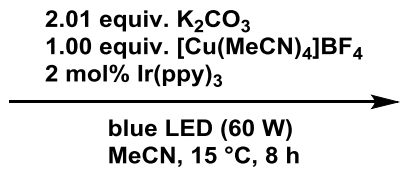

$67 \%$

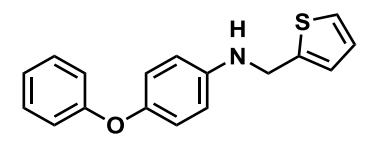

$1 \mathrm{v}$

Under ambient atmosphere, a $4 \mathrm{~mL}$ glass-vial was charged with tetrafluorothianthrenium salt TFT-1 (218 mg, $0.401 \mathrm{mmol}, 1.00$ equiv.), [Cu(MeCN)$)_{4} \mathrm{BF}_{4}\left(126 \mathrm{mg}, 0.401 \mathrm{mmol}, 1.00\right.$ equiv.), $\operatorname{lr}(\mathrm{ppy})_{3}(4 \mathrm{mg}, 6 \mu \mathrm{mol}$, $2 \mathrm{~mol} \%$ ), $\mathrm{K}_{2} \mathrm{CO}_{3}$ (111 mg, $0.803 \mathrm{mmol}, 2.01$ equiv.), and acetonitrile (2 $\left.\mathrm{mL}, \mathrm{c}=0.2 \mathrm{M}\right) .2$ -

Thiophenemethylamine ( $62 \mu \mathrm{L}, 68 \mathrm{mg}, 0.60 \mathrm{mmol}, 1.5$ equiv.) was added, and the suspension was degassed by bubbling through argon with a cannula for $5 \mathrm{~min}$. While stirring, the suspension was irradiated for $8 \mathrm{~h}$ at $15^{\circ} \mathrm{C}$ using a blue LED $(60 \mathrm{~W})$. The solvent was removed under reduced pressure. The residue was purified by chromatography on silica gel eluting with hexanes / EtOAc (1:0-9:1 (v/v)) to afford $75 \mathrm{mg}(67 \%)$ of $1 \mathrm{v}$ as off-white solid.

$\mathbf{R} \boldsymbol{f}=0.49$ (hexanes / EtOAc, 9:1 (v/v)).

\section{NMR Spectroscopy:}

${ }^{1} \mathbf{H}$ NMR $\left(500 \mathrm{MHz}, \mathrm{CDCl}_{3}, 25^{\circ} \mathrm{C}, \delta\right): 7.30-7.26(\mathrm{~m}, 2 \mathrm{H}), 7.24(\mathrm{dd}, J=5.1,1.2 \mathrm{~Hz}), 7.04-6.98$ (m, 3H), $6.95-6.92(\mathrm{~m}, 4 \mathrm{H}), 6.69-6.66(\mathrm{~m}, 2 \mathrm{H}), 4.51(\mathrm{~s}, 2 \mathrm{H}), 3.99(\mathrm{~s}, 1 \mathrm{H})$.

${ }^{13} \mathrm{C}$ NMR $\left\{{ }^{1} \mathrm{H}\right\}\left(126 \mathrm{MHz}, \mathrm{CDCl}_{3}, 25^{\circ} \mathrm{C}, \delta\right): 159.1,148.4,144.3,143.0,129.6,127.0,125.2,124.8$, $122.2,121.2,117.4,114.4,44.2$.

HRMS-ESI (m/z) calc'd. for $\mathrm{C}_{17} \mathrm{H}_{16} \mathrm{NOS}^{+}[\mathrm{M}+\mathrm{H}]^{+}$, 282.094711; found, 282.094870; deviation: -0.56 ppm.

Aniline 1w
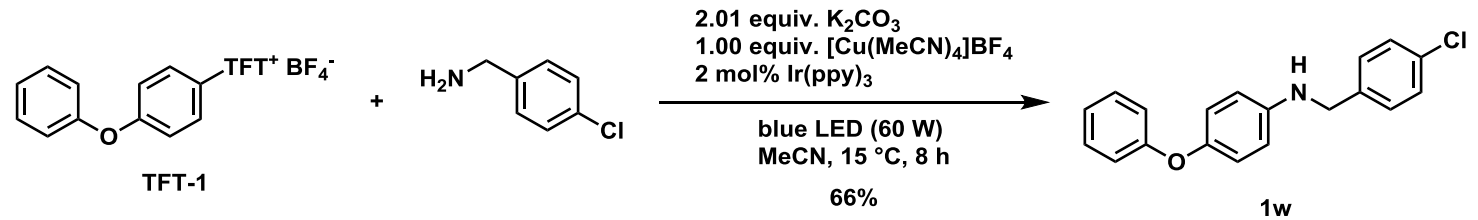

Under ambient atmosphere, a $4 \mathrm{~mL}$ glass-vial was charged with tetrafluorothianthrenium salt TFT-1 (218 mg, $0.401 \mathrm{mmol}, 1.00$ equiv.), [Cu(MeCN)$\left.)_{4}\right] \mathrm{BF}_{4}$ (126 mg, $0.401 \mathrm{mmol}, 1.00$ equiv.), $\operatorname{Ir}(\mathrm{ppy})_{3}(4 \mathrm{mg}, 6 \mu \mathrm{mol}$, 
2 mol\%), $\mathrm{K}_{2} \mathrm{CO}_{3}$ (111 mg, $0.803 \mathrm{mmol}, 2.01$ equiv.), and acetonitrile (2 mL, $\mathrm{C}=0.2 \mathrm{M}$ ). 4-Chlorobenzylamine ( $73 \mu \mathrm{L}, 85 \mathrm{mg}, 0.60 \mathrm{mmol}, 1.5$ equiv.) was added, and the suspension was degassed by bubbling through argon with a cannula for $5 \mathrm{~min}$. While stirring, the suspension was irradiated for $8 \mathrm{~h}$ at $15^{\circ} \mathrm{C}$ using a blue LED $(60 \mathrm{~W})$. The solvent was removed under reduced pressure. The residue was purified by chromatography on silica gel eluting with hexanes / EtOAc (1:0-9:1 (v/v)) to afford $82 \mathrm{mg}(66 \%)$ of $\mathbf{1} \mathbf{w}$ as yellow oil.

$\mathbf{R}_{\boldsymbol{f}}=0.11$ (hexanes / EtOAc, 95:5 (v/v)).

\section{NMR Spectroscopy:}

${ }^{1} \mathrm{H}$ NMR $\left(500 \mathrm{MHz}, \mathrm{CDCl}_{3}, 25^{\circ} \mathrm{C}, \delta\right): 7.32(\mathrm{~s}, 4 \mathrm{H}), 7.29-7.26(\mathrm{~m}, 2 \mathrm{H}), 7.02-6.99(\mathrm{~m}, 1 \mathrm{H}), 6.94$ $-6.88(\mathrm{~m}, 4 \mathrm{H}), 6.62-6.59(\mathrm{~m}, 2 \mathrm{H}), 4.30(\mathrm{~s}, 2 \mathrm{H}), 3.98(\mathrm{br} \mathrm{s}, 1 \mathrm{H})$.

${ }^{13} \mathrm{C}$ NMR $\left\{{ }^{1} \mathrm{H}\right\}\left(126 \mathrm{MHz}, \mathrm{CDCl}_{3}, 25^{\circ} \mathrm{C}, \delta\right): 159.1,148.2,144.6,138.0,133.1,129.6,128.93$, 128.89, 122.2, 121.3, 117.3, 114.0, 48.3.

HRMS-ESI (m/z) calc'd. for $\mathrm{C}_{19} \mathrm{H}_{17} \mathrm{NOCl}^{+}[\mathrm{M}+\mathrm{H}]^{+}, 310.099317$; found, 310.099490; deviation: -0.56 ppm.

\section{C-N cross-coupling: Scope of arenes}

\section{Thianthrenium salt TT-2}
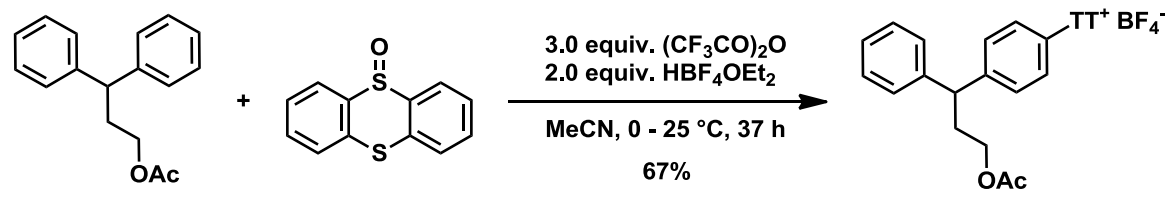

TT-2

Under ambient atmosphere, a $50 \mathrm{~mL}$ Schlenk-tube equipped with a magnetic stir bar was charged with 4-((3acetoxy-1-phenyl)yl)benzene (400 mg, $1.57 \mathrm{mmol}, 1.00$ equiv.), thianthrene S-oxide (377 mg, $1.62 \mathrm{mmol}$, 1.03 equiv.), and $\mathrm{MeCN}(7.9 \mathrm{~mL}, \mathrm{C}=0.20 \mathrm{M})$. After cooling to $0^{\circ} \mathrm{C}$, trifluoroacetic anhydride $(0.66 \mathrm{~mL}, 1.0 \mathrm{~g}$, $4.7 \mathrm{mmol}, 3.0$ equiv.) was added while stirring. $\mathrm{HBF}_{4} \cdot \mathrm{OEt}_{2}(0.43 \mathrm{ml}, 0.51 \mathrm{~g}, 3.2 \mathrm{mmol}, 2.0$ equiv.) was added dropwise. The mixture was stirred at $0^{\circ} \mathrm{C}$ for $1 \mathrm{~h}$, then at ambient temperature for $36 \mathrm{~h}$. The reaction mixture was added to a saturated aqueous $\mathrm{NaHCO}_{3}$ solution $(50 \mathrm{~mL})$, and the aqueous phase was extracted with $\mathrm{DCM}(3 \times 20 \mathrm{~mL})$. The organic phase was washed with aqueous $\mathrm{NaBF}_{4}$ solution $(30 \mathrm{~mL}, 10 \%)$, dried over $\mathrm{MgSO}_{4}$, and the solvent was removed under reduced pressure. The residue was purified by chromatography on silica gel eluting with DCM / MeOH (1:0-94:6 (v/v)) to afford $588 \mathrm{mg}(67 \%)$ of TT-2 as white foam.

$\mathbf{R}_{\boldsymbol{f}}=0.43(\mathrm{DCM} / \mathrm{MeOH}, 94: 6(\mathrm{v} / \mathrm{v}))$.

\section{NMR Spectroscopy:}

${ }^{1} \mathrm{H}$ NMR $\left(500 \mathrm{MHz}, \mathrm{CDCl}_{3}, 25^{\circ} \mathrm{C}, \delta\right): 8.50(\mathrm{~d}, J=7.8 \mathrm{~Hz}, 2 \mathrm{H}), 7.79(\mathrm{q}, J=8.0 \mathrm{~Hz}, 4 \mathrm{H}), 7.72(\mathrm{t}, J=$ $7.4 \mathrm{~Hz}, 2 \mathrm{H}), 7.31(\mathrm{~d}, J=8.7 \mathrm{~Hz}, 2 \mathrm{H}), 7.28-7.20(\mathrm{~m}, 2 \mathrm{H}), 7.20-7.15(\mathrm{~m}, 1 \mathrm{H}), 7.12(\mathrm{t}, J=6.8 \mathrm{~Hz}$, $4 \mathrm{H}), 4.03(\mathrm{t}, J=7.8 \mathrm{~Hz}, 1 \mathrm{H}), 3.98-3.87(\mathrm{~m}, 2 \mathrm{H}), 2.36-2.21(\mathrm{~m}, 2 \mathrm{H}), 1.96(\mathrm{~s}, 3 \mathrm{H})$.

${ }^{13} \mathrm{C}$ NMR $\left\{{ }^{1} \mathrm{H}\right\}\left(126 \mathrm{MHz}, \mathrm{CDCl}_{3}, 25{ }^{\circ} \mathrm{C}, \delta\right): 171.0,150.4,141.8,136.6,136.5,135.4,135.0,130.4$, 
$130.4,130.2,129.0,128.5,127.9,127.2,121.7,118.7,118.7,62.3,47.4,33.9,21.0$.

${ }^{19} \mathrm{~F}$ NMR $\left\{{ }^{1} \mathrm{H}\right\}\left(471 \mathrm{MHz}, \mathrm{CDCl}_{3}, 25^{\circ} \mathrm{C}, \delta\right):-151.0,-151.1$.

HRMS-ESI (m/z) calc'd. for $\mathrm{C}_{29} \mathrm{H}_{25} \mathrm{O}_{2} \mathrm{~S}_{2}{ }^{+}\left[\mathrm{M}-\mathrm{BF}_{4}\right]^{+}$, 469.129050; found, 469.128980; deviation: +0.15 ppm.

Morpholine 2
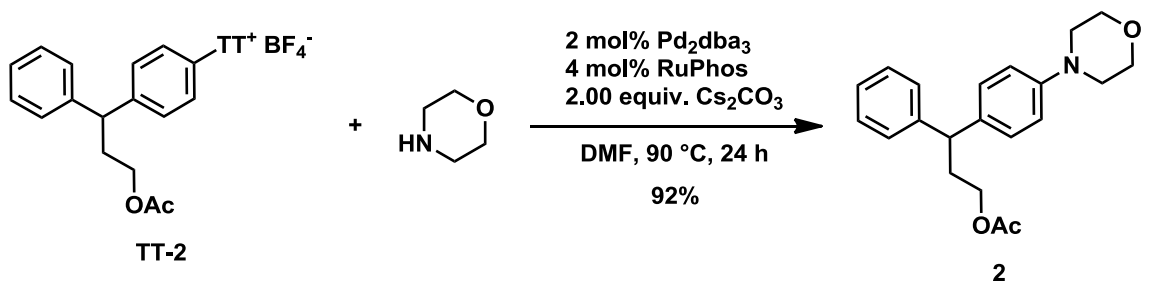

In an argon-filled glovebox, a $4 \mathrm{~mL}$ glass-vial equipped with a magnetic stir bar was charged with TT-2

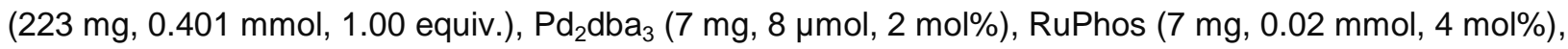
$\mathrm{Cs}_{2} \mathrm{CO}_{3}$ (261 mg, $0.801 \mathrm{mmol}, 2.00$ equiv.), and DMF (1 mL, c = $\left.0.4 \mathrm{M}\right)$. Morpholine (53 $\mu \mathrm{L}, 53 \mathrm{mg}$, $0.61 \mathrm{mmol}, 1.5$ equiv.) was added, and the sealed vial was taken out from the glovebox. The suspension was stirred at $90{ }^{\circ} \mathrm{C}$ for $24 \mathrm{~h}$. The solvent was removed under reduced pressure, and the residue was purified by chromatography on silica gel eluting with hexanes / EtOAc (1:0-6:4 (v/v)) to afford $125 \mathrm{mg}(92 \%)$ of 2 as yellow oil.

$\mathbf{R}_{\boldsymbol{f}}=0.38($ hexanes / EtOAc, 6:4 (v/v)).

\section{NMR Spectroscopy:}

${ }^{1} \mathrm{H}$ NMR $\left(500 \mathrm{MHz}, \mathrm{CDCl}_{3}, 25^{\circ} \mathrm{C}, \delta\right): 7.32-7.25(\mathrm{~m}, 2 \mathrm{H}), 7.25-7.21(\mathrm{~m}, 2 \mathrm{H}), 7.21-7.10(\mathrm{~m}$, $3 \mathrm{H}), 6.84(\mathrm{~d}, J=8.7 \mathrm{~Hz}, 2 \mathrm{H}), 4.07-3.96(\mathrm{~m}, 3 \mathrm{H}), 3.88-3.80(\mathrm{~m}, 4 \mathrm{H}), 3.16-3.08(\mathrm{~m}, 4 \mathrm{H}), 2.36$ (q, $J=7.1 \mathrm{~Hz}, 2 \mathrm{H}), 2.02(\mathrm{~s}, 3 \mathrm{H})$.

${ }^{13} \mathrm{C}$ NMR $\left\{{ }^{1} \mathrm{H}\right\}\left(126 \mathrm{MHz}, \mathrm{CDCl}_{3}, 25{ }^{\circ} \mathrm{C}, \delta\right): 171.1,149.8,144.6,135.5,128.6,128.6,127.8,126.4$, 115.9, 67.0, 63.1, 49.5, 46.8, 34.4, 21.1.

HRMS-ESI (m/z) calc'd. for $\mathrm{C}_{21} \mathrm{H}_{25} \mathrm{NO}_{3} \mathrm{Na}^{+}[\mathrm{M}+\mathrm{Na}]^{+}$, 362.172662; found, 362.172590; deviation: +0.20 ppm.

\section{Thianthrenium salt TT-3}
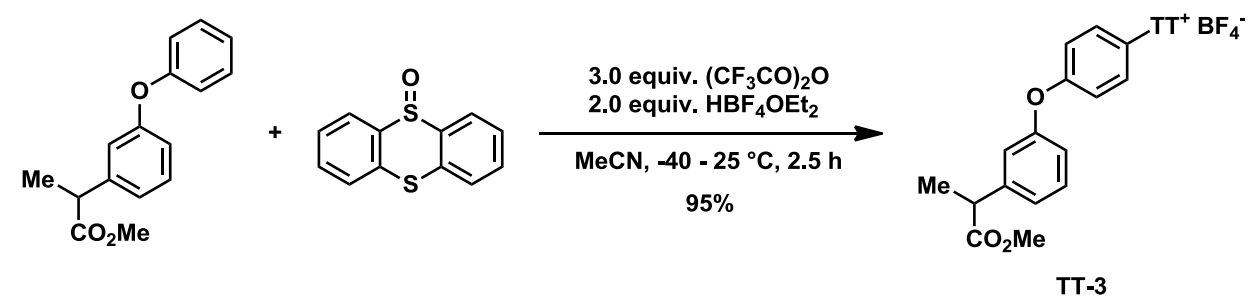

Under ambient atmosphere, a $50 \mathrm{~mL}$ Schlenk-tube equipped with a magnetic stir bar was charged with fenoprofen methyl ester ( $260 \mathrm{mg}, 1.01 \mathrm{mmol}, 1.00$ equiv.), thianthrene S-oxide (236 mg, $1.02 \mathrm{mmol}$, 1.00 equiv.), and $\mathrm{MeCN}(5.1 \mathrm{~mL}, \mathrm{C}=0.20 \mathrm{M})$. After cooling to $-40^{\circ} \mathrm{C}$, trifluoroacetic anhydride $(0.42 \mathrm{~mL}$, 
$0.63 \mathrm{~g}, 3.0 \mathrm{mmol}, 3.0$ equiv.) was added while stirring. $\mathrm{HBF}_{4} \cdot \mathrm{OEt}_{2}(0.28 \mathrm{ml}, 0.33 \mathrm{~g}, 2.1 \mathrm{mmol}, 2.0$ equiv.) was added dropwise. The mixture was stirred at $-40^{\circ} \mathrm{C}$ for $30 \mathrm{~min}$, then at ambient temperature for $2 \mathrm{~h}$. The reaction mixture was added to a saturated aqueous $\mathrm{NaHCO}_{3}$ solution $(50 \mathrm{~mL})$, and the aqueous phase was extracted with DCM $(3 \times 20 \mathrm{~mL})$. The organic phase was washed with aqueous $\mathrm{NaBF}_{4}$ solution $(30 \mathrm{~mL}, 10 \%)$, dried over $\mathrm{MgSO}_{4}$, and the solvent was removed under reduced pressure. The residue was purified by chromatography on silica gel eluting with $\mathrm{DCM} / \mathrm{MeOH}(1: 0-9: 1(\mathrm{v} / \mathrm{v}))$ to afford $536 \mathrm{mg}(95 \%)$ of TT-3 as white foam.

$\mathbf{R}_{\boldsymbol{f}}=0.39(\mathrm{DCM} / \mathrm{MeOH}, 94: 6(\mathrm{v} / \mathrm{v}))$.

NMR Spectroscopy:

${ }^{1} \mathrm{H}$ NMR $\left(500 \mathrm{MHz}, \mathrm{CDCl}_{3}, 25^{\circ} \mathrm{C}, \delta\right): 8.47(\mathrm{~d}, J=6.8 \mathrm{~Hz}, 2 \mathrm{H}), 7.86-7.77(\mathrm{~m}, 4 \mathrm{H}), 7.75-7.69(\mathrm{~m}$, 2H), $7.29(\mathrm{t}, J=7.9 \mathrm{~Hz}, 1 \mathrm{H}), 7.24-7.18(\mathrm{~m}, 2 \mathrm{H}), 7.12(\mathrm{~d}, J=7.7 \mathrm{~Hz}, 1 \mathrm{H}), 6.96-6.89(\mathrm{~m}, 3 \mathrm{H})$, $6.84(\mathrm{dd}, J=8.1,1.5 \mathrm{~Hz}, 1 \mathrm{H}), 3.68(\mathrm{q}, J=7.2 \mathrm{~Hz}, 1 \mathrm{H}), 3.62(\mathrm{~s}, 3 \mathrm{H}), 1.44(\mathrm{~d}, J=7.2 \mathrm{~Hz}, 3 \mathrm{H})$.

${ }^{13} \mathrm{C}$ NMR $\left\{{ }^{1} \mathrm{H}\right\}\left(126 \mathrm{MHz}, \mathrm{CDCl}_{3}, 25{ }^{\circ} \mathrm{C}, \delta\right): 174.5,162.3,154.4,143.2,136.3,135.0,135.0,130.5$, $130.5,130.4,124.8,119.8,119.2,119.1,119.0,115.8,52.3,45.2,18.6$.

${ }^{19} \mathrm{~F}$ NMR $\left\{{ }^{1} \mathrm{H}\right\}\left(471 \mathrm{MHz}, \mathrm{CDCl}_{3}, 25^{\circ} \mathrm{C}, \delta\right):-151.1,-151.1$.

HRMS-ESI (m/z) calc'd. for $\mathrm{C}_{28} \mathrm{H}_{23} \mathrm{O}_{3} \mathrm{~S}_{2}{ }^{+}\left[\mathrm{M}-\mathrm{BF}_{4}\right]^{+}$, 471.108315; found, 471.108290; deviation: +0.05 ppm.

Aniline 3
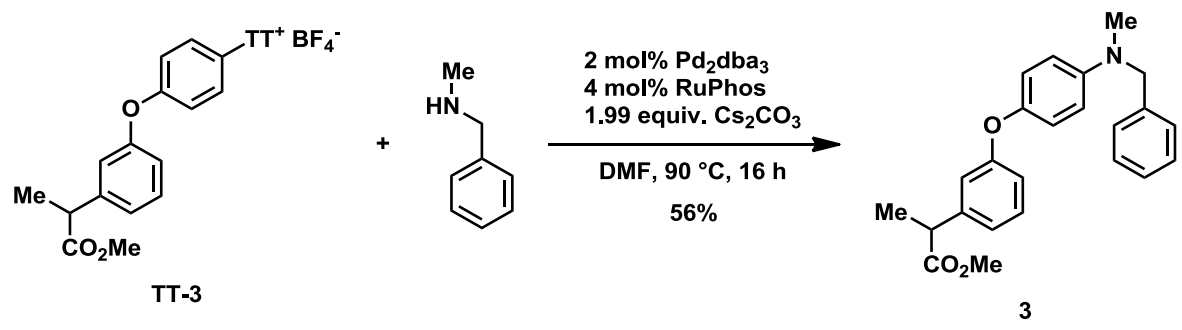

In an argon-filled glovebox, a $4 \mathrm{~mL}$ glass-vial equipped with a magnetic stir bar was charged with TT-3

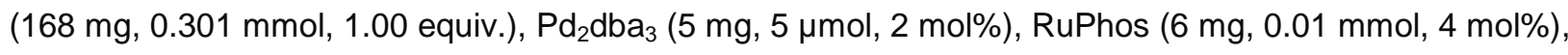
$\mathrm{Cs}_{2} \mathrm{CO}_{3}$ (195 mg, $0.598 \mathrm{mmol}, 1.99$ equiv.), and DMF (1.5 mL, $\left.\mathrm{C}=0.20 \mathrm{M}\right)$. N-Methylbenzylamine $(59 \mu \mathrm{L}$, $55 \mathrm{mg}, 0.46 \mathrm{mmol}, 1.5$ equiv.) was added, and the sealed vial was taken out from the glovebox. The suspension was stirred at $90{ }^{\circ} \mathrm{C}$ for $16 \mathrm{~h}$. The suspension was added to a aqueous saturated $\mathrm{NaHCO}_{3}$ solution $(20 \mathrm{~mL})$, and the aqueous phase was extracted with DCM $(3 \times 20 \mathrm{~mL})$. The organic phase was dried over $\mathrm{MgSO}_{4}$, and the solvent was removed under reduced pressure. The residue was purified by chromatography on silica gel eluting with hexanes / EtOAc $(1: 0-7: 3(\mathrm{v} / \mathrm{v}))$ to afford $63 \mathrm{mg}(56 \%)$ of 3 as yellowish oil.

$\mathbf{R}_{\boldsymbol{f}}=0.53$ (hexanes / EtOAc, 8:2(v/v)).

\section{NMR Spectroscopy:}


${ }^{1}$ H NMR $\left(500 \mathrm{MHz}, \mathrm{CDCl}_{3}, 25^{\circ} \mathrm{C}, \delta\right): 7.37-7.31(\mathrm{~m}, 2 \mathrm{H}), 7.27$ (d, $\left.J=6.6 \mathrm{~Hz}, 3 \mathrm{H}\right), 7.21$ (t, $J=7.9$ $\mathrm{Hz}, 1 \mathrm{H}), 6.97-6.90(\mathrm{~m}, 4 \mathrm{H}), 6.79(\mathrm{~d}, J=8.2 \mathrm{~Hz}, 1 \mathrm{H}), 6.74(\mathrm{~d}, J=9.0 \mathrm{~Hz}, 2 \mathrm{H}), 4.51(\mathrm{~s}, 2 \mathrm{H}), 3.72$ - $3.64(\mathrm{~m}, 4 \mathrm{H}), 3.01(\mathrm{~s}, 3 \mathrm{H}), 1.48(\mathrm{~d}, J=7.2 \mathrm{~Hz}, 3 \mathrm{H})$.

${ }^{13} \mathrm{C}$ NMR $\left\{{ }^{1} \mathrm{H}\right\}\left(126 \mathrm{MHz}, \mathrm{CDCl}_{3}, 25^{\circ} \mathrm{C}, \delta\right): 174.9,159.3,147.1,146.9,142.3,139.1,129.7,128.7$, $127.1,127.0,121.1,121.1,116.7,115.7,113.8,57.5,52.2,45.5,39.0,18.7$.

HRMS-ESI (m/z) calc'd. for $\mathrm{C}_{24} \mathrm{H}_{26} \mathrm{NO}_{3}{ }^{+}[\mathrm{M}+\mathrm{H}]^{+}$, 376.190719; found, 376.191210; deviation: -1.31 ppm.

\section{Thianthrenium salt TT-4}

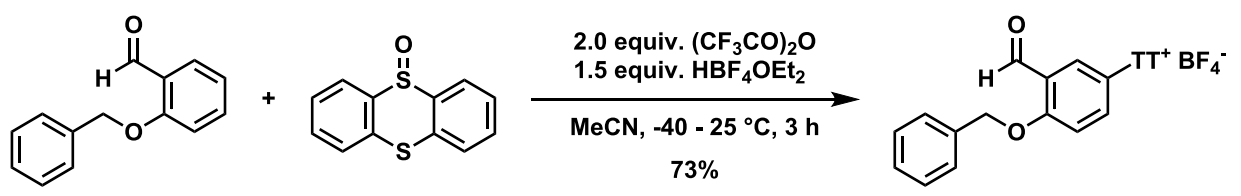

Under ambient atmosphere, a $50 \mathrm{~mL}$ Schlenk-tube equipped with a magnetic stir bar was charged with 2-benzyloxybenzaldehyde (300 mg, $1.41 \mathrm{mmol}, 1.00$ equiv.), thianthrene S-oxide (328 mg, $1.41 \mathrm{mmol}$, 0.999 equiv.), and $\mathrm{MeCN}(7.1 \mathrm{~mL}, \mathrm{C}=0.20 \mathrm{M})$. After cooling to $-40{ }^{\circ} \mathrm{C}$, trifluoroacetic anhydride $(0.39 \mathrm{~mL}$, $0.59 \mathrm{~g}, 2.8 \mathrm{mmol}, 2.0$ equiv.) was added while stirring. $\mathrm{HBF}_{4} \cdot \mathrm{OEt}_{2}(0.29 \mathrm{ml}, 0.35 \mathrm{~g}, 2.1 \mathrm{mmol}, 1.5$ equiv.) was added dropwise. The mixture was stirred at $-40{ }^{\circ} \mathrm{C}$ for $1 \mathrm{~h}$, then at ambient temperature for $2 \mathrm{~h}$. The reaction mixture was added to a saturated aqueous $\mathrm{NaHCO}_{3}$ solution $(50 \mathrm{~mL})$, and the aqueous phase was extracted with $\mathrm{DCM}(3 \times 20 \mathrm{~mL})$. The organic phase was washed with aqueous $\mathrm{NaBF}_{4}$ solution $(30 \mathrm{~mL}, 10 \%)$, dried over $\mathrm{MgSO}_{4}$, and the solvent was removed under reduced pressure. The residue was purified by chromatography on silica gel eluting with $\mathrm{DCM} / \mathrm{MeOH}(1: 0-9: 1(\mathrm{v} / \mathrm{v}))$ to afford $532 \mathrm{mg}(73 \%)$ of the intermediate as white solid.

$\mathbf{R}_{\boldsymbol{f}}=0.15(\mathrm{DCM} / \mathrm{MeOH}, 96: 4(\mathrm{v} / \mathrm{v}))$.

\section{NMR Spectroscopy:}

${ }^{1} \mathrm{H}$ NMR $\left(500 \mathrm{MHz}, \mathrm{DMSO}-\mathrm{d}_{6}, 25^{\circ} \mathrm{C}, \delta\right): 10.26(\mathrm{~s}, 1 \mathrm{H}), 8.61-8.55(\mathrm{~m}, 2 \mathrm{H}), 8.10-8.04(\mathrm{~m}, 2 \mathrm{H})$, $7.96-7.89(\mathrm{~m}, 2 \mathrm{H}), 7.89-7.83(\mathrm{~m}, 2 \mathrm{H}), 7.49(\mathrm{~s}, 4 \mathrm{H}), 7.47(\mathrm{~s}, 1 \mathrm{H}), 7.42-7.36(\mathrm{~m}, 2 \mathrm{H}), 7.36-$ $7.31(\mathrm{~m}, 1 \mathrm{H}), 5.33(\mathrm{~s}, 2 \mathrm{H})$.

${ }^{13} \mathrm{C}$ NMR $\left\{{ }^{1} \mathrm{H}\right\}\left(75 \mathrm{MHz}\right.$, DMSO-d $\left.\mathrm{d}_{6}, 25^{\circ} \mathrm{C}, \delta\right): 187.5,162.9,135.5,135.4,135.1,134.8,130.2$, 129.6, 128.6, 128.3, 128.0, 127.7, 125.1, 119.6, 116.5, 116.3, 70.7.

${ }^{19} \mathrm{~F}$ NMR $\left\{{ }^{1} \mathrm{H}\right\}\left(471 \mathrm{MHz}, \mathrm{DMSO}-\mathrm{d}_{6}, 25^{\circ} \mathrm{C}, \delta\right):-148.2,-148.3$.

HRMS-ESI (m/z) calc'd. for $\mathrm{C}_{26} \mathrm{H}_{19} \mathrm{O}_{2} \mathrm{~S}_{2}{ }^{+}\left[\mathrm{M}-\mathrm{BF}_{4}\right]^{+}, 427.082100$; found, 427.082540; deviation: -1.03 ppm.

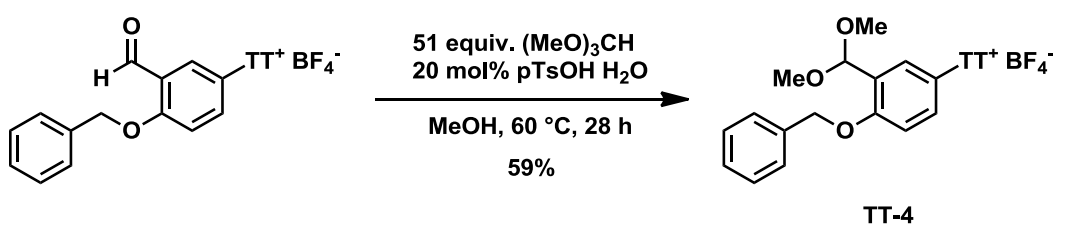


Under ambient atmosphere, a $50 \mathrm{~mL}$ round-bottom flask equipped with a magnetic stir bar was charged with the intermediate ( $400 \mathrm{mg}, 778 \mu \mathrm{mol}, 1.00$ equiv.), $p$-toluenesulfonic acid monohydrate (15 mg, $79 \mu \mathrm{mol}$, $10 \mathrm{~mol} \%)$, trimethyl orthoformate $(4.3 \mathrm{~mL}, 4.2 \mathrm{~g}, 39 \mathrm{mmol}, 51$ equiv.), and methanol $(1.6 \mathrm{~mL}, \mathrm{C}=0.49 \mathrm{M})$. The solution was stirred at $60^{\circ} \mathrm{C}$ for $20 \mathrm{~h}$. $p$-Toluenesulfonic acid monohydrate (15 mg, $79 \mu \mathrm{mol}, 10 \mathrm{~mol} \%$ ) was added, and the solution was stirred at $60^{\circ} \mathrm{C}$ for $8 \mathrm{~h}$. The solvent and volatile components were removed under reduced pressure. DCM $(20 \mathrm{~mL})$ was added, and the resulting suspension was added to a saturated aqueous $\mathrm{NaHCO}_{3}$ solution ( $30 \mathrm{~mL}$ ). The layers were separated, and the aqueous phase was extracted with $\mathrm{DCM}(3 \times 20 \mathrm{~mL})$. The organic phase was dried over $\mathrm{MgSO}_{4}$, and the solvent was removed under reduced pressure. The residue was purified by chromatography on silica gel eluting with $\mathrm{DCM} / \mathrm{MeOH}(1: 0-9: 1(\mathrm{v} / \mathrm{v}))$ to afford $258 \mathrm{mg}(59 \%)$ of TT-4 as white solid.

$\mathbf{R} \boldsymbol{f}=0.29(\mathrm{DCM} / \mathrm{MeOH}, 94: 6(\mathrm{v} / \mathrm{v}))$.

\section{NMR Spectroscopy:}

${ }^{1}$ H NMR (500 MHz, DMSO-d $\left.6,25^{\circ} \mathrm{C}, \delta\right): 8.55(\mathrm{~d}, J=8.1 \mathrm{~Hz}, 2 \mathrm{H}), 8.06(\mathrm{~d}, J=7.9 \mathrm{~Hz}, 2 \mathrm{H}), 7.91$ (td, $J=7.7,1.5 \mathrm{~Hz}, 2 \mathrm{H}), 7.84(\mathrm{td}, J=7.7,1.4 \mathrm{~Hz}, 2 \mathrm{H}), 7.45-7.40(\mathrm{~m}, 2 \mathrm{H}), 7.40-7.35(\mathrm{~m}, 2 \mathrm{H}), 7.35$

$-7.27(\mathrm{~m}, 2 \mathrm{H}), 7.27-7.22(\mathrm{~m}, 1 \mathrm{H}), 7.22-7.18(\mathrm{~m}, 1 \mathrm{H}), 5.48(\mathrm{~s}, 1 \mathrm{H}), 5.20(\mathrm{~s}, 2 \mathrm{H}), 3.15(\mathrm{~s}, 6 \mathrm{H})$.

${ }^{13} \mathrm{C}$ NMR $\left\{{ }^{1} \mathrm{H}\right\}\left(75 \mathrm{MHz}, \mathrm{DMSO}-\mathrm{d}_{6}, 25^{\circ} \mathrm{C}, \delta\right): 158.9,136.0,135.2,135.0,134.6,130.6,130.1$, 129.6, 128.6, 128.5, 128.1, 127.4, 126.9, 119.9, 114.7, 114.6, 98.0, 70.0, 53.8.

${ }^{19}$ F NMR $\left\{{ }^{1} \mathrm{H}\right\}\left(471 \mathrm{MHz}, \mathrm{DMSO}-\mathrm{d}_{6}, 25^{\circ} \mathrm{C}, \delta\right):-148.2,-148.3$.

HRMS-ESI (m/z) calc'd. for $\mathrm{C}_{28} \mathrm{H}_{25} \mathrm{O}_{3} \mathrm{~S}_{2}{ }^{+}\left[\mathrm{M}-\mathrm{BF}_{4}\right]^{+}$, 473.123965; found, 473.123980; deviation: -0.03 ppm.

Aniline 4

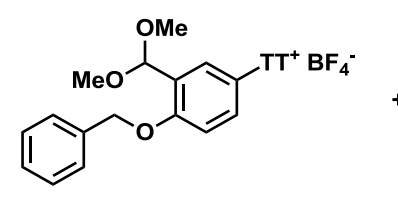

TT-4

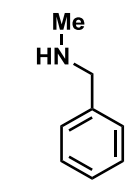

1

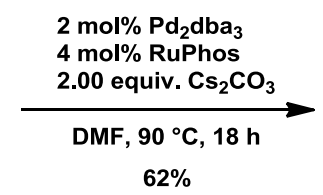

$62 \%$

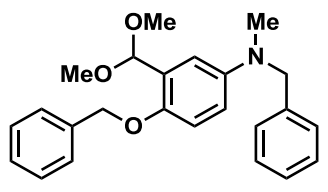

4

In an argon-filled glovebox, a $4 \mathrm{~mL}$ glass-vial equipped with a magnetic stir bar was charged with TT-4 (168 mg, 0.300 mmol, 1.00 equiv.), $\mathrm{Pd}_{2} \mathrm{dba}_{3}$ (5 mg, 5 umol, 2 mol\%), RuPhos (6 mg, $0.01 \mathrm{mmol}, 4 \mathrm{~mol} \%$ ), $\mathrm{Cs}_{2} \mathrm{CO}_{3}$ (195 mg, $0.598 \mathrm{mmol}, 2.00$ equiv.), and DMF (0.75 mL, c = $\left.0.40 \mathrm{M}\right)$. N-Methylbenzylamine $(58 \mu \mathrm{L}$, $54 \mathrm{mg}, 0.45 \mathrm{mmol}, 1.5$ equiv.) was added, and the sealed vial was taken out from the glovebox. The suspension was stirred at $90{ }^{\circ} \mathrm{C}$ for $18 \mathrm{~h}$. The suspension was added to a aqueous saturated $\mathrm{NaHCO}_{3}$ solution $(20 \mathrm{~mL})$, and the aqueous phase was extracted with DCM $(3 \times 20 \mathrm{~mL})$. The organic phase was dried over $\mathrm{MgSO}_{4}$, and the solvent was removed under reduced pressure. The residue was purified by chromatography on silica gel eluting with hexanes / EtOAc $(1: 0-7: 3(\mathrm{v} / \mathrm{v}))$ to afford $70 \mathrm{mg}(62 \%)$ of 4 as yellow oil.

$\mathbf{R} \boldsymbol{f}=0.50$ (hexanes / EtOAc, 7:3 (v/v)). 


\section{NMR Spectroscopy:}

${ }^{1}$ H NMR (500 MHz, $\left.\mathrm{CDCl}_{3}, 25^{\circ} \mathrm{C}, \delta\right): 7.44(\mathrm{~d}, J=7.1 \mathrm{~Hz}, 2 \mathrm{H}), 7.41-7.36(\mathrm{~m}, 2 \mathrm{H}), 7.34-7.28(\mathrm{~m}$, $3 \mathrm{H}), 7.26-7.21(\mathrm{~m}, 3 \mathrm{H}), 7.05(\mathrm{~d}, J=2.9 \mathrm{~Hz}, 1 \mathrm{H}), 6.86(\mathrm{~d}, J=8.9 \mathrm{~Hz}, 1 \mathrm{H}), 6.69(\mathrm{dd}, J=8.9,3.1$ $\mathrm{Hz}, 1 \mathrm{H}), 5.68(\mathrm{~s}, 1 \mathrm{H}), 5.04(\mathrm{~s}, 2 \mathrm{H}), 4.46(\mathrm{~s}, 2 \mathrm{H}), 3.37(\mathrm{~s}, 6 \mathrm{H}), 2.94(\mathrm{~s}, 3 \mathrm{H})$.

${ }^{13} \mathrm{C}$ NMR $\left\{{ }^{1} \mathrm{H}\right\}\left(126 \mathrm{MHz}, \mathrm{CDCl}_{3}, 25{ }^{\circ} \mathrm{C}, \delta\right): 148.6,145.0,139.3,137.9,128.6,128.5,127.8,127.4$, $127.3,126.9,114.7,114.4,112.3,100.0,71.6,57.8,54.3,39.1$.

HRMS-ESI (m/z) calc'd. for $\mathrm{C}_{24} \mathrm{H}_{28} \mathrm{NO}_{3}{ }^{+}[\mathrm{M}+\mathrm{H}]^{+}$, 378.206369; found, 378.206070; deviation: +0.79 ppm.

\section{Thianthrenium salt TT-5}
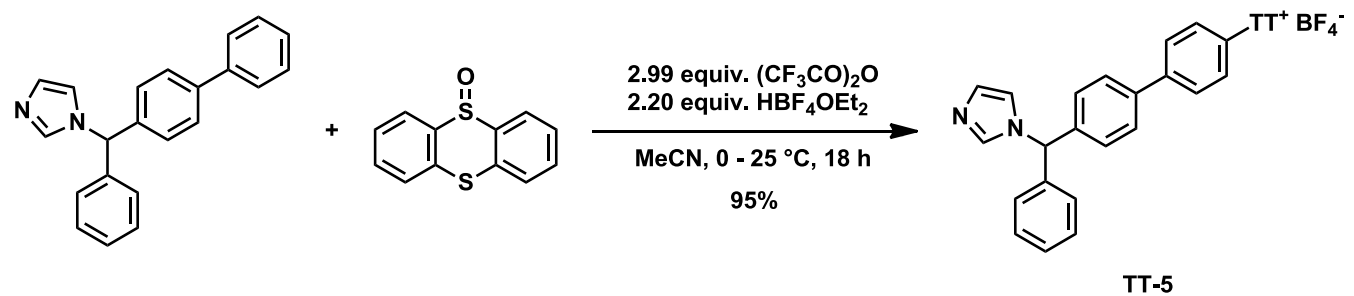

Under ambient atmosphere, a $100 \mathrm{~mL}$ Schlenk-tube equipped with a magnetic stir bar was charged with bifonazole ( $1.00 \mathrm{~g}, 3.22 \mathrm{mmol}, 1.00$ equiv.), thianthrene S-oxide (748 mg, $3.22 \mathrm{mmol}, 0.999$ equiv.), and $\operatorname{MeCN}(15 \mathrm{~mL}, \mathrm{C}=0.21 \mathrm{M})$. After cooling to $0{ }^{\circ} \mathrm{C}$, trifluoroacetic anhydride $(1.34 \mathrm{~mL}, 2.02 \mathrm{~g}, 9.64 \mathrm{mmol}$, 2.99 equiv.) was added while stirring. $\mathrm{HBF}_{4} \cdot \mathrm{OEt}_{2}(964 \mu \mathrm{l}, 1.15 \mathrm{~g}, 7.08 \mathrm{mmol}, 2.20$ equiv.) was added dropwise. The mixture was stirred and allowed to warm to ambient temperature over $18 \mathrm{~h}$. The reaction mixture was concentrated under reduced pressure, and subsequently diluted with DCM $(100 \mathrm{~mL})$. The solution was poured onto a saturated aqueous $\mathrm{NaHCO}_{3}$ solution $(100 \mathrm{~mL})$, and the layers were separated. The organic phase was washed with aqueous $\mathrm{NaBF}_{4}$ solution $(2 \times 100 \mathrm{~mL}, 10 \%)$, and with water (100 mL). The organic phase was dried over $\mathrm{MgSO}_{4}$, and the solvent was removed under reduced pressure. The residue was purified by chromatography on silica gel eluting with $\mathrm{DCM} / \mathrm{MeOH}(1: 0-10: 1(\mathrm{v} / \mathrm{v}))$ to afford $1.88 \mathrm{~g}(95 \%)$ of TT-5 as off-white foam.

$\mathbf{R}_{\boldsymbol{f}}=0.30(\mathrm{DCM} / \mathrm{MeOH}, 20: 1(\mathrm{v} / \mathrm{v}))$.

\section{NMR Spectroscopy:}

${ }^{1} \mathrm{H}$ NMR (500 MHz, DMSO-d $\left.6,25^{\circ} \mathrm{C}, \delta\right): 8.61$ (dd, $J=7.9,1.4 \mathrm{~Hz}, 2 \mathrm{H}$ ), 8.08 (dd, $J=7.9,1.4 \mathrm{~Hz}$, 2H), 7.94 (td, $J=7.7,1.5 \mathrm{~Hz}, 2 \mathrm{H}$ ), 7.88 (td, $J=7.7,1.4 \mathrm{~Hz}, 2 \mathrm{H}$ ), 7.84 (d, $J=8.8 \mathrm{~Hz}, 2 \mathrm{H}$ ), 7.68 (d, $J=8.1 \mathrm{~Hz}, 2 \mathrm{H}), 7.42-7.38(\mathrm{~m}, 2 \mathrm{H}), 7.38-7.33(\mathrm{~m}, 1 \mathrm{H}), 7.29(\mathrm{~d}, J=8.8 \mathrm{~Hz}, 2 \mathrm{H}), 7.24(\mathrm{~d}, J=8.1$ $\mathrm{Hz}, 2 \mathrm{H}), 7.18(\mathrm{~d}, J=7.0 \mathrm{~Hz}, 2 \mathrm{H}), 6.96(\mathrm{~s}, 1 \mathrm{H})$. The three protons of the imidazolyl group did not give detectable nmr signals.

${ }^{13} \mathrm{C}$ NMR $\left\{{ }^{1} \mathrm{H}\right\}\left(126 \mathrm{MHz}\right.$, DMSO-d $\left.6,25^{\circ} \mathrm{C}, \delta\right): 143.5,140.4,139.3,137.4,135.7,135.4,134.9$, $130.3,129.7,128.9,128.8,128.6,128.6,128.2,127.9,127.5,123.7,119.3,63.5$. The three carbon atoms of the imidazolyl group did not give detectable nmr signals. They are detectable when trifluoroacetic acid is added to the sample: 
${ }^{13} \mathbf{C}$ NMR $\left\{{ }^{1} \mathrm{H}\right\}\left(101 \mathrm{MHz}, 0.6 \mathrm{~mL} \mathrm{MeCN-d_{3 }}+1\right.$ drop of TFA, $\left.25^{\circ} \mathrm{C}, \delta\right): 145.3,139.9,138.3,137.7$, $137.3,136.3,136.2,136.1,131.7,131.0,130.5,130.4,130.0,129.9,129.7,129.3,129.1,124.0$, 123.0, 121.6, 119.5, 67.5 .

${ }^{19}$ F NMR $\left\{{ }^{1} \mathrm{H}\right\}\left(471 \mathrm{MHz}, \mathrm{DMSO}-\mathrm{d}_{6}, 25^{\circ} \mathrm{C}, \delta\right):-148.2,-148.2$.

HRMS-ESI (m/z) calc'd. for $\mathrm{C}_{34} \mathrm{H}_{25} \mathrm{~N}_{2} \mathrm{~S}_{2}{ }^{+}\left[\mathrm{M}-\mathrm{BF}_{4}\right]^{+}$, 525.145368; found, 525.145640; deviation: -0.52 ppm.

\section{Morpholine 5}
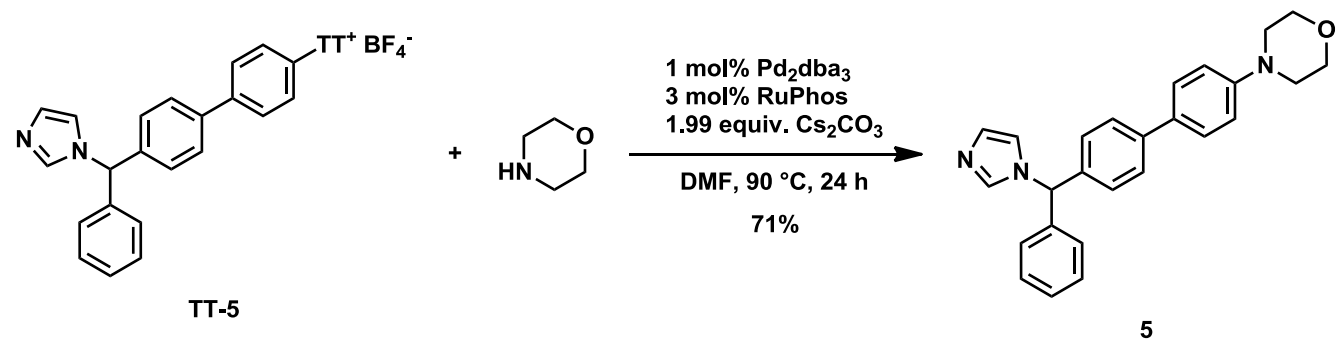

In an argon-filled glovebox, a $4 \mathrm{~mL}$ glass-vial equipped with a magnetic stir bar was charged with TT-5

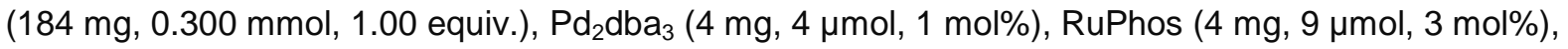
$\mathrm{Cs}_{2} \mathrm{CO}_{3}$ (195 mg, $0.598 \mathrm{mmol}, 1.99$ equiv.), and DMF (2.5 mL, c = $0.12 \mathrm{M}$ ). Morpholine (39 $\mu \mathrm{L}, 39 \mathrm{mg}$, $0.45 \mathrm{mmol}, 1.5$ equiv.) was added, and the sealed vial was taken out from the glovebox. The suspension was stirred at $90^{\circ} \mathrm{C}$ for $24 \mathrm{~h}$. The solvent was removed under reduced pressure, and the residue was purified by chromatography on silica gel eluting with $\mathrm{DCM} / \mathrm{MeOH}(1: 0-19: 1(\mathrm{v} / \mathrm{v}))$ to afford $85 \mathrm{mg}(71 \%)$ of 5 as orange oil.

$\mathbf{R}_{\boldsymbol{f}}=0.30(\mathrm{DCM} / \mathrm{MeOH}, 95: 5(\mathrm{v} / \mathrm{v}))$.

\section{NMR Spectroscopy:}

${ }^{1} \mathrm{H}$ NMR $\left(500 \mathrm{MHz}, \mathrm{CDCl}_{3}, 25^{\circ} \mathrm{C}, \delta\right): 7.55-7.51(\mathrm{~m}, 4 \mathrm{H}), 7.46(\mathrm{~s}, 1 \mathrm{H}), 7.39-7.35(\mathrm{~m}, 3 \mathrm{H}), 7.15$ - $7.12(\mathrm{~m}, 5 \mathrm{H}), 6.99-6.97(\mathrm{~m}, 2 \mathrm{H}), 6.89(\mathrm{~s}, 1 \mathrm{H}), 6.54(\mathrm{~s}, 1 \mathrm{H}), 3.89-3.87(\mathrm{~m}, 4 \mathrm{H}), 3.22-3.20(\mathrm{~m}$, $4 \mathrm{H})$.

${ }^{13} \mathrm{C}$ NMR $\left\{{ }^{1} \mathrm{H}\right\}\left(126 \mathrm{MHz}, \mathrm{CDCl}_{3}, 25{ }^{\circ} \mathrm{C}, \delta\right): 150.9,141.0,139.4,137.3,131.6,129.0,128.6,128.5$, $128.2,127.9,127.0,115.8,67.0,65.0,49.2$.

HRMS-ESI (m/z) calc'd. for $\mathrm{C}_{26} \mathrm{H}_{26} \mathrm{~N}_{3} \mathrm{O}^{+}[\mathrm{M}+\mathrm{H}]^{+}$, 396.207036; found, 396.207200; deviation: -0.41 ppm.

\section{Thianthrenium salt TT-6}
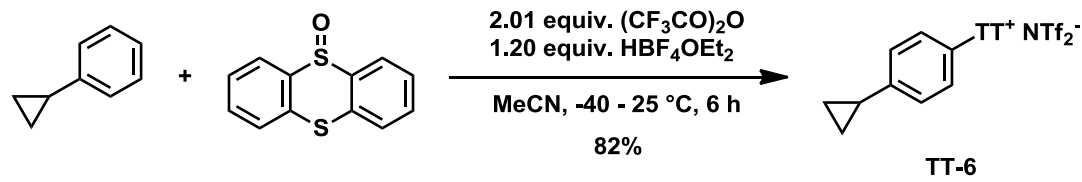

Under ambient atmosphere, a $100 \mathrm{~mL}$ Schlenk-tube equipped with a magnetic stir bar was charged with cyclopropylbenzene (532 $\mu \mathrm{l}, 500 \mathrm{mg}, 4.23 \mathrm{mmol}, 1.00$ equiv.), thianthrene S-oxide (983 mg, $4.23 \mathrm{mmol}$, 
1.00 equiv.), and $\mathrm{MeCN}(15 \mathrm{~mL}, \mathrm{C}=0.28 \mathrm{M})$. After cooling to $-40^{\circ} \mathrm{C}$, trifluoroacetic anhydride $(1.18 \mathrm{~mL}$, $1.78 \mathrm{~g}, 8.49 \mathrm{mmol}, 2.01$ equiv.) was added while stirring. $\mathrm{HBF}_{4} \cdot \mathrm{OEt}_{2}(691 \mu \mathrm{l}, 822 \mathrm{mg}, 5.08 \mathrm{mmol}, 1.20$ equiv.) was added dropwise over $1 \mathrm{~min}$. The mixture was stirred at $-40^{\circ} \mathrm{C}$ for $4 \mathrm{~h}$, then at ambient temperature for $1 \mathrm{~h}$. The reaction mixture was concentrated under reduced pressure, and subsequently diluted with DCM $(100 \mathrm{~mL})$. The solution was poured onto a saturated aqueous $\mathrm{NaHCO}_{3}$ solution $(100 \mathrm{~mL})$, and the layers were separated. The organic phase was washed with aqueous $\mathrm{LiNtf}_{2}$ solution $(2 \times 100 \mathrm{~mL}, 10 \%)$, and with water $(100 \mathrm{~mL})$. The organic phase was dried over $\mathrm{MgSO}_{4}$, and the solvent was removed under reduced pressure. The residue was purified by chromatography on silica gel eluting with DCM / MeOH (1:0-20:1 (v/v)) to afford $1.94 \mathrm{~g}(82 \%)$ of TT-6 as yellow oil.

$\mathbf{R}_{\boldsymbol{f}}=0.70(\mathrm{DCM} / \mathrm{MeOH}, 20: 1(\mathrm{v} / \mathrm{v}))$.

\section{NMR Spectroscopy:}

${ }^{1} \mathrm{H}$ NMR $\left(500 \mathrm{MHz}, \mathrm{CDCl}_{3}, 25^{\circ} \mathrm{C}, \delta\right): 8.50-8.40(\mathrm{~m}, 2 \mathrm{H}), 7.87-7.73(\mathrm{~m}, 6 \mathrm{H}), 7.10(\mathrm{~d}, J=8.8 \mathrm{~Hz}$, 2H), $7.07-7.01(\mathrm{~m}, 2 \mathrm{H}), 1.86(\mathrm{tt}, J=8.4,4.9 \mathrm{~Hz}, 1 \mathrm{H}), 1.09-1.02(\mathrm{~m}, 2 \mathrm{H}), 0.73-0.67(\mathrm{~m}, 2 \mathrm{H})$.

${ }^{13} \mathrm{C}$ NMR $\left\{{ }^{1} \mathrm{H}\right\}\left(126 \mathrm{MHz}, \mathrm{CDCl}_{3}, 25{ }^{\circ} \mathrm{C}, \delta\right): 151.6,136.6,135.0,134.9,130.5,130.4,128.1,128.0$, 120.1 (q, $J=321.6 \mathrm{~Hz}), 119.1,118.9,15.7,11.1$.

${ }^{19} \mathrm{~F}$ NMR $\left\{{ }^{1} \mathrm{H}\right\}\left(471 \mathrm{MHz}, \mathrm{CDCl}_{3}, 25^{\circ} \mathrm{C}, \delta\right):-78.6$.

HRMS-ESI (m/z) calc'd. for $\mathrm{C}_{21} \mathrm{H}_{17} \mathrm{~S}_{2}^{+}\left[\mathrm{M}-\mathrm{NTf}_{2}\right]^{+}$, 333.076740; found, 333.076620; deviation: -0.4 ppm.

Isonicotinamide 6

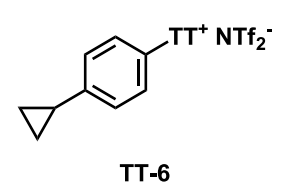

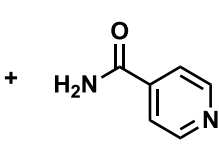

TT-6

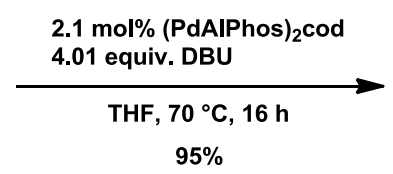

$95 \%$

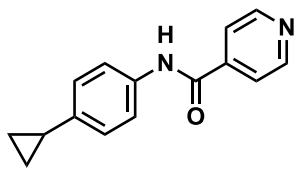

6

In an argon-filled glovebox, a $4 \mathrm{~mL}$ glass-vial equipped with a magnetic stir bar was charged with TT-6 (184 mg, $0.300 \mathrm{mmol}, 1.00$ equiv.), (PdAlphos) $)_{2} \operatorname{cod}(12 \mathrm{mg}, 6.2 \mu \mathrm{mol}, 2.1 \mathrm{~mol} \%$ ), and THF (0.5 mL, $\mathrm{c}=$ $0.6 \mathrm{M}$ ). Isonicotinamide (55 mg, $0.45 \mathrm{mmol}, 1.5$ equiv.) and DBU (180 $\mu \mathrm{L}, 183 \mathrm{mg}, 1.20 \mathrm{mmol}, 4.01$ equiv.) were added, and the sealed vial was taken out from the glovebox. The solution was stirred at $70{ }^{\circ} \mathrm{C}$ for $16 \mathrm{~h}$. The solvent was removed under reduced pressure, and the residue was purified by chromatography on silica gel eluting with hexanes / EtOAc (1:0-2:8 (v/v)) to afford $68 \mathrm{mg}(95 \%)$ of 6 as white solid.

$\mathbf{R} \boldsymbol{f}=0.23$ (hexanes / EtOAc, 15:85 (v/v)).

\section{NMR Spectroscopy:}

${ }^{1} \mathrm{H}$ NMR $\left(500 \mathrm{MHz}, \mathrm{DMSO}-\mathrm{d}_{6}, 25^{\circ} \mathrm{C}, \delta\right): 10.41(\mathrm{~s}, 1 \mathrm{H}), 8.78-8.77(\mathrm{~m}, 2 \mathrm{H}), 7.85-7.84(\mathrm{~m}, 2 \mathrm{H})$, $7.65-7.63(\mathrm{~m}, 2 \mathrm{H}), 7.08-7.06(\mathrm{~m}, 2 \mathrm{H}), 1.89(\mathrm{tt}, J=8.4,5.0 \mathrm{~Hz}, 1 \mathrm{H}), 0.94-0.90(\mathrm{~m}, 2 \mathrm{H}), 0.66-$ $0.63(\mathrm{~m}, 2 \mathrm{H})$.

${ }^{13} \mathrm{C}$ NMR $\left\{{ }^{1} \mathrm{H}\right\}\left(126 \mathrm{MHz}, \mathrm{DMSO}-\mathrm{d}_{6}, 25^{\circ} \mathrm{C}, \delta\right): 163.7,150.3,142.0,139.6,136.0,125.6,121.6$, 
120.6, 14.7, 9.3.

HRMS-ESI (m/z) calc'd. for $\mathrm{C}_{15} \mathrm{H}_{15} \mathrm{~N}_{2} \mathrm{O}^{+}[\mathrm{M}+\mathrm{H}]^{+}$, 239.117888; found, 239.117940; deviation: -0.22 ppm.

Thianthrenium salt TT-7

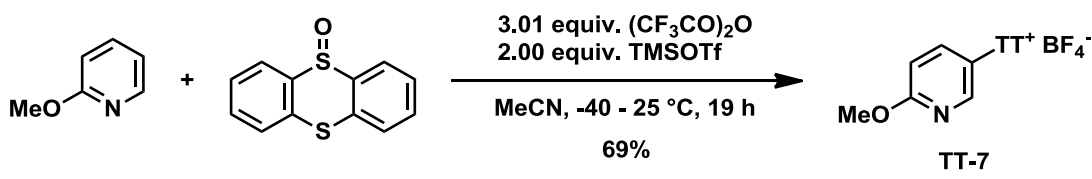

A flame-dried, $100 \mathrm{~mL}$ argon-filled Schlenk-tube equipped with a magnetic stir bar was charged with 2-methoxypyridine ( $525 \mu \mathrm{L}, 546 \mathrm{mg}, 5.00 \mathrm{mmol}, 1.00$ equiv.), thianthrene S-oxide (1.16 g, $4.99 \mathrm{mmol}$, 0.998 equiv.), and dry $\mathrm{MeCN}(20 \mathrm{~mL}, \mathrm{C}=0.25 \mathrm{M})$. After cooling to $-40^{\circ} \mathrm{C}$, trifluoroacetic anhydride $(2.09 \mathrm{~mL}$, $3.16 \mathrm{~g}, 15.0 \mathrm{mmol}, 3.01$ equiv.) was added while stirring. A solution of trimethylsilyl trifluoromethanesulfonate ( $1.81 \mathrm{~mL}, 2.22 \mathrm{~g}, 10.0 \mathrm{mmol}, 2.00$ equiv.) in $5 \mathrm{~mL}$ of dry MeCN was added dropwise over $5 \mathrm{~min}$. The mixture was stirred at $-40{ }^{\circ} \mathrm{C}$ for $1 \mathrm{~h}$, then at ambient temperature for $18 \mathrm{~h}$. The reaction mixture was concentrated under reduced pressure, and subsequently diluted with DCM $(100 \mathrm{~mL})$. The solution was washed with aqueous $\mathrm{NaBF}_{4}$ solution $(3 \times 100 \mathrm{~mL}, 10 \%)$, and with water $(100 \mathrm{~mL})$. The organic phase was dried over $\mathrm{MgSO}_{4}$, and the solvent was removed under reduced pressure. The residue was purified by chromatography on silica gel eluting with DCM / MeOH (1:0-20:1 (v/v)) to afford $1.42 \mathrm{~g}(69 \%)$ of TT-7 as yellow foam.

$\mathbf{R}_{\boldsymbol{f}}=0.18(\mathrm{DCM} / \mathrm{MeOH}, 10: 1(\mathrm{v} / \mathrm{v}))$.

\section{NMR Spectroscopy:}

${ }^{1} \mathrm{H}$ NMR $\left(500 \mathrm{MHz}, \mathrm{CDCl}_{3}, 25^{\circ} \mathrm{C}, \delta\right): 8.53(\mathrm{dd}, J=7.9,1.1 \mathrm{~Hz}, 2 \mathrm{H}), 7.93(\mathrm{~d}, J=2.8 \mathrm{~Hz}, 1 \mathrm{H}), 7.88$ - $7.78(\mathrm{~m}, 4 \mathrm{H}), 7.78-7.71(\mathrm{~m}, 2 \mathrm{H}), 7.61(\mathrm{dd}, J=9.1,2.8 \mathrm{~Hz}, 1 \mathrm{H}), 6.79(\mathrm{~d}, J=9.1 \mathrm{~Hz}, 1 \mathrm{H}), 3.88$ $(\mathrm{s}, 3 \mathrm{H})$.

${ }^{13} \mathrm{C}$ NMR $\left\{{ }^{1} \mathrm{H}\right\}\left(126 \mathrm{MHz}, \mathrm{CDCl}_{3}, 25^{\circ} \mathrm{C}, \delta\right): 166.9,147.6,138.4,136.4,135.1,135.1,130.6,130.4$, $118.5,133.9,112.8,54.7$.

${ }^{19}$ F NMR $\left\{{ }^{1} \mathrm{H}\right\}\left(471 \mathrm{MHz}, \mathrm{CDCl}_{3}, 25^{\circ} \mathrm{C}, \delta\right):-150.0,-150.0$.

HRMS-ESI (m/z) calc'd. for $\mathrm{C}_{18} \mathrm{H}_{14} \mathrm{NOS}_{2}{ }^{+}\left[\mathrm{M}-\mathrm{BF}_{4}\right]^{+}$, 324.051134; found, 324.050980; deviation: +0.48 ppm.

\section{Oxazolidinone 7}

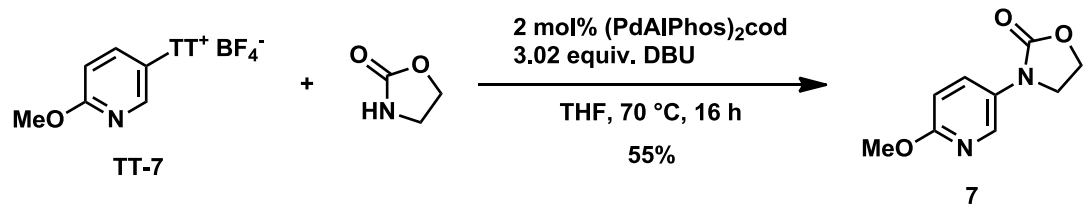

In an argon-filled glovebox, a $4 \mathrm{~mL}$ glass-vial equipped with a magnetic stir bar was charged with TT-7 (123 mg, $0.299 \mathrm{mmol}, 1.00$ equiv.), (PdAlphos) ${ }_{2} \operatorname{cod}(9 \mathrm{mg}, 4 \mu \mathrm{mol}, 2 \mathrm{~mol} \%$ ), and THF ( $1 \mathrm{~mL}, \mathrm{C}=0.3 \mathrm{M}$ ). 2-Oxazolidinone (52 mg, $0.60 \mathrm{mmol}, 2.0$ equiv.) and DBU (135 $\mu \mathrm{L}, 137 \mathrm{mg}, 0.903 \mathrm{mmol}, 3.02$ equiv.) were added, and the sealed vial was taken out from the glovebox. The solution was stirred at $70{ }^{\circ} \mathrm{C}$ for $16 \mathrm{~h}$. The 
solvent was removed under reduced pressure, and the residue was purified by chromatography on silica gel eluting with hexanes / EtOAc (1:0-19:1 (v/v)) to afford $32 \mathrm{mg}(55 \%)$ of 7 as white solid.

$\mathbf{R} \boldsymbol{f}=0.49$ (hexanes / EtOAc, 95:5 (v/v)).

\section{NMR Spectroscopy:}

${ }^{1} \mathrm{H}$ NMR $\left(500 \mathrm{MHz}, \mathrm{CDCl}_{3}, 25^{\circ} \mathrm{C}, \delta\right): 8.06-8.03(\mathrm{~m}, 2 \mathrm{H}), 6.76-6.75(\mathrm{~m}, 2 \mathrm{H}), 4.48(\mathrm{t}, \mathrm{J}=7.6 \mathrm{~Hz}$, $2 \mathrm{H}), 4.02$ (t, $J=7.6 \mathrm{~Hz}, 2 \mathrm{H}), 3.90(\mathrm{~s}, 3 \mathrm{H})$.

${ }^{13} \mathrm{C}$ NMR $\left\{{ }^{1} \mathrm{H}\right\}\left(126 \mathrm{MHz}, \mathrm{CDCl}_{3}, 25{ }^{\circ} \mathrm{C}, \delta\right): 161.0,155.6,136.5,130.6,129.4,111.0,61.7,53.7$, 45.3.

HRMS-ESI (m/z) calc'd. for $\mathrm{C}_{9} \mathrm{H}_{11} \mathrm{~N}_{2} \mathrm{O}_{3}{ }^{+}[\mathrm{M}+\mathrm{H}]^{+}$, 195.076417; found, 195.076640; deviation: -1.14 ppm.

Thianthrenium salt TT-8
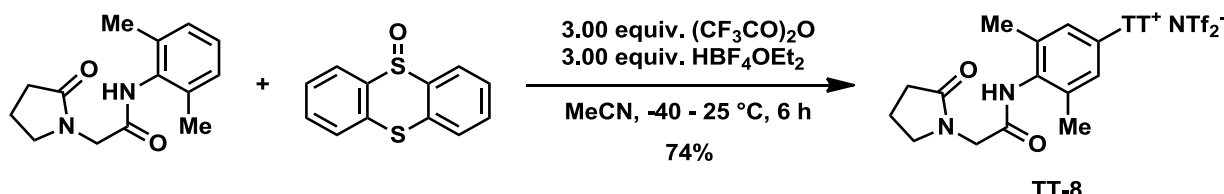

Under ambient atmosphere, a $100 \mathrm{~mL}$ Schlenk-tube equipped with a magnetic stir bar was charged with nefiracetam (550 mg, $2.23 \mathrm{mmol}, 1.00$ equiv.), thianthrene S-oxide (519 mg, $2.23 \mathrm{mmol}, 1.00$ equiv.), and $\mathrm{MeCN}(10 \mathrm{~mL}, \mathrm{C}=0.22 \mathrm{M})$. After cooling to $-40^{\circ} \mathrm{C}$, trifluoroacetic anhydride $(931 \mu \mathrm{L}, 1.41 \mathrm{~g}, 6.70 \mathrm{mmol}$, 3.00 equiv.) was added while stirring. $\mathrm{HBF}_{4} \cdot \mathrm{OEt}_{2}(912 \mu \mathrm{l}, 1.09 \mathrm{~g}, 6.70 \mathrm{mmol}, 3.00$ equiv.) was added dropwise. The mixture was stirred at $-40{ }^{\circ} \mathrm{C}$ for $1 \mathrm{~h}$, then at ambient temperature for $5 \mathrm{~h}$. The reaction mixture was concentrated under reduced pressure, and subsequently diluted with DCM (100 mL). The solution was poured onto a saturated aqueous $\mathrm{NaHCO}_{3}$ solution $(100 \mathrm{~mL})$, and the layers were separated. The organic phase was washed with aqueous $\mathrm{LiNtf}_{2}$ solution $(2 \times 100 \mathrm{~mL}, 10 \%)$, and with water $(100 \mathrm{~mL})$. The organic phase was dried over $\mathrm{MgSO}_{4}$, and the solvent was removed under reduced pressure. The residue was purified by chromatography on silica gel eluting with $\mathrm{DCM} / \mathrm{MeOH}(1: 0-10: 1(\mathrm{v} / \mathrm{v}))$ to afford $1.22 \mathrm{~g}(74 \%)$ of TT-8 as colorless foam.

$\mathbf{R}_{\boldsymbol{f}}=0.10(\mathrm{DCM} / \mathrm{MeOH}, 10: 1(\mathrm{v} / \mathrm{v}))$.

\section{NMR Spectroscopy:}

${ }^{1} \mathrm{H}$ NMR $\left(400 \mathrm{MHz}, \mathrm{CDCl}_{3}, 25^{\circ} \mathrm{C}, \delta\right): 8.36-8.28(\mathrm{~m}, 3 \mathrm{H}), 7.90-7.79(\mathrm{~m}, 4 \mathrm{H}), 7.76(\mathrm{td}, J=7.5$, $1.7 \mathrm{~Hz}, 2 \mathrm{H}), 6.86(\mathrm{~s}, 2 \mathrm{H}), 4.07(\mathrm{~s}, 2 \mathrm{H}), 3.52(\mathrm{t}, J=7.1 \mathrm{~Hz}, 2 \mathrm{H}), 2.39(\mathrm{t}, J=8.1 \mathrm{~Hz}, 2 \mathrm{H}), 2.12$ (s, $6 \mathrm{H}), 2.11-2.03(\mathrm{~m}, 2 \mathrm{H})$.

${ }^{13} \mathrm{C}$ NMR $\left\{{ }^{1} \mathrm{H}\right\}\left(101 \mathrm{MHz}, \mathrm{CDCl}_{3}, 25{ }^{\circ} \mathrm{C}, \delta\right): 176.7,167.1,139.7,139.6,136.6,135.1,134.6,130.6$, 130.4, 127.5, 120.5, 119.9 (q, $J=321.5 \mathrm{~Hz}$ ), 118.8, 48.7, 47.0, 30.4, 18.9, 18.0.

${ }^{19} \mathrm{~F}$ NMR $\left\{{ }^{1} \mathrm{H}\right\}\left(471 \mathrm{MHz}, \mathrm{CDCl}_{3}, 25^{\circ} \mathrm{C}, \delta\right):-78.7$.

HRMS-ESI (m/z) calc'd. for $\mathrm{C}_{26} \mathrm{H}_{25} \mathrm{~N}_{2} \mathrm{O}_{2} \mathrm{~S}_{2}^{+}\left[\mathrm{M}-\mathrm{NTf}_{2}\right]^{+}, 461.135198$; found, 461.135260; deviation: -0.14 ppm. 
Aminopyridine 8a

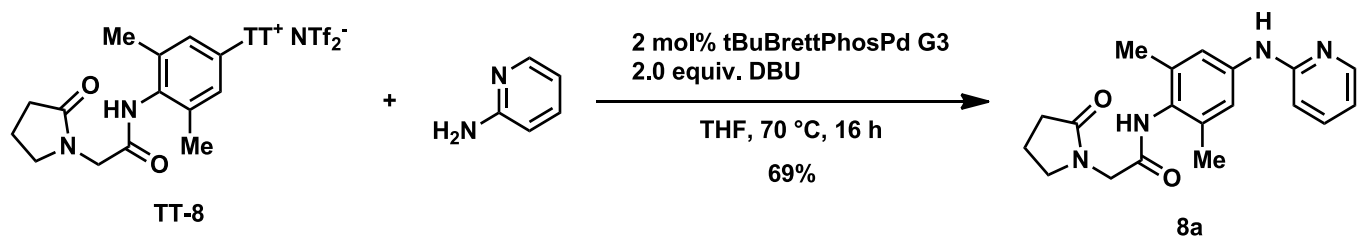

In an argon-filled glovebox, a $4 \mathrm{~mL}$ glass-vial equipped with a magnetic stir bar was charged with TT-8 (120 mg, 0.162 mmol, 1.00 equiv.), tBuBrettPhosPd G3 (3 mg, $4 \mu \mathrm{mol}, 2 \mathrm{~mol} \%)$, and THF (0.5 mL, $\mathrm{c}=0.3 \mathrm{M})$. 2-Aminopyridine (23 mg, $0.24 \mathrm{mmol}, 1.5$ equiv.) and DBU (48 $\mu \mathrm{L}, 49 \mathrm{mg}, 0.32 \mathrm{mmol}, 2.0$ equiv.) were added, and the sealed vial was taken out from the glovebox. The solution was stirred at $70^{\circ} \mathrm{C}$ for $16 \mathrm{~h}$. The solvent was removed under reduced pressure, and the residue was purified by chromatography on silica gel eluting with $\mathrm{DCM} / \mathrm{MeOH}(1: 0-9: 1(\mathrm{v} / \mathrm{v}))$ to afford $38 \mathrm{mg}(69 \%)$ of $8 \mathrm{a}$ as white solid.

$\mathbf{R}_{\boldsymbol{f}}=0.47(\mathrm{DCM} / \mathrm{MeOH}, 9: 1(\mathrm{v} / \mathrm{v}))$.

\section{NMR Spectroscopy:}

${ }^{1} \mathrm{H}$ NMR $\left(500 \mathrm{MHz}, \mathrm{DMSO}-\mathrm{d}_{6}, 25^{\circ} \mathrm{C}, \delta\right): 9.12(\mathrm{~s}, 1 \mathrm{H}), 8.87(\mathrm{~s}, 1 \mathrm{H}), 8.15-8.13(\mathrm{~m}, 1 \mathrm{H}), 7.55-$ $7.51(\mathrm{~m}, 1 \mathrm{H}), 7.37(\mathrm{~s}, 1 \mathrm{H}), 6.81-6.79(\mathrm{~m}, 1 \mathrm{H}), 6.72-6.70(\mathrm{~m}, 1 \mathrm{H}), 4.00(\mathrm{~s}, 2 \mathrm{H}), 3.46(\mathrm{t}, J=7.1$ Hz. 2H), 2.27 (t, $J=8.1 \mathrm{~Hz}, 2 \mathrm{H}), 1.98(\mathrm{p}, J=7.5 \mathrm{~Hz}, 2 \mathrm{H})$.

${ }^{13} \mathrm{C}$ NMR $\left\{{ }^{1} \mathrm{H}\right\}\left(101 \mathrm{MHz}, \mathrm{DMSO}-\mathrm{d}_{6}, 25^{\circ} \mathrm{C}, \delta\right): 174.7,166.6,156.0,147.3,140.0,137.2,135.3$, $127.5,117.1,114.1,110.6,47.4,45.2,30.0,18.4,17.4$.

HRMS-ESI (m/z) calc'd. for $\mathrm{C}_{19} \mathrm{H}_{23} \mathrm{~N}_{4} \mathrm{O}_{2}{ }^{+}[\mathrm{M}+\mathrm{H}]^{+}$, 339.181550; found, 339.181420; deviation: +0.38 ppm.

Phthalimide 8b
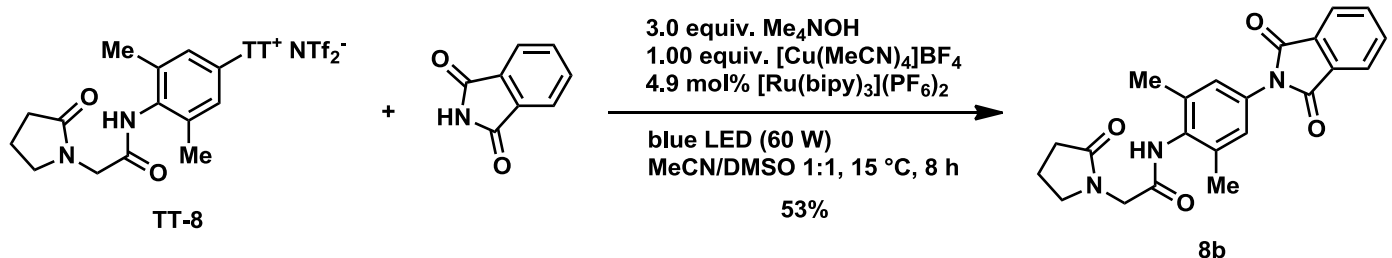

Under ambient atmosphere, a $4 \mathrm{~mL}$ glass-vial was charged with phthalimide (177 $\mathrm{mg}, 1.20 \mathrm{mmol}$, 3.00 equiv.) and tetramethylammonium hydroxide (25\% in methanol, $438 \mathrm{mg}, 0.51 \mathrm{~mL}, 1.2 \mathrm{mmol}, 3.0$ equiv.), and the solvent was removed under reduced pressure. Thianthrenium salt TT-8 (297 mg, $0.400 \mathrm{mmol}$, 1.00 equiv.), $\left[\mathrm{Cu}\left(\mathrm{MeCN}_{4}\right]_{\mathrm{BF}}\right.$ (126 mg, $0.401 \mathrm{mmol}, 1.00$ equiv.), [Ru(bipy) $\left.{ }_{3}\right]\left(\mathrm{PF}_{6}\right)_{2}(17 \mathrm{mg}, 0.020 \mathrm{mmol}$, $4.9 \mathrm{~mol} \%)$, acetonitrile $(1 \mathrm{~mL})$ and dimethylsulfoxide $\left(1 \mathrm{~mL}, \mathrm{c}_{\text {total }}=0.20 \mathrm{M}\right)$ were added, and the suspension was degassed by bubbling through argon with a cannula for $5 \mathrm{~min}$. While stirring, the suspension was irradiated for $8 \mathrm{~h}$ at $15^{\circ} \mathrm{C}$ using a blue LED $(60 \mathrm{~W})$. The suspension was added to water $(30 \mathrm{~mL})$, and the aqueous phase was extracted with DCM $(3 \times 20 \mathrm{~mL})$. The organic phase was dried over $\mathrm{MgSO}_{4}$, and the solvent was removed under reduced pressure. The residue was purified by chromatography on silica gel eluting with $\mathrm{DCM} / \mathrm{MeOH}(1: 0-20: 1(\mathrm{v} / \mathrm{v}))$ to afford $83 \mathrm{mg}(53 \%)$ of $8 \mathrm{~b}$ as pink solid. 
$\mathbf{R}_{\boldsymbol{f}}=0.39(\mathrm{DCM} / \mathrm{MeOH}, 95: 5(\mathrm{v} / \mathrm{v}))$.

\section{NMR Spectroscopy:}

${ }^{1} \mathrm{H}$ NMR $\left(500 \mathrm{MHz}, \mathrm{DMSO}_{-} \mathrm{d}_{6}, 25^{\circ} \mathrm{C}, \delta\right): 9.44(\mathrm{~s}, 1 \mathrm{H}), 7.97-7.94(\mathrm{~m}, 2 \mathrm{H}), 7.92-7.90(\mathrm{~m}, 2 \mathrm{H})$, $7.16(\mathrm{~s}, 2 \mathrm{H}), 4.08(\mathrm{~s}, 2 \mathrm{H}), 3.48(\mathrm{t}, J=7.0 \mathrm{~Hz}, 2 \mathrm{H}), 2.29$ (t, $J=8.1 \mathrm{~Hz}, 2 \mathrm{H}), 2.18(\mathrm{~s}, 6 \mathrm{H}), 2.03-$ $1.96(\mathrm{~m}, 2 \mathrm{H})$.

${ }^{13} \mathrm{C}$ NMR $\left\{{ }^{1} \mathrm{H}\right\}\left(126 \mathrm{MHz}\right.$, DMSO-d $\left.6,25^{\circ} \mathrm{C}, \delta\right): 174.6,167.1,166.6,136.0,134.7,131.5,129.9$, 126.6, 123.4, 47.4, 45.2, 30.0, 18.1, 17.5 .

HRMS-ESI (m/z) calc'd. for $\mathrm{C}_{22} \mathrm{H}_{21} \mathrm{~N}_{3} \mathrm{O}_{4} \mathrm{Na}^{+}[\mathrm{M}+\mathrm{Na}]^{+}$, 414.142425; found, 414.142450; deviation: -0.06 ppm.

Aniline 9
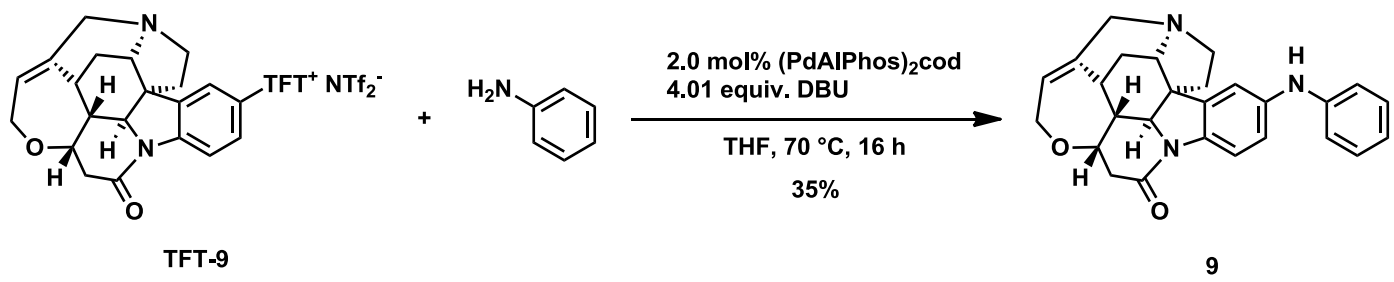

In an argon-filled glovebox, a $4 \mathrm{~mL}$ glass-vial equipped with a magnetic stir bar was charged with TFT-9 (271 mg, $0.300 \mathrm{mmol}, 1.00$ equiv.), (PdAlphos) ${ }_{2} \operatorname{cod}(12 \mathrm{mg}, 6.2 \mu \mathrm{mol}, 2.0 \mathrm{~mol} \%$ ), and THF ( $1 \mathrm{~mL}, \mathrm{c}=0.3 \mathrm{M}$ ). Aniline ( $82 \mu \mathrm{L}, 84 \mathrm{mg}, 0.90 \mathrm{mmol}, 3.0$ equiv.) and DBU (180 $\mu \mathrm{L}, 183 \mathrm{mg}, 1.20 \mathrm{mmol}, 4.01$ equiv.) were added, and the sealed vial was taken out from the glovebox. The solution was stirred at $70{ }^{\circ} \mathrm{C}$ for $16 \mathrm{~h}$. The solvent was removed under reduced pressure, and the residue was purified by chromatography on silica gel eluting with DCM / MeOH (1:0-9:1 (v/v)) to afford $45 \mathrm{mg}(35 \%)$ of 9 as white solid.

$\mathbf{R}_{\boldsymbol{f}}=0.21(\mathrm{DCM} / \mathrm{MeOH}, 95: 5(\mathrm{v} / \mathrm{v}))$.

\section{NMR Spectroscopy:}

${ }^{1} \mathrm{H}$ NMR $\left(500 \mathrm{MHz}, \mathrm{CDCl}_{3}, 25^{\circ} \mathrm{C}, \delta\right): 7.98-7.97(\mathrm{~m}, 1 \mathrm{H}), 7.24-7.21(\mathrm{~m}, 2 \mathrm{H}), 7.05-7.03(\mathrm{~m}$, $1 \mathrm{H}), 6.99-6.97(\mathrm{~m}, 2 \mathrm{H}), 6.92-6.92(\mathrm{~m}, 1 \mathrm{H}), 6.90-6.87(\mathrm{~m}, 1 \mathrm{H}), 6.09(\mathrm{br} \mathrm{s}, 1 \mathrm{H}), 5.87(\mathrm{~s}, 1 \mathrm{H})$, $4.29-4.27(\mathrm{~m}, 1 \mathrm{H}), 4.20-4.16(\mathrm{~m}, 2 \mathrm{H}), 4.07-4.03(\mathrm{~m}, 2 \mathrm{H}), 3.55-3.52(\mathrm{~m}, 1 \mathrm{H}), 3.21(\mathrm{~s}, 1 \mathrm{H})$, $3.14-3.09(\mathrm{~m}, 1 \mathrm{H}), 3.05-2.99(\mathrm{~m}, 2 \mathrm{H}), 2.67-2.63(\mathrm{~m}, 1 \mathrm{H}), 2.43-2.38(\mathrm{~m}, 1 \mathrm{H}), 2.02-1.99(\mathrm{~m}$, $2 \mathrm{H}), 1.55(\mathrm{~d}, J=14.7 \mathrm{~Hz}, 1 \mathrm{H}), 1.30-1.28(\mathrm{~m}, 1 \mathrm{H})$.

${ }^{13} \mathrm{C}$ NMR $\left\{{ }^{1} \mathrm{H}\right\}\left(126 \mathrm{MHz}, \mathrm{CDCl}_{3}, 25^{\circ} \mathrm{C}, \delta\right): 168.6,143.5,140.3,136.8,136.4,132.2,131.5,129.5$, $120.8,119.1,117.2,117.2,112.6,77.67,64.5,61.1,59.9,52.8,52.2,50.8,47.8,42.3,42.0,31.2$, 26.2.

HRMS-ESI (m/z) calc'd. for $\mathrm{C}_{27} \mathrm{H}_{28} \mathrm{~N}_{3} \mathrm{O}_{2}{ }^{+}[\mathrm{M}+\mathrm{H}]^{+}$, 426.217601; found, 426.217320; deviation: +0.66 ppm. 
Thianthrenium salt TT-10

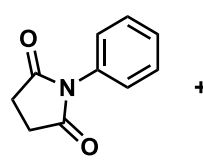<smiles>O=S1c2ccccc2Sc2ccccc21</smiles>
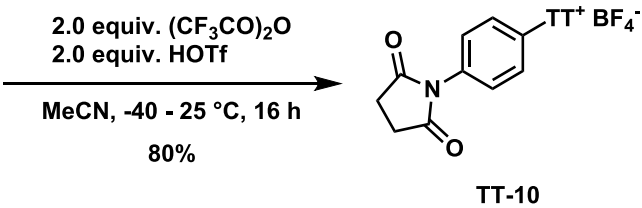

Under ambient atmosphere, a $100 \mathrm{~mL}$ Schlenk-tube equipped with a magnetic stir bar was charged with $\mathrm{N}$-phenyl succinimide (452 mg, $2.58 \mathrm{mmol}, 1.00$ equiv.), thianthrene S-oxide (900 mg, $3.87 \mathrm{mmol}$,

1.50 equiv.), and $\mathrm{MeCN}(12 \mathrm{~mL}, \mathrm{C}=0.22 \mathrm{M})$. After cooling to $-40^{\circ} \mathrm{C}$, trifluoroacetic anhydride $(0.72 \mathrm{~mL}, 1.1 \mathrm{~g}$, $5.2 \mathrm{mmol}, 2.0$ equiv.) was added while stirring. HOTf ( $0.46 \mathrm{~mL}, 0.78 \mathrm{~g}, 5.2 \mathrm{mmol}, 2.0$ equiv.) was added in one portion. The mixture was stirred at $-40^{\circ} \mathrm{C}$ for $2 \mathrm{~h}$, then at ambient temperature for $14 \mathrm{~h}$. The reaction mixture was concentrated under reduced pressure, and subsequently diluted with DCM $(50 \mathrm{~mL})$. The solution was washed with aqueous $\mathrm{NaBF}_{4}$ solution $(3 \times 50 \mathrm{~mL}, 10 \%)$, and with water $(50 \mathrm{~mL})$. The organic phase was dried over $\mathrm{MgSO}_{4}$, and the solvent was removed under reduced pressure. The residue was purified by chromatography on silica gel eluting with DCM / MeOH (1:0-10:1 (v/v)) to afford $989 \mathrm{mg}(80 \%)$ of TT-10 as colorless foam.

$\mathbf{R}_{\boldsymbol{f}}=0.38(\mathrm{DCM} / \mathrm{MeOH}, 95: 5(\mathrm{v} / \mathrm{v}))$.

\section{NMR Spectroscopy:}

${ }^{1} \mathrm{H}$ NMR $\left(500 \mathrm{MHz}, \mathrm{MeCN}-\mathrm{d}_{3}, 25^{\circ} \mathrm{C}, \delta\right): 8.41$ (dd, $\left.J=7.9,1.5 \mathrm{~Hz}, 2 \mathrm{H}\right), 7.98(\mathrm{dd}, J=7.9,1.4 \mathrm{~Hz}$, 2H), 7.91 (td, $J=7.7,1.4 \mathrm{~Hz}, 2 \mathrm{H}$ ), 7.84 (td, $J=7.7,1.4 \mathrm{~Hz}, 2 \mathrm{H}), 7.45-7.40$ (m, 2H), $7.24-7.19$ $(\mathrm{m}, 2 \mathrm{H}), 2.75(\mathrm{~s}, 4 \mathrm{H})$.

${ }^{13} \mathrm{C}$ NMR $\left\{{ }^{1} \mathrm{H}\right\}\left(126 \mathrm{MHz}, \mathrm{MeCN}-\mathrm{d}_{3}, 25^{\circ} \mathrm{C}, \delta\right): 177.4,138.0,137.7,136.3,136.3,131.8,131.0$, $129.7,129.5,124.2,119.3,29.2$.

${ }^{19} \mathrm{~F}$ NMR $\left\{{ }^{1} \mathrm{H}\right\}\left(471 \mathrm{MHz}, \mathrm{MeCN}-\mathrm{d}_{3}, 25^{\circ} \mathrm{C}, \delta\right):-151.6,-151.7$.

HRMS-ESI (m/z) calc'd. for $\mathrm{C}_{22} \mathrm{H}_{16} \mathrm{NO}_{2} \mathrm{~S}_{2}{ }^{+}\left[\mathrm{M}-\mathrm{BF}_{4}\right]^{+}$, 390.061699; found, 390.061750; deviation: -0.13 ppm.

\section{Pyrazole 10}
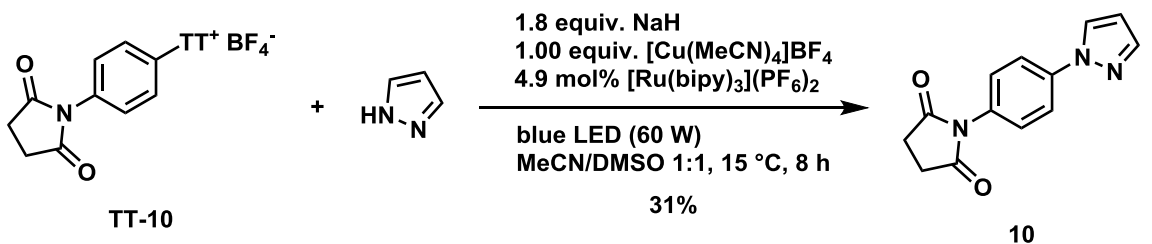

Under ambient atmosphere, a $4 \mathrm{~mL}$ glass-vial was charged with pyrazole ( $82 \mathrm{mg}, 1.2 \mathrm{mmol}, 3.0$ equiv.), THF $(1 \mathrm{~mL})$, and $\mathrm{NaH}$ (60\% dispersion in mineral oil, $29 \mathrm{mg}, 0.73 \mathrm{mmol}, 1.8$ equiv.) in three portions, and the solvent was removed under reduced pressure. Thianthrenium salt TT-10 (191 mg, 0.400 mmol, 1.00 equiv.), $\left[\mathrm{Cu}(\mathrm{MeCN})_{4}\right] \mathrm{BF}_{4}$ (126 mg, $0.401 \mathrm{mmol}, 1.00$ equiv.), [Ru(bipy) $\left.{ }_{3}\right]\left(\mathrm{PF}_{6}\right)_{2}(17 \mathrm{mg}, 20 \mu \mathrm{mol}, 4.9 \mathrm{~mol} \%)$, acetonitrile $(1 \mathrm{~mL})$ and dimethylsulfoxide $\left(1 \mathrm{~mL}, \mathrm{C}_{\text {total }}=0.2 \mathrm{M}\right)$ were added, and the suspension was degassed 
by bubbling through argon with a cannula for $5 \mathrm{~min}$. While stirring, the suspension was irradiated for $8 \mathrm{~h}$ at $15^{\circ} \mathrm{C}$ using a blue LED $(60 \mathrm{~W})$. The suspension was added to water $(30 \mathrm{~mL})$, and the aqueous phase was extracted with DCM $(3 \times 20 \mathrm{~mL})$. The organic phase was dried over $\mathrm{MgSO}_{4}$, and the solvent was removed under reduced pressure. The residue was purified by chromatography on silica gel eluting with hexanes / EtOAc $(1: 0-3: 1(v / v))$ to afford $30 \mathrm{mg}(31 \%)$ of $\mathbf{1 0}$ as white solid.

$\mathbf{R}_{\boldsymbol{f}}=0.36($ hexanes / EtOAc, 7:3 (v/v)).

\section{NMR Spectroscopy:}

${ }^{1} \mathrm{H}$ NMR $\left(500 \mathrm{MHz}, \mathrm{CDCl}_{3}, 25^{\circ} \mathrm{C}, \delta\right): 7.94-7.93(\mathrm{~m}, 1 \mathrm{H}), 7.82-7.80(\mathrm{~m}, 2 \mathrm{H}), 7.73-7.73(\mathrm{~m}$, $1 \mathrm{H}), 7.42-7.40(\mathrm{~m}, 2 \mathrm{H}), 6.48-6.47(\mathrm{~m}, 1 \mathrm{H}), 2.90(\mathrm{~s}, 4 \mathrm{H})$.

${ }^{13} \mathrm{C}$ NMR $\left\{{ }^{1} \mathrm{H}\right\}\left(126 \mathrm{MHz}, \mathrm{CDCl}_{3}, 25{ }^{\circ} \mathrm{C}, \delta\right): 176.1,141.6,140.0,129.9,127.6,126.9,119.7,108.2$, 28.5

HRMS-EI (m/z) calc'd. for $\mathrm{C}_{13} \mathrm{H}_{11} \mathrm{~N}_{3} \mathrm{O}_{2} \mathrm{Na}^{+}[\mathrm{M}+\mathrm{Na}]^{+}$, 264.074345; found, 264.074250; deviation: +0.36 ppm.

Thianthrenium salt TT-11
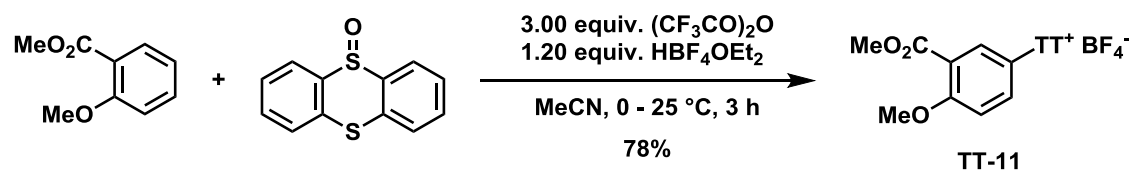

Under ambient atmosphere, a $50 \mathrm{~mL}$ round-bottom flask was charged with methyl-2-methoxybenzoate (897 mg, $5.40 \mathrm{mmol}, 1.00$ equiv.), thianthrene S-oxide (1.26 g, $5.40 \mathrm{mmol}, 1.00$ equiv.) and dry MeCN (22 mL, $\mathrm{C}=0.25 \mathrm{M})$. After cooling to $0^{\circ} \mathrm{C}$, trifluoroacetic anhydride $(2.24 \mathrm{~mL}, 3.44 \mathrm{~g}, 16.2 \mathrm{mmol}, 3.00$ equiv.) was added in one portion at $0{ }^{\circ} \mathrm{C}$, followed by $\mathrm{HBF}_{4} \cdot \mathrm{OEt}_{2}\left(886 \mu \mathrm{L}, 6.48 \mathrm{mmol}, 1.20\right.$ equiv.) addition at $0{ }^{\circ} \mathrm{C}$ in one portion. The flask was sealed with a rubber septum, which was equipped with a balloon. The mixture was allowed to stir at $0{ }^{\circ} \mathrm{C}$ for $1 \mathrm{~h}$, followed by warming the reaction mixture to $25^{\circ} \mathrm{C}$ over a period of $1 \mathrm{~h}$. After stirring at $25^{\circ} \mathrm{C}$ for $1 \mathrm{~h}$ further, the reaction mixture was concentrated under reduced pressure, and diluted with $20 \mathrm{~mL}$ DCM. The DCM solution was poured onto a saturated aqueous $\mathrm{NaHCO}_{3}$ solution (ca. $20 \mathrm{~mL}$ ). After stirring at $25^{\circ} \mathrm{C}$ for $2 \mathrm{~h}$, the mixture was poured onto a separatory funnel, and the layers were separated. The DCM layer was collected, and the aqueous layer was further extracted with DCM ( $\times$ ca. $10 \mathrm{~mL})$. The combined DCM solution was washed with aqueous $\mathrm{NaBF}_{4}$ solution $(2 \times \mathrm{ca} .20 \mathrm{~mL}, 5 \% \mathrm{w} / \mathrm{w})$. The DCM layer was dried over $\mathrm{Na}_{2} \mathrm{SO}_{4}$, filtered, and the solvent was removed under reduced pressure. The residue was purified by chromatography on silica gel eluting with DCM / $\operatorname{PrOH}(50: 1(\mathrm{v} / \mathrm{v}))$. The product was dissolved in $5 \mathrm{~mL} \mathrm{DCM}$, and precipitated with $20 \mathrm{~mL} \mathrm{Et}_{2} \mathrm{O}$. The suspension was decanted, and the solid was dried in vacuo to afford $1.97 \mathrm{~g}(78 \%)$ of TT-11 as colorless solid.

$\mathbf{R}_{\boldsymbol{f}}=0.38(\mathrm{DCM} / \mathrm{MeOH}, 15: 1(\mathrm{v} / \mathrm{v}))$.

\section{NMR Spectroscopy:}

${ }^{1} \mathrm{H}$ NMR $\left(500 \mathrm{MHz}, \mathrm{CD}_{2} \mathrm{Cl}_{2}, 25^{\circ} \mathrm{C}, \delta\right): 8.38(\mathrm{~d}, J=7.9 \mathrm{~Hz}, 2 \mathrm{H}), 7.91(\mathrm{~d}, J=7.8 \mathrm{~Hz}, 2 \mathrm{H}), 7.86(\mathrm{t}, J$ 
$=7.6 \mathrm{~Hz}, 2 \mathrm{H}), 7.79(\mathrm{t}, J=7.6 \mathrm{~Hz}, 2 \mathrm{H}), 7.55(\mathrm{~d}, J=2.8 \mathrm{~Hz}, 1 \mathrm{H}), 7.41(\mathrm{dd}, J=9.2,2.8 \mathrm{~Hz}, 1 \mathrm{H}), 7.12$ $(\mathrm{d}, J=9.2 \mathrm{~Hz}, 1 \mathrm{H}), 3.90(\mathrm{~s}, 3 \mathrm{H}), 3.79(\mathrm{~s}, 3 \mathrm{H})$.

${ }^{13} \mathrm{C}$ NMR $\left\{{ }^{1} \mathrm{H}\right\}\left(126 \mathrm{MHz}, \mathrm{CD}_{2} \mathrm{Cl}_{2}, 25^{\circ} \mathrm{C}, \delta\right): 164.8,163.2,137.1,135.7,135.0,134.1,132.0,131.2$, $130.9,123.2,119.3,115.3,113.6,57.3,53.1$.

${ }^{19} \mathrm{~F}$ NMR $\left\{{ }^{1} \mathrm{H}\right\}\left(471 \mathrm{MHz}, \mathrm{CD}_{2} \mathrm{Cl}_{2}, 25{ }^{\circ} \mathrm{C}, \delta\right):-151.3,-151.4$.

HRMS-ESI (m/z) calc'd. for $\mathrm{C}_{21} \mathrm{H}_{17} \mathrm{O}_{3} \mathrm{~S}_{2}{ }^{+}\left[\mathrm{M}-\mathrm{BF}_{4}\right]^{+}$, 381.061365; found, 381.061490; deviation: -0.33 ppm.

\section{Triazole 11}

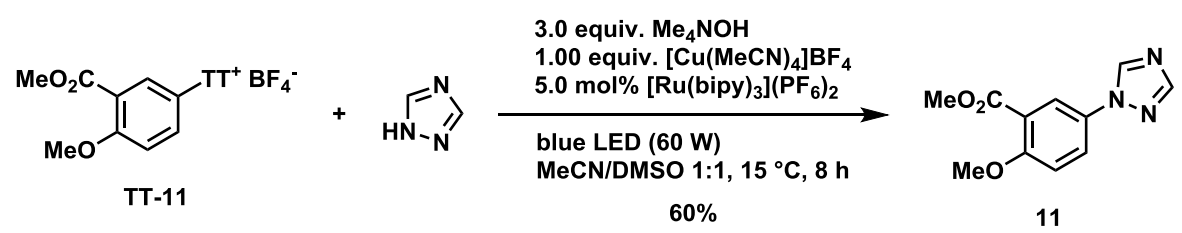

Under ambient atmosphere, a $4 \mathrm{~mL}$ glass-vial was charged with 1,2,4-triazole (83 mg, $1.2 \mathrm{mmol}$, 3.0 equiv.) and tetramethylammonium hydroxide (25\% in methanol, $438 \mathrm{mg}, 0.51 \mathrm{~mL}, 1.2 \mathrm{mmol}, 3.0$ equiv.), and the solvent was removed under reduced pressure. Thianthrenium salt TT-11 (187 mg, 0.399 mmol, 1.00 equiv.), $\left[\mathrm{Cu}(\mathrm{MeCN})_{4}\right] \mathrm{BF}_{4}$ (126 mg, $0.401 \mathrm{mmol}, 1.00$ equiv.), [Ru(bipy) $\left.{ }_{3}\right]\left(\mathrm{PF}_{6}\right)_{2}(17 \mathrm{mg}, 20 \mu \mathrm{mol}, 5.0 \mathrm{~mol} \%)$, acetonitrile $(1.0 \mathrm{~mL})$ and dimethylsulfoxide $\left(1.0 \mathrm{~mL}, \mathrm{c}_{\text {total }}=0.20 \mathrm{M}\right)$ were added, and the suspension was degassed by bubbling through argon with a cannula for $5 \mathrm{~min}$. While stirring, the suspension was irradiated for $8 \mathrm{~h}$ at $15^{\circ} \mathrm{C}$ using a blue LED $(60 \mathrm{~W})$. The suspension was added to water $(30 \mathrm{~mL})$, and the aqueous phase was extracted with DCM $(3 \times 20 \mathrm{~mL})$. The organic phase was dried over $\mathrm{MgSO}_{4}$, and the solvent was removed under reduced pressure. The residue was purified by chromatography on silica gel eluting with hexanes / EtOAc (4:1 (v/v)) to afford $56 \mathrm{mg}(60 \%)$ of 11 as white solid.

$\mathbf{R}_{\boldsymbol{f}}=0.21$ (hexanes / EtOAc, 7:3 (v/v)).

\section{NMR Spectroscopy:}

${ }^{1} \mathrm{H}$ NMR $\left(500 \mathrm{MHz}, \mathrm{CDCl}_{3}, 25^{\circ} \mathrm{C}, \delta\right): 9.25(\mathrm{~s}, 1 \mathrm{H}), 8.21(\mathrm{~s}, 1 \mathrm{H}), 8.10-8.09(\mathrm{~m}, 1 \mathrm{H}), 8.03-8.00$ $(\mathrm{m}, 1 \mathrm{H}), 7.36-7.34(\mathrm{~m}, 1 \mathrm{H}), 3.89(\mathrm{~s}, 3 \mathrm{H}), 3.83(\mathrm{~s}, 3 \mathrm{H})$.

${ }^{13} \mathrm{C}$ NMR $\left\{{ }^{1} \mathrm{H}\right\}\left(126 \mathrm{MHz}, \mathrm{CDCl}_{3}, 25{ }^{\circ} \mathrm{C}, \delta\right): 165.3,157.3,152.3,142.2,129.6,124.4,121.8,120.8$, $113.8,56.3,52.2$.

HRMS-ESI (m/z) calc'd. for $\mathrm{C}_{11} \mathrm{H}_{11} \mathrm{~N}_{3} \mathrm{O}_{3} \mathrm{Na}^{+}[\mathrm{M}+\mathrm{Na}]^{+}$, 256.069260; found, 256.069290; deviation: -0.12 ppm.

Thianthrenium salt TT-12
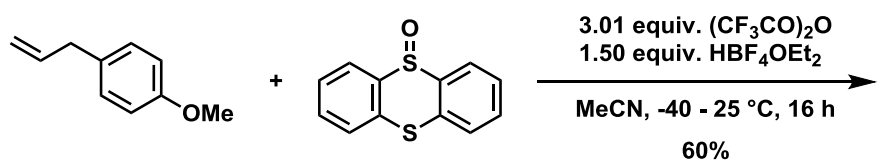<smiles></smiles>

TT-12

Under ambient atmosphere, a $100 \mathrm{~mL}$ Schlenk-tube equipped with a magnetic stir bar was charged with 
1-allyl-4-methoxybenzene ( $772 \mu \mathrm{L}, 741 \mathrm{mg}, 5.00 \mathrm{mmol}, 1.00$ equiv.), thianthrene S-oxide $(1.20 \mathrm{~g}, 5.17 \mathrm{mmol}$, 1.03 equiv.), and $\mathrm{MeCN}(20 \mathrm{~mL}, \mathrm{C}=0.25 \mathrm{M})$. After cooling to $-40^{\circ} \mathrm{C}$, trifluoroacetic anhydride $(2.09 \mathrm{~mL}$, $3.16 \mathrm{~g}, 15.0 \mathrm{mmol}, 3.01$ equiv.) was added while stirring. $\mathrm{HBF}_{4} \cdot \mathrm{OEt}_{2}(1.02 \mathrm{~mL}, 1.21 \mathrm{~g}, 7.50 \mathrm{mmol}$, 1.50 equiv.) was added dropwise. The mixture was stirred at $-40^{\circ} \mathrm{C}$ for $1 \mathrm{~h}$, then at ambient temperature for $15 \mathrm{~h}$. The reaction mixture was concentrated under reduced pressure, and subsequently diluted with DCM $(100 \mathrm{~mL})$. The solution was poured onto a saturated aqueous $\mathrm{NaHCO}_{3}$ solution $(100 \mathrm{~mL})$, and the layers were separated. The organic phase was washed with aqueous $\mathrm{NaBF}_{4}$ solution $(2 \times 100 \mathrm{~mL}, 10 \%)$, and with water $(100 \mathrm{~mL})$. The organic phase was dried over $\mathrm{MgSO}_{4}$, and the solvent was removed under reduced pressure. The residue was purified by chromatography on silica gel eluting with DCM / MeOH (1:0-20:1 (v/v)) to afford $1.36 \mathrm{~g}(60 \%)$ of TT-12 as off-white solid.

$\mathbf{R} \boldsymbol{f}=0.29(\mathrm{DCM} / \mathrm{MeOH}, 95: 5(\mathrm{v} / \mathrm{v}))$.

\section{NMR Spectroscopy:}

${ }^{1} \mathrm{H}$ NMR $\left(500 \mathrm{MHz}, \mathrm{CDCl}_{3}, 25^{\circ} \mathrm{C}, \delta\right): 8.38-8.36(\mathrm{~m}, 2 \mathrm{H}), 7.84-7.82(\mathrm{~m}, 2 \mathrm{H}), 7.80-7.72(\mathrm{~m}$, $4 \mathrm{H}$ ), $7.38-7.36(\mathrm{~m}, 1 \mathrm{H}), 7.04-7.03(\mathrm{~m}, 1 \mathrm{H}), 6.32$ (apt d, $J=2.0 \mathrm{~Hz}, 1 \mathrm{H}$ ), 5.70 (ddt, $J=16.8$, 10.1, 6.6 Hz, 1H), $5.01-4.98(\mathrm{~m}, 1 \mathrm{H}), 4.91-4.86(\mathrm{~m}, 1 \mathrm{H}) .3 .95(\mathrm{~s}, 3 \mathrm{H}), 3.17(\mathrm{~d}, J=6.6 \mathrm{~Hz}, 2 \mathrm{H})$.

${ }^{13} \mathrm{C}$ NMR $\left\{{ }^{1} \mathrm{H}\right\}\left(126 \mathrm{MHz}, \mathrm{CDCl}_{3}, 25{ }^{\circ} \mathrm{C}, \delta\right): 156.7,137.4,136.3,135.8,135.4,134.8,133.8,130.3$, $130.2,128.5,117.4,117.2,114.0,108.5,57.2,38.8$.

${ }^{19} \mathrm{~F}$ NMR $\left\{{ }^{1} \mathrm{H}\right\}\left(471 \mathrm{MHz}, \mathrm{CDCl}_{3}, 25^{\circ} \mathrm{C}, \delta\right):-152.5,-152.6$.

HRMS-ESI (m/z) calc'd. for $\mathrm{C}_{22} \mathrm{H}_{19} \mathrm{OS}_{2}{ }^{+}\left[\mathrm{M}-\mathrm{BF}_{4}\right]^{+}$, 363.086910; found, 363.087185; deviation: +0.8 ppm.

\section{Triazole 12}

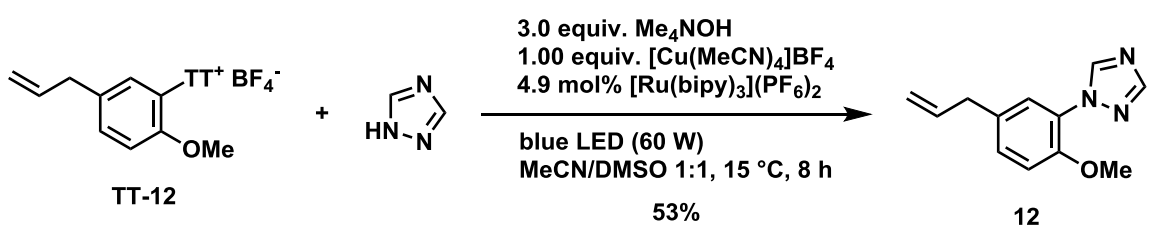

Under ambient atmosphere, a $4 \mathrm{~mL}$ glass-vial was charged with 1,2,4-triazole (83 mg, $1.2 \mathrm{mmol}, 3.0$ equiv.) and tetramethylammonium hydroxide ( $25 \%$ in methanol, $438 \mathrm{mg}, 0.51 \mathrm{~mL}, 1.2 \mathrm{mmol}, 3.0$ equiv.), and the solvent was removed under reduced pressure. Thianthrenium salt TT-12 (180 mg, $0.400 \mathrm{mmol}, 1.00$ equiv.), $\left[\mathrm{Cu}(\mathrm{MeCN})_{4}\right] \mathrm{BF}_{4}$ (126 mg, $0.401 \mathrm{mmol}, 1.00$ equiv.), [Ru(bipy) $\left.)_{3}\right]\left(\mathrm{PF}_{6}\right)_{2}(17 \mathrm{mg}, 20 \mu \mathrm{mol}, 4.9 \mathrm{~mol} \%)$, acetonitrile $(1 \mathrm{~mL})$ and dimethylsulfoxide $\left(1 \mathrm{~mL}, \mathrm{C}_{\text {total }}=0.2 \mathrm{M}\right)$ were added, and the suspension was degassed by bubbling through argon with a cannula for $5 \mathrm{~min}$. While stirring, the suspension was irradiated for $8 \mathrm{~h}$ at $15^{\circ} \mathrm{C}$ using a blue LED $(60 \mathrm{~W})$. The suspension was added to water $(30 \mathrm{~mL})$, and the aqueous phase was extracted with DCM $(3 \times 20 \mathrm{~mL})$. The organic phase was dried over $\mathrm{MgSO}_{4}$, and the solvent was removed under reduced pressure. The residue was purified by chromatography on silica gel eluting with hexanes / EtOAc $(1: 0-4: 1(v / v))$ to afford $46 \mathrm{mg}(53 \%)$ of 12 as white solid. 
$\mathbf{R}_{\boldsymbol{f}}=0.11$ (hexanes / EtOAc, 8:2(v/v)).

\section{NMR Spectroscopy:}

${ }^{1} \mathrm{H}$ NMR $\left(500 \mathrm{MHz}, \mathrm{CDCl}_{3}, 25^{\circ} \mathrm{C}, \delta\right): 8.74(\mathrm{~s}, 1 \mathrm{H}), 8.06(\mathrm{~s}, 1 \mathrm{H}), 7.61(\mathrm{~s}, 1 \mathrm{H}), 7.18-7.16(\mathrm{~m}, 1 \mathrm{H})$, $7.01-7.00(\mathrm{~m}, 1 \mathrm{H}), 5.99-5.91(\mathrm{~m}, 1 \mathrm{H}), 5.12-5.08(\mathrm{~m}, 2 \mathrm{H}), 3.91-3.90(\mathrm{~s}, 3 \mathrm{H}), 3.39(\mathrm{~d}, J=6.6$ $\mathrm{Hz}, 2 \mathrm{H})$.

${ }^{13} \mathrm{C}$ NMR $\left\{{ }^{1} \mathrm{H}\right\}\left(126 \mathrm{MHz}, \mathrm{CDCl}_{3}, 25{ }^{\circ} \mathrm{C}, \delta\right): 151.4,149.4,144.7,137.0,133.5,129.1,126.3,124.6$, $116.5,112.4,56.2,39.3$.

HRMS-EI (m/z) calc'd. for $\mathrm{C}_{12} \mathrm{H}_{13} \mathrm{~N}_{3} \mathrm{O}^{+}[\mathrm{M}]^{+}$, 215.105311; found, 215.105620; deviation: -1.44 ppm.

\section{Thianthrenium salt TT-13}

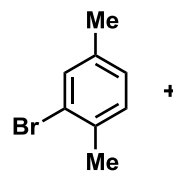<smiles>O=S1c2ccccc2Sc2ccccc21</smiles>
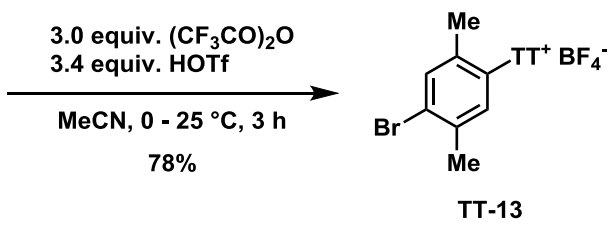

Under ambient atmosphere, a $50 \mathrm{~mL}$ Schlenk-tube equipped with a magnetic stir bar was charged with 1-bromo-2,5-dimethylbenzene (159 mg, 859 umol, 1.00 equiv.), thianthrene S-oxide (200 mg, $861 \mu \mathrm{mol}$, 1.00 equiv.), and $\mathrm{MeCN}(3.0 \mathrm{~mL}, \mathrm{C}=0.29 \mathrm{M})$. After cooling to $0{ }^{\circ} \mathrm{C}$, trifluoroacetic anhydride $(0.36 \mathrm{~mL}, 0.54 \mathrm{~g}$, $2.6 \mathrm{mmol}, 3.0$ equiv.) was added while stirring. HOTf $(0.26 \mathrm{ml}, 0.44 \mathrm{~g}, 2.9 \mathrm{mmol}, 3.4$ equiv.) was added dropwise. The mixture was stirred at $0{ }^{\circ} \mathrm{C}$ for $1 \mathrm{~h}$, then at ambient temperature for $2 \mathrm{~h}$. The reaction mixture was added to a saturated aqueous $\mathrm{NaHCO}_{3}$ solution $(30 \mathrm{~mL})$, and the aqueous phase was extracted with $\mathrm{DCM}(3 \times 20 \mathrm{~mL})$. The organic phase was washed with aqueous $\mathrm{NaBF}_{4}$ solution $(30 \mathrm{~mL}, 10 \%)$, dried over $\mathrm{MgSO}_{4}$, and the solvent was removed under reduced pressure. The residue was purified by chromatography on silica gel eluting with DCM / MeOH (1:0-95:5 (v/v)) to afford $329 \mathrm{mg}(78 \%)$ of TT-13 as yellowish foam. $\mathbf{R}_{\boldsymbol{f}}=0.40(\mathrm{DCM} / \mathrm{MeOH}, 94: 6(\mathrm{v} / \mathrm{v}))$.

\section{NMR Spectroscopy:}

${ }^{1} \mathrm{H}$ NMR $\left(500 \mathrm{MHz}, \mathrm{CDCl}_{3}, 25^{\circ} \mathrm{C}, \delta\right): 8.44(\mathrm{~d}, J=8.0 \mathrm{~Hz}, 2 \mathrm{H}), 7.87$ (dd, $\left.J=8.0,1.4 \mathrm{~Hz}, 2 \mathrm{H}\right), 7.80$ (td, $J=7.7,1.4 \mathrm{~Hz}, 2 \mathrm{H}), 7.71$ (td, $J=7.7,1.4 \mathrm{~Hz}, 2 \mathrm{H}), 7.52(\mathrm{~s}, 1 \mathrm{H}), 6.65(\mathrm{~s}, 1 \mathrm{H}), 2.62(\mathrm{~s}, 3 \mathrm{H}), 2.21$ (s, 3H).

${ }^{13} \mathrm{C}$ NMR $\left\{{ }^{1} \mathrm{H}\right\}\left(126 \mathrm{MHz}, \mathrm{CDCl}_{3}, 25{ }^{\circ} \mathrm{C}, \delta\right): 138.9,138.0,137.4,137.4,135.2,135.0,131.7,130.8$, 130.4, 130.2, 120.0, 117.8, 23.0, 19.8.

${ }^{19}$ F NMR $\left\{{ }^{1} \mathrm{H}\right\}\left(471 \mathrm{MHz}, \mathrm{CDCl}_{3}, 25^{\circ} \mathrm{C}, \delta\right):-151.0,-151.1$.

HRMS-ESI (m/z) calc'd. for $\mathrm{C}_{20} \mathrm{H}_{16} \mathrm{BrS}_{2}{ }^{+}\left[\mathrm{M}-\mathrm{BF}_{4}\right]^{+}$, 398.987145; found, 398.987120; deviation: +0.06 ppm. 
Triazole 13

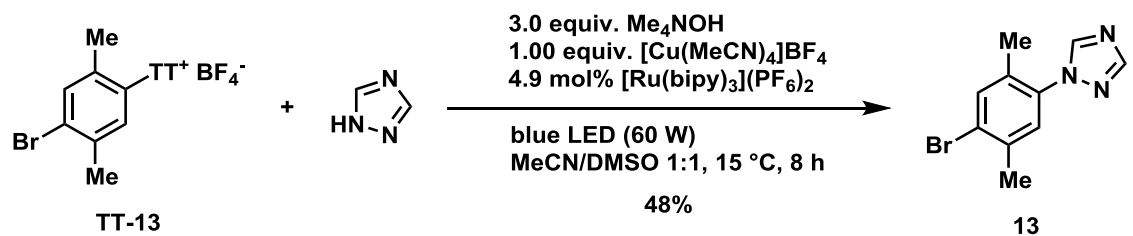

Under ambient atmosphere, a $4 \mathrm{~mL}$ glass-vial was charged with 1,2,4-triazole (83 mg, $1.2 \mathrm{mmol}$, 3.0 equiv.) and tetramethylammonium hydroxide (25\% in methanol, $438 \mathrm{mg}, 0.51 \mathrm{~mL}, 1.2 \mathrm{mmol}, 3.0$ equiv.), and the solvent was removed under reduced pressure. Thianthrenium salt TT-13 (195 mg, $0.400 \mathrm{mmol}, 1.00$ equiv.), $\left[\mathrm{Cu}(\mathrm{MeCN})_{4}\right] \mathrm{BF}_{4}$ (126 mg, $0.401 \mathrm{mmol}, 1.00$ equiv.), [Ru(bipy) $\left.{ }_{3}\right]\left(\mathrm{PF}_{6}\right)_{2}(17 \mathrm{mg}, 20 \mu \mathrm{mol}, 4.9 \mathrm{~mol} \%)$, acetonitrile $(1.0 \mathrm{~mL})$ and dimethylsulfoxide $\left(1.0 \mathrm{~mL}, \mathrm{c}_{\text {total }}=0.20 \mathrm{M}\right)$ were added, and the suspension was degassed by bubbling through argon with a cannula for $5 \mathrm{~min}$. While stirring, the suspension was irradiated for $8 \mathrm{~h}$ at $15^{\circ} \mathrm{C}$ using a blue LED (60 W). The suspension was added to water $(30 \mathrm{~mL})$, and the aqueous phase was extracted with $\mathrm{DCM}(3 \times 20 \mathrm{~mL})$. The organic phase was dried over $\mathrm{MgSO}_{4}$, and the solvent was removed under reduced pressure. The residue was purified by chromatography on silica gel eluting with hexanes / EtOAc (10:0-0:10 (v/v)) to afford $48 \mathrm{mg}(48 \%)$ of 13 as brownish solid.

$\mathbf{R} \boldsymbol{f}=0.29($ hexanes / EtOAc, 60:40 (v/v)).

\section{NMR Spectroscopy:}

${ }^{1} \mathrm{H}$ NMR $\left(500 \mathrm{MHz}, \mathrm{CDCl}_{3}, 25^{\circ} \mathrm{C}, \delta\right): 8.23(\mathrm{~s}, 1 \mathrm{H}), 8.09(\mathrm{~s}, 1 \mathrm{H}), 7.52(\mathrm{~s}, 1 \mathrm{H}), 7.18(\mathrm{~s}, 1 \mathrm{H}), 2.38(\mathrm{~s}$, $3 \mathrm{H}), 2.16(\mathrm{~s}, 3 \mathrm{H})$.

${ }^{13} \mathrm{C}$ NMR $\left\{{ }^{1} \mathrm{H}\right\}\left(126 \mathrm{MHz}, \mathrm{CDCl}_{3}, 25{ }^{\circ} \mathrm{C}, \delta\right): 152.4,143.8,136.9,135.3,135.0,132.8,127.9,125.8$, 22.4, 17.3.

HRMS-El (m/z) calc'd. for $\mathrm{C}_{10} \mathrm{H}_{10} \mathrm{~N}_{3} \mathrm{Br}^{+}[\mathrm{M}]^{+}$, 251.005272; found, 251.005550; deviation: -1.11 ppm.

\section{Thianthrenium salt TT-14}
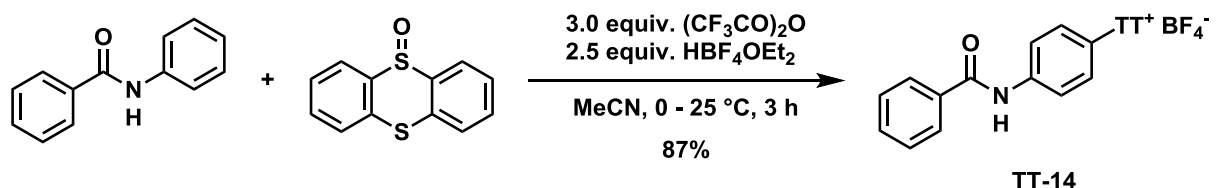

Under ambient atmosphere, a $250 \mathrm{~mL}$ Schlenk-tube equipped with a magnetic stir bar was charged with benzanilide (1.27 g, $6.44 \mathrm{mmol}, 1.00$ equiv.), thianthrene S-oxide ( $1.50 \mathrm{~g}, 6.46 \mathrm{mmol}, 1.00$ equiv.), and MeCN $(50 \mathrm{~mL}, \mathrm{c}=0.13 \mathrm{M})$. After cooling to $0{ }^{\circ} \mathrm{C}$, trifluoroacetic anhydride $(2.7 \mathrm{~mL}, 4.1 \mathrm{~g}, 19 \mathrm{mmol}, 3.0$ equiv.) was added while stirring. $\mathrm{HBF}_{4} \cdot \mathrm{OEt}_{2}(2.2 \mathrm{~mL}, 2.6 \mathrm{~g}, 16 \mathrm{mmol}, 2.5$ equiv.) was added dropwise. The mixture was stirred at $0{ }^{\circ} \mathrm{C}$ for $1 \mathrm{~h}$, then at ambient temperature for $2 \mathrm{~h}$. The reaction mixture was concentrated under reduced pressure, and subsequently diluted with DCM $(100 \mathrm{~mL})$. The solution was poured onto a saturated aqueous $\mathrm{NaHCO}_{3}$ solution $(100 \mathrm{~mL}$ ), and the layers were separated. The organic phase was washed with aqueous $\mathrm{NaBF}_{4}$ solution $(2 \times 100 \mathrm{~mL}, 10 \%)$, and with water $(100 \mathrm{~mL})$. The organic phase was dried over 
$\mathrm{MgSO}_{4}$, and the solvent was removed under reduced pressure. The residue was purified by chromatography on silica gel eluting with DCM / MeOH (1:0-20:1 (v/v)) to afford $2.79 \mathrm{~g}(87 \%)$ of TT-14 as off-white solid.

$\mathbf{R}_{\boldsymbol{f}}=0.29(\mathrm{DCM} / \mathrm{MeOH}, 94: 6(\mathrm{v} / \mathrm{v}))$.

\section{NMR Spectroscopy:}

${ }^{1}$ H NMR (500 MHz, DMSO-d $\left.\mathrm{d}_{6}, 25^{\circ} \mathrm{C}, \delta\right): 10.63(\mathrm{~s}, 1 \mathrm{H}), 8.54$ (dd, $\left.J=7.9,1.5 \mathrm{~Hz}, 2 \mathrm{H}\right), 8.07$ (dd, $J$ $=7.9,1.4 \mathrm{~Hz}, 2 \mathrm{H}), 7.97-7.89(\mathrm{~m}, 6 \mathrm{H}), 7.85(\mathrm{td}, J=7.7,1.4 \mathrm{~Hz}, 2 \mathrm{H}), 7.63-7.58(\mathrm{~m}, 1 \mathrm{H}), 7.55-$ $7.50(\mathrm{~m}, 2 \mathrm{H}), 7.27(\mathrm{~d}, J=9.2 \mathrm{~Hz}, 2 \mathrm{H})$.

${ }^{13} \mathrm{C}$ NMR $\left\{{ }^{1} \mathrm{H}\right\}\left(126 \mathrm{MHz}, \mathrm{CDCl}_{3}, 25^{\circ} \mathrm{C}, \delta\right): 166.1,143.2,135.2,135.2,134.7,134.1,132.1,130.3$, $129.6,129.2,128.5,127.8,121.3,119.6,117.2$.

${ }^{19} \mathrm{~F}$ NMR $\left\{{ }^{1} \mathrm{H}\right\}\left(471 \mathrm{MHz}, \mathrm{DMSO}-\mathrm{d}_{6}, 25^{\circ} \mathrm{C}, \delta\right):-148.5,-148.5$.

HRMS-ESI (m/z) calc'd. for $\mathrm{C}_{25} \mathrm{H}_{18} \mathrm{NOS}_{2}{ }^{+}\left[\mathrm{M}-\mathrm{BF}_{4}\right]^{+}$, 412.082434; found, 412.082120; deviation: +0.76 ppm.

\section{Triazole 14}

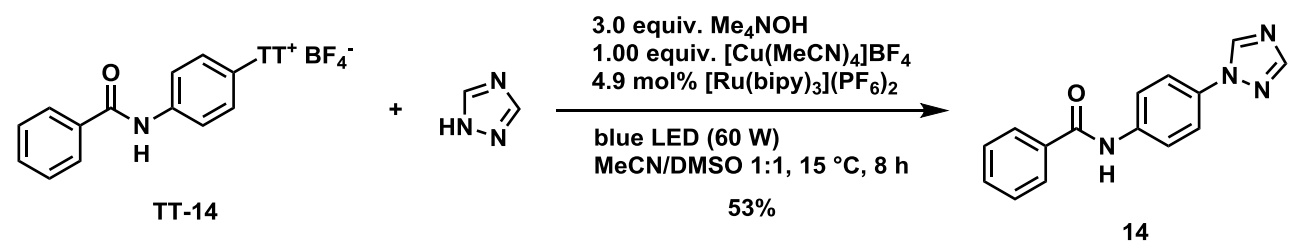

Under ambient atmosphere, a $4 \mathrm{~mL}$ glass-vial was charged with 1,2,4-triazole (83 $\mathrm{mg}, 1.2 \mathrm{mmol}, 3.0$ equiv.) and tetramethylammonium hydroxide ( $25 \%$ in methanol, $438 \mathrm{mg}, 0.51 \mathrm{~mL}, 1.2 \mathrm{mmol}, 3.0$ equiv.), and the solvent was removed under reduced pressure. Thianthrenium salt TT-14 (200 mg, 0.401 mmol, 1.00 equiv.), $\left[\mathrm{Cu}(\mathrm{MeCN})_{4}\right] \mathrm{BF}_{4}$ (126 mg, $0.401 \mathrm{mmol}, 1.00$ equiv.), [Ru(bipy) $\left.)_{3}\right]\left(\mathrm{PF}_{6}\right)_{2}(17 \mathrm{mg}, 20 \mu \mathrm{mol}, 4.9 \mathrm{~mol} \%)$, acetonitrile $(1 \mathrm{~mL})$ and dimethylsulfoxide $\left(1 \mathrm{~mL}, \mathrm{C}_{\text {total }}=0.2 \mathrm{M}\right)$ were added, and the suspension was degassed by bubbling through argon with a cannula for $5 \mathrm{~min}$. While stirring, the suspension was irradiated for $8 \mathrm{~h}$ at $15^{\circ} \mathrm{C}$ using a blue LED $(60 \mathrm{~W})$. The suspension was added to water $(30 \mathrm{~mL})$, and the aqueous phase was extracted with DCM $(3 \times 20 \mathrm{~mL})$. The organic phase was dried over $\mathrm{MgSO}_{4}$, and the solvent was removed under reduced pressure. The residue was purified by chromatography on silica gel eluting with hexanes / EtOAc $(9: 1-6: 4(v / v))$ to afford 56 mg (53\%) of 14 as off-white solid.

$\mathbf{R}_{\boldsymbol{f}}=0.42(\mathrm{EtOAc})$.

\section{NMR Spectroscopy:}

${ }^{1} \mathrm{H}$ NMR $\left(500 \mathrm{MHz}, \mathrm{DMSO}-\mathrm{d}_{6}, 25^{\circ} \mathrm{C}, \delta\right): 10.46(\mathrm{~s}, 1 \mathrm{H}), 9.24(\mathrm{~s}, 1 \mathrm{H}), 8.23(\mathrm{~s}, 1 \mathrm{H}), 8.00-7.96(\mathrm{~m}$, $4 \mathrm{H}), 7.87-7.83(\mathrm{~m}, 2 \mathrm{H}), 7.63-7.59(\mathrm{~m}, 1 \mathrm{H}), 7.57-7.52(\mathrm{~m}, 2 \mathrm{H})$.

${ }^{13} \mathrm{C}$ NMR $\left\{{ }^{1} \mathrm{H}\right\}\left(126 \mathrm{MHz}, \mathrm{DMSO}-\mathrm{d}_{6}, 25^{\circ} \mathrm{C}, \delta\right): 165.7,152.2,142.0,138.7,134.7,132.3,131.7$, 128.4, 127.7, 121.1, 119.9.

HRMS- $\mathrm{Cl}(\mathbf{m} / \mathbf{z})$ calc'd. for $\mathrm{C}_{15} \mathrm{H}_{13} \mathrm{~N}_{4} \mathrm{O}^{+}[\mathrm{M}+\mathrm{H}]^{+}$, 265.108385; found, 265.108630; deviation: -0.92 ppm. 
Thianthrenium salt TT-15
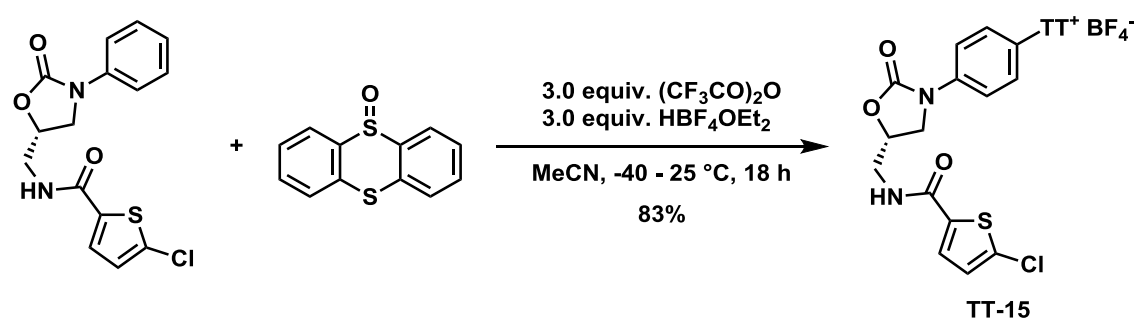

Under ambient atmosphere, a $100 \mathrm{~mL}$ Schlenk-tube equipped with a magnetic stir bar was charged with (S)-5-chloro- $N$-((2-oxo-3-phenyloxazolidin-5-yl)methyl)thiophene-2-carboxamide (800 mg, $2.38 \mathrm{mmol}$, 1.00 equiv.), thianthrene S-oxide (569 mg, $2.45 \mathrm{mmol}, 1.03$ equiv.), and $\mathrm{MeCN}$ ( $15 \mathrm{~mL}, \mathrm{c}=0.16 \mathrm{M}$ ). After cooling to $-40^{\circ} \mathrm{C}$, trifluoroacetic anhydride $(0.99 \mathrm{~mL}, 1.5 \mathrm{~g}, 7.1 \mathrm{mmol}, 3.0$ equiv.) was added while stirring. $\mathrm{HBF}_{4} \cdot \mathrm{OEt}_{2}\left(0.97 \mathrm{~mL}, 1.2 \mathrm{~g}, 7.1 \mathrm{mmol}, 3.0\right.$ equiv.) was added dropwise. The mixture was stirred at $-40{ }^{\circ} \mathrm{C}$ for $1 \mathrm{~h}$, then at ambient temperature for $17 \mathrm{~h}$. The reaction mixture was concentrated under reduced pressure, and subsequently diluted with DCM $(100 \mathrm{~mL})$. The solution was poured onto a saturated aqueous $\mathrm{NaHCO}_{3}$ solution $(100 \mathrm{~mL})$, and the layers were separated. The organic phase was washed with aqueous $\mathrm{NaBF}_{4}$ solution $(2 \times 100 \mathrm{~mL}, 10 \%)$, and with water $(100 \mathrm{~mL})$. The organic phase was dried over $\mathrm{MgSO}_{4}$, and the solvent was removed under reduced pressure. The residue was purified by chromatography on silica gel eluting with DCM / MeOH (1:0-19:1 (v/v)) to afford $1.25 \mathrm{~g}(83 \%)$ of TT-15 as white solid.

$\mathbf{R}_{\boldsymbol{f}}=0.20(\mathrm{DCM} / \mathrm{MeOH}, 95: 5(\mathrm{v} / \mathrm{v}))$.

\section{NMR Spectroscopy:}

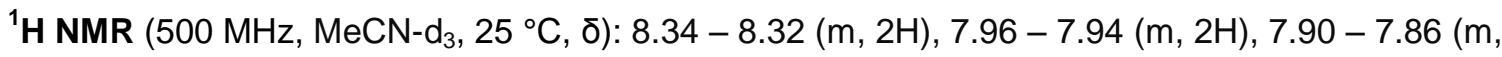
$2 \mathrm{H}), 7.82-7.79(\mathrm{~m}, 2 \mathrm{H}), 7.63-7.60(\mathrm{~m}, 2 \mathrm{H}), 7.35(\mathrm{~d}, J=4.1 \mathrm{~Hz}, 1 \mathrm{H}), 7.33-7.31(\mathrm{~m}, 1 \mathrm{H}), 7.15-$ $7.12(\mathrm{~m}, 2 \mathrm{H}), 6.96(\mathrm{~d}, J=4.1 \mathrm{~Hz}, 1 \mathrm{H}), 4.83-4.78(\mathrm{~m}, 1 \mathrm{H}), 4.05(\mathrm{t}, J=9.1 \mathrm{~Hz}, 1 \mathrm{H}), 3.80(\mathrm{dd}, J=$ 9.3, $6.1 \mathrm{~Hz}, 1 \mathrm{H}), 3.71-3.59(\mathrm{~m}, 2 \mathrm{H})$.

${ }^{13} \mathrm{C}$ NMR $\left\{{ }^{1} \mathrm{H}\right\}\left(126 \mathrm{MHz}, \mathrm{MeCN}-\mathrm{d}_{3}, 25^{\circ} \mathrm{C}, \delta\right): 162.3,155.1,144.0,139.1,137.3,136.1,135.8$, $135.5,131.6,130.9,130.1,128.8,128.7,120.1,119.8,117.3,73.0,48.4,42.9$.

${ }^{19} \mathrm{~F}$ NMR $\left\{{ }^{1} \mathrm{H}\right\}\left(471 \mathrm{MHz}, \mathrm{MeCN}-\mathrm{d}_{3}, 25^{\circ} \mathrm{C}, \delta\right):-151.5,-151.5$.

HRMS-ESI (m/z) calc'd. for $\mathrm{C}_{27} \mathrm{H}_{20} \mathrm{CIN}_{2} \mathrm{O}_{3} \mathrm{~S}_{3}{ }^{+}\left[\mathrm{M}-\mathrm{BF}_{4}\right]^{+}$, 551.031470; found, 551.031913; deviation: +0.8 ppm.

\section{Triazole 15}
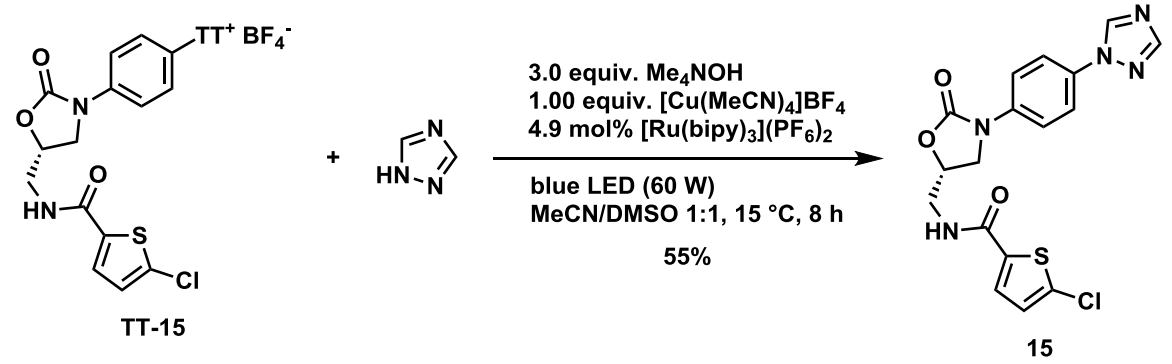
Under ambient atmosphere, a $4 \mathrm{~mL}$ glass-vial was charged with 1,2,4-triazole (83 mg, $1.2 \mathrm{mmol}, 3.0$ equiv.) and tetramethylammonium hydroxide ( $25 \%$ in methanol, $438 \mathrm{mg}, 0.51 \mathrm{~mL}, 1.2 \mathrm{mmol}, 3.0$ equiv.), and the solvent was removed under reduced pressure. Thianthrenium salt TT-15 (256 mg, $0.401 \mathrm{mmol}, 1.00$ equiv.), $\left[\mathrm{Cu}(\mathrm{MeCN})_{4}\right] \mathrm{BF}_{4}$ (126 mg, $0.401 \mathrm{mmol}, 1.00$ equiv.), [Ru(bipy) $\left.)_{3}\right]\left(\mathrm{PF}_{6}\right)_{2}(17 \mathrm{mg}, 20 \mu \mathrm{mol}, 4.9 \mathrm{~mol} \%)$, acetonitrile $(1 \mathrm{~mL})$ and dimethylsulfoxide $\left(1 \mathrm{~mL}, \mathrm{c}_{\text {total }}=0.2 \mathrm{M}\right)$ were added, and the suspension was degassed by bubbling through argon with a cannula for $5 \mathrm{~min}$. While stirring, the suspension was irradiated for $8 \mathrm{~h}$ at $15^{\circ} \mathrm{C}$ using a blue LED $(60 \mathrm{~W})$. The suspension was added to water $(30 \mathrm{~mL})$, and the aqueous phase was extracted with DCM $(3 \times 20 \mathrm{~mL})$. The organic phase was dried over $\mathrm{MgSO}_{4}$, and the solvent was removed under reduced pressure. The residue was purified by chromatography on silica gel eluting with hexanes / EtOAc $(1: 0-2: 1(\mathrm{v} / \mathrm{v}))$ to afford $89 \mathrm{mg}(55 \%)$ of 15 as white solid.

$\mathbf{R}_{\boldsymbol{f}}=0.13($ hexanes / EtOAc, $2: 8(\mathrm{v} / \mathrm{v}))$.

\section{NMR Spectroscopy:}

${ }^{1} \mathrm{H}$ NMR $\left(500 \mathrm{MHz}, \mathrm{DMSO}-\mathrm{d}_{6}, 25^{\circ} \mathrm{C}, \delta\right): 9.25(\mathrm{~s}, 1 \mathrm{H}), 8.99-8.97(\mathrm{~m}, 1 \mathrm{H}), 8.22(\mathrm{~s}, 1 \mathrm{H}), 7.88-$ $7.86(\mathrm{~m}, 2 \mathrm{H}), 7.73-7.71(\mathrm{~m}, 2 \mathrm{H}), 7.69(\mathrm{~d}, J=4.1 \mathrm{~Hz}, 1 \mathrm{H}), 7.19(\mathrm{~d}, J=4.0 \mathrm{~Hz}, 1 \mathrm{H}), 4.90-4.84$ (m, 1H), $4.23(\mathrm{t}, J=9.0 \mathrm{~Hz}, 1 \mathrm{H}), 3.91-3.88(\mathrm{~m}, 1 \mathrm{H}), 3.63(\mathrm{t}, J=5.6 \mathrm{~Hz}, 2 \mathrm{H})$.

${ }^{13} \mathrm{C}$ NMR $\left\{{ }^{1} \mathrm{H}\right\}\left(126 \mathrm{MHz}\right.$, DMSO-d $\left.6,25^{\circ} \mathrm{C}, \delta\right): 160.8,154.1,152.3,142.1,138.4,137.8,133.3$, $132.3,128.4,128.1,120.0,118.8,71.5,47.4,42.2$.

HRMS-ESI (m/z) calc'd. for $\mathrm{C}_{17} \mathrm{H}_{14} \mathrm{~N}_{5} \mathrm{O}_{3} \mathrm{SCINa}^{+}[\mathrm{M}+\mathrm{Na}]^{+}$, 426.039808; found, 426.039950; deviation: $0.33 \mathrm{ppm}$.

Thianthrenium salt TT-16<smiles>CCOc1ccc(C(C)(C)COCc2cccc(Oc3ccccc3)c2)cc1</smiles><smiles>O=S1c2ccccc2Sc2ccccc21</smiles>
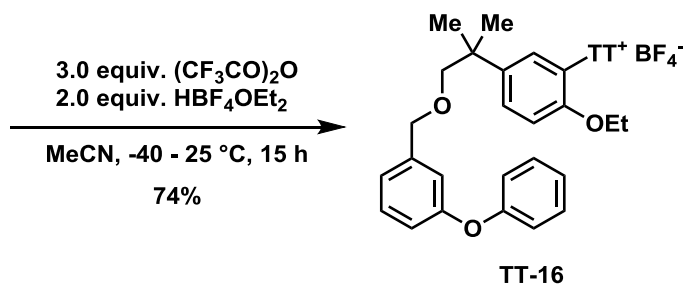

Under ambient atmosphere, a $50 \mathrm{~mL}$ Schlenk-tube equipped with a magnetic stir bar was charged with etofenprox (400 mg, $1.06 \mathrm{mmol}, 1.00$ equiv.), thianthrene S-oxide (247 mg, $1.06 \mathrm{mmol}, 1.00$ equiv.), and $\operatorname{MeCN}(5.3 \mathrm{~mL}, \mathrm{C}=0.20 \mathrm{M})$. After cooling to $-40^{\circ} \mathrm{C}$, trifluoroacetic anhydride $(0.44 \mathrm{~mL}, 0.66 \mathrm{~g}, 3.2 \mathrm{mmol}$, 3.0 equiv.) was added while stirring. $\mathrm{HBF}_{4} \cdot \mathrm{OEt}_{2}(0.29 \mathrm{ml}, 0.35 \mathrm{~g}, 2.1 \mathrm{mmol}, 2.0$ equiv.) was added dropwise. The mixture was stirred at $-40{ }^{\circ} \mathrm{C}$ for $1 \mathrm{~h}$, then at ambient temperature for $14 \mathrm{~h}$. The reaction mixture was added to a saturated aqueous $\mathrm{NaHCO}_{3}$ solution $(50 \mathrm{~mL})$, and the aqueous phase was extracted with DCM (3 $\times 20 \mathrm{~mL}$ ). The organic phase was washed with aqueous $\mathrm{NaBF}_{4}$ solution (30 mL, 10\%), dried over $\mathrm{MgSO}_{4}$, and the solvent was removed under reduced pressure. The residue was purified by chromatography on silica gel eluting with DCM / MeOH (1:0-9:1 (v/v)) to afford $533 \mathrm{mg}(74 \%)$ of TT-16 as off-white foam.

$\mathbf{R}_{\boldsymbol{f}}=0.19(\mathrm{DCM} / \mathrm{MeOH}, 96: 4(\mathrm{v} / \mathrm{v}))$. 


\section{NMR Spectroscopy:}

${ }^{1} \mathrm{H}$ NMR $\left(500 \mathrm{MHz}, \mathrm{CDCl}_{3}, 25^{\circ} \mathrm{C}, \delta\right): 8.35(\mathrm{~d}, J=7.6 \mathrm{~Hz}, 2 \mathrm{H}), 7.78-7.73(\mathrm{~m}, 4 \mathrm{H}), 7.72-7.66(\mathrm{~m}$, 2H), $7.54(\mathrm{dd}, J=8.8,2.3 \mathrm{~Hz}, 1 \mathrm{H}), 7.38-7.32(\mathrm{~m}, 2 \mathrm{H}), 7.32-7.27(\mathrm{~m}, 1 \mathrm{H}), 7.16-7.09(\mathrm{~m}, 1 \mathrm{H})$, $7.03-6.96(\mathrm{~m}, 3 \mathrm{H}), 6.94-6.90(\mathrm{~m}, 2 \mathrm{H}), 6.82-6.78(\mathrm{~m}, 1 \mathrm{H}), 6.60-6.54(\mathrm{~m}, 1 \mathrm{H}), 4.31(\mathrm{~s}, 2 \mathrm{H})$, $4.17(\mathrm{q}, J=7.0 \mathrm{~Hz}, 2 \mathrm{H}), 3.21(\mathrm{~s}, 2 \mathrm{H}), 1.50(\mathrm{t}, J=7.0 \mathrm{~Hz}, 3 \mathrm{H}), 1.09(\mathrm{~s}, 6 \mathrm{H})$.

${ }^{13} \mathrm{C}$ NMR $\left\{{ }^{1} \mathrm{H}\right\}\left(126 \mathrm{MHz}, \mathrm{CDCl}_{3}, 25{ }^{\circ} \mathrm{C}, \delta\right): 157.3,157.1,155.7,141.2,140.6,137.4,135.4,135.4$, 134.6, 134.6, 133.8, 130.1, 129.9, 129.9, 129.8, 127.0, 123.5, 122.0, 118.9, 117.9, 117.5, 113.9, $107.2,79.4,72.6,66.2,38.7,25.9,14.5$.

${ }^{19} \mathrm{~F}$ NMR $\left\{{ }^{1} \mathrm{H}\right\}\left(471 \mathrm{MHz}, \mathrm{CDCl}_{3}, 25^{\circ} \mathrm{C}, \delta\right):-152.5,-152.6$.

HRMS-ESI (m/z) calc'd. for $\mathrm{C}_{37} \mathrm{H}_{35} \mathrm{O}_{3} \mathrm{~S}_{2}{ }^{+}\left[\mathrm{M}-\mathrm{BF}_{4}\right]^{+}$, 591.202215; found, 591.202520; deviation: -0.52 ppm.

\section{Triazole 16}
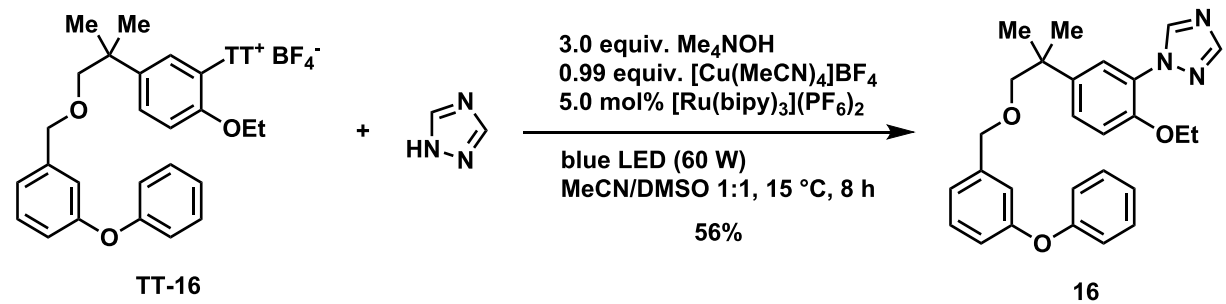

Under ambient atmosphere, a $4 \mathrm{~mL}$ glass-vial was charged with 1,2,4-triazole (62 mg, $0.90 \mathrm{mmol}, 3.0$ equiv.) and tetramethylammonium hydroxide (25\% in methanol, $328 \mathrm{mg}, 0.38 \mathrm{~mL}, 0.90 \mathrm{mmol}, 3.0$ equiv.), and the solvent was removed under reduced pressure. Thianthrenium salt TT-16 (204 mg, $0.301 \mathrm{mmol}, 1.00$ equiv.), $\left[\mathrm{Cu}(\mathrm{MeCN})_{4}\right] \mathrm{BF}_{4}$ (94 mg, $0.30 \mathrm{mmol}, 0.99$ equiv.), [Ru(bipy) $\left.)_{3}\right]\left(\mathrm{PF}_{6}\right)_{2}(13 \mathrm{mg}, 15 \mu \mathrm{mol}, 5.0 \mathrm{~mol} \%$ ), acetonitrile $(0.75 \mathrm{~mL})$ and dimethylsulfoxide $\left(0.75 \mathrm{~mL}, \mathrm{c}_{\text {total }}=0.20 \mathrm{M}\right)$ were added, and the suspension was degassed by bubbling through argon with a cannula for $5 \mathrm{~min}$. While stirring, the suspension was irradiated for $8 \mathrm{~h}$ at $15^{\circ} \mathrm{C}$ using a blue LED $(60 \mathrm{~W})$. The suspension was added to water $(30 \mathrm{~mL})$, and the aqueous phase was extracted with DCM $(3 \times 20 \mathrm{~mL})$. The organic phase was dried over $\mathrm{MgSO}_{4}$, and the solvent was removed under reduced pressure. The residue was purified by chromatography on silica gel eluting with hexanes / EtOAc (1:0-6:4 (v/v)) to afford $75 \mathrm{mg}(56 \%)$ of 16 as colorless oil.

$\mathbf{R}_{\boldsymbol{f}}=0.33$ (hexanes / EtOAc, 6:4 (v/v)).

\section{NMR Spectroscopy:}

${ }^{1} \mathrm{H}$ NMR $\left(500 \mathrm{MHz}, \mathrm{CDCl}_{3}, 25^{\circ} \mathrm{C}, \delta\right): 8.84(\mathrm{~s}, 1 \mathrm{H}), 8.09(\mathrm{~s}, 1 \mathrm{H}), 7.84(\mathrm{~d}, J=2.5 \mathrm{~Hz}, 1 \mathrm{H}), 7.38-$ $7.31(\mathrm{~m}, 3 \mathrm{H}), 7.30-7.24(\mathrm{~m}, 1 \mathrm{H}), 7.15-7.09(\mathrm{~m}, 1 \mathrm{H}), 7.05-6.96(\mathrm{~m}, 4 \mathrm{H}), 6.96-6.92(\mathrm{~m}, 1 \mathrm{H})$, $6.92-6.88(\mathrm{~m}, 1 \mathrm{H}), 4.48(\mathrm{~s}, 2 \mathrm{H}), 4.13(\mathrm{q}, J=7.0 \mathrm{~Hz}, 2 \mathrm{H}), 3.48(\mathrm{~s}, 2 \mathrm{H}), 1.44(\mathrm{t}, J=6.9 \mathrm{~Hz}, 3 \mathrm{H})$, $1.37(\mathrm{~s}, 6 \mathrm{H})$.

${ }^{13} \mathrm{C}$ NMR $\left\{{ }^{1} \mathrm{H}\right\}\left(126 \mathrm{MHz}, \mathrm{CDCl}_{3}, 25{ }^{\circ} \mathrm{C}, \delta\right): 157.4,157.3,151.2,148.3,140.9,140.9,129.8,129.7$, 126.7, 126.0, 123.3, 122.3, 122.1, 119.0, 117.9, 117.8, 112.8, 80.0, 72.9, 64.8, 38.9, 26.2, 14.9. 
HRMS-ESI (m/z) calc'd. for $\mathrm{C}_{27} \mathrm{H}_{29} \mathrm{~N}_{3} \mathrm{O}_{3} \mathrm{Na}^{+}[\mathrm{M}+\mathrm{Na}]^{+}$, 466.210110; found, 466.210130; deviation: -0.04 ppm.

Triazole 17
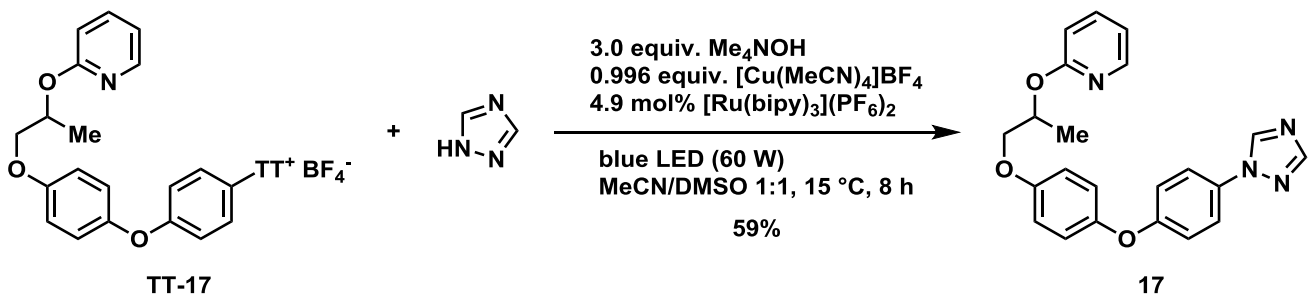

Under ambient atmosphere, a $4 \mathrm{~mL}$ glass-vial was charged with 1,2,4-triazole (69 mg, $1.0 \mathrm{mmol}, 3.0$ equiv.) and tetramethylammonium hydroxide ( $25 \%$ in methanol, $363 \mathrm{mg}, 0.42 \mathrm{~mL}, 1.0 \mathrm{mmol}, 3.0$ equiv.), and the solvent was removed under reduced pressure. Thianthrenium salt TT-17 (207 mg, $0.332 \mathrm{mmol}, 1.00$ equiv.), $\left[\mathrm{Cu}(\mathrm{MeCN})_{4}\right] \mathrm{BF}_{4}\left(104 \mathrm{mg}, 0.331 \mathrm{mmol}, 0.996\right.$ equiv.), [Ru(bipy) $\left.{ }_{3}\right]\left(\mathrm{PF}_{6}\right)_{2}(14 \mathrm{mg}, 16 \mu \mathrm{mol}, 4.9 \mathrm{~mol} \%)$, acetonitrile $(0.83 \mathrm{~mL})$ and dimethylsulfoxide $\left(0.83 \mathrm{~mL}, \mathrm{c}_{\text {total }}=0.20 \mathrm{M}\right)$ were added, and the suspension was degassed by bubbling through argon with a cannula for $5 \mathrm{~min}$. While stirring, the suspension was irradiated for $8 \mathrm{~h}$ at $15^{\circ} \mathrm{C}$ using a blue LED $(60 \mathrm{~W})$. The suspension was added to water $(30 \mathrm{~mL})$, and the aqueous phase was extracted with $\mathrm{DCM}(3 \times 20 \mathrm{~mL})$. The organic phase was dried over $\mathrm{MgSO}_{4}$, and the solvent was removed under reduced pressure. The residue was purified by chromatography on silica gel eluting with hexanes / EtOAc (1:0-0:1 (v/v)) to afford $81 \mathrm{mg}(59 \%)$ of 17 as white solid.

$\mathbf{R} \boldsymbol{f}=0.55$ (hexanes / EtOAc, 2:8 (v/v)).

\section{NMR Spectroscopy:}

${ }^{1} \mathrm{H}$ NMR $\left(500 \mathrm{MHz}, \mathrm{CDCl}_{3}, 25^{\circ} \mathrm{C}, \delta\right): 8.48(\mathrm{~s}, 1 \mathrm{H}), 8.18-8.13(\mathrm{~m}, 1 \mathrm{H}), 8.09(\mathrm{~s}, 1 \mathrm{H}), 7.62-7.53$ $(\mathrm{m}, 3 \mathrm{H}), 7.09-7.02(\mathrm{~m}, 2 \mathrm{H}), 7.02-6.93(\mathrm{~m}, 4 \mathrm{H}), 6.89-6.83(\mathrm{~m}, 1 \mathrm{H}), 6.75(\mathrm{~d}, J=8.4 \mathrm{~Hz}, 1 \mathrm{H})$, $5.66-5.55(\mathrm{~m}, 1 \mathrm{H}), 4.21$ (dd, $J=9.9,5.3 \mathrm{~Hz}, 1 \mathrm{H}), 4.10$ (dd, $J=9.9,4.8 \mathrm{~Hz}, 1 \mathrm{H}), 1.50$ (d, $J=6.5$ $\mathrm{Hz}, 3 \mathrm{H})$.

${ }^{13} \mathrm{C}$ NMR $\left\{{ }^{1} \mathrm{H}\right\}\left(126 \mathrm{MHz}, \mathrm{CDCl}_{3}, 25{ }^{\circ} \mathrm{C}, \delta\right): 163.2,158.6,155.8,152.5,149.5,146.8,140.9,138.7$, $131.7,121.9,121.1,118.3,116.8,116.0,111.7,71.1,69.2,17.1$.

HRMS-ESI (m/z) calc'd. for $\mathrm{C}_{22} \mathrm{H}_{21} \mathrm{~N}_{4} \mathrm{O}_{3}{ }^{+}[\mathrm{M}+\mathrm{H}]^{+}$, 389.160815; found, 389.160770; deviation: +0.12 ppm.

Tetrafluorothianthrenium salt TFT-18<smiles>Cn1cnc2ccccc2c1=O</smiles>

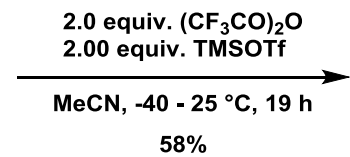

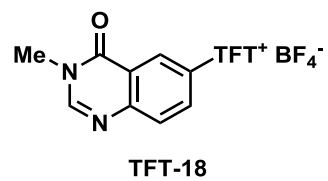

A flame-dried, $100 \mathrm{~mL}$ argon-filled Schlenk-tube equipped with a magnetic stir bar was charged with 3-methylquinazolin-4(3H)-one (510 mg, $3.18 \mathrm{mmol}, 1.00$ equiv.), tetrafluorothianthrene S-oxide (1.00 g, $3.29 \mathrm{mmol}, 1.03$ equiv.), and dry $\mathrm{MeCN}(12 \mathrm{~mL}, \mathrm{c}=0.27 \mathrm{M})$. After cooling to $-40{ }^{\circ} \mathrm{C}$, trifluoroacetic anhydride 
( $0.89 \mathrm{~mL}, 1.3 \mathrm{~g}, 6.4 \mathrm{mmol}, 2.0$ equiv.) was added while stirring. A solution of trimethylsilyl trifluoromethanesulfonate ( $1.15 \mathrm{~mL}, 1.41 \mathrm{~g}, 6.35 \mathrm{mmol}, 2.00$ equiv.) in $5 \mathrm{~mL}$ of dry MeCN was added dropwise over $5 \mathrm{~min}$. The mixture was stirred at $-40^{\circ} \mathrm{C}$ for $1 \mathrm{~h}$, then at ambient temperature for $18 \mathrm{~h}$. The reaction mixture was concentrated under reduced pressure, and subsequently diluted with $\mathrm{DCM}(100 \mathrm{~mL})$. The solution was washed with aqueous $\mathrm{NaBF}_{4}$ solution $(3 \times 100 \mathrm{~mL}, 10 \%)$, and with water $(100 \mathrm{~mL})$. The organic phase was dried over $\mathrm{MgSO}_{4}$, and the solvent was removed under reduced pressure. The residue was purified by chromatography on silica gel eluting with DCM / MeOH $(10: 1(\mathrm{v} / \mathrm{v}))$ to afford $990 \mathrm{mg}(58 \%)$ of TFT-18 as off-white foam.

$\mathbf{R}_{\boldsymbol{f}}=0.12(\mathrm{DCM} / \mathrm{MeOH}, 10: 1(\mathrm{v} / \mathrm{v}))$.

\section{NMR Spectroscopy:}

${ }^{1} \mathrm{H}$ NMR $\left(500 \mathrm{MHz}, \mathrm{MeCN}_{-} \mathrm{d}_{3}, 25^{\circ} \mathrm{C}, \delta\right): 8.47$ (dd, $\left.J=9.0,7.2 \mathrm{~Hz}, 2 \mathrm{H}\right), 8.22(\mathrm{~s}, 1 \mathrm{H}), 8.04(\mathrm{~d}, J=$ $2.6 \mathrm{~Hz}, 1 \mathrm{H}), 7.97(\mathrm{dd}, J=9.9,7.0 \mathrm{~Hz}, 2 \mathrm{H}), 7.75-7.73(\mathrm{~m}, 1 \mathrm{H}), 7.50-7.48(\mathrm{~m}, 1 \mathrm{H}), 3.46(\mathrm{~s}, 3 \mathrm{H})$.

${ }^{13} \mathrm{C}$ NMR $\left\{{ }^{1} \mathrm{H}\right\}\left(126 \mathrm{MHz}, \mathrm{MeCN}-\mathrm{d}_{3}, 25^{\circ} \mathrm{C}, \delta\right): 160.6,154.8(\mathrm{dd}, J=262.3,13.1 \mathrm{~Hz}), 152.5,152.4$, $151.6(\mathrm{dd}, J=255.9,13.6 \mathrm{~Hz}), 135.4(\mathrm{dd}, J=8.6,3.9 \mathrm{~Hz}), 133.2,131.0,128.7,125.6$ (dd, $J=$ 22.1, $2.6 \mathrm{~Hz}$ ), 121.3 (d, $J=21.9 \mathrm{~Hz}), 121.3,121.1,115.5$ (dd, $J=7.2,3.4 \mathrm{~Hz}), 34.7$.

${ }^{19}$ F NMR $\left\{{ }^{1} \mathrm{H}\right\}\left(471 \mathrm{MHz}, \mathrm{MeCN}-\mathrm{d}_{3}, 25^{\circ} \mathrm{C}, \delta\right):-124.8(\mathrm{~d}, J=20.2 \mathrm{~Hz}),-133.3(\mathrm{~d}, J=20.3 \mathrm{~Hz}),-$ 151.4, - 151.4 .

HRMS-ESI (m/z) calc'd. for $\mathrm{C}_{21} \mathrm{H}_{11} \mathrm{~F}_{4} \mathrm{~N}_{2} \mathrm{OS}_{2}{ }^{+}\left[\mathrm{M}-\mathrm{BF}_{4}\right]^{+}$, 447.024347; found, 447.024750; deviation: $-0.90 \mathrm{ppm}$.

\section{Aniline 18}
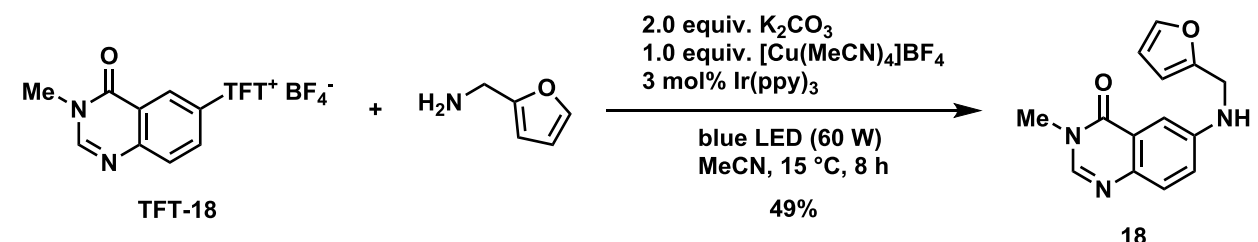

18

Under ambient atmosphere, a $4 \mathrm{~mL}$ glass-vial was charged with tetrafluorothianthrenium salt TFT-18 (160 mg, 0.299 mmol, 1.00 equiv.), [Cu(MeCN) $]_{B} \mathrm{BF}_{4}\left(94 \mathrm{mg}, 0.30 \mathrm{mmol}, 1.0\right.$ equiv.), $\operatorname{lr}(\mathrm{ppy})_{3}(6 \mathrm{mg}, 9 \mu \mathrm{mol}, 3 \mathrm{~mol} \%)$, $\mathrm{K}_{2} \mathrm{CO}_{3}(83 \mathrm{mg}, 0.60 \mathrm{mmol}, 2.0$ equiv.), and acetonitrile $(2 \mathrm{~mL}, \mathrm{C}=0.1 \mathrm{M})$. Furfurylamine $(40 \mu \mathrm{L}, 44 \mathrm{mg}$, $0.45 \mathrm{mmol}, 1.5$ equiv.) was added, and the suspension was degassed by bubbling through argon with a cannula for $5 \mathrm{~min}$. While stirring, the suspension was irradiated for $8 \mathrm{~h}$ at $15^{\circ} \mathrm{C}$ using a blue LED (60 W). The solvent was removed under reduced pressure. The residue was purified by chromatography on silica gel eluting with hexanes / EtOAc (1:0-1:5 (v/v)) to afford $74 \mathrm{mg}(49 \%)$ of 18 as off-white solid.

$\mathbf{R}_{\boldsymbol{f}}=0.21$ (hexanes / EtOAc, $2: 8(\mathrm{v} / \mathrm{v})$ ).

\section{NMR Spectroscopy:}


${ }^{1} \mathrm{H}$ NMR $\left(500 \mathrm{MHz}, \mathrm{CDCl}_{3}, 25^{\circ} \mathrm{C}, \delta\right): 7.84(\mathrm{~s}, 1 \mathrm{H}), 7.54-7.52(\mathrm{~m}, 1 \mathrm{H}), 7.43-7.42(\mathrm{~m}, 1 \mathrm{H}), 7.37$

$-7.36(\mathrm{~m}, 1 \mathrm{H}), 7.10-7.07(\mathrm{~m}, 1 \mathrm{H}), 6.32-6.28(\mathrm{~m}, 2 \mathrm{H}), 4.41(\mathrm{~s}, 3 \mathrm{H}), 3.56(\mathrm{~s}, 3 \mathrm{H})$.

${ }^{13} \mathrm{C}$ NMR $\left\{{ }^{1} \mathrm{H}\right\}\left(126 \mathrm{MHz}, \mathrm{CDCl}_{3}, 25{ }^{\circ} \mathrm{C}, \delta\right): 161.7,151.8,146.9,143.4,142.3,141.0,128.7,123.2$, 122.2, 110.5, 107.7, 105.4, 41.4, 34.1.

HRMS-ESI (m/z) calc'd. for $\mathrm{C}_{14} \mathrm{H}_{14} \mathrm{~N}_{3} \mathrm{O}_{2}{ }^{+}[\mathrm{M}+\mathrm{H}]^{+}$, 256.108051; found, 256.108070; deviation: -0.07 ppm.

Aniline 19
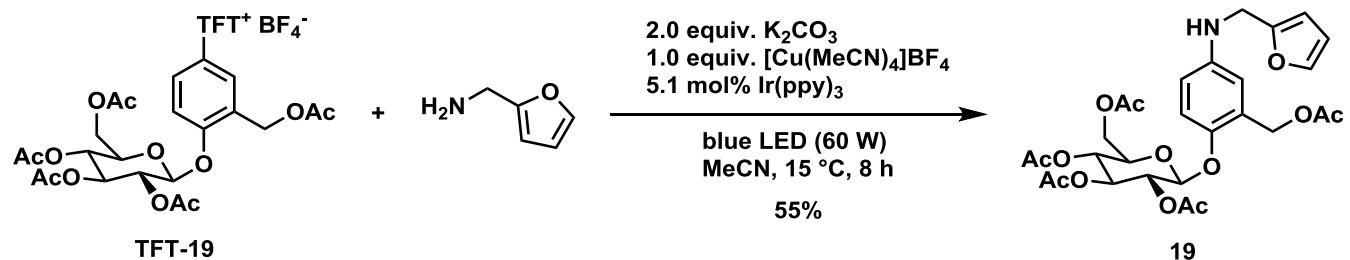

Under ambient atmosphere, a $4 \mathrm{~mL}$ glass-vial was charged with tetrafluorothianthrenium salt TFT-19 (261 mg,

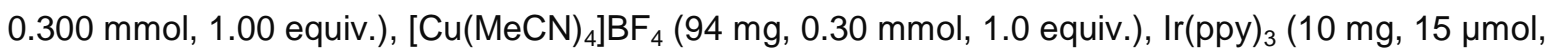

$5.1 \mathrm{~mol} \%), \mathrm{K}_{2} \mathrm{CO}_{3}$ (83 mg, $0.60 \mathrm{mmol}, 2.0$ equiv.), and acetonitrile $(1.5 \mathrm{~mL}, \mathrm{c}=0.20 \mathrm{M})$. Furfurylamine $(40 \mu \mathrm{L}$, $44 \mathrm{mg}, 0.45 \mathrm{mmol}, 1.5$ equiv.) was added, and the suspension was degassed by bubbling through argon with a cannula for $5 \mathrm{~min}$. While stirring, the suspension was irradiated for $8 \mathrm{~h}$ at $15^{\circ} \mathrm{C}$ using a blue LED (60 W). The solvent was removed under reduced pressure. The residue was purified by chromatography on silica gel eluting with hexanes / EtOAc (1:0-2:8 (v/v)) to afford $97 \mathrm{mg}(55 \%)$ of 19 as orange oil.

$\mathbf{R}_{\boldsymbol{f}}=0.28($ hexanes / EtOAc, 1:1 (v/v)).

\section{NMR Spectroscopy:}

${ }^{1} \mathrm{H}$ NMR $\left(500 \mathrm{MHz}, \mathrm{CDCl}_{3}, 25^{\circ} \mathrm{C}, \delta\right): 7.38(\mathrm{~s}, 1 \mathrm{H}), 7.00(\mathrm{~d}, J=8.7 \mathrm{~Hz}, 1 \mathrm{H}), 6.67(\mathrm{~d}, J=2.9 \mathrm{~Hz}$, $1 \mathrm{H}), 6.57(\mathrm{dd}, J=8.7,1.8 \mathrm{~Hz}, 1 \mathrm{H}), 6.36-6.32(\mathrm{~m}, 1 \mathrm{H}), 6.26-6.22(\mathrm{~m}, 1 \mathrm{H}), 5.30-5.25(\mathrm{~m}, 2 \mathrm{H})$, $5.21-5.15(\mathrm{~m}, 1 \mathrm{H}), 5.09(\mathrm{~d}, J=12.8 \mathrm{~Hz}, 1 \mathrm{H}), 5.01(\mathrm{~d}, J=12.7 \mathrm{~Hz}, 1 \mathrm{H}), 4.92-4.88(\mathrm{~m}, 1 \mathrm{H}), 4.30$ $(\mathrm{s}, 3 \mathrm{H}), 4.27(\mathrm{~d}, J=5.2 \mathrm{~Hz}, 1 \mathrm{H}), 4.22-4.17(\mathrm{~m}, 1 \mathrm{H}), 4.01(\mathrm{~s}, 1 \mathrm{H}), 3.82-3.75(\mathrm{~m}, 1 \mathrm{H}), 2.13(\mathrm{~s}$, $3 \mathrm{H}), 2.11(\mathrm{~s}, 3 \mathrm{H}), 2.10(\mathrm{~s}, 3 \mathrm{H}), 2.06(\mathrm{~s}, 3 \mathrm{H}), 2.05(\mathrm{~s}, 3 \mathrm{H})$.

${ }^{13} \mathrm{C}$ NMR $\left\{{ }^{1} \mathrm{H}\right\}\left(126 \mathrm{MHz}, \mathrm{CDCl}_{3}, 25{ }^{\circ} \mathrm{C}, \delta\right): 170.9,170.7,170.4,169.5,152.6,147.1,144.4,142.1$, 127.8, 121.3, 119.4, 114.1, 113.4, 110.5, 107.2, 101.0, 72.9, 72.0, 71.3, 68.5, 62.0, 61.4, 41.9, $21.1,20.8,20.8,20.7$.

HRMS-ESI (m/z) calc'd. for $\mathrm{C}_{28} \mathrm{H}_{33} \mathrm{NO}_{13} \mathrm{Na}^{+}[\mathrm{M}+\mathrm{Na}]^{+}$, 614.184413; found, 614.184490; deviation: -0.13 ppm.

Tetrafluorothianthrenium salt TFT-20
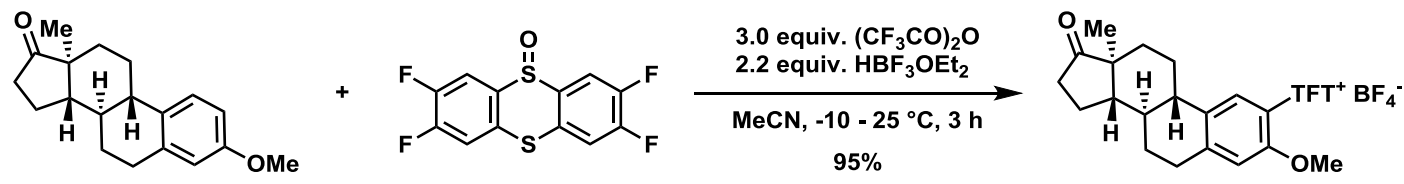

TFT-20 
Under ambient atmosphere, a $100 \mathrm{~mL}$ Schlenk-tube equipped with a magnetic stir bar was charged with estrone methyl ether (500 mg, $1.76 \mathrm{mmol}, 1.00$ equiv.), tetrafluorothianthrene S-oxide (552 mg, $1.81 \mathrm{mmol}$, 1.03 equiv.), and $\mathrm{MeCN}(10 \mathrm{~mL}, \mathrm{C}=0.18 \mathrm{M})$. After cooling to $-10^{\circ} \mathrm{C}$, trifluoroacetic anhydride $(0.73 \mathrm{~mL}, 1.1 \mathrm{~g}$, $5.3 \mathrm{mmol}, 3.0$ equiv.) was added while stirring. $\mathrm{HBF}_{4} \cdot \mathrm{OEt}_{2}(0.53 \mathrm{~mL}, 0.63 \mathrm{~g}, 3.9 \mathrm{mmol}, 2.2$ equiv.) was added dropwise. The mixture was stirred at $-10^{\circ} \mathrm{C}$ for $2 \mathrm{~h}$, then at ambient temperature for $1 \mathrm{~h}$. The reaction mixture was concentrated under reduced pressure, and subsequently diluted with DCM $(35 \mathrm{~mL})$. The solution was poured onto a saturated aqueous $\mathrm{NaHCO}_{3}$ solution $(25 \mathrm{~mL})$, and the layers were separated. The organic phase was washed with aqueous $\mathrm{NaBF}_{4}$ solution $(2 \times 35 \mathrm{~mL}, 10 \%)$, and with water $(30 \mathrm{~mL})$. The organic phase was dried over $\mathrm{MgSO}_{4}$, and the solvent was removed under reduced pressure. The residue was purified by chromatography on silica gel eluting with $\mathrm{DCM} / \mathrm{MeOH}(1: 0-10: 1(\mathrm{v} / \mathrm{v}))$ to afford $1.10 \mathrm{~g}(95 \%)$ of TFT-20 as colorless foam.

$\mathbf{R}_{\boldsymbol{f}}=0.19(\mathrm{DCM} / \mathrm{MeOH}, 20: 1(\mathrm{v} / \mathrm{v}))$.

\section{NMR Spectroscopy:}

${ }^{1} \mathrm{H}$ NMR $\left(500 \mathrm{MHz}, \mathrm{CDCl}_{3}, 25^{\circ} \mathrm{C}, \delta\right): 8.31-8.28(\mathrm{~m}, 2 \mathrm{H}), 7.78$ (ddd, $\left.\mathrm{J}=20.6,8.9,6.8 \mathrm{~Hz}, 2 \mathrm{H}\right)$, $6.87(\mathrm{~s}, 1 \mathrm{H}), 6.55(\mathrm{~s}, 1 \mathrm{H}), 3.94(\mathrm{~s}, 3 \mathrm{H}), 2.98-2.86(\mathrm{~m}, 2 \mathrm{H}), 2.48-2.42(\mathrm{~m}, 1 \mathrm{H}), 2.10-2.05(2 \mathrm{H})$, $2.03-1.97(\mathrm{~m}, 2 \mathrm{H}), 1.86-1.83(\mathrm{~m}, 1 \mathrm{H}), 1.78-1.74(\mathrm{~m}, 1 \mathrm{H}), 1.62-1.53(\mathrm{~m}, 1 \mathrm{H}), 1.50-1.34(\mathrm{~m}$, $4 \mathrm{H}), 1.30-1.22(\mathrm{~m}, 1 \mathrm{H}), 0.83(\mathrm{~s}, 3 \mathrm{H})$.

${ }^{13} \mathrm{C}$ NMR $\left\{{ }^{1} \mathrm{H}\right\}\left(126 \mathrm{MHz}, \mathrm{CDCl}_{3}, 25^{\circ} \mathrm{C}, \delta\right): 220.1,155.9,153.5$ (ddd, $\left.J=259.1,21.9,13.0 \mathrm{~Hz}\right)$, 150.7 (ddd, $J=259.1,21.9,13.0 \mathrm{~Hz}$ ), 147.5, 134.5, $133.94-113.85(\mathrm{~m}), 126.0,124.5$ (dd, $J=$ $39.3,21.6 \mathrm{~Hz}), 119.3(\mathrm{dd}, J=21.1,16.7 \mathrm{~Hz}), 114.4-113.6(\mathrm{~m}), 103.9,57.3,50.1,47.8,43.4$, $37.6,35.8,31.3,30.0,25.7,25.6,21.5,13.8$.

${ }^{19} \mathrm{~F}$ NMR $\left\{{ }^{1} \mathrm{H}\right\}\left(471 \mathrm{MHz}, \mathrm{CDCl}_{3}, 25^{\circ} \mathrm{C}, \delta\right):-122.9(\mathrm{dd}, J=226.0,21.8 \mathrm{~Hz}),-130.5(\mathrm{dd}, J=203.3$, $21.3 \mathrm{~Hz}),-150.7,-150.7$.

HRMS-ESI (m/z) calc'd. for $\mathrm{C}_{31} \mathrm{H}_{27} \mathrm{~F}_{4} \mathrm{O}_{2} \mathrm{~S}_{2}{ }^{+}\left[\mathrm{M}-\mathrm{BF}_{4}\right]^{+}, 571.138130$; found 571.138314; deviation: +0.3 ppm.

Aniline 20
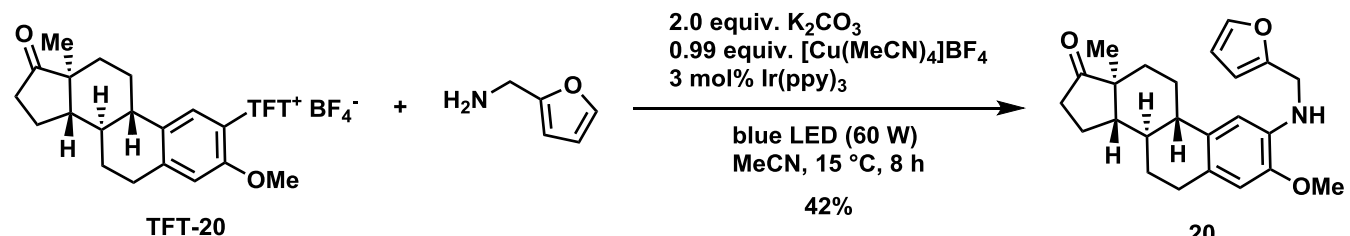

20

Under ambient atmosphere, a $4 \mathrm{~mL}$ glass-vial was charged with tetrafluorothianthrenium salt TFT-20 (198 mg, $0.301 \mathrm{mmol}, 1.00$ equiv.), [Cu(MeCN$\left.)_{4}\right]_{B F}$ (94 mg, $0.30 \mathrm{mmol}, 0.99$ equiv.), $\operatorname{lr}(\mathrm{ppy})_{3}(6 \mathrm{mg}, 9$ umol, $3 \mathrm{~mol} \%)$, $\mathrm{K}_{2} \mathrm{CO}_{3}$ (83 mg, $0.60 \mathrm{mmol}, 2.0$ equiv.), and acetonitrile $(2 \mathrm{~mL}, \mathrm{C}=0.2 \mathrm{M})$. Furfurylamine $(40 \mu \mathrm{L}, 44 \mathrm{mg}$, $0.45 \mathrm{mmol}, 1.5$ equiv.) was added, and the suspension was degassed by bubbling through argon with a cannula for $5 \mathrm{~min}$. While stirring, the suspension was irradiated for $8 \mathrm{~h}$ at $15^{\circ} \mathrm{C}$ using a blue LED $(60 \mathrm{~W})$. The 
solvent was removed under reduced pressure. The residue was purified by chromatography on silica gel eluting with hexanes / EtOAc $(1: 0-6: 1(\mathrm{v} / \mathrm{v}))$ to afford $48 \mathrm{mg}(42 \%)$ of 20 as off-white solid.

$\mathbf{R}_{\boldsymbol{f}}=0.38($ hexanes / EtOAc, 8:2 (v/v)).

\section{NMR Spectroscopy:}

${ }^{1} \mathrm{H}$ NMR $\left(500 \mathrm{MHz}, \mathrm{CDCl}_{3}, 25^{\circ} \mathrm{C}, \delta\right): 7.38-7.37(\mathrm{~m}, 1 \mathrm{H}), 6.62(\mathrm{~s}, 1 \mathrm{H}), 6.51(\mathrm{~s}, 1 \mathrm{H}), 6.33-6.32$ (m, 1H), $6.25-6.24(\mathrm{~m}, 1 \mathrm{H}), 4.46(\mathrm{br} \mathrm{s}, 1 \mathrm{H}), 4.32(\mathrm{~s}, 1 \mathrm{H}), 3.81(\mathrm{~s}, 3 \mathrm{H}), 2.89-2.77(\mathrm{~m}, 2 \mathrm{H}), 2.53$ $-2.47(\mathrm{~m}, 1 \mathrm{H}), 2.37-2.31(\mathrm{~m}, 1 \mathrm{H}), 2.27-2.23(\mathrm{~m}, 1 \mathrm{H}), 2.18-2.10(\mathrm{~m}, 1 \mathrm{H}), 2.08-1.94(\mathrm{~m}, 3 \mathrm{H})$, $1.67-1.37(\mathrm{~m}, 6 \mathrm{H}), 0.91(\mathrm{~s}, 3 \mathrm{H})$.

${ }^{13} \mathrm{C}$ NMR $\left\{{ }^{1} \mathrm{H}\right\}\left(126 \mathrm{MHz}, \mathrm{CDCl}_{3}, 25{ }^{\circ} \mathrm{C}, \delta\right): 221.2,153.2,145.6,141.9,135.7,131.8,124.9,110.4$, $110.3,107.8,107.0,55.6,50.6,48.2,44.5,41.6,38.7,36.0,31.8,29.3,27.0,26.2,21.7,14.0$.

HRMS-ESI (m/z) calc'd. for $\mathrm{C}_{24} \mathrm{H}_{30} \mathrm{NO}_{3}{ }^{+}[\mathrm{M}+\mathrm{H}]^{+}$, 380.222019; found, 380.222030; deviation: -0.03 ppm.

Thianthrenium salt TT-21

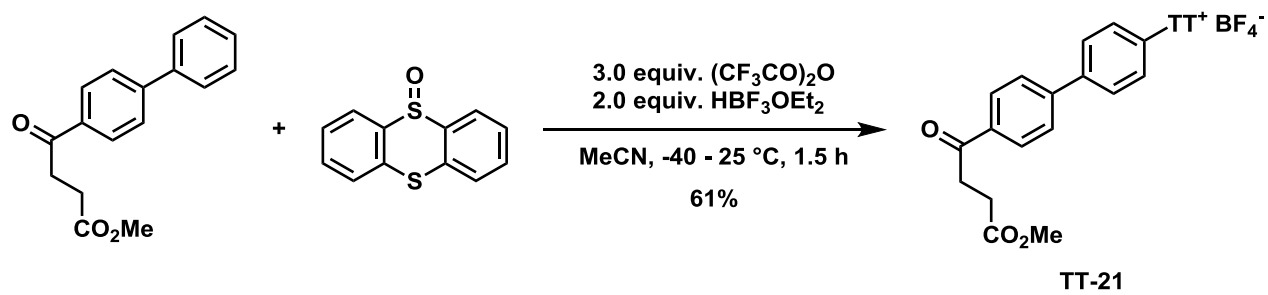

Under ambient atmosphere, a $50 \mathrm{~mL}$ Schlenk-tube equipped with a magnetic stir bar was charged with fenbufen methyl ester (443 mg, $1.65 \mathrm{mmol}, 1.00$ equiv.), thianthrene S-oxide (384 mg, $1.65 \mathrm{mmol}$, 1.00 equiv.), and $\mathrm{MeCN}(8.3 \mathrm{~mL}, \mathrm{c}=0.20 \mathrm{M})$. After cooling to $-40^{\circ} \mathrm{C}$, trifluoroacetic anhydride $(0.69 \mathrm{~mL}, 1.0 \mathrm{~g}$, $5.0 \mathrm{mmol}, 3.0$ equiv. $)$ was added while stirring. $\mathrm{HBF}_{4} \cdot \mathrm{OEt}_{2}(0.45 \mathrm{ml}, 0.54 \mathrm{~g}, 3.3 \mathrm{mmol}, 2.0$ equiv. $)$ was added dropwise. The mixture was stirred at $-40{ }^{\circ} \mathrm{C}$ for $30 \mathrm{~min}$, then at ambient temperature for $1 \mathrm{~h}$. The reaction mixture was added to a saturated aqueous $\mathrm{NaHCO}_{3}$ solution $(50 \mathrm{~mL})$, and the aqueous phase was extracted with $\mathrm{DCM}(3 \times 20 \mathrm{~mL})$. The organic phase was washed with aqueous $\mathrm{NaBF}_{4}$ solution $(30 \mathrm{~mL}, 10 \%)$, dried over $\mathrm{MgSO}_{4}$, and the solvent was removed under reduced pressure. The residue was purified by chromatography on silica gel eluting with $\mathrm{DCM} / \mathrm{MeOH}(1: 0-9: 1(\mathrm{v} / \mathrm{v}))$ to afford $570 \mathrm{mg}(61 \%)$ of TT-21 as yellowish foam.

$\mathbf{R}_{\boldsymbol{f}}=0.27(\mathrm{DCM} / \mathrm{MeOH}, 94: 6(\mathrm{v} / \mathrm{v}))$.

\section{NMR Spectroscopy:}

${ }^{1} \mathrm{H}$ NMR $\left(500 \mathrm{MHz}, \mathrm{CDCl}_{3}, 25^{\circ} \mathrm{C}, \delta\right): 8.57$ (d, $\left.J=7.9 \mathrm{~Hz}, 2 \mathrm{H}\right), 7.99(\mathrm{~d}, J=8.5 \mathrm{~Hz}, 2 \mathrm{H}), 7.87-$ $7.80(\mathrm{~m}, 5 \mathrm{H}), 7.81-7.74(\mathrm{~m}, 2 \mathrm{H}), 7.61(\mathrm{~d}, J=8.7 \mathrm{~Hz}, 2 \mathrm{H}), 7.53(\mathrm{~d}, J=8.5 \mathrm{~Hz}, 2 \mathrm{H}), 7.24$ (d, $J=$ $8.7 \mathrm{~Hz}, 2 \mathrm{H}), 3.68(\mathrm{~s}, 3 \mathrm{H}), 3.30(\mathrm{t}, J=6.5 \mathrm{~Hz}, 2 \mathrm{H}), 2.75(\mathrm{t}, J=6.5 \mathrm{~Hz}, 2 \mathrm{H})$.

${ }^{13} \mathrm{C}$ NMR $\left\{{ }^{1} \mathrm{H}\right\}\left(126 \mathrm{MHz}, \mathrm{CDCl}_{3}, 25{ }^{\circ} \mathrm{C}, \delta\right): 197.6,173.6,170.3,148.9,146.1,134.0,128.8,128.2$, $128.2,126.0,113.0,98.9,80.8,68.2,66.3,51.9,42.7,40.8,36.4,35.5,33.4,30.3,28.3,28.2$, 
19.9.

${ }^{19} \mathrm{~F}$ NMR $\left\{{ }^{1} \mathrm{H}\right\}\left(471 \mathrm{MHz}, \mathrm{CDCl}_{3}, 25^{\circ} \mathrm{C}, \delta\right):-150.7,-150.8$.

HRMS-ESI (m/z) calc'd. for $\mathrm{C}_{29} \mathrm{H}_{23} \mathrm{O}_{3} \mathrm{~S}_{2}{ }^{+}\left[\mathrm{M}-\mathrm{BF}_{4}\right]^{+}$, 483.108315; found 483.108380; deviation: -0.13 ppm.

Aniline 21
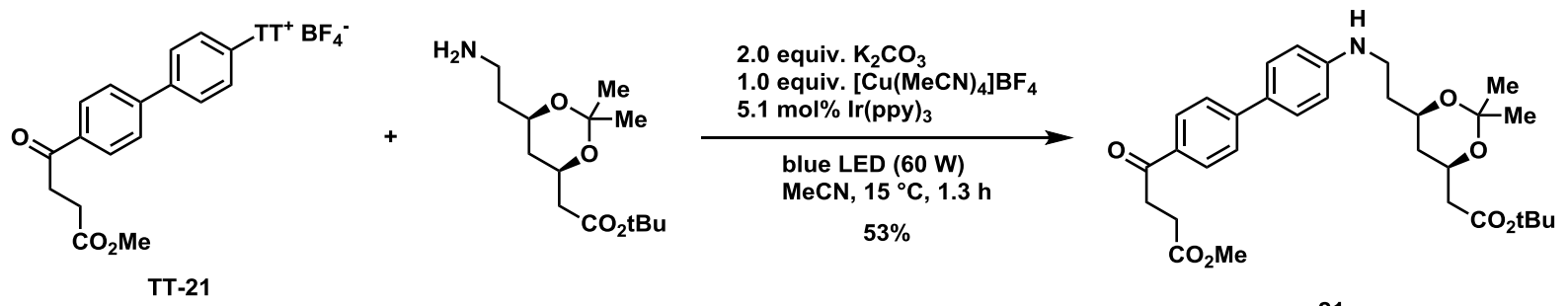

21

Under ambient atmosphere, a $4 \mathrm{~mL}$ glass-vial was charged with thianthrenium salt TT-21 (171 mg, 0.300 mmol, 1.00 equiv.), [Cu(MeCN) ${ }_{4} \mathrm{BF}_{4}$ (94 mg, $0.30 \mathrm{mmol}, 1.0$ equiv.), $\operatorname{lr}(\mathrm{ppy})_{3}(10 \mathrm{mg}, 15 \mu \mathrm{mol}$, $5.1 \mathrm{~mol} \%), \mathrm{K}_{2} \mathrm{CO}_{3}$ (83 mg, $0.60 \mathrm{mmol}, 2.0$ equiv.), and acetonitrile $(1.5 \mathrm{~mL}, \mathrm{c}=0.20 \mathrm{M})$. Tert-butyl 2((4R,6R)-6-(2-aminoethyl)-2,2-dimethyl-1,3-dioxan-4-yl)acetate (123 mg, $0.450 \mathrm{mmol}, 1.50$ equiv.) was added, and the suspension was degassed by bubbling through argon with a cannula for $5 \mathrm{~min}$. While stirring, the suspension was irradiated for $1 \mathrm{~h} 20 \mathrm{~min}$ at $15^{\circ} \mathrm{C}$ using a blue LED $(60 \mathrm{~W})$. The suspension was added to an aqueous saturated $\mathrm{NaHCO}_{3}$ solution $(20 \mathrm{~mL})$, and the aqueous phase was extracted with $\mathrm{DCM}(3 \times 20 \mathrm{~mL})$. The organic phase was dried over $\mathrm{MgSO}_{4}$, and the solvent was removed under reduced pressure. The residue was purified by chromatography on silica gel eluting with hexanes / EtOAc $(1: 0-2: 8(\mathrm{v} / \mathrm{v}))$ to afford $85 \mathrm{mg}(53 \%)$ of 21 as yellow solid.

$\mathbf{R} \boldsymbol{f}=0.50($ hexanes / EtOAc, 6:4 (v/v)).

\section{NMR Spectroscopy:}

${ }^{1} \mathrm{H}$ NMR $\left(500 \mathrm{MHz}, \mathrm{CDCl}_{3}, 25^{\circ} \mathrm{C}, \delta\right): 8.00(\mathrm{~d}, J=8.5 \mathrm{~Hz}, 2 \mathrm{H}), 7.62(\mathrm{~d}, J=8.5 \mathrm{~Hz}, 2 \mathrm{H}), 7.49(\mathrm{~d}, J$ $=8.7 \mathrm{~Hz}, 2 \mathrm{H}), 6.66(\mathrm{~d}, J=8.7 \mathrm{~Hz}, 2 \mathrm{H}), 4.27(\mathrm{dtd}, J=11.5,6.6,2.4 \mathrm{~Hz}, 1 \mathrm{H}), 4.05$ (dddd, $J=11.6$, 7.1, 4.7, $2.5 \mathrm{~Hz}, 1 \mathrm{H}), 3.71(\mathrm{~s}, 3 \mathrm{H}), 3.36-3.29(\mathrm{~m}, 3 \mathrm{H}), 3.29-3.21(\mathrm{~m}, 1 \mathrm{H}), 2.78(\mathrm{t}, J=6.7 \mathrm{~Hz}$, 2H), $2.44(\mathrm{dd}, J=15.2,6.9 \mathrm{~Hz}, 1 \mathrm{H}), 2.31(\mathrm{dd}, J=15.2,6.2 \mathrm{~Hz}, 1 \mathrm{H}), 1.84-1.76(\mathrm{~m}, 2 \mathrm{H}), 1.57$ (dt, $J=12.7,2.5 \mathrm{~Hz}, 1 \mathrm{H}), 1.46(\mathrm{~s}, 3 \mathrm{H}), 1.45(\mathrm{~s}, 9 \mathrm{H}), 1.42(\mathrm{~s}, 3 \mathrm{H}), 1.31-1.27(\mathrm{~m}, 1 \mathrm{H})$.

${ }^{13} \mathrm{C}$ NMR $\left\{{ }^{1} \mathrm{H}\right\}\left(126 \mathrm{MHz}, \mathrm{CDCl}_{3}, 25{ }^{\circ} \mathrm{C}, \delta\right): 197.6,173.6,170.4,148.9,146.1,134.1,128.8,128.3$, $126.0,113.1,98.9,80.8,68.2,66.3,52.0,42.8,40.9,36.4,35.5,33.4,30.3,28.2,19.9$.

HRMS-ESI (m/z) calc'd. for $\mathrm{C}_{31} \mathrm{H}_{41} \mathrm{NO}_{7} \mathrm{Na}^{+}[\mathrm{M}+\mathrm{Na}]^{+}$, 562.277523; found, 562.277710; deviation: -0.33 ppm. 
Thianthrenium salt TT-22

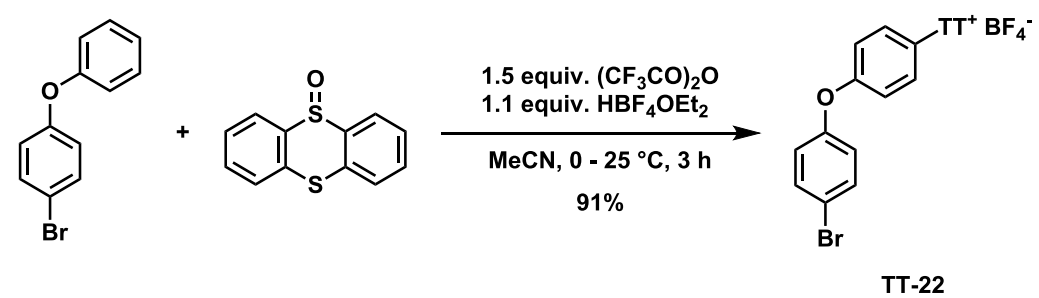

Under ambient atmosphere, a $50 \mathrm{~mL}$ Schlenk-tube equipped with a magnetic stir bar was charged with bromodiphenylether (2.491 g, $10.00 \mathrm{mmol}, 1.000$ equiv.), thianthrene S-oxide (2.323 g, $10.00 \mathrm{mmol}$, 0.9999 equiv.), and $\mathrm{MeCN}(15 \mathrm{~mL}, \mathrm{C}=0.67 \mathrm{M})$. $\mathrm{HBF}_{4} \cdot \mathrm{OEt}_{2}(1.5 \mathrm{ml}, 1.8 \mathrm{~g}, 11 \mathrm{mmol}, 1.1$ equiv.) was added. After cooling to $0{ }^{\circ} \mathrm{C}$, trifluoroacetic anhydride $(2.1 \mathrm{~mL}, 3.2 \mathrm{~g}, 15 \mathrm{mmol}, 1.5$ equiv.) was added while stirring. The mixture was allowed to warm to ambient temperature, and then was stirred for $3 \mathrm{~h}$. The reaction mixture was concentrated under reduced pressure, and subsequently diluted with DCM (30 mL). The solution was washed with water $(40 \mathrm{~mL})$, and with aqueous $\mathrm{NaBF}_{4}$ solution $(3 \times 40 \mathrm{~mL}, 10 \%)$. The organic phase was dried over $\mathrm{MgSO}_{4}$, and the solvent was removed under reduced pressure giving a colorless foam. The foam was dissolved in DCM $(5 \mathrm{~mL})$, and precipitated by addition of MTBE $(100 \mathrm{~mL})$ resulting in a viscous oil. Drying under high vacuum afforded $5.475 \mathrm{~g}(91 \%)$ of TT-22 in a purity of $92 \%$ (containing $8 \% \mathrm{MTBE}$ ) as white solid. Further drying afforded an analytically pure sample.

$\mathbf{R} \boldsymbol{f}=0.47(\mathrm{DCM} / \mathrm{MeOH}, 94: 6(\mathrm{v} / \mathrm{v}))$.

\section{NMR Spectroscopy:}

${ }^{1} \mathrm{H}$ NMR $\left(500 \mathrm{MHz}, \mathrm{DMSO}-\mathrm{d}_{6}, 25^{\circ} \mathrm{C}, \delta\right): 8.53(\mathrm{dd}, J=8.1,1.4 \mathrm{~Hz}, 2 \mathrm{H}), 8.07$ (dd, $J=8.0,1.3 \mathrm{~Hz}$, 2H), $7.93-7.89(\mathrm{~m}, 2 \mathrm{H}), 7.84$ (ddd, $J=7.9,7.5,1.4 \mathrm{~Hz}, 2 \mathrm{H}), 7.62-7.58(\mathrm{~m}, 2 \mathrm{H}), 7.30-7.26$ (m, $2 \mathrm{H}), 7.16-7.12(\mathrm{~m}, 2 \mathrm{H}), 7.06-7.02(\mathrm{~m}, 2 \mathrm{H})$.

${ }^{13} \mathrm{C}$ NMR $\left\{{ }^{1} \mathrm{H}\right\}\left(126 \mathrm{MHz}, \mathrm{DMSO}-\mathrm{d}_{6}, 25^{\circ} \mathrm{C}, \delta\right): 160.4,153.9,135.3,135.2,134.8,133.3,130.8$, $130.3,129.7,122.3,119.6,119.3,117.8,117.2$.

${ }^{19}$ F NMR $\left\{{ }^{1} \mathrm{H}\right\}\left(471 \mathrm{MHz}, \mathrm{DMSO}-\mathrm{d}_{6}, 25^{\circ} \mathrm{C}, \delta\right):-148.2,-148.2$.

HRMS-ESI (m/z) calc'd. for $\mathrm{C}_{24} \mathrm{H}_{16} \mathrm{OS}_{2} \mathrm{Br}^{+}\left[\mathrm{M}-\mathrm{BF}_{4}\right]^{+}$, 462.982061; found, 462.981640; deviation: +0.91 ppm.

Aniline 22

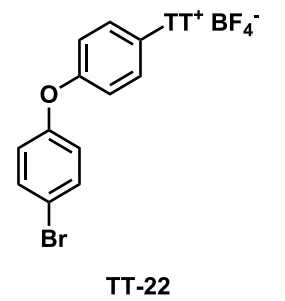

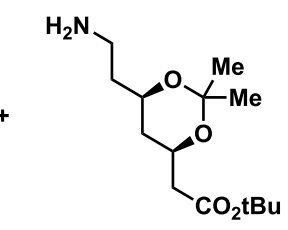

TT-22

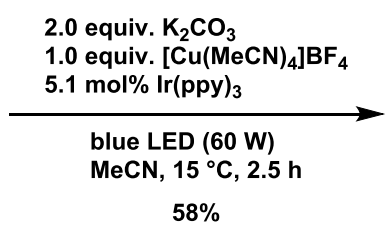

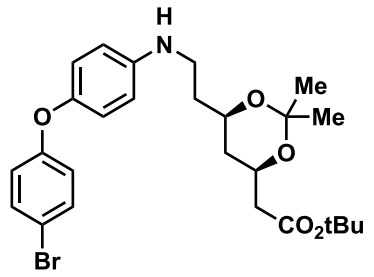

22

Under ambient atmosphere, a $4 \mathrm{~mL}$ glass-vial was charged with thianthrenium salt TT-22 (165 mg, 
0.299 mmol, 1.00 equiv.), [Cu(MeCN) $]_{B} \mathrm{BF}_{4}(94 \mathrm{mg}, 0.30 \mathrm{mmol}, 1.0$ equiv.), Ir(ppy) 3 (10 mg, $15 \mu \mathrm{mol}$, $5.1 \mathrm{~mol} \%$ ), $\mathrm{K}_{2} \mathrm{CO}_{3}$ (83 mg, $0.60 \mathrm{mmol}, 2.0$ equiv.), and acetonitrile (1.5 mL, $\mathrm{c}=0.20 \mathrm{M}$ ). Tert-butyl 2((4R,6R)-6-(2-aminoethyl)-2,2-dimethyl-1,3-dioxan-4-yl)acetate (123 mg, $0.450 \mathrm{mmol}, 1.50$ equiv.) was added, and the suspension was degassed by bubbling through argon with a cannula for $5 \mathrm{~min}$. While stirring, the suspension was irradiated for $2.5 \mathrm{~h}$ at $15^{\circ} \mathrm{C}$ using a blue LED $(60 \mathrm{~W})$. The suspension was added to an aqueous saturated $\mathrm{NaHCO}_{3}$ solution $(20 \mathrm{~mL})$, and the aqueous phase was extracted with $\mathrm{DCM}(3 \times 20 \mathrm{~mL})$. The organic phase was dried over $\mathrm{MgSO}_{4}$, and the solvent was removed under reduced pressure. The residue was purified by chromatography on silica gel eluting with hexanes / EtOAc $(1: 0-7: 3(\mathrm{v} / \mathrm{v}))$ to afford $91 \mathrm{mg}(58 \%)$ of 22 as brown oil.

$\mathbf{R} \boldsymbol{f}=0.34$ (hexanes / EtOAc, 8:2 (v/v)).

\section{NMR Spectroscopy:}

${ }^{1} \mathrm{H}$ NMR $\left(500 \mathrm{MHz}, \mathrm{CDCl}_{3}, 25^{\circ} \mathrm{C}, \delta\right): 7.38-7.32(\mathrm{~m}, 2 \mathrm{H}), 6.90-6.84(\mathrm{~m}, 2 \mathrm{H}), 6.82-6.76(\mathrm{~m}$, $2 \mathrm{H}), 6.61-6.54(\mathrm{~m}, 2 \mathrm{H}), 4.31-4.22(\mathrm{~m}, 1 \mathrm{H}), 4.08-3.99(\mathrm{~m}, 2 \mathrm{H}), 3.29-3.14(\mathrm{~m}, 2 \mathrm{H}), 2.44(\mathrm{dd}$, $J=15.2,6.9 \mathrm{~Hz}, 1 \mathrm{H}), 2.31(\mathrm{dd}, J=15.2,6.2 \mathrm{~Hz}, 1 \mathrm{H}), 1.78(\mathrm{q}, J=6.3 \mathrm{~Hz}, 2 \mathrm{H}), 1.60-1.54(\mathrm{~m}, 1 \mathrm{H})$, $1.46(\mathrm{~s}, 3 \mathrm{H}), 1.45(\mathrm{~s}, 9 \mathrm{H}), 1.40(\mathrm{~s}, 3 \mathrm{H}), 1.32-1.25(\mathrm{~m}, 1 \mathrm{H})$.

${ }^{13} \mathrm{C}$ NMR $\left\{{ }^{1} \mathrm{H}\right\}\left(126 \mathrm{MHz}, \mathrm{CDCl}_{3}, 25{ }^{\circ} \mathrm{C}, \delta\right): 170.4,158.6,147.0,145.7,132.5,121.4,118.8,114.2$, $113.8,98.9,80.8,68.2,66.3,42.8,41.4,36.5,35.7,30.3,28.2,19.9$.

HRMS-ESI (m/z) calc'd. for $\mathrm{C}_{26} \mathrm{H}_{35} \mathrm{NO}_{5} \mathrm{Br}^{+}[\mathrm{M}+\mathrm{H}]^{+}, 520.169324$; found, 520.169660; deviation: -0.65 ppm.

\section{Morpholine 23}

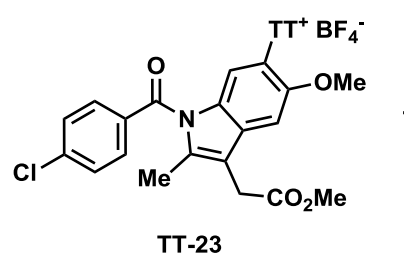

TT-23

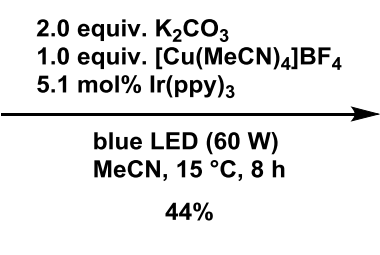

$44 \%$

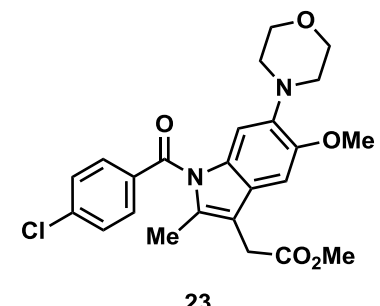

23

Under ambient atmosphere, a $4 \mathrm{~mL}$ glass-vial was charged with thianthrenium salt TT-23 $(202 \mathrm{mg}$, 0.300 mmol, 1.00 equiv.), [Cu(MeCN) ]BF $_{4}\left(94 \mathrm{mg}, 0.30 \mathrm{mmol}, 1.0\right.$ equiv.), Ir(ppy) ${ }_{3}(10 \mathrm{mg}, 15 \mu \mathrm{mol}$, $5.1 \mathrm{~mol} \%), \mathrm{K}_{2} \mathrm{CO}_{3}$ (83 mg, $0.60 \mathrm{mmol}, 2.0$ equiv.), and acetonitrile $(2 \mathrm{~mL}, \mathrm{c}=0.1 \mathrm{M})$. Morpholine (39 $\mathrm{mg}$, $39 \mu \mathrm{L}, 0.45 \mathrm{mmol}, 1.5$ equiv.) was added, and the suspension was degassed by bubbling through argon with a cannula for $5 \mathrm{~min}$. While stirring, the suspension was irradiated for $8 \mathrm{~h}$ at $15^{\circ} \mathrm{C}$ using a blue LED $(60 \mathrm{~W})$. The solvent was removed under reduced pressure. The residue was purified by chromatography on silica gel eluting with hexanes / EtOAc (1:0-4:6 (v/v)) to afford $60 \mathrm{mg}(44 \%)$ of 23 as yellow solid.

$\mathbf{R} \boldsymbol{f}=0.29$ (hexanes / EtOAc, 1:1 (v/v)).

\section{NMR Spectroscopy:}


${ }^{1} \mathrm{H}$ NMR $\left(500 \mathrm{MHz}, \mathrm{CDCl}_{3}, 25^{\circ} \mathrm{C}, \delta\right): 7.68-7.63(\mathrm{~m}, 2 \mathrm{H}), 7.51-7.44(\mathrm{~m}, 2 \mathrm{H}), 6.92(\mathrm{~s}, 1 \mathrm{H}), 6.70$ (s, 1H), $3.91(\mathrm{~s}, 3 \mathrm{H}), 3.83(\mathrm{t}, J=4.6 \mathrm{~Hz}, 4 \mathrm{H}), 3.69(\mathrm{~s}, 3 \mathrm{H}), 3.65(\mathrm{~s}, 2 \mathrm{H}), 2.88-2.80(\mathrm{~m}, 4 \mathrm{H}), 2.30$ $(\mathrm{s}, 3 \mathrm{H})$.

${ }^{13} \mathrm{C}$ NMR $\left\{{ }^{1} \mathrm{H}\right\}\left(126 \mathrm{MHz}, \mathrm{CDCl}_{3}, 25{ }^{\circ} \mathrm{C}, \delta\right): 171.5,168.5,149.9,139.2,138.7,134.3,133.8,131.2$, $130.8,129.2,124.9,112.6,105.1,100.3,67.3,55.9,52.3,51.6,30.4,13.7$.

HRMS-ESI (m/z) calc'd. for $\mathrm{C}_{24} \mathrm{H}_{26} \mathrm{CIN}_{2} \mathrm{O}_{5}{ }^{+}[\mathrm{M}+\mathrm{H}]^{+}$, 457.152475; found, 457.152640; deviation: -0.36 ppm.

Thianthrenium salt TT-24
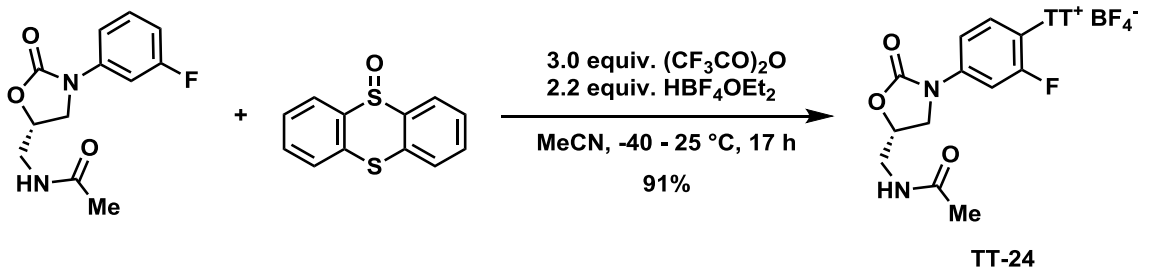

Under ambient atmosphere, a $100 \mathrm{~mL}$ Schlenk-tube equipped with a magnetic stir bar was charged with (S)-N-((3-(3-fluorophenyl)-2-oxooxazolidin-5-yl)methyl)acetamide (500 mg, $1.98 \mathrm{mmol}, 1.00$ equiv.), thianthrene S-oxide (461 mg, $1.98 \mathrm{mmol}, 1.00$ equiv.), and $\mathrm{MeCN}(10 \mathrm{~mL}, \mathrm{c}=0.20 \mathrm{M})$. After cooling to $-40{ }^{\circ} \mathrm{C}$, trifluoroacetic anhydride (0.83 mL, $1.3 \mathrm{~g}, 6.0 \mathrm{mmol}, 3.0$ equiv.) was added while stirring. $\mathrm{HBF}_{4} \cdot \mathrm{OEt}_{2}(0.59 \mathrm{~mL}$, $0.70 \mathrm{~g}, 4.3 \mathrm{mmol}, 2.2$ equiv.) was added dropwise. The mixture was stirred at $-40{ }^{\circ} \mathrm{C}$ for $2 \mathrm{~h}$, then at ambient temperature for $15 \mathrm{~h}$. The reaction mixture was concentrated under reduced pressure, and subsequently diluted with $\mathrm{DCM}(50 \mathrm{~mL})$. The solution was poured onto a saturated aqueous $\mathrm{NaHCO}_{3}$ solution $(25 \mathrm{~mL})$, and the layers were separated. The organic phase was washed with aqueous $\mathrm{NaBF}_{4}$ solution $(2 \times 50 \mathrm{~mL}, 10 \%)$, and with water $(50 \mathrm{~mL})$. The organic phase was dried over $\mathrm{MgSO}_{4}$, and the solvent was removed under reduced pressure. The residue was purified by chromatography on silica gel eluting with $\mathrm{DCM} / \mathrm{MeOH}(1: 0-$ 19:1 (v/v)) to afford $998 \mathrm{mg}(91 \%)$ of TT-24 as colorless foam.

$\mathbf{R}_{\boldsymbol{f}}=0.58(\mathrm{DCM} / \mathrm{MeOH}, 95: 5(\mathrm{v} / \mathrm{v}))$.

\section{NMR Spectroscopy:}

${ }^{1} \mathrm{H}$ NMR $\left(500 \mathrm{MHz}, \mathrm{DMSO}-\mathrm{d}_{6}, 25^{\circ} \mathrm{C}, \delta\right): 8.43-8.41(\mathrm{~m}, 2 \mathrm{H}), 8.21-8.19(\mathrm{~m}, 1 \mathrm{H}), 8.11-8.08(\mathrm{~m}$, $2 \mathrm{H}), 7.94-7.90(\mathrm{~m}, 2 \mathrm{H}), 7.86-7.83(\mathrm{~m}, 2 \mathrm{H}), 7.77-7.74(\mathrm{~m}, 1 \mathrm{H}), 7.48-7.46(\mathrm{~m}, 1 \mathrm{H}), 6.95(\mathrm{t}, J$ $=8.9 \mathrm{~Hz}, 1 \mathrm{H}), 4.78-4.73(\mathrm{~m}, 1 \mathrm{H}), 4.09(\mathrm{t}, J=9.1 \mathrm{~Hz}, 1 \mathrm{H}), 3.70(\mathrm{dd}, J=9.3,6.4 \mathrm{~Hz}, 1 \mathrm{H}), 3.40(\mathrm{t}$, $J=5.4 \mathrm{~Hz}, 2 \mathrm{H}), 1.80$ (s, 3H).

${ }^{13} \mathrm{C}$ NMR $\left\{{ }^{1} \mathrm{H}\right\}\left(126 \mathrm{MHz}, \mathrm{DMSO}-\mathrm{d}_{6}, 25^{\circ} \mathrm{C}, \delta\right): 170.0,159.7(\mathrm{~d}, J=249.1 \mathrm{~Hz}), 153.7,145.0(\mathrm{~d}, J=$ $11.4 \mathrm{~Hz}$ ), 135.7 (d, $J=4.2 \mathrm{~Hz}), 135.0,134.8,130.8,130.3,129.8,117.8$ (d, $J=9.5 \mathrm{~Hz}), 114.1$, $106.6(\mathrm{~d}, J=25.2 \mathrm{~Hz}), 103.6$ (d, $J=14.2 \mathrm{~Hz}), 72.2,47.1$, 41.2, 22.4.

${ }^{19} \mathrm{~F}$ NMR $\left\{{ }^{1} \mathrm{H}\right\}\left(471 \mathrm{MHz}, \mathrm{DMSO}-\mathrm{d}_{6}, 25^{\circ} \mathrm{C}, \delta\right):-107.0,-148.2,-148.3$.

HRMS-ESI (m/z) calc'd. for $\mathrm{C}_{24} \mathrm{H}_{20} \mathrm{FN}_{2} \mathrm{O}_{3} \mathrm{~S}_{2}{ }^{+}\left[\mathrm{M}-\mathrm{BF}_{4}\right]^{+}$, 467.089391; found, 467.088950; deviation: 
+0.94 ppm.

Morpholine 24
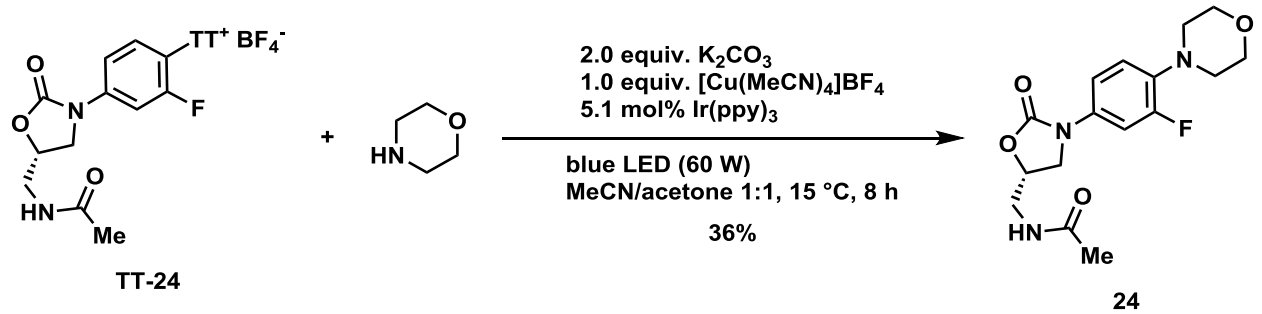

Under ambient atmosphere, a $4 \mathrm{~mL}$ glass-vial was charged with thianthrenium salt TT-24 (166 mg, 0.299 mmol, 1.00 equiv.), [Cu(MeCN) ${ }_{4} \mathrm{BF}_{4}$ (94 mg, $0.30 \mathrm{mmol}, 1.0$ equiv.), Ir(ppy) ${ }_{3}(10 \mathrm{mg}, 15 \mu \mathrm{mol}$, $5.1 \mathrm{~mol} \%), \mathrm{K}_{2} \mathrm{CO}_{3}$ (83 mg, $0.60 \mathrm{mmol}, 2.0$ equiv.), and acetone $(1 \mathrm{~mL})$ and acetonitrile $\left(1 \mathrm{~mL}, \mathrm{c}_{\text {total }}=0.1 \mathrm{M}\right)$. Morpholine ( $79 \mu \mathrm{L}, 79 \mathrm{mg}, 0.90 \mathrm{mmol}, 3.0$ equiv.) was added, and the suspension was degassed by bubbling through argon with a cannula for $5 \mathrm{~min}$. While stirring, the suspension was irradiated for $8 \mathrm{~h}$ at $15^{\circ} \mathrm{C}$ using a blue LED $(60 \mathrm{~W})$. The solvent was removed under reduced pressure. The residue was purified by chromatography on silica gel eluting with hexanes / EtOAc (1:0-1:5 (v/v)) to afford $37 \mathrm{mg}(36 \%)$ of 24 as colorless solid.

$\mathbf{R} \boldsymbol{f}=0.13($ hexanes / EtOAc, 2:8 (v/v)).

\section{NMR Spectroscopy:}

${ }^{1} \mathrm{H}$ NMR $\left(500 \mathrm{MHz}, \mathrm{CDCl}_{3}, 25^{\circ} \mathrm{C}, \delta\right): 7.43-7.40(\mathrm{~m}, 1 \mathrm{H}), 7.07-7.05(\mathrm{~m}, 1 \mathrm{H}), 6.92-6.88(\mathrm{~m}$, $1 \mathrm{H}), 6.41$ (br s, 1H), $4.79-4.75(\mathrm{~m}, 1 \mathrm{H}), 4.01(\mathrm{t}, J=8.9 \mathrm{~Hz}, 1 \mathrm{H}), 3.86-3.84(\mathrm{~m}, 4 \mathrm{H}), 3.76-3.73$ (m, 1H), $3.65-3.59(m, 2 H), 3.04-3.03(m, 4 H), 2.01(s, 3 H)$.

${ }^{13} \mathrm{C}$ NMR $\left\{{ }^{1} \mathrm{H}\right\}\left(126 \mathrm{MHz}, \mathrm{CDCl}_{3}, 25^{\circ} \mathrm{C}, \delta\right): 171.3,155.6(\mathrm{~d}, J=246.6 \mathrm{~Hz}), 154.5,136.7(\mathrm{~d}, J=8.9$ $\mathrm{Hz}$ ), 133.0 (d, $J=10.4 \mathrm{~Hz}), 118.9$ (d, $J=4.3 \mathrm{~Hz}), 114.1$ (d, $J=3.3 \mathrm{~Hz}), 107.7$ (d, $J=26.3 \mathrm{~Hz}$ ), $72.1,67.1,51.1,47.7,42.0,23.2$

${ }^{19} \mathrm{~F}$ NMR $\left\{{ }^{1} \mathrm{H}\right\}\left(471 \mathrm{MHz}, \mathrm{CDCl}_{3}, 25{ }^{\circ} \mathrm{C}, \delta\right):-120.1$.

HRMS-ESI (m/z) calc'd. for $\mathrm{C}_{16} \mathrm{H}_{21} \mathrm{FN}_{3} \mathrm{O}_{4}{ }^{+}[\mathrm{M}+\mathrm{H}]^{+}$, 338.151060; found, 338.151230; deviation: -0.50 ppm.

Thianthrenium salt TT-25<smiles>CC(=O)c1ccc(-c2ccccc2)c(F)c1</smiles><smiles>O=S1c2ccccc2Sc2ccccc21</smiles>

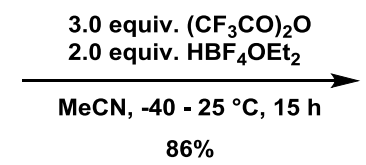<smiles>CC(=O)OC(C)c1ccc(-c2ccc([In+][Ba])cc2)c(F)c1</smiles>

Under ambient atmosphere, a $50 \mathrm{~mL}$ Schlenk-tube equipped with a magnetic stir bar was charged with flurbiprofen methyl ester (400 mg, $1.55 \mathrm{mmol}, 1.00$ equiv.), thianthrene S-oxide (360 mg, $1.55 \mathrm{mmol}$, 1.00 equiv. $)$, and $\mathrm{MeCN}(7.7 \mathrm{~mL}, \mathrm{C}=0.20 \mathrm{M})$. After cooling to $-40^{\circ} \mathrm{C}$, trifluoroacetic anhydride $(0.65 \mathrm{~mL}$, 
$0.98 \mathrm{~g}, 4.7 \mathrm{mmol}, 3.0$ equiv.) was added while stirring. $\mathrm{HBF}_{4} \cdot \mathrm{OEt}_{2}(0.42 \mathrm{ml}, 0.50 \mathrm{~g}, 3.1 \mathrm{mmol}, 2.0$ equiv.) was added dropwise. The mixture was stirred at $-40^{\circ} \mathrm{C}$ for $1 \mathrm{~h}$, then at ambient temperature for $14 \mathrm{~h}$. The reaction mixture was added to a saturated aqueous $\mathrm{NaHCO}_{3}$ solution $(50 \mathrm{~mL})$, and the aqueous phase was extracted with $\mathrm{DCM}(3 \times 20 \mathrm{~mL})$. The organic phase was washed with aqueous $\mathrm{NaBF}_{4}$ solution $(30 \mathrm{~mL}, 10 \%)$, dried over $\mathrm{MgSO}_{4}$, and the solvent was removed under reduced pressure. The residue was purified by chromatography on silica gel eluting with $\mathrm{DCM} / \mathrm{MeOH}(1: 0-9: 1(\mathrm{v} / \mathrm{v}))$ to afford $746 \mathrm{mg}(86 \%)$ of TT-25 as white solid.

$\mathbf{R}_{\boldsymbol{f}}=0.35(\mathrm{DCM} / \mathrm{MeOH}, 15: 1(\mathrm{v} / \mathrm{v}))$.

\section{NMR Spectroscopy:}

${ }^{1}$ H NMR (500 MHz, DMSO-d $\left.6,25^{\circ} \mathrm{C}, \delta\right): 8.62(\mathrm{dd}, J=7.9,1.4 \mathrm{~Hz}, 2 \mathrm{H}), 8.09(\mathrm{~d}, J=7.1 \mathrm{~Hz}, 2 \mathrm{H})$, $7.94(\mathrm{td}, J=7.7,1.5 \mathrm{~Hz}, 2 \mathrm{H}), 7.88(\mathrm{td}, J=7.7,1.4 \mathrm{~Hz}, 2 \mathrm{H}), 7.72(\mathrm{dd}, J=8.7,1.6 \mathrm{~Hz}, 2 \mathrm{H}), 7.47$ (t, $J=8.2 \mathrm{~Hz}, 1 \mathrm{H}), 7.35-7.30(\mathrm{~m}, 2 \mathrm{H}), 7.30-7.19(\mathrm{~m}, 2 \mathrm{H}), 3.90(\mathrm{q}, J=7.1 \mathrm{~Hz}, 1 \mathrm{H}), 3.60(\mathrm{~s}, 3 \mathrm{H})$, $1.41(\mathrm{~d}, J=7.2 \mathrm{~Hz}, 3 \mathrm{H})$.

${ }^{13} \mathrm{C}$ NMR $\left\{{ }^{1} \mathrm{H}\right\}\left(126 \mathrm{MHz}, \mathrm{DMSO}-\mathrm{d}_{6}, 25^{\circ} \mathrm{C}, \delta\right): 173.6,158.8(\mathrm{~d}, J=247.4 \mathrm{~Hz}), 144.0(\mathrm{~d}, J=7.9$ $\mathrm{Hz}), 139.0,135.7,135.4,134.8,130.9$ (d, $J=3.1 \mathrm{~Hz}), 130.6(\mathrm{~d}, J=3.0 \mathrm{~Hz}), 130.3,129.6,128.4$, $124.6(\mathrm{~d}, J=12.8 \mathrm{~Hz}), 124.3,124.2(\mathrm{~d}, J=16.0 \mathrm{~Hz}), 119.1,115.4(\mathrm{~d}, J=23.0 \mathrm{~Hz}), 52.0,43.8$, 18.3.

${ }^{19} \mathrm{~F}$ NMR $\left\{{ }^{1} \mathrm{H}\right\}\left(471 \mathrm{MHz}, \mathrm{DMSO}-\mathrm{d}_{6}, 25^{\circ} \mathrm{C}, \delta\right):-117.8,-148.2,-148.3$.

HRMS-ESI (m/z) calc'd. for $\mathrm{C}_{28} \mathrm{H}_{22} \mathrm{FO}_{2} \mathrm{~S}_{2}{ }^{+}\left[\mathrm{M}-\mathrm{BF}_{4}\right]^{+}$, 473.103979; found, 473.103320; deviation: +1.39 ppm.

\section{Aniline 25a}
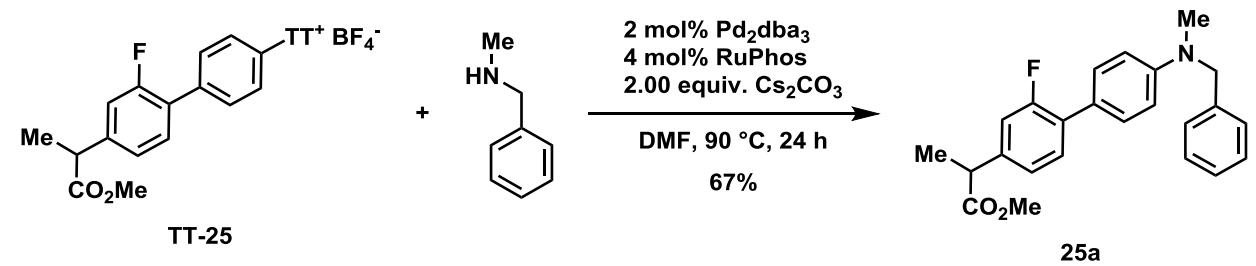

In an argon-filled glovebox, a $4 \mathrm{~mL}$ glass-vial equipped with a magnetic stir bar was charged with TT-25 (224 mg, 0.400 mmol, 1.00 equiv.), $\mathrm{Pd}_{2} \mathrm{dba}_{3}$ (7 mg, 8 umol, 2 mol\%), RuPhos (7 mg, 0.02 mmol, 4 mol\%), $\mathrm{Cs}_{2} \mathrm{CO}_{3}$ (261 mg, $0.801 \mathrm{mmol}, 2.00$ equiv.), and DMF (1 mL, c = 0.4 M). N-Methylbenzylamine (77 $\mu \mathrm{L}, 72 \mathrm{mg}$, $0.60 \mathrm{mmol}, 1.5$ equiv.) was added, and the sealed vial was taken out from the glovebox. The suspension was stirred at $90{ }^{\circ} \mathrm{C}$ for $24 \mathrm{~h}$. The solvent was removed under reduced pressure. The residue was purified by chromatography on silica gel eluting with hexanes / EtOAc (1:0-6:4 (v/v)) to afford $101 \mathrm{mg}(67 \%)$ of 25a as yellowish oil.

$\mathbf{R}_{\boldsymbol{f}}=0.35$ (hexanes / EtOAc, 9:1 (v/v)).

\section{NMR Spectroscopy:}


${ }^{1}$ H NMR (500 MHz, $\left.\mathrm{CDCl}_{3}, 25^{\circ} \mathrm{C}, \delta\right): 7.43(\mathrm{~d}, J=7.0 \mathrm{~Hz}, 2 \mathrm{H}), 7.40-7.31(\mathrm{~m}, 3 \mathrm{H}), 7.30-7.22(\mathrm{~m}$, $3 \mathrm{H}), 7.14-7.04(\mathrm{~m}, 2 \mathrm{H}), 6.83(\mathrm{~d}, J=8.3 \mathrm{~Hz}, 2 \mathrm{H}), 4.59(\mathrm{~s}, 2 \mathrm{H}), 3.74(\mathrm{q}, J=7.2 \mathrm{~Hz}, 1 \mathrm{H}), 3.70$ (s, $3 \mathrm{H}), 3.09(\mathrm{~s}, 3 \mathrm{H}), 1.53(\mathrm{~d}, J=7.2 \mathrm{~Hz}, 3 \mathrm{H})$.

${ }^{13} \mathrm{C}$ NMR $\left\{{ }^{1} \mathrm{H}\right\}\left(126 \mathrm{MHz}, \mathrm{CDCl}_{3}, 25^{\circ} \mathrm{C}, \delta\right): 174.7,159.8(\mathrm{~d}, J=246.9 \mathrm{~Hz}), 140.5(\mathrm{~d}, J=7.6 \mathrm{~Hz})$, $130.3(\mathrm{~d}, J=4.2 \mathrm{~Hz}), 129.9$ (d, $J=3.1 \mathrm{~Hz}), 128.8,128.0(\mathrm{~d}, J=13.4 \mathrm{~Hz}), 127.1,126.9,123.5$ (d, $J$ $=3.3 \mathrm{~Hz}), 115.3(\mathrm{~d}, J=24.3 \mathrm{~Hz}), 112.3,56.7,52.3,45.0,38.8,18.6$.

${ }^{19} \mathrm{~F}$ NMR $\left\{{ }^{1} \mathrm{H}\right\}\left(471 \mathrm{MHz}, \mathrm{CDCl}_{3}, 25^{\circ} \mathrm{C}, \delta\right):-117.8$.

HRMS-ESI (m/z) calc'd. for $\mathrm{C}_{24} \mathrm{H}_{25} \mathrm{FNO}_{2}{ }^{+}[\mathrm{M}+\mathrm{H}]^{+}$, 378.186382; found, 378.186440; deviation: -0.15 ppm.

Aminoisoxazole 25b
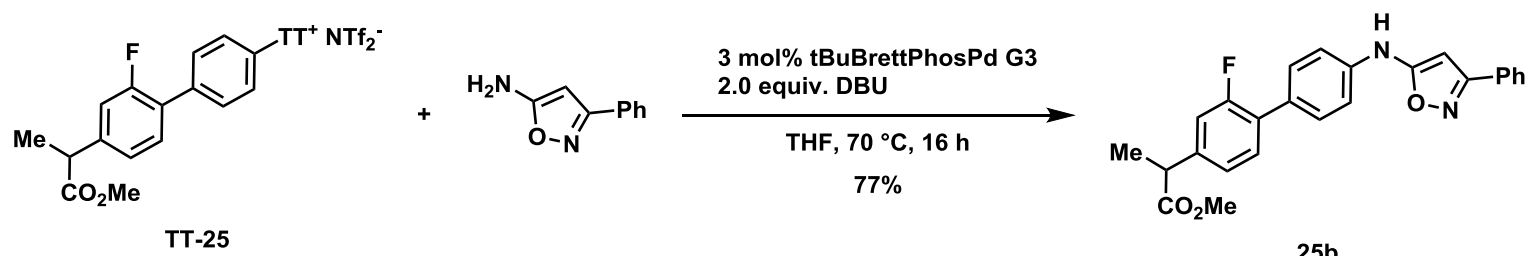

In an argon-filled glovebox, a $4 \mathrm{~mL}$ glass-vial equipped with a magnetic stir bar was charged with TT-25 (226 mg, $0.300 \mathrm{mmol}, 1.00$ equiv.), tBuBrettPhosPd G3 ( $8 \mathrm{mg}, 9 \mu \mathrm{mol}, 3 \mathrm{~mol} \%$ ), and THF ( $1 \mathrm{~mL}, \mathrm{c}=0.3 \mathrm{M}$ ). 5-Amino-3-phenylisoxazole (72 mg, $0.45 \mathrm{mmol}, 1.5$ equiv.) and DBU (90 $\mu \mathrm{L}, 92 \mathrm{mg}, 0.60 \mathrm{mmol}, 2.0$ equiv.) were added, and the sealed vial was taken out from the glovebox. The solution was stirred at $70{ }^{\circ} \mathrm{C}$ for $16 \mathrm{~h}$. The solvent was removed under reduced pressure, and the residue was purified by chromatography on silica gel eluting with hexanes / EtOAc(1:0-3:1 (v/v)) to afford $97 \mathrm{mg}(77 \%)$ of $25 \mathrm{~b}$ as white solid.

$\mathbf{R} \boldsymbol{f}=0.28$ (hexanes / EtOAc, 75:25 (v/v)).

\section{NMR Spectroscopy:}

${ }^{1} \mathrm{H}$ NMR $\left(500 \mathrm{MHz}, \mathrm{DMSO}_{-} \mathrm{d}_{6}, 25^{\circ} \mathrm{C}, \delta\right): 10.24(\mathrm{~s}, 1 \mathrm{H}), 7.90-7.88(\mathrm{~m}, 2 \mathrm{H}), 7.55-7.47(\mathrm{~m}, 6 \mathrm{H})$, $7.37-7.36(\mathrm{~m}, 2 \mathrm{H}), 7.24-7.19(\mathrm{~m}, 2 \mathrm{H}), 6.35(\mathrm{~s}, 1 \mathrm{H}), 3.89$ (q, J = 7.1 Hz, 1H), $3.62(\mathrm{~s}, 3 \mathrm{H}), 1.43$ $(\mathrm{d}, J=7.1 \mathrm{~Hz}, 3 \mathrm{H})$.

${ }^{13} \mathrm{C}$ NMR $\left\{{ }^{1} \mathrm{H}\right\}\left(126 \mathrm{MHz}, \mathrm{DMSO}-\mathrm{d}_{6}, 25^{\circ} \mathrm{C}, \delta\right): 173.8,165.6,162.8,158.9(\mathrm{~d}, J=245.9 \mathrm{~Hz}), 141.8$ $(\mathrm{d}, J=7.7 \mathrm{~Hz}), 139.5,130.4$ (d, $J=3.9 \mathrm{~Hz}), 130.0,129.7$ (d, $J=3.2 \mathrm{~Hz}), 128.9,127.7,126.6$, $123.9(\mathrm{~d}, J=3.2 \mathrm{~Hz}), 116.5,115.2(\mathrm{~d}, J=23.4 \mathrm{~Hz}), 79.4,52.0,43.8$, 18.3.

${ }^{19} \mathrm{~F}$ NMR $\left\{{ }^{1} \mathrm{H}\right\}\left(471 \mathrm{MHz}, \mathrm{DMSO}-\mathrm{d}_{6}, 25^{\circ} \mathrm{C}, \delta\right):-118.2$.

HRMS-ESI (m/z) calc'd. for $\mathrm{C}_{25} \mathrm{H}_{20} \mathrm{FN}_{2} \mathrm{O}_{3}{ }^{-}[\mathrm{M}-\mathrm{H}]^{-}$, 415.146346; found, 415.146420; deviation: -0.18 ppm. 
Triazole 25c
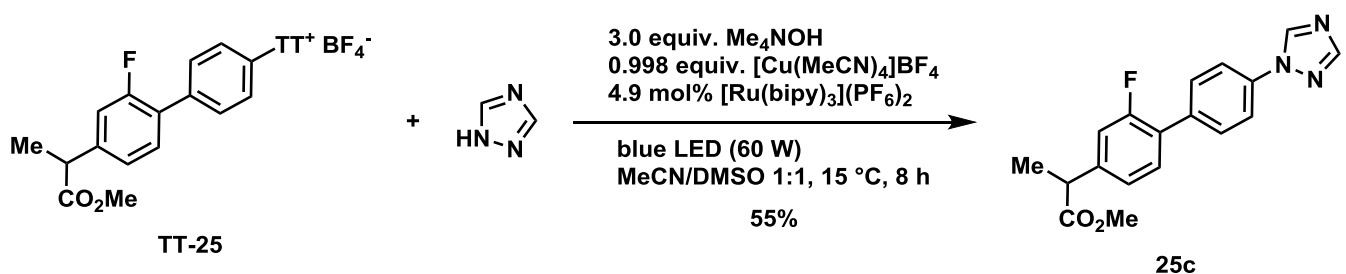

Under ambient atmosphere, a $4 \mathrm{~mL}$ glass-vial was charged with 1,2,4-triazole (74 mg, $1.1 \mathrm{mmol}, 3.0$ equiv.) and tetramethylammonium hydroxide (25\% in methanol, $390 \mathrm{mg}, 0.45 \mathrm{~mL}, 1.1 \mathrm{mmol}, 3.0$ equiv.), and the solvent was removed under reduced pressure. Thianthrenium salt TT-25 (200 mg, 0.357 mmol, 1.00 equiv.), [Cu(MeCN)$\left.)_{4}\right] \mathrm{BF}_{4}$ (112 mg, $0.356 \mathrm{mmol}, 0.998$ equiv.), [Ru(bipy) $)_{3}\left(\mathrm{PF}_{6}\right)_{2}(15 \mathrm{mg}, 17 \mu \mathrm{mol}, 4.9 \mathrm{~mol} \%)$, acetonitrile $(0.89 \mathrm{~mL})$ and dimethylsulfoxide $\left(0.89 \mathrm{~mL}, \mathrm{c}_{\text {total }}=0.20 \mathrm{M}\right)$ were added, and the suspension was degassed by bubbling through argon with a cannula for $5 \mathrm{~min}$. While stirring, the suspension was irradiated for $8 \mathrm{~h}$ at $15^{\circ} \mathrm{C}$ using a blue LED (60 W). The suspension was added to water (30 mL), and the aqueous phase was extracted with $\mathrm{DCM}(3 \times 20 \mathrm{~mL})$. The organic phase was washed with a saturated aqueous $\mathrm{NaCl}$ solution (20 mL), dried over $\mathrm{MgSO}_{4}$, and the solvent was removed under reduced pressure. The residue was purified by chromatography on silica gel eluting with hexanes / EtOAc $(9: 1-0: 1(\mathrm{v} / \mathrm{v}))$ to afford $69 \mathrm{mg}(55 \%)$ of $25 \mathrm{c}$ as yellowish solid.

$\mathbf{R} \boldsymbol{f}=0.46$ (hexanes / EtOAc, 2:8 (v/v)).

\section{NMR Spectroscopy:}

${ }^{1} \mathrm{H}$ NMR $\left(500 \mathrm{MHz}, \mathrm{CDCl}_{3}, 25^{\circ} \mathrm{C}, \delta\right): 8.59(\mathrm{~s}, 1 \mathrm{H}), 8.11(\mathrm{~s}, 1 \mathrm{H}), 7.78-7.71(\mathrm{~m}, 2 \mathrm{H}), 7.69-7.62$ $(\mathrm{m}, 2 \mathrm{H}), 7.40(\mathrm{t}, J=8.0 \mathrm{~Hz}, 1 \mathrm{H}), 7.19-7.11(\mathrm{~m}, 2 \mathrm{H}), 3.76(\mathrm{q}, J=7.2 \mathrm{~Hz}, 1 \mathrm{H}), 3.69(\mathrm{~s}, 3 \mathrm{H}), 1.53$ (d, $J=7.0 \mathrm{~Hz}, 3 \mathrm{H})$.

${ }^{13} \mathrm{C}$ NMR $\left\{{ }^{1} \mathrm{H}\right\}\left(126 \mathrm{MHz}, \mathrm{CDCl}_{3}, 25{ }^{\circ} \mathrm{C}, \delta\right): 174.3,159.7(\mathrm{~d}, J=249.0 \mathrm{~Hz}), 152.7,142.6(\mathrm{~d}, J=7.7$ $\mathrm{Hz}), 140.9,136.3,135.5,130.6$ (d, $J=3.6 \mathrm{~Hz}), 130.3$ (d, $J=3.2 \mathrm{~Hz}), 126.4$ (d, $J=13.1 \mathrm{~Hz}), 123.8$ (d, $J=3.2 \mathrm{~Hz}), 120.0,115.5(\mathrm{~d}, J=23.3 \mathrm{~Hz}), 52.3,45.0,18.5$.

${ }^{19} \mathrm{~F} \mathrm{NMR}\left\{{ }^{1} \mathrm{H}\right\}\left(471 \mathrm{MHz}, \mathrm{CDCl}_{3}, 25^{\circ} \mathrm{C}, \delta\right):-117.4$.

HRMS-ESI (m/z) calc'd. for $\mathrm{C}_{18} \mathrm{H}_{17} \mathrm{FN}_{3} \mathrm{O}_{2}{ }^{+}[\mathrm{M}+\mathrm{H}]^{+}$, 326.129930; found, 326.129910; deviation: +0.06 ppm.

Aniline 25d
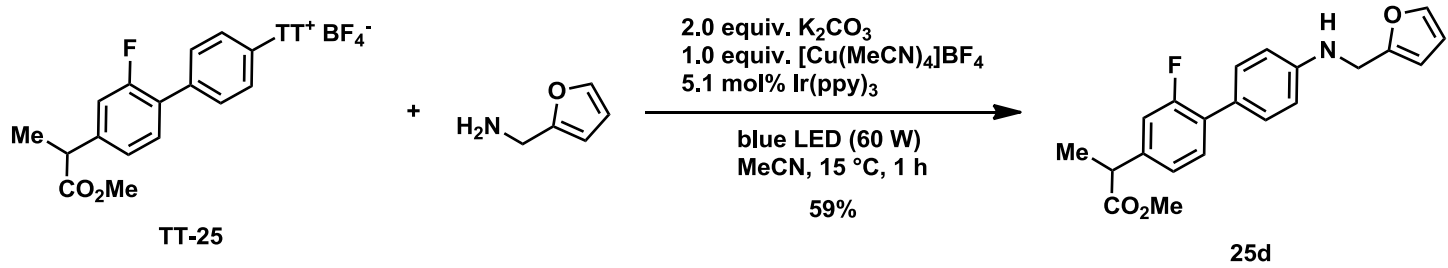

Under ambient atmosphere, a $4 \mathrm{~mL}$ glass-vial was charged with thianthrenium salt TT-25 (168 mg, 
0.300 mmol, 1.00 equiv.), [Cu(MeCN) $)_{4} \mathrm{BF}_{4}\left(94 \mathrm{mg}, 0.30 \mathrm{mmol}, 1.0\right.$ equiv.), Ir(ppy) ${ }_{3}(10 \mathrm{mg}, 15 \mu \mathrm{mol}$,

$5.1 \mathrm{~mol} \%), \mathrm{K}_{2} \mathrm{CO}_{3}$ (83 mg, $0.60 \mathrm{mmol}, 2.0$ equiv.), and acetonitrile $(1.5 \mathrm{~mL}, \mathrm{c}=0.20 \mathrm{M})$. Furfurylamine $(40 \mu \mathrm{L}$, $44 \mathrm{mg}, 0.45 \mathrm{mmol}, 1.5$ equiv.) was added, and the suspension was degassed by bubbling through argon with a cannula for $5 \mathrm{~min}$. While stirring, the suspension was irradiated for $1 \mathrm{~h}$ at $15^{\circ} \mathrm{C}$ using a blue LED (60 W). The suspension was added to an aqueous saturated $\mathrm{NaHCO}_{3}$ solution $(20 \mathrm{~mL})$, and the aqueous phase was extracted with DCM $(3 \times 20 \mathrm{~mL})$. The organic phase was dried over $\mathrm{MgSO}_{4}$, and the solvent was removed under reduced pressure. The residue was purified by chromatography on silica gel eluting with hexanes / EtOAc $(1: 0-2: 8(v / v))$ to afford $63 \mathrm{mg}(59 \%)$ of $\mathbf{2 5 d}$ as yellowish oil.

$\mathbf{R} \boldsymbol{f}=0.38$ (hexanes / EtOAc, 8:2 (v/v)).

\section{NMR Spectroscopy:}

${ }^{1} \mathrm{H}$ NMR $\left(500 \mathrm{MHz}, \mathrm{CDCl}_{3}, 25^{\circ} \mathrm{C}, \delta\right): 7.42-7.37(\mathrm{~m}, 3 \mathrm{H}), 7.35(\mathrm{t}, J=8.2 \mathrm{~Hz}, 1 \mathrm{H}), 7.13-7.05(\mathrm{~m}$, 2H), $6.76-6.71(\mathrm{~m}, 2 \mathrm{H}), 6.34(\mathrm{dd}, J=3.2,1.8 \mathrm{~Hz}, 1 \mathrm{H}), 6.27(\mathrm{~d}, J=3.2 \mathrm{~Hz}, 1 \mathrm{H}), 4.36$ (d, J = 4.7 $\mathrm{Hz}, 2 \mathrm{H}), 4.16(\mathrm{~s}, 1 \mathrm{H}), 3.74(\mathrm{q}, J=7.2 \mathrm{~Hz}, 1 \mathrm{H}), 3.70(\mathrm{~s}, 3 \mathrm{H}), 1.53(\mathrm{~d}, J=7.2 \mathrm{~Hz}, 3 \mathrm{H})$.

${ }^{13} \mathrm{C}$ NMR $\left\{{ }^{1} \mathrm{H}\right\}\left(126 \mathrm{MHz}, \mathrm{CDCl}_{3}, 25{ }^{\circ} \mathrm{C}, \delta\right): 174.7,159.8(\mathrm{~d}, \mathrm{~J}=247.4 \mathrm{~Hz}), 152.6,147.3,142.1$, 140.7 (d, $J=7.7 \mathrm{~Hz}), 130.4$ (d, $J=4.2 \mathrm{~Hz}), 130.0$ (d, $J=3.2 \mathrm{~Hz}), 128.0$ (d, $J=13.6 \mathrm{~Hz}), 125.0$, $123.5(\mathrm{~d}, J=3.2 \mathrm{~Hz}), 115.3(\mathrm{~d}, J=23.9 \mathrm{~Hz}), 113.1,110.5,107.2,52.3,45.0,41.5,18.6$.

${ }^{19} \mathrm{~F} \mathrm{NMR}\left\{{ }^{1} \mathrm{H}\right\}\left(471 \mathrm{MHz}, \mathrm{CDCl}_{3}, 25^{\circ} \mathrm{C}, \delta\right):-117.8$.

HRMS-ESI (m/z) calc'd. for $\mathrm{C}_{21} \mathrm{H}_{20} \mathrm{NO}_{3} \mathrm{FNa}^{+}[\mathrm{M}+\mathrm{Na}]^{+}$, 376.131941; found, 376.131630; deviation: +0.83 ppm.

\section{Development of Reaction Conditions}

Table S1: Optimization of Pd-catalyzed C-N cross-coupling of aryl thianthrenium salts with morpholine; Ligand screening.

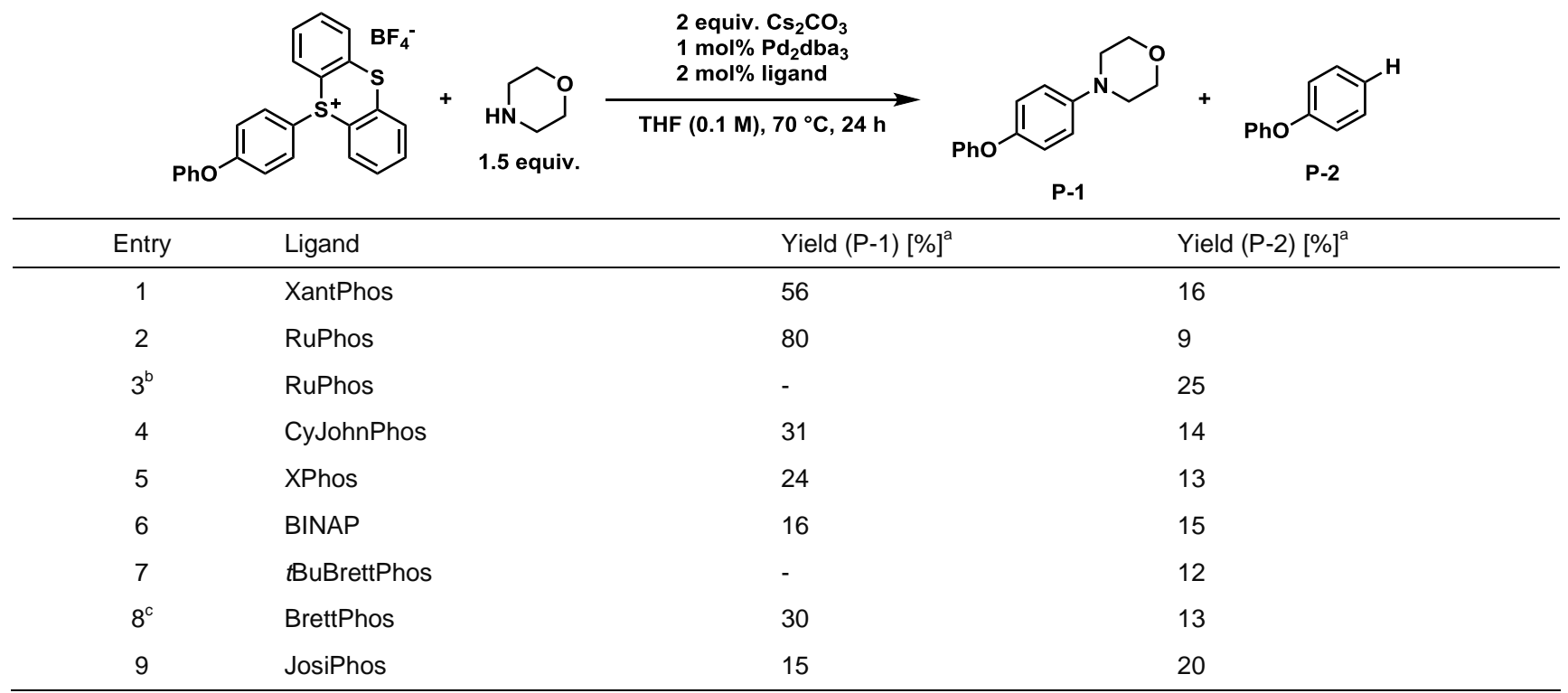


(a) GC yield; (b) TFT instead of TT; (c) $\mathrm{T}=85^{\circ} \mathrm{C}$.

Table S2: Optimization of Pd-catalyzed C-N cross-coupling of aryl thianthrenium salts with morpholine;

Catalyst screening.

\begin{tabular}{|c|c|c|c|c|}
\hline & $\mathrm{BF}_{4}$ & $\begin{array}{r}2 \mathrm{eq} \\
1 \mathrm{~m} \\
2 \mathrm{~m} \\
\text { THF }(0 .\end{array}$ & Pho & Pho \\
\hline Entry & $\mathrm{Pd}$ & Ligand & Yield (P-1) [\%] $]^{a}$ & Yield (P-2) [\%] $]^{\mathrm{a}}$ \\
\hline 1 & $\mathrm{Pd}_{2} \mathrm{dba}_{3}$ & - & 8 & 12 \\
\hline 2 & $\mathrm{Pd}_{2} \mathrm{dba}_{3}$ & RuPhos & 57 & 11 \\
\hline $3^{\mathrm{b}}$ & $\mathrm{Pd}(\mathrm{OAc})_{2}$ & RuPhos & 49 & 10 \\
\hline 4 & RuPhos-Pd G2 & - & 14 & 13 \\
\hline 5 & RuPhos-Pd G2 & RuPhos & 58 & 10 \\
\hline 6 & RuPhos-Pd G3 & - & 17 & 14 \\
\hline 7 & RuPhos-Pd G3 & RuPhos & 44 & 12 \\
\hline $8^{c}$ & tBuBrettPhos-Pd G3 & - & - & 10 \\
\hline 9 & tBuBrettPhos-Pd G3 & tBuBrettPhos & - & 10 \\
\hline $10^{\mathrm{b}}$ & $\mathrm{Pd}\left(\mathrm{PPh}_{3}\right)_{4}$ & - & 15 & 15 \\
\hline $11^{\mathrm{b}}$ & $\mathrm{Pd}\left(\mathrm{PPh}_{3}\right)_{4}$ & RuPhos & 19 & 13 \\
\hline
\end{tabular}

(a) GC yield; (b) T $=85^{\circ} \mathrm{C}$.

Table S3: Optimization of Pd-catalyzed C-N cross-coupling of aryl thianthrenium salts with morpholine; Base screening.

\begin{tabular}{|c|c|c|c|}
\hline & BF & $\begin{array}{l}2 \text { equiv. base } \\
1 \mathrm{~mol} \% \mathrm{Pd}_{2} \mathrm{dba}_{3} \\
2 \mathrm{~mol} \% \mathrm{RuPhos} \\
\mathrm{HF}(0.1 \mathrm{M}), 70^{\circ} \mathrm{C}, 16 \mathrm{~h}\end{array}$ & P-2 \\
\hline Entry & Base & Yield (P-1) [\% $]^{\mathrm{a}}$ & Yield (P-2) [\%] $]^{a}$ \\
\hline 1 & $\mathrm{Cs}_{2} \mathrm{CO}_{3}$ & 57 & 11 \\
\hline 2 & $\mathrm{~K}_{2} \mathrm{CO}_{3}$ & 23 & 16 \\
\hline 3 & NaHMDS & 9 & 10 \\
\hline 4 & tBuOK & 6 & 10 \\
\hline 5 & DBU & - & 17 \\
\hline
\end{tabular}

(a) GC yield. 
Table S4: Optimization of Pd-catalyzed C-N cross-coupling of aryl thianthrenium salts with morpholine;

Concentration screening.

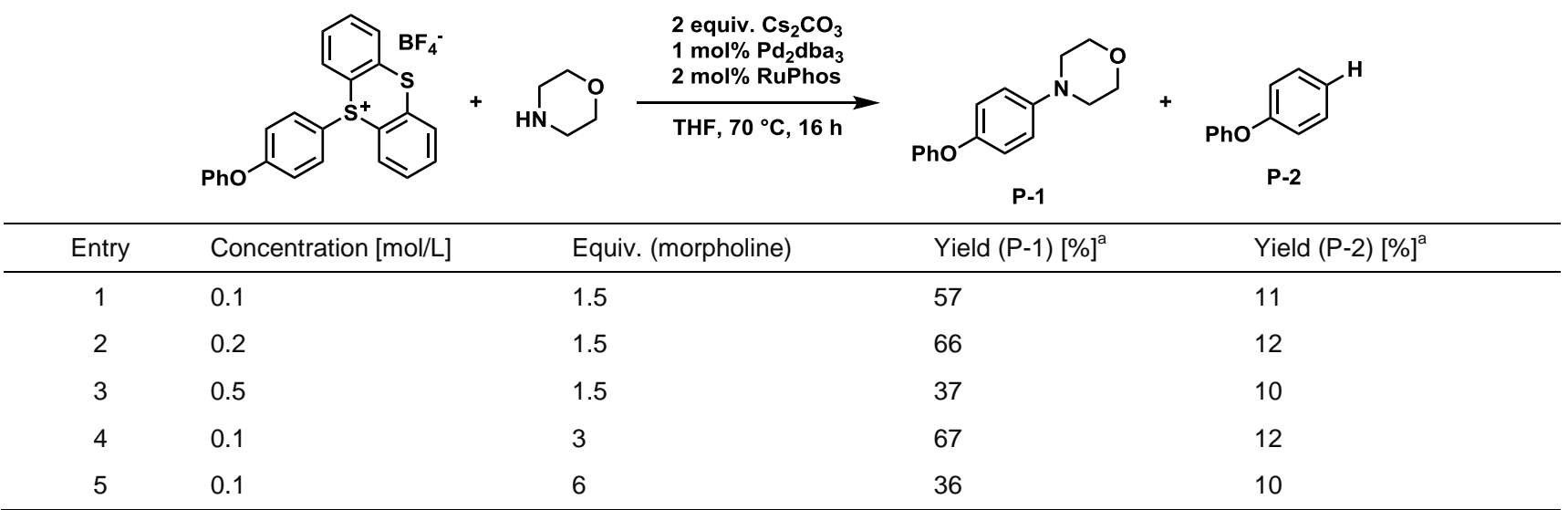

(a) GC yield.

Table S5: Optimization of Pd-catalyzed C-N cross-coupling of aryl thianthrenium salts with aniline.

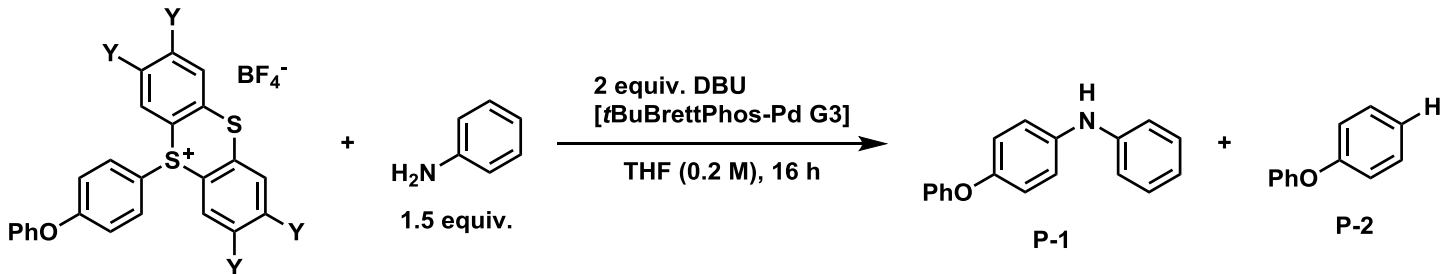

\begin{tabular}{clllll}
\hline Entry & $\mathrm{Y}$ & tBuBrettPhos-Pd G3 [mol\%] & $\mathrm{T}\left[{ }^{\circ} \mathrm{C}\right]$ & ${\text { Yield }(\mathrm{P}-1)[\%]^{\mathrm{a}}}$ & ${\text { Yield }(\mathrm{P}-2)[\%]^{\mathrm{a}}}^{\mathrm{a}}$ \\
\hline 1 & $\mathrm{H}$ & 1 & 25 & 52 & 22 \\
2 & $\mathrm{H}$ & 3 & 25 & $>99$ & $<5$ \\
3 & $\mathrm{H}$ & 1 & 65 & $>98$ & $<5$ \\
4 & $\mathrm{H}$ & 3 & 65 & $>99\left(98^{\mathrm{b}}\right)$ & $<5$ \\
5 & $\mathrm{~F}$ & 3 & 65 & 44 & $<5$ \\
\hline
\end{tabular}

(a) GC yield; (b) isolated yield.

Table S6: Optimization of $\mathrm{Pd}$-catalyzed $\mathrm{C}-\mathrm{N}$ cross-coupling of aryl thianthrenium salts with oxazolidinone.

\begin{tabular}{|c|c|c|c|c|}
\hline & $\mathrm{BF}_{4}^{-}$ & $\begin{array}{l}2 \mathrm{e} \\
3 \mathrm{~m} \\
\end{array}$ & Pho & $\mathrm{PhO}_{\mathrm{P}-2}$ \\
\hline Entry & Equiv. (oxazolidinone) & $\mathrm{T}\left[{ }^{\circ} \mathrm{C}\right]$ & Yield $(P-1)[\%]^{a}$ & Yield (P-2) [\% $]^{\mathrm{a}}$ \\
\hline 1 & 1 & 25 & 53 & 16 \\
\hline 2 & 1 & 65 & 68 & 21 \\
\hline 3 & 2 & 65 & 54 & 7 \\
\hline $4^{b}$ & 1 & 70 & $85^{c}$ & n.d. \\
\hline
\end{tabular}


(a) GC yield; (b) 1 mol\% (PdAlPhos) ${ }_{2}$ cod instead of tBuBrettPhos; (c) isolated yield.

Table S7: Development of photoredox conditions for cross-coupling of aryl thianthrenium salts with azide; Screening of $\mathrm{Cu}(\mathrm{I})$ sources.

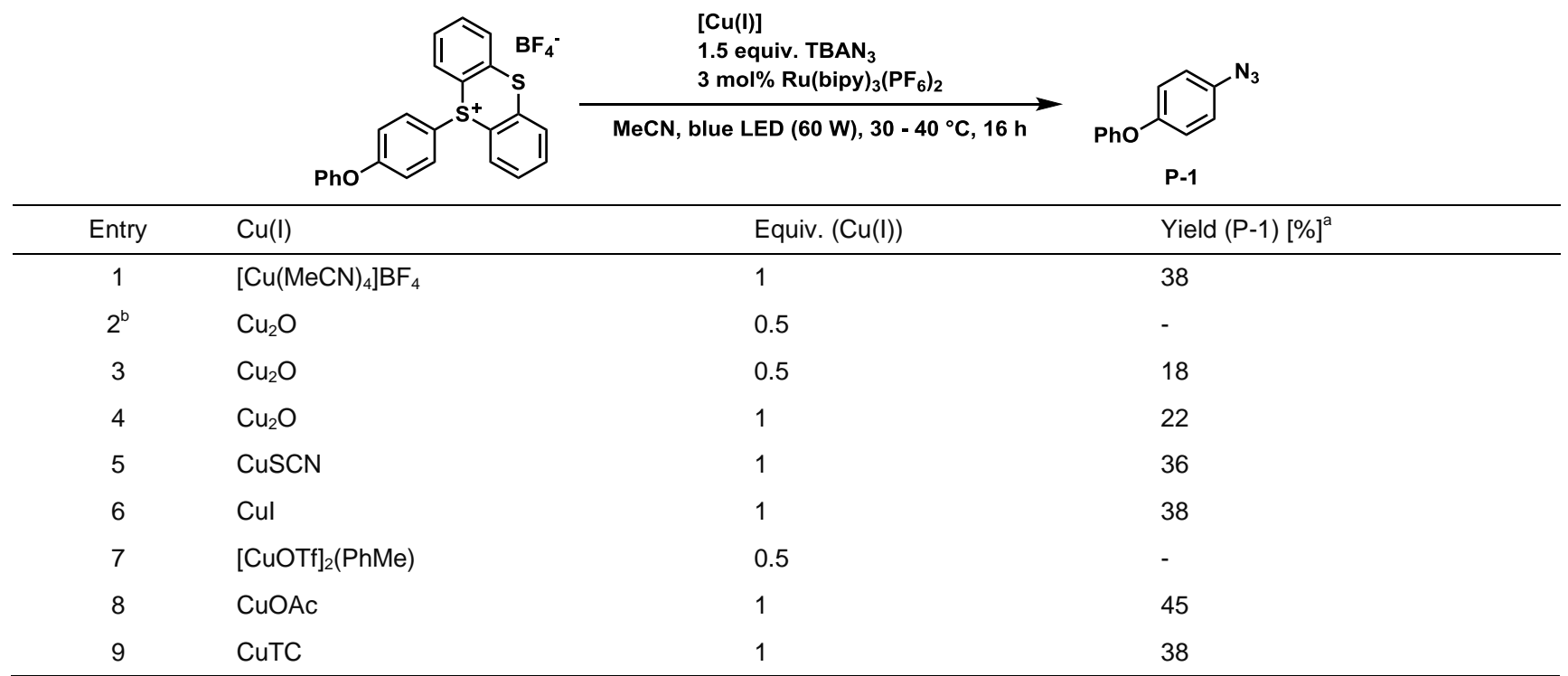

(a) isolated yield; (b) $\mathrm{NaN}_{3}$ instead of $\mathrm{TBAN}_{3}$.

Table S8: Testing of photoredox conditions for cross-coupling of aryl thianthrenium salts with azide; Screening of photoredox catalysts.

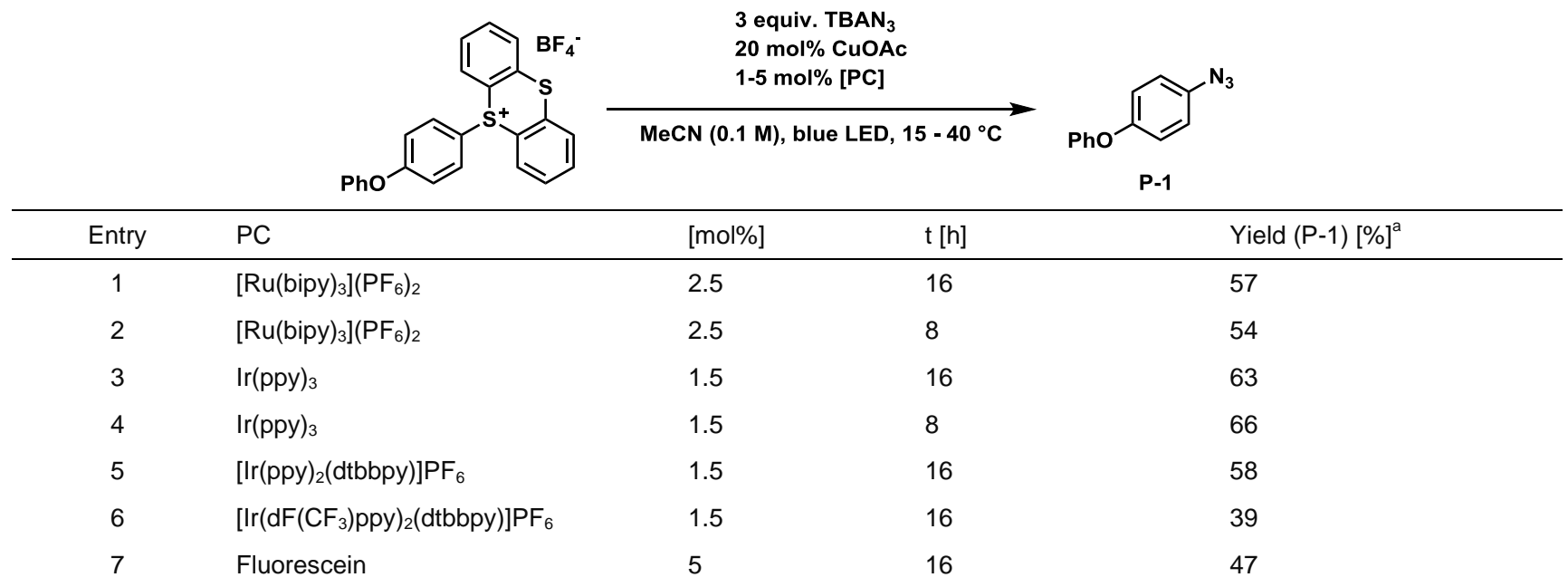

(a) isolated yield. 
Table S9: Testing of photoredox conditions for cross-coupling of aryl thianthrenium salts with morpholine.

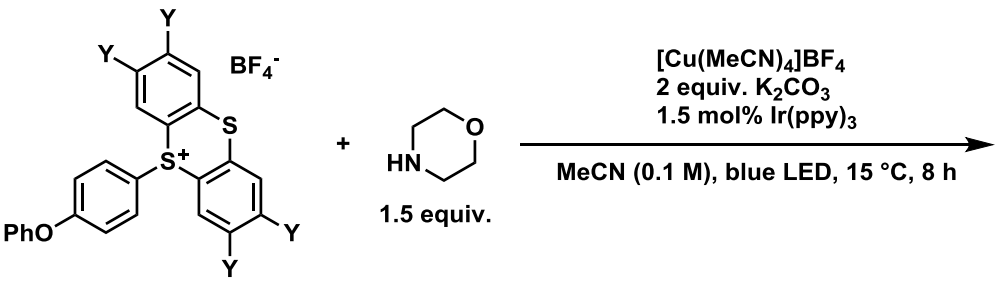

\begin{tabular}{|c|c|c|c|c|}
\hline Entry & $\mathrm{Y}$ & Equiv. ([Cu(MeCN $\left.\left.)_{4}\right] \mathrm{BF}_{4}\right)$ & 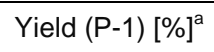 & Yield (P-2) [\%] $]^{\mathrm{a}}$ \\
\hline 1 & $\mathrm{~F}$ & 2 & 27 & 21 \\
\hline 2 & $\mathrm{~F}$ & 1 & 52 & 22 \\
\hline 3 & $\mathrm{~F}$ & 0.2 & 21 & 31 \\
\hline 4 & $\mathrm{~F}$ & 0.1 & 5 & 56 \\
\hline 5 & $\mathrm{H}$ & 2 & 48 & 8 \\
\hline 6 & $\mathrm{H}$ & 1 & 60 & 22 \\
\hline 7 & $\mathrm{H}$ & 0.2 & 11 & 39 \\
\hline 8 & $\mathrm{H}$ & 0.1 & 3 & 47 \\
\hline $9^{b}$ & $\mathrm{H}$ & 1 & - & 52 \\
\hline
\end{tabular}

(a) GC yield; (b) $\mathrm{Cu}_{2} \mathrm{O}$ instead of $\left[\mathrm{Cu}(\mathrm{MeCN})_{4}\right] \mathrm{BF}_{4}$.

\section{Bromination and lodination of Arenes}

Table S10: Qualitative selectivity for bromination and iodination of arenes; Determined by ${ }^{1} \mathrm{H}$ NMR of the residue after aqueous work-up.

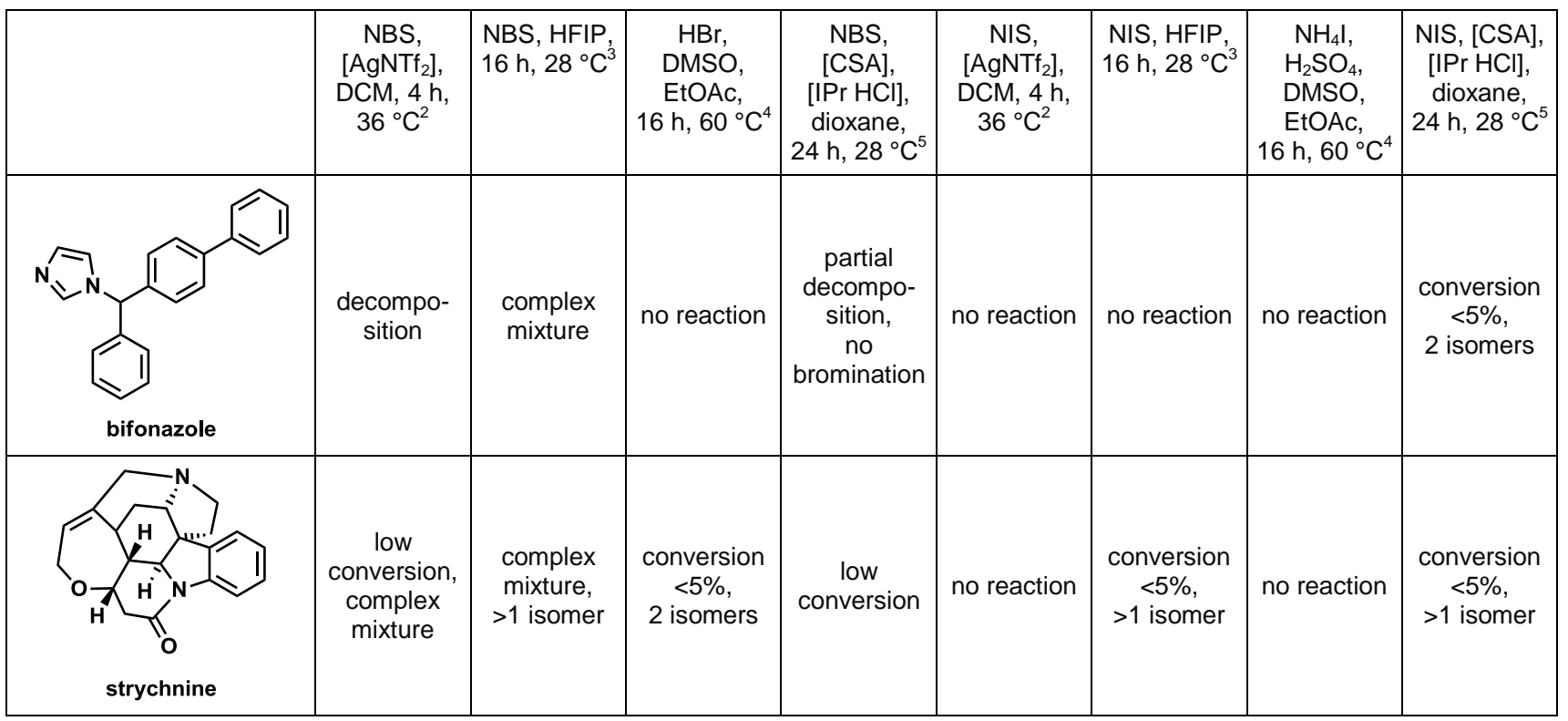

\footnotetext{
2 J. Org. Chem. 2016, 81, 772-780.

${ }^{3}$ J. Org. Chem. 2018, 83, 930-938.

${ }^{4}$ Org. Lett. 2015, 17, 2886-2889.

${ }^{5}$ Synlett 2015, 26, 2831-2834.
} 


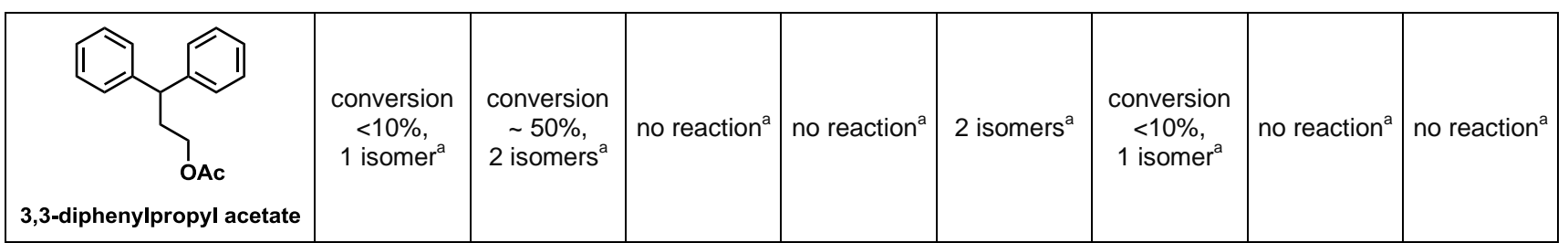

(a) 3 d reaction time. 


\section{SPECTROSCOPIC DATA}

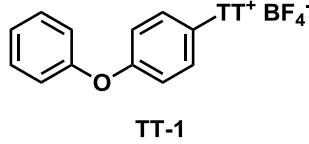

${ }^{1} \mathrm{H}$ NMR of thianthrenium salt TT-1

$\mathrm{MeCN}-\mathrm{d}_{3}, 25^{\circ} \mathrm{C}$

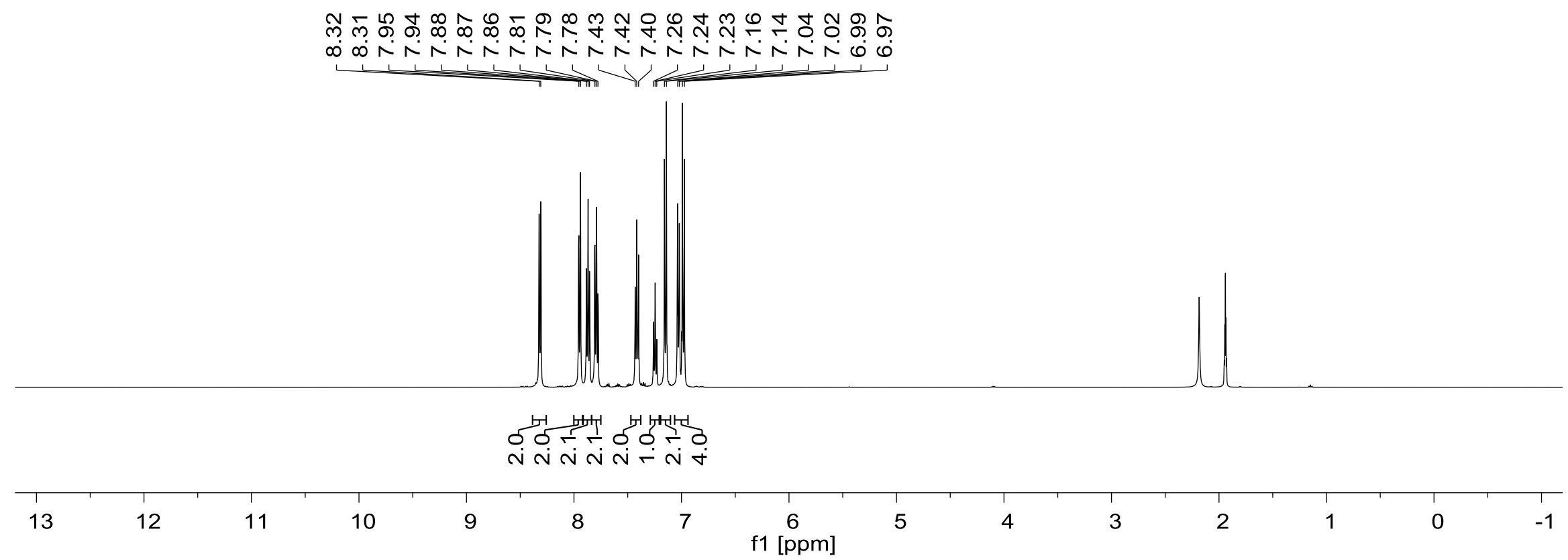




\section{${ }^{13} \mathrm{C}$ NMR of thianthrenium salt TT-1}

$\mathrm{MeCN}-\mathrm{d}_{3}, 25^{\circ} \mathrm{C}$
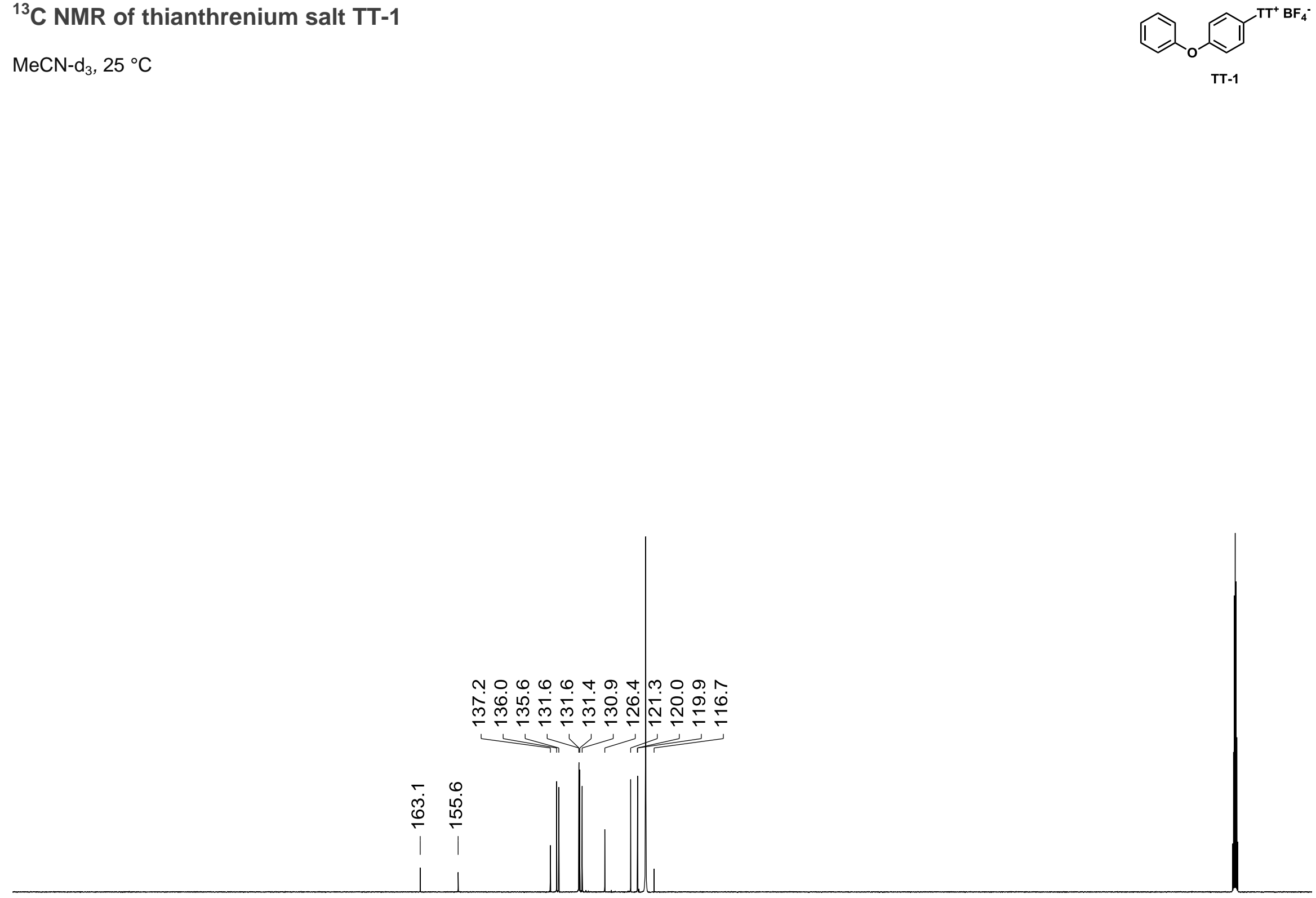

$\begin{array}{llllllllllllllllllllllllllllllll}240 & 230 & 220 & 210 & 200 & 190 & 180 & 170 & 160 & 150 & 140 & 130 & 120 & 110 & 100 & 90 & 80 & 70 & 60 & 50 & 40 & 30 & 20 & 10 & 0 & -10\end{array}$ f1 [ppm] 
${ }^{19} \mathrm{~F}$ NMR of thianthrenium salt TT-1

$\mathrm{MeCN}-\mathrm{d}_{3}, 25^{\circ} \mathrm{C}$ 
${ }^{1} \mathrm{H}$ NMR of tetrafluorothianthrenium salt TFT-1

$\mathrm{MeCN}-\mathrm{d}_{3}, 25^{\circ} \mathrm{C}$
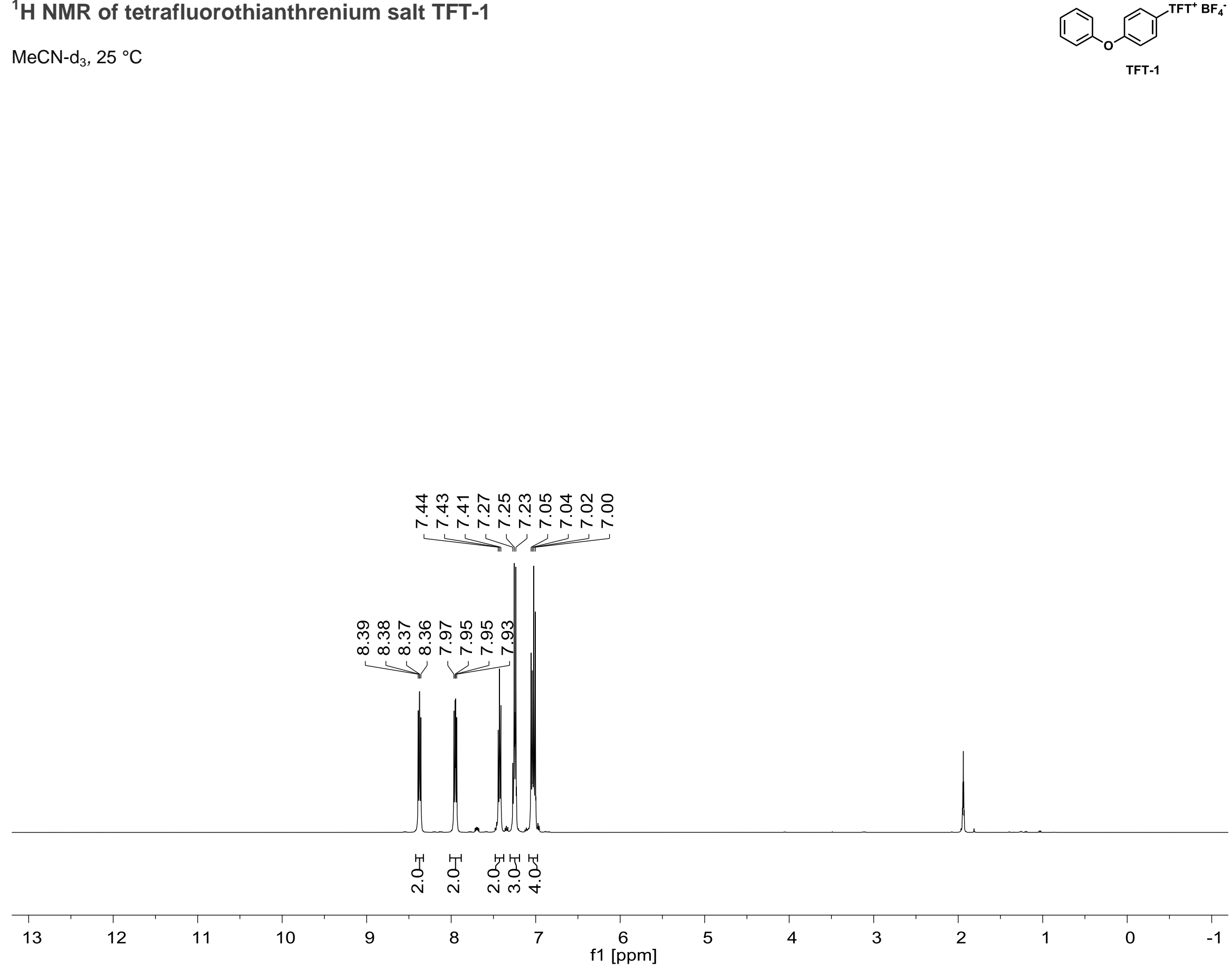


\section{${ }^{13} \mathrm{C}$ NMR of tetrafluorothianthrenium salt TFT-1}

$\mathrm{MeCN}-\mathrm{d} 3,25^{\circ} \mathrm{C}$

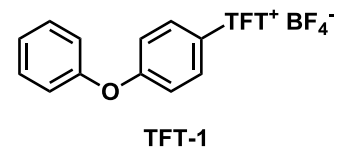

이 の

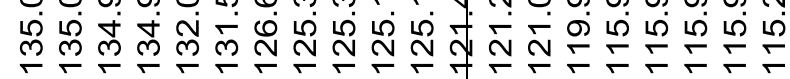

เ $\infty$ ก

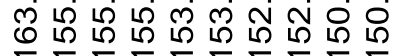
य 1

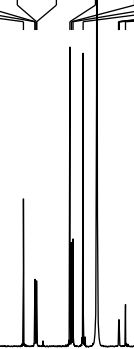


${ }^{19} \mathrm{~F}$ NMR of tetrafluorothianthrenium salt TFT-1

$\mathrm{MeCN}-\mathrm{d}_{3}, 25^{\circ} \mathrm{C}$
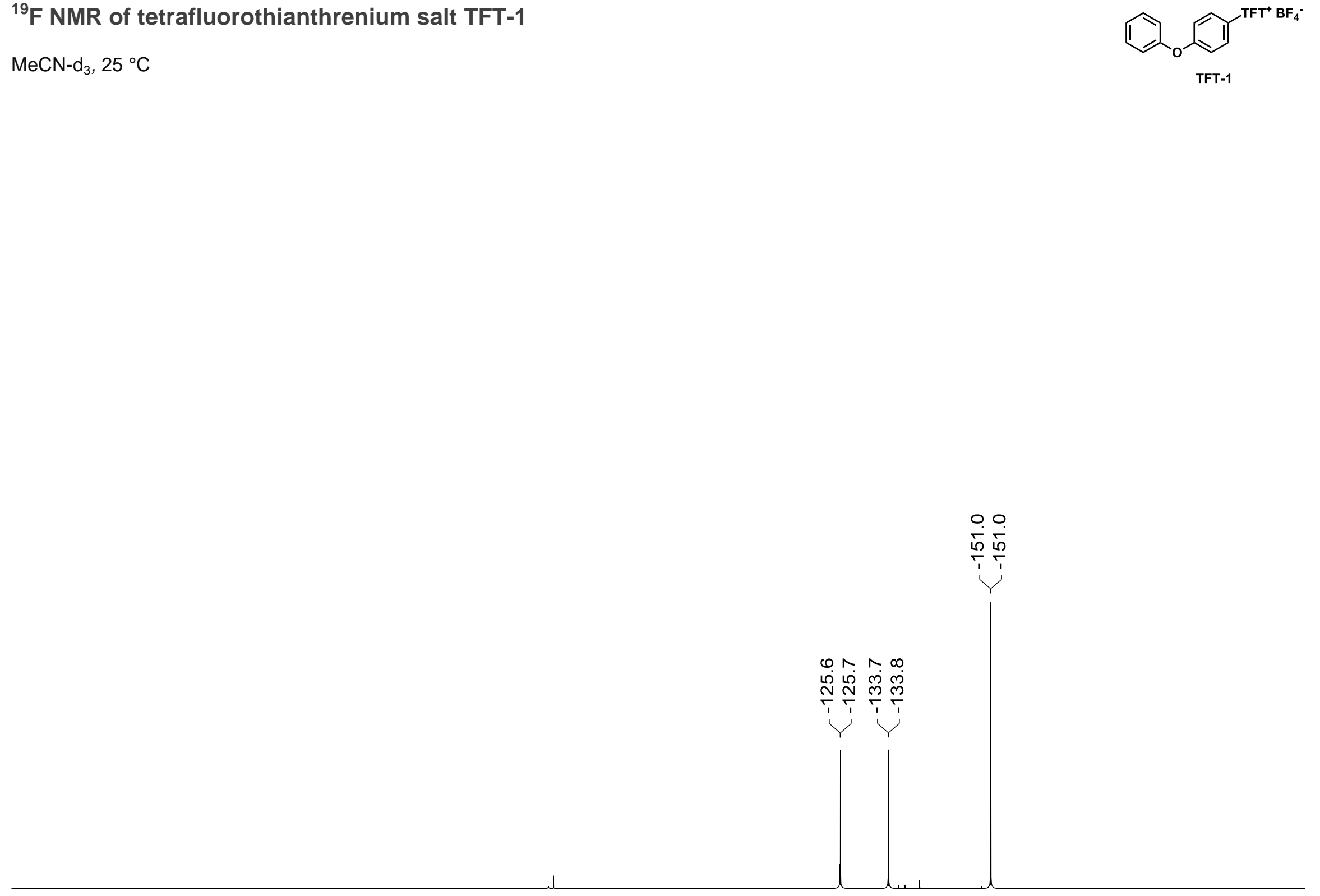


\section{${ }^{1} \mathrm{H}$ NMR of aniline 1a}

$\mathrm{CDCl}_{3}, 25^{\circ} \mathrm{C}$

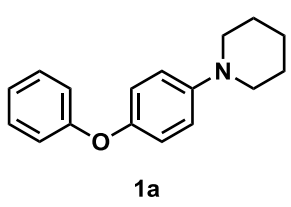

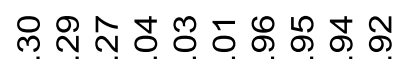

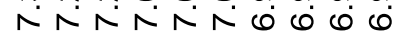

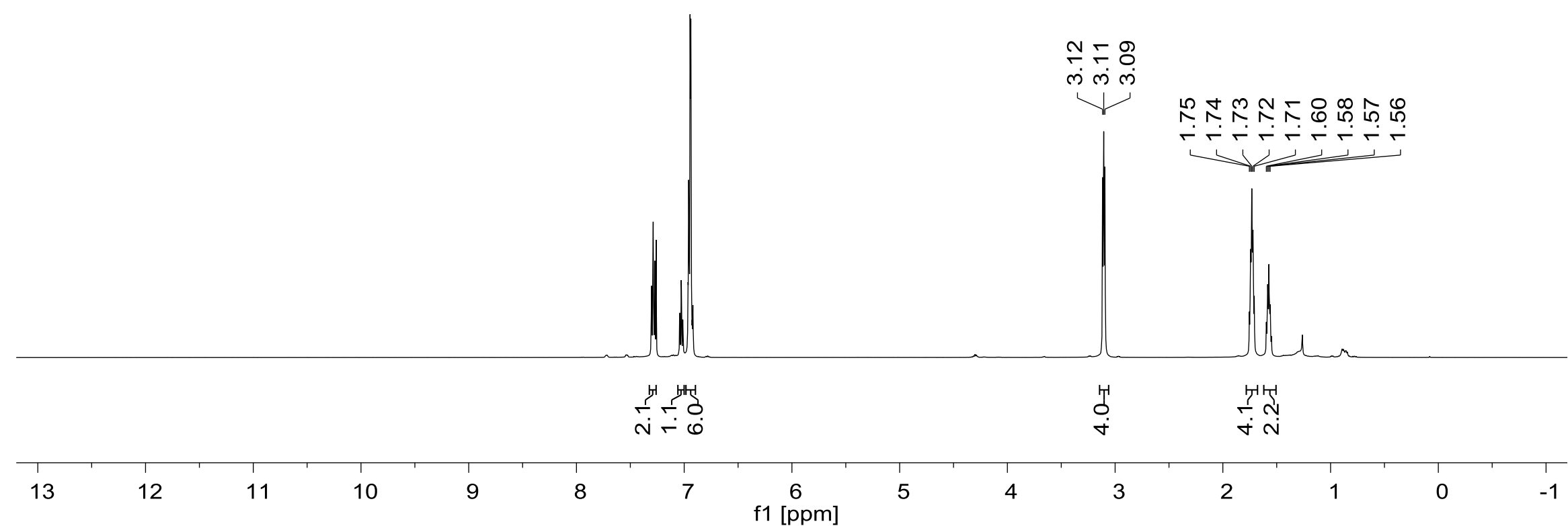




\section{${ }^{13} \mathrm{C}$ NMR of aniline $1 \mathrm{a}$}

$\mathrm{CDCl}_{3}, 25^{\circ} \mathrm{C}$

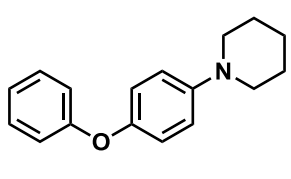

1

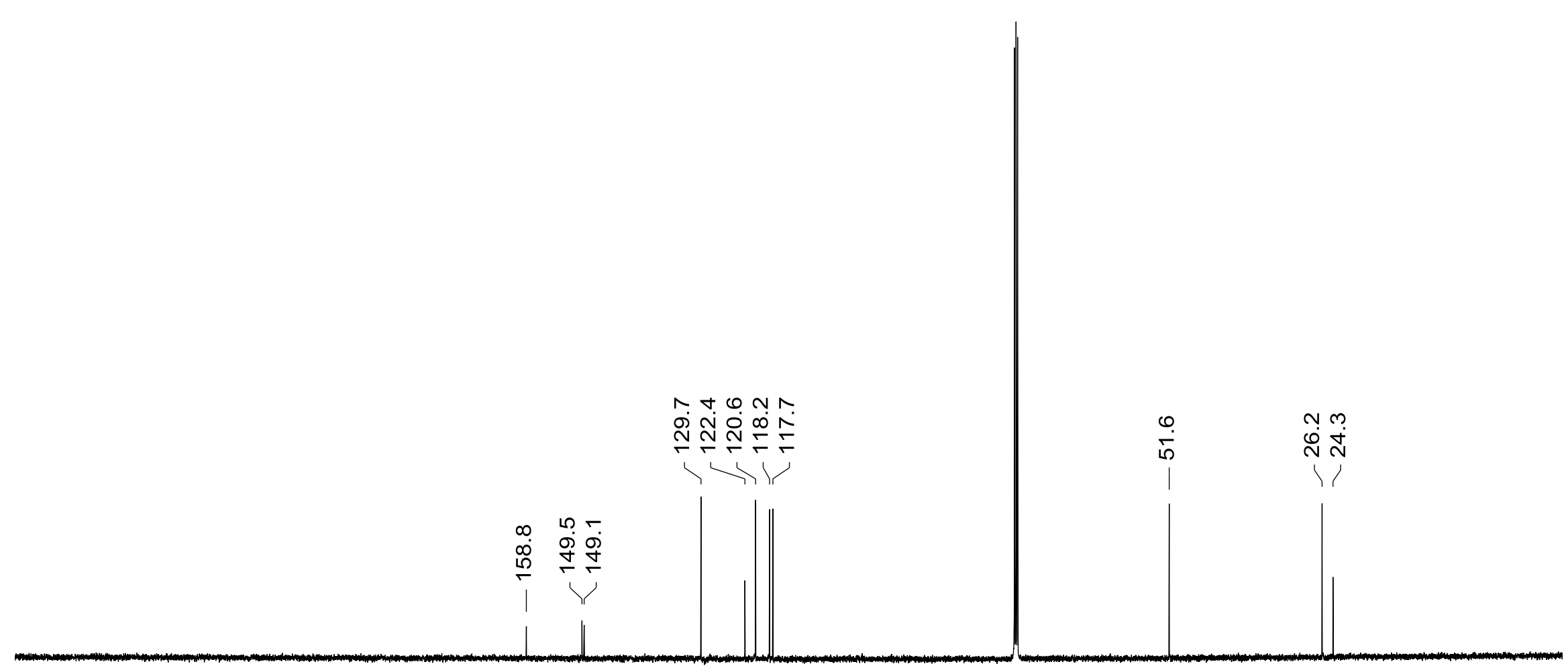




\section{${ }^{1} \mathrm{H}$ NMR of morpholine $1 \mathrm{~b}$}

$\mathrm{CDCl}_{3}, 25^{\circ} \mathrm{C}$

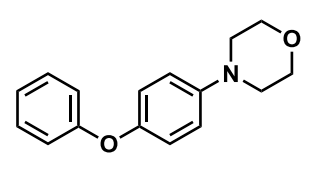

1b

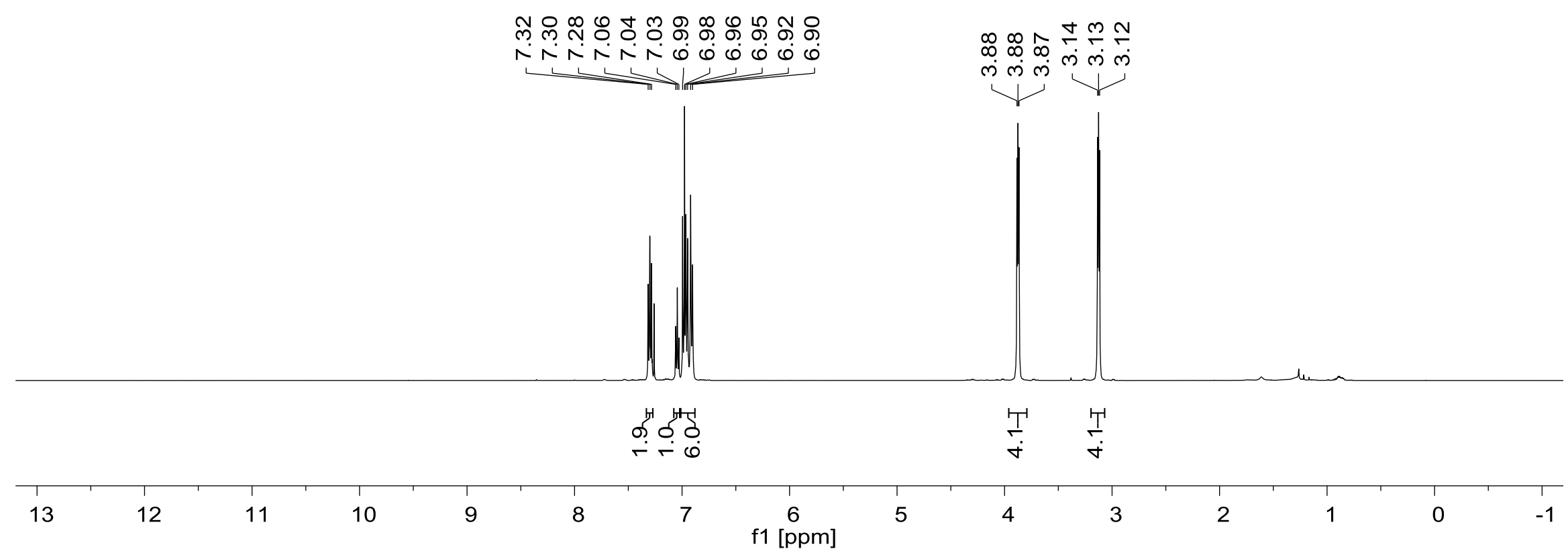




\section{${ }^{13} \mathrm{C}$ NMR of morpholine $1 \mathrm{~b}$}

$\mathrm{CDCl}_{3}, 25^{\circ} \mathrm{C}$

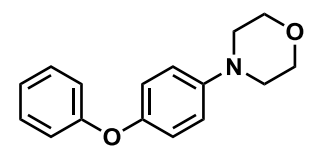

$1 \mathrm{~b}$

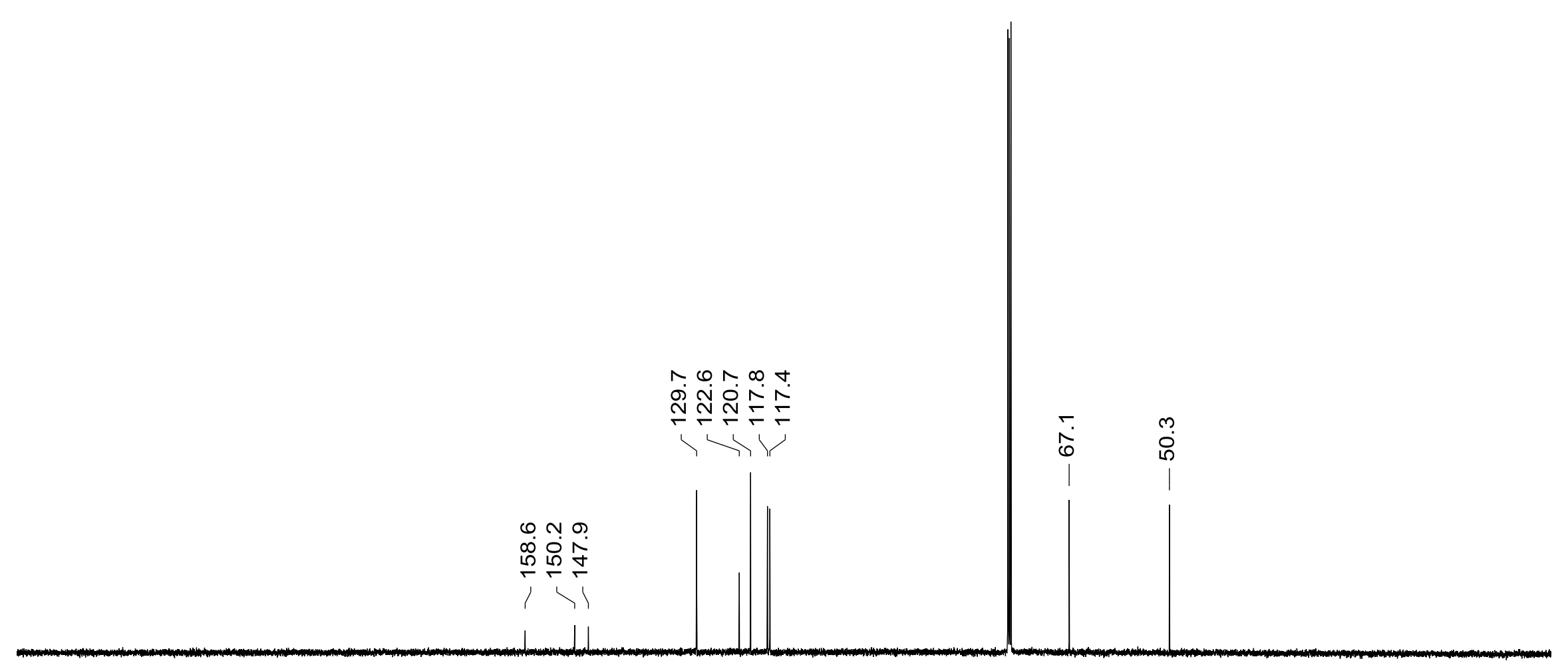

$\begin{array}{llllllllllllllllllllllllllllllll}240 & 230 & 220 & 210 & 200 & 190 & 180 & 170 & 160 & 150 & 140 & 130 & 120 & 110 & 100 & 90 & 80 & 70 & 60 & 50 & 40 & 30 & 20 & 10 & 0 & -10\end{array}$ f1 [ppm] 


\section{${ }^{1} \mathrm{H}$ NMR of piperazine $1 \mathrm{c}$}

$\mathrm{CDCl}_{3}, 25^{\circ} \mathrm{C}$

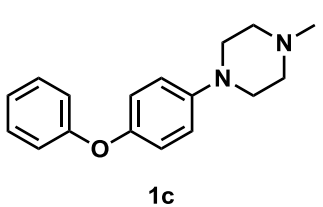

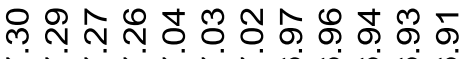

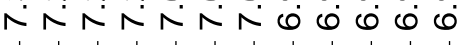

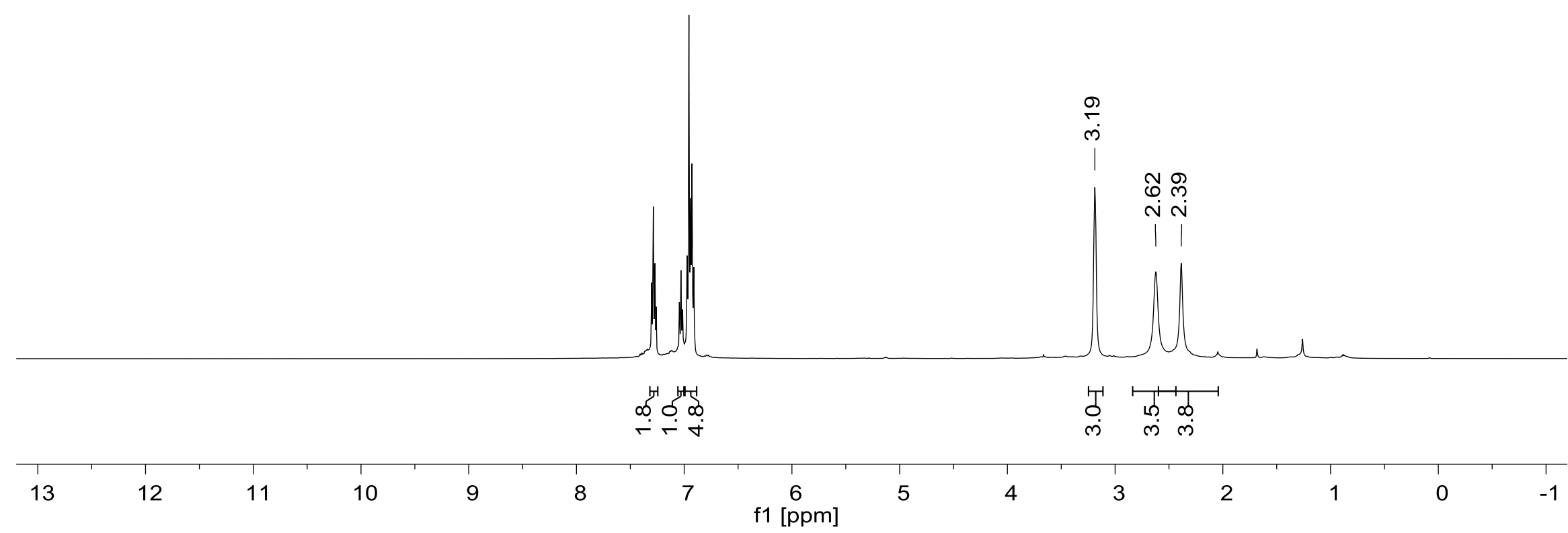




\section{${ }^{13} \mathrm{C}$ NMR of piperazine $1 \mathrm{c}$}

$\mathrm{CDCl}_{3}, 25^{\circ} \mathrm{C}$
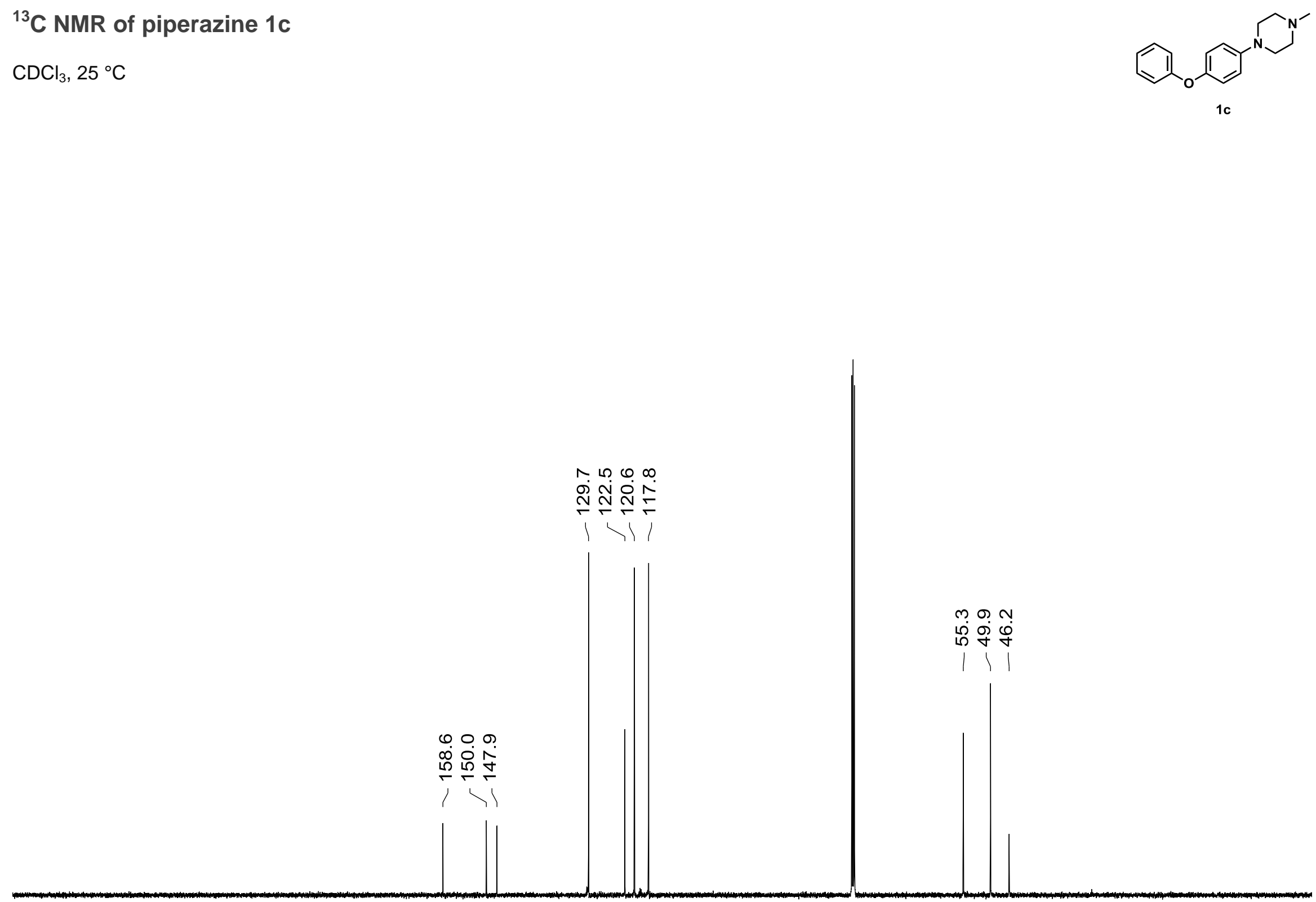


\section{${ }^{1} \mathrm{H}$ NMR of aniline $1 \mathrm{~d}$}

$\mathrm{CDCl}_{3}, 25^{\circ} \mathrm{C}$
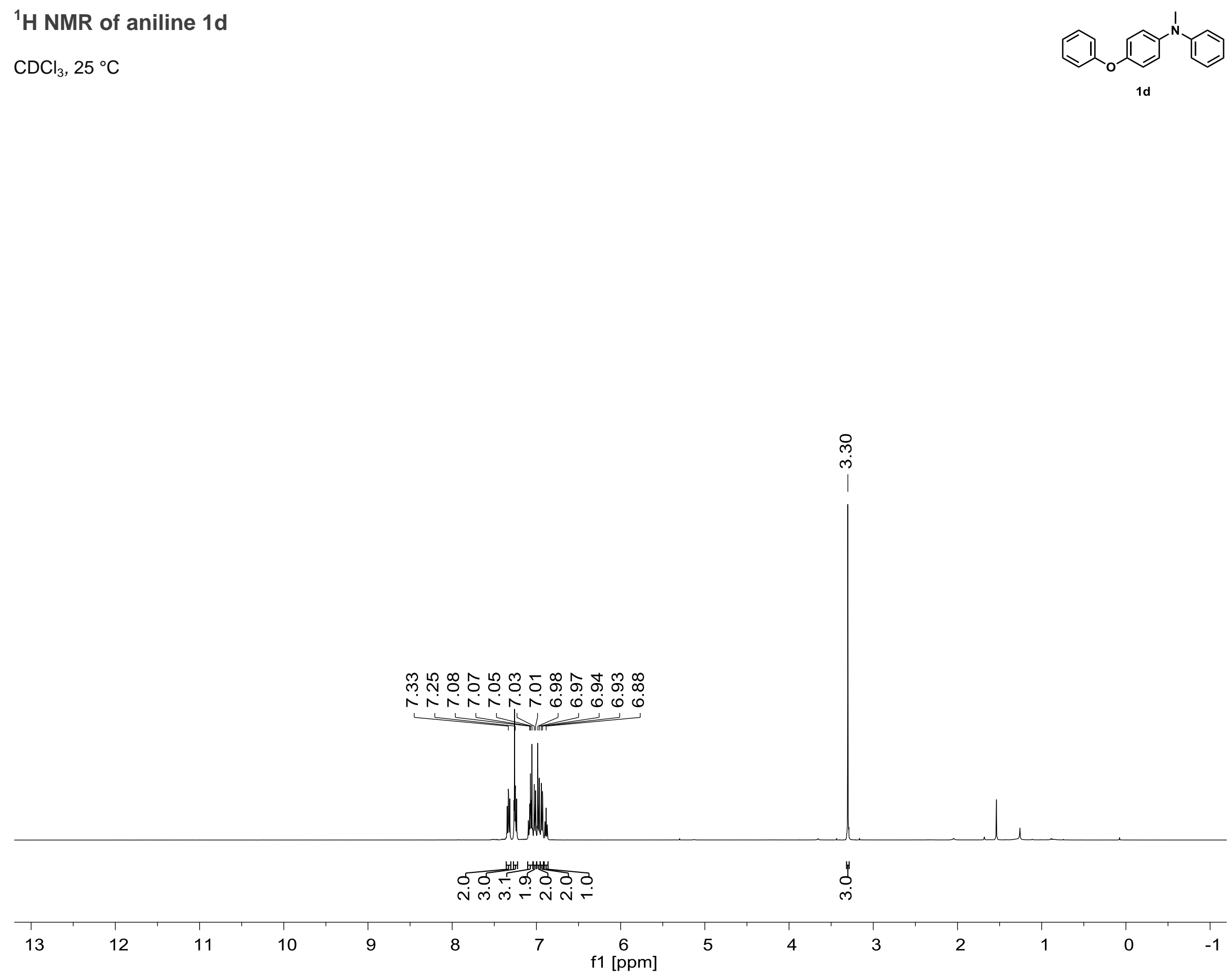


\section{${ }^{13} \mathrm{C}$ NMR of aniline $1 \mathrm{~d}$}

$\mathrm{CDCl}_{3}, 25^{\circ} \mathrm{C}$
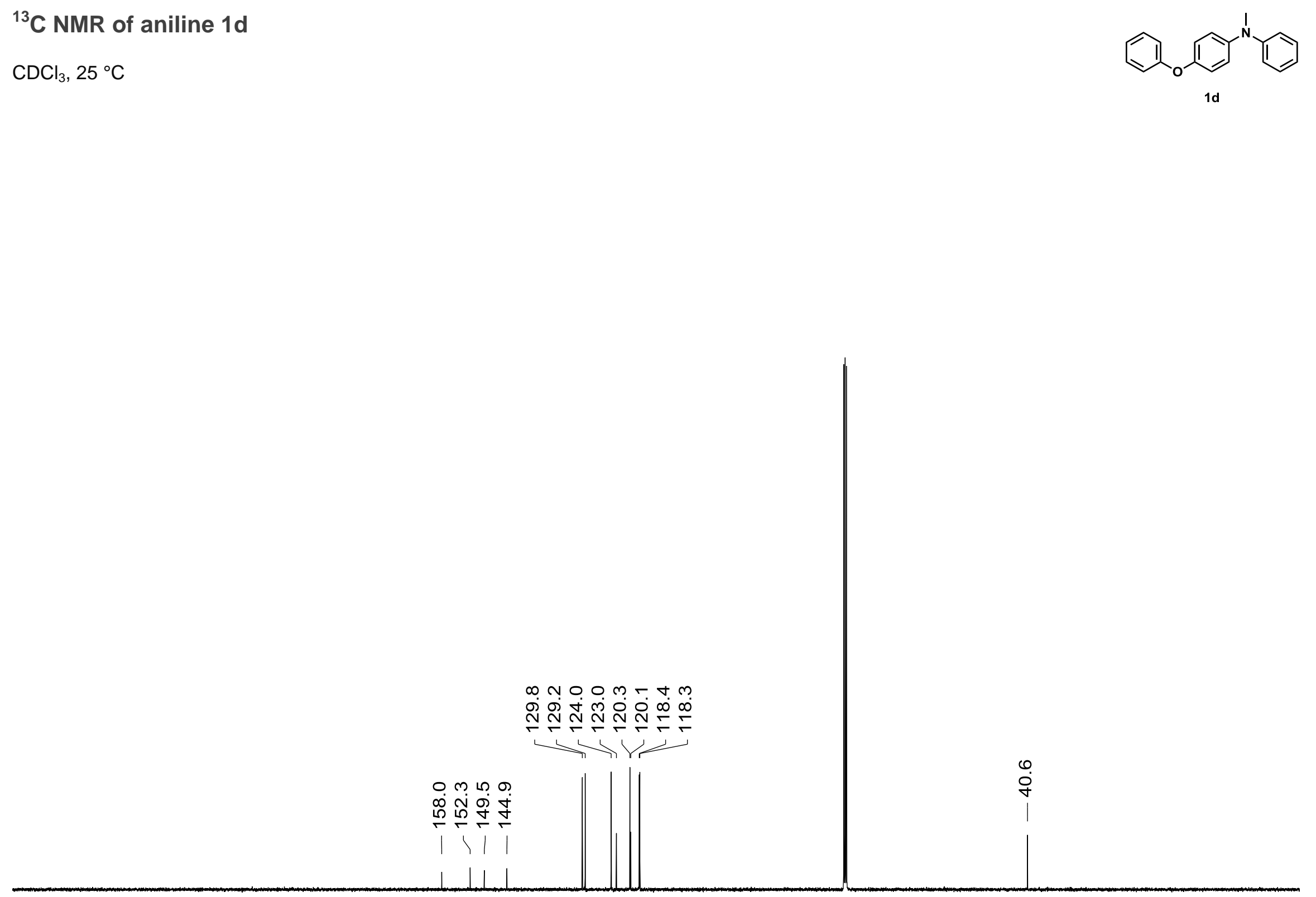

$\begin{array}{lllllllllllllllllllllllllllllllllll}240 & 230 & 220 & 210 & 200 & 190 & 180 & 170 & 160 & 150 & 140 & 130 & 120 & 110 & 100 & 90 & 80 & 70 & 60 & 50 & 40 & 30 & 20 & 10 & 0 & -10\end{array}$ f1 [ppm] 


\section{${ }^{1} \mathrm{H}$ NMR of aniline $1 \mathrm{e}$}

$\mathrm{CDCl}_{3}, 25^{\circ} \mathrm{C}$

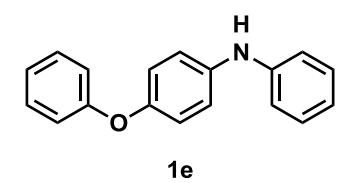

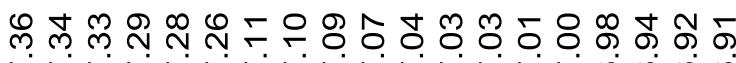

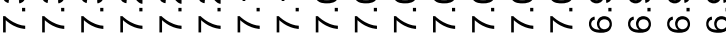

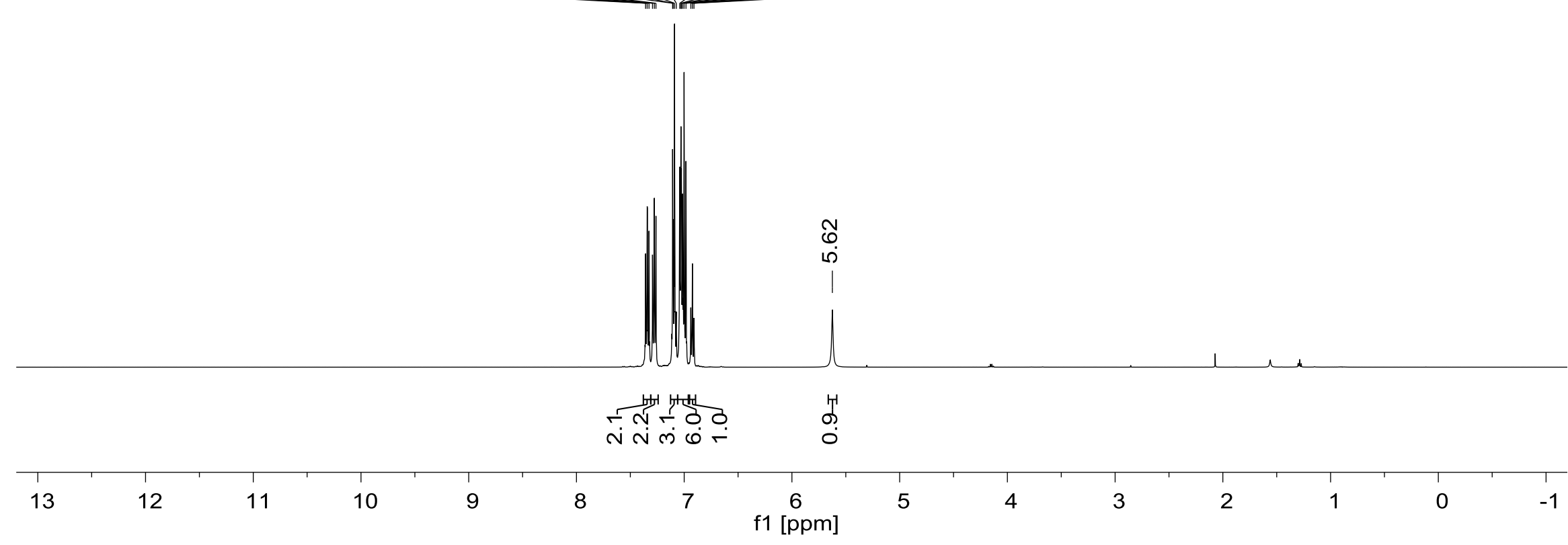




\section{${ }^{13} \mathrm{C}$ NMR of aniline $1 \mathrm{e}$}

$\mathrm{CDCl}_{3}, 25^{\circ} \mathrm{C}$
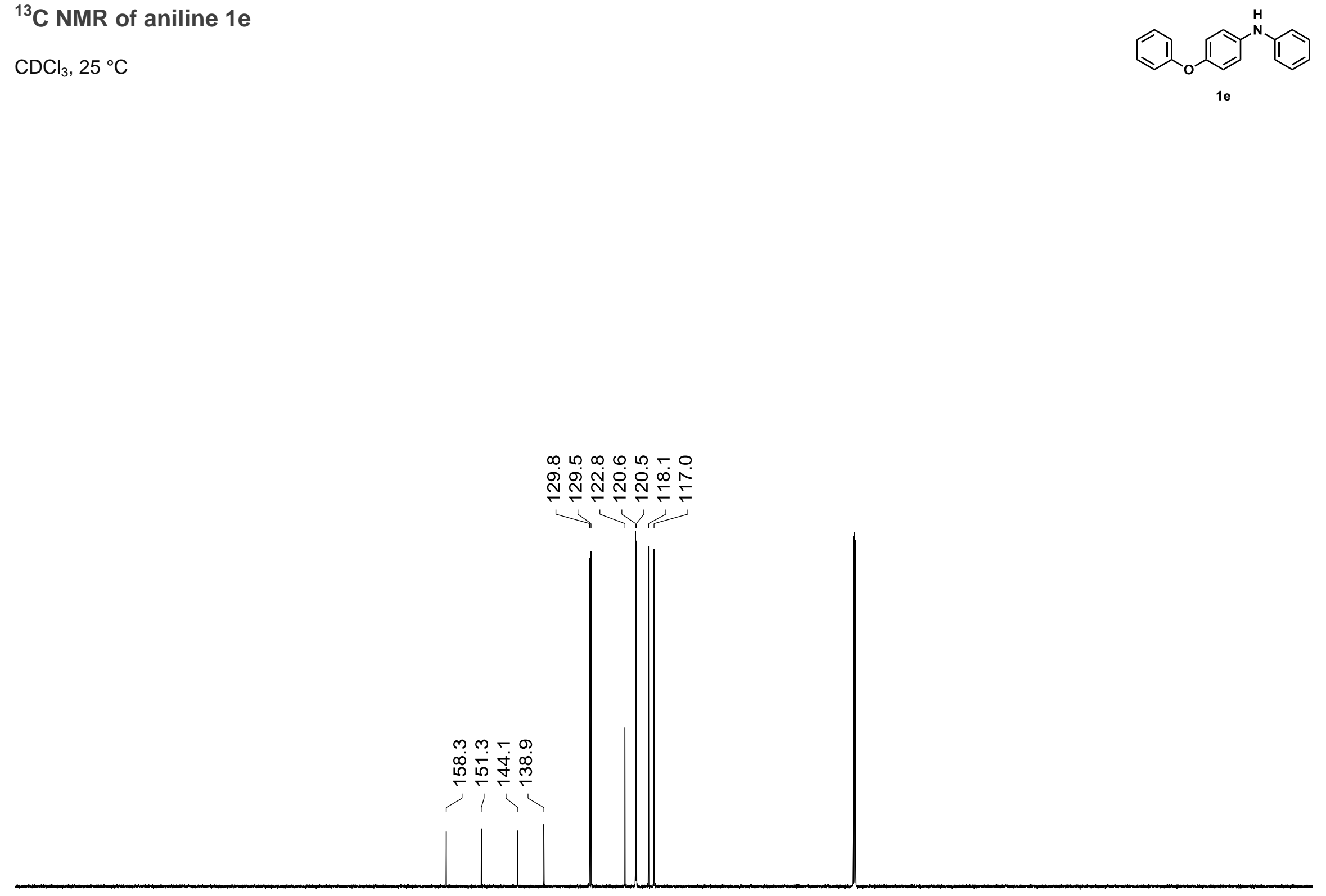


\section{${ }^{1} \mathrm{H}$ NMR of aminopyridine $1 \mathrm{f}$}

$\mathrm{CDCl}_{3}, 25^{\circ} \mathrm{C}$

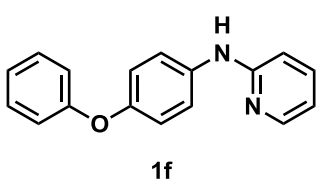

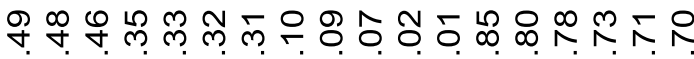

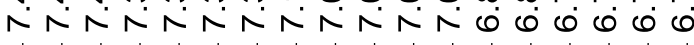

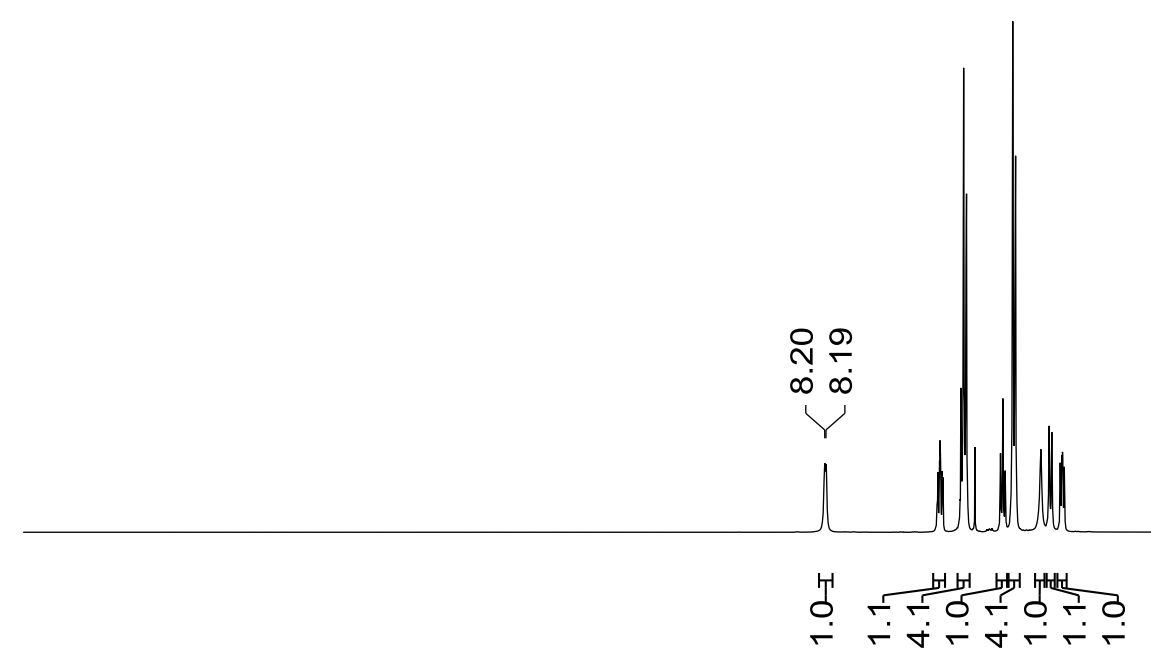

$\begin{array}{rlllllll}13 & 12 & 11 & 10 & 9 & 8 & 7 & 6 \\ \mathrm{f} 1[\mathrm{ppm}\end{array}$

6
f1 $[\mathrm{ppm}]$

3

2 


\section{${ }^{13} \mathrm{C}$ NMR of aminopyridine $1 \mathrm{f}$}

$\mathrm{CDCl}_{3}, 25^{\circ} \mathrm{C}$

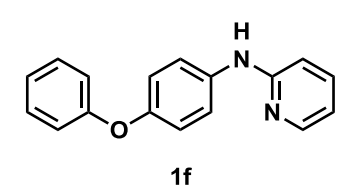

$\infty$ ํ $\infty$ ○ $\infty$ m

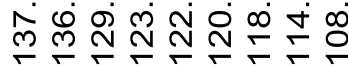

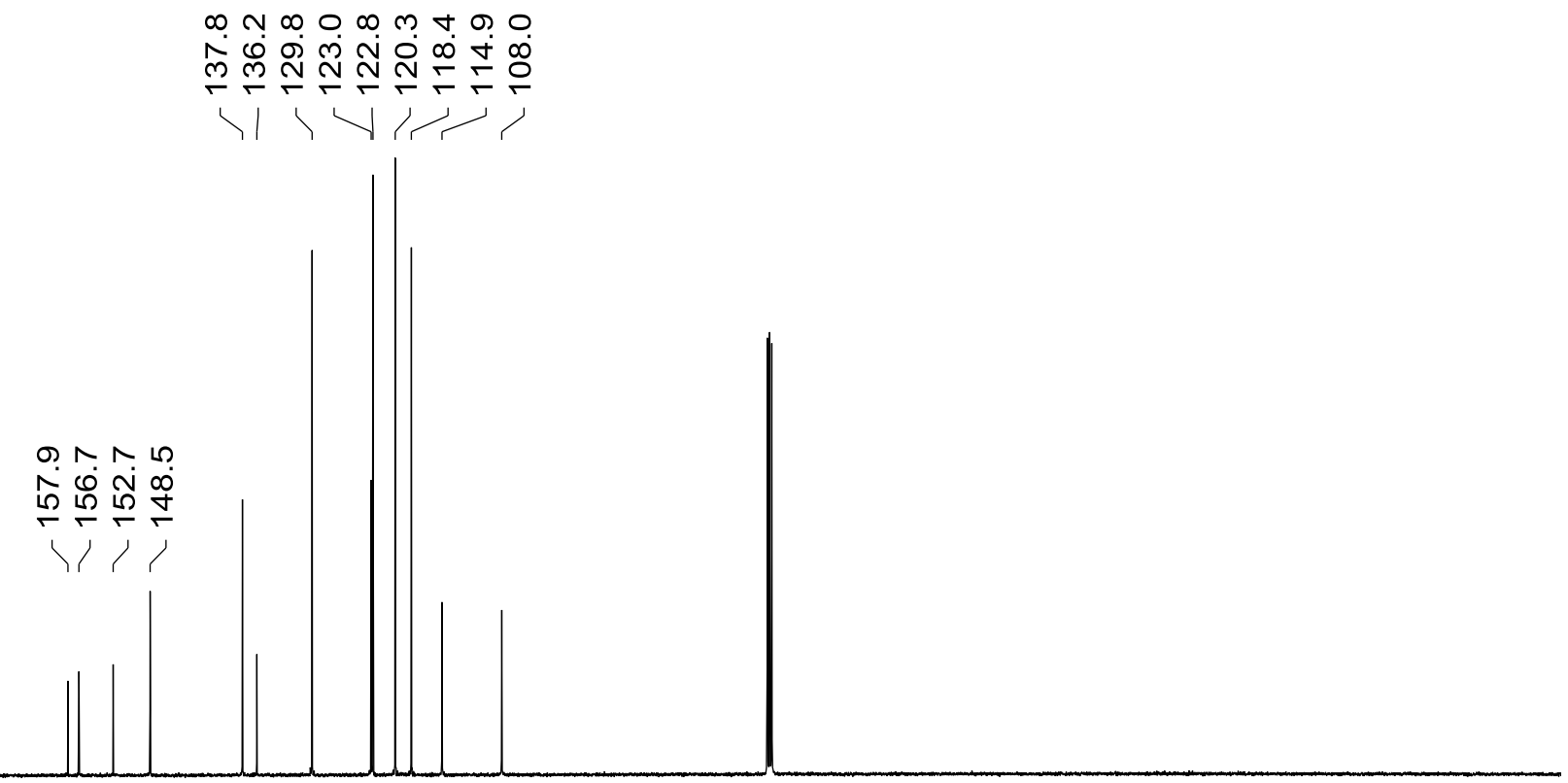




\section{${ }^{1} \mathrm{H}$ NMR of aminothiazole $1 \mathrm{~g}$}

$\mathrm{CDCl}_{3}, 25^{\circ} \mathrm{C}$
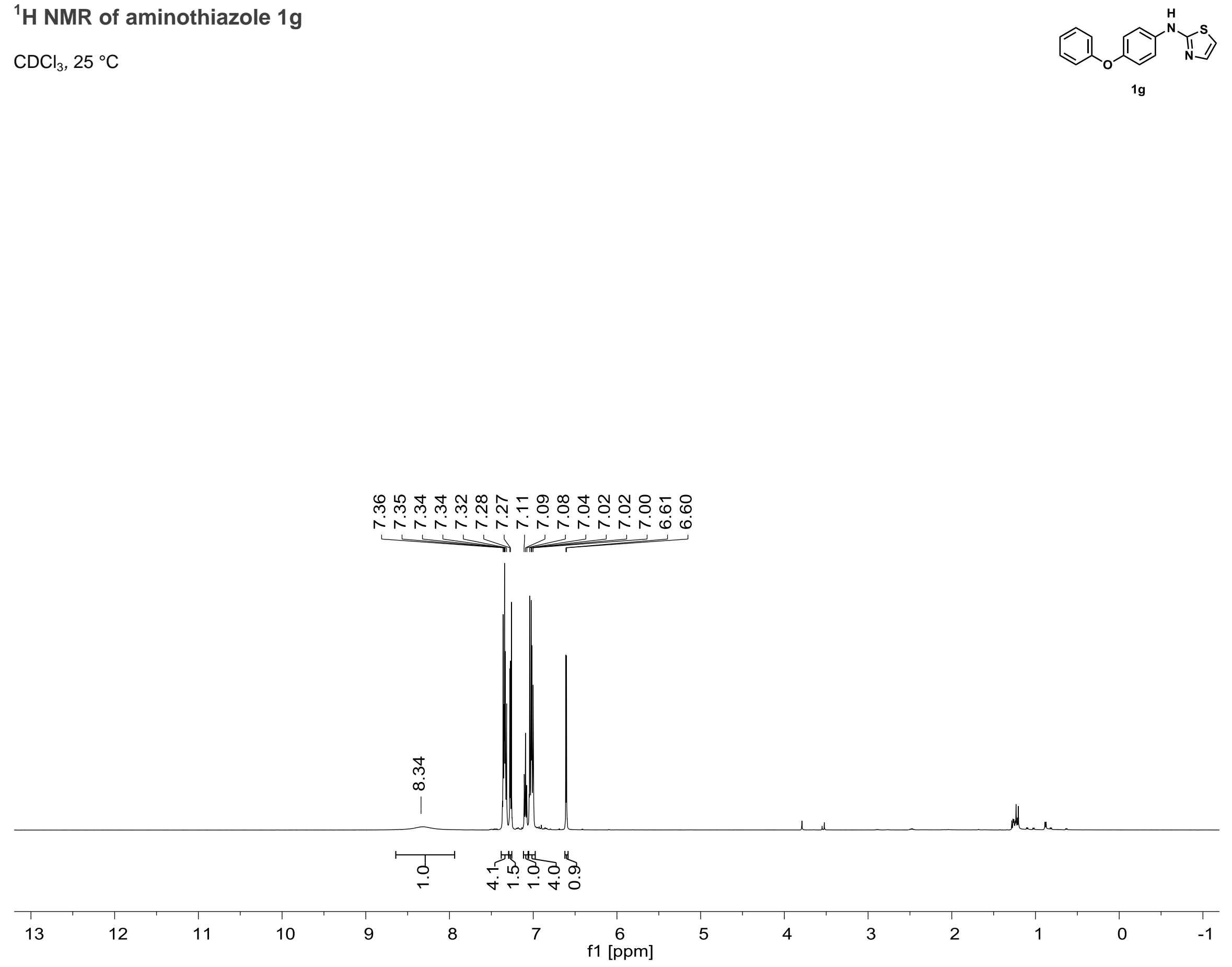


\section{${ }^{13} \mathrm{C}$ NMR of aminothiazole $1 \mathrm{~g}$}

$\mathrm{CDCl}_{3}, 25^{\circ} \mathrm{C}$
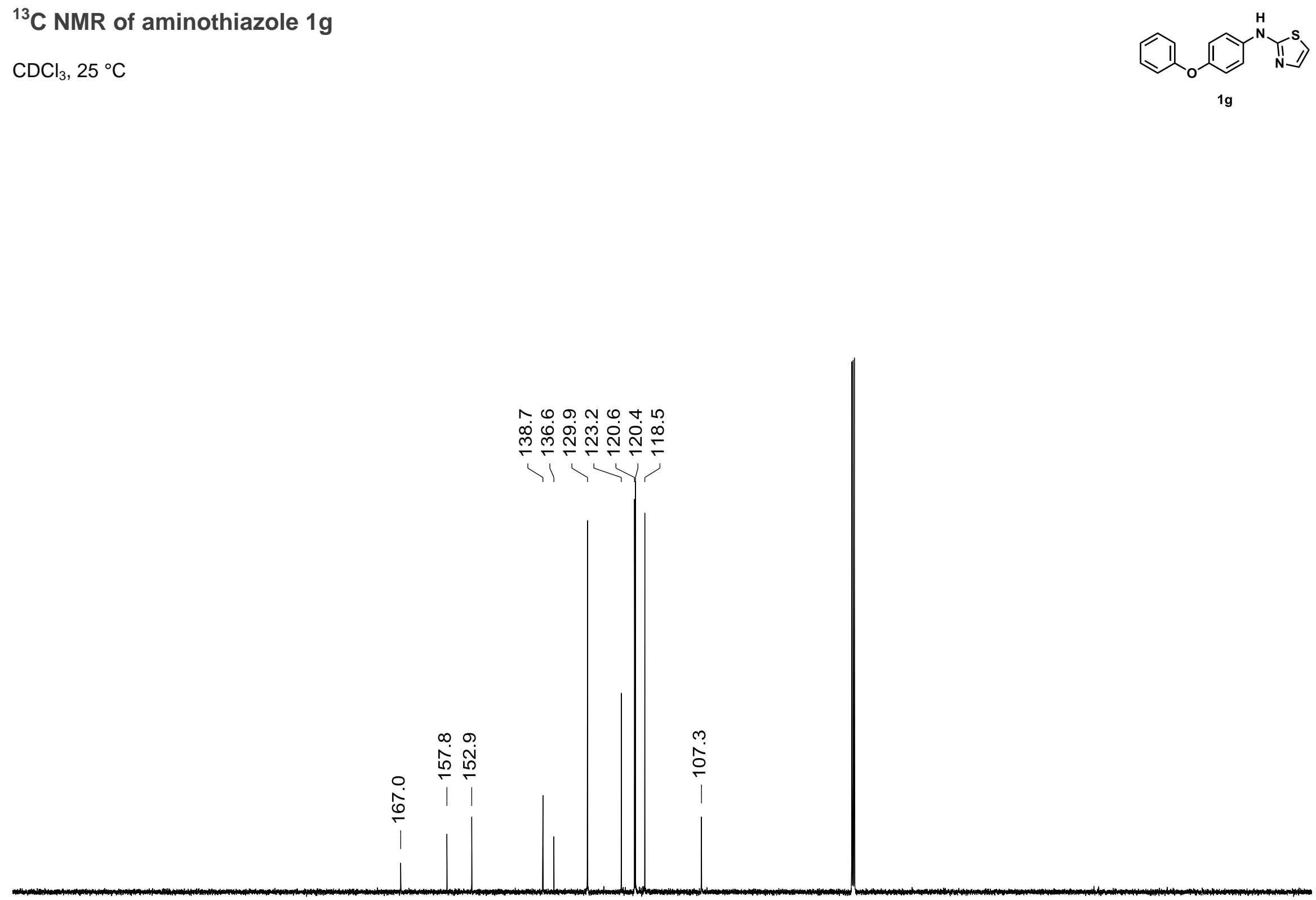


\section{${ }^{1} \mathrm{H}$ NMR of isonicotinamide $1 \mathrm{~h}$}

$\mathrm{CDCl}_{3}, 25^{\circ} \mathrm{C}$

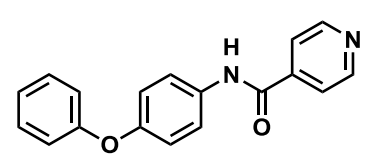

$1 \mathrm{~h}$

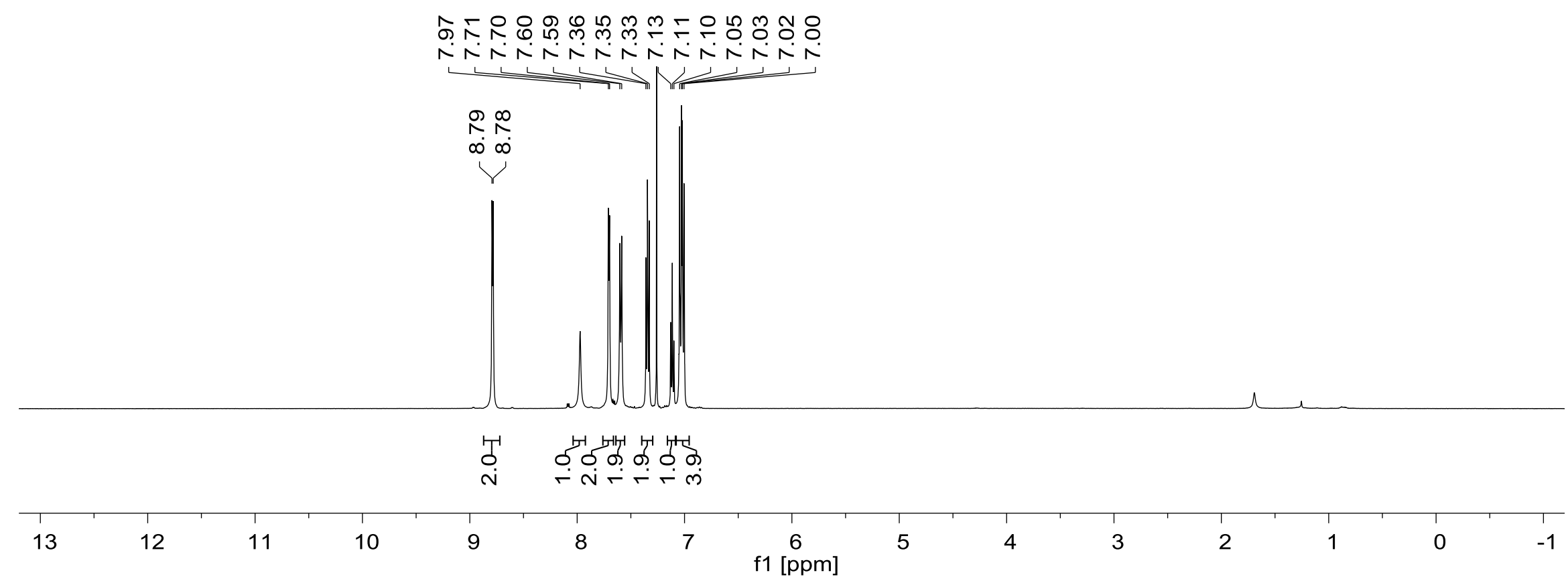




\section{${ }^{13} \mathrm{C}$ NMR of isonicotinamide $1 \mathrm{~h}$}

$\mathrm{CDCl}_{3}, 25^{\circ} \mathrm{C}$

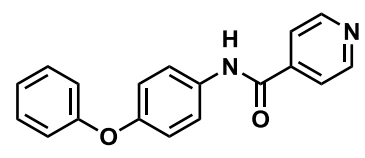

$1 \mathrm{~h}$

0 . ㅇ m

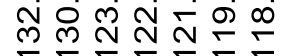

m

ชู

$r-$

$\mid>1$

पराए

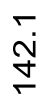

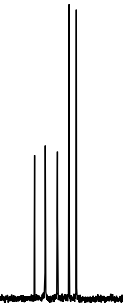


${ }^{1} \mathrm{H}$ NMR of oxazolidinone $1 \mathrm{i}$

$\mathrm{CDCl}_{3}, 25^{\circ} \mathrm{C}$

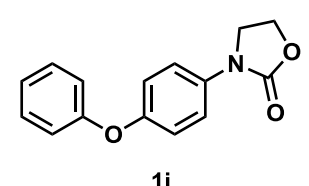

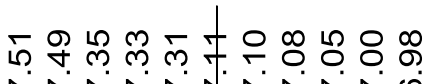

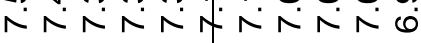

$\longrightarrow$

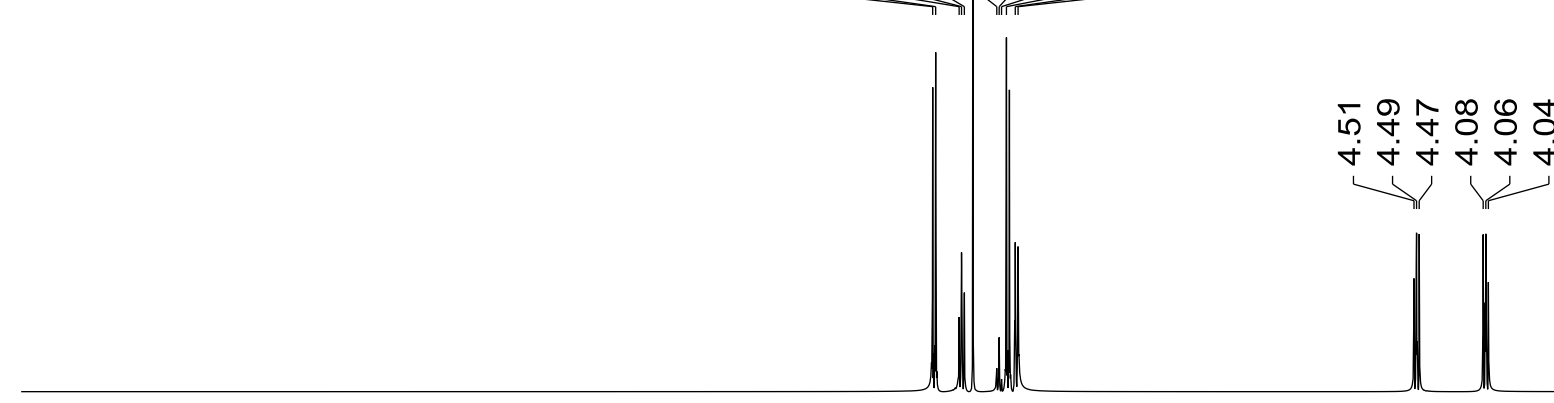

7




\section{${ }^{13} \mathrm{C}$ NMR of oxazolidinone $1 \mathrm{i}$}

$\mathrm{CDCl}_{3}, 25^{\circ} \mathrm{C}$
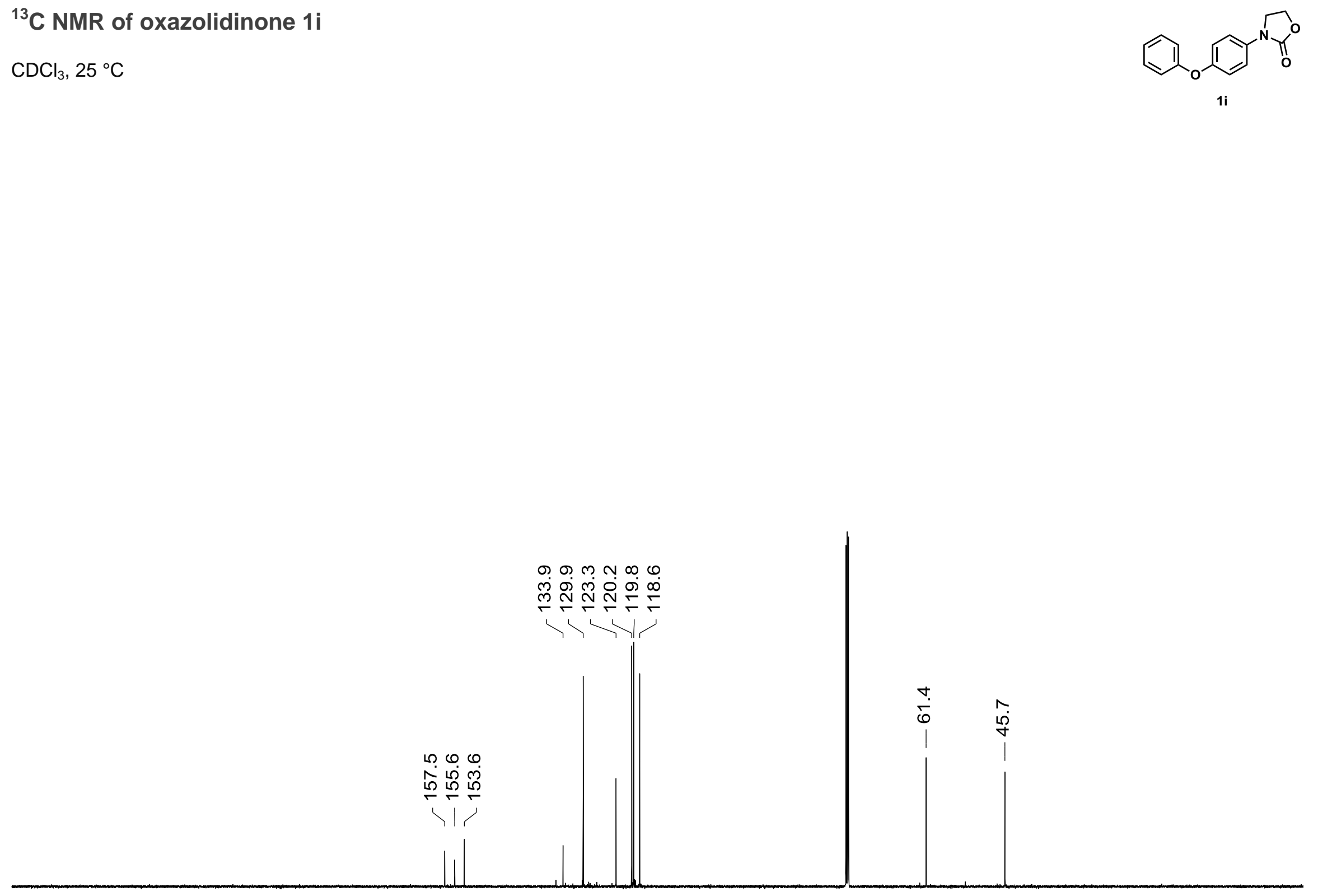

$\begin{array}{lllllllllllllllllllllllll}240 & 230 & 220 & 210 & 200 & 190 & 180 & 170 & 160 & 150 & 140 & 130 & \begin{array}{c}120 \\ \mathrm{f} 1\end{array}\left[\begin{array}{ll}110 \\ {[\mathrm{ppm}]}\end{array}\right. & 100 & 90 & 80 & 70 & 60 & 50 & 40 & 30 & 20 & 10 & 0 & -10\end{array}$ 


\section{${ }^{1} \mathrm{H}$ NMR of urea $1 \mathrm{j}$}

DMSO- $\mathrm{d}_{6}, 25^{\circ} \mathrm{C}$
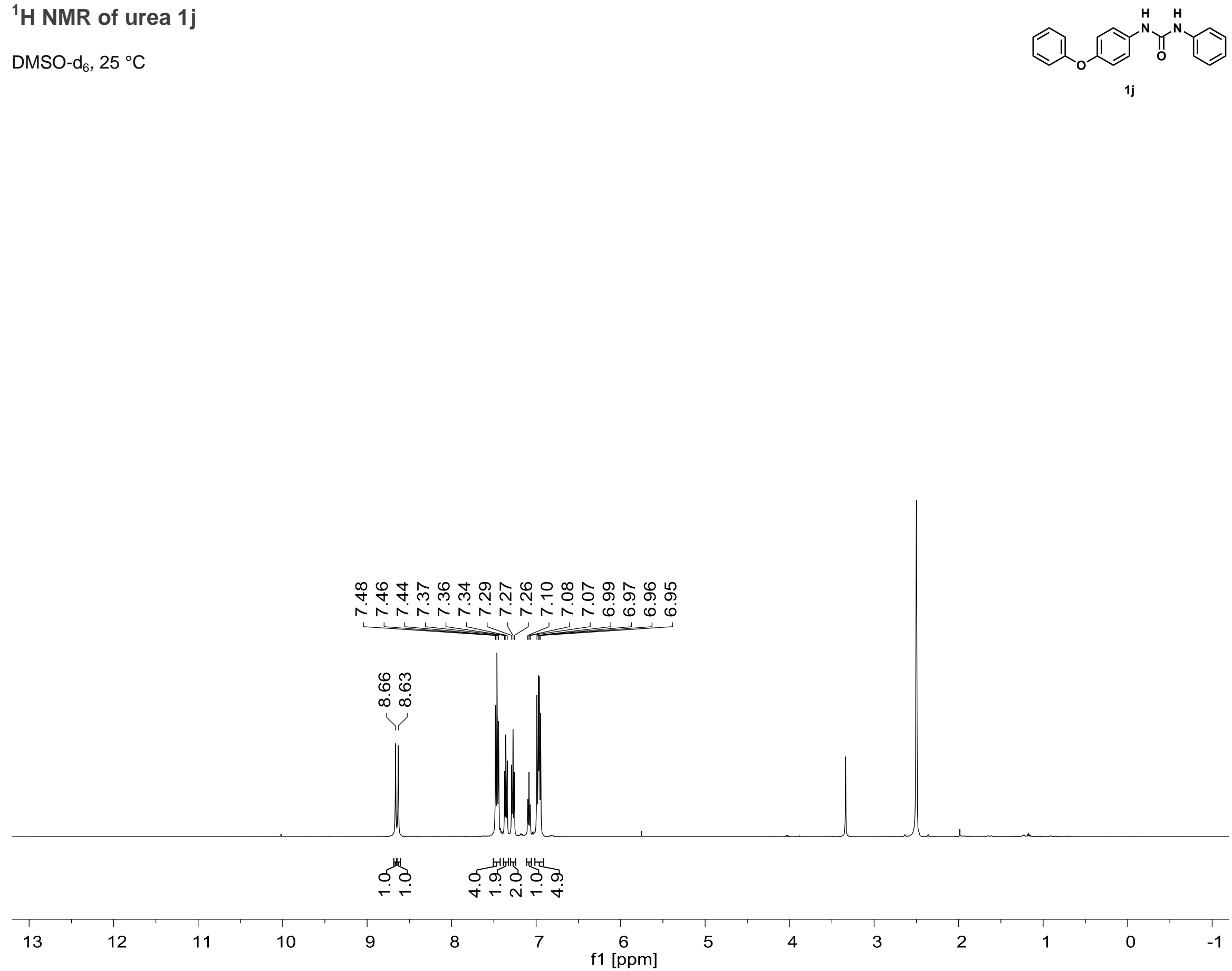


\section{${ }^{13} \mathrm{C}$ NMR of urea $1 \mathrm{j}$}

DMSO- $\mathrm{d}_{6}, 25^{\circ} \mathrm{C}$

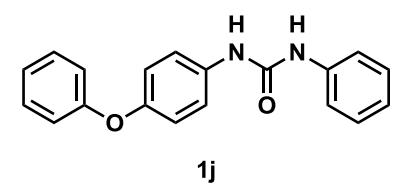

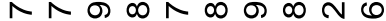

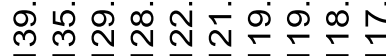

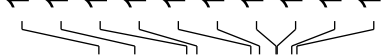

N 60

ำ กำ 


\section{${ }^{1} \mathrm{H}$ NMR of pyrazole $1 \mathrm{k}$}

$\mathrm{CDCl}_{3}, 25^{\circ} \mathrm{C}$

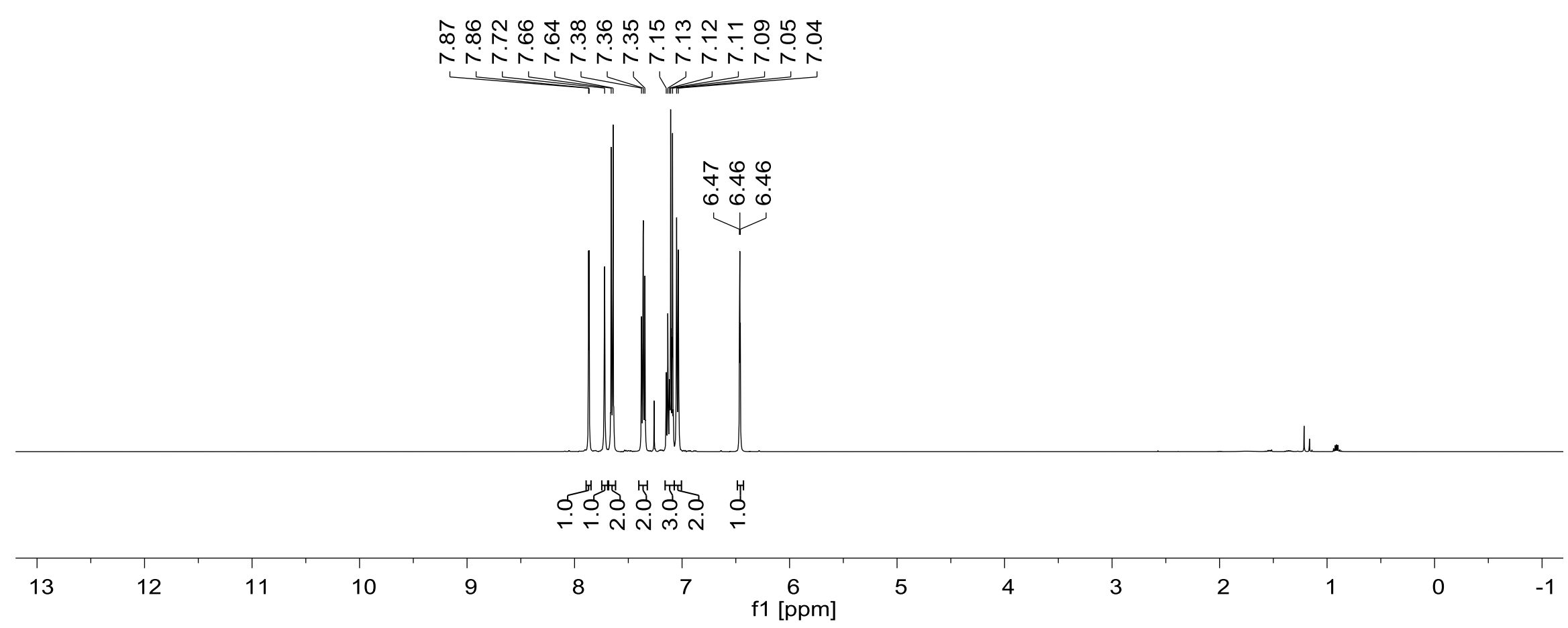




\section{${ }^{13} \mathrm{C}$ NMR of pyrazole $1 \mathrm{k}$}

$\mathrm{CDCl}_{3}, 25 \stackrel{\circ}{\circ}$

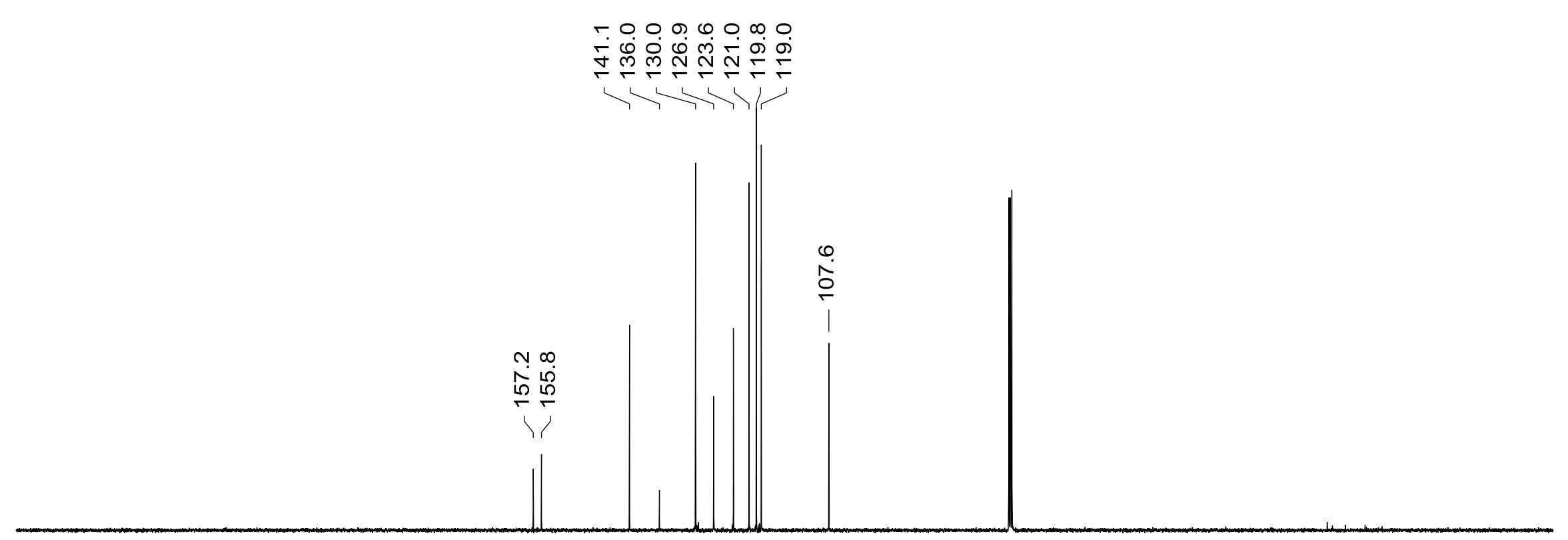




\section{${ }^{1} \mathrm{H}$ NMR of pyrazolopyridine 1 I}

$\mathrm{CDCl}_{3}, 25^{\circ} \mathrm{C}$

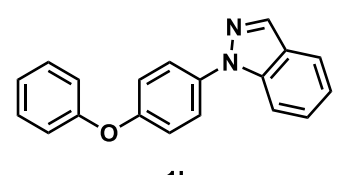

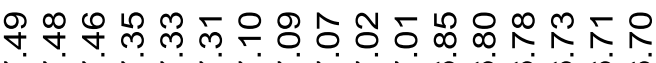

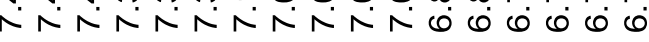

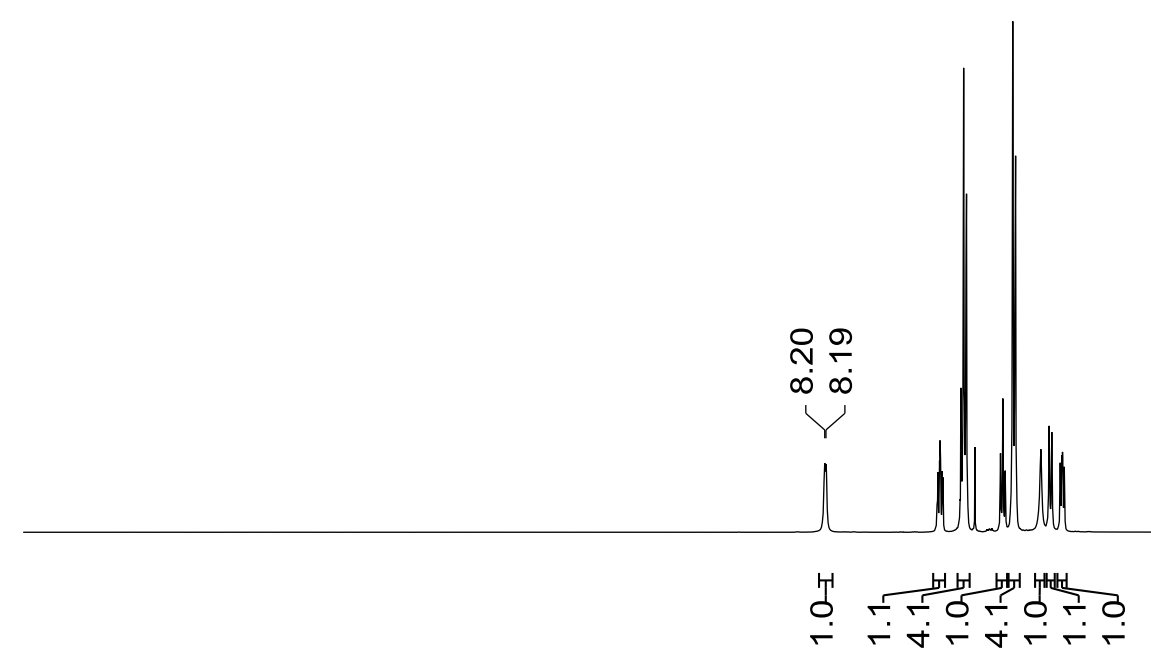




\section{${ }^{13} \mathrm{C}$ NMR of pyrazolopyridine $1 \mathrm{I}$}

$\mathrm{CDCl}_{3}, 25^{\circ} \mathrm{C}$

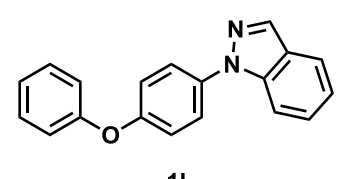

$\infty$ ก $\infty$ ○ $\infty$ m

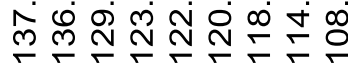

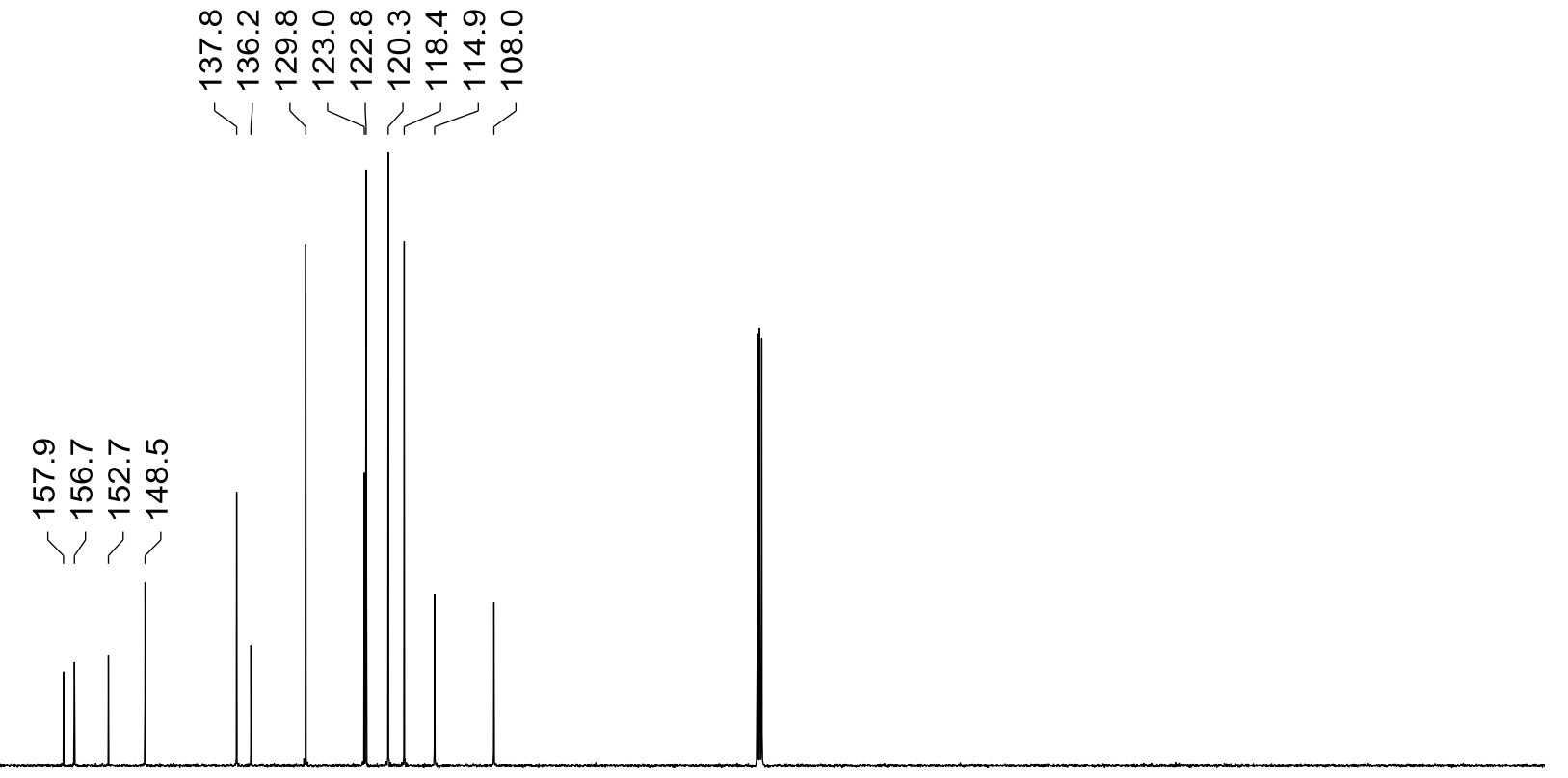




\section{${ }^{1} \mathrm{H}$ NMR of imidazole $1 \mathrm{~m}$}

$\mathrm{CDCl}_{3}, 25^{\circ} \mathrm{C}$

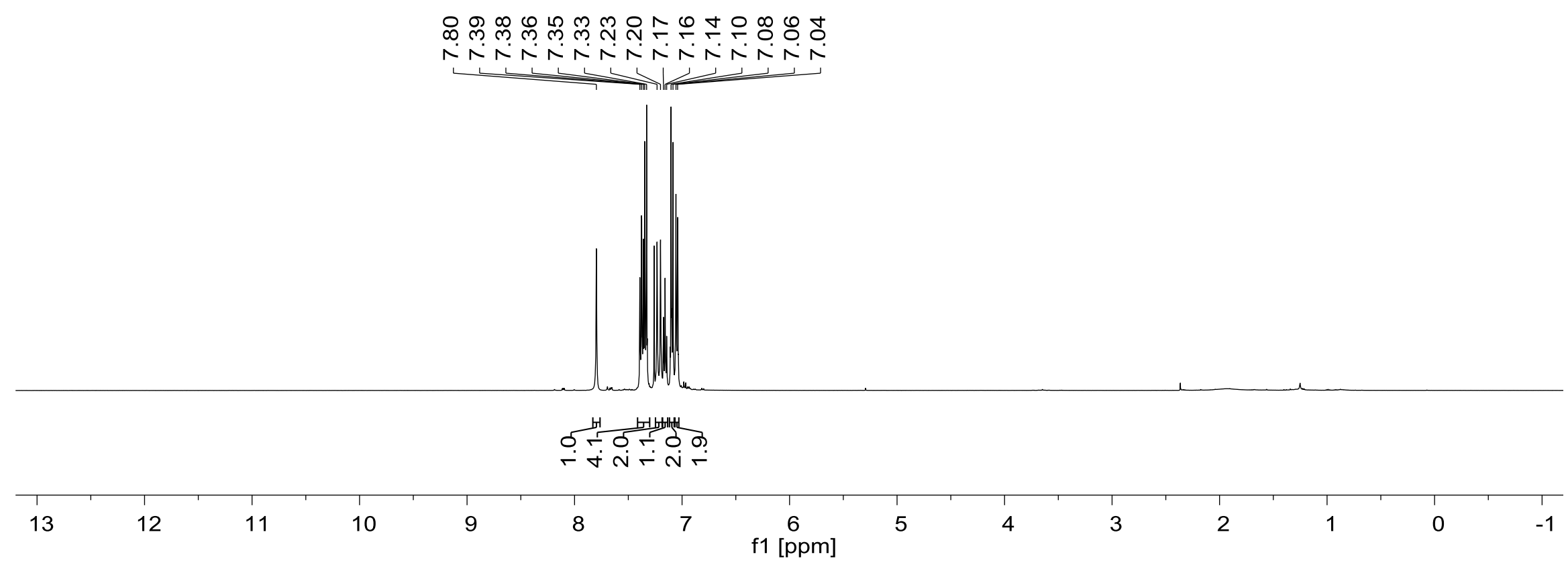




\section{${ }^{13} \mathrm{C}$ NMR of imidazole $1 \mathrm{~m}$}

$\mathrm{CDCl}_{3}, 25^{\circ} \mathrm{C}$

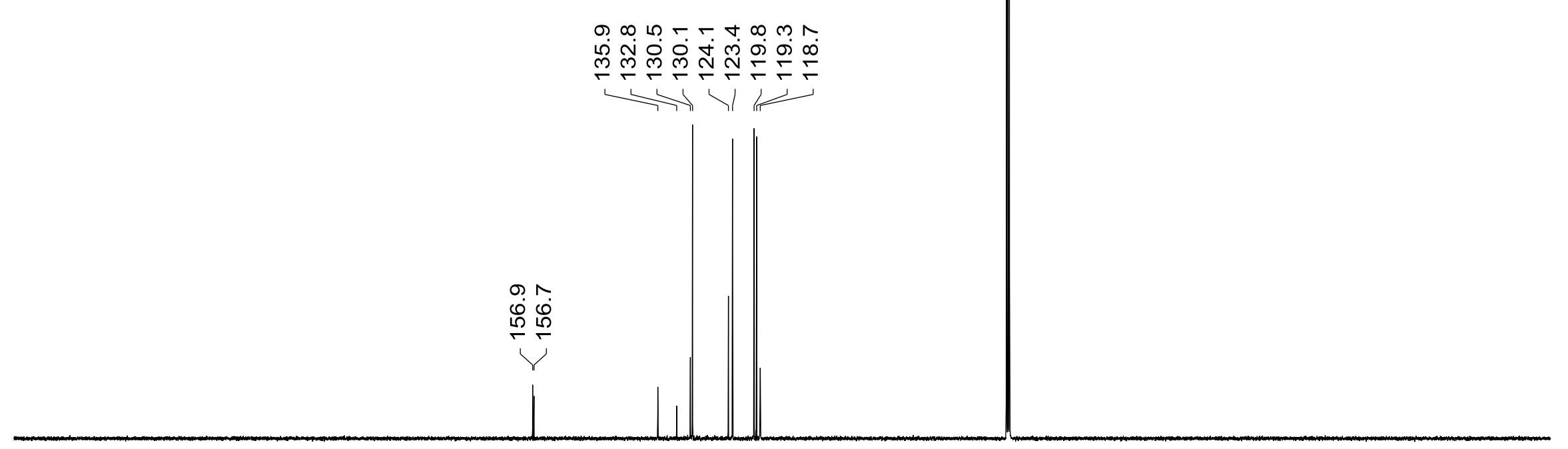




\section{${ }^{1} \mathrm{H}$ NMR of benzimidazole $1 \mathrm{n}$}

$\mathrm{CDCl}_{3}, 25^{\circ} \mathrm{C}$

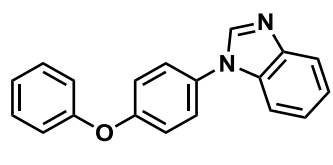

1n

ำ

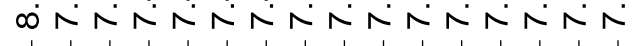

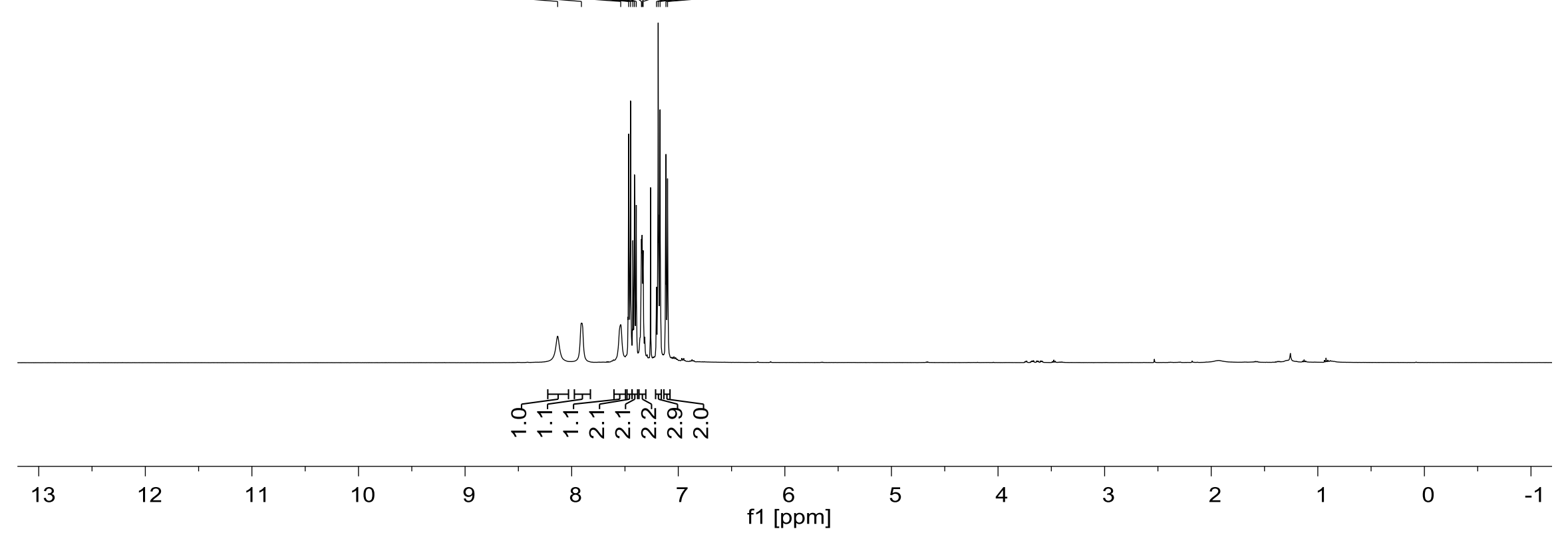




\section{${ }^{13} \mathrm{C}$ NMR of benzimidazole $1 \mathrm{n}$}

$\mathrm{CDCl}_{3}, 25^{\circ} \mathrm{C}$

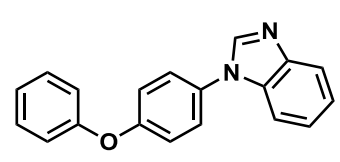

$1 \mathrm{n}$

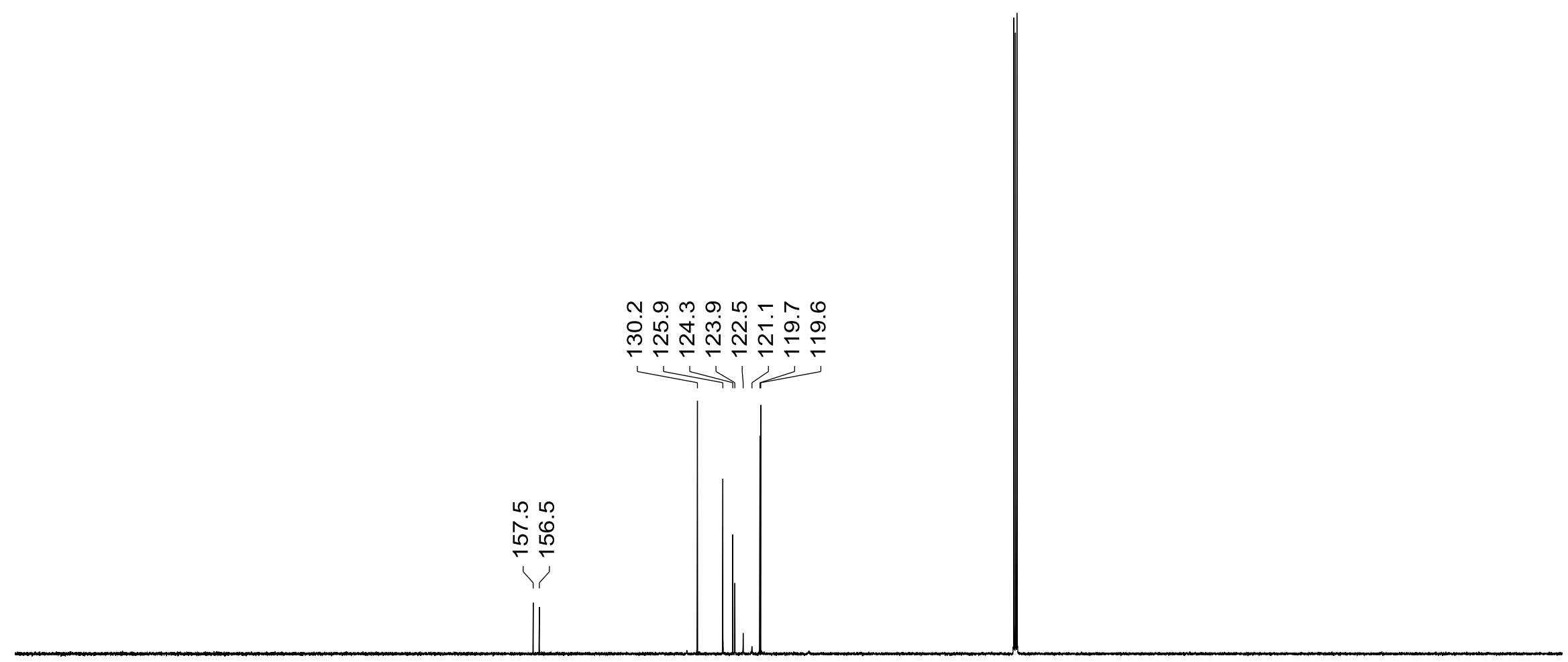

$\begin{array}{llllllllllllllllllllllllllllll}240 & 230 & 220 & 210 & 200 & 190 & 180 & 170 & 160 & 150 & 140 & 130 & 120 & 110 & 100 & 90 & 80 & 70 & 60 & 50 & 40 & 30 & 20 & 10 & 0 & -10\end{array}$ f1 [ppm] 


\section{${ }^{1} \mathrm{H}$ NMR of triazole 10}

$\mathrm{CDCl}_{3}, 25^{\circ} \mathrm{C}$

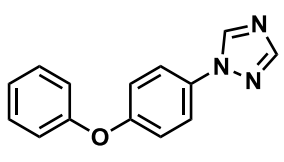

10

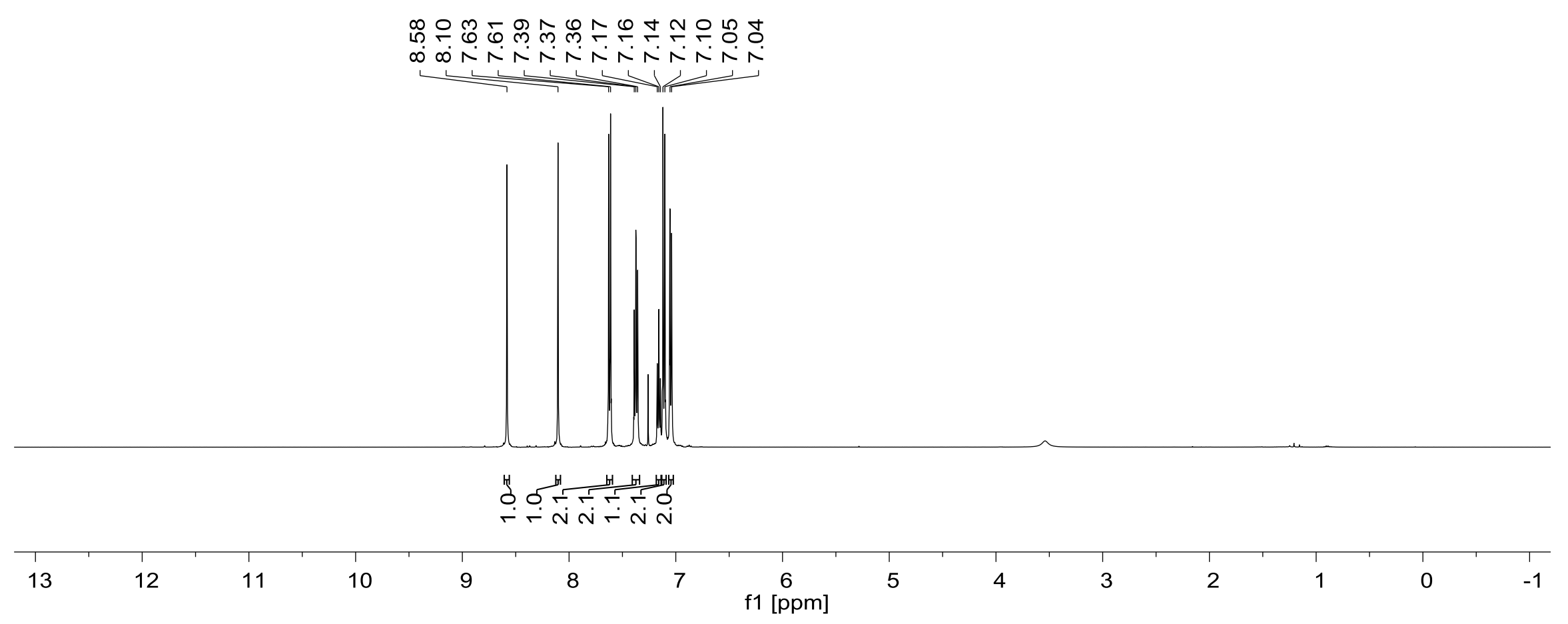




\section{${ }^{13} \mathrm{C}$ NMR of triazole 10}

$\mathrm{CDCl}_{3}, 25^{\circ} \mathrm{C}$

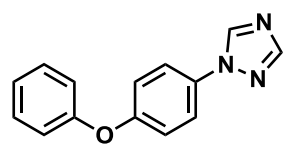

10

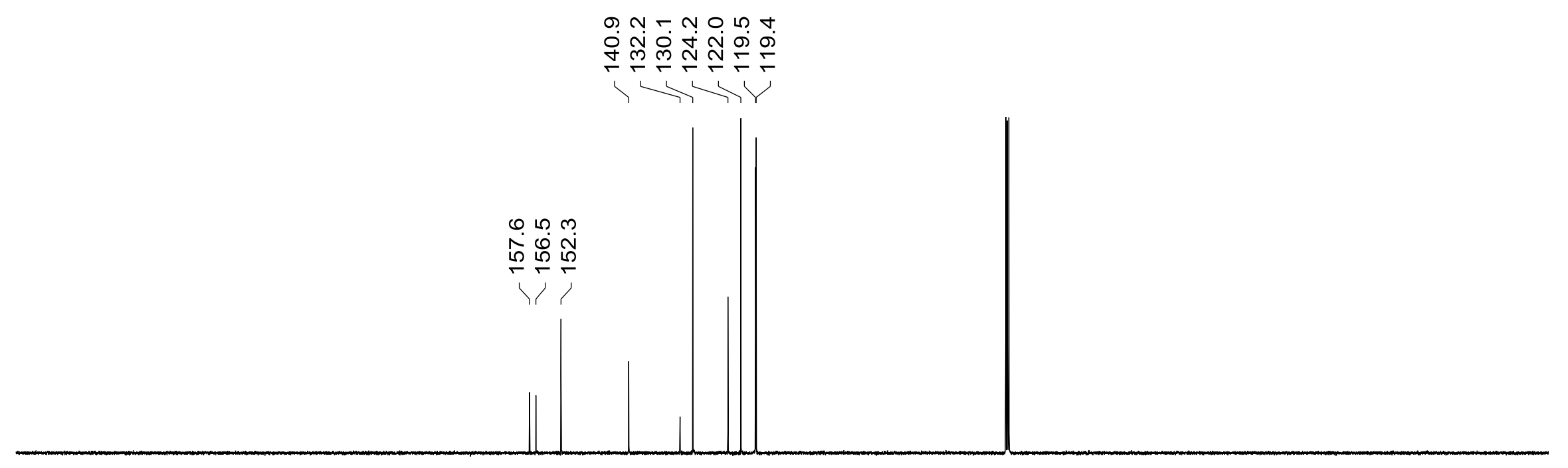




\section{${ }^{1} \mathrm{H}$ NMR of phthalimide $1 \mathrm{p}$}

$\mathrm{CDCl}_{3}, 25^{\circ} \mathrm{C}$

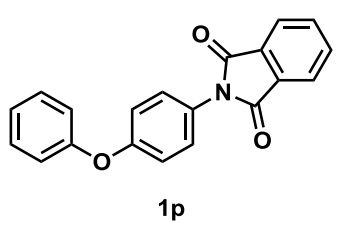

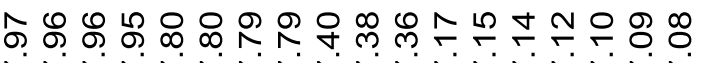

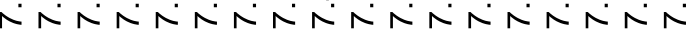
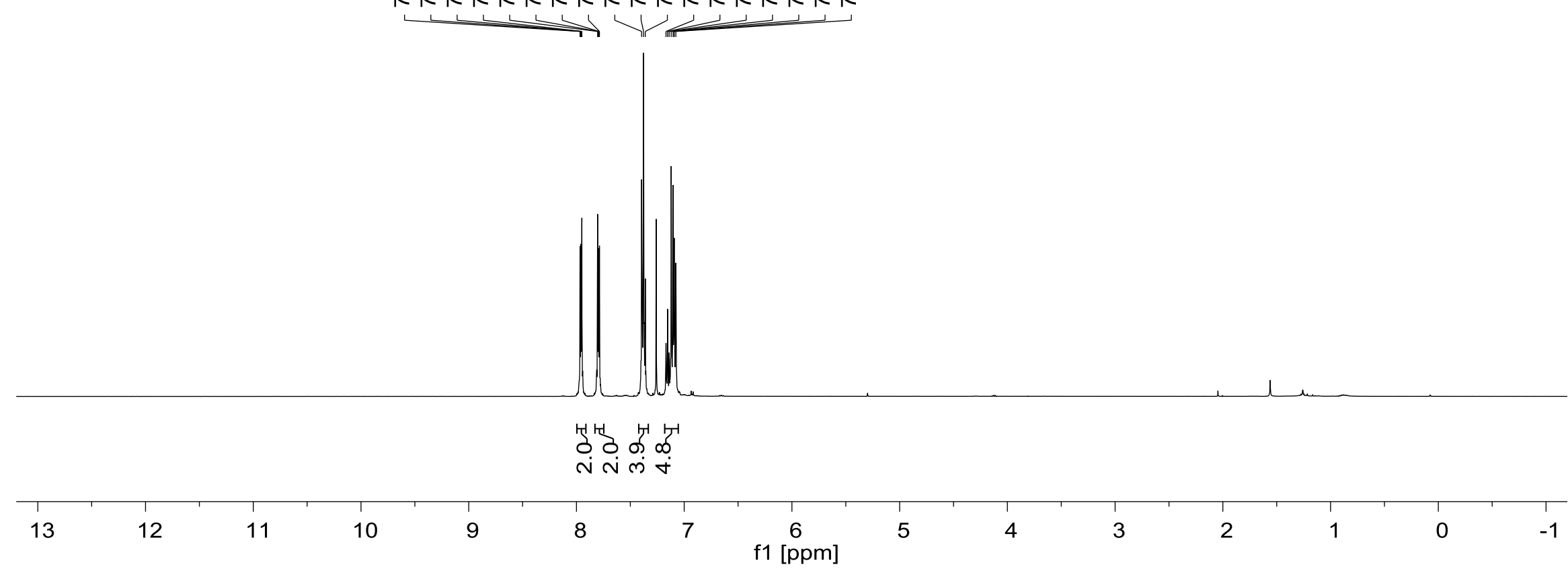


\section{${ }^{13} \mathrm{C}$ NMR of phthalimide $1 \mathrm{p}$}

$\mathrm{CDCl}_{3}, 25^{\circ} \mathrm{C}$
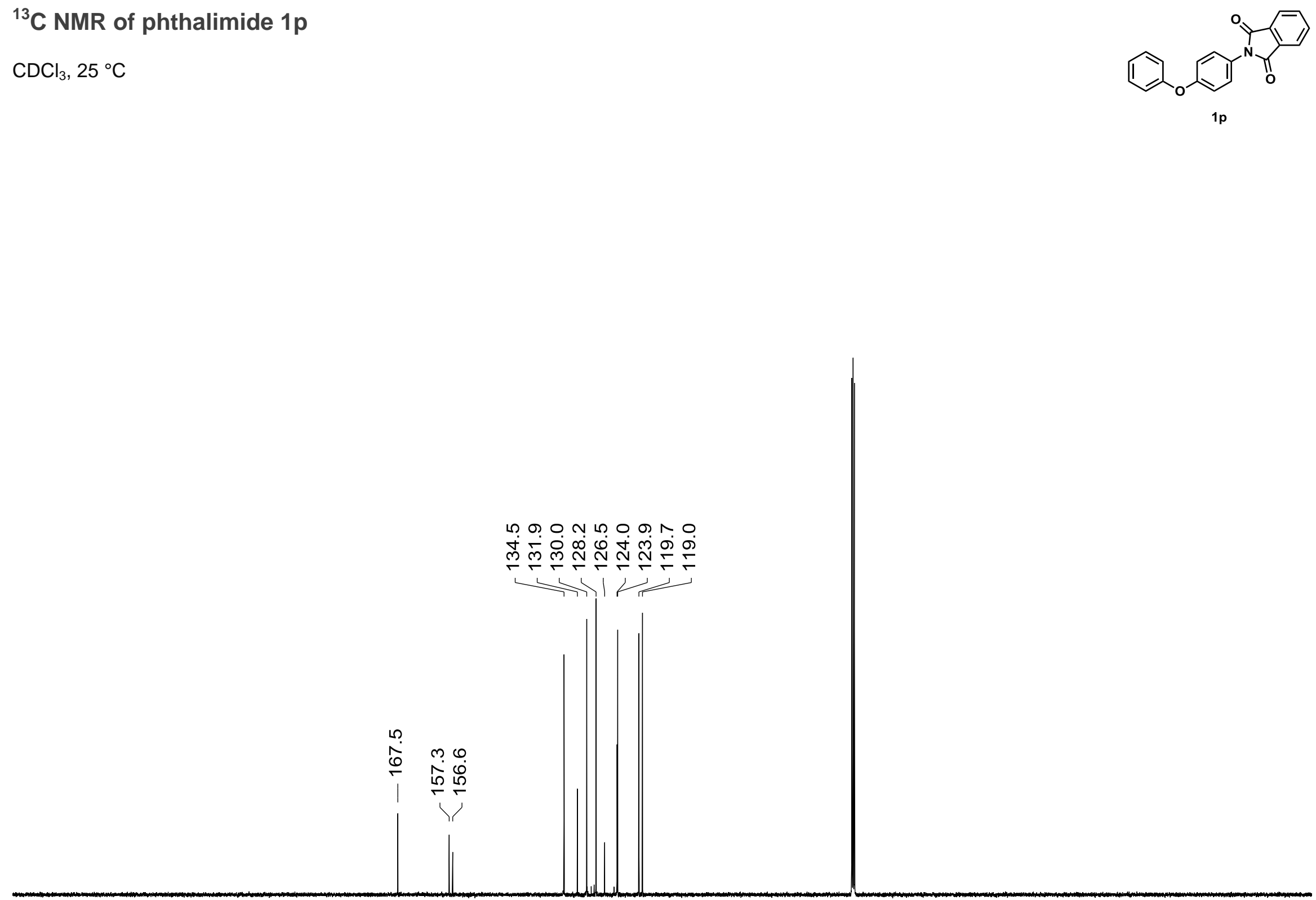


\section{${ }^{1} \mathrm{H}$ NMR of hydantoin $1 \mathrm{q}$}

$\mathrm{CDCl}_{3}, 25^{\circ} \mathrm{C}$
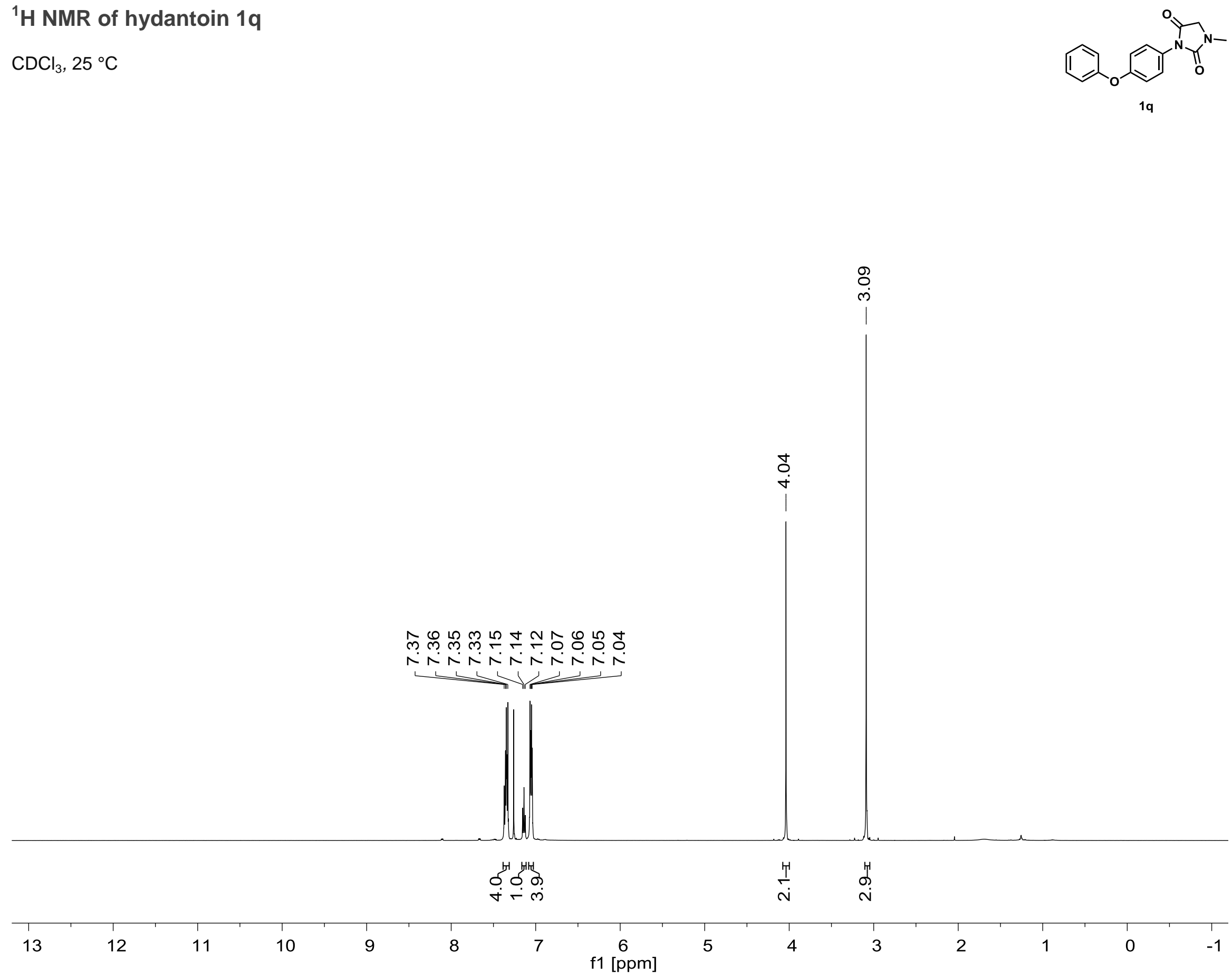


\section{${ }^{13} \mathrm{C}$ NMR of hydantoin $1 \mathrm{q}$}

$\mathrm{CDCl}_{3}, 25^{\circ} \mathrm{C}$
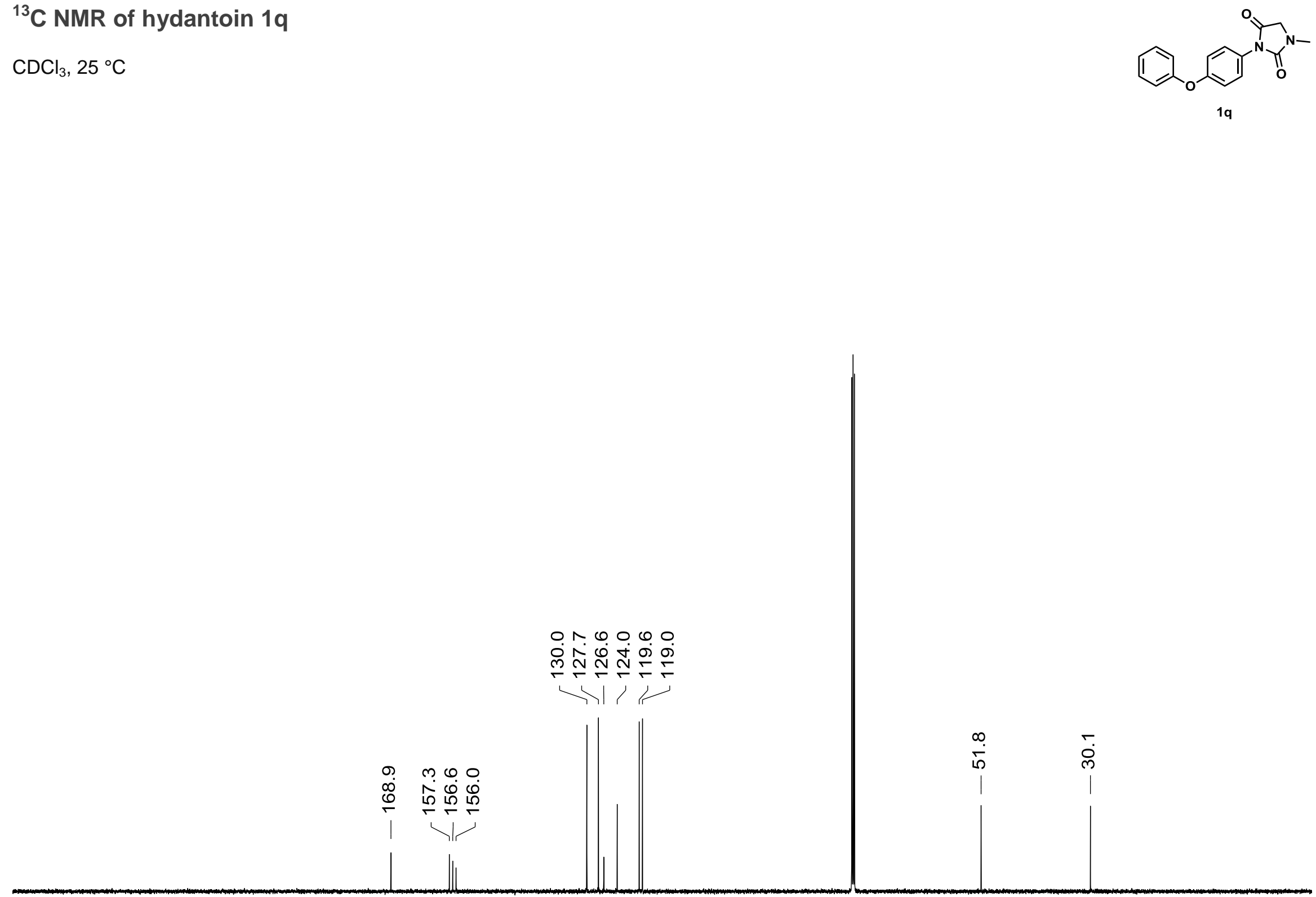

$\begin{array}{lllllllllllllllllllllllllllllllllll}240 & 230 & 220 & 210 & 200 & 190 & 180 & 170 & 160 & 150 & 140 & 130 & 120 & 110 & 100 & 90 & 80 & 70 & 60 & 50 & 40 & 30 & 20 & 10 & 0 & -10\end{array}$ f1 [ppm] 


\section{${ }^{1} \mathrm{H}$ NMR of pyridine $1 \mathrm{r}$}

$\mathrm{CDCl}_{3}, 25^{\circ} \mathrm{C}$

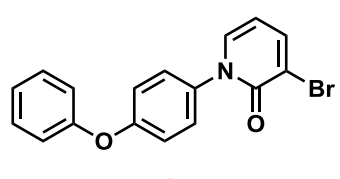

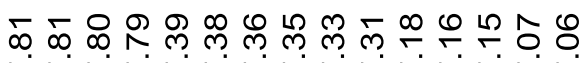

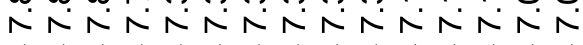

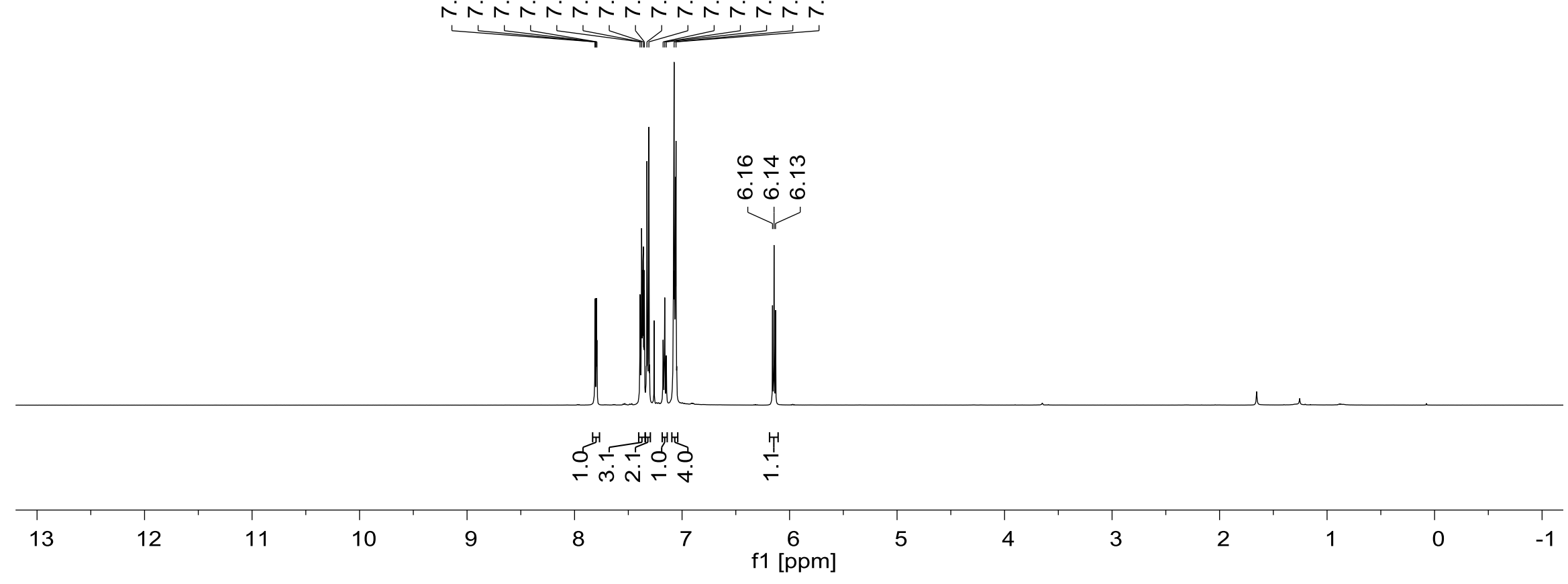




\section{${ }^{13} \mathrm{C}$ NMR of pyridine $1 \mathrm{r}$}

$\mathrm{CDCl}_{3}, 25^{\circ} \mathrm{C}$
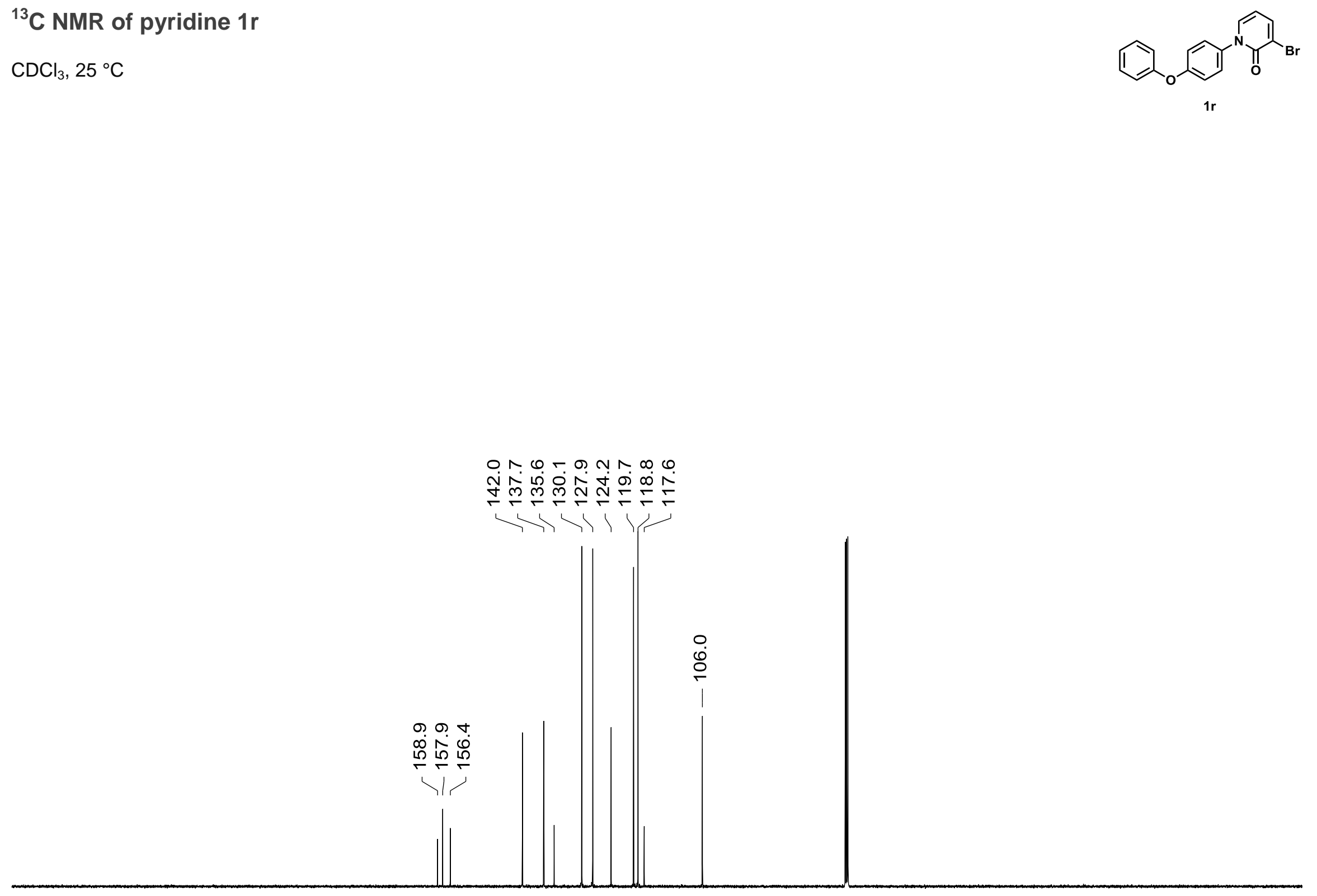
${ }^{1} \mathrm{H}$ NMR of azide $1 \mathrm{~s}$

$\mathrm{CD}_{2} \mathrm{Cl}_{2}, 25^{\circ} \mathrm{C}$

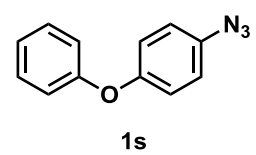

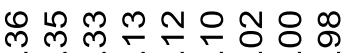

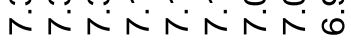

舟

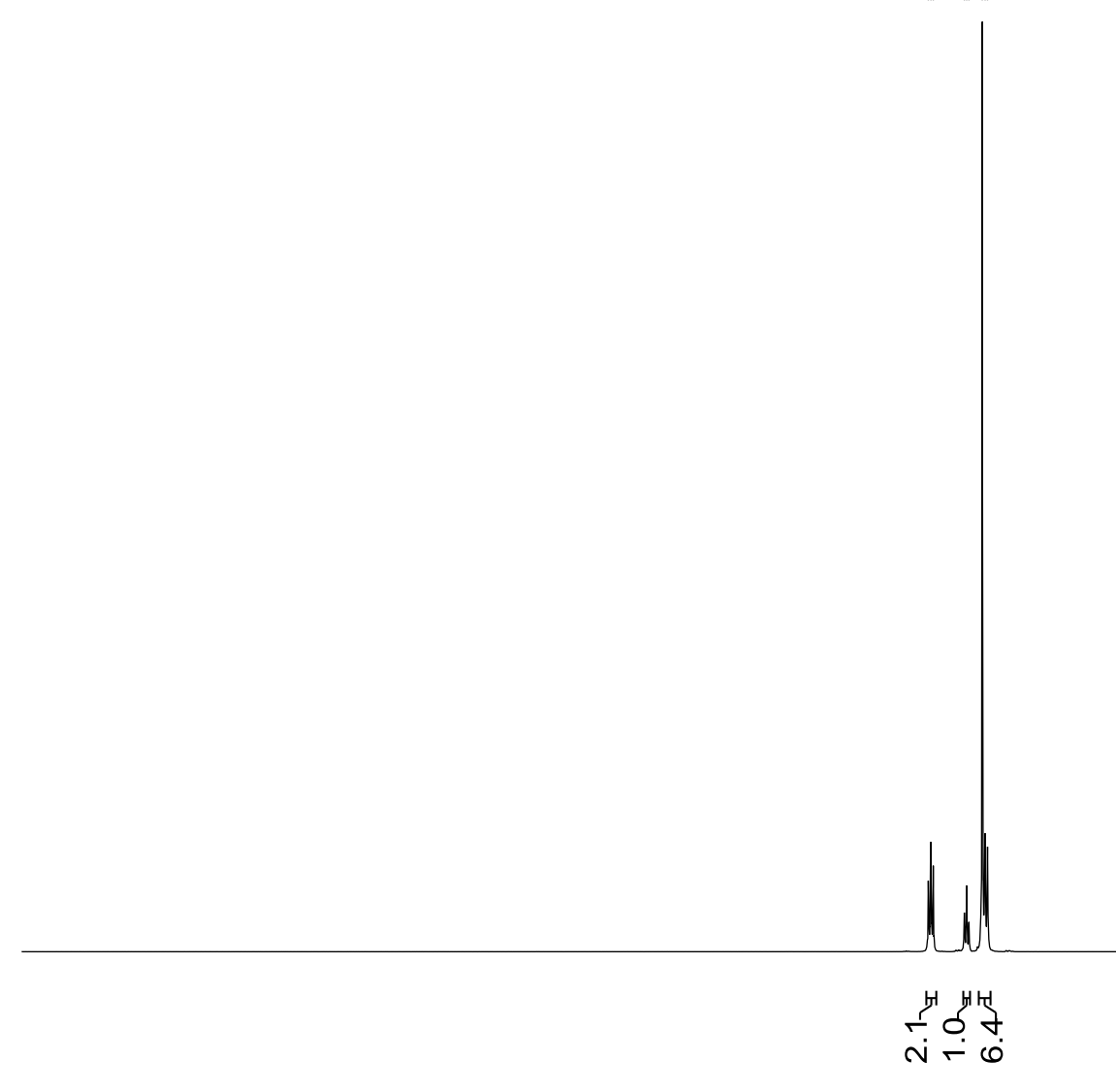

$13 \quad 12$




\section{${ }^{13} \mathrm{C}$ NMR of azide $1 \mathrm{~s}$}

$\mathrm{CD}_{2} \mathrm{Cl}_{2}, 25^{\circ} \mathrm{C}$

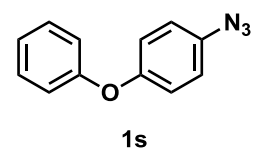

$\omega+\sigma a \infty$ -

மூ

它官

i

\रो। 


\section{${ }^{1} \mathrm{H}$ NMR of aniline $1 \mathrm{t}$}

$\mathrm{CDCl}_{3}, 25^{\circ} \mathrm{C}$
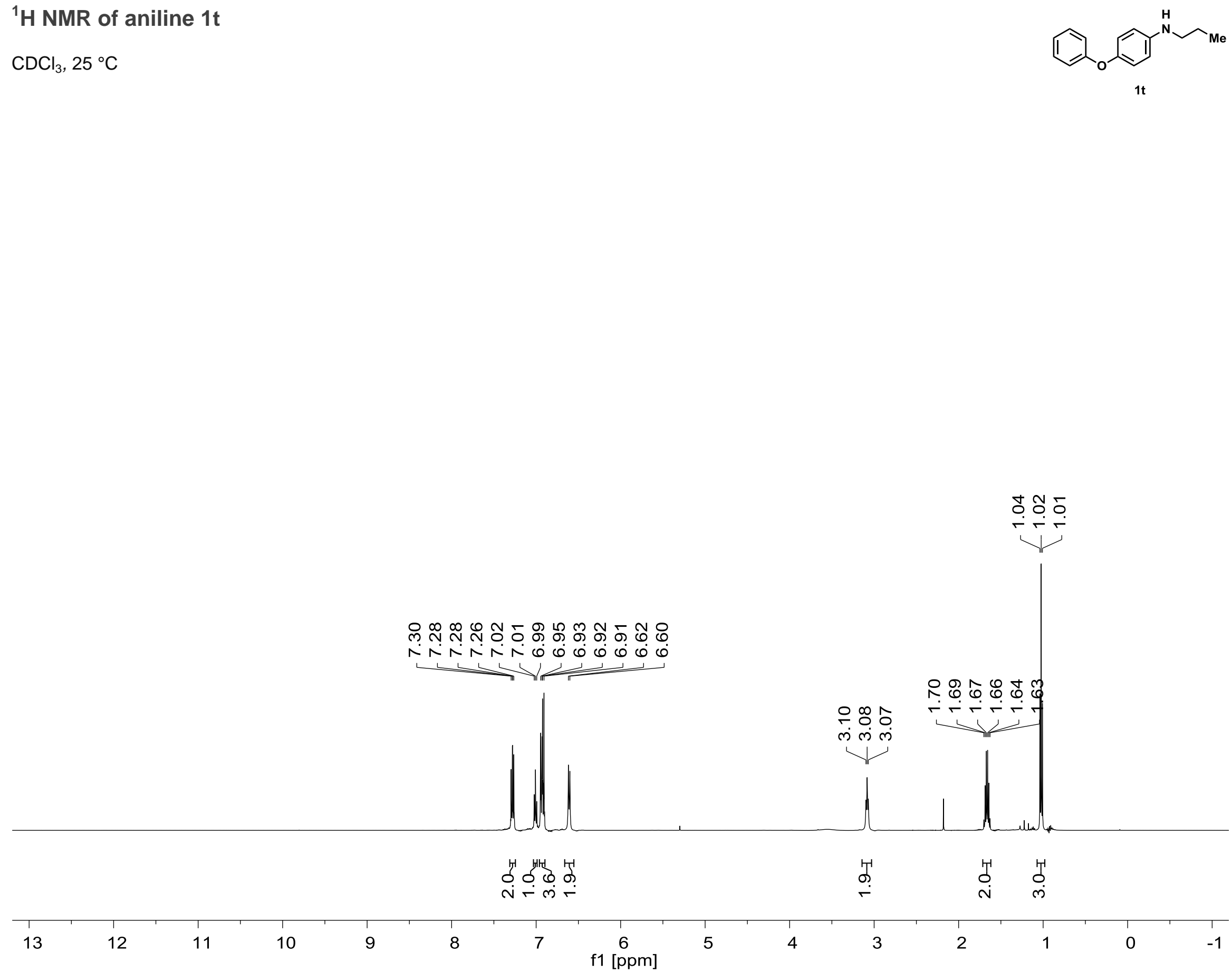


\section{${ }^{13} \mathrm{C}$ NMR of aniline $1 \mathrm{t}$}

$\mathrm{CDCl}_{3}, 25^{\circ} \mathrm{C}$
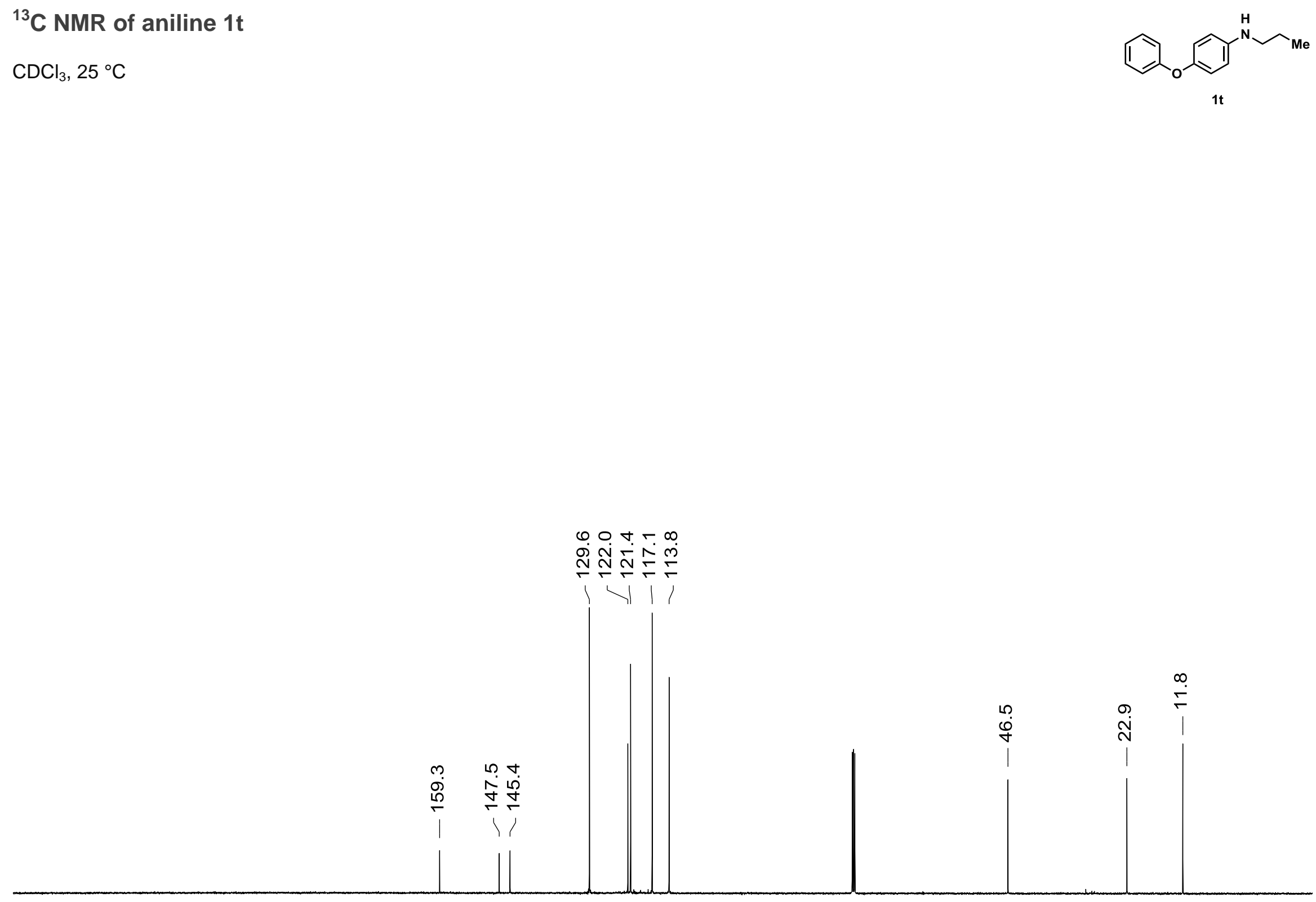

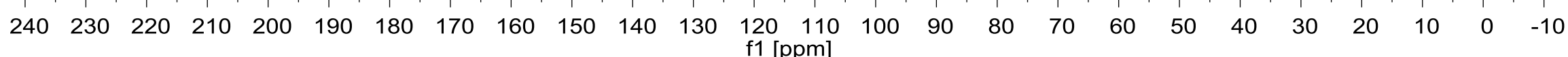




\section{${ }^{1} \mathrm{H}$ NMR of aniline $1 \mathrm{u}$}

$\mathrm{CDCl}_{3}, 25^{\circ} \mathrm{C}$

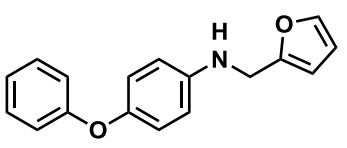

14

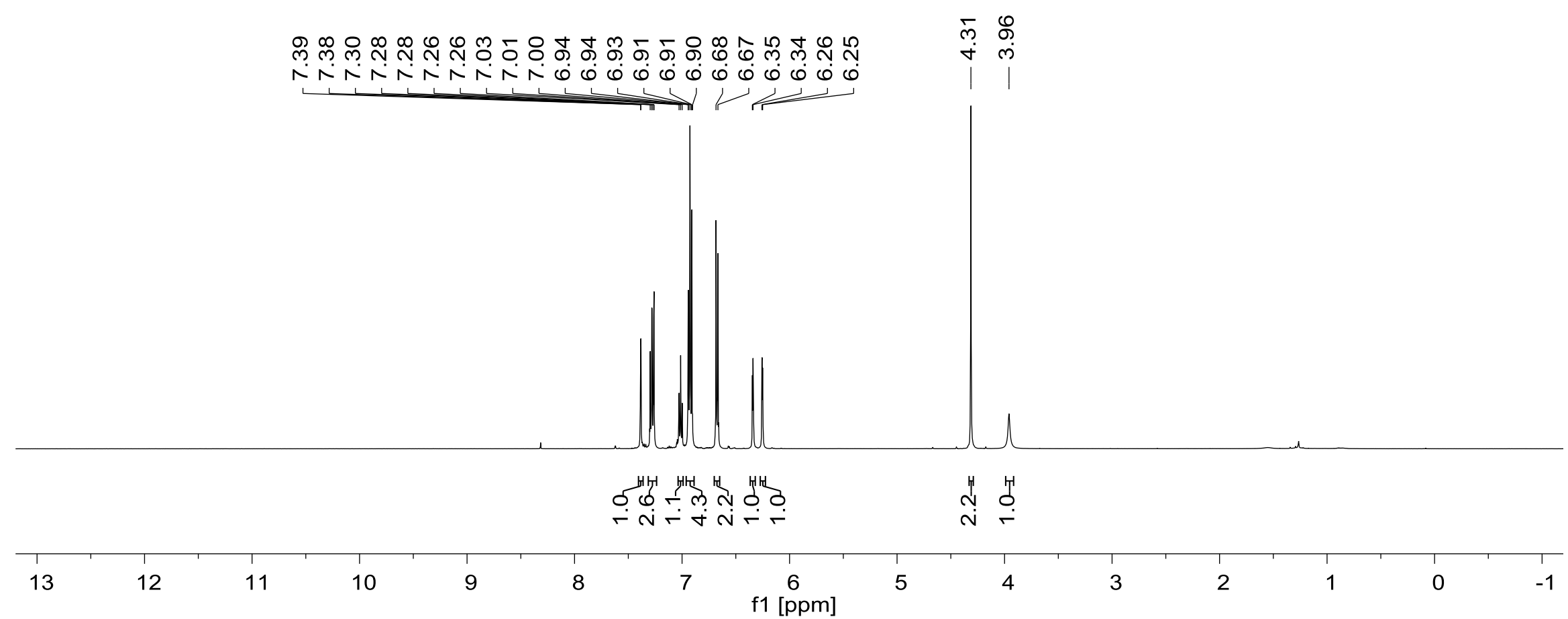




\section{${ }^{13} \mathrm{C}$ NMR of aniline $1 \mathrm{u}$}

$\mathrm{CDCl}_{3}, 25^{\circ} \mathrm{C}$

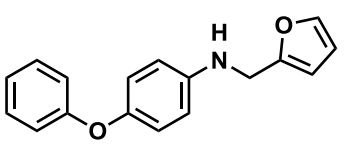

1u

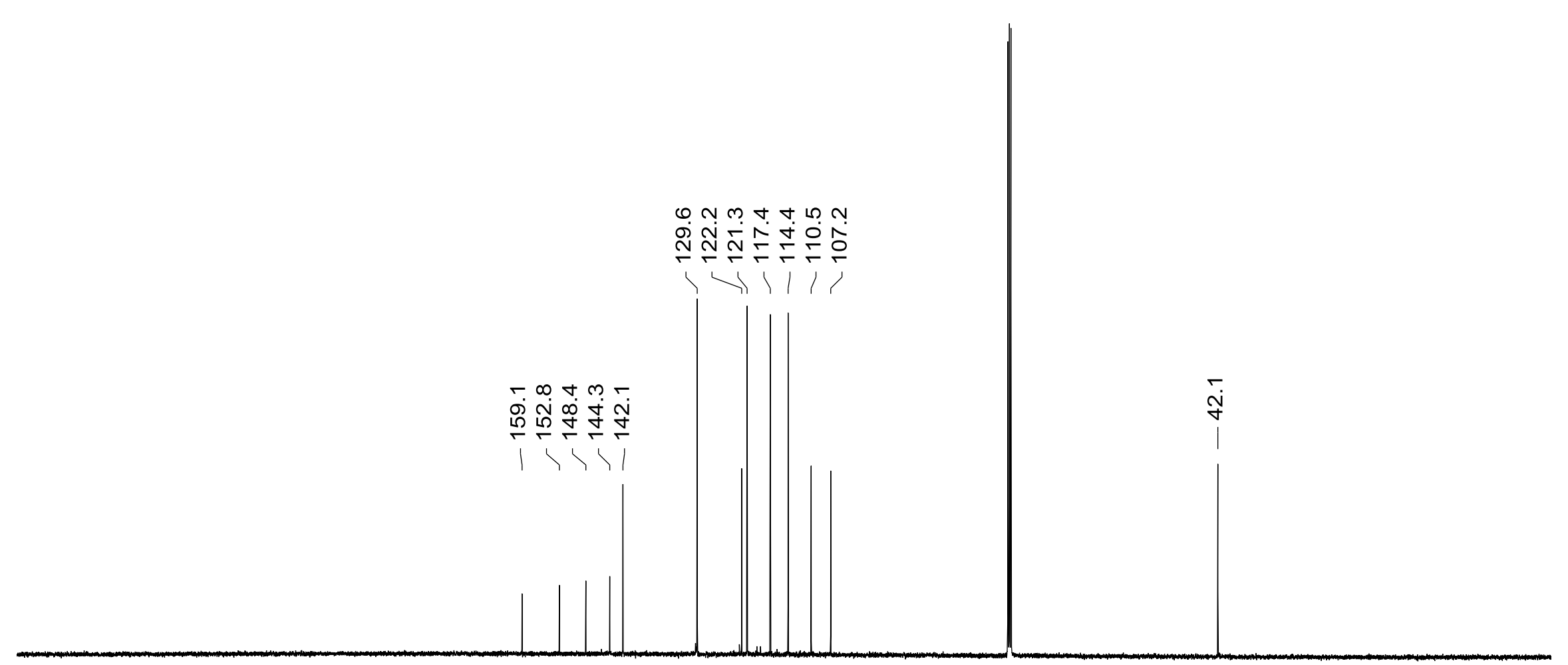

$\begin{array}{lllllllllllllllllllllllllllllllll}240 & 230 & 220 & 210 & 200 & 190 & 180 & 170 & 160 & 150 & 140 & 130 & 120 & 110 & 100 & 90 & 80 & 70 & 60 & 50 & 40 & 30 & 20 & 10 & 0 & -10\end{array}$ 


\section{${ }^{1} \mathrm{H}$ NMR of aniline $1 \mathrm{v}$}

$\mathrm{CDCl}_{3}, 25^{\circ} \mathrm{C}$

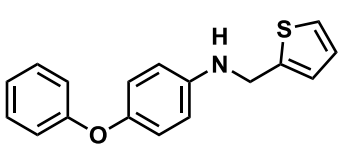

1v

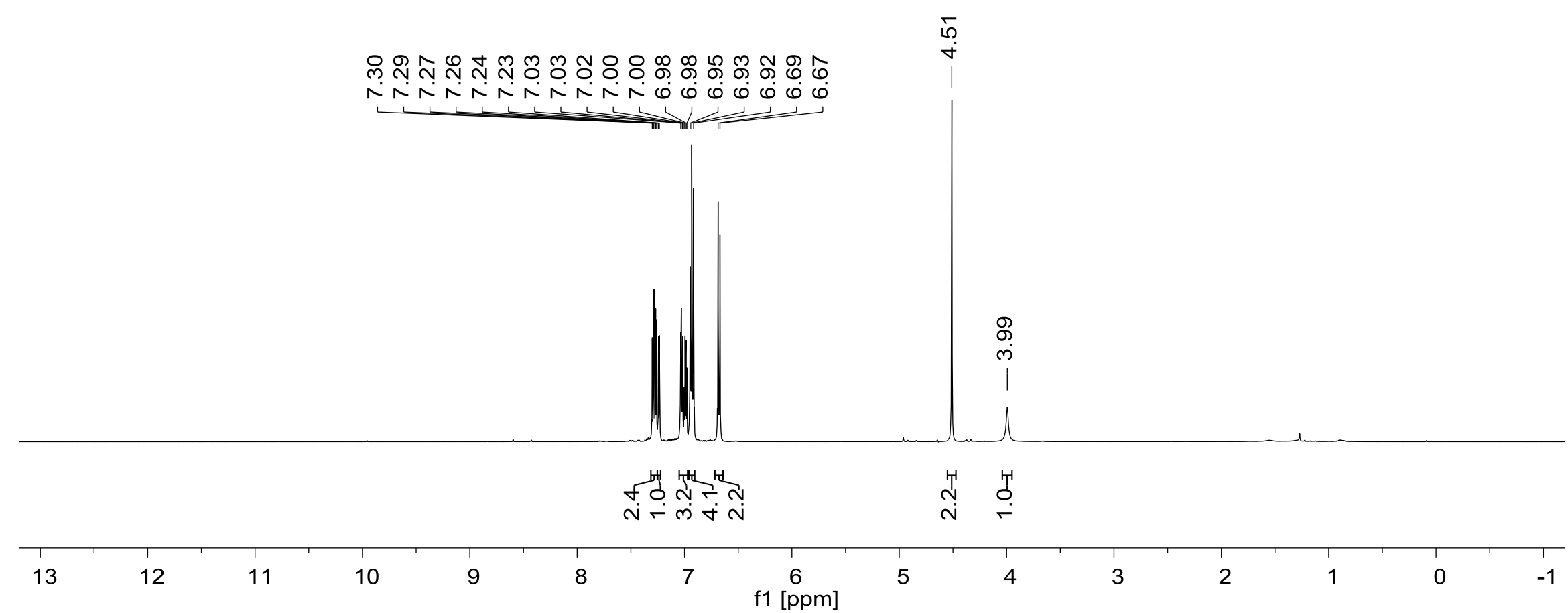




\section{${ }^{13} \mathrm{C}$ NMR of aniline $1 \mathrm{v}$}

$\mathrm{CDCl}_{3}, 25^{\circ} \mathrm{C}$

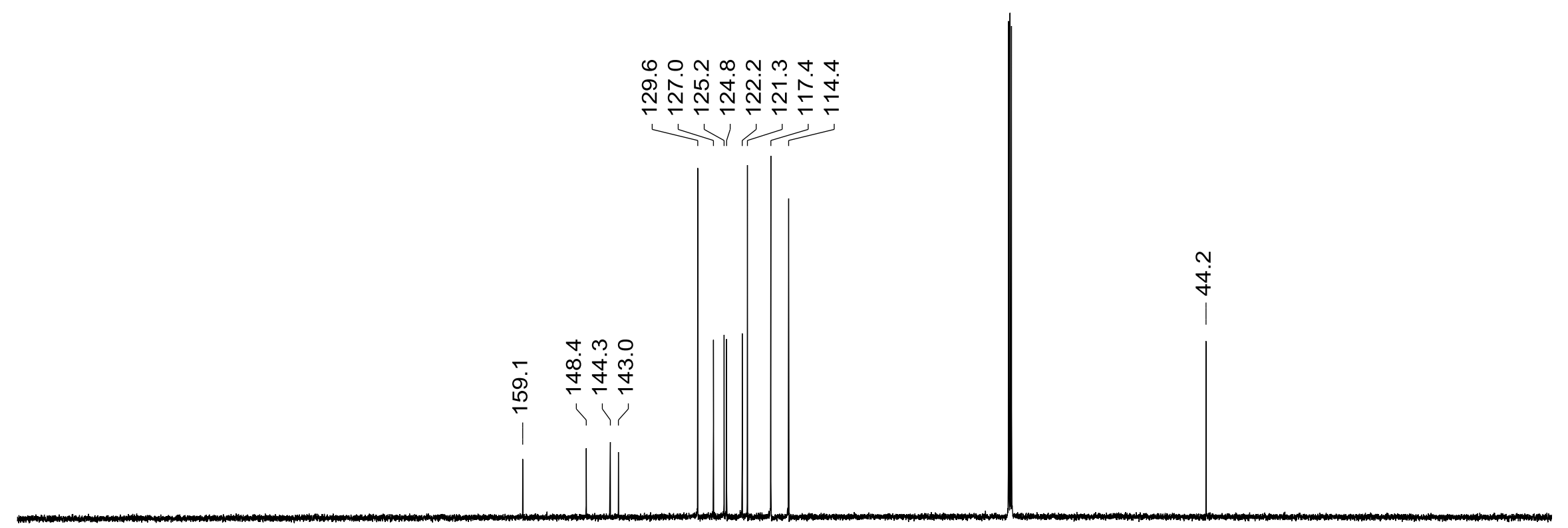




\section{${ }^{1} \mathrm{H}$ NMR of aniline $1 \mathrm{w}$}

$\mathrm{CDCl}_{3}, 25^{\circ} \mathrm{C}$

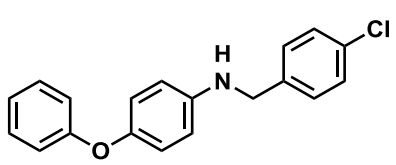

$1 w$

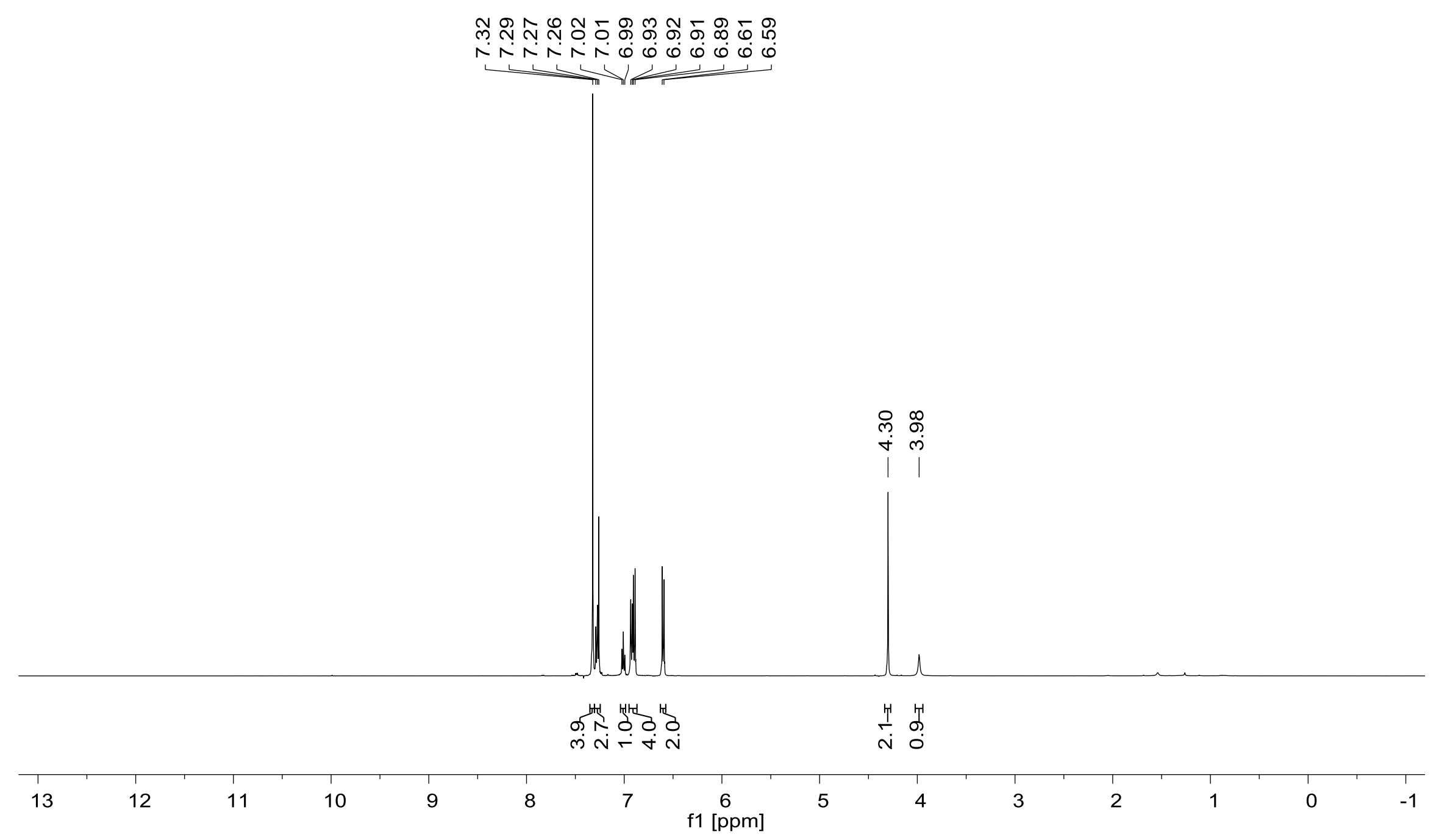




\section{${ }^{13} \mathrm{C}$ NMR of aniline $1 \mathrm{w}$}

$\mathrm{CDCl}_{3}, 25^{\circ} \mathrm{C}$
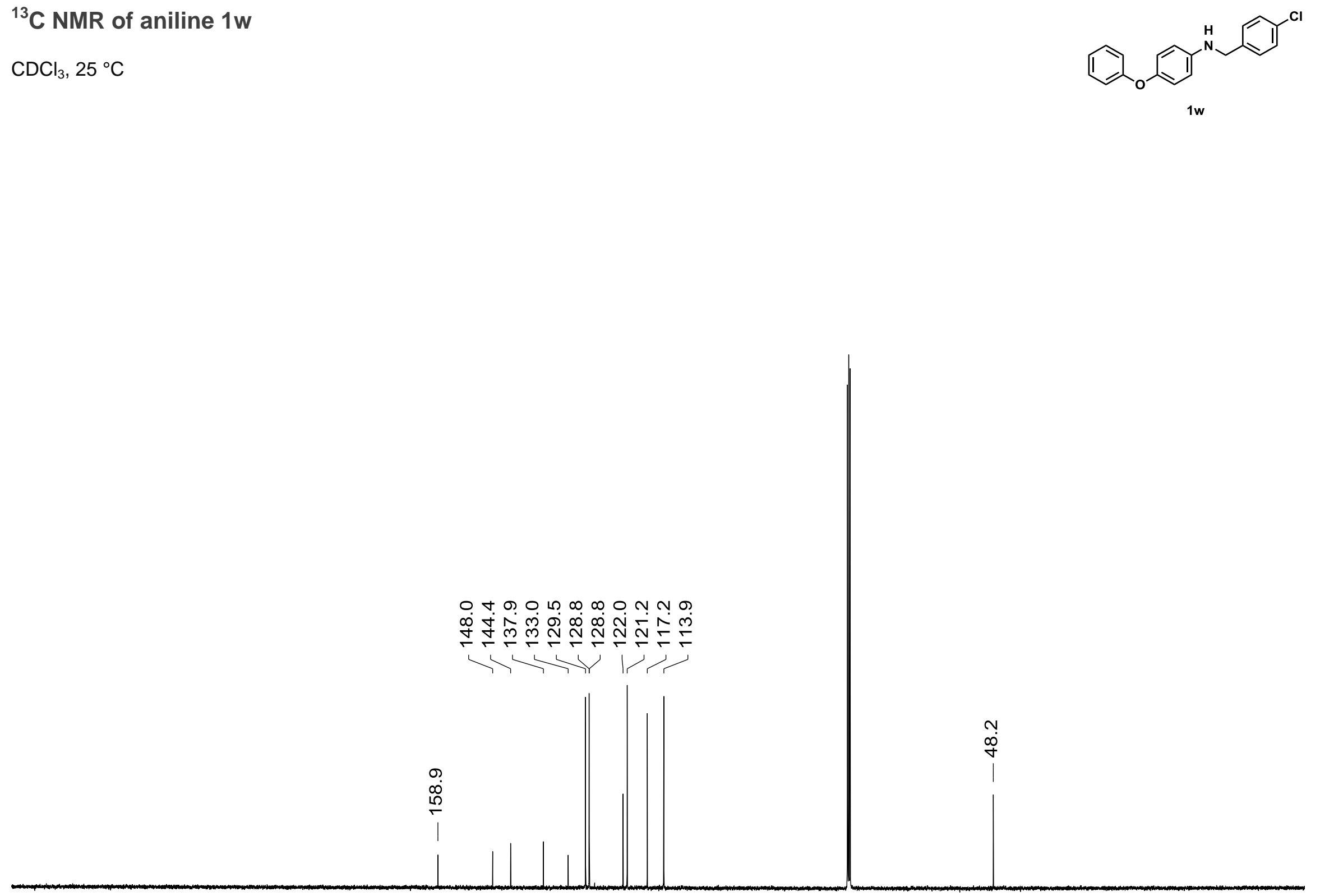


\section{${ }^{1} \mathrm{H}$ NMR of thianthrenium salt TT-2}

$\mathrm{CDCl}_{3}, 25^{\circ} \mathrm{C}$
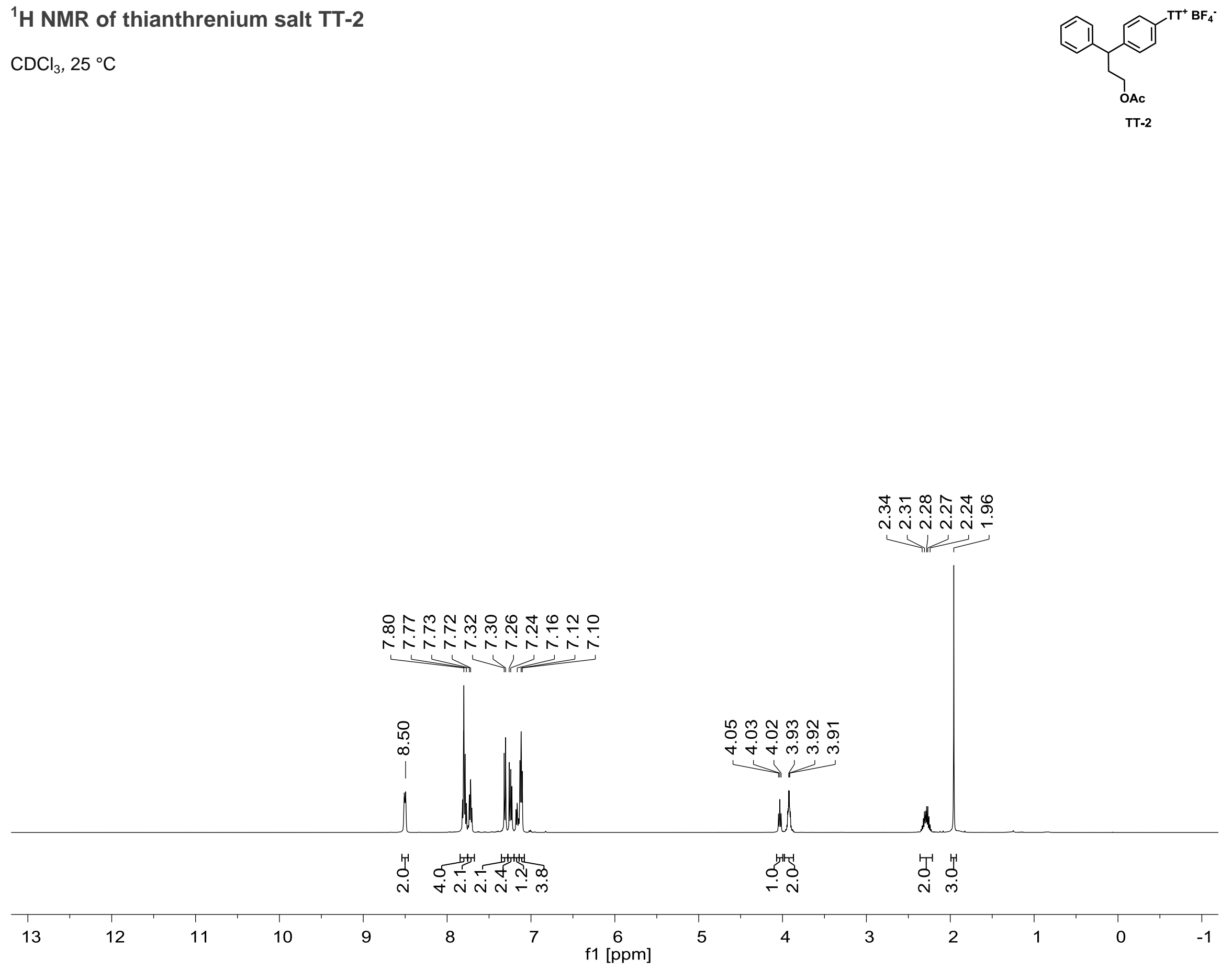
${ }^{13} \mathrm{C}$ NMR of thianthrenium salt TT-2

$\mathrm{CDCl}_{3}, 25^{\circ} \mathrm{C}$

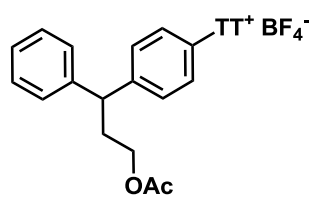

TT-2

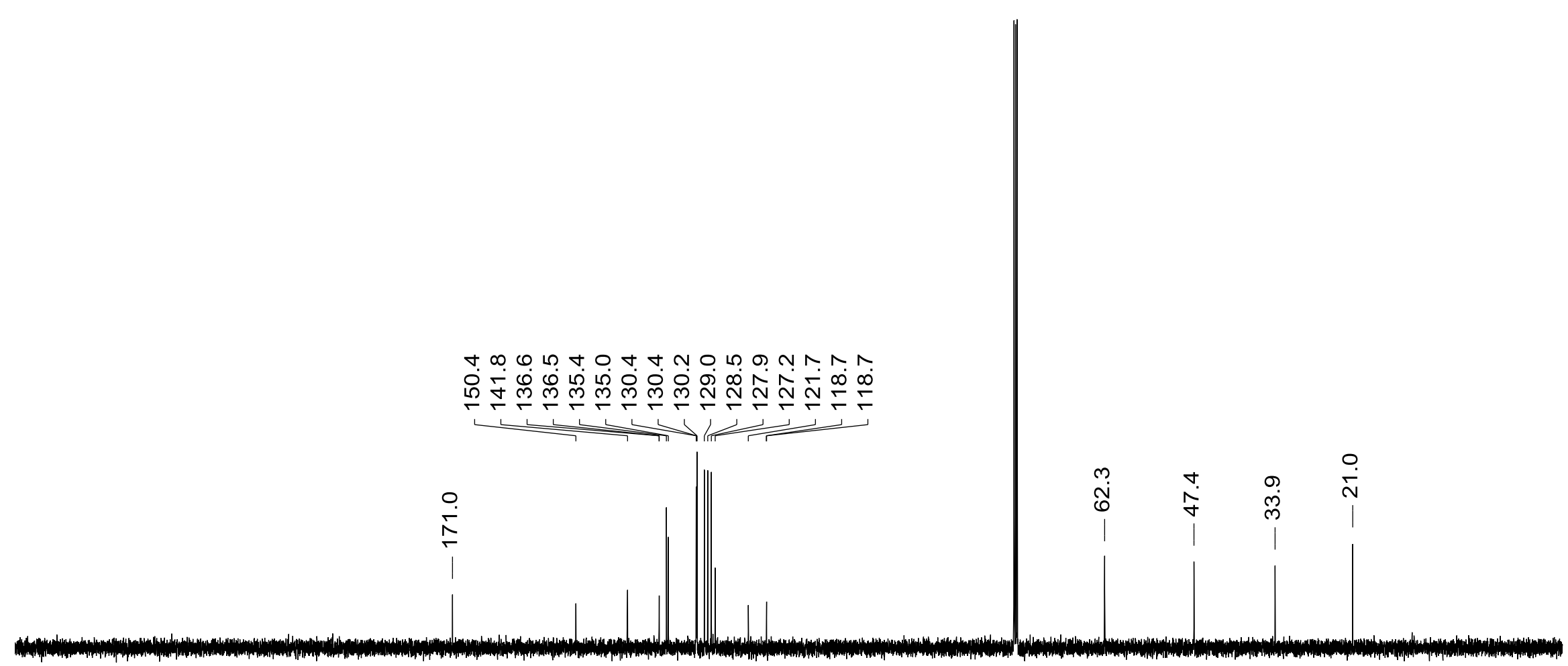

$\begin{array}{lllllllllllllllllllllllllllllllllll}240 & 230 & 220 & 210 & 200 & 190 & 180 & 170 & 160 & 150 & 140 & 130 & 120 & 110 & 100 & 90 & 80 & 70 & 60 & 50 & 40 & 30 & 20 & 10 & 0 & -10\end{array}$




\section{${ }^{19} \mathrm{~F}$ NMR of thianthrenium salt TT-2}

$\mathrm{CDCl}_{3}, 25^{\circ} \mathrm{C}$

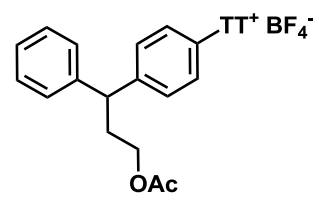

TT-2

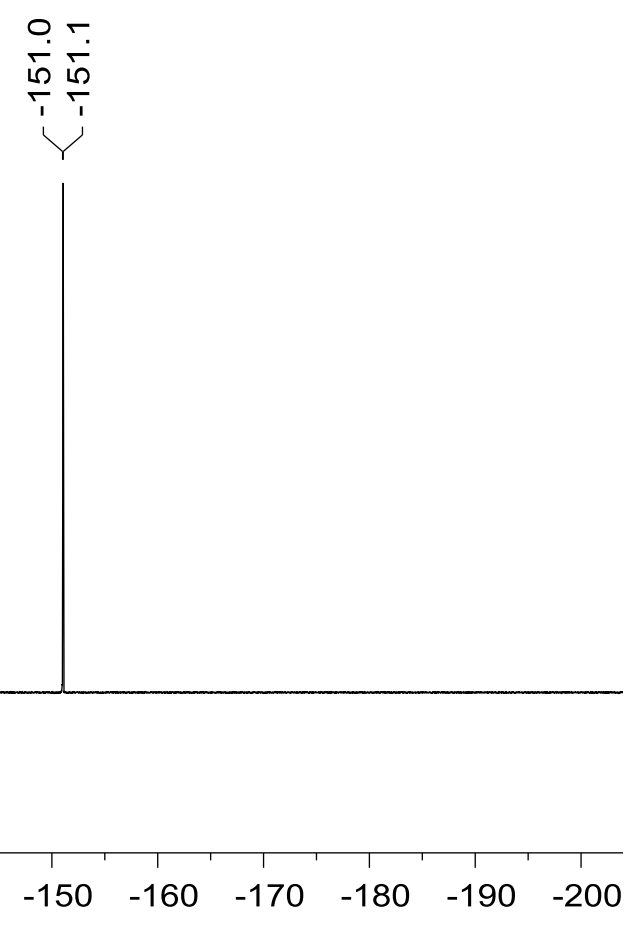

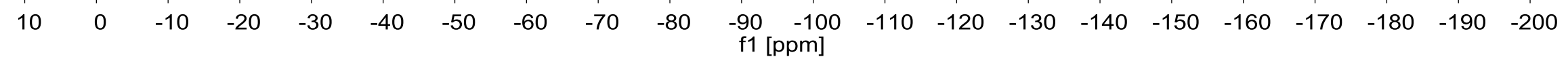




\section{${ }^{1} \mathrm{H}$ NMR of morpholine 2}

$\mathrm{CDCl}_{3}, 25^{\circ} \mathrm{C}$

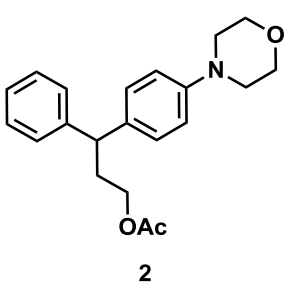

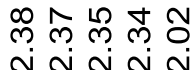

กับ

ป⿻一𠃋ㅇำ

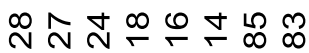

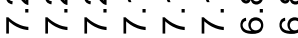

$\longrightarrow$

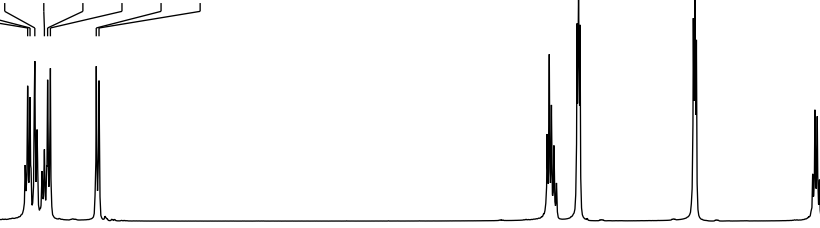

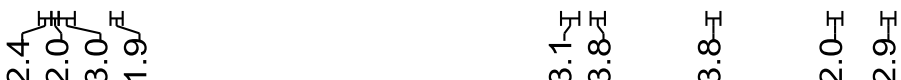

8

ก

लं

3




\section{${ }^{13} \mathrm{C}$ NMR of morpholine 2}

$\mathrm{CDCl}_{3}, 25^{\circ} \mathrm{C}$

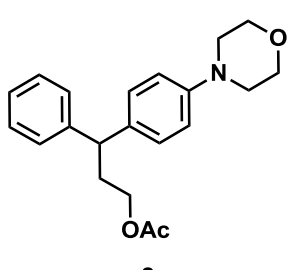

2

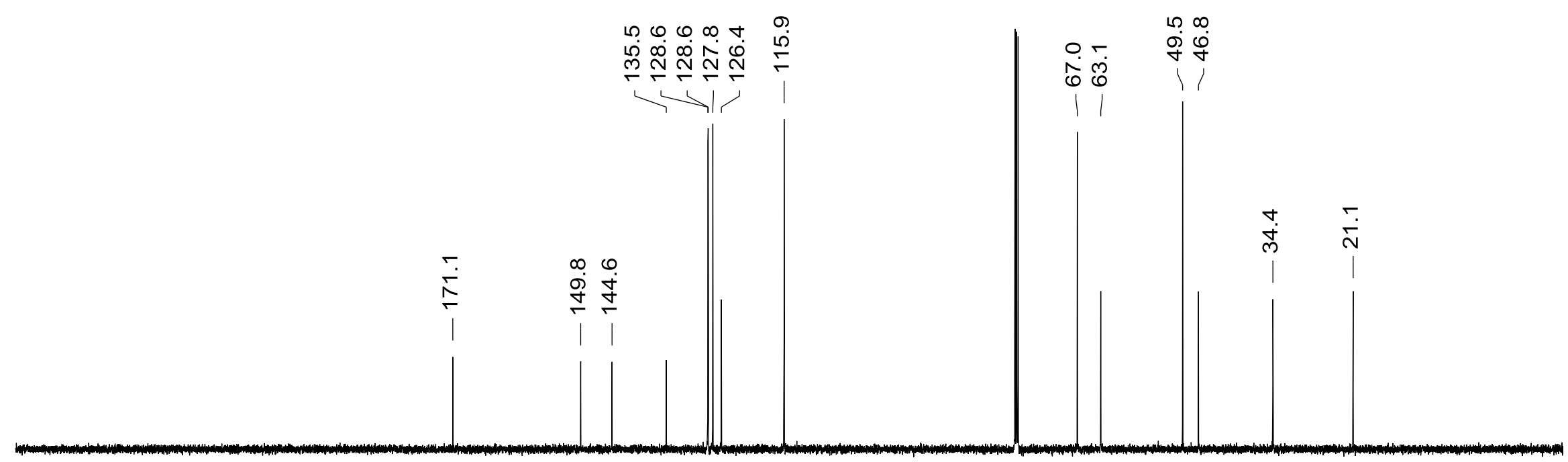

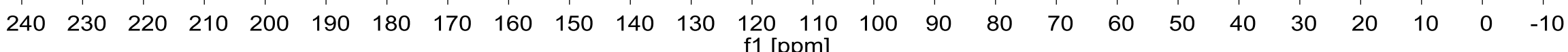




\section{${ }^{1} \mathrm{H}$ NMR of thianthrenium salt TT-3}

$\mathrm{CDCl}_{3}, 25^{\circ} \mathrm{C}$
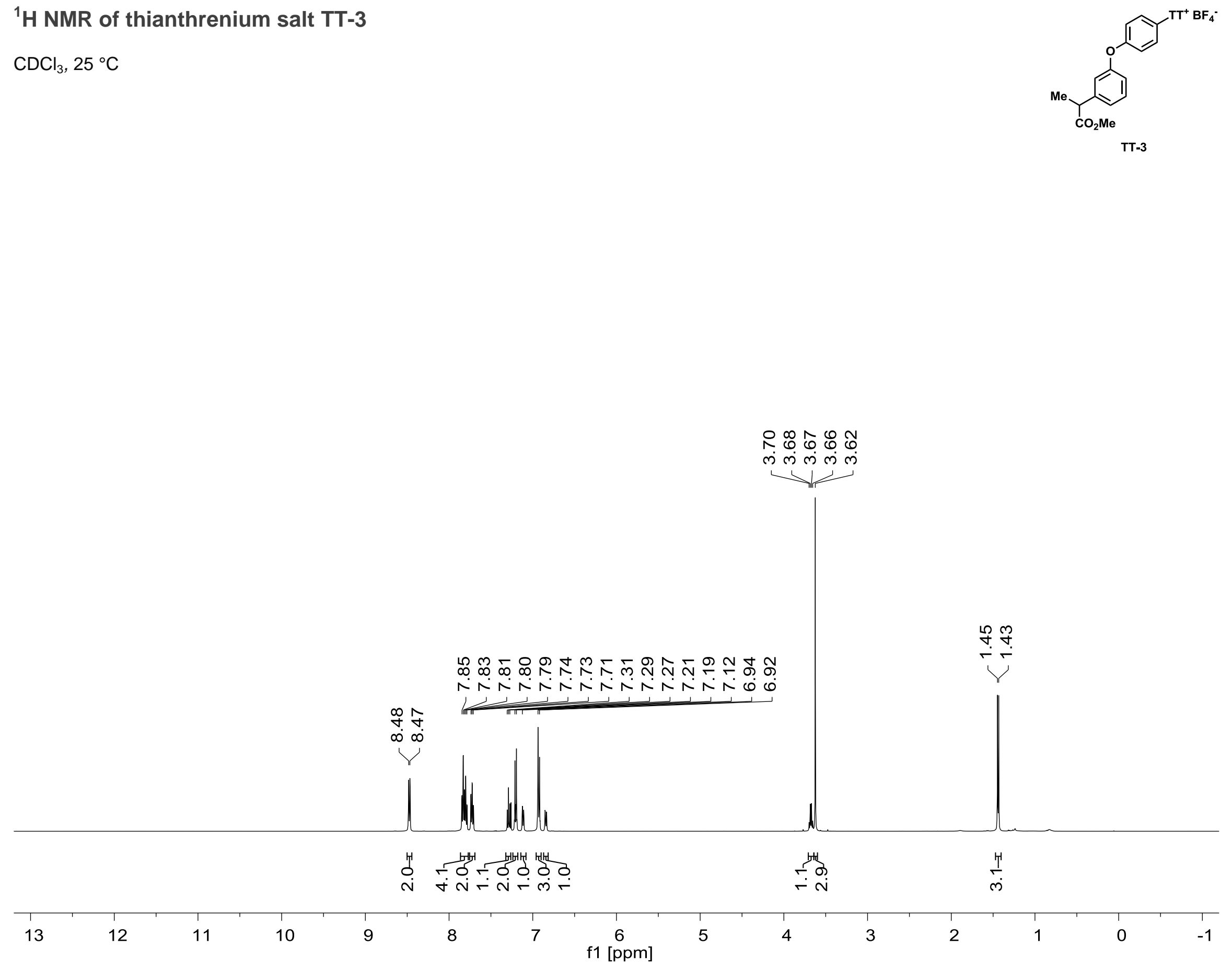
${ }^{13} \mathrm{C}$ NMR of thianthrenium salt TT-3

$\mathrm{CDCl}_{3}, 25^{\circ} \mathrm{C}$

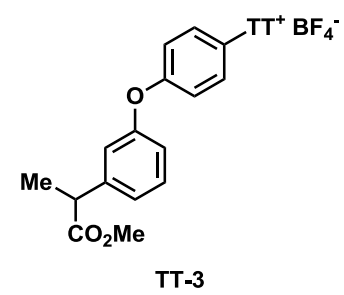

N m 0 o n L

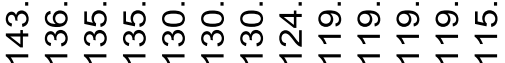

$\underset{1}{1}$

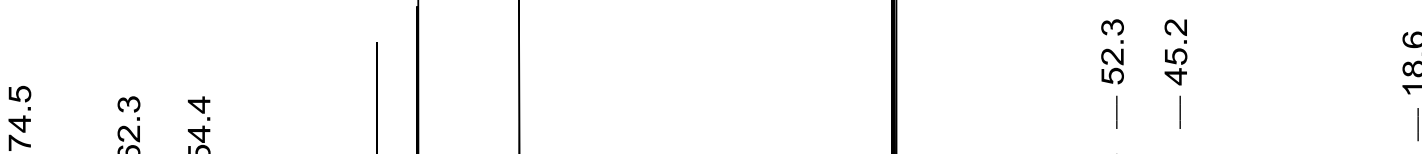

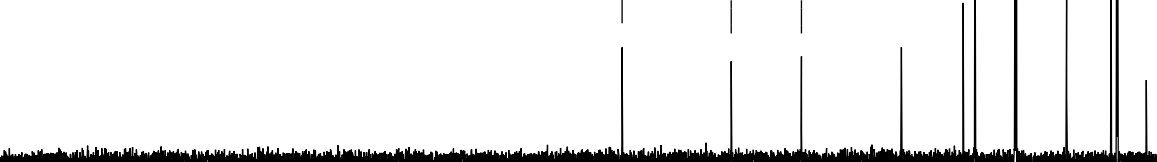

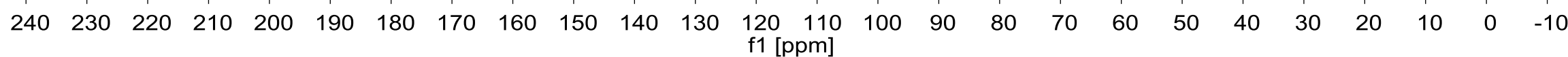




\section{${ }^{19} \mathrm{~F}$ NMR of thianthrenium salt TT-3}

$\mathrm{CDCl}_{3}, 25^{\circ} \mathrm{C}$
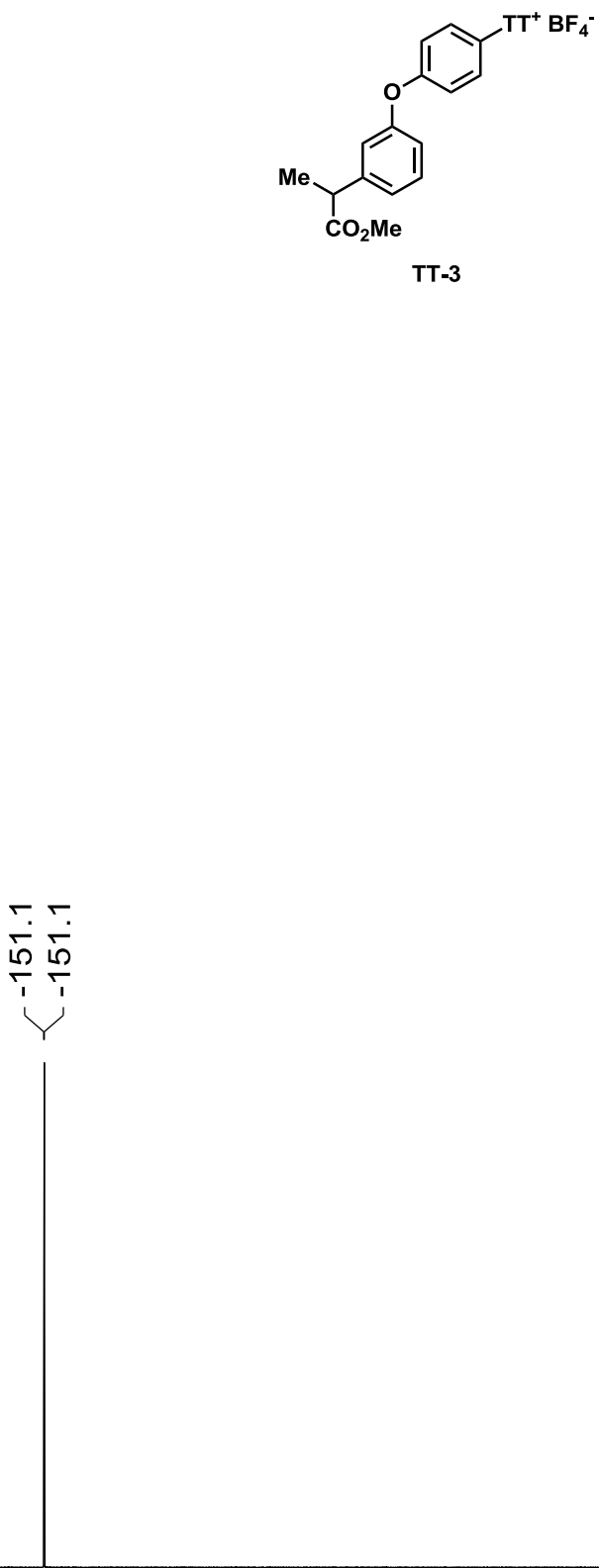

\begin{tabular}{lllllllllllllllllllllllllll}
\hline 10 & 0 & -10 & -20 & -30 & -40 & -50 & -60 & -70 & -80 & -90 & -100 & -110 & -120 & -130 & -140 & -150 & -160 & -170 & -180 & -190 & -200
\end{tabular}




\section{${ }^{1} \mathrm{H}$ NMR of aniline 3}

$\mathrm{CDCl}_{3}, 25^{\circ} \mathrm{C}$
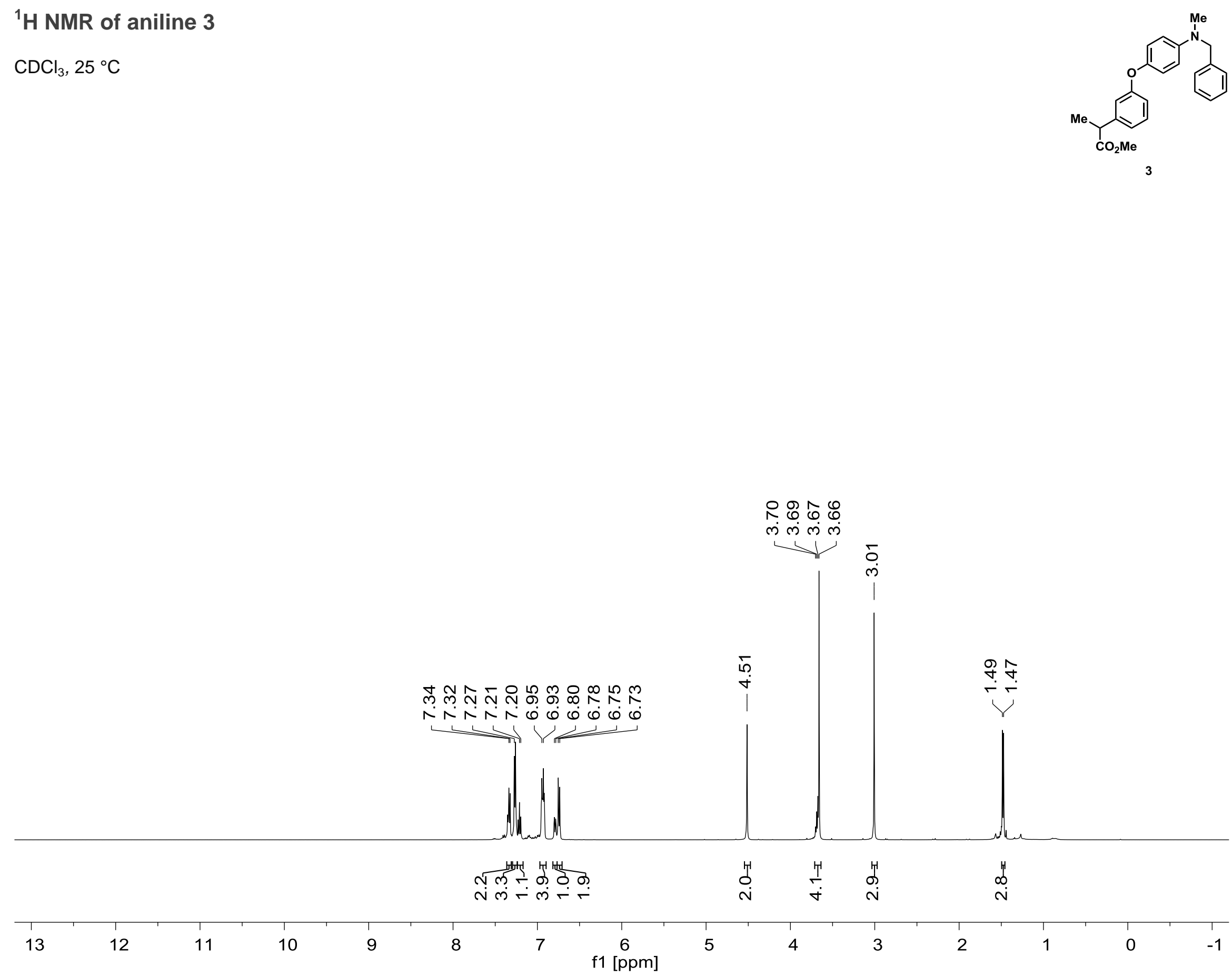


\section{${ }^{13} \mathrm{C}$ NMR of aniline 3}

$\mathrm{CDCl}_{3}, 25^{\circ} \mathrm{C}$
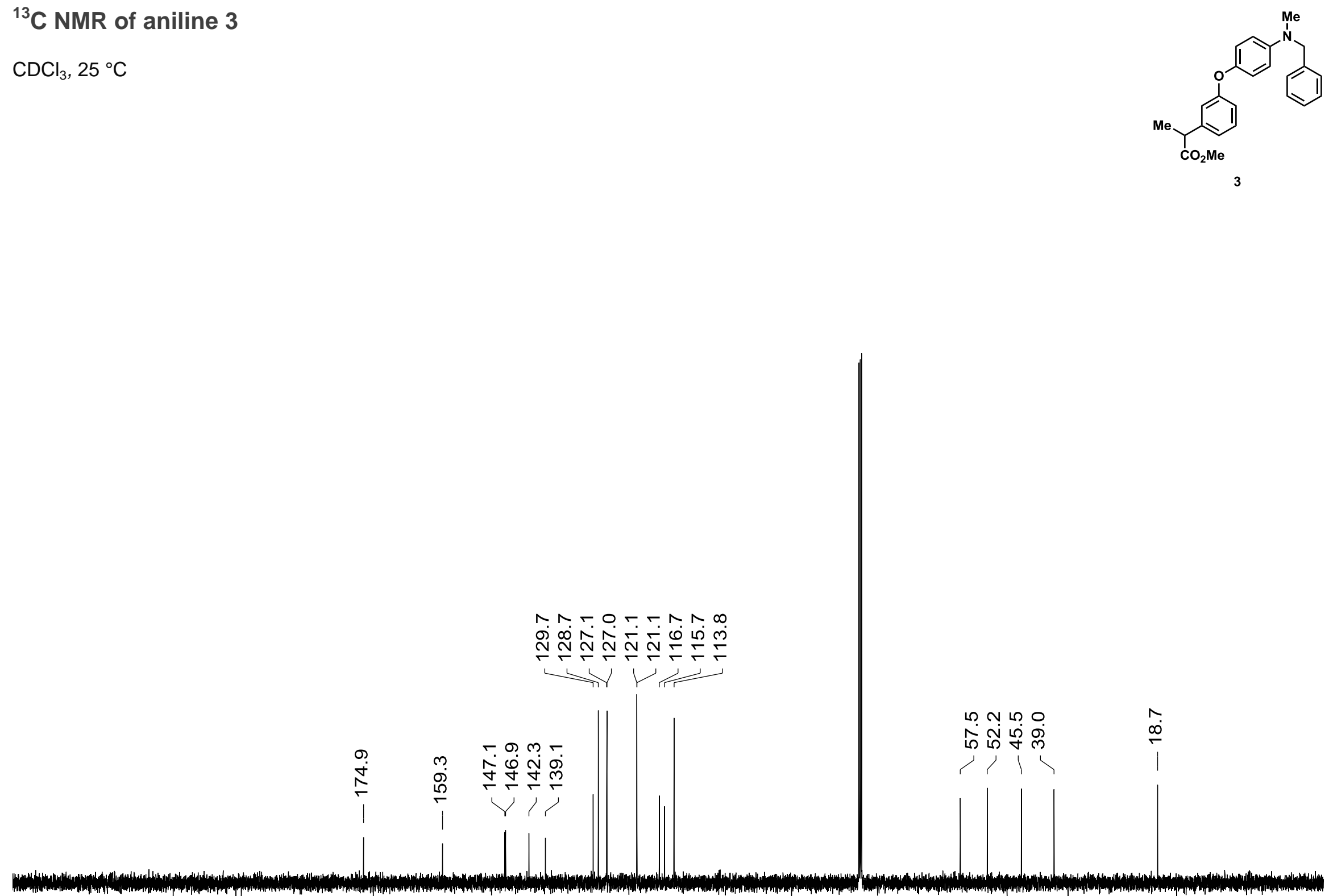

$\begin{array}{llllllllllllllllllllllllllllll}240 & 230 & 220 & 210 & 200 & 190 & 180 & 170 & 160 & 150 & 140 & 130 & 120 & 110 & 100 & 90 & 80 & 70 & 60 & 50 & 40 & 30 & 20 & 10 & 0 & -10\end{array}$ f1 [ppm] 


\section{${ }^{1} \mathrm{H}$ NMR of thianthrenium salt TT-4 (intermediate)}

DMSO- $\mathrm{d}_{6}, 25^{\circ} \mathrm{C}$

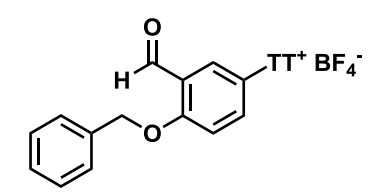

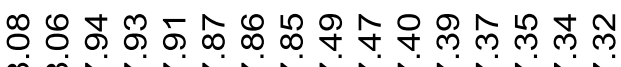

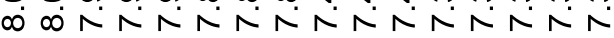

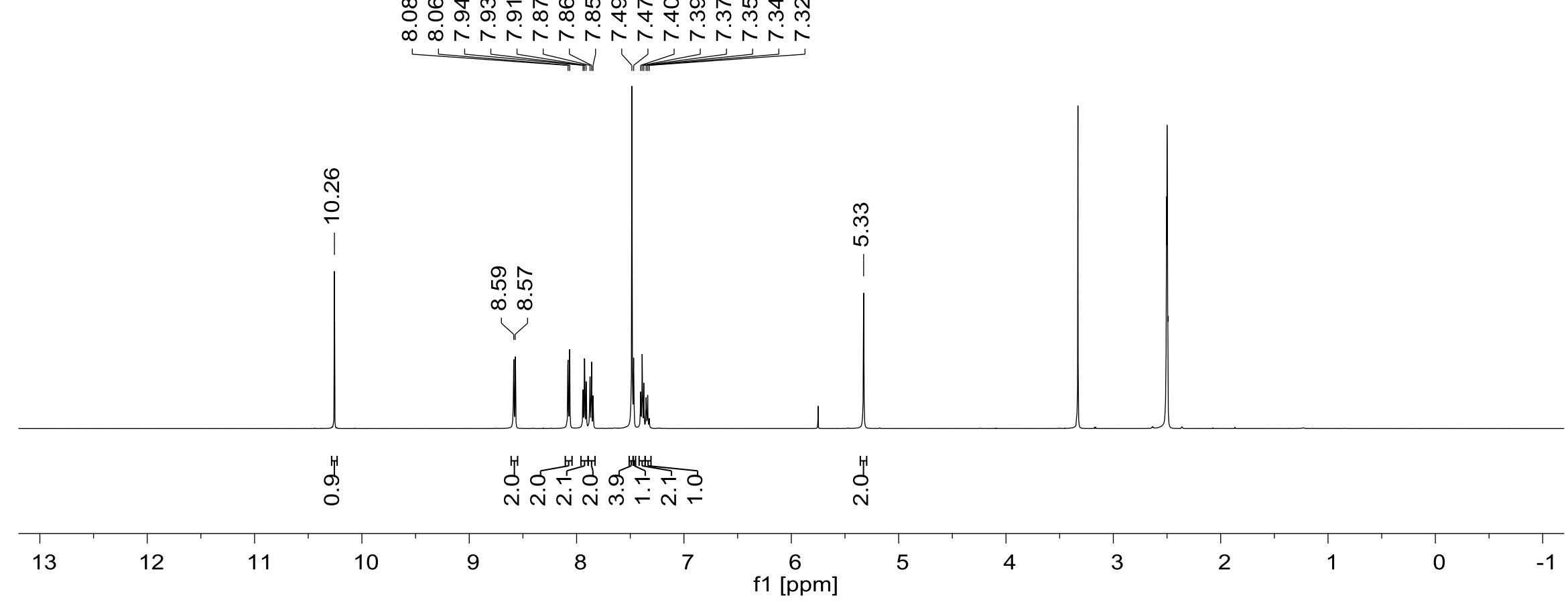




\section{${ }^{13} \mathrm{C}$ NMR of thianthrenium salt TT-4 (intermediate)}

DMSO- $d_{6}, 25^{\circ} \mathrm{C}$

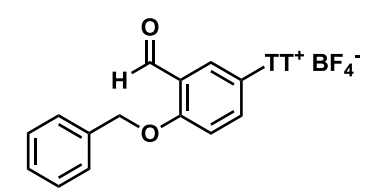

L

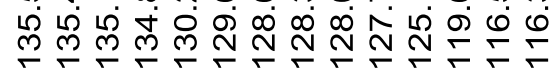

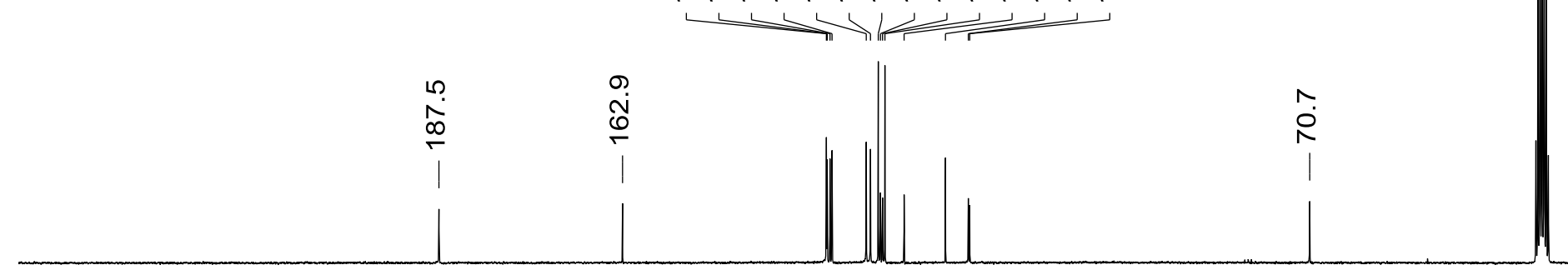

$\begin{array}{lllllllllllllllllllllllllllllll}240 & 230 & 220 & 210 & 200 & 190 & 180 & 170 & 160 & 150 & 140 & 130 & 120 & 110 & 100 & 90 & 80 & 70 & 60 & 50 & 40 & 30 & 20 & 10 & 0 & -10\end{array}$ f1 [ppm] 


\section{${ }^{19} \mathrm{~F}$ NMR of thianthrenium salt TT-4 (intermediate)}

DMSO- $\mathrm{d}_{6}, 25^{\circ} \mathrm{C}$

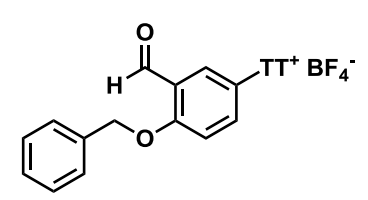

No

$\stackrel{\infty}{\leftarrow} \underset{+}{\stackrel{\infty}{+}}$

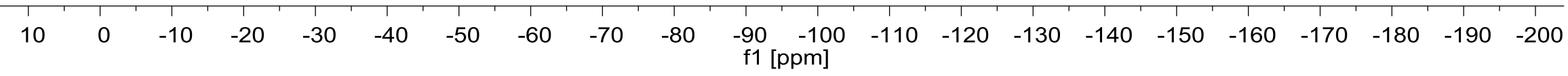




\section{${ }^{1} \mathrm{H}$ NMR of thianthrenium salt TT-4}

DMSO- $\mathrm{d}_{6}, 25^{\circ} \mathrm{C}$
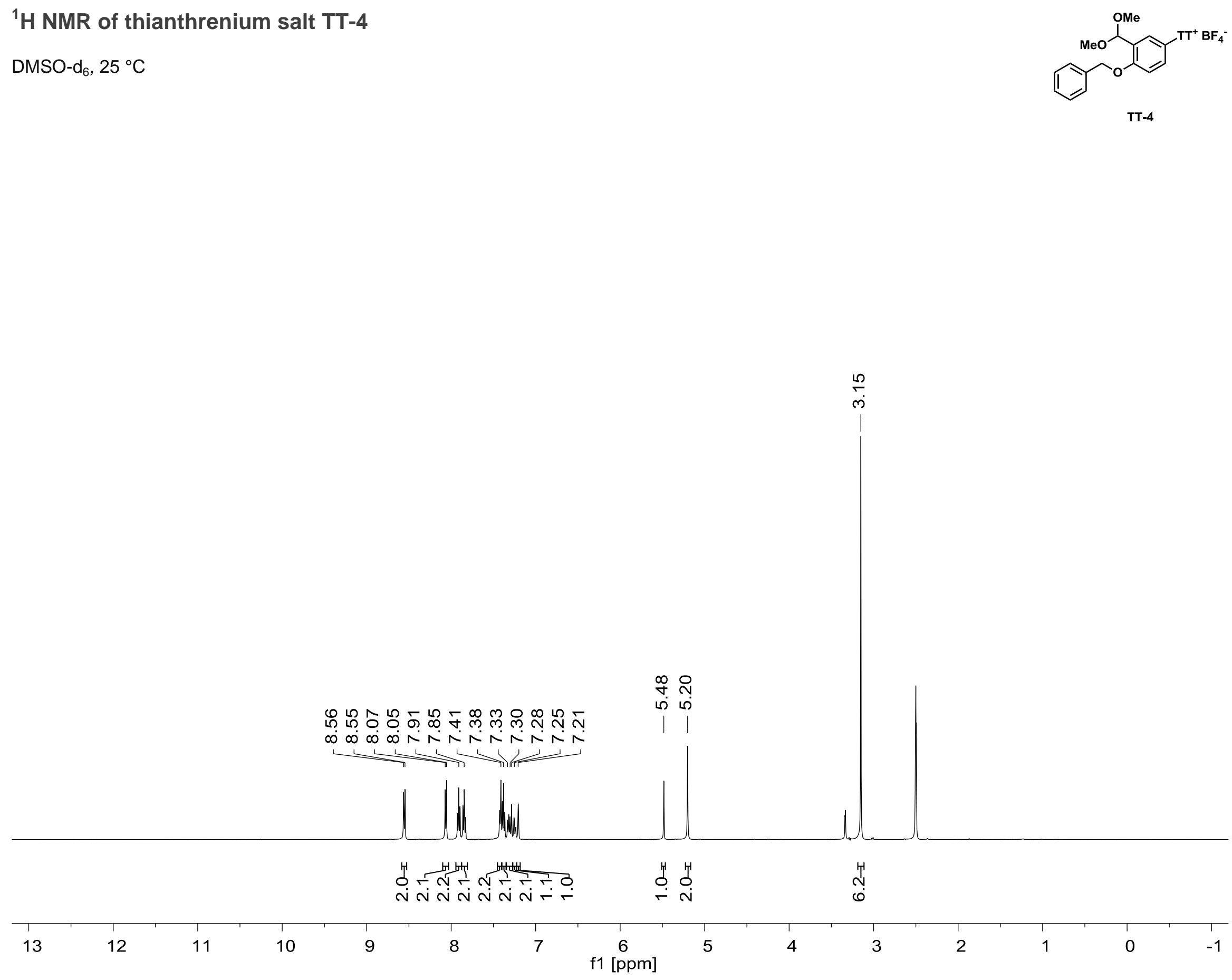


\section{${ }^{13} \mathrm{C}$ NMR of thianthrenium salt TT-4}

DMSO- $\mathrm{d}_{6}, 25^{\circ} \mathrm{C}$
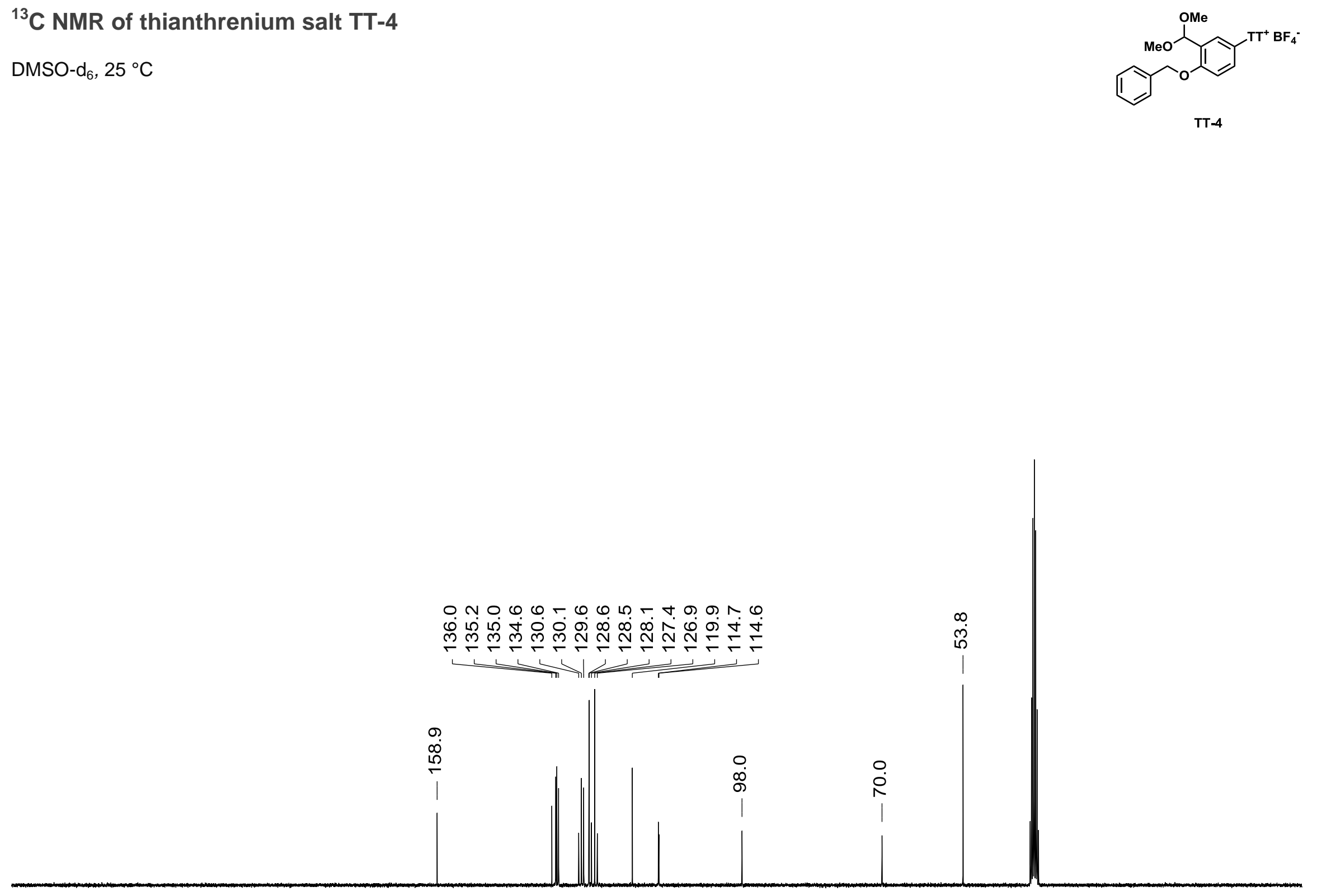

$\begin{array}{lllllllllllllllllllllllll}240 & 230 & 220 & 210 & 200 & 190 & 180 & 170 & 160 & 150 & 140 & 130 & \begin{array}{c}120 \\ \mathrm{f} 1\end{array}\left[\begin{array}{ll}110 \\ {[\mathrm{ppm}]}\end{array}\right. & 100 & 90 & 80 & 70 & 60 & 50 & 40 & 30 & 20 & 10 & 0 & -10\end{array}$




\section{${ }^{19} \mathrm{~F}$ NMR of thianthrenium salt TT-4}

DMSO- $\mathrm{d}_{6}, 25^{\circ} \mathrm{C}$

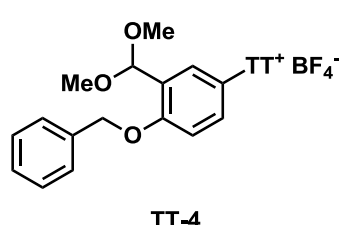

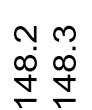




\section{${ }^{1} \mathrm{H}$ NMR of aniline 4}

$\mathrm{CDCl}_{3}, 25^{\circ} \mathrm{C}$
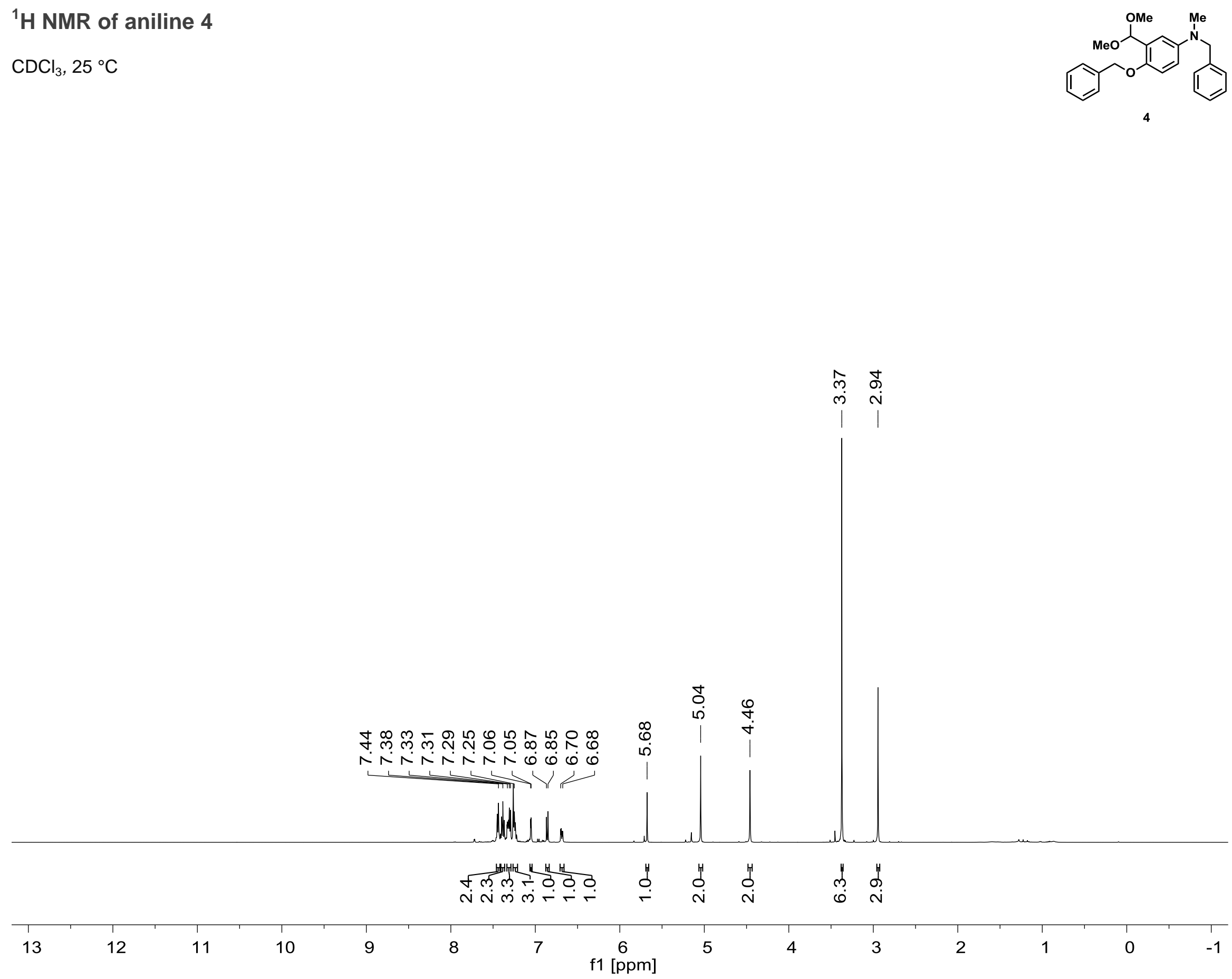
${ }^{13} \mathrm{C}$ NMR of aniline 4

$\mathrm{CDCl}_{3}, 25^{\circ} \mathrm{C}$
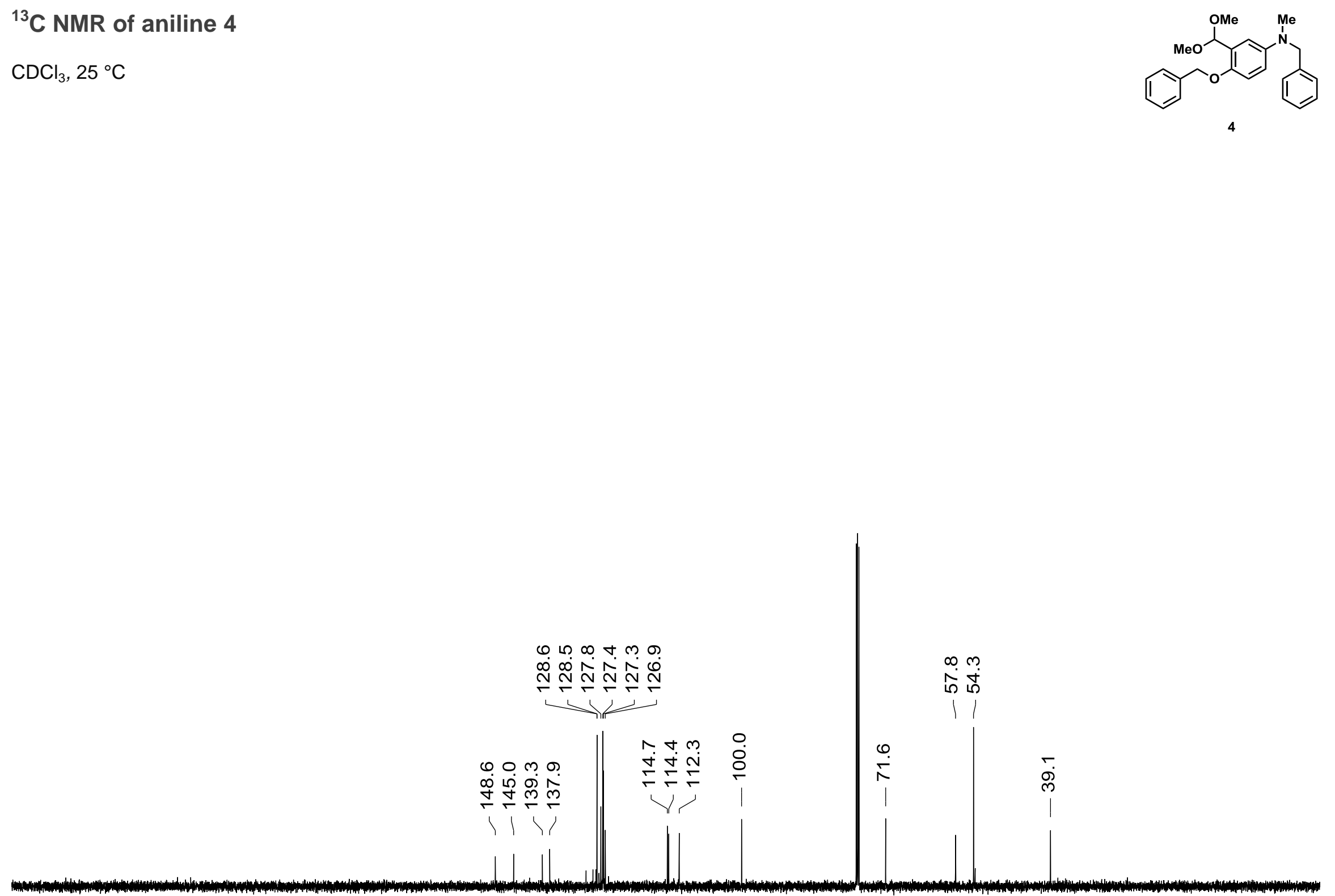


\section{${ }^{1} \mathrm{H}$ NMR of thianthrenium salt TT-5}

DMSO- $\mathrm{d}_{6}, 25^{\circ} \mathrm{C}$
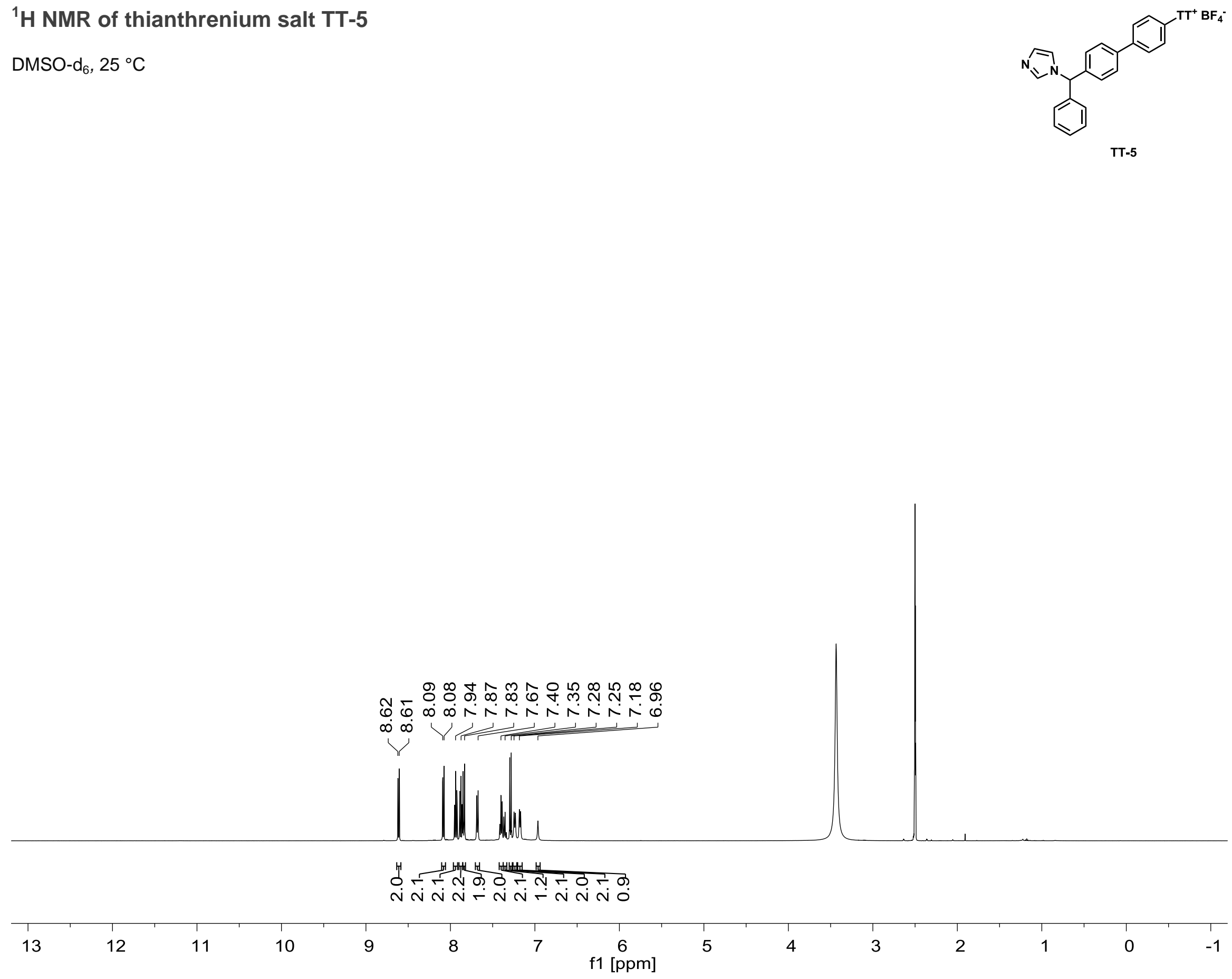


\section{${ }^{13} \mathrm{C}$ NMR of thianthrenium salt TT-5}

DMSO- $\mathrm{d}_{6}, 25^{\circ} \mathrm{C}$

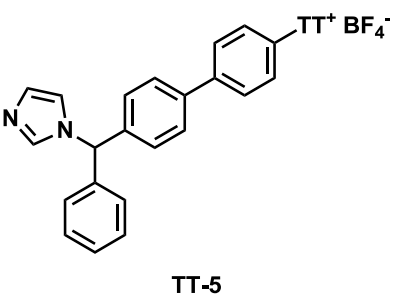

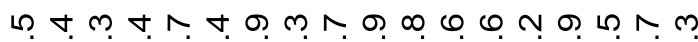

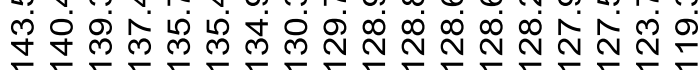




\section{${ }^{13} \mathrm{C}$ NMR of thianthrenium salt TT-5}

$\mathrm{MeCN}-\mathrm{d}_{3}+\mathrm{TFA}, 25^{\circ} \mathrm{C}$

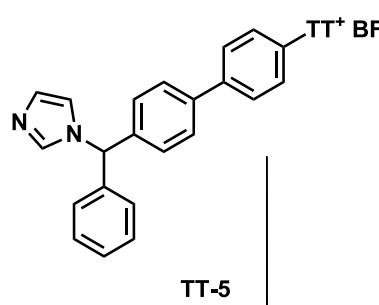

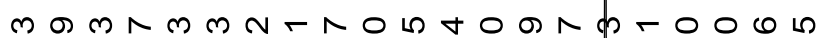

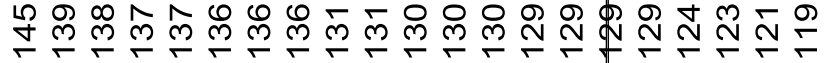

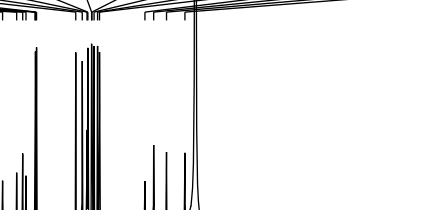




\section{${ }^{19} \mathrm{~F}$ NMR of thianthrenium salt TT-5}

DMSO- $\mathrm{d}_{6}, 25^{\circ} \mathrm{C}$

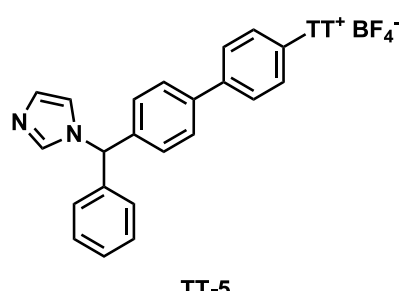

กุ

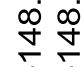




\section{${ }^{1} \mathrm{H}$ NMR of morpholine 5}

$\mathrm{CDCl}_{3}, 25^{\circ} \mathrm{C}$
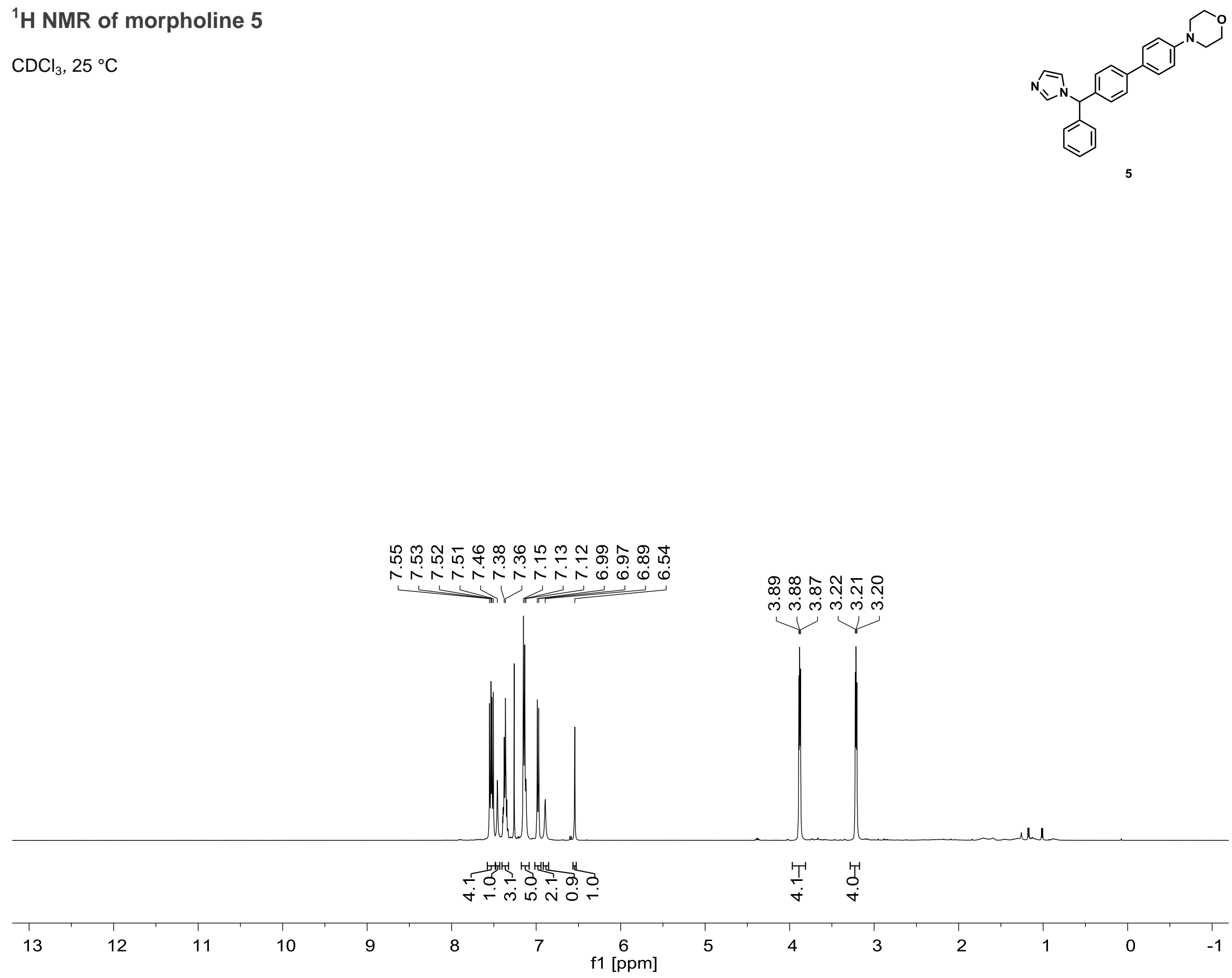


\section{${ }^{13} \mathrm{C}$ NMR of morpholine 5}

$\mathrm{CDCl}_{3}, 25^{\circ} \mathrm{C}$
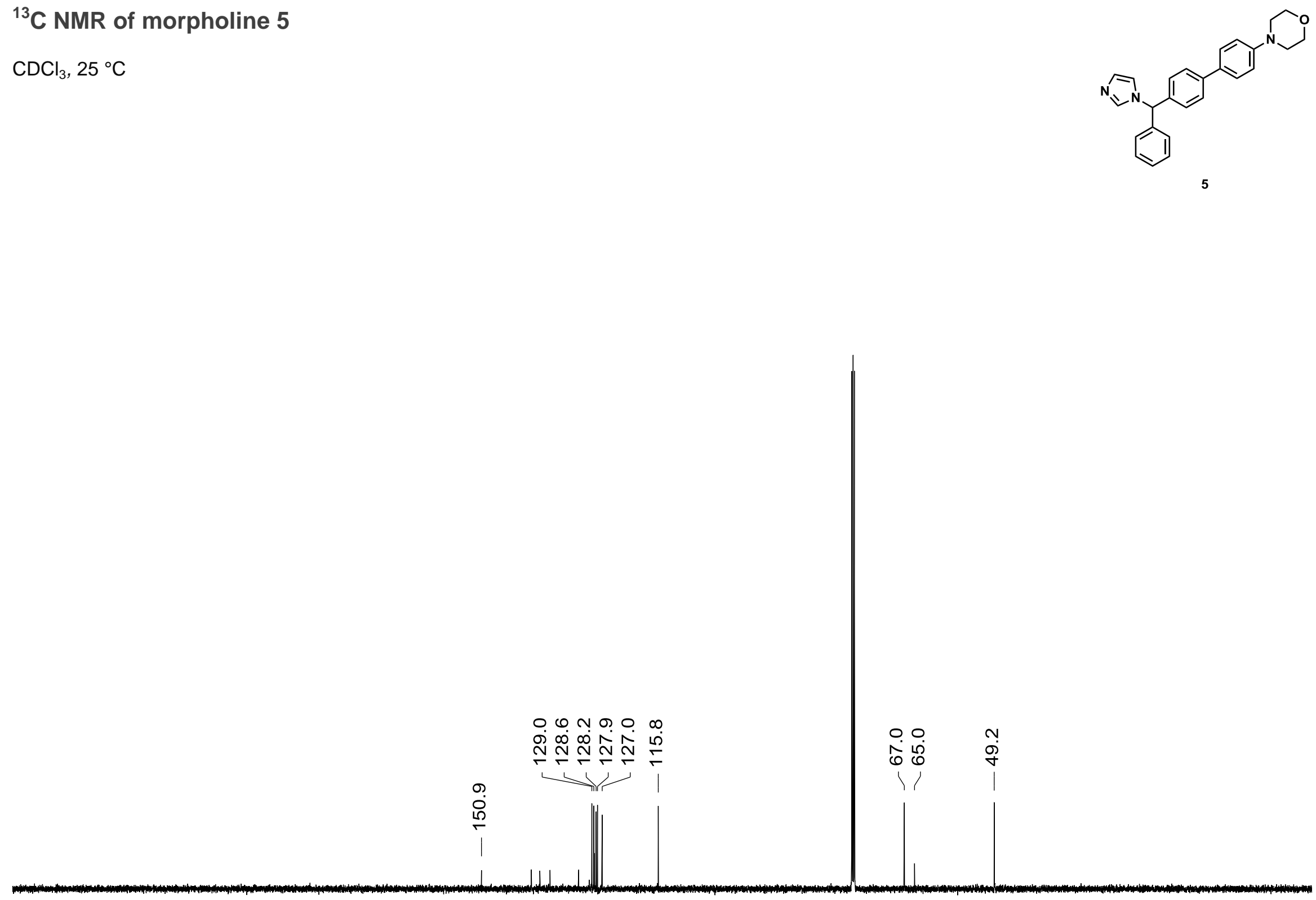


\section{${ }^{1} \mathrm{H}$ NMR of thianthrenium salt TT-6}

$\mathrm{CDCl}_{3}, 25^{\circ} \mathrm{C}$

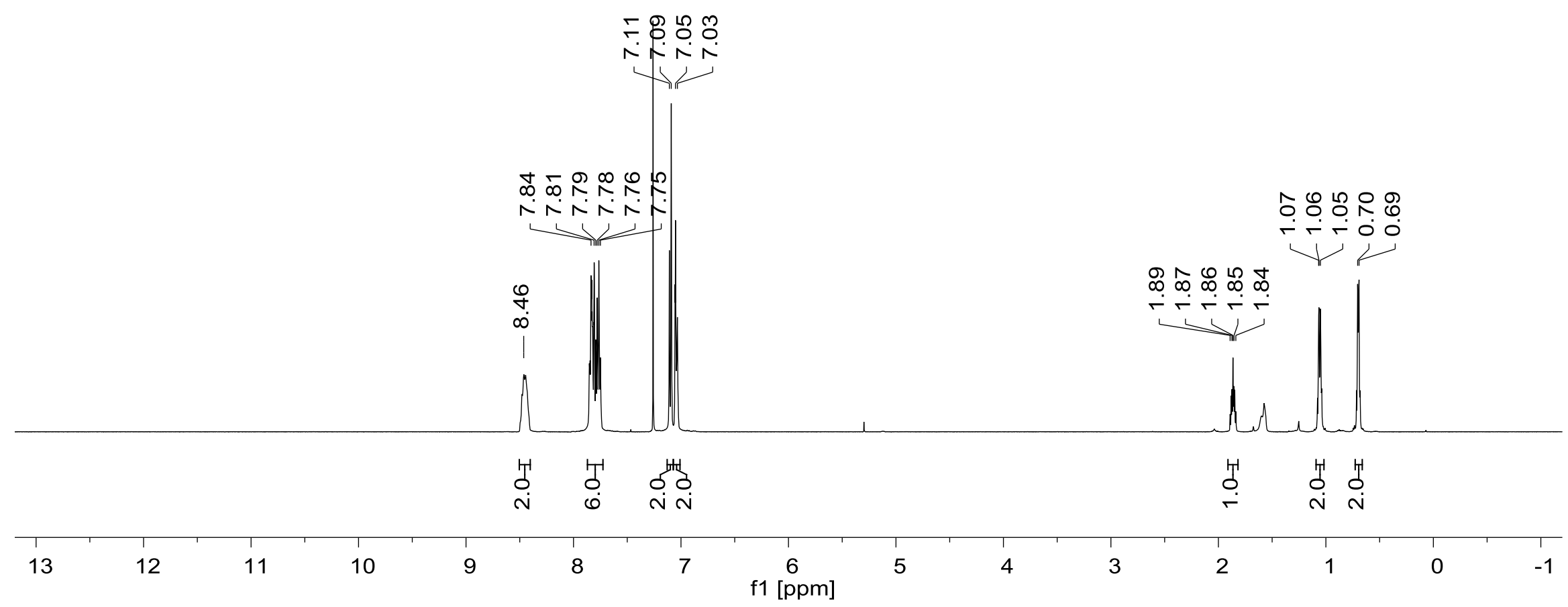


${ }^{13} \mathrm{C}$ NMR of thianthrenium salt TT-6

$\mathrm{CDCl}_{3}, 25^{\circ} \mathrm{C}$

ம 0 0 0 L

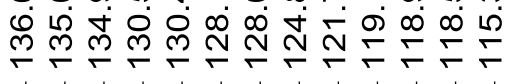

计

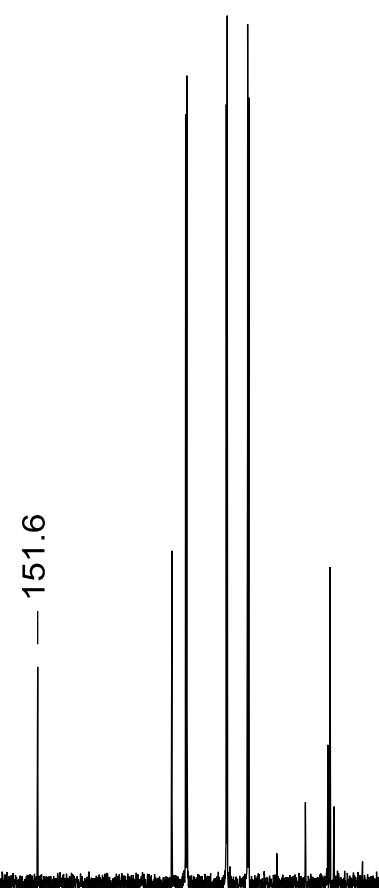

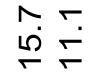

।

TT-6

$\begin{array}{llllllllllllllllllllllllllll}240 & 230 & 220 & 210 & 200 & 190 & 180 & 170 & 160 & 150 & 140 & 130 & 120 & 110 & 100 & 90 & 80 & 70 & 60 & 50 & 40 & 30 & 20 & 10 & 0 & -10\end{array}$ f1 [ppm] 


\section{${ }^{19} \mathrm{~F}$ NMR of thianthrenium salt TT-6}

$\mathrm{CDCl}_{3}, 25^{\circ} \mathrm{C}$ 


\section{${ }^{1} \mathrm{H}$ NMR of isonicotinamide 6}

DMSO- $\mathrm{d}_{6}, 25^{\circ} \mathrm{C}$
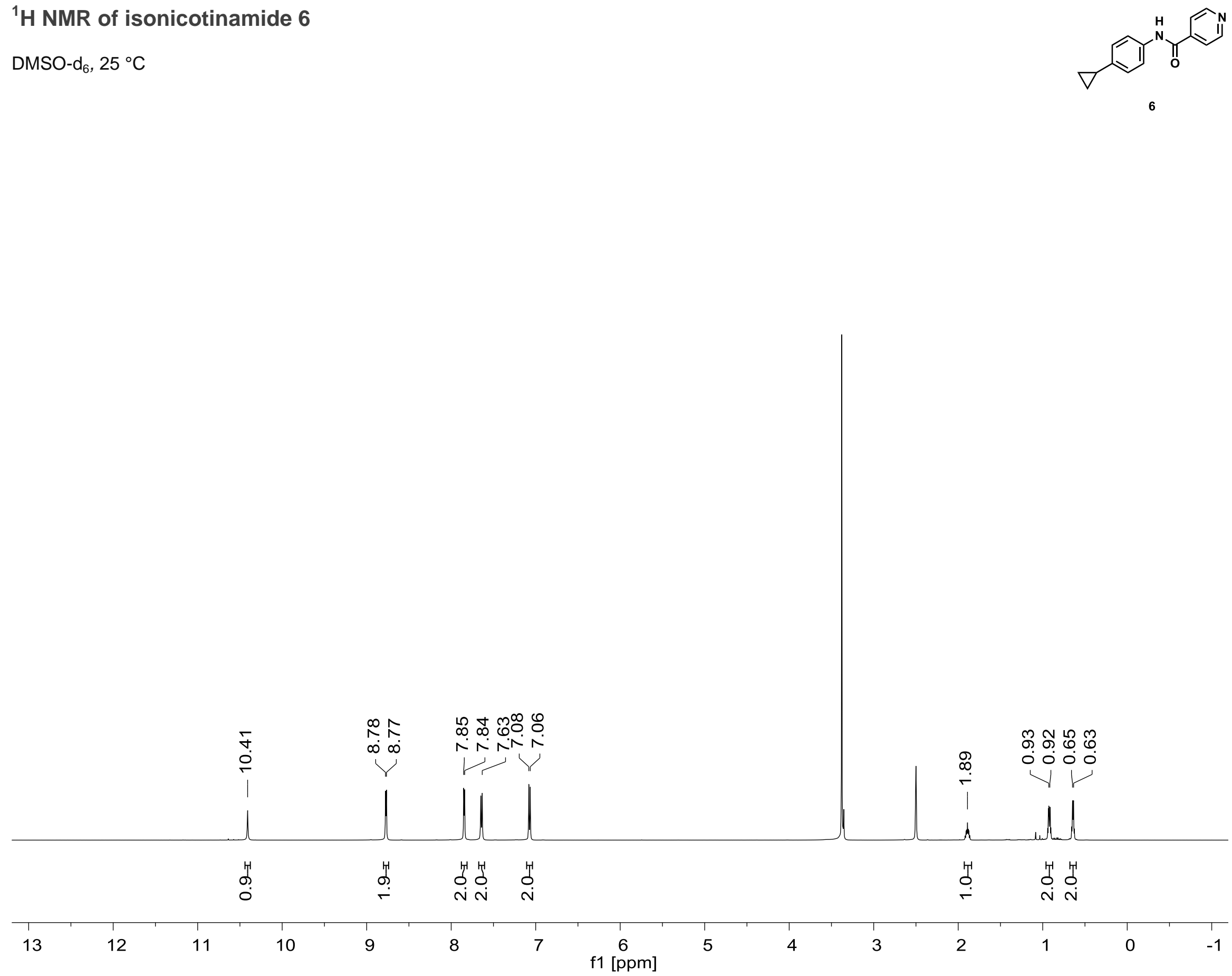


\section{${ }^{13} \mathrm{C}$ NMR of isonicotinamide 6}

DMSO- $\mathrm{d}_{6}, 25^{\circ} \mathrm{C}$

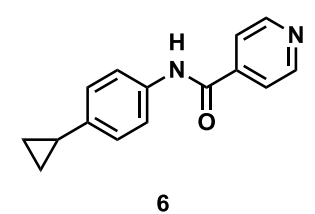

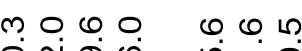

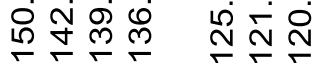

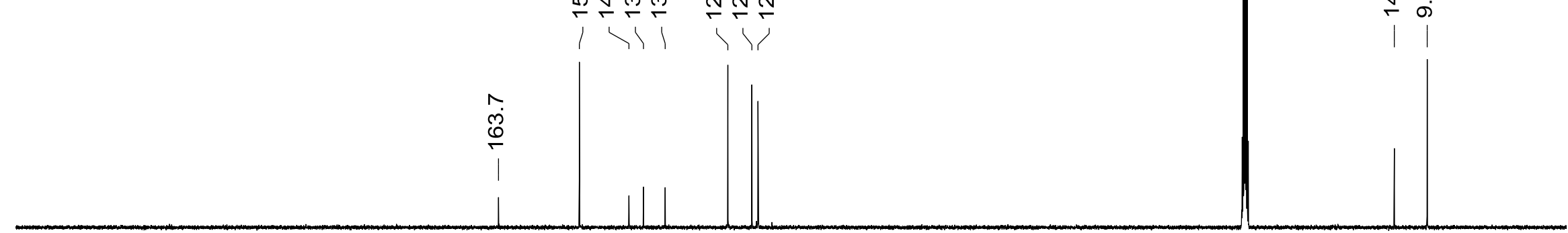




\section{${ }^{1} \mathrm{H}$ NMR of thianthrenium salt TT-7}

$\mathrm{CDCl}_{3}, 25^{\circ} \mathrm{C}$

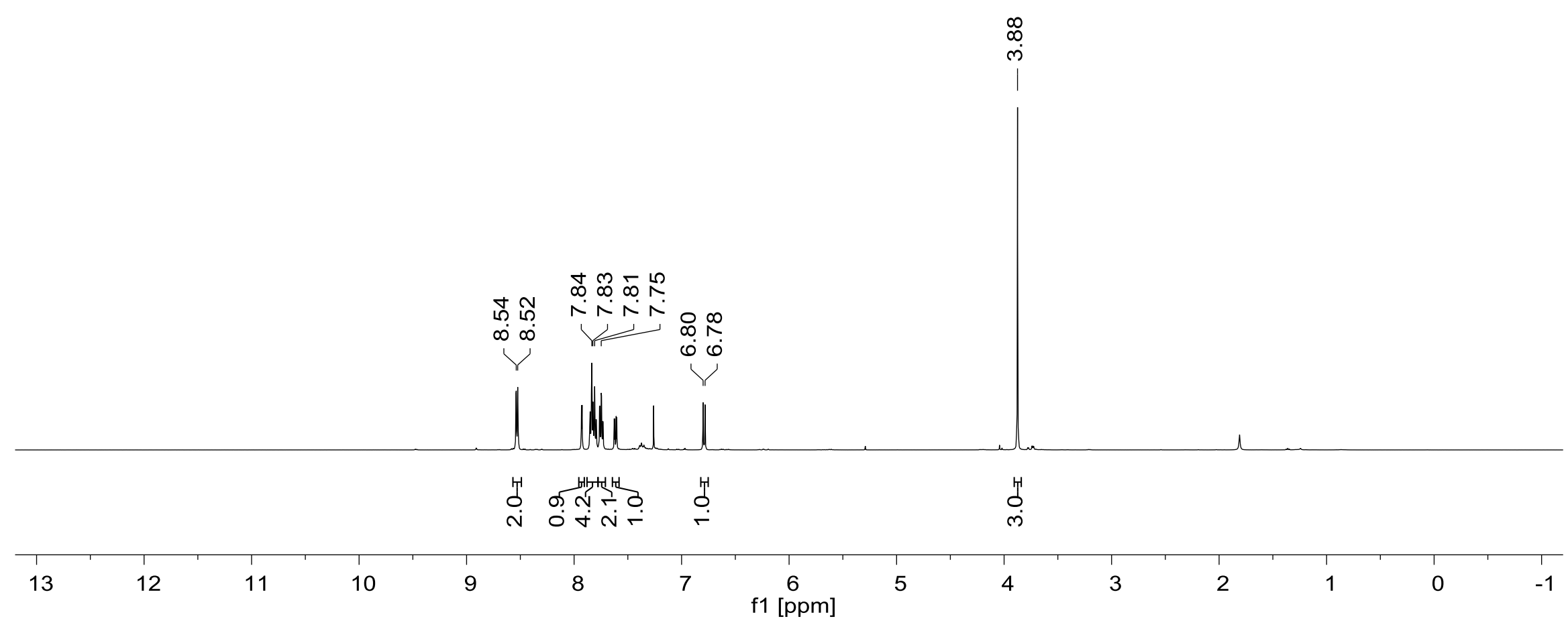




\section{${ }^{13} \mathrm{C}$ NMR of thianthrenium salt TT-7}

$\mathrm{CDCl}_{3}, 25^{\circ} \mathrm{C}$

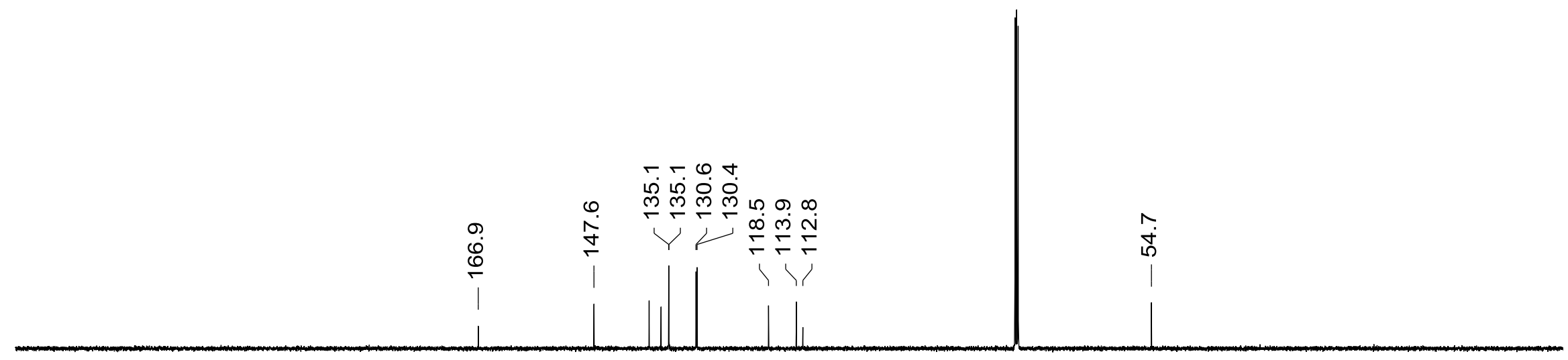


${ }^{19} \mathrm{~F}$ NMR of thianthrenium salt TT-7

$\mathrm{CDCl}_{3}, 25^{\circ} \mathrm{C}$

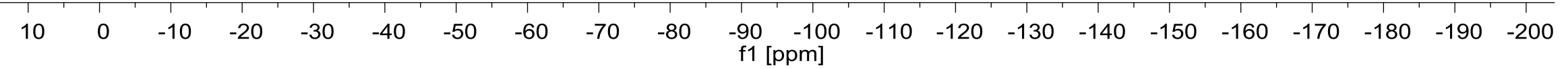




\section{${ }^{1} \mathrm{H}$ NMR of oxazolidinone 7}

$\mathrm{CDCl}_{3}, 25^{\circ} \mathrm{C}$
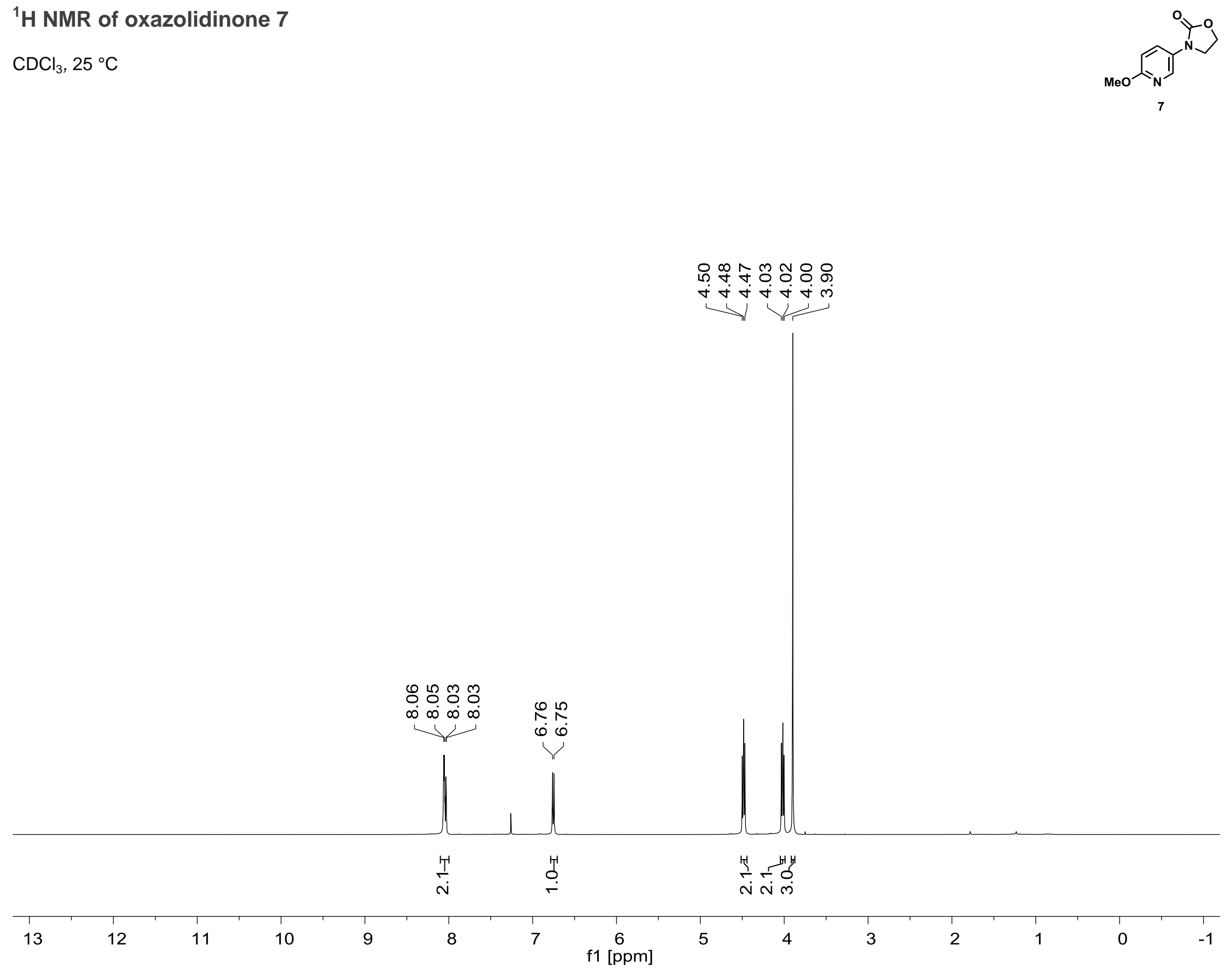


\section{${ }^{13} \mathrm{C}$ NMR of oxazolidinone 7}

$\mathrm{CDCl}_{3}, 25^{\circ} \mathrm{C}$
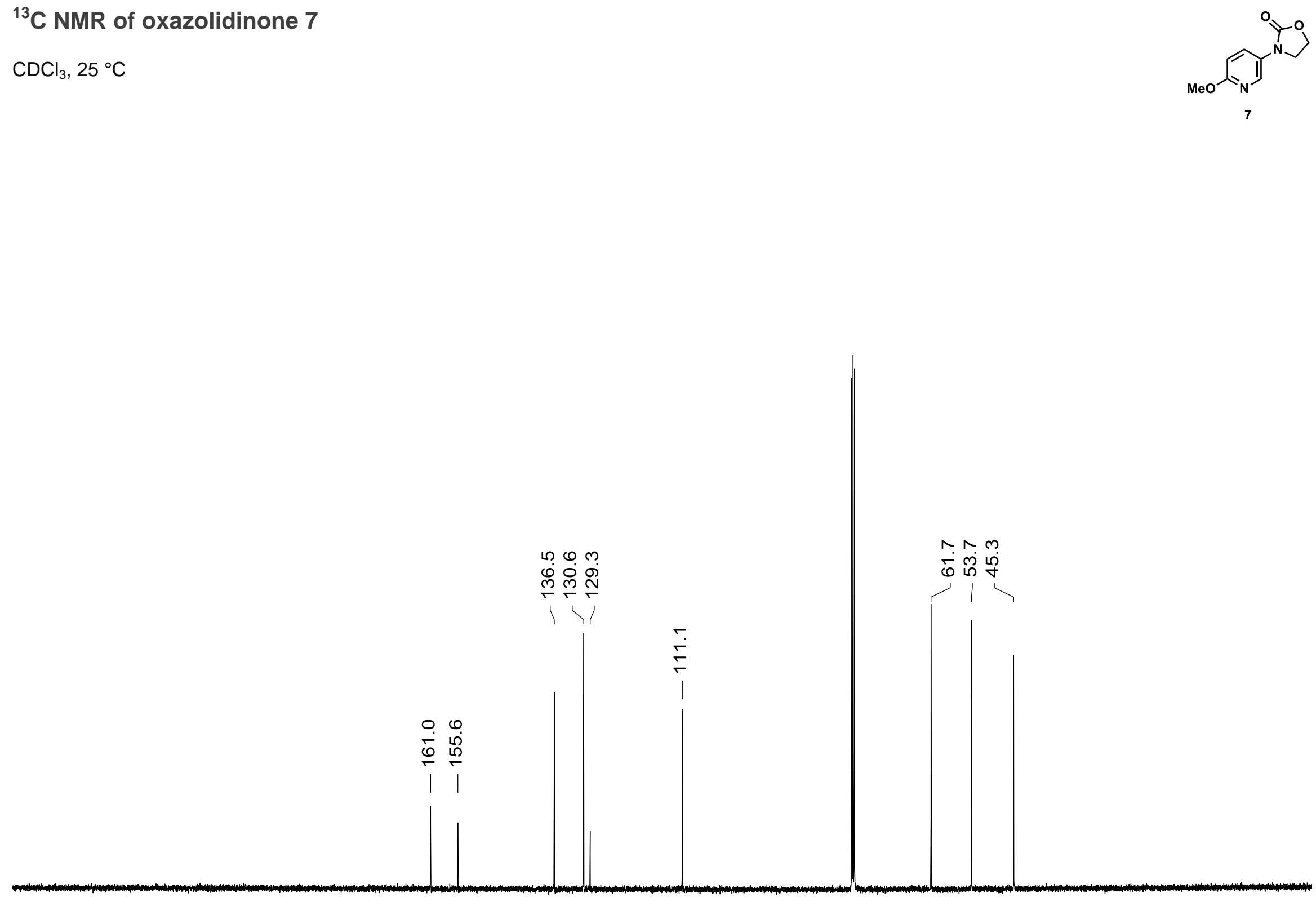


\section{${ }^{1} \mathrm{H}$ NMR of thianthrenium salt TT-8}

$\mathrm{CDCl}_{3}, 25^{\circ} \mathrm{C}$
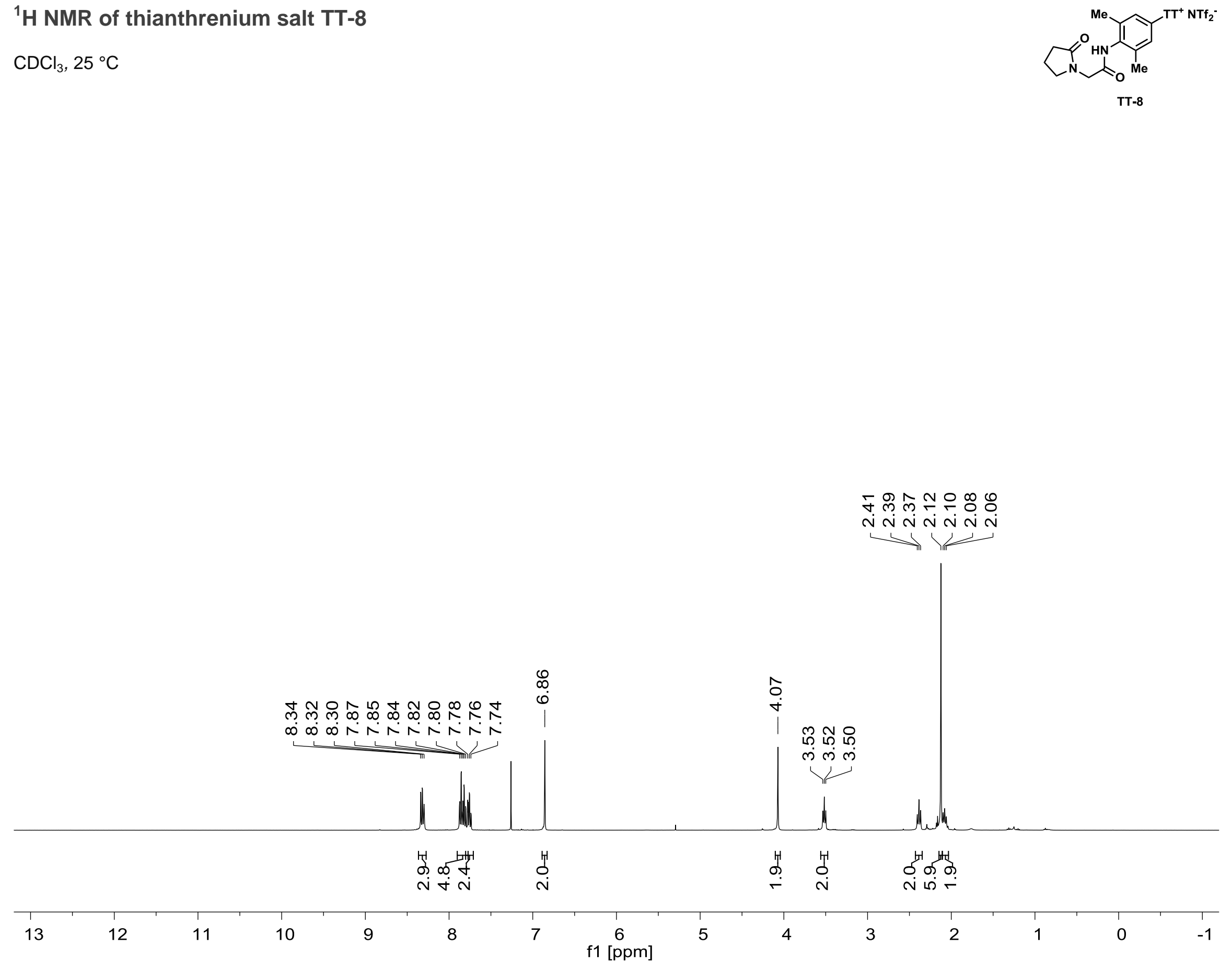


\section{${ }^{13} \mathrm{C}$ NMR of thianthrenium salt TT-8}

$\mathrm{CDCl}_{3}, 25^{\circ} \mathrm{C}$
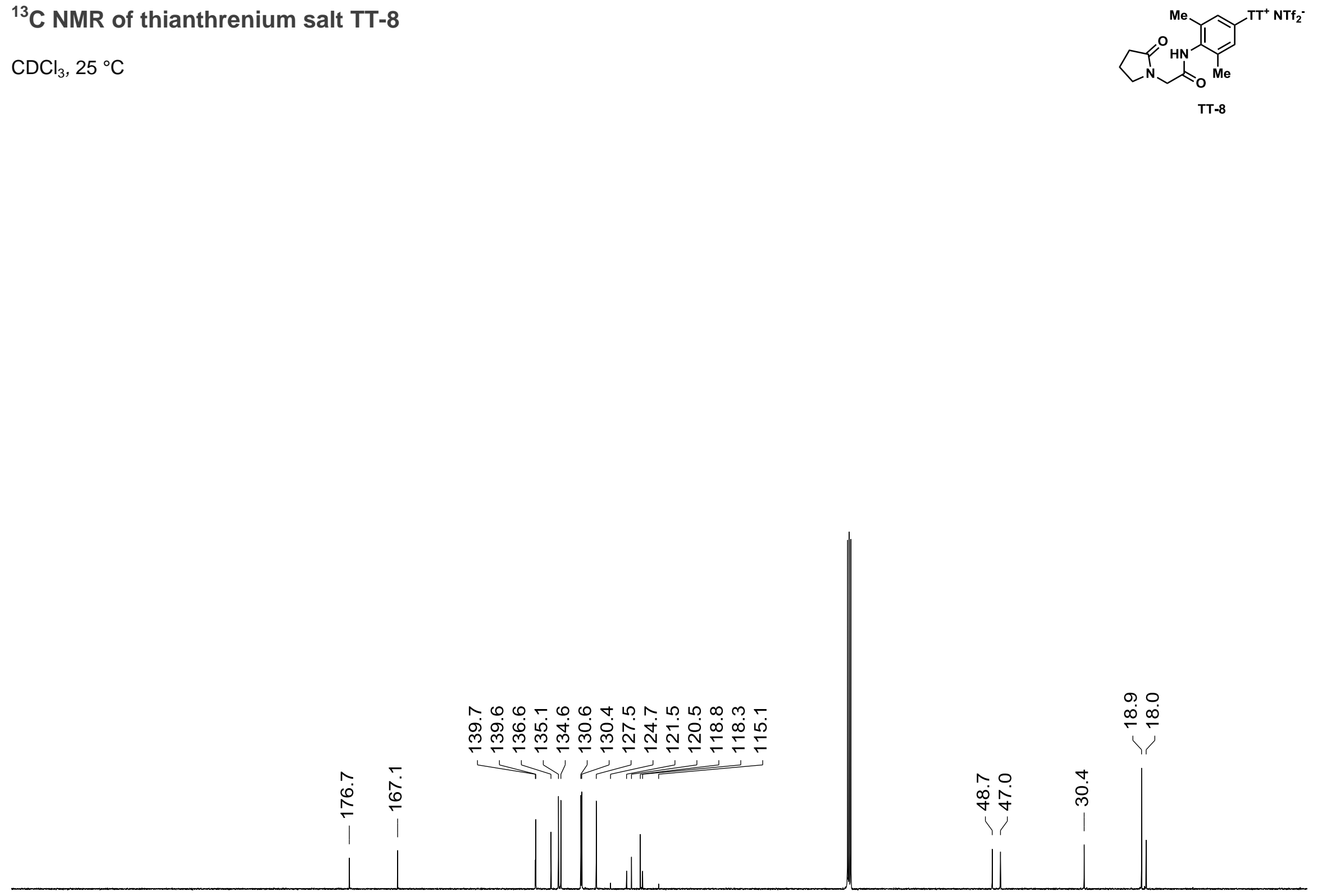

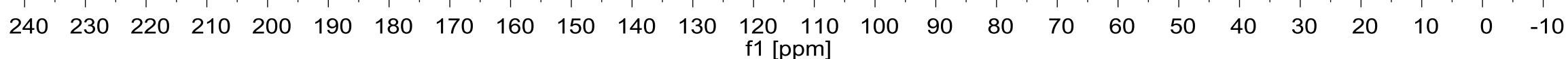




\section{${ }^{19} \mathrm{~F}$ NMR of thianthrenium salt TT-8}

$\mathrm{CDCl}_{3}, 25^{\circ} \mathrm{C}$

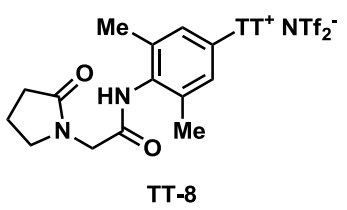

\begin{tabular}{lllllllllllllllllllllllllll}
\hline 10 & 0 & -10 & -20 & -30 & -40 & -50 & -60 & -70 & -80 & -90 & -100 & -110 & -120 & -130 & -140 & -150 & -160 & -170 & -180 & -190 & -200
\end{tabular}




\section{${ }^{1} \mathrm{H}$ NMR of aminopyridine $8 \mathrm{a}$}

DMSO- $\mathrm{d}_{6}, 25^{\circ} \mathrm{C}$
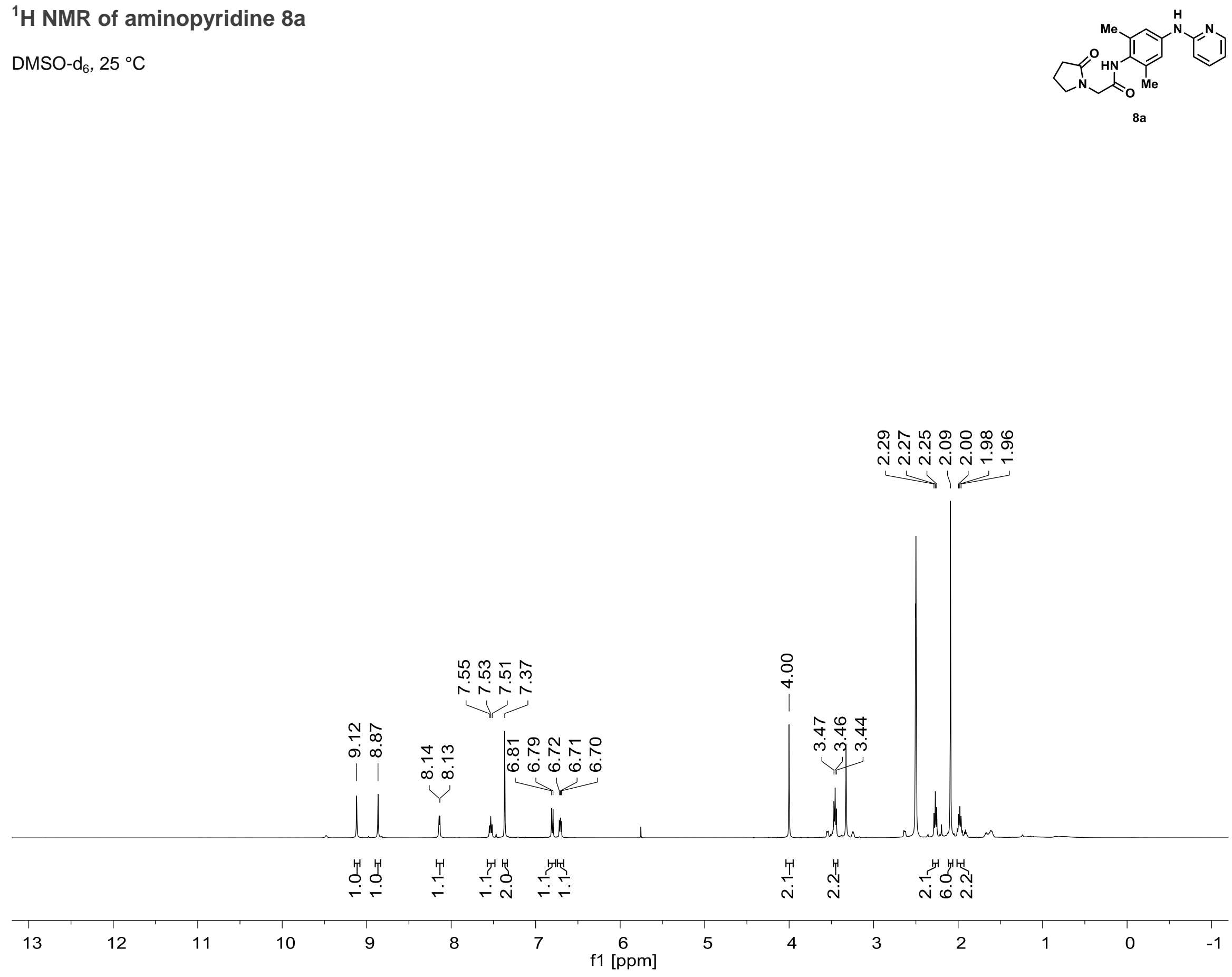


\section{${ }^{13} \mathrm{C}$ NMR of aminopyridine 8a}

DMSO-d $6,25^{\circ} \mathrm{C}$
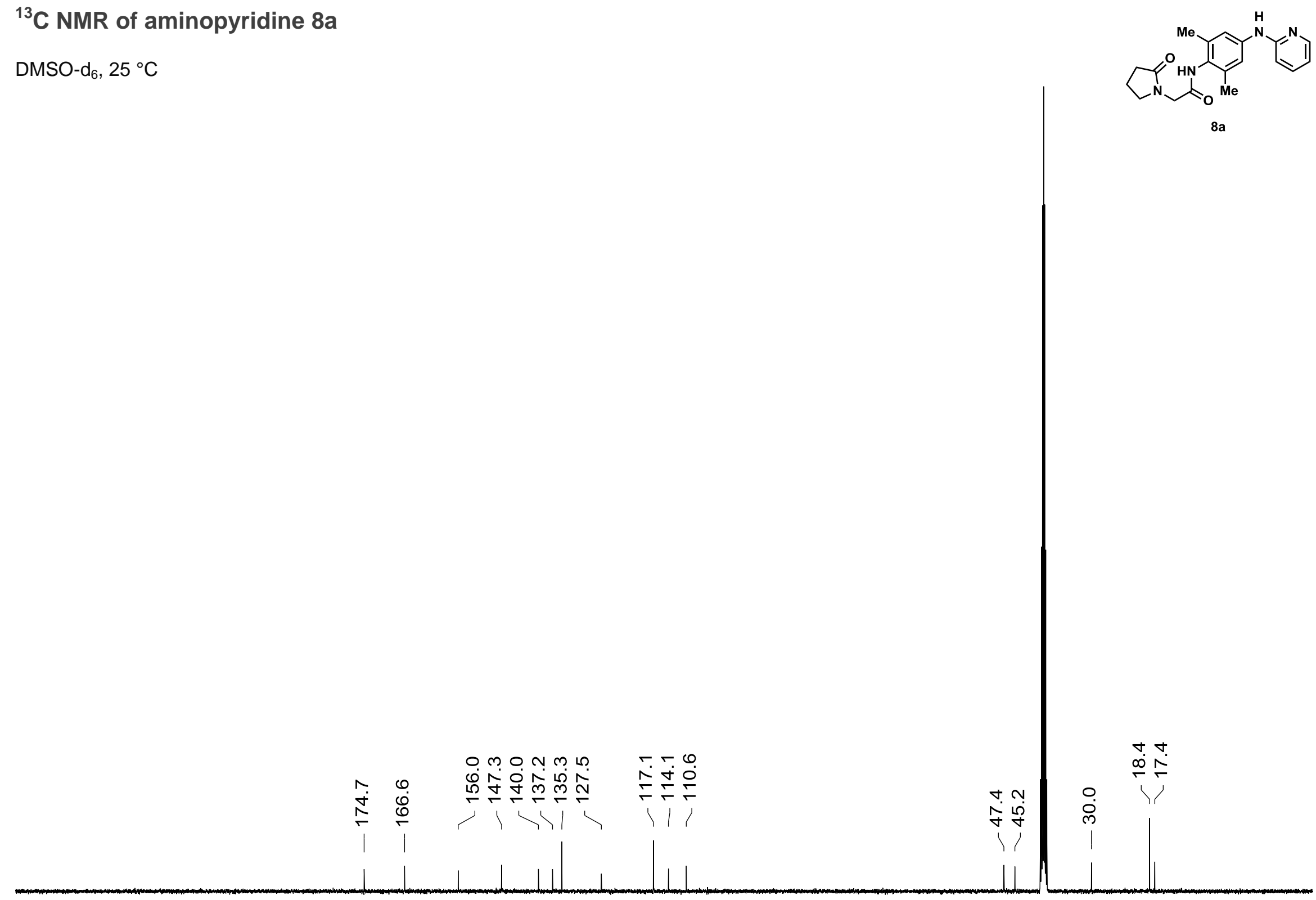


\section{${ }^{1} \mathrm{H}$ NMR of phthalimide $8 \mathrm{~b}$}

DMSO- $\mathrm{d}_{6}, 25^{\circ} \mathrm{C}$
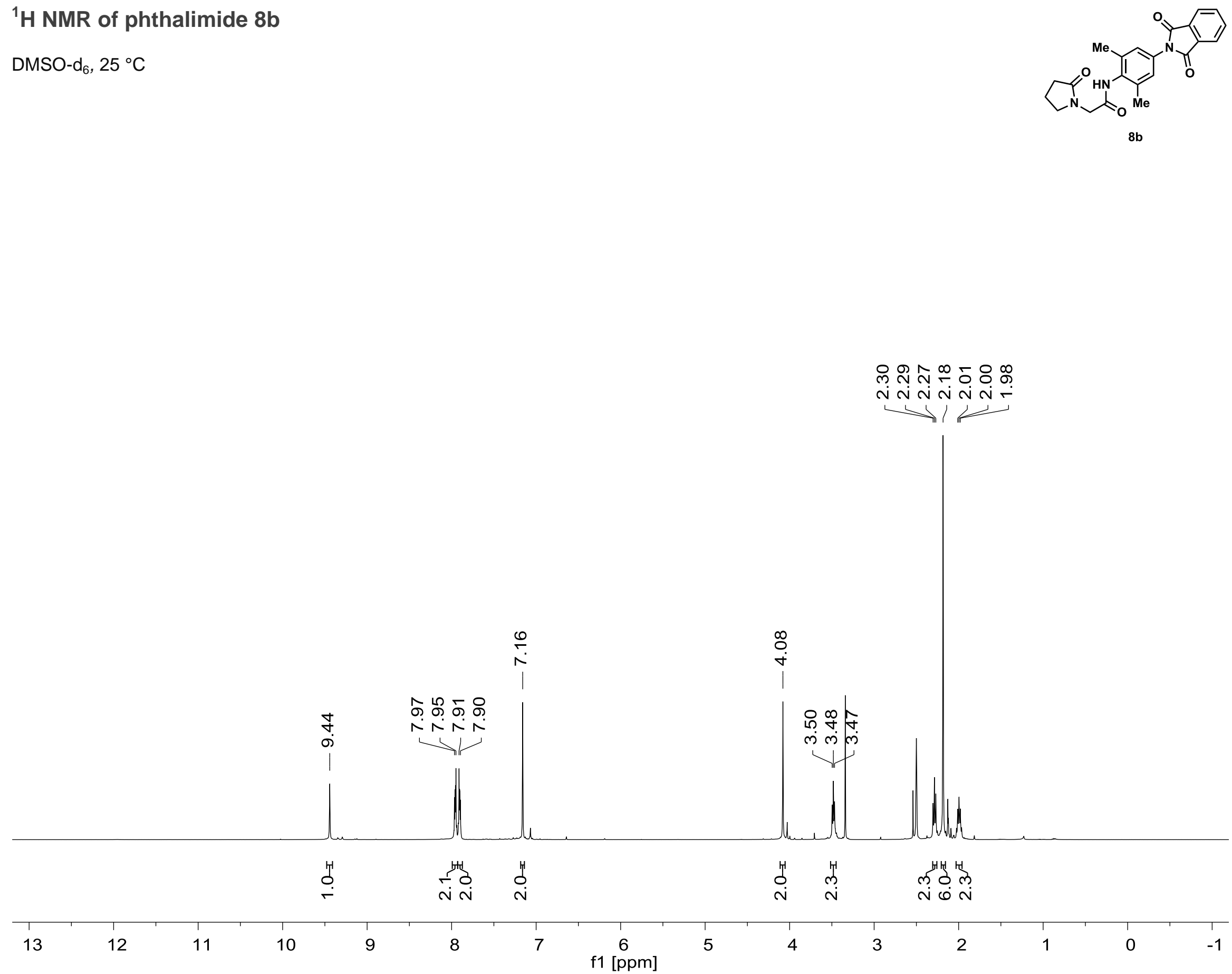


\section{${ }^{13} \mathrm{C}$ NMR of phthalimide $8 \mathrm{~b}$}

DMSO- $\mathrm{d}_{6}, 25^{\circ} \mathrm{C}$

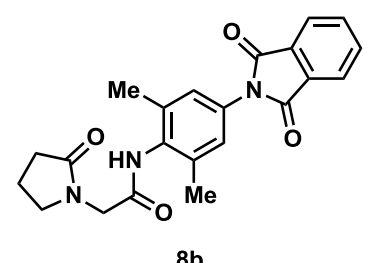

$8 \mathbf{b}$

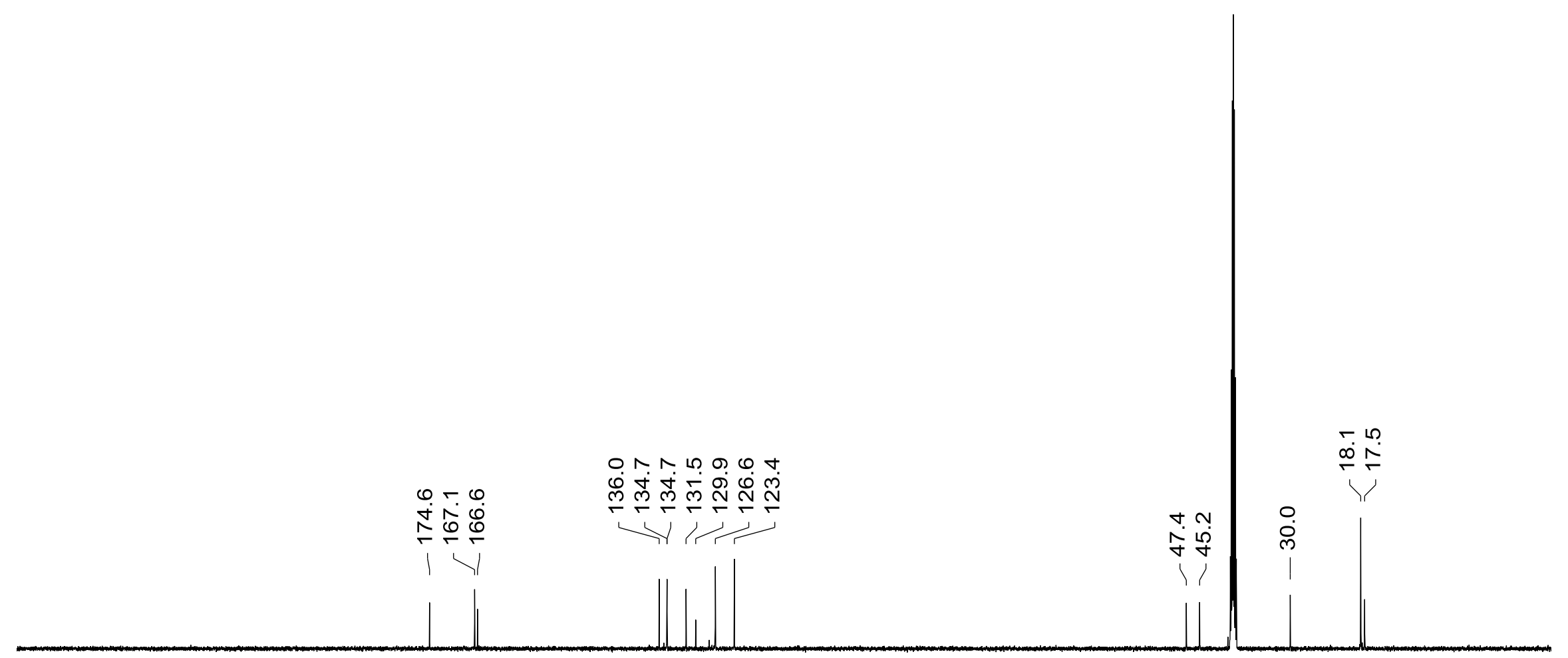




\section{${ }^{1} \mathrm{H}$ NMR of aniline 9}

$\mathrm{CDCl}_{3}, 25^{\circ} \mathrm{C}$

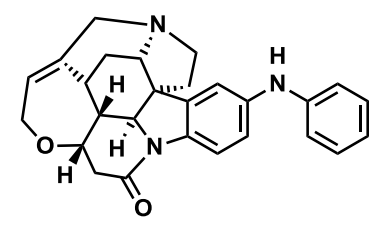

9

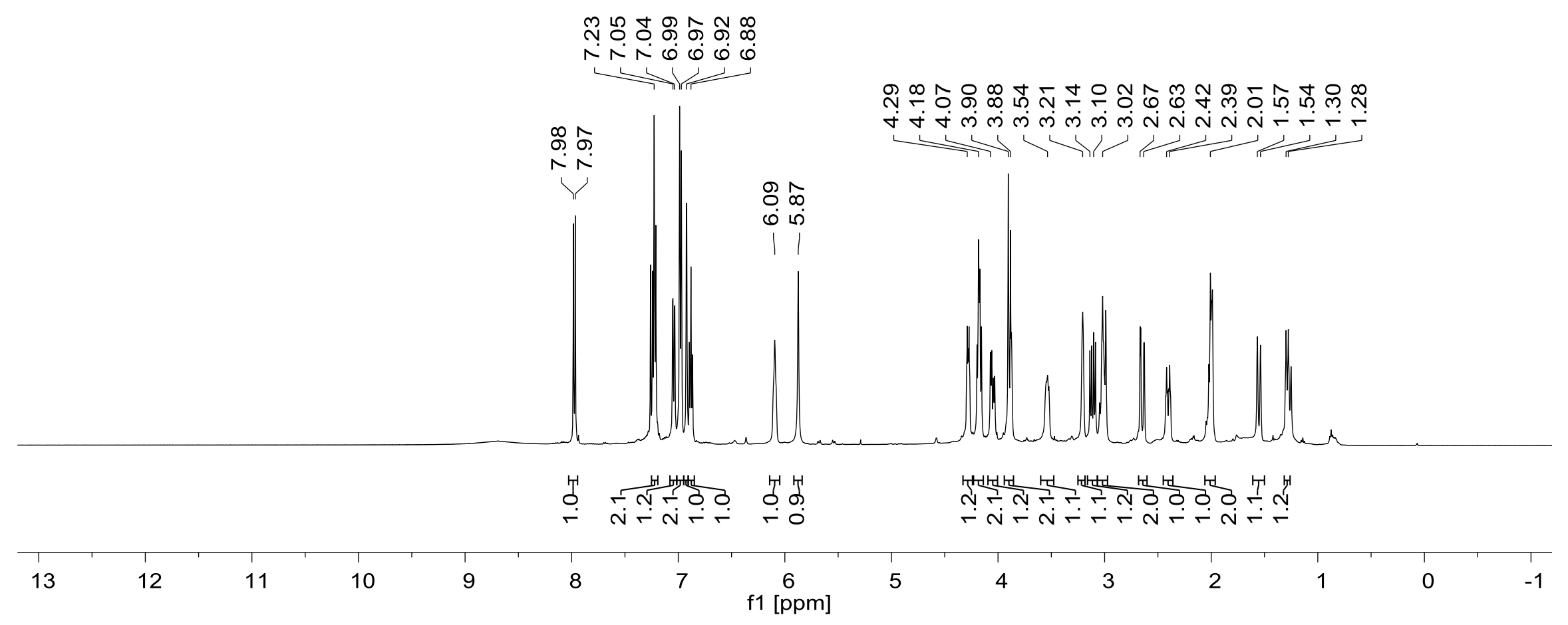




\section{${ }^{13} \mathrm{C}$ NMR of aniline 9}

$\mathrm{CDCl}_{3}, 25^{\circ} \mathrm{C}$

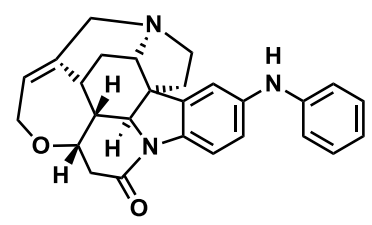

9

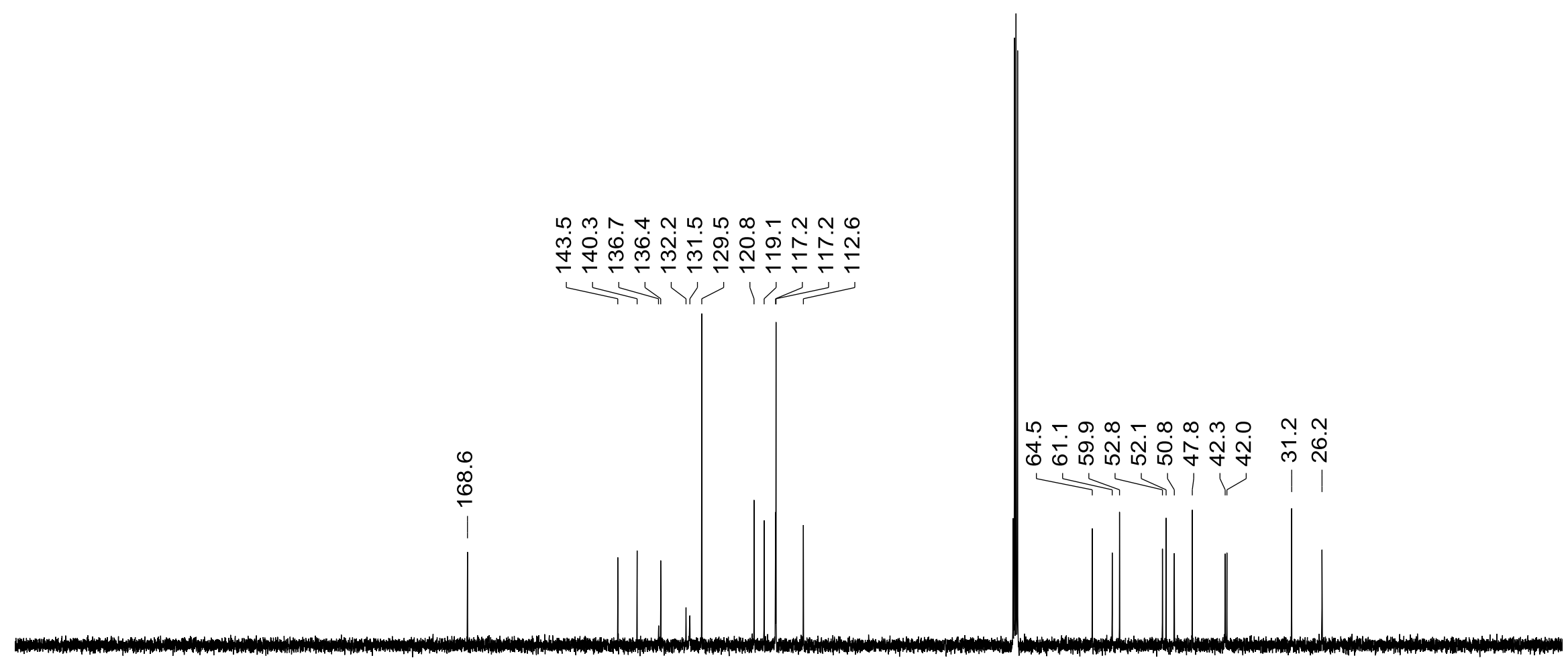

$\begin{array}{lllllllllllllllllllllllllllllllllll}240 & 230 & 220 & 210 & 200 & 190 & 180 & 170 & 160 & 150 & 140 & 130 & 120 & 110 & 100 & 90 & 80 & 70 & 60 & 50 & 40 & 30 & 20 & 10 & 0 & -10\end{array}$ f1 [ppm] 


\section{${ }^{1} \mathrm{H}$ NMR of thianthrenium salt TT-10}

$\mathrm{MeCN}-\mathrm{d}_{3}, 25^{\circ} \mathrm{C}$
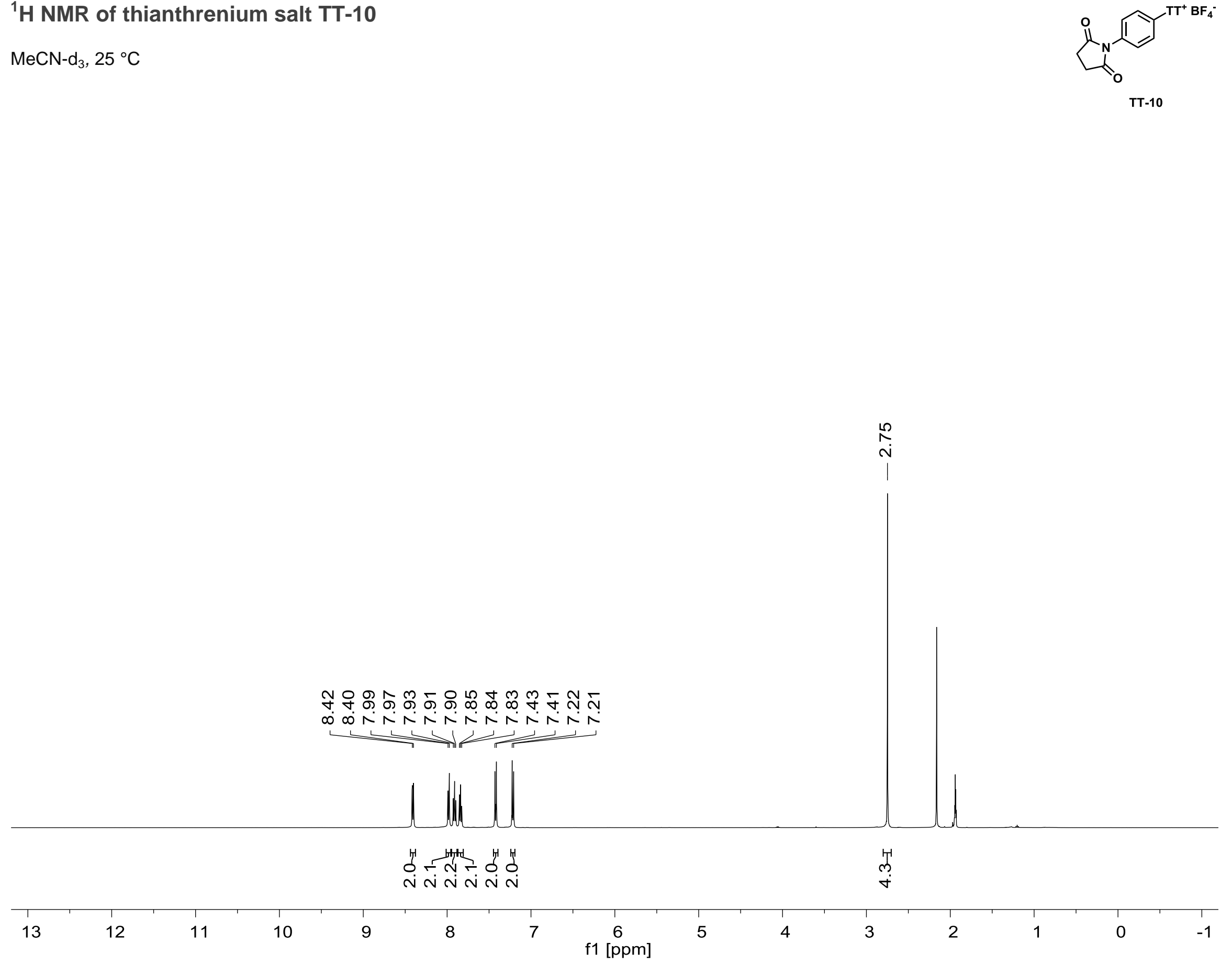


\section{${ }^{13} \mathrm{C}$ NMR of thianthrenium salt TT-10}

$\mathrm{MeCN}-\mathrm{d}_{3}, 25^{\circ} \mathrm{C}$
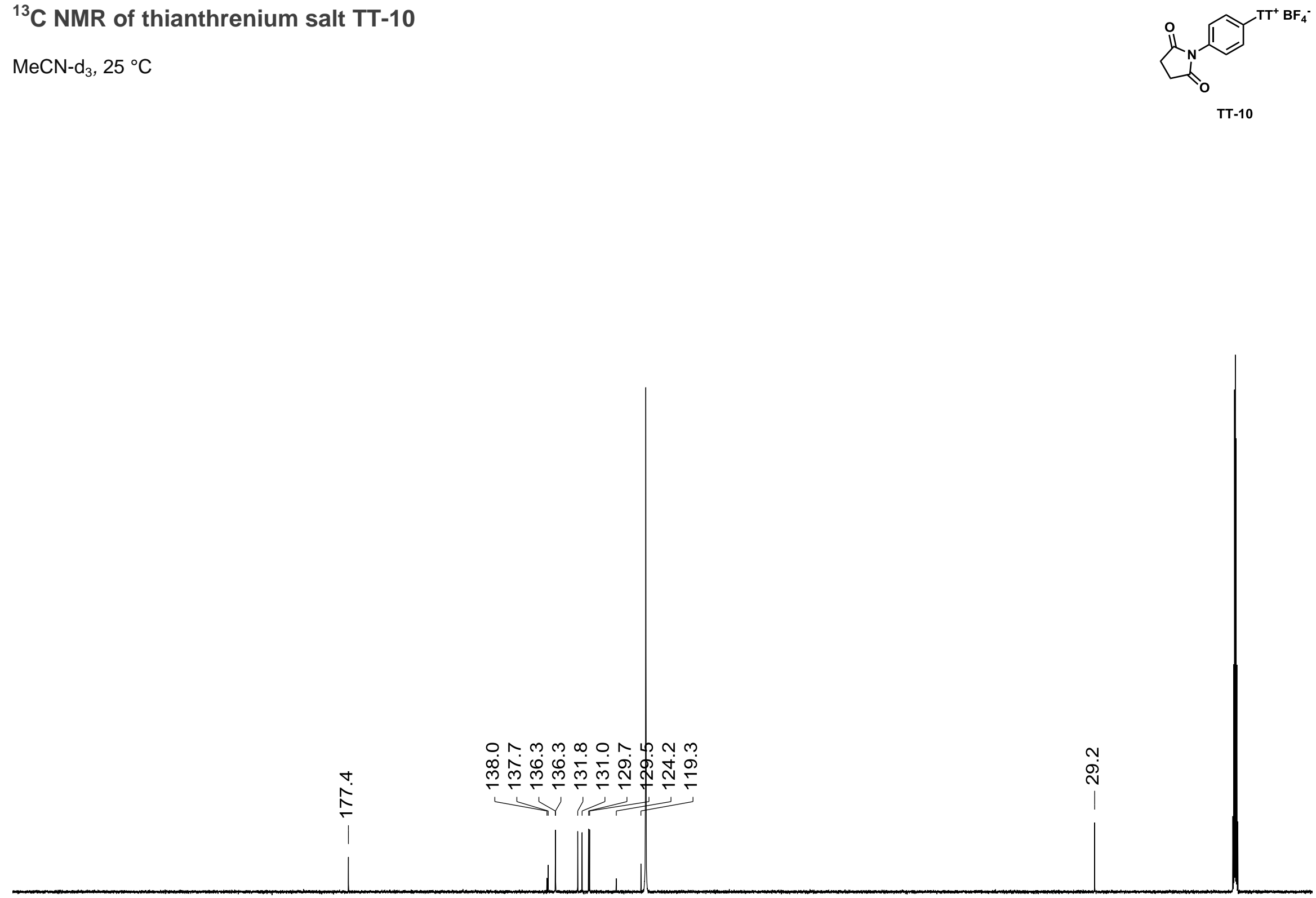

$\begin{array}{lllllllllllllllllllllllllllllll}240 & 230 & 220 & 210 & 200 & 190 & 180 & 170 & 160 & 150 & 140 & 130 & 120 & 110 & 100 & 90 & 80 & 70 & 60 & 50 & 40 & 30 & 20 & 10 & 0 & -10\end{array}$ 
${ }^{19} \mathrm{~F}$ NMR of thianthrenium salt TT-10

$\mathrm{MeCN}-\mathrm{d}_{3}, 25^{\circ} \mathrm{C}$

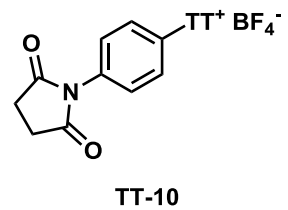

$\stackrel{1}{\frac{1}{5}} \frac{1}{5}$

\begin{tabular}{llllllllllllllllllllll}
\hline 10 & 0 & -10 & -20 & -30 & -40 & -50 & -60 & -70 & -80 & -90 & -100 & -110 & -120 & -130 & -140 & -150 & -160 & -170 & -180 & -190 & -200
\end{tabular}




\section{${ }^{1} \mathrm{H}$ NMR of pyrazole 10}

$\mathrm{CDCl}_{3}, 25^{\circ} \mathrm{C}$
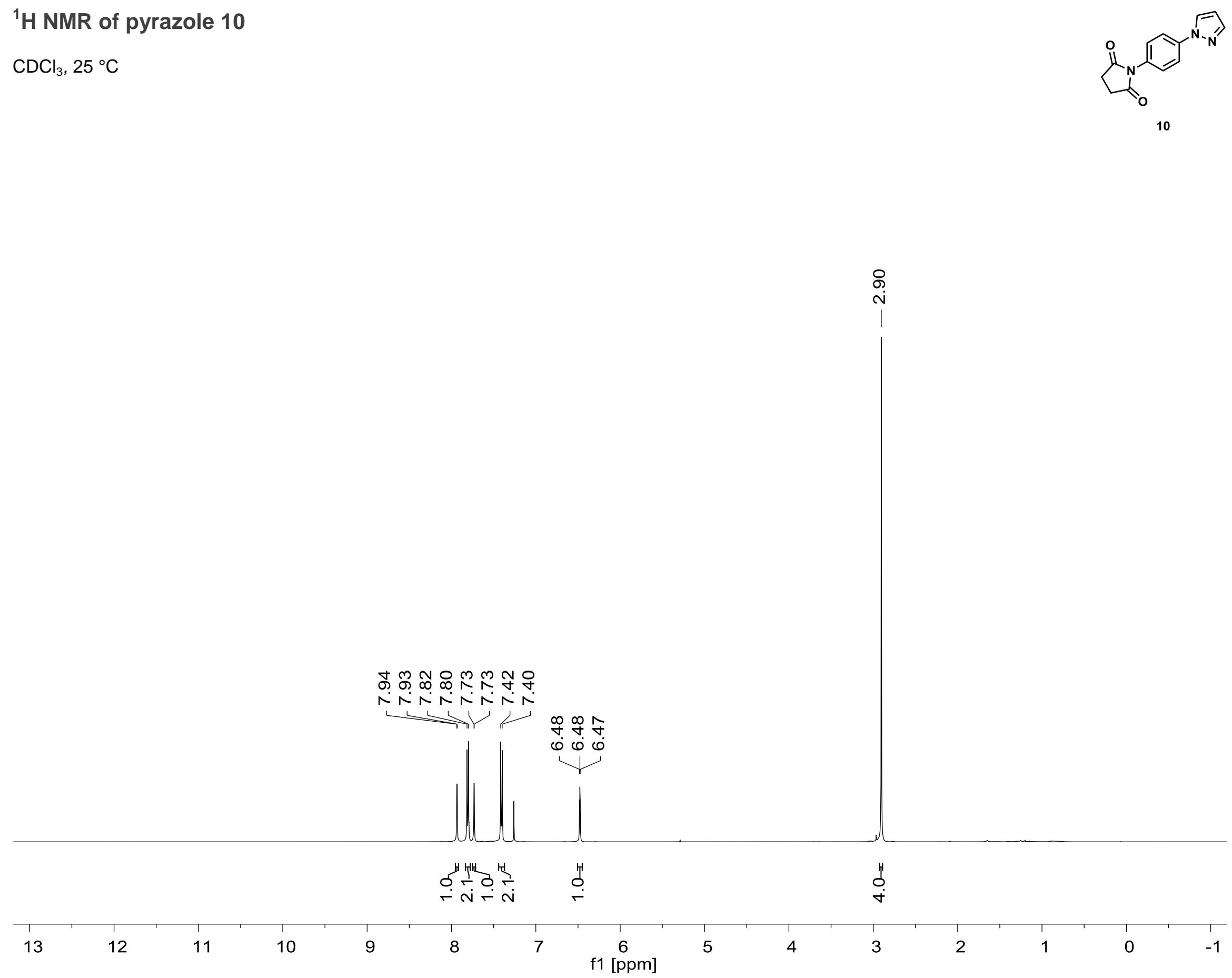


\section{${ }^{13} \mathrm{C}$ NMR of pyrazole 10}

$\mathrm{CDCl}_{3}, 25^{\circ} \mathrm{C}$

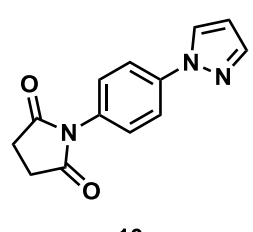

10

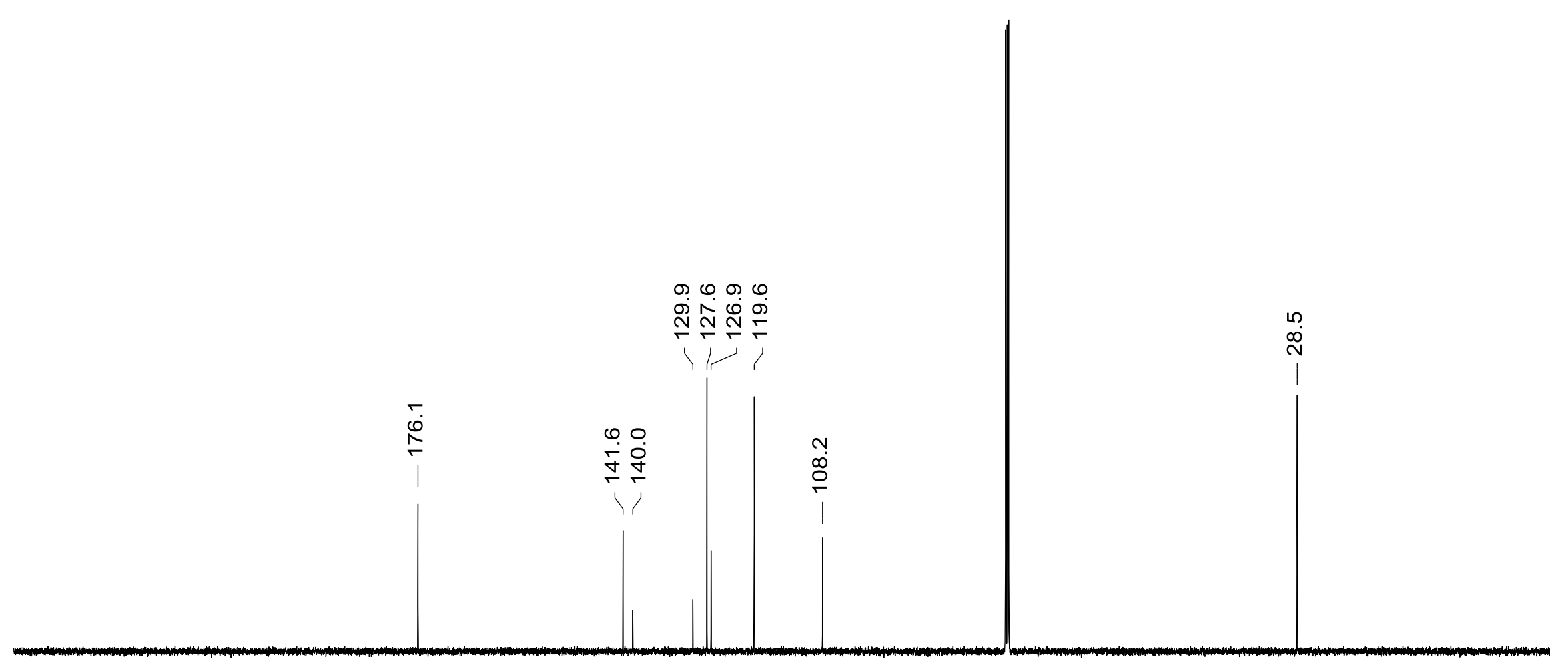

$\begin{array}{lllllllllllllllllllllllllllllll}240 & 230 & 220 & 210 & 200 & 190 & 180 & 170 & 160 & 150 & 140 & 130 & 120 & 110 & 100 & 90 & 80 & 70 & 60 & 50 & 40 & 30 & 20 & 10 & 0 & -10\end{array}$ f1 [ppm] 


\section{${ }^{1} \mathrm{H}$ NMR of thianthrenium salt TT-11}

$\mathrm{CD}_{2} \mathrm{Cl}_{2}, 25^{\circ} \mathrm{C}$

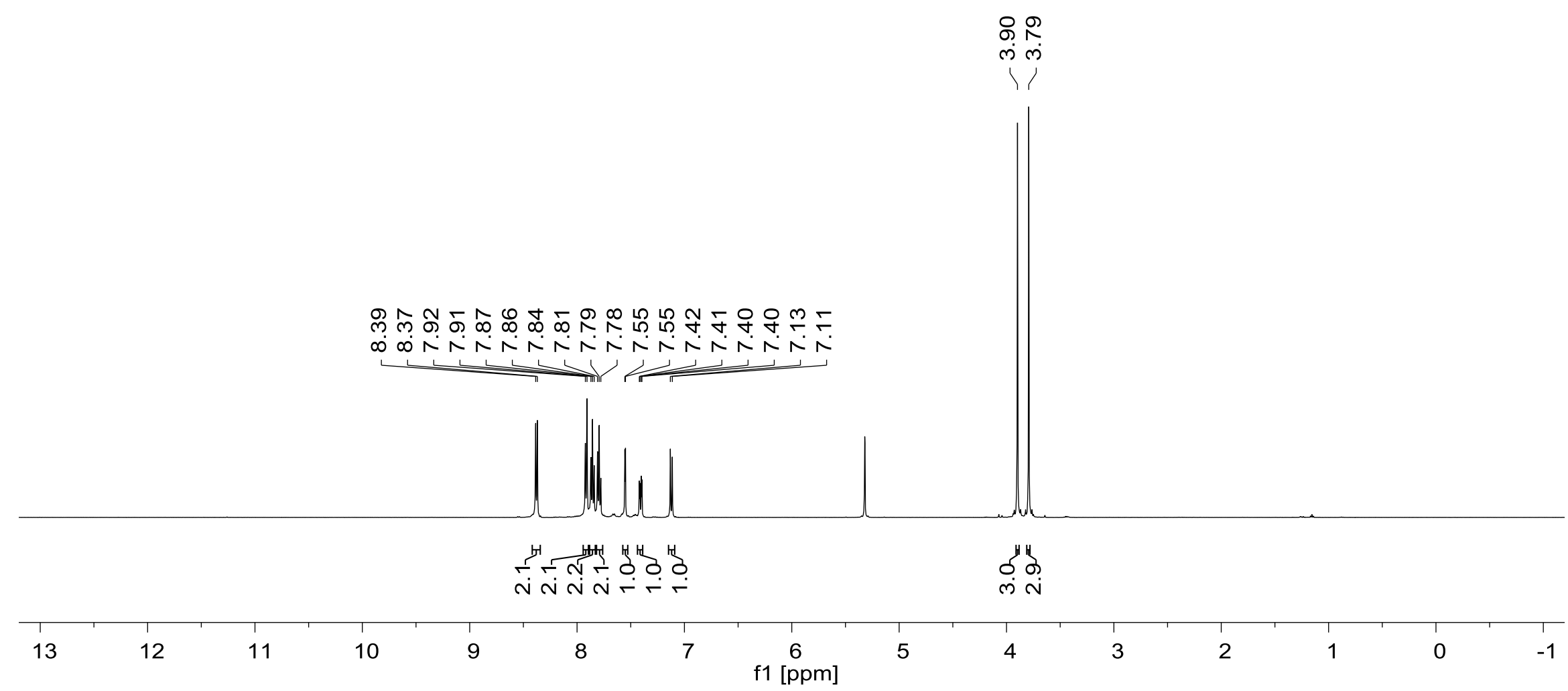




\section{${ }^{13} \mathrm{C}$ NMR of thianthrenium salt TT-11}

$\mathrm{CD}_{2} \mathrm{Cl}_{2}, 25^{\circ} \mathrm{C}$

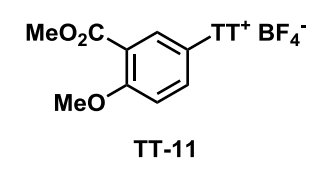

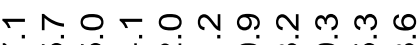

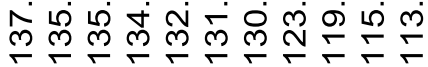

$\infty$ ?

車

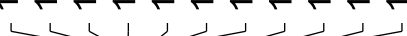

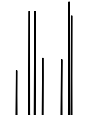


${ }^{19} \mathrm{~F}$ NMR of thianthrenium salt TT-11

$\mathrm{CD}_{2} \mathrm{Cl}_{2}, 25^{\circ} \mathrm{C}$

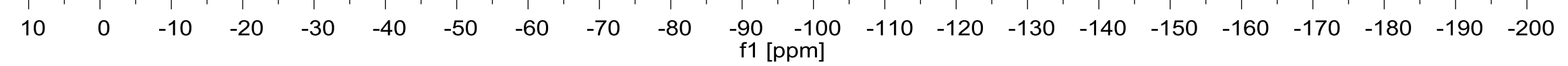


${ }^{1} \mathrm{H}$ NMR of triazole 11

$\mathrm{CDCl}_{3}, 25^{\circ} \mathrm{C}$
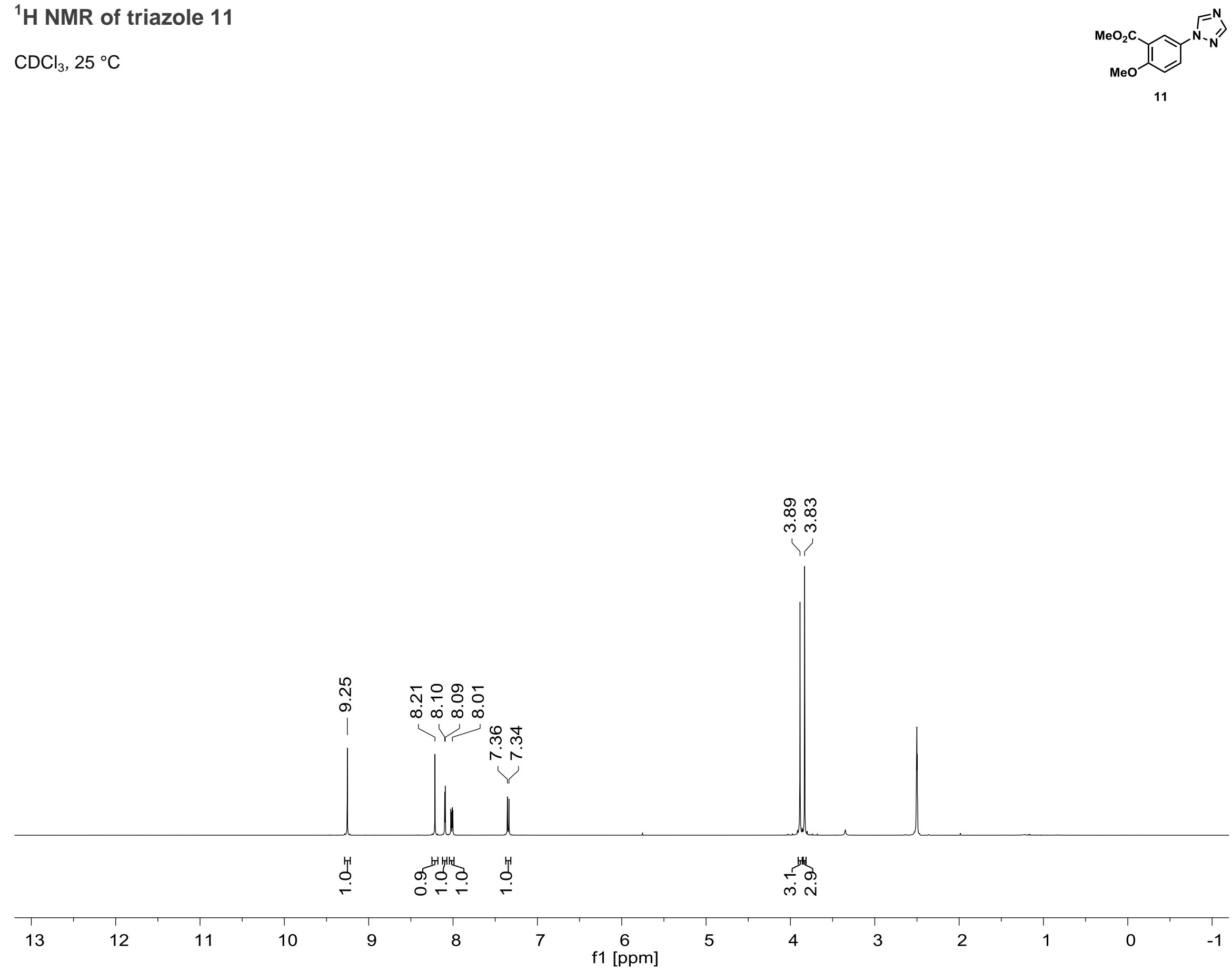


\section{${ }^{13} \mathrm{C}$ NMR of triazole 11}

$\mathrm{CDCl}_{3}, 25^{\circ} \mathrm{C}$
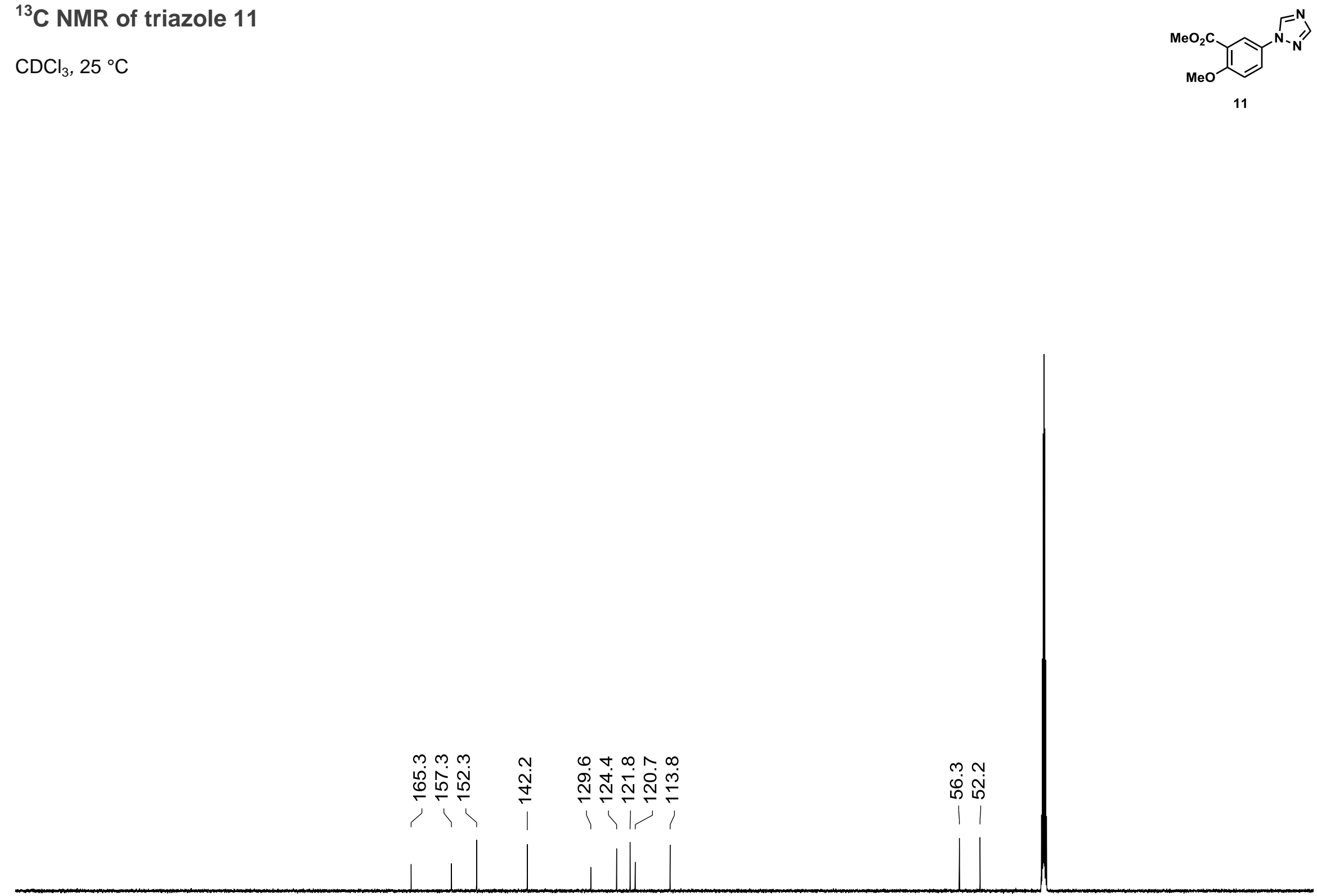

$\begin{array}{llllllllllllllllllllllllllll}240 & 230 & 220 & 210 & 200 & 190 & 180 & 170 & 160 & 150 & 140 & 130 & 120 & 110 & 100 & 90 & 80 & 70 & 60 & 50 & 40 & 30 & 20 & 10 & 0 & -10\end{array}$ f1 [ppm] 


\section{${ }^{1} \mathrm{H}$ NMR of thianthrenium salt TT-12}

$\mathrm{CDCl}_{3}, 25^{\circ} \mathrm{C}$

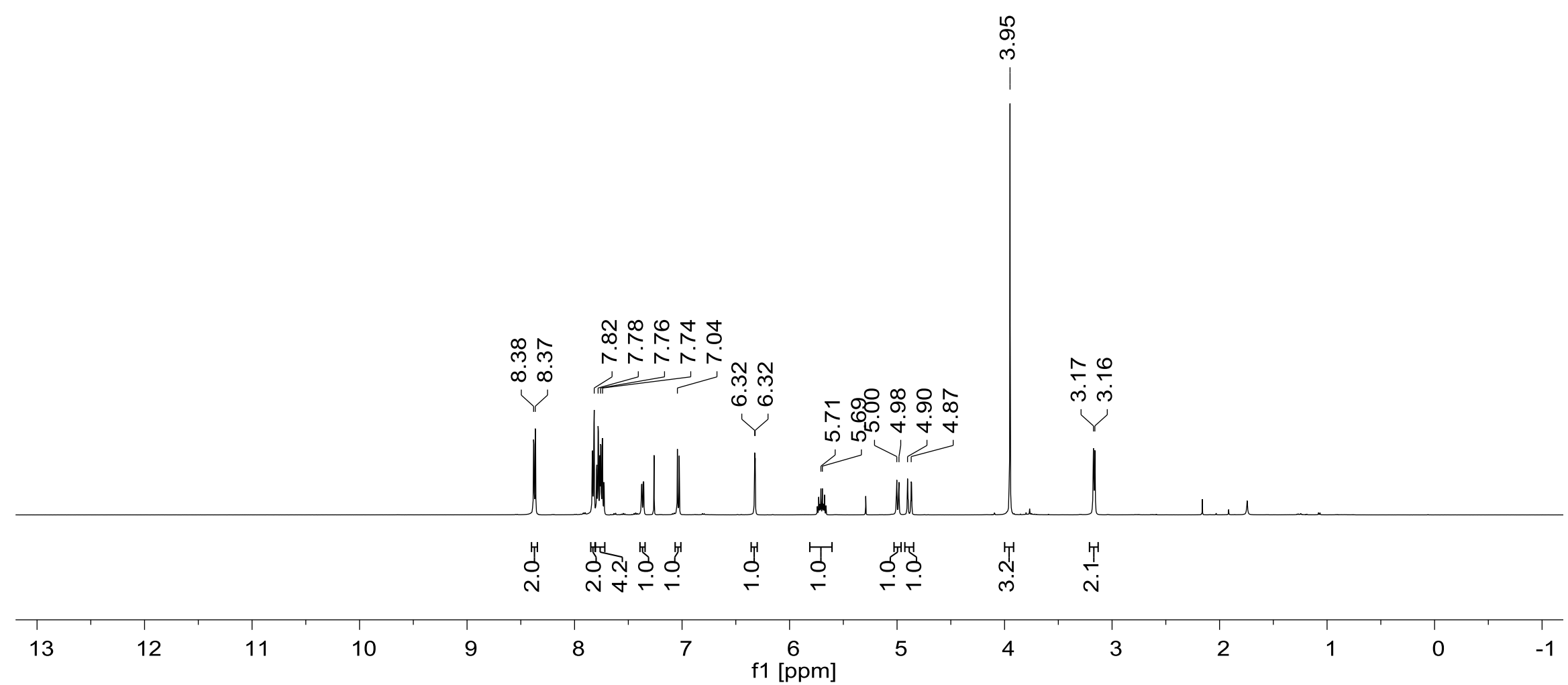




\section{${ }^{13} \mathrm{C}$ NMR of thianthrenium salt TT-12}

$\mathrm{CDCl}_{3}, 25^{\circ} \mathrm{C}$

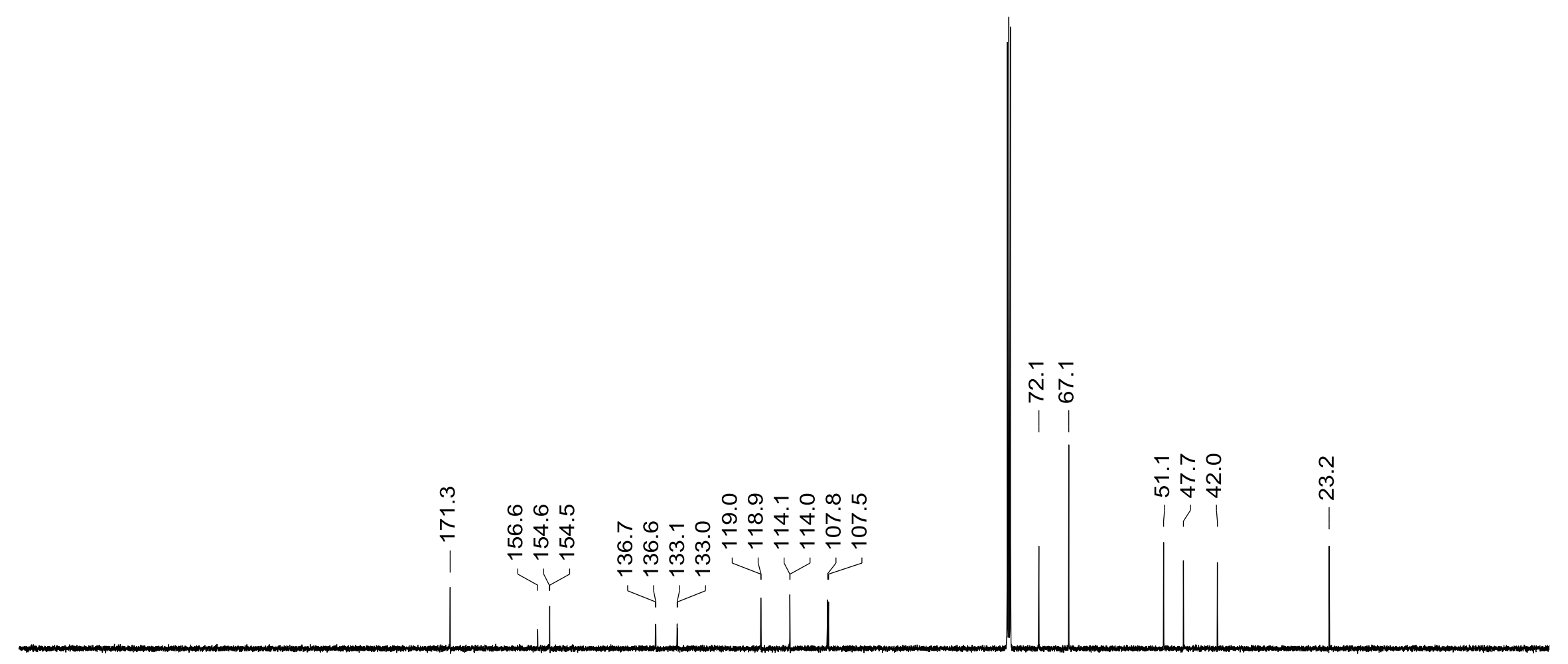


${ }^{19} \mathrm{~F}$ NMR of thianthrenium salt TT-12

$\mathrm{CDCl}_{3}, 25^{\circ} \mathrm{C}$

\begin{tabular}{lllllllllllllllllllllllllllll}
\hline 10 & 0 & -10 & -20 & -30 & -40 & -50 & -60 & -70 & -80 & -90 & -100 & -110 & -120 & -130 & -140 & -150 & -160 & -170 & -180 & -190 & -200
\end{tabular}




\section{${ }^{1} \mathrm{H}$ NMR of triazole 12}

$\mathrm{CDCl}_{3}, 25^{\circ} \mathrm{C}$

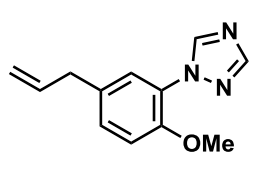

12

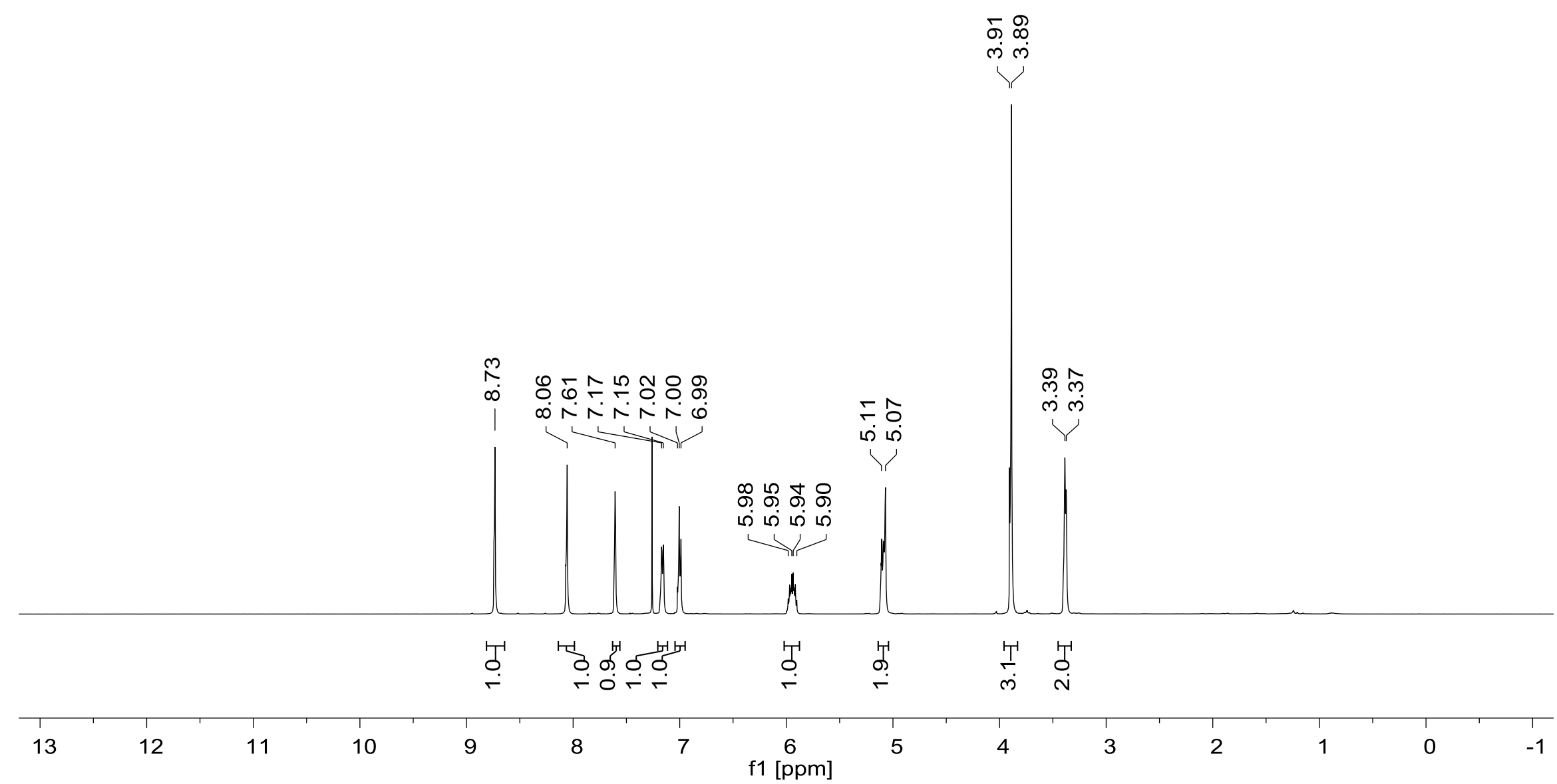




\section{${ }^{13} \mathrm{C}$ NMR of triazole 12}

$\mathrm{CDCl}_{3}, 25^{\circ} \mathrm{C}$

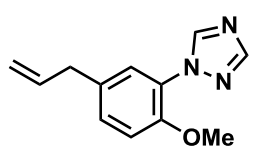

12

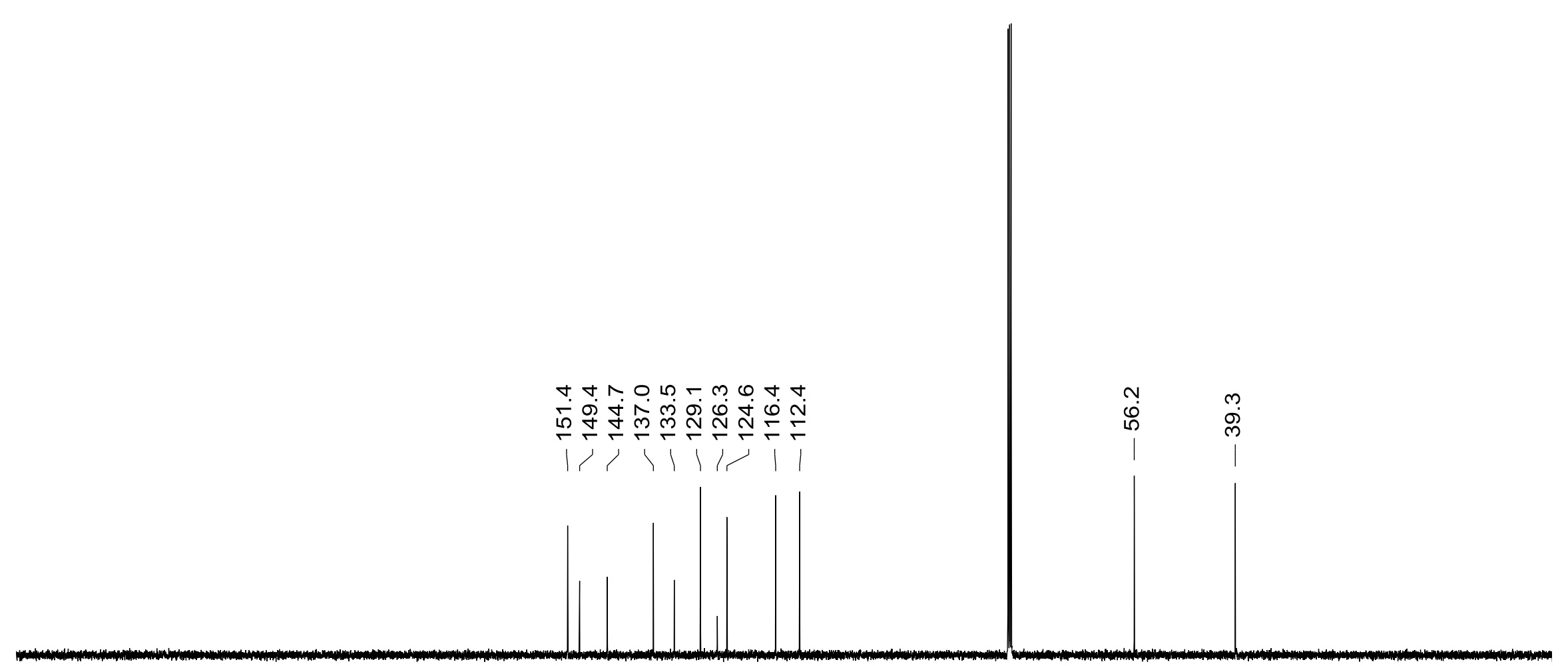

$\begin{array}{lllllllllllllllllllllllllllllllllllllll}240 & 230 & 220 & 210 & 200 & 190 & 180 & 170 & 160 & 150 & 140 & 130 & 120 & 110 & 100 & 90 & 80 & 70 & 60 & 50 & 40 & 30 & 20 & 10 & 0 & -10\end{array}$ f1 [ppm] 
${ }^{1} \mathrm{H}$ NMR of thianthrenium salt TT-13

$\mathrm{CDCl}_{3}, 25^{\circ} \mathrm{C}$
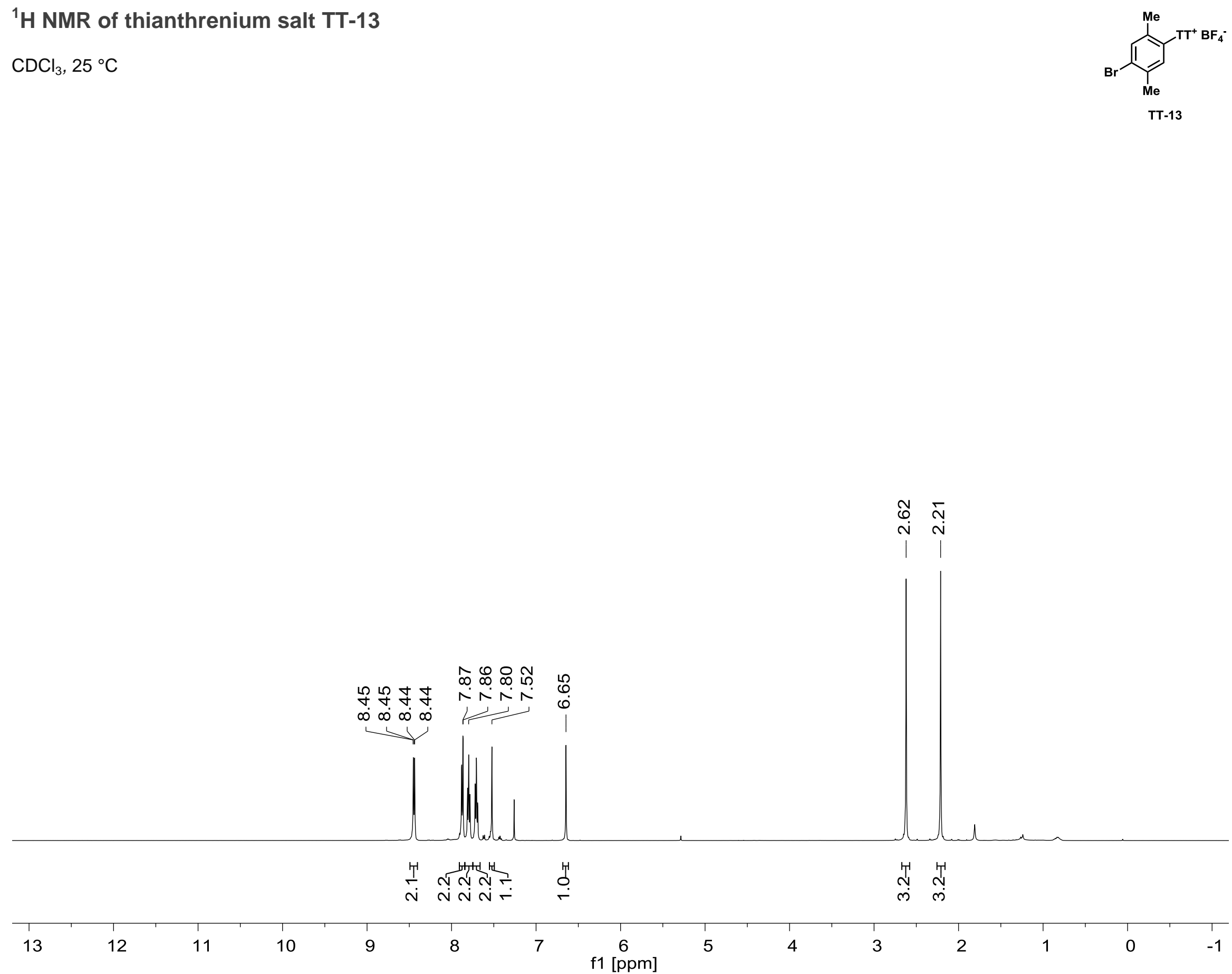
${ }^{13} \mathrm{C}$ NMR of thianthrenium salt TT-13

$\mathrm{CDCl}_{3}, 25^{\circ} \mathrm{C}$

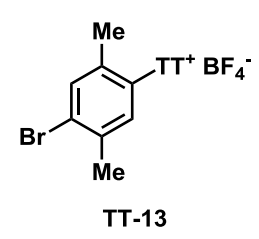

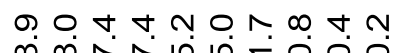

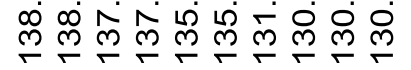

U

$\stackrel{\infty}{\circ \infty}$

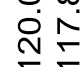

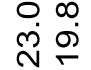

11

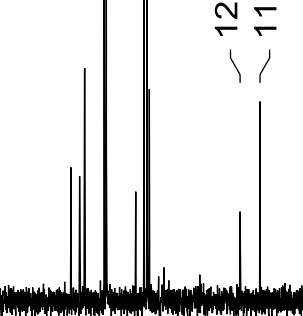


${ }^{19} \mathrm{~F}$ NMR of thianthrenium salt TT-13

$\mathrm{CDCl}_{3}, 25^{\circ} \mathrm{C}$

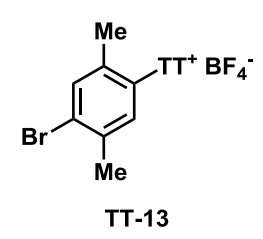

은 $\frac{1}{5}$

$\frac{1}{1}$

\begin{tabular}{lllllllllllllllllllllll}
\hline 10 & 0 & -10 & -20 & -30 & -40 & -50 & -60 & -70 & -80 & -90 & -100 & -110 & -120 & -130 & -140 & -150 & -160 & -170 & -180 & -190 & -200
\end{tabular}




\section{${ }^{1} \mathrm{H}$ NMR of triazole 13}

$\mathrm{CDCl}_{3}, 25^{\circ} \mathrm{C}$
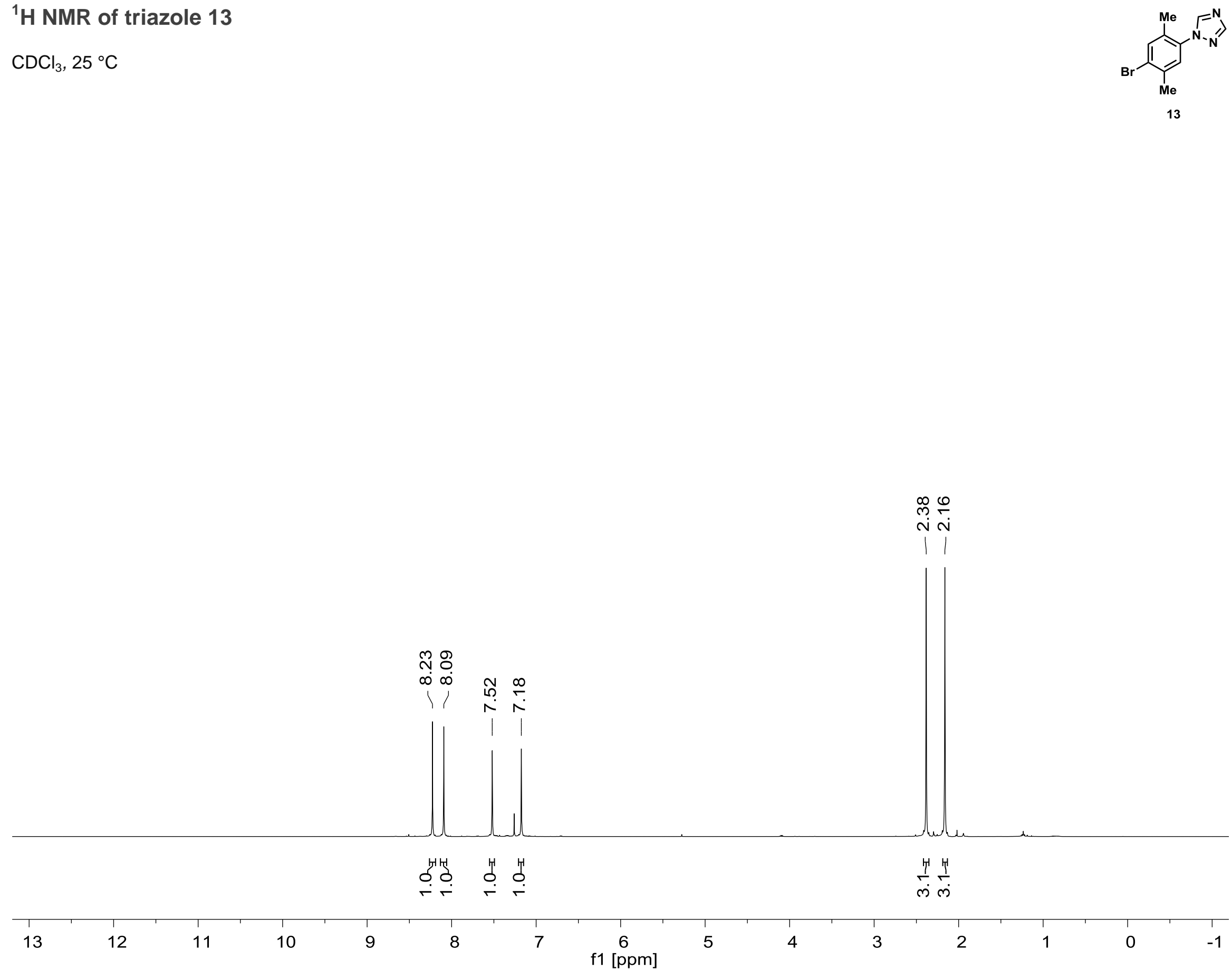


\section{${ }^{13} \mathrm{C}$ NMR of triazole 13}

$\mathrm{CDCl}_{3}, 25^{\circ} \mathrm{C}$

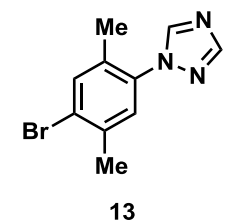

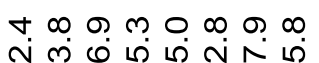

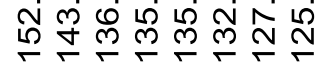
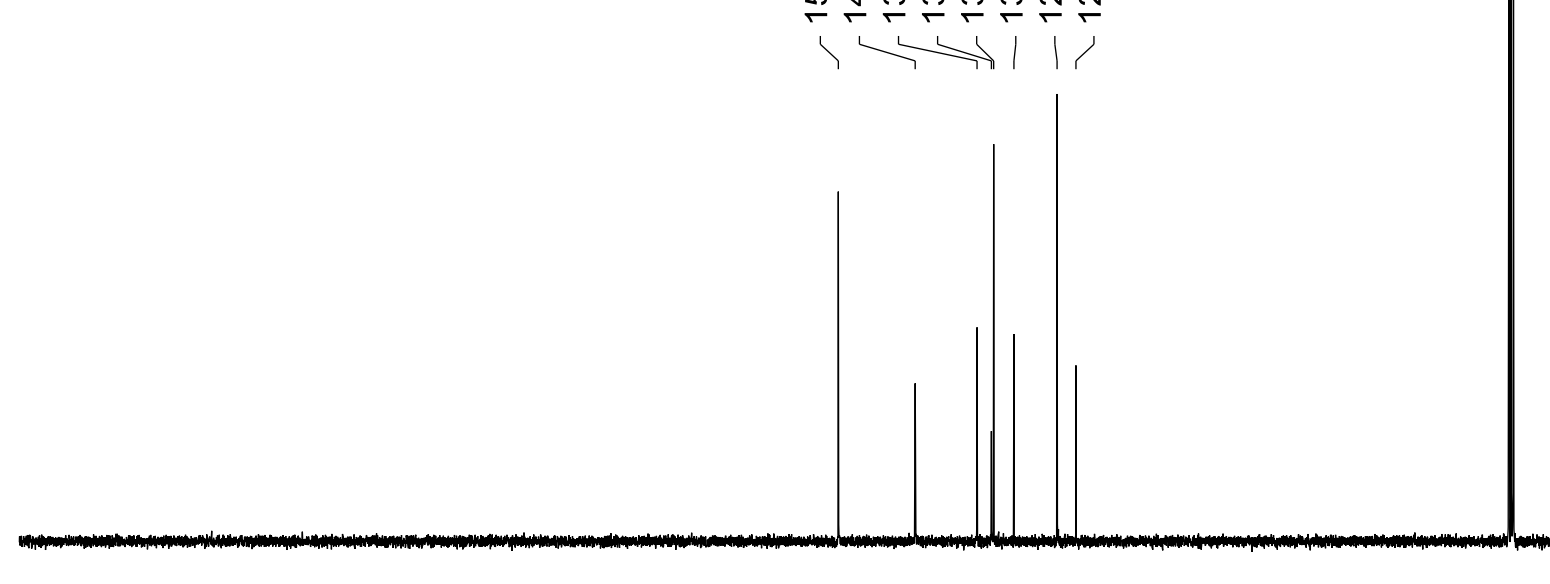

+

กั่ 


\section{${ }^{1} \mathrm{H}$ NMR of thianthrenium salt TT-14}

DMSO- $\mathrm{d}_{6}, 25^{\circ} \mathrm{C}$

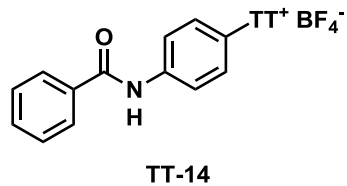

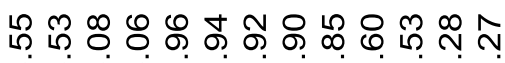

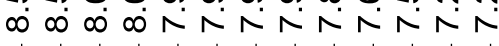

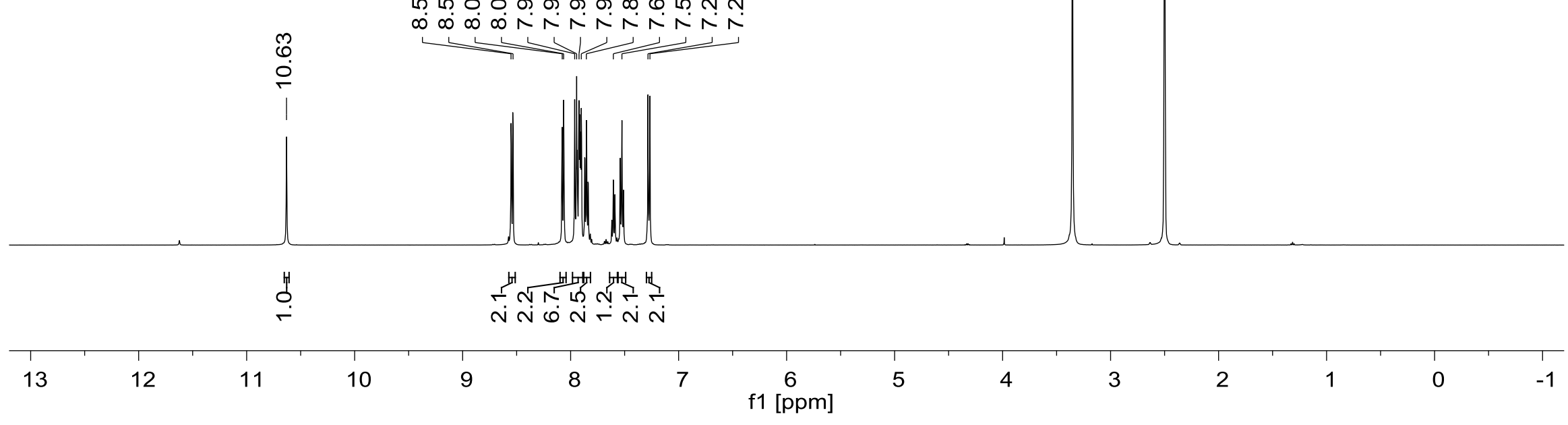




\section{${ }^{13} \mathrm{C}$ NMR of thianthrenium salt TT-14}

$\mathrm{CDCl}_{3}, 25^{\circ} \mathrm{C}$

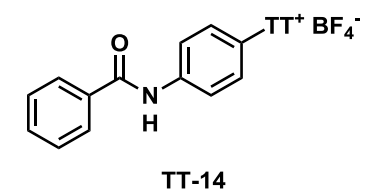

ก ก

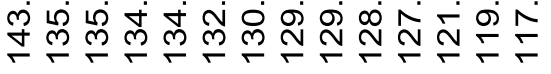

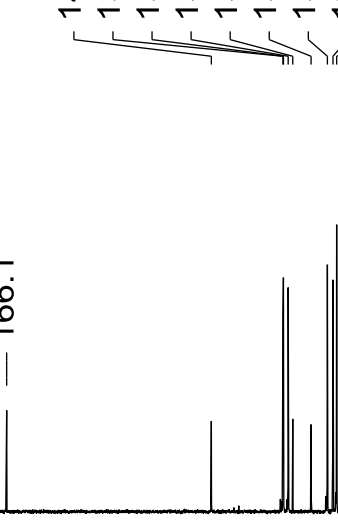


${ }^{19} \mathrm{~F}$ NMR of thianthrenium salt TT-14

DMSO- $d_{6}, 25^{\circ} \mathrm{C}$

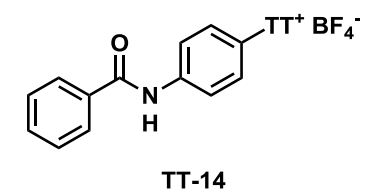

10

$\stackrel{\infty}{\sim} \underset{\sim}{\stackrel{\infty}{+}}$

is

$\begin{array}{lllllllllllllllllllllll}10 & 0 & -10 & -20 & -30 & -40 & -50 & -60 & -70 & -80 & -90 & -100 & -110 & -120 & -130 & -140 & -150 & -160 & -170 & -180 & -190 & -200\end{array}$




\section{${ }^{1} \mathrm{H}$ NMR of triazole 14}

DMSO- $\mathrm{d}_{6}, 25^{\circ} \mathrm{C}$
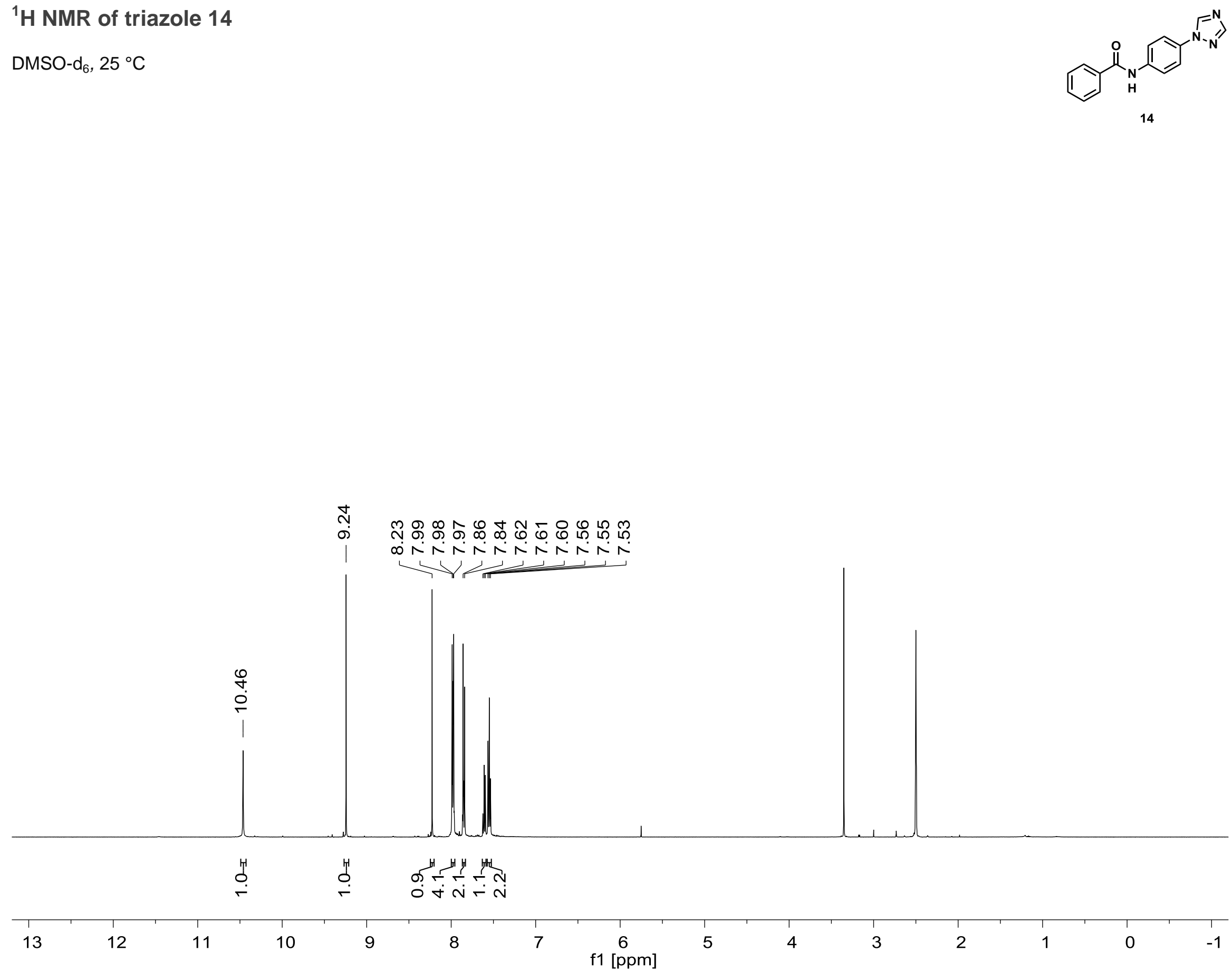


\section{${ }^{13} \mathrm{C}$ NMR of triazole 14}

DMSO-d $6,25^{\circ} \mathrm{C}$
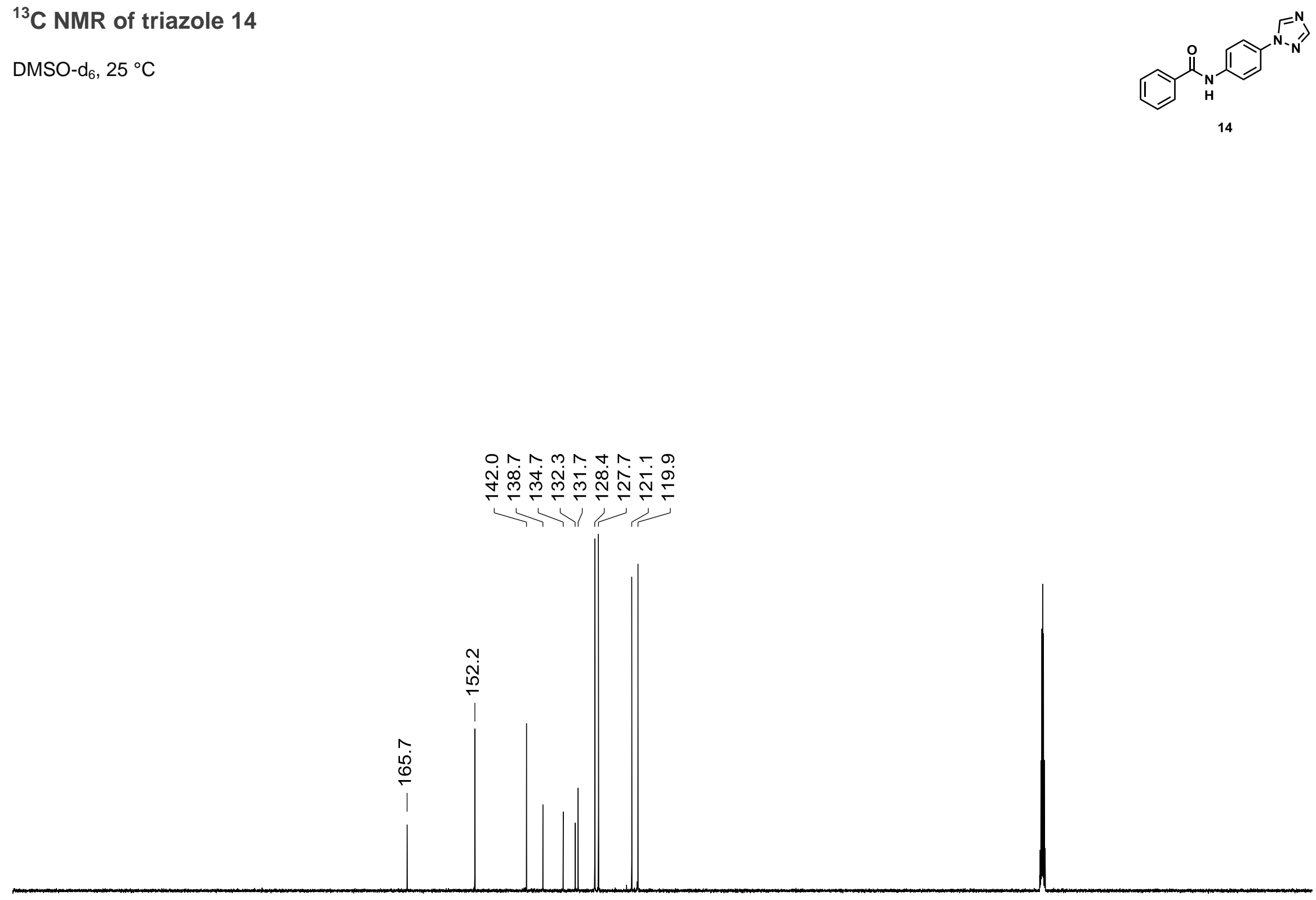

$\begin{array}{lllllllllllllllllllllllllllllllll}240 & 230 & 220 & 210 & 200 & 190 & 180 & 170 & 160 & 150 & 140 & 130 & 120 & 110 & 100 & 90 & 80 & 70 & 60 & 50 & 40 & 30 & 20 & 10 & 0 & -10\end{array}$ f1 [ppm] 


\section{${ }^{1} \mathrm{H}$ NMR of thianthrenium salt TT-15}

$\mathrm{MeCN}-\mathrm{d}_{3}, 25^{\circ} \mathrm{C}$

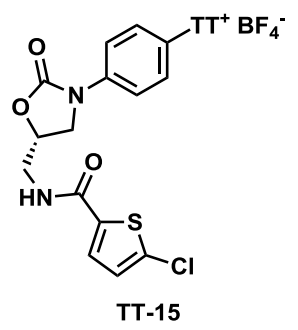

లా లె

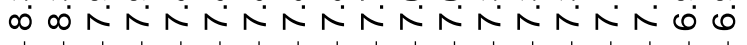

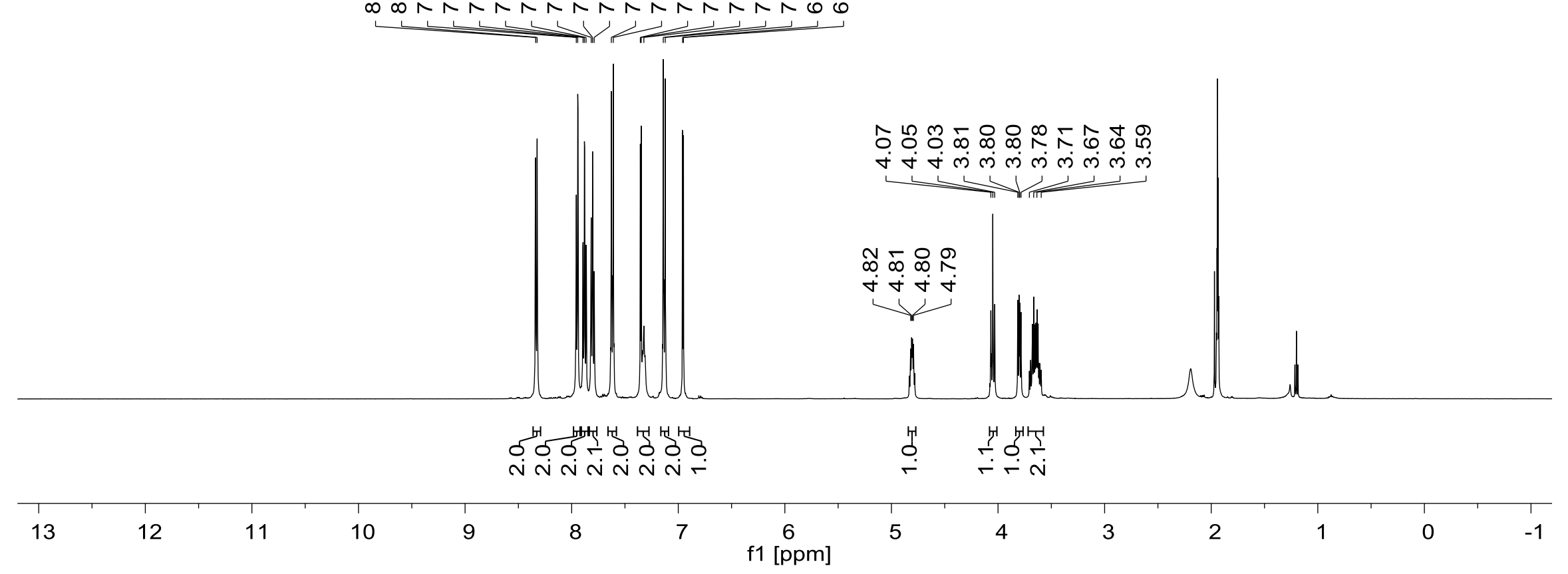




\section{${ }^{13} \mathrm{C}$ NMR of thianthrenium salt TT-15}

$\mathrm{MeCN}-\mathrm{d}_{3}, 25^{\circ} \mathrm{C}$
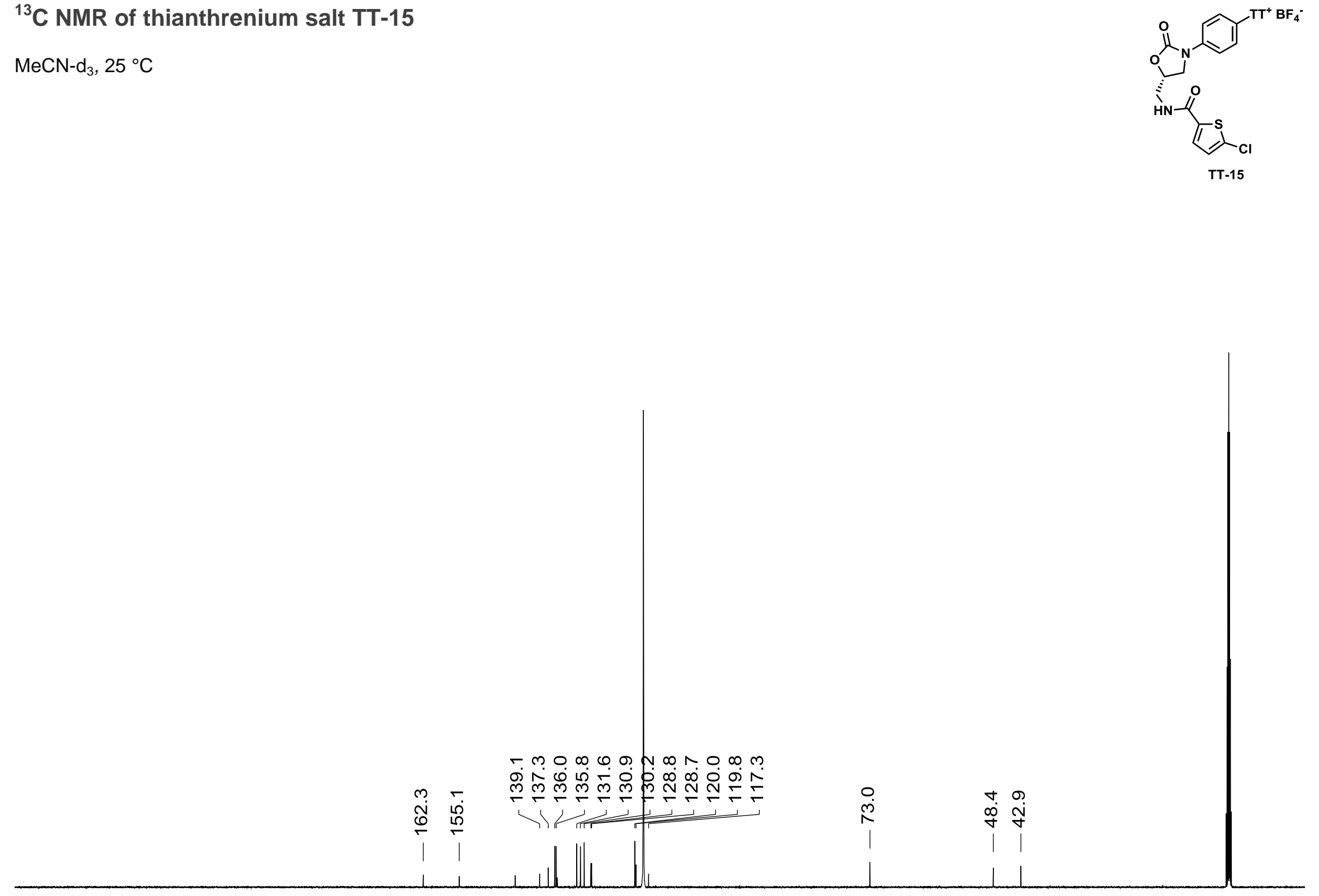

$\begin{array}{lllllllllllllllllllllllllllllllll}240 & 230 & 220 & 210 & 200 & 190 & 180 & 170 & 160 & 150 & 140 & 130 & 120 & 110 & 100 & 90 & 80 & 70 & 60 & 50 & 40 & 30 & 20 & 10 & 0 & -10\end{array}$ 
${ }^{19} \mathrm{~F}$ NMR of thianthrenium salt TT-15

$\mathrm{MeCN}-\mathrm{d}_{3}, 25^{\circ} \mathrm{C}$

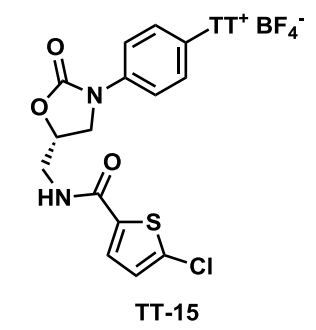

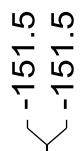

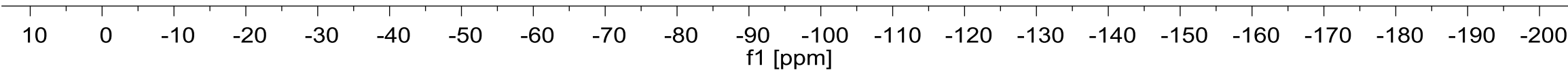




\section{${ }^{1} \mathrm{H}$ NMR of triazole 15}

DMSO-d $6,25^{\circ} \mathrm{C}$
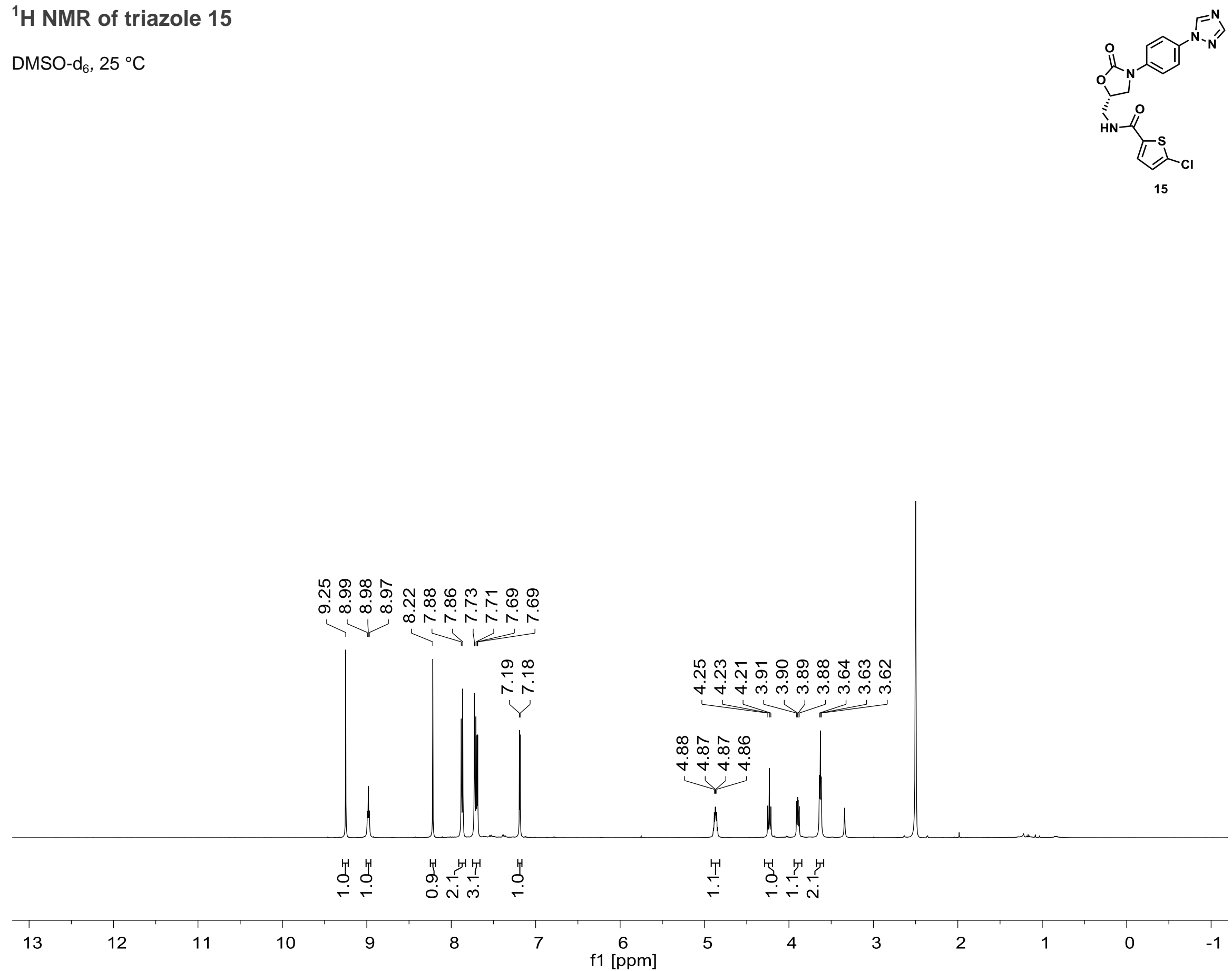


\section{${ }^{13} \mathrm{C}$ NMR of triazole 15}

DMSO- $\mathrm{d}_{6}, 25^{\circ} \mathrm{C}$

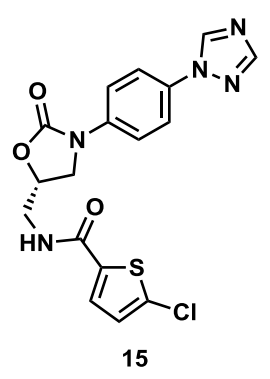

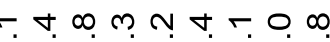

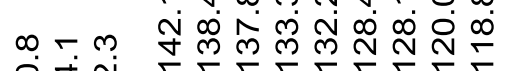

o

$\div$
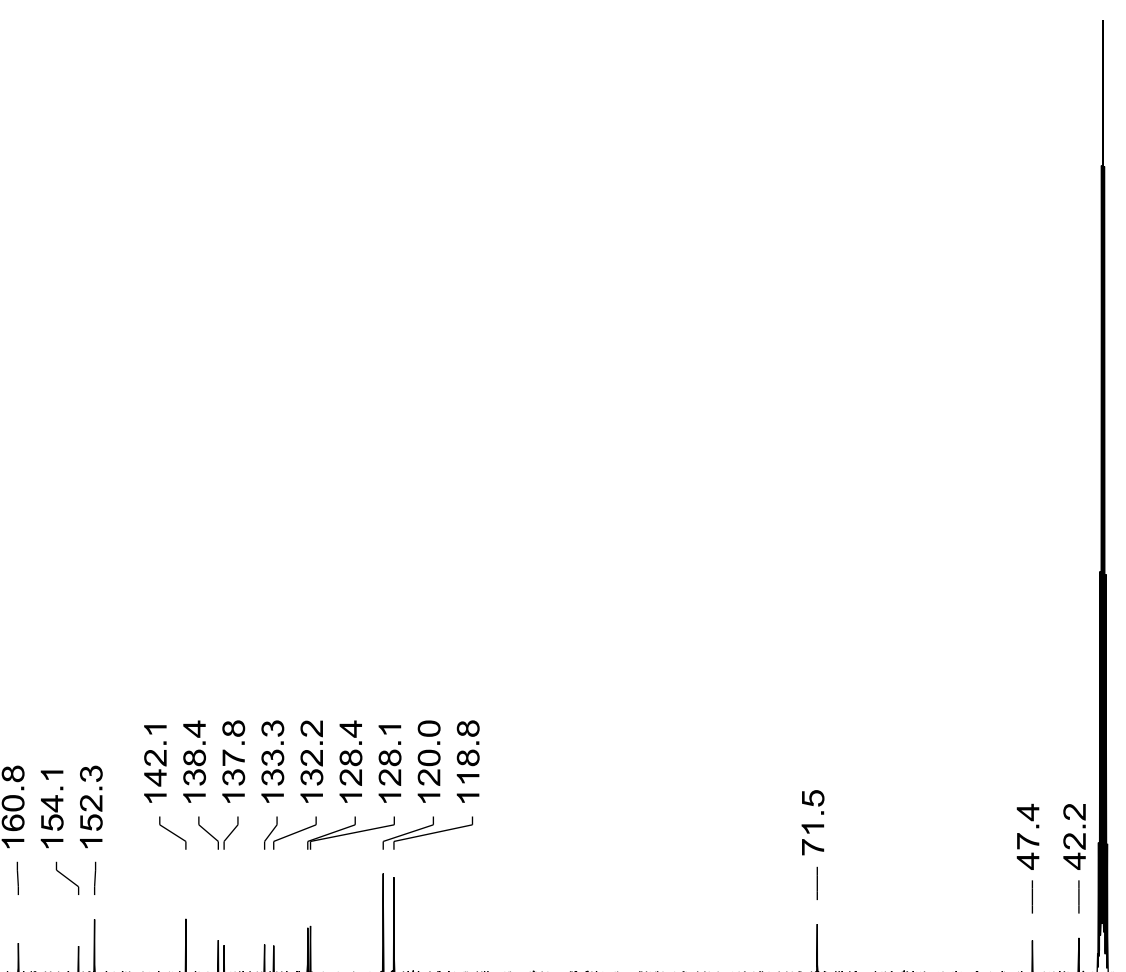

$\begin{array}{llllllllllllllllllllllllllllllll}240 & 230 & 220 & 210 & 200 & 190 & 180 & 170 & 160 & 150 & 140 & 130 & 120 & 110 & 100 & 90 & 80 & 70 & 60 & 50 & 40 & 30 & 20 & 10 & 0 & -10\end{array}$ f1 [ppm] 


\section{${ }^{1} \mathrm{H}$ NMR of thianthrenium salt TT-16}

$\mathrm{CDCl}_{3}, 25^{\circ} \mathrm{C}$
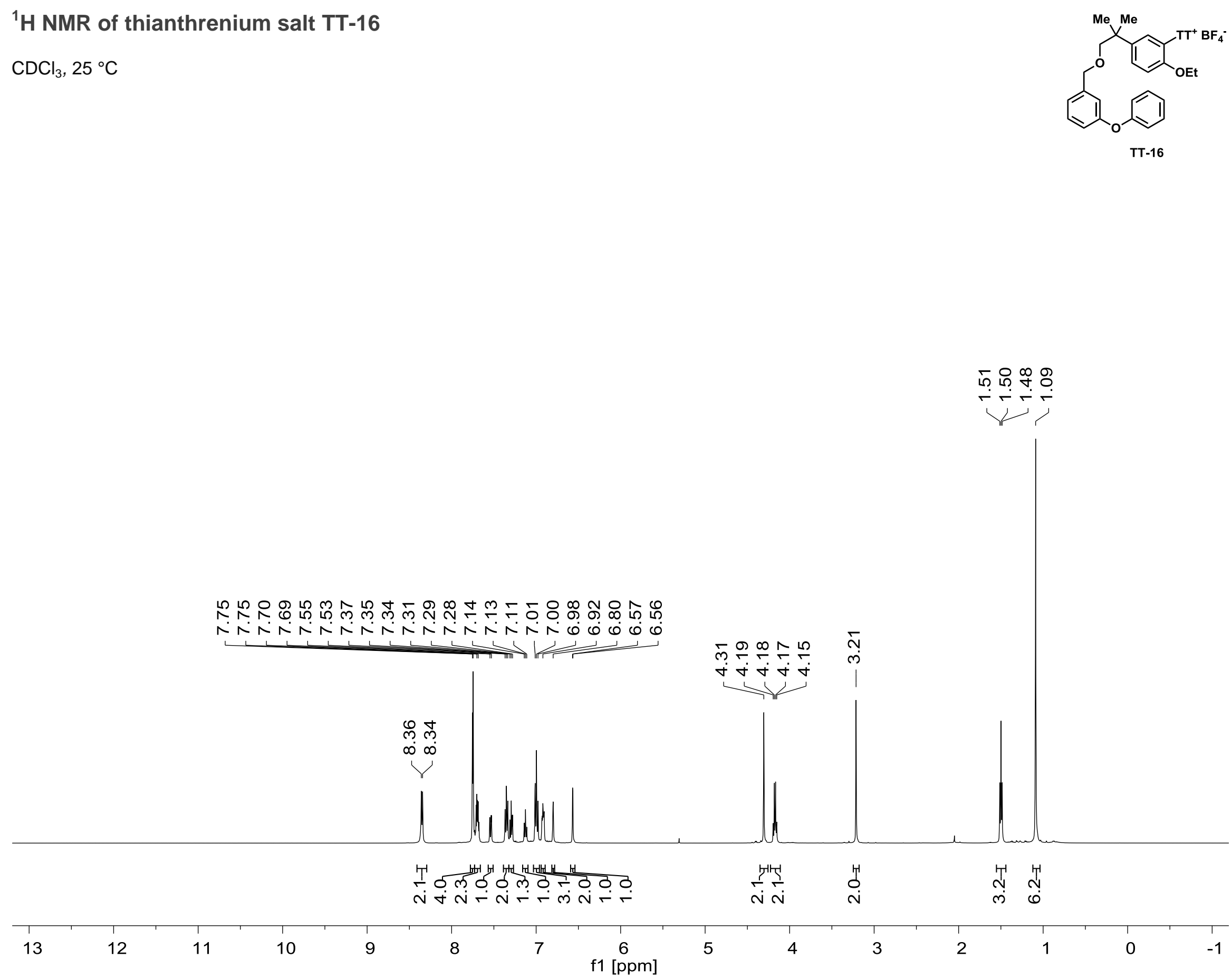
${ }^{13} \mathrm{C}$ NMR of thianthrenium salt TT-16

$\mathrm{CDCl}_{3}, 25^{\circ} \mathrm{C}$

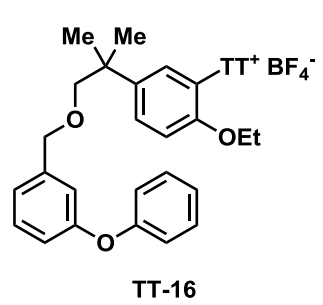

ก

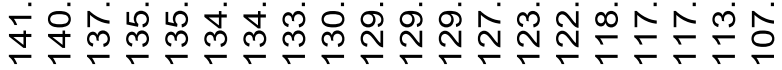
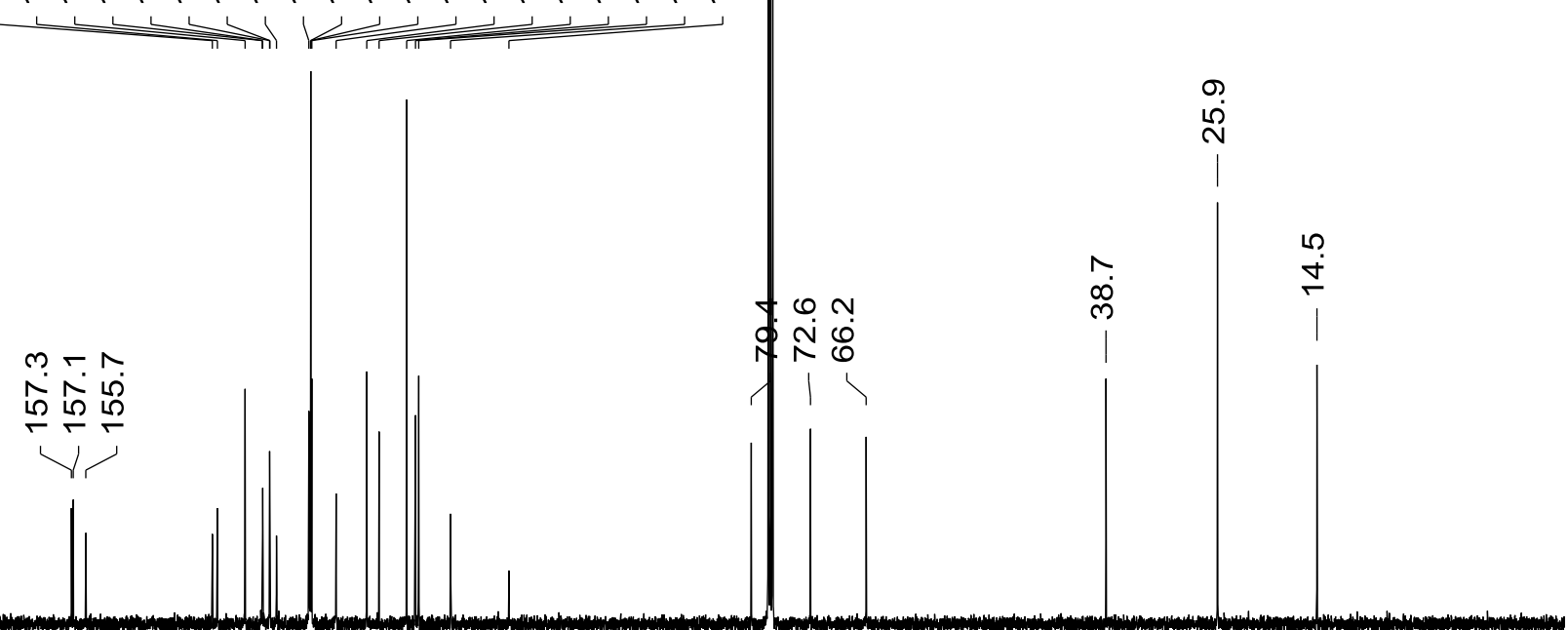

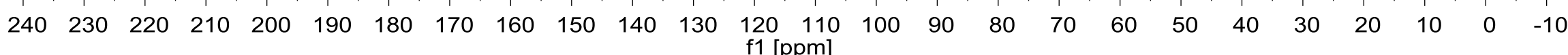


${ }^{19} \mathrm{~F}$ NMR of thianthrenium salt TT-16

$\mathrm{CDCl}_{3}, 25^{\circ} \mathrm{C}$

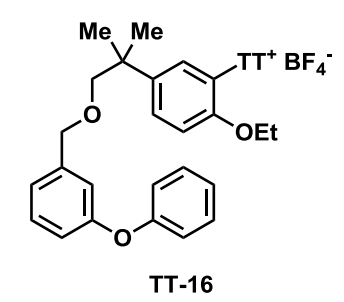

$\ln 6$

กิ่ กิ่ 


\section{${ }^{1} \mathrm{H}$ NMR of triazole 16}

$\mathrm{CDCl}_{3}, 25^{\circ} \mathrm{C}$

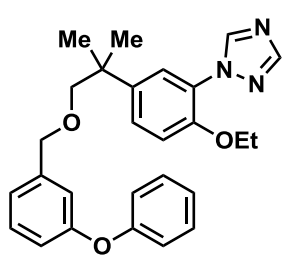

16

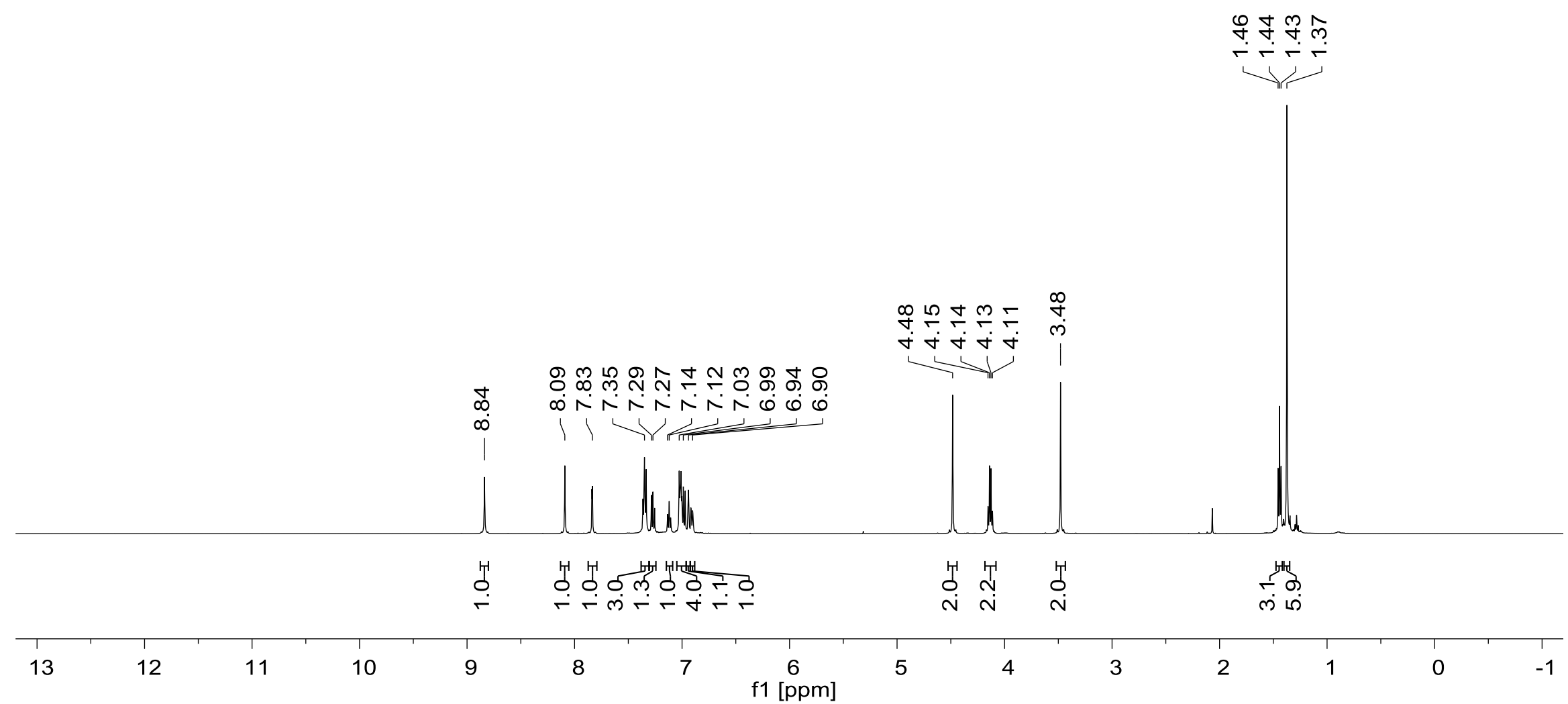




\section{${ }^{13} \mathrm{C}$ NMR of triazole 16}

$\mathrm{CDCl}_{3}, 25^{\circ} \mathrm{C}$

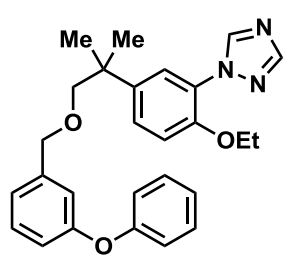

16
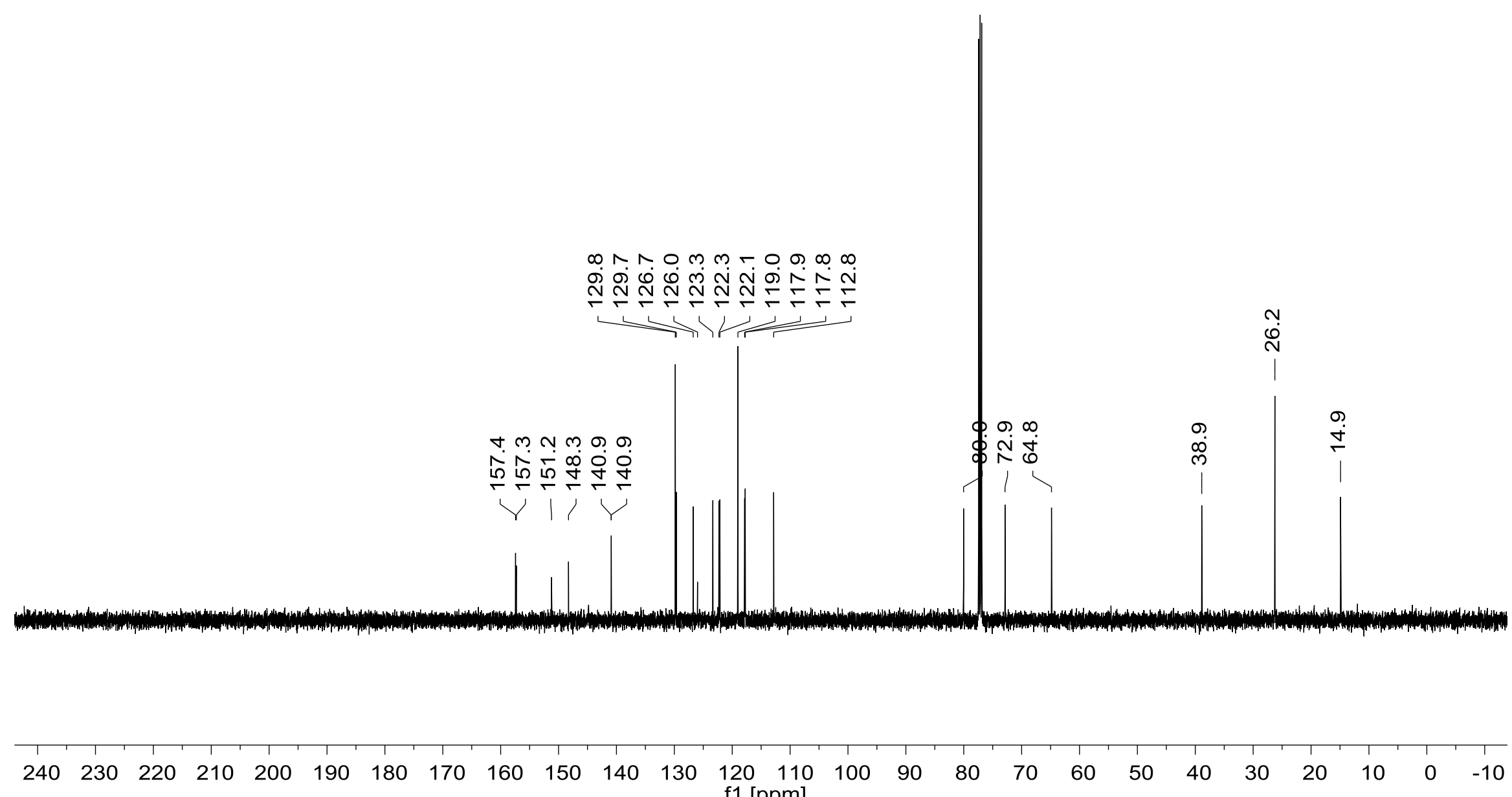


\section{${ }^{1} \mathrm{H}$ NMR of triazole 17}

$\mathrm{CDCl}_{3}, 25^{\circ} \mathrm{C}$

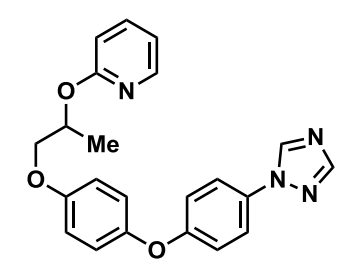

17

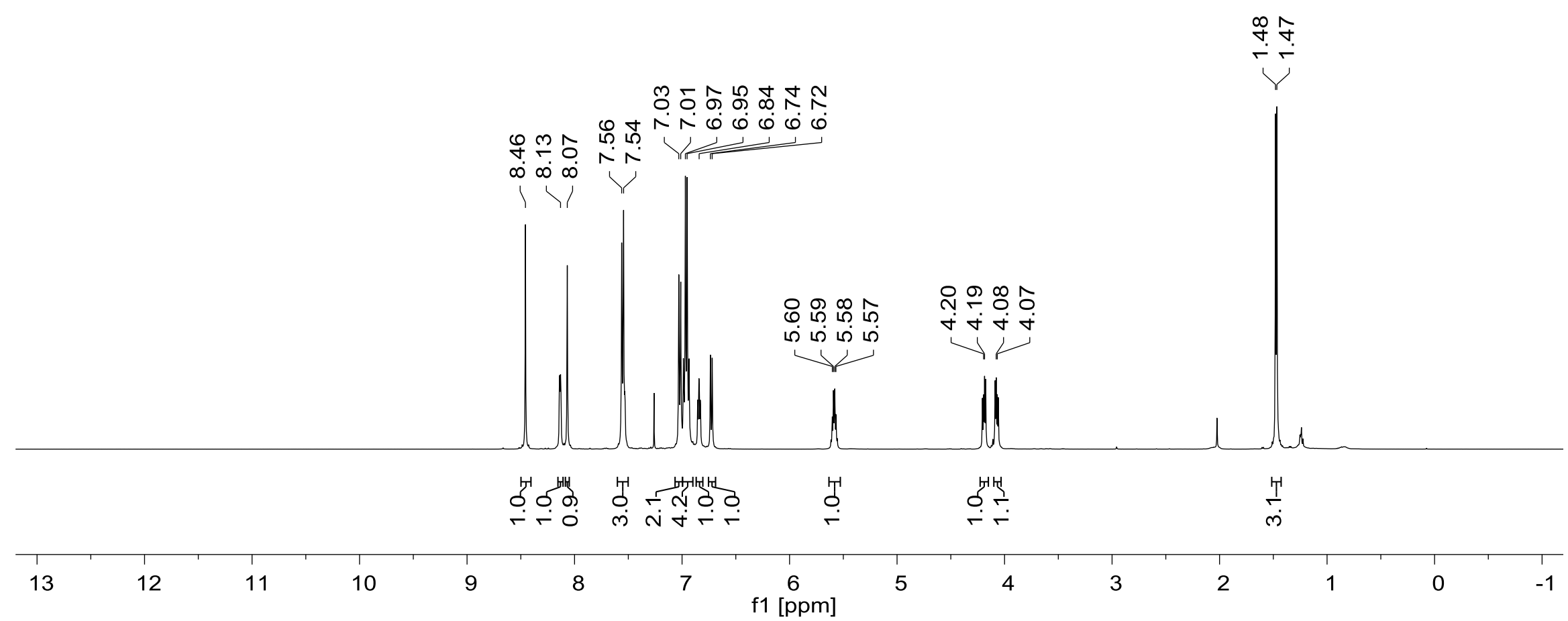




\section{${ }^{13} \mathrm{C}$ NMR of triazole 17}

$\mathrm{CDCl}_{3}, 25^{\circ} \mathrm{C}$

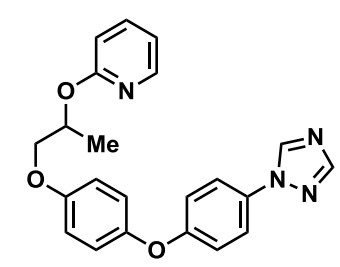

17
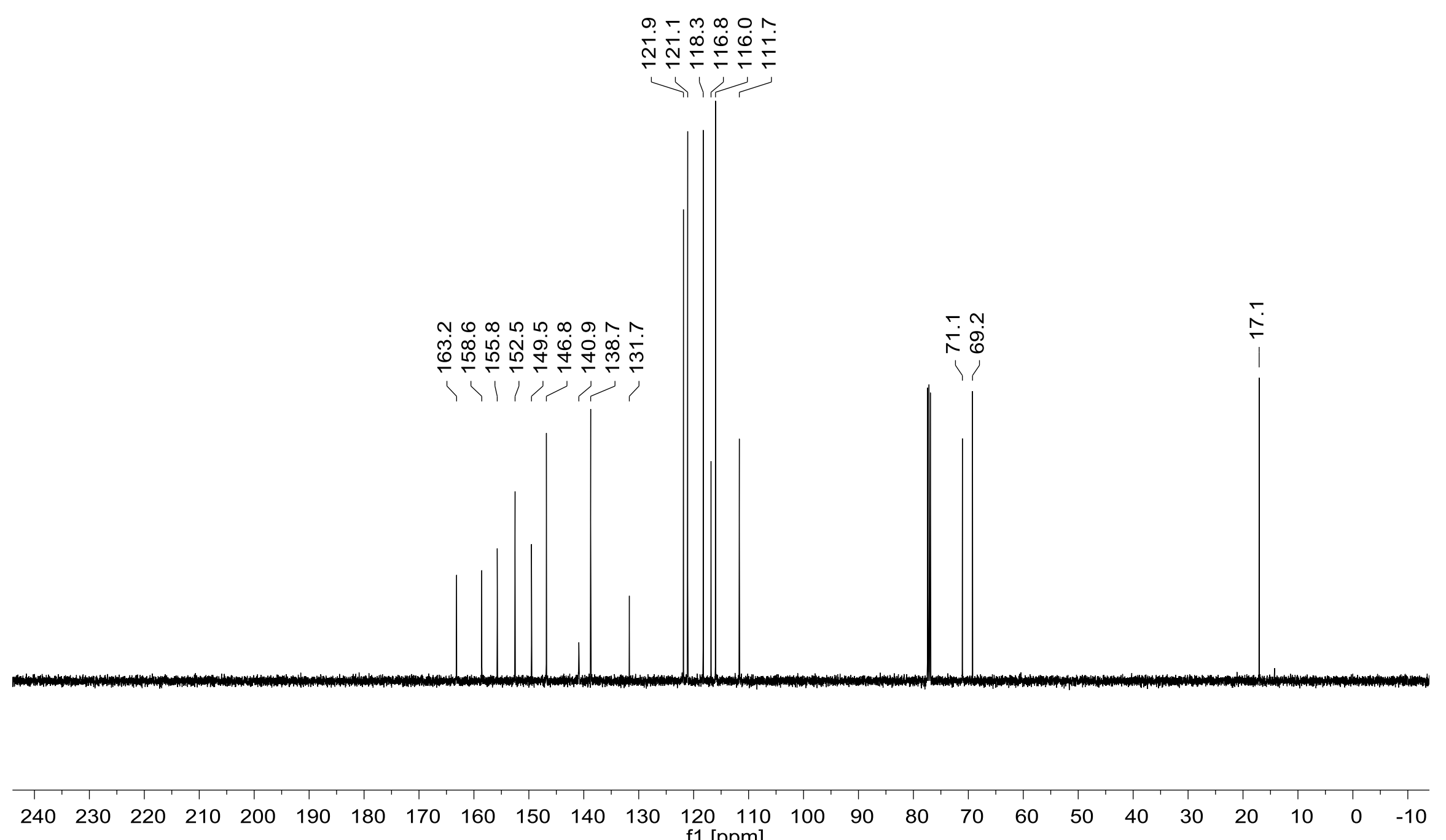


\section{${ }^{1} \mathrm{H}$ NMR of tetrafluorothianthrenium salt TFT-18}

$\mathrm{MeCN}-\mathrm{d}_{3}, 25^{\circ} \mathrm{C}$

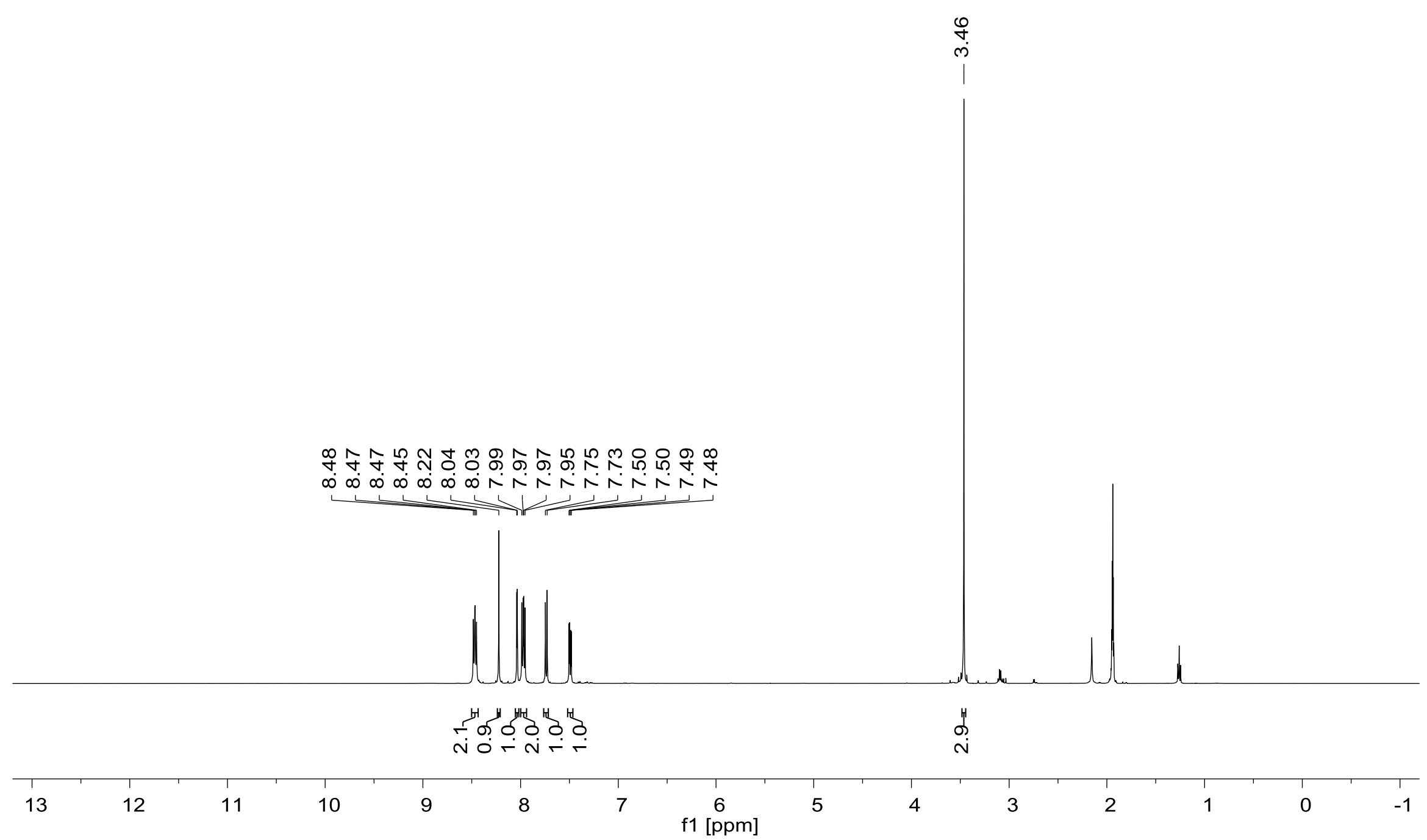




\section{${ }^{13} \mathrm{C}$ NMR of tetrafluorothianthrenium salt TFT-18}

$\mathrm{MeCN}-\mathrm{d}_{3}, 25^{\circ} \mathrm{C}$

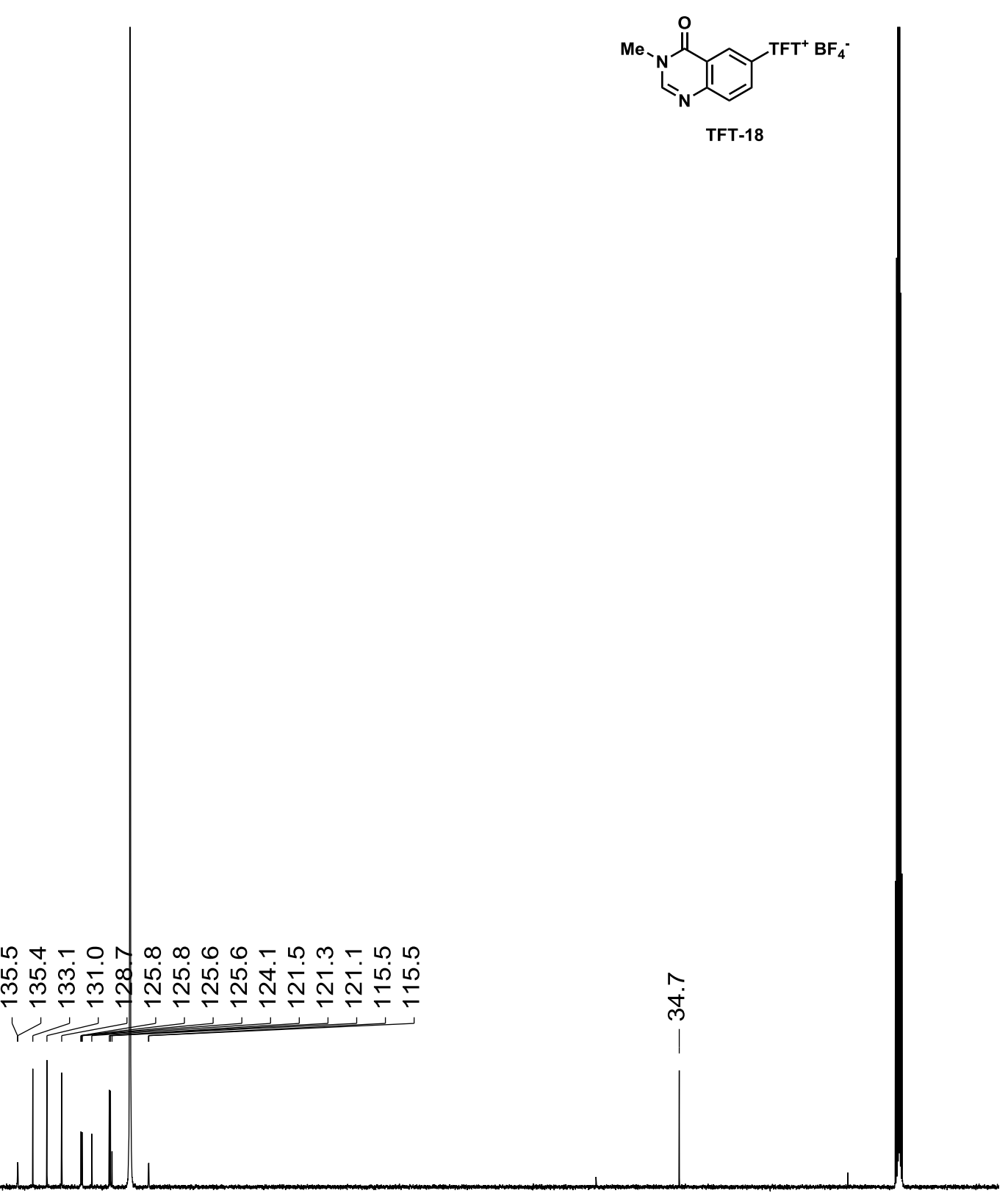

$\begin{array}{llllllllllllllllllllllllll}240 & 230 & 220 & 210 & 200 & 190 & 180 & 170 & 160 & 150 & 140 & 130 & \begin{array}{c}120 \\ \mathrm{f} 1\end{array}[\mathrm{ppm}] & 100 & 90 & 80 & 70 & 60 & 50 & 40 & 30 & 20 & 10 & 0 & -10\end{array}$ 
${ }^{19} \mathrm{~F}$ NMR of tetrafluorothianthrenium salt TFT-18

$\mathrm{MeCN}-\mathrm{d}_{3}, 25{ }^{\circ} \mathrm{C}$
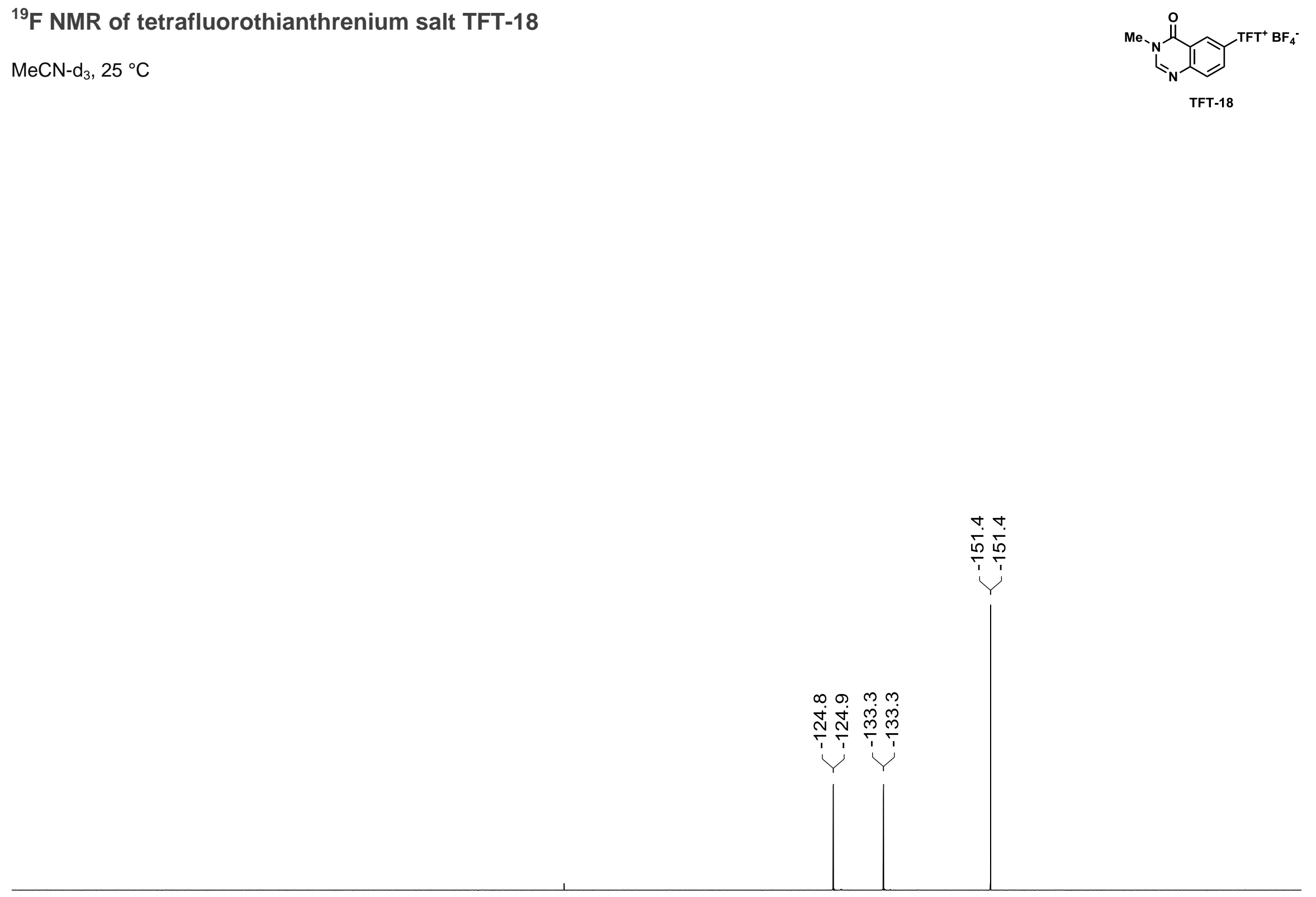

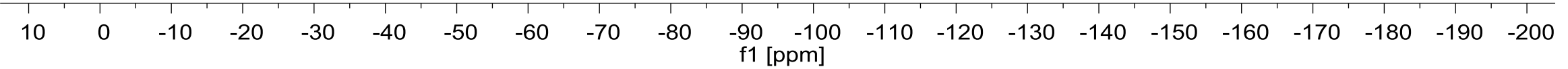




\section{${ }^{1} \mathrm{H}$ NMR of aniline 18}

$\mathrm{CDCl}_{3}, 25^{\circ} \mathrm{C}$
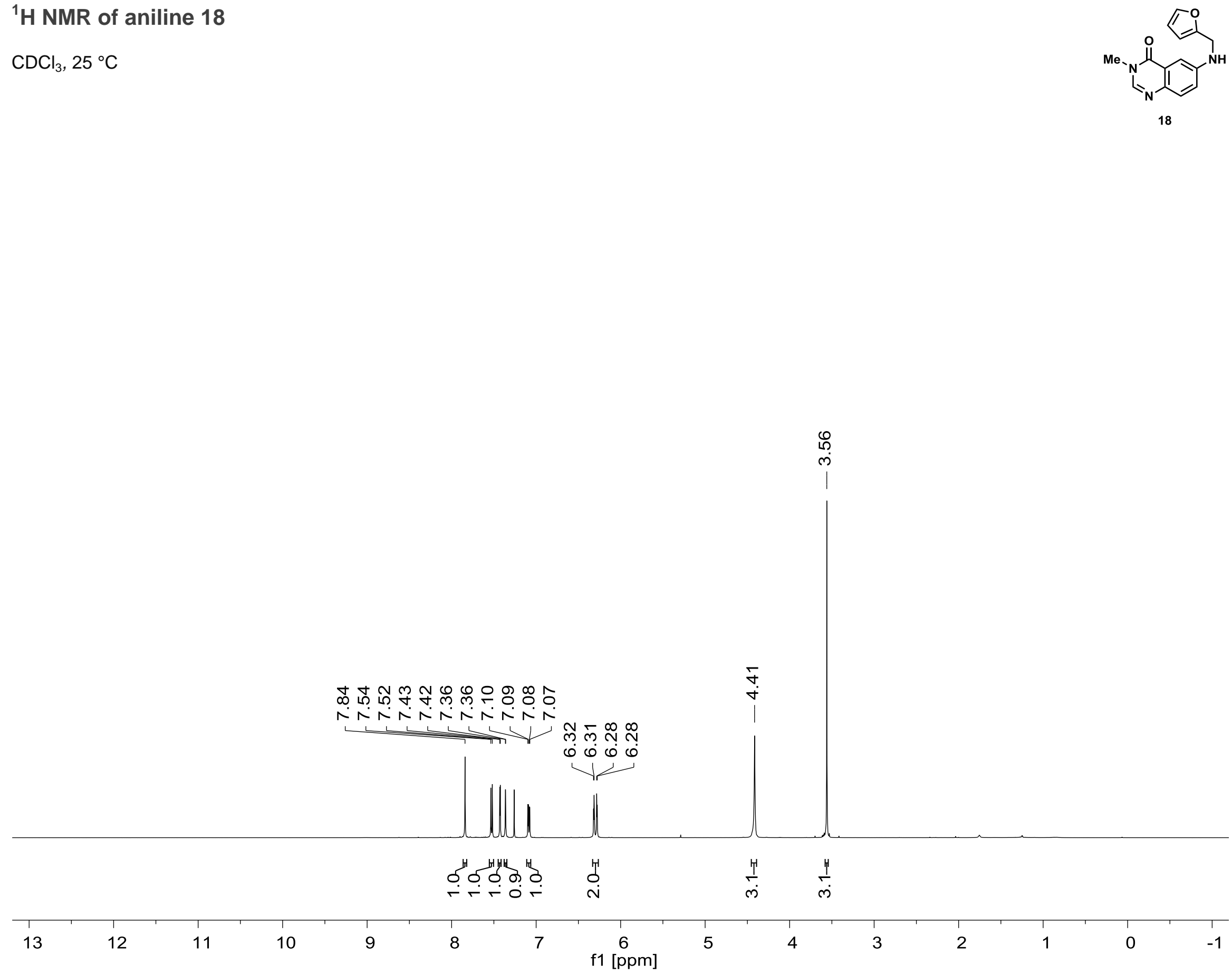


\section{${ }^{13} \mathrm{C}$ NMR of aniline 18}

$\mathrm{CDCl}_{3}, 25^{\circ} \mathrm{C}$
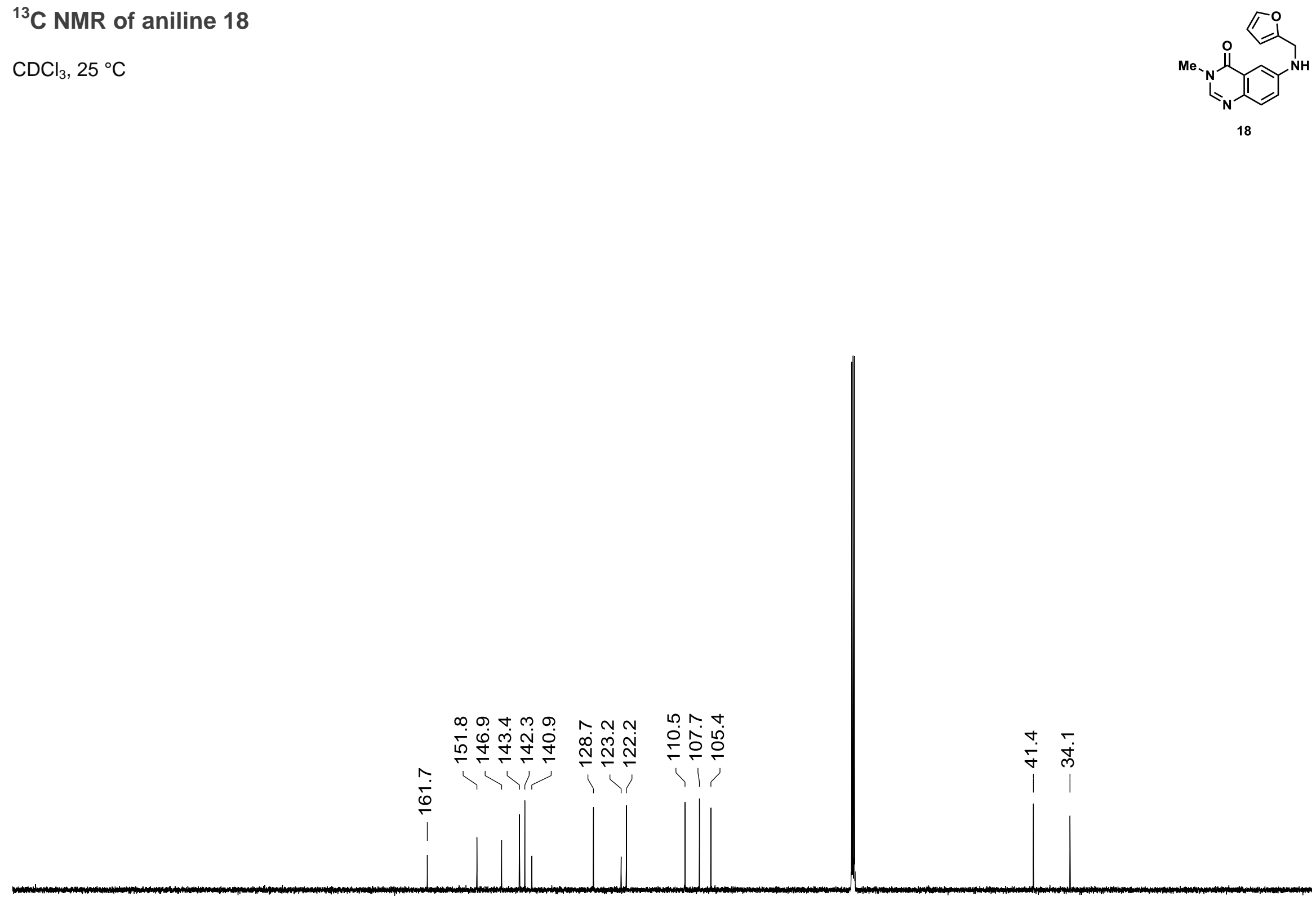


\section{${ }^{1} \mathrm{H}$ NMR of aniline 19}

$\mathrm{CDCl}_{3}, 25^{\circ} \mathrm{C}$
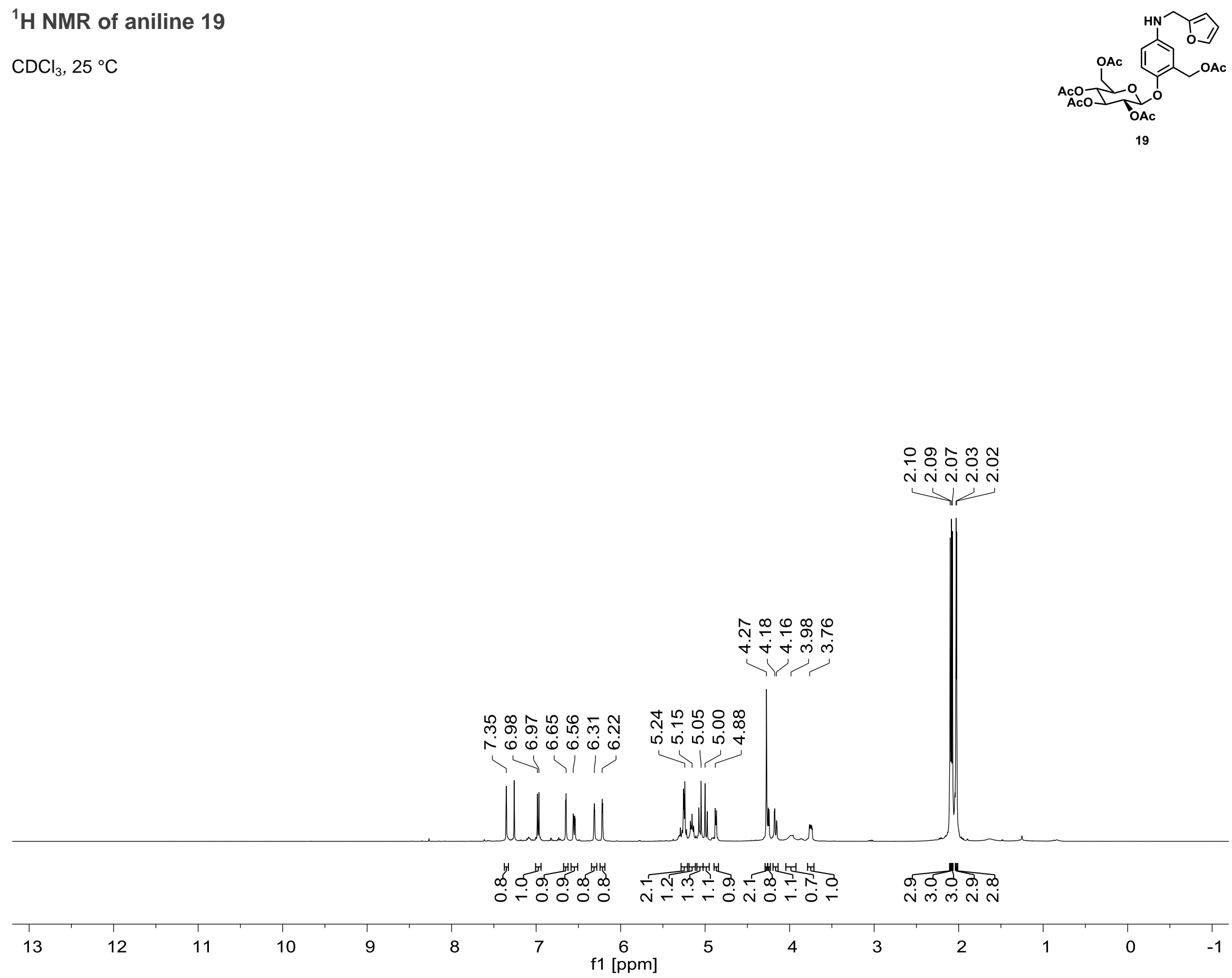


\section{${ }^{13} \mathrm{C}$ NMR of aniline 19}

$\mathrm{CDCl}_{3}, 25^{\circ} \mathrm{C}$
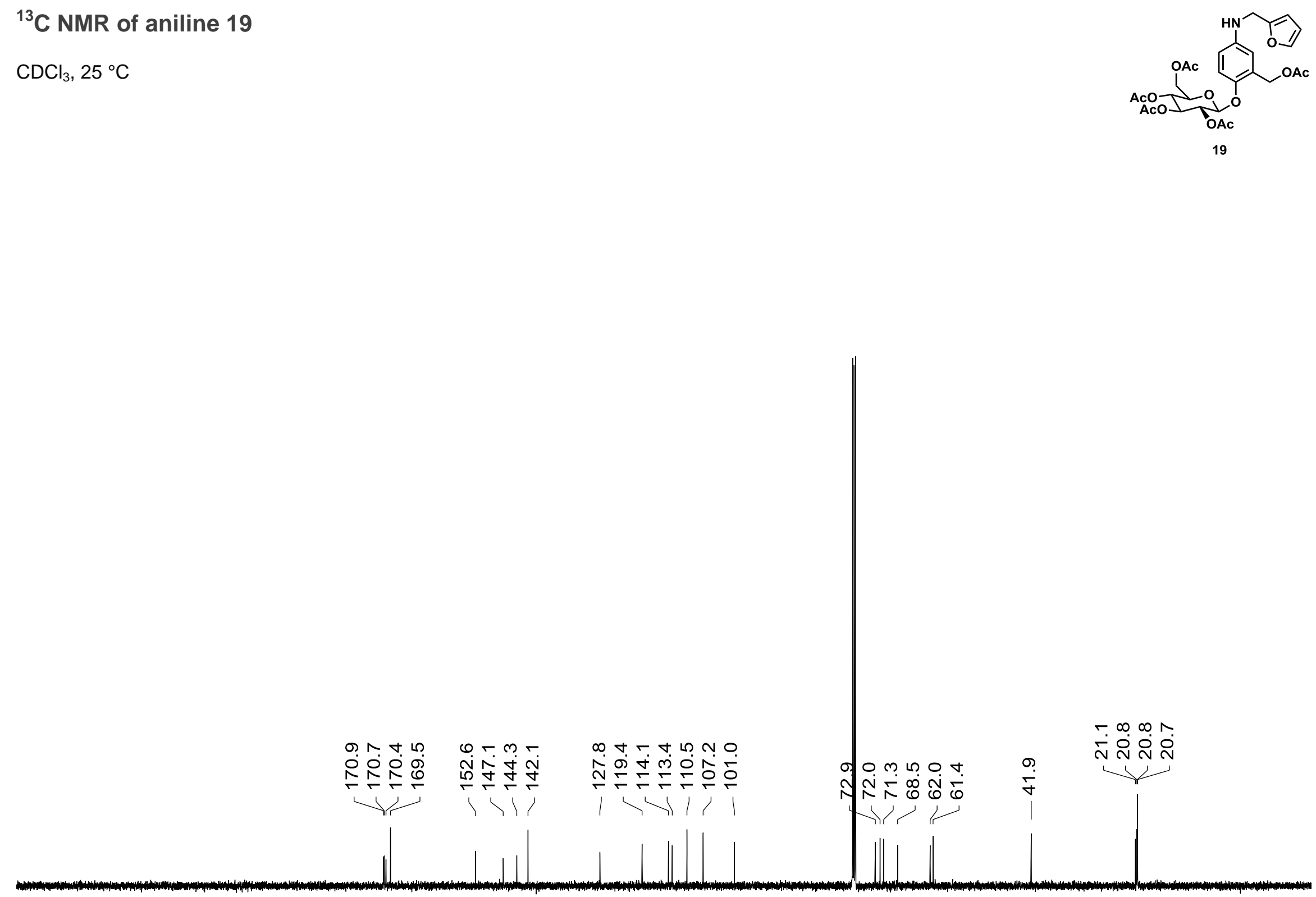

$\begin{array}{lllllllllllllllllllllllllllllllllll}240 & 230 & 220 & 210 & 200 & 190 & 180 & 170 & 160 & 150 & 140 & 130 & 120 & 110 & 100 & 90 & 80 & 70 & 60 & 50 & 40 & 30 & 20 & 10 & 0 & -10\end{array}$ f1 [ppm] 


\section{${ }^{1} \mathrm{H}$ NMR of tetrafluorothianthrenium salt TFT-20}

$\mathrm{CDCl}_{3}, 25^{\circ} \mathrm{C}$
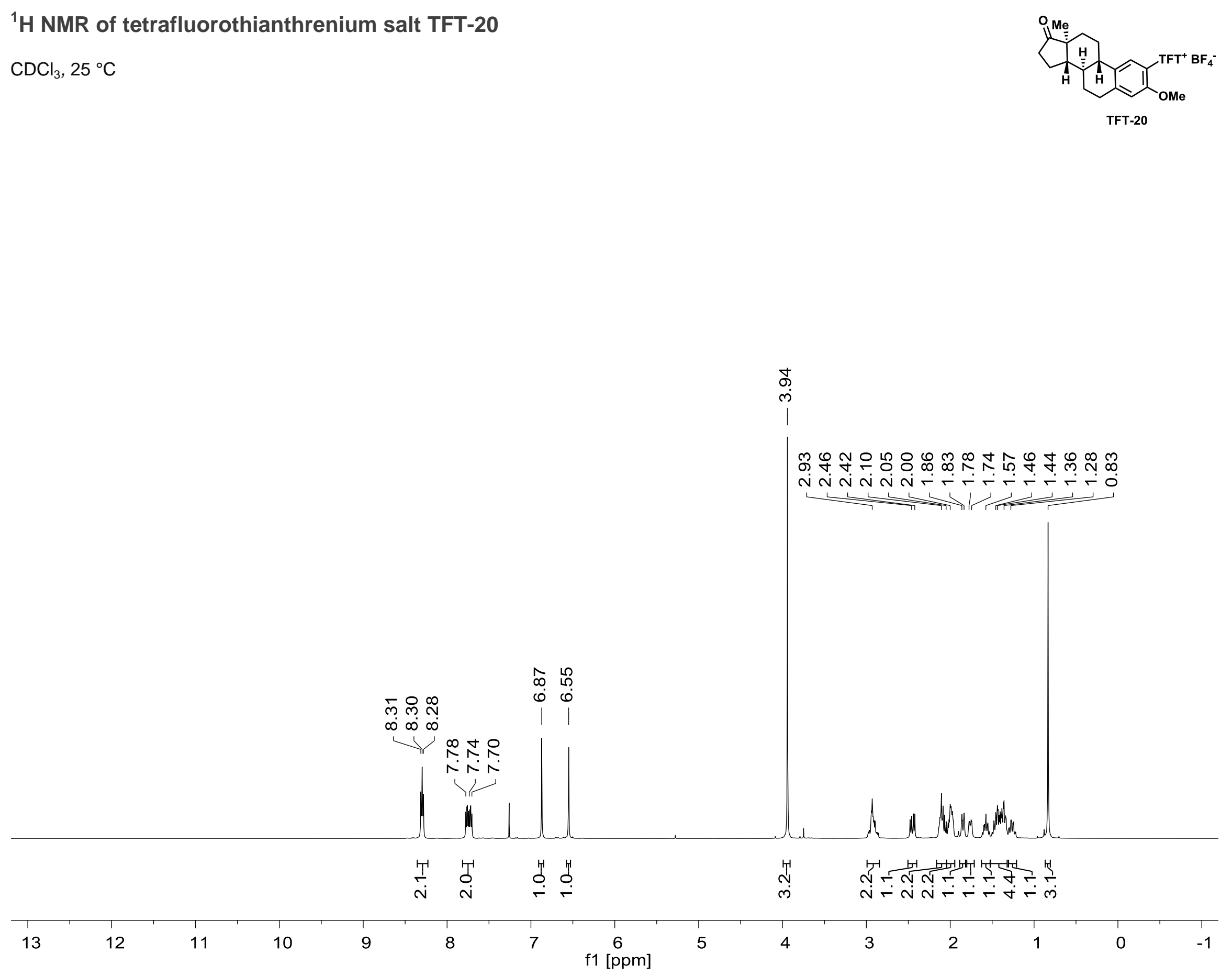


\section{${ }^{13} \mathrm{C}$ NMR of tetrafluorothianthrenium salt TFT-20}

$\mathrm{CDCl}_{3}, 25^{\circ} \mathrm{C}$
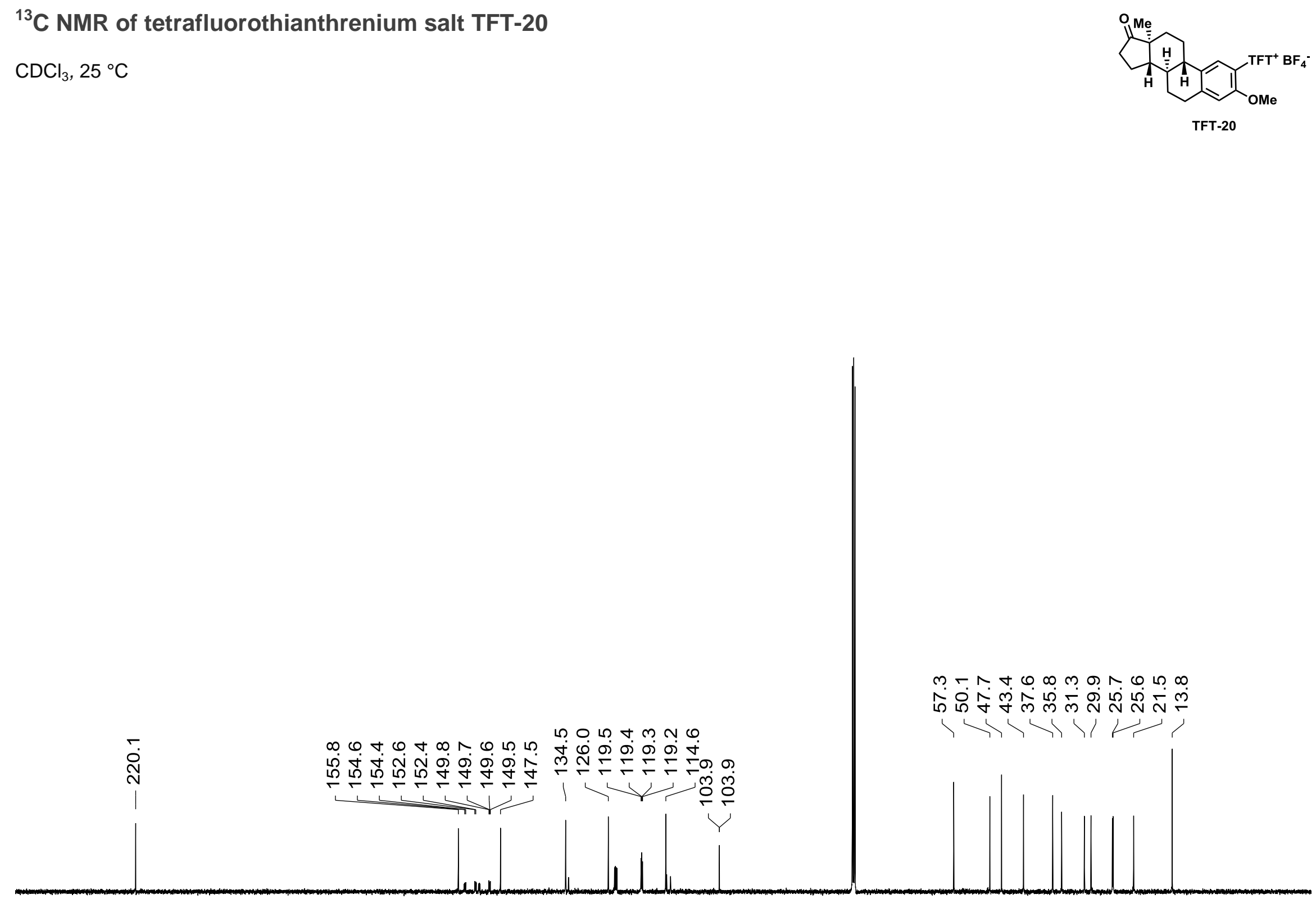

$\begin{array}{llllllllllllllllllllllllllllllllll}240 & 230 & 220 & 210 & 200 & 190 & 180 & 170 & 160 & 150 & 140 & 130 & 120 & 110 & 100 & 90 & 80 & 70 & 60 & 50 & 40 & 30 & 20 & 10 & 0 & -10\end{array}$ f1 [ppm] 


\section{${ }^{19} \mathrm{~F}$ NMR of tetrafluorothianthrenium salt TFT-20}

$\mathrm{CDCl}_{3}, 25^{\circ} \mathrm{C}$
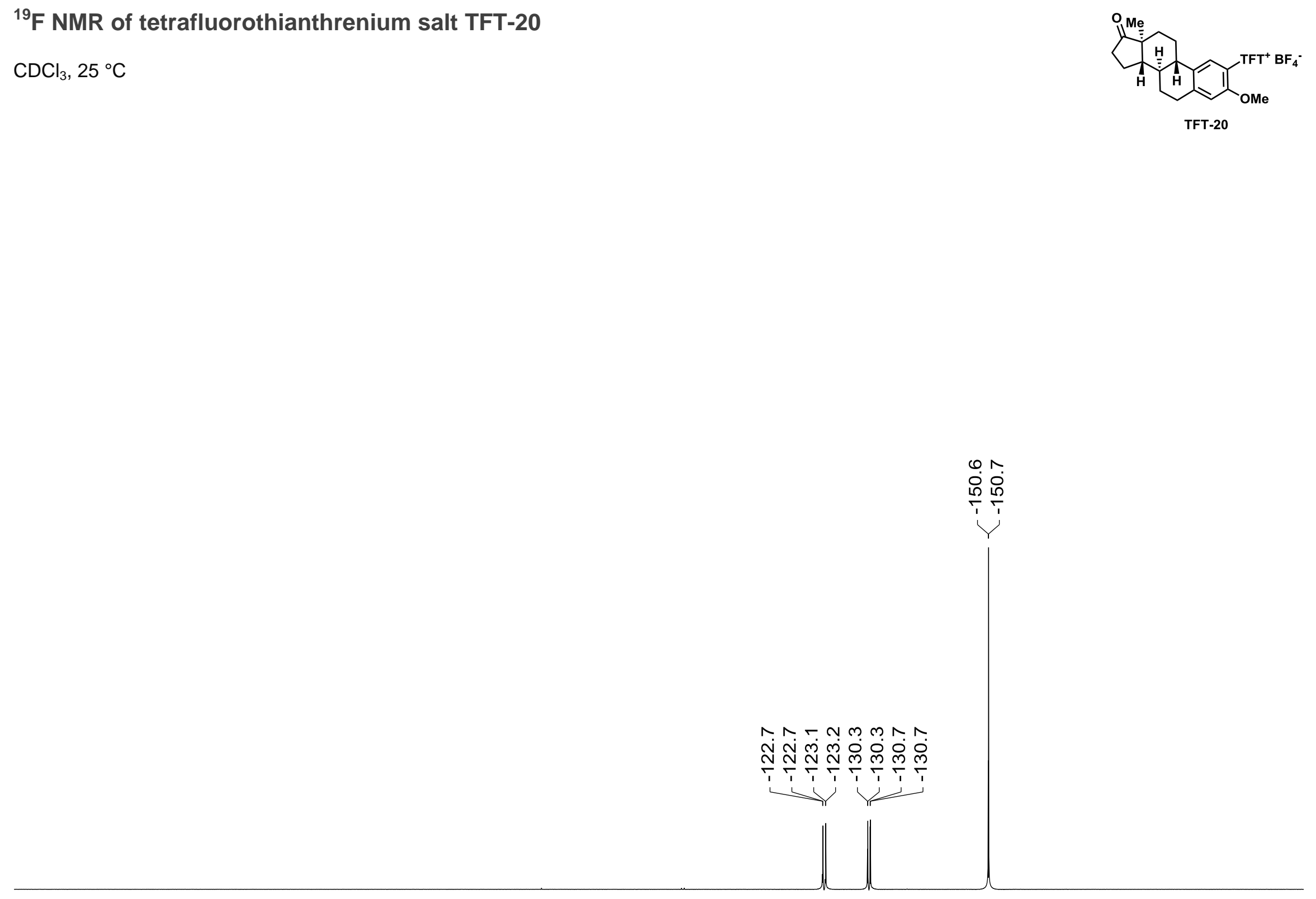

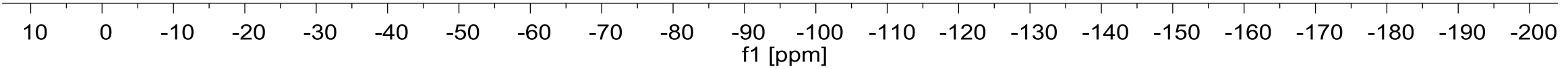




\section{${ }^{1} \mathrm{H}$ NMR of aniline 20}

$\mathrm{CDCl}_{3}, 25^{\circ} \mathrm{C}$

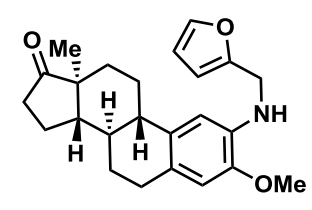

20

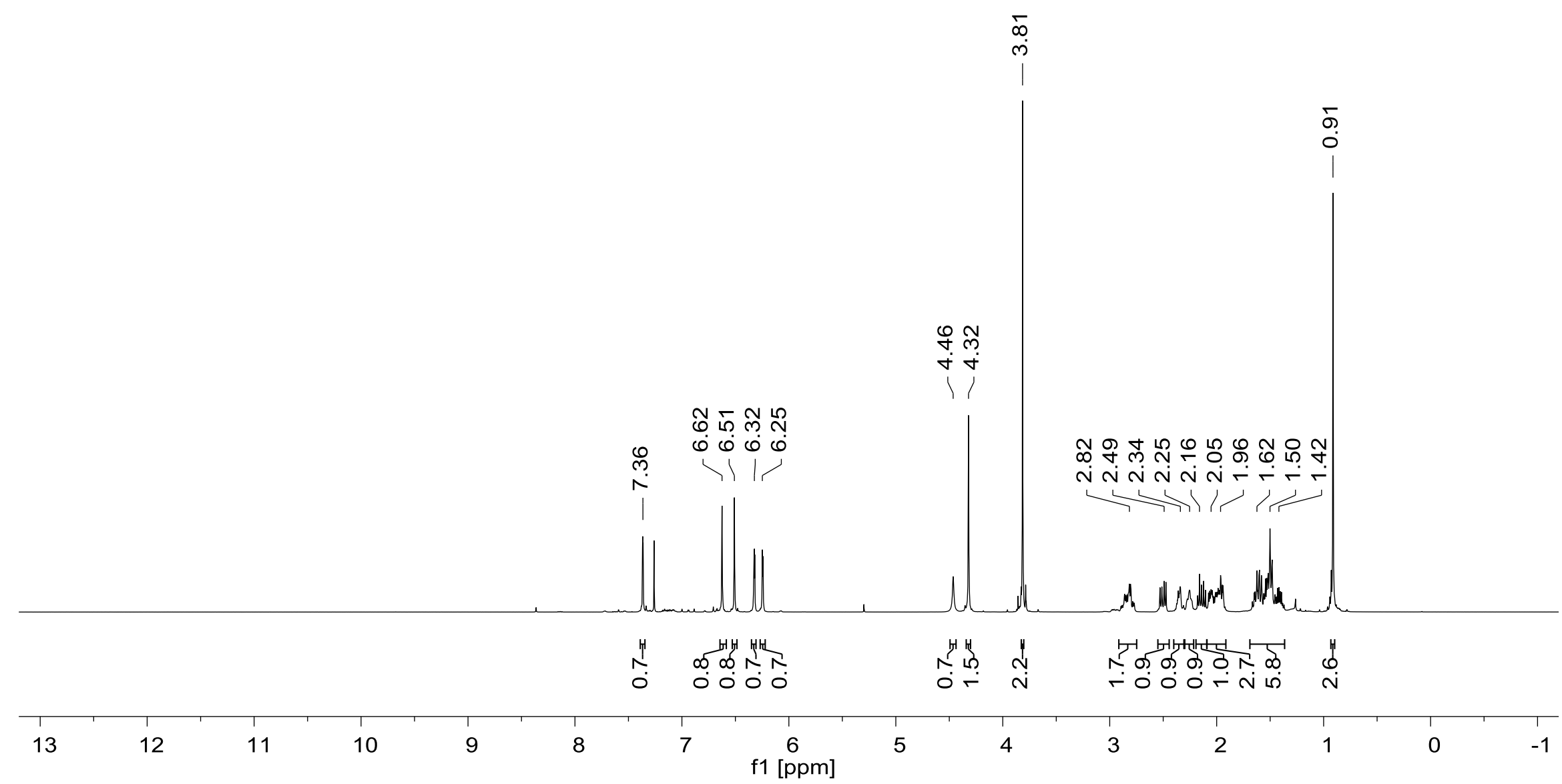




\section{${ }^{13} \mathrm{C}$ NMR of aniline 20}

$\mathrm{CDCl}_{3}, 25^{\circ} \mathrm{C}$

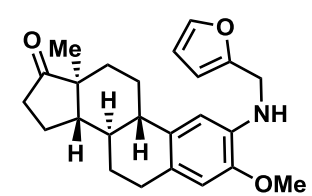

20

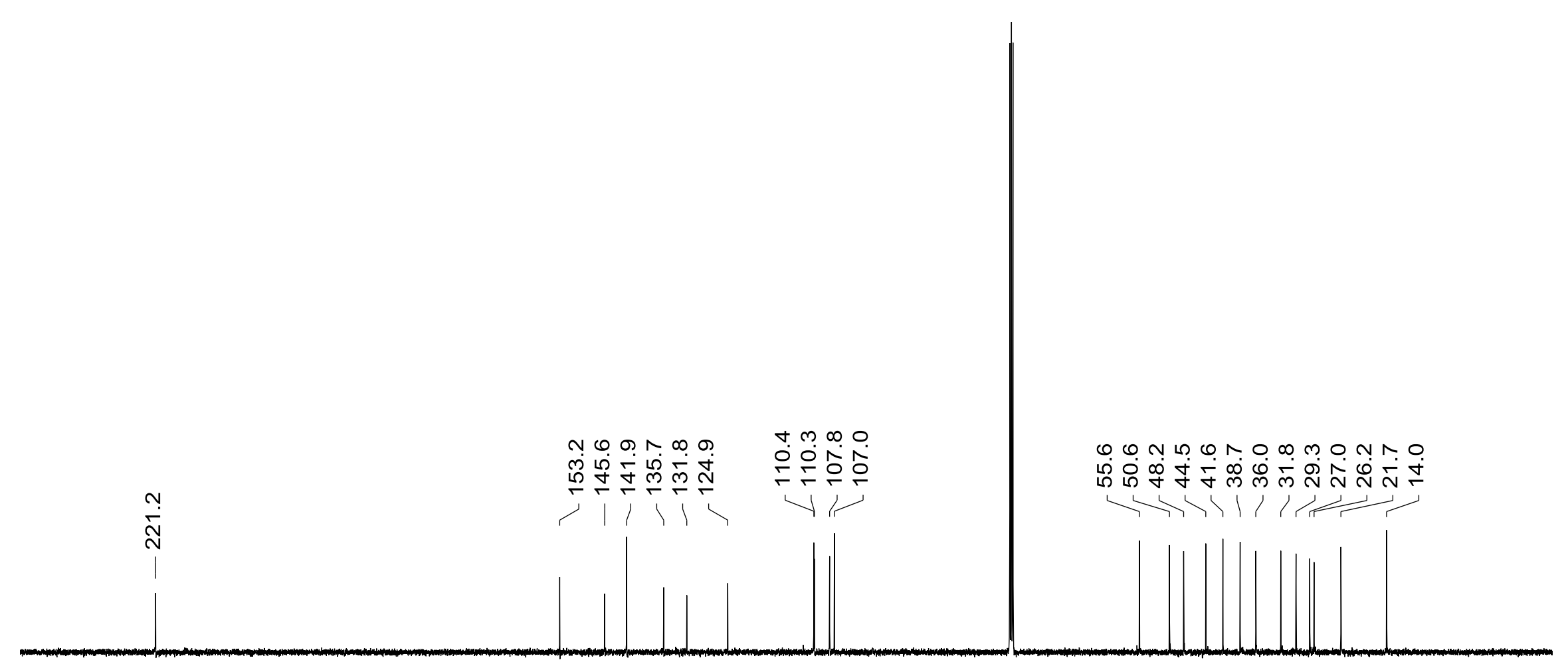

$\begin{array}{llllllllllllllllllllllllllllllllll}240 & 230 & 220 & 210 & 200 & 190 & 180 & 170 & 160 & 150 & 140 & 130 & 120 & 110 & 100 & 90 & 80 & 70 & 60 & 50 & 40 & 30 & 20 & 10 & 0 & -10\end{array}$ f1 [ppm] 


\section{${ }^{1} \mathrm{H}$ NMR of thianthrenium salt TT-21}

$\mathrm{CDCl}_{3}, 25^{\circ} \mathrm{C}$
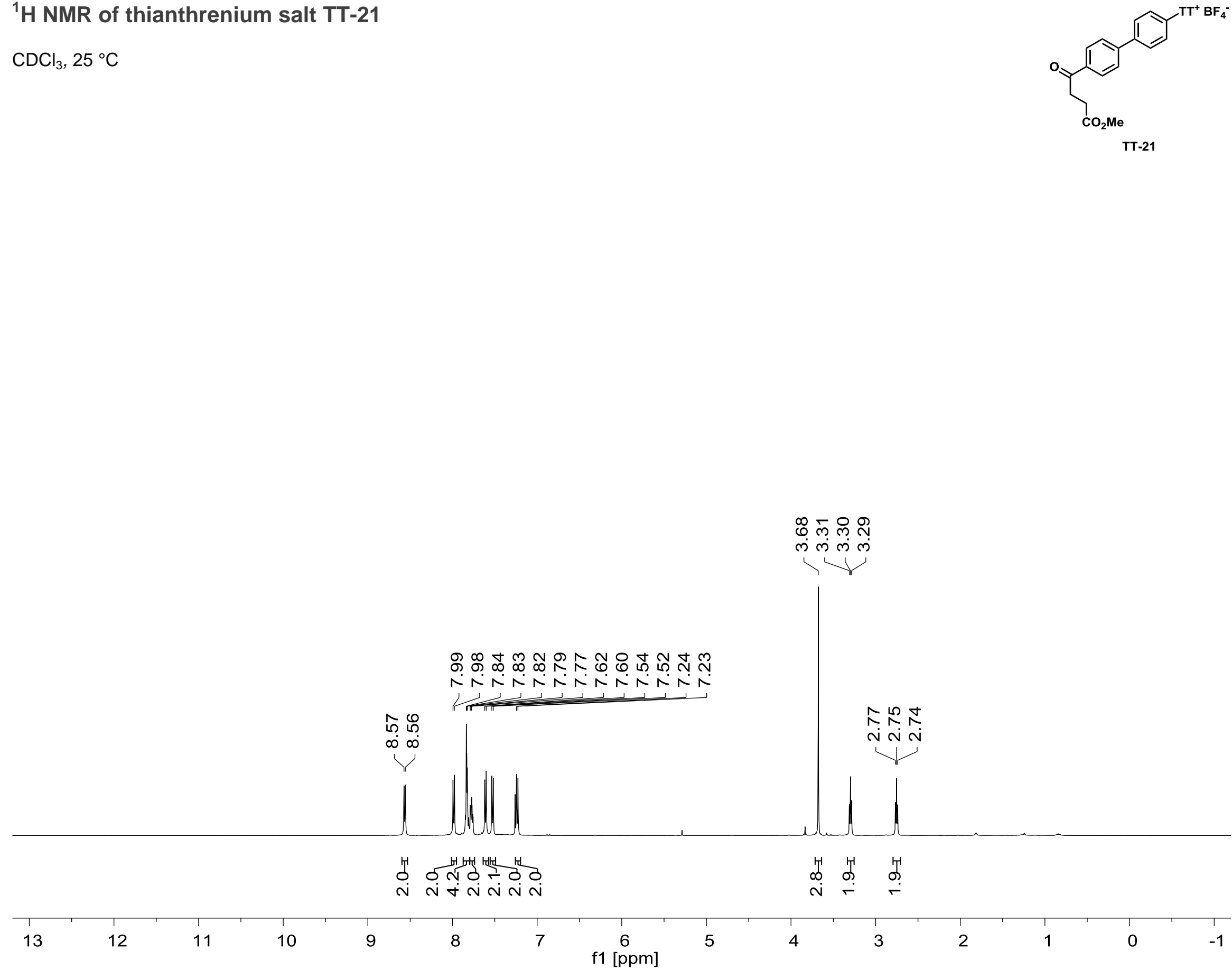


\section{${ }^{13} \mathrm{C}$ NMR of thianthrenium salt TT-21}

$\mathrm{CDCl}_{3}, 25^{\circ} \mathrm{C}$

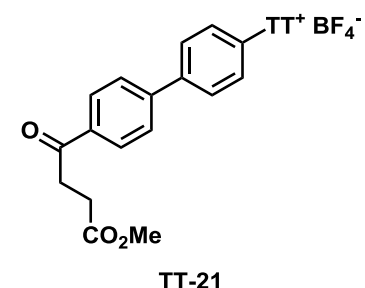

0.0 .

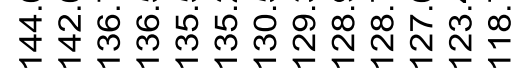

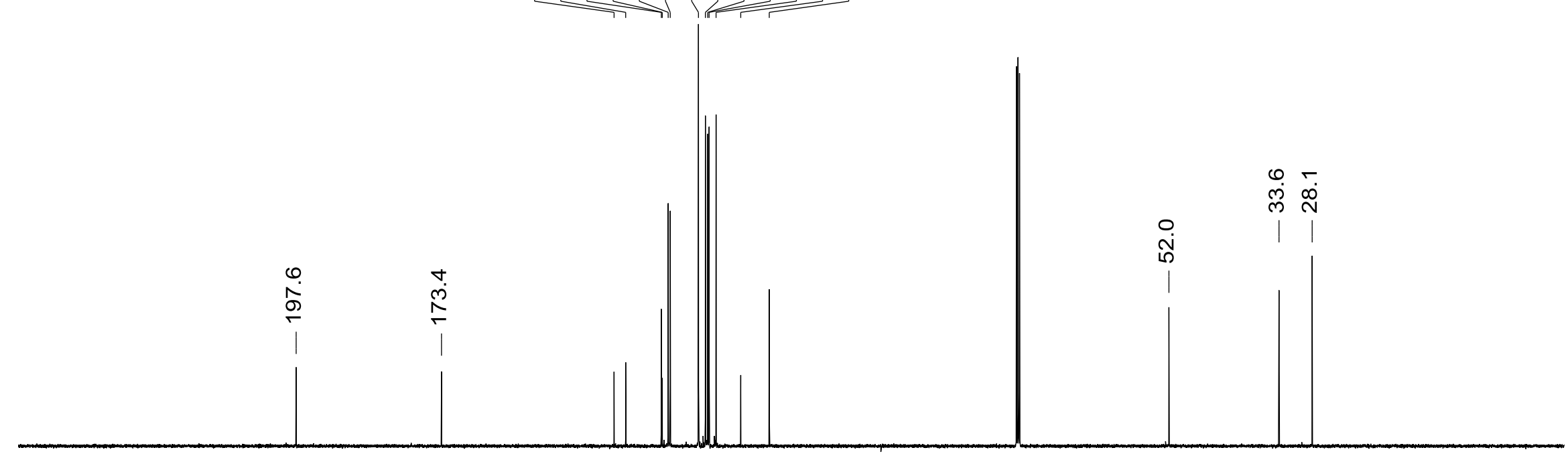

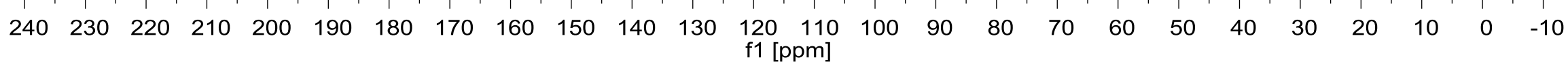


${ }^{19} \mathrm{~F}$ NMR of thianthrenium salt TT-21

$\mathrm{CDCl}_{3}, 25^{\circ} \mathrm{C}$

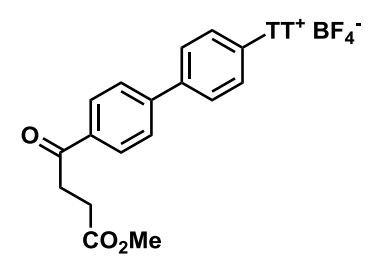

TT-21

ก.

$\stackrel{1}{\circ} \stackrel{1}{\circ}$

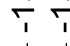

$Y$

$\begin{array}{lllllllllllllllllllllll}10 & 0 & -10 & -20 & -30 & -40 & -50 & -60 & -70 & -80 & -90 & -100 & -110 & -120 & -130 & -140 & -150 & -160 & -170 & -180 & -190 & -200\end{array}$




\section{${ }^{1} \mathrm{H}$ NMR of aniline 21}

$\mathrm{CDCl}_{3}, 25^{\circ} \mathrm{C}$
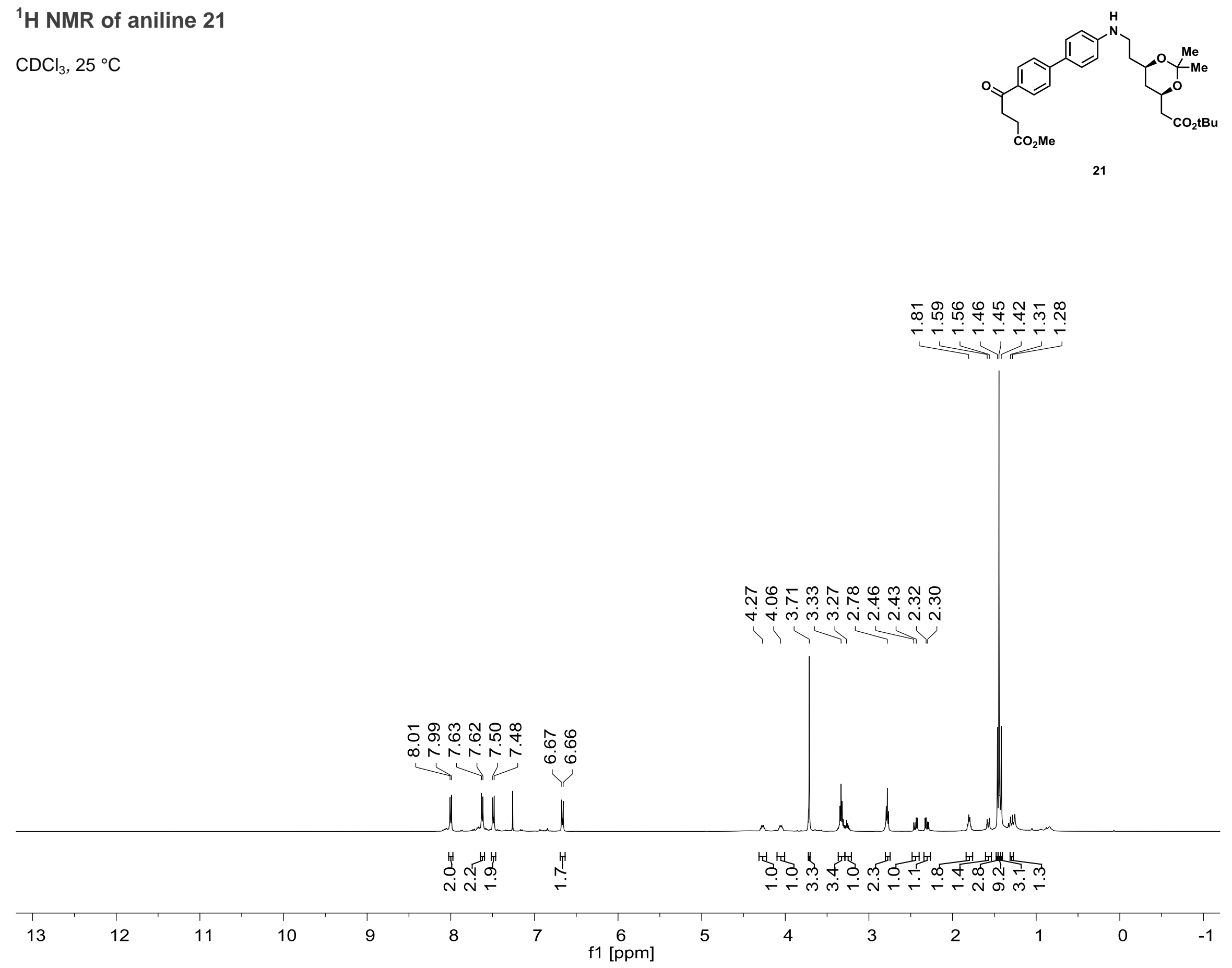
${ }^{13} \mathrm{C}$ NMR of aniline 21

$\mathrm{CDCl}_{3}, 25^{\circ} \mathrm{C}$
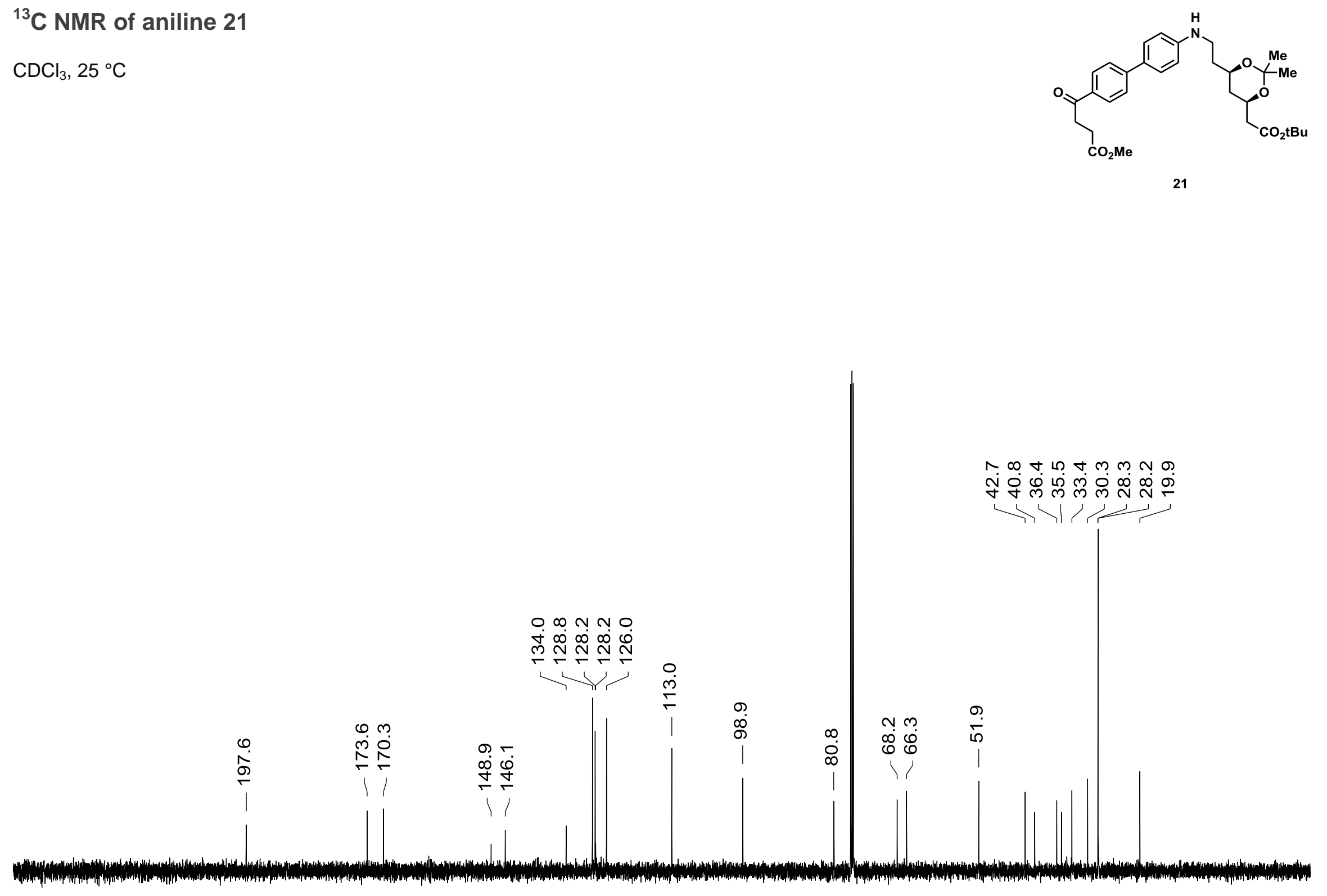

$\begin{array}{llllllllllllllllllllllllll}240 & 230 & 220 & 210 & 200 & 190 & 180 & 170 & 160 & 150 & 140 & 130 & 120 & 110 & 100 & 90 & 80 & 70 & 60 & 50 & 40 & 30 & 20 & 10 & 0 & -10\end{array}$ f1 [ppm] 


\section{${ }^{1} \mathrm{H}$ NMR of thianthrenium salt TT-22}

DMSO- $\mathrm{d}_{6}, 25^{\circ} \mathrm{C}$

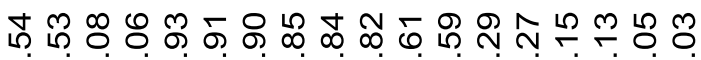

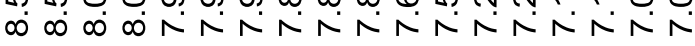

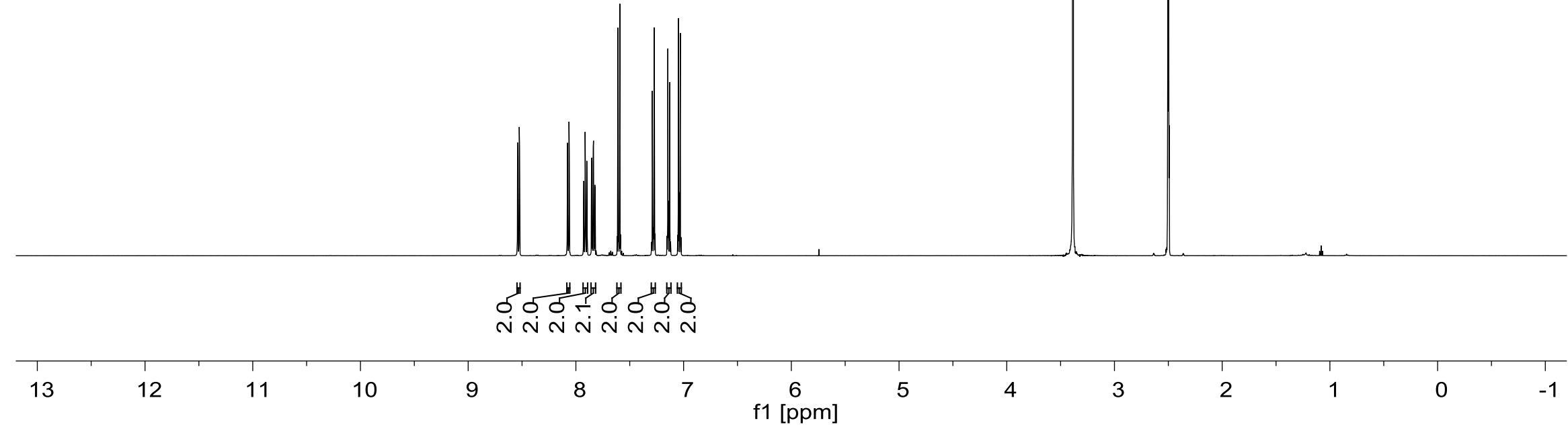




\section{${ }^{13} \mathrm{C}$ NMR of thianthrenium salt TT-22}

DMSO- $\mathrm{d}_{6}, 25^{\circ} \mathrm{C}$

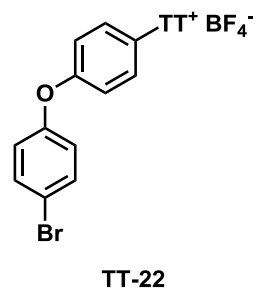

m

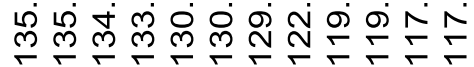

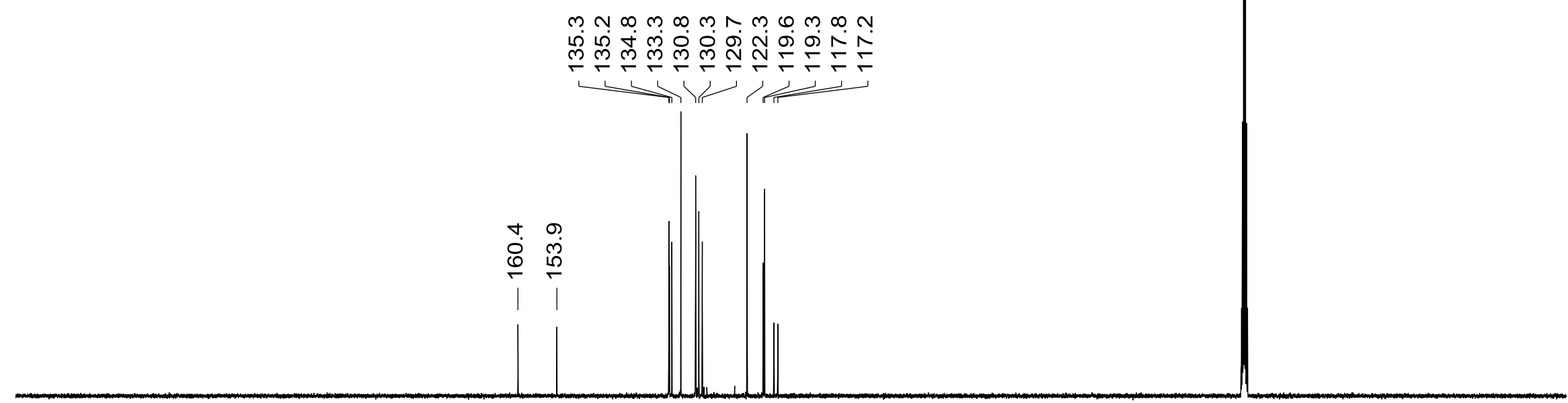

$\begin{array}{lllllllllllllllllllllllllllll}240 & 230 & 220 & 210 & 200 & 190 & 180 & 170 & 160 & 150 & 140 & 130 & 120 & 110 & 100 & 90 & 80 & 70 & 60 & 50 & 40 & 30 & 20 & 10 & 0 & -10\end{array}$ f1 [ppm] 


\section{${ }^{19} \mathrm{~F}$ NMR of thianthrenium salt TT-22}

DMSO- $\mathrm{d}_{6}, 25^{\circ} \mathrm{C}$

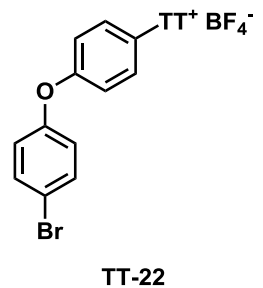

กิ

$\stackrel{\substack{+\infty \\ \infty}}{\infty}$

$\stackrel{+}{\sim}$

\begin{tabular}{lllllllllllllllllllllll}
\hline 10 & 0 & -10 & -20 & -30 & -40 & -50 & -60 & -70 & -80 & -90 & -100 & -110 & -120 & -130 & -140 & -150 & -160 & -170 & -180 & -190 & -200
\end{tabular}




\section{${ }^{1} \mathrm{H}$ NMR of aniline 22}

$\mathrm{CDCl}_{3}, 25^{\circ} \mathrm{C}$

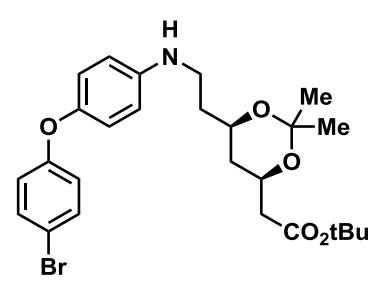

22

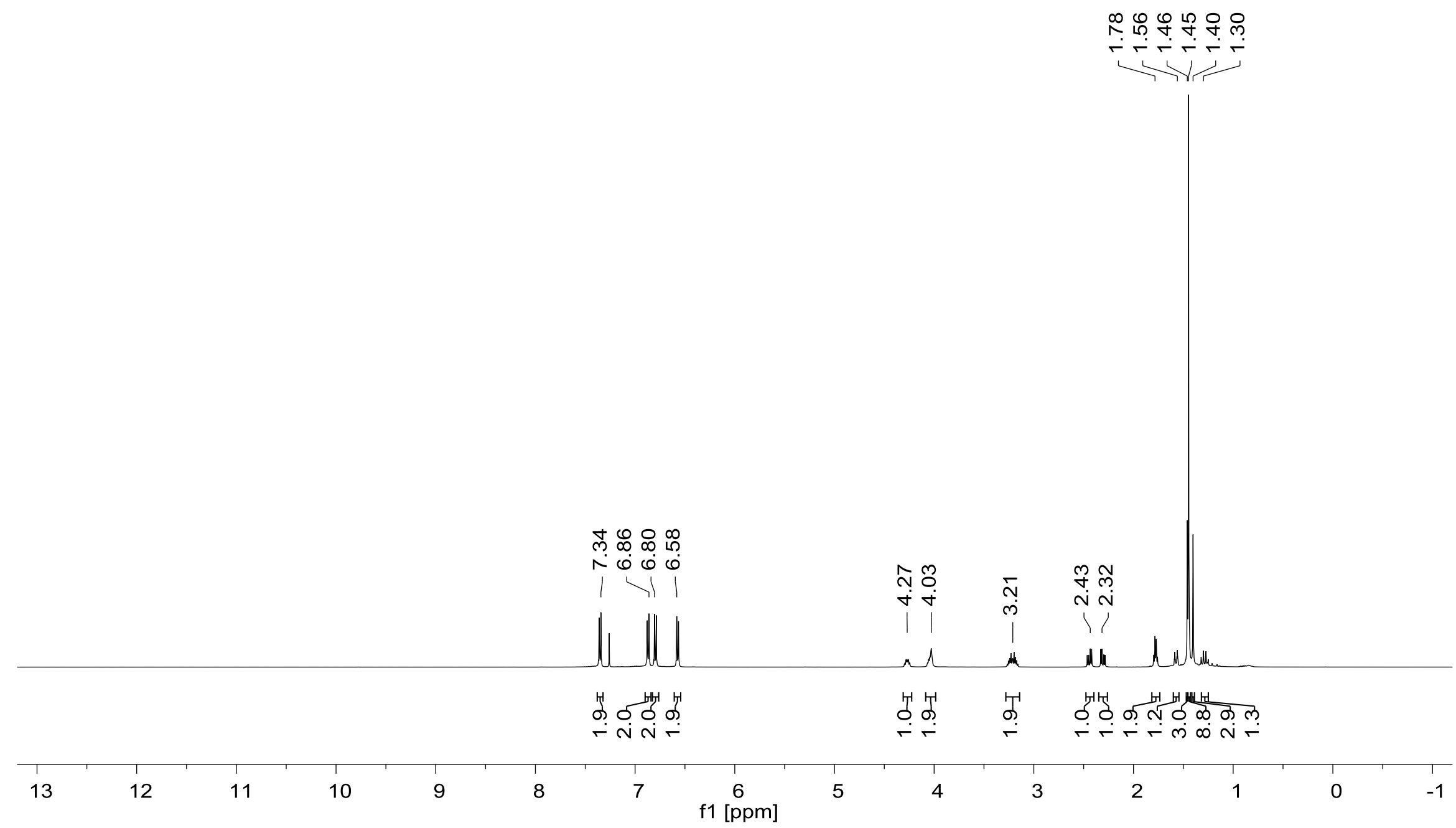




\section{${ }^{13} \mathrm{C}$ NMR of aniline 22}

$\mathrm{CDCl}_{3}, 25^{\circ} \mathrm{C}$

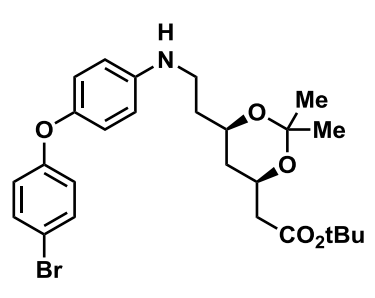

22

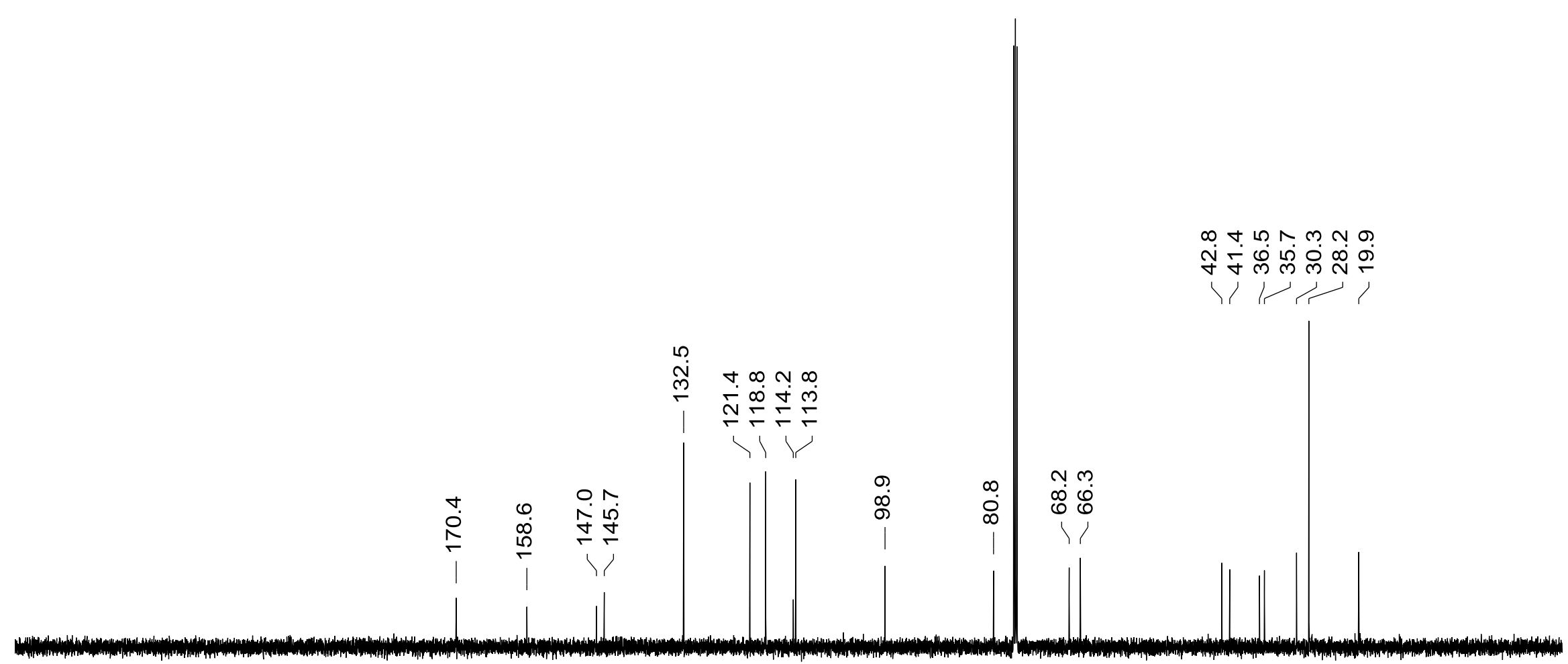

$\begin{array}{lllllllllllllllllllllllllllllll}240 & 230 & 220 & 210 & 200 & 190 & 180 & 170 & 160 & 150 & 140 & 130 & 120 & 110 & 100 & 90 & 80 & 70 & 60 & 50 & 40 & 30 & 20 & 10 & 0 & -10\end{array}$ f1 [ppm] 


\section{${ }^{1} \mathrm{H}$ NMR of morpholine 23}

$\mathrm{CDCl}_{3}, 25^{\circ} \mathrm{C}$
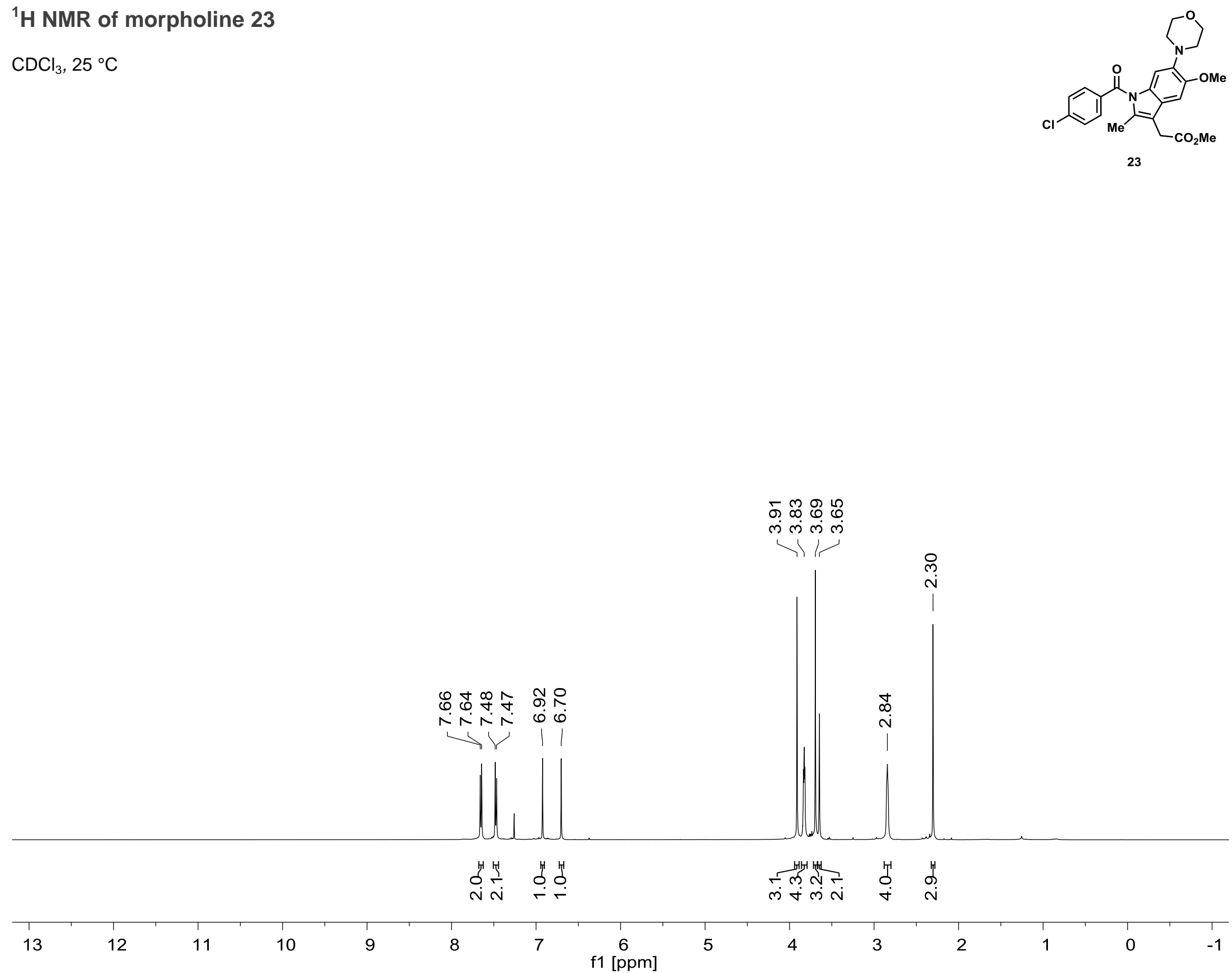


\section{${ }^{13} \mathrm{C}$ NMR of morpholine 23}

$\mathrm{CDCl}_{3}, 25^{\circ} \mathrm{C}$

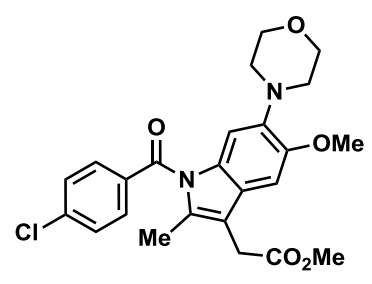

23
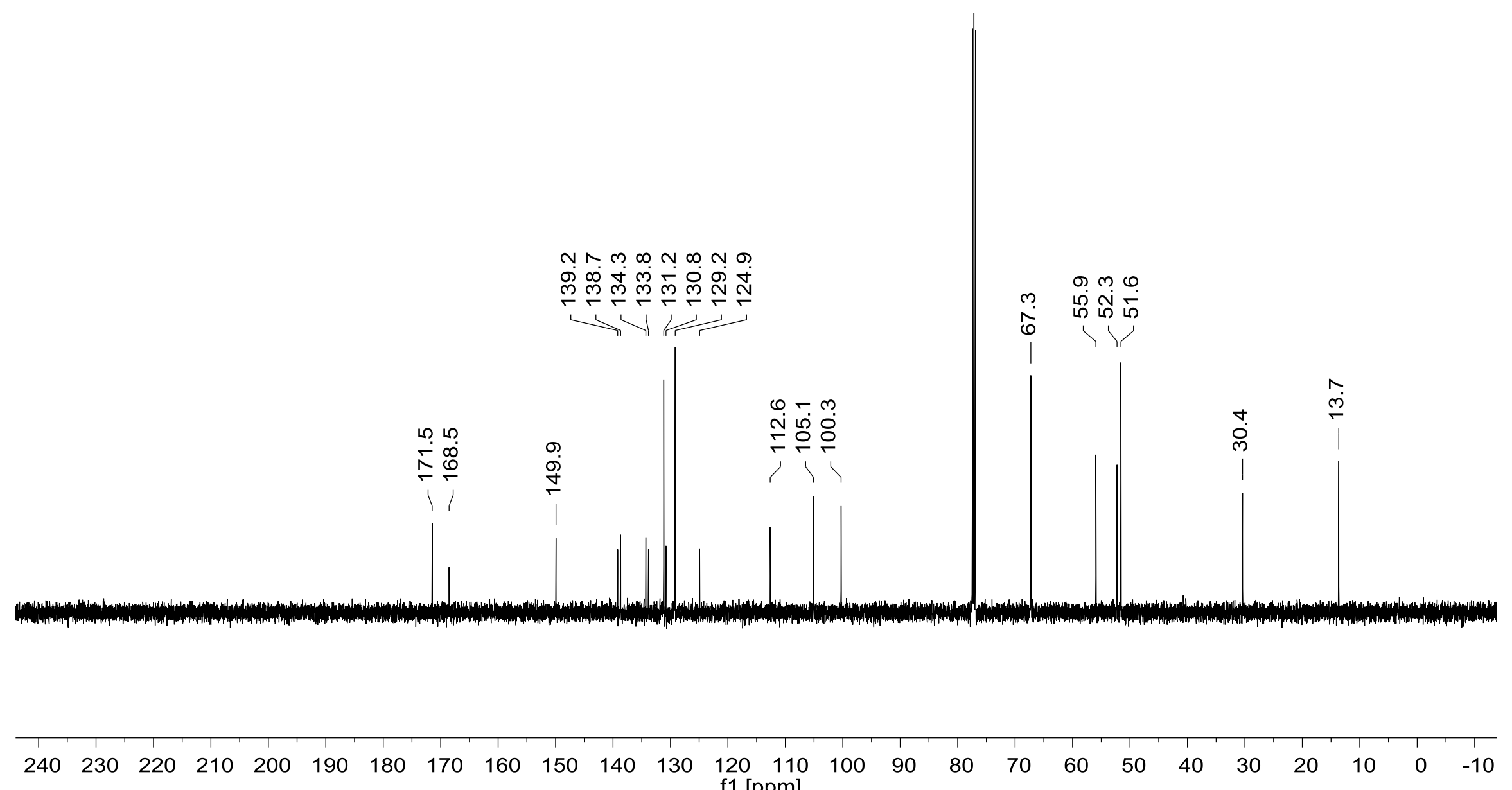


\section{${ }^{1} \mathrm{H}$ NMR of thianthrenium salt TT-24}

DMSO- $\mathrm{d}_{6}, 25^{\circ} \mathrm{C}$

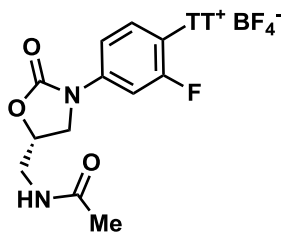

TT-24

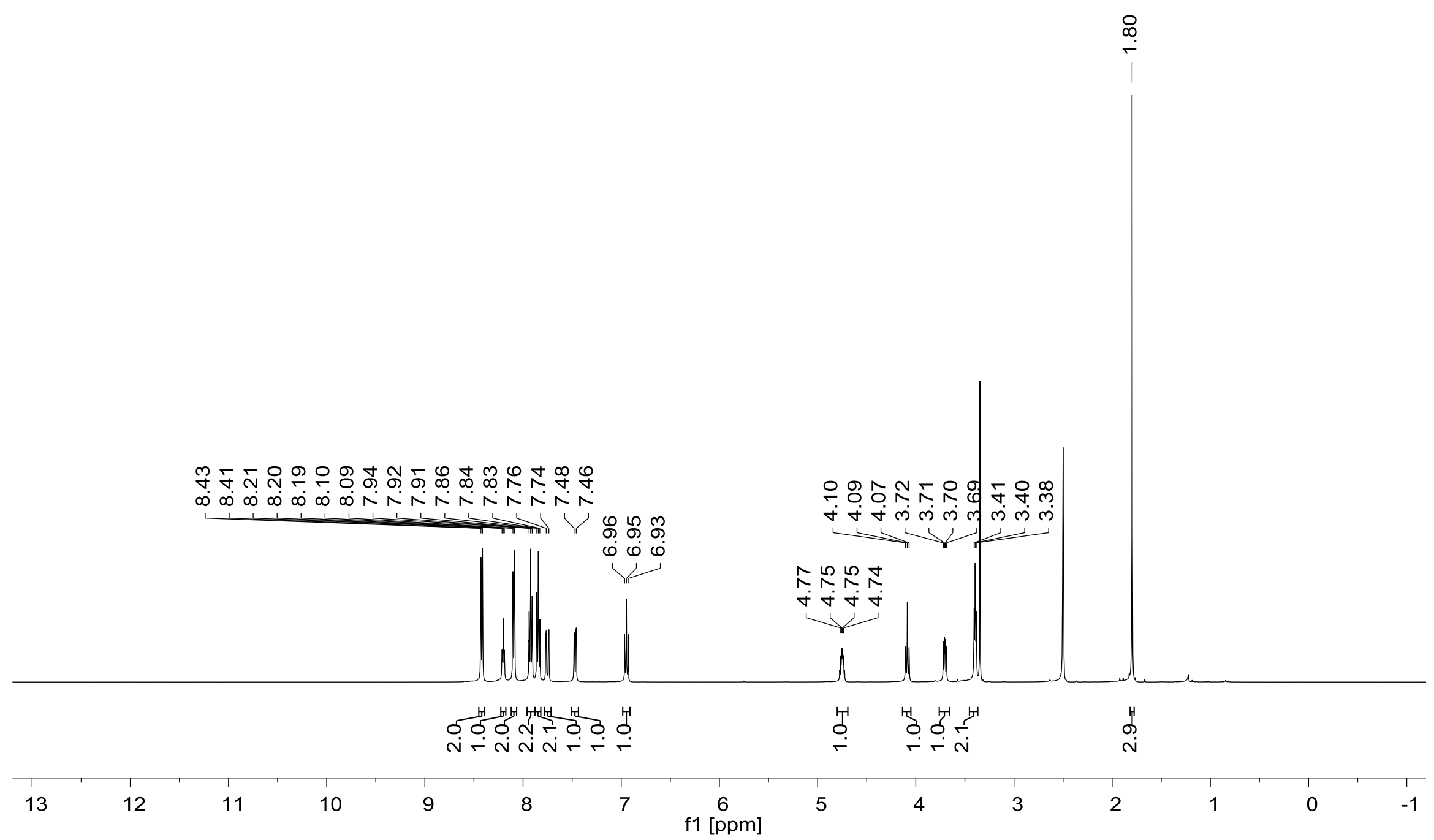




\section{${ }^{13} \mathrm{C}$ NMR of thianthrenium salt TT-24}

DMSO- $\mathrm{d}_{6}, 25^{\circ} \mathrm{C}$

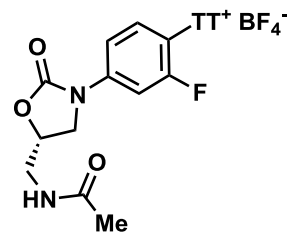

TT-24

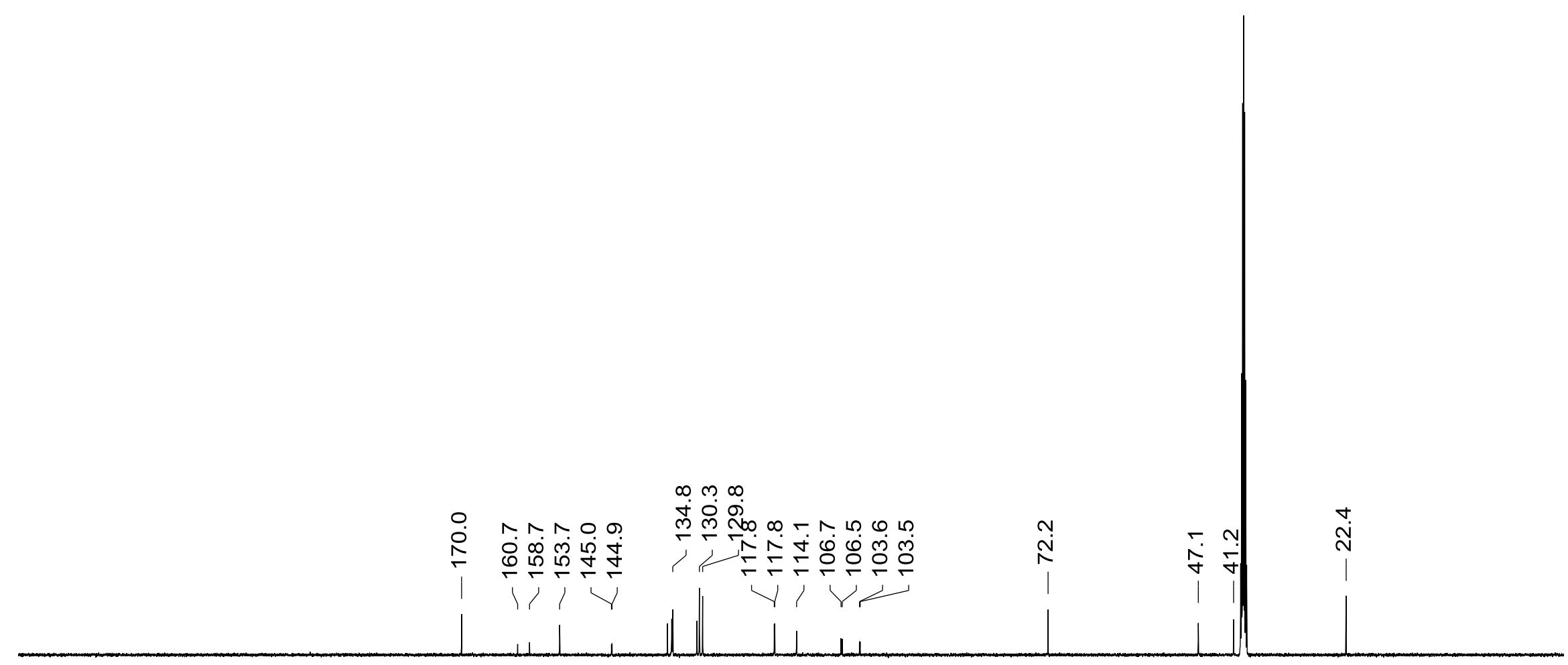

$\begin{array}{llllllllllllllllllllllllllllllllll}240 & 230 & 220 & 210 & 200 & 190 & 180 & 170 & 160 & 150 & 140 & 130 & 120 & 110 & 100 & 90 & 80 & 70 & 60 & 50 & 40 & 30 & 20 & 10 & 0 & -10\end{array}$ f1 [ppm] 
${ }^{19} \mathrm{~F}$ NMR of thianthrenium salt TT-24

DMSO- $\mathrm{d}_{6}, 25^{\circ} \mathrm{C}$

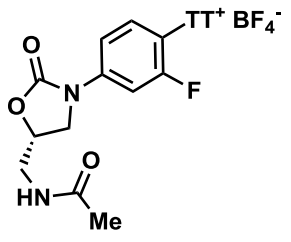

TT-24

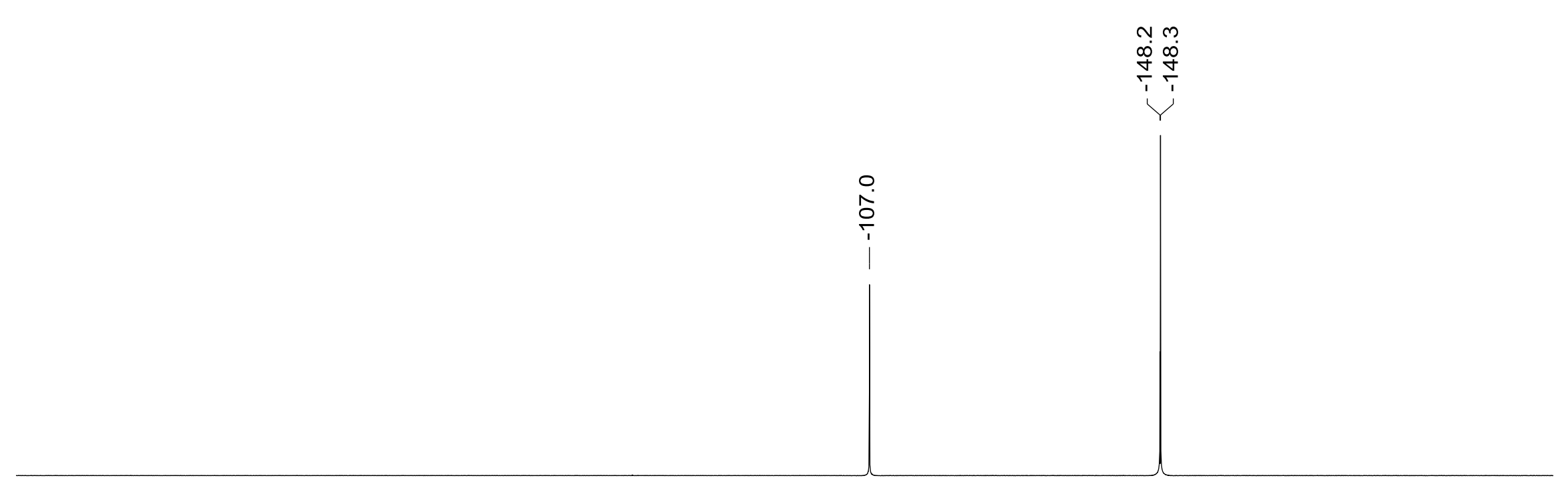

\begin{tabular}{lllllllllllllllllllllll}
\hline 10 & 0 & -10 & -20 & -30 & -40 & -50 & -60 & -70 & -80 & -90 & -100 & -110 & -120 & -130 & -140 & -150 & -160 & -170 & -180 & -190 & -200
\end{tabular}




\section{${ }^{1} \mathrm{H}$ NMR of morpholine 24}

$\mathrm{CDCl}_{3}, 25^{\circ} \mathrm{C}$
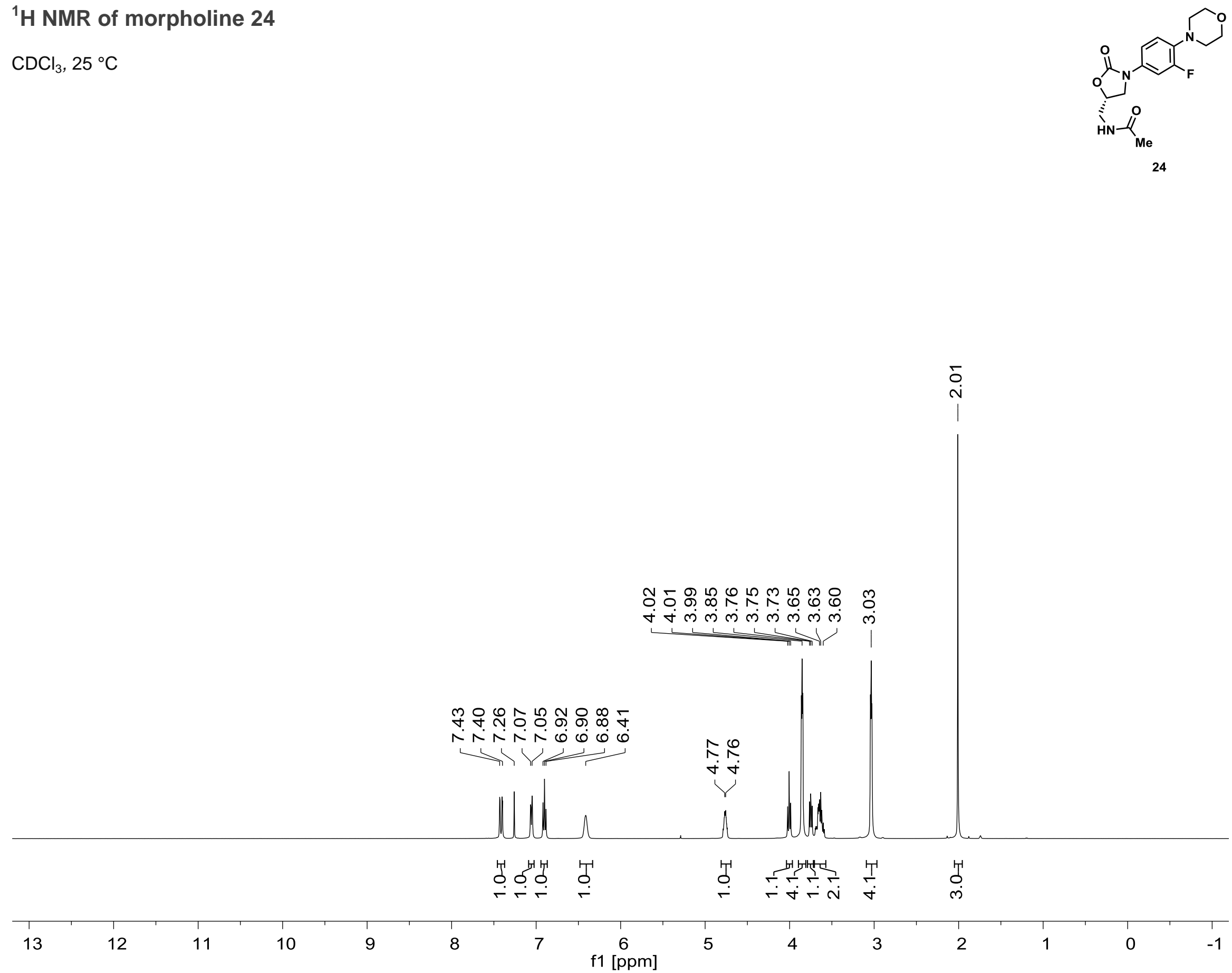


\section{${ }^{13} \mathrm{C}$ NMR of morpholine 24}

$\mathrm{CDCl}_{3}, 25^{\circ} \mathrm{C}$
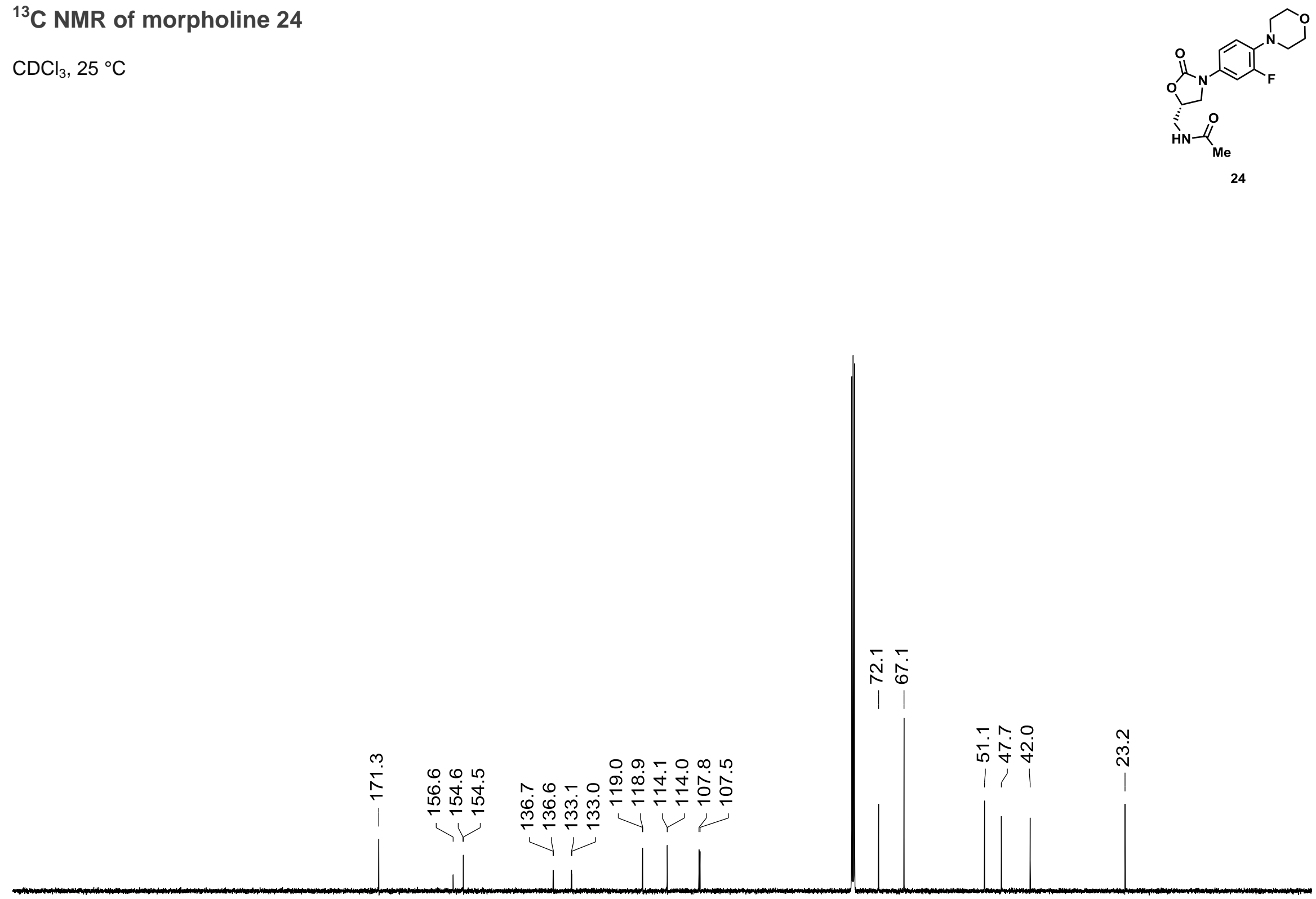


\section{${ }^{19} \mathrm{~F}$ NMR of morpholine 24}

$\mathrm{CDCl}_{3}, 25^{\circ} \mathrm{C}$
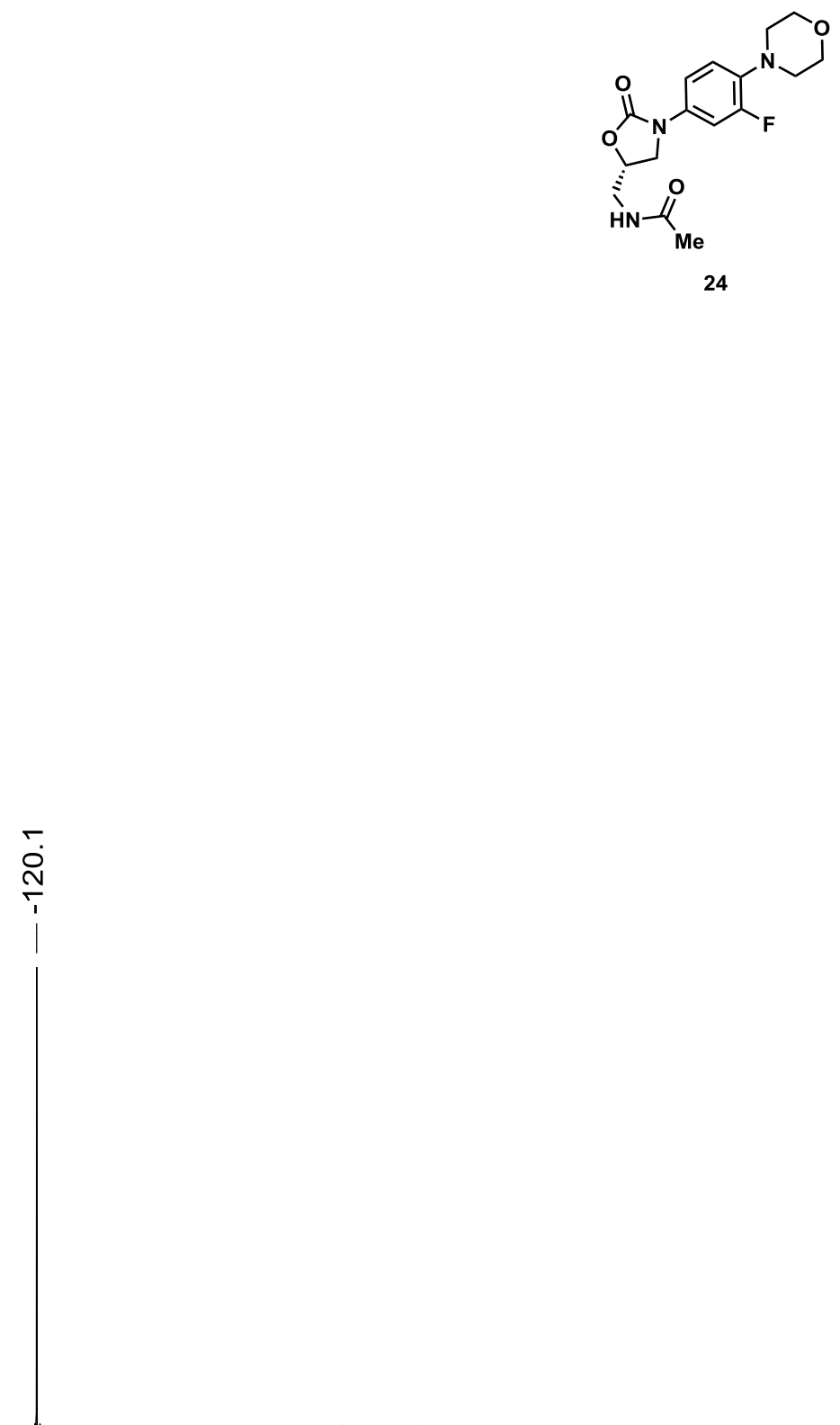

\begin{tabular}{lllllllllllllllllllllll}
\hline 10 & 0 & -10 & -20 & -30 & -40 & -50 & -60 & -70 & -80 & -90 & -100 & -110 & -120 & -130 & -140 & -150 & -160 & -170 & -180 & -190 & -200
\end{tabular}




\section{${ }^{1} \mathrm{H}$ NMR of thianthrenium salt TT-25}

DMSO- $\mathrm{d}_{6}, 25^{\circ} \mathrm{C}$
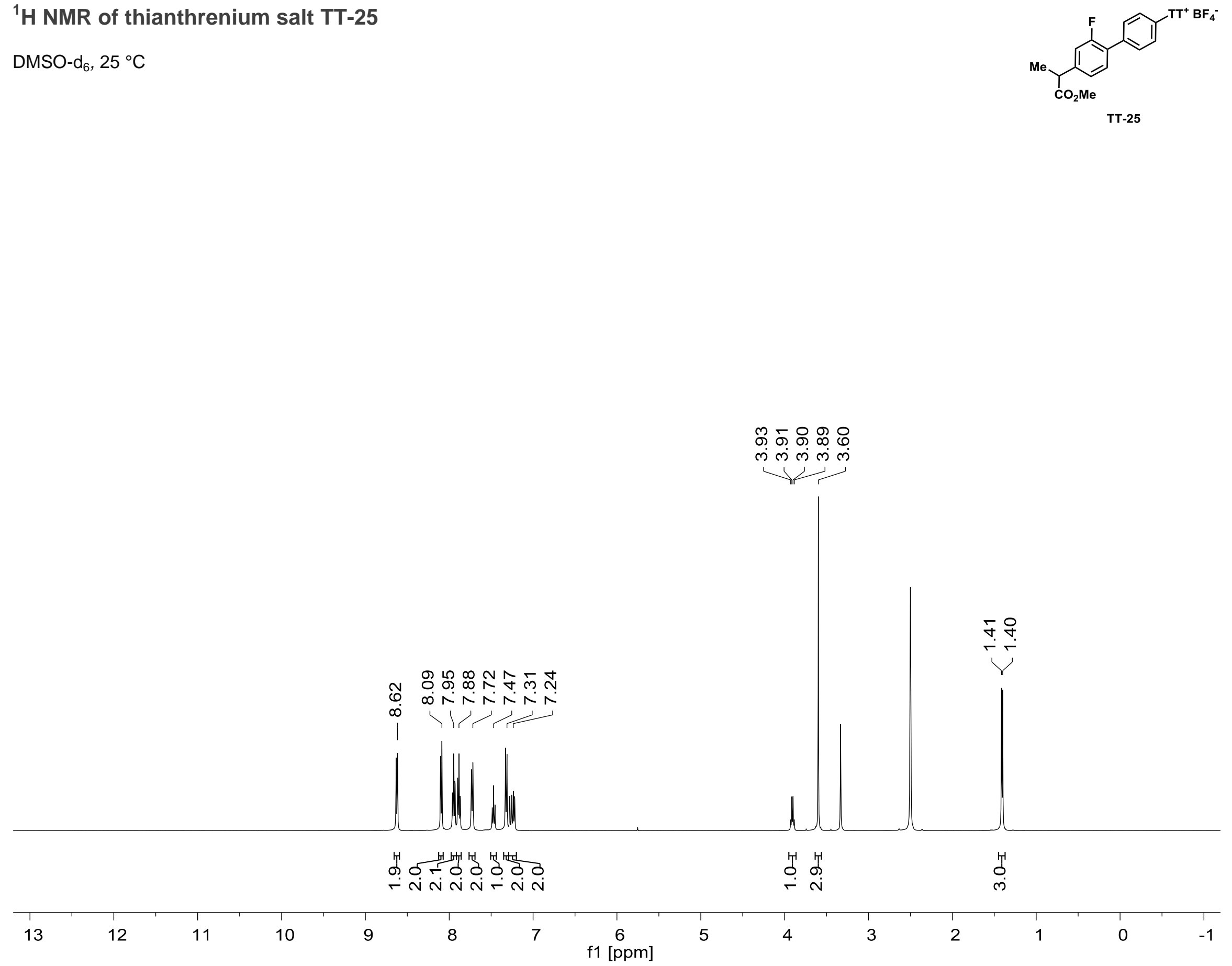


\section{${ }^{13} \mathrm{C}$ NMR of thianthrenium salt TT-25}

DMSO- $\mathrm{d}_{6}, 25^{\circ} \mathrm{C}$

0 ○.

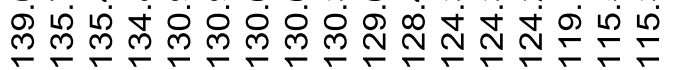
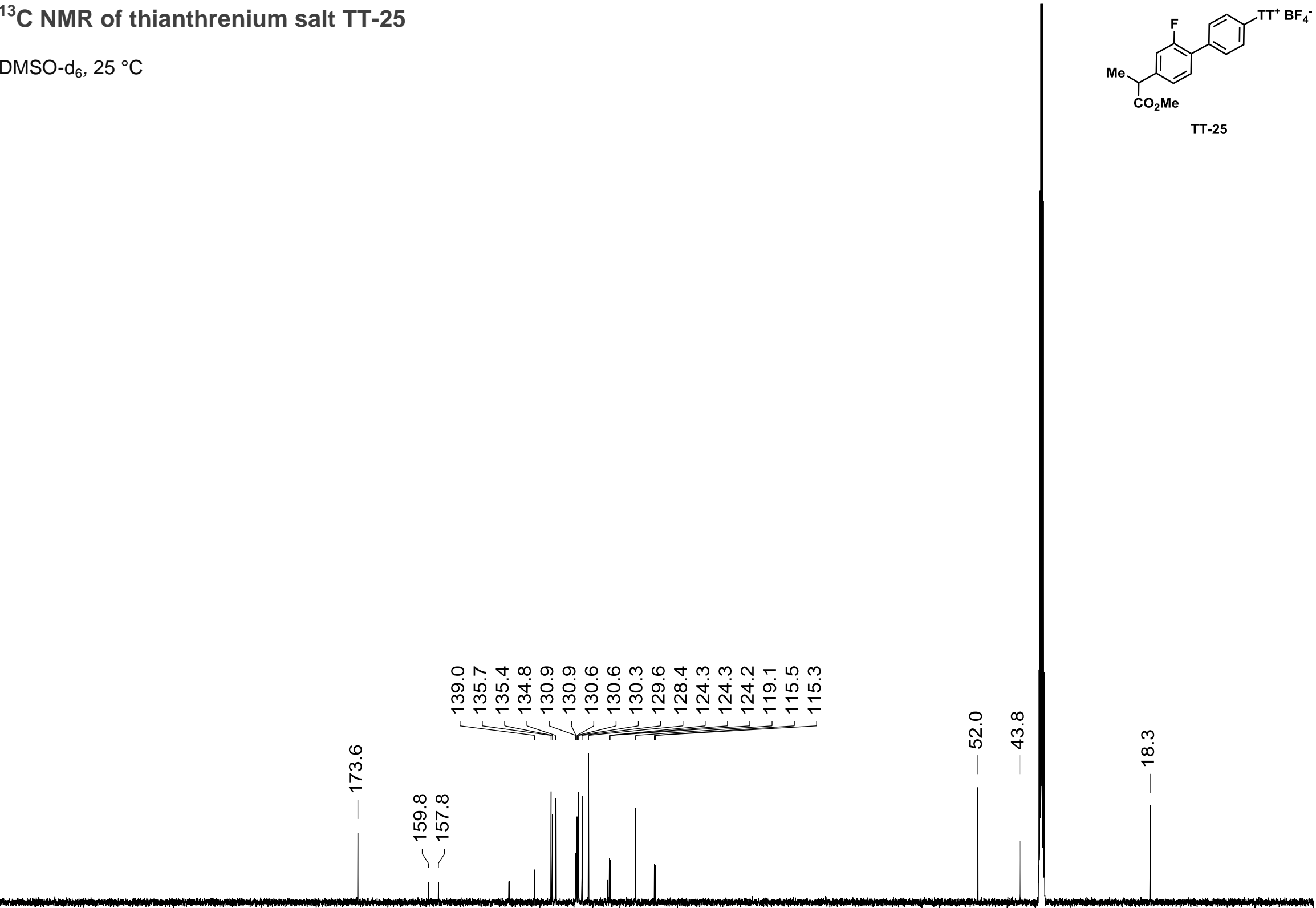

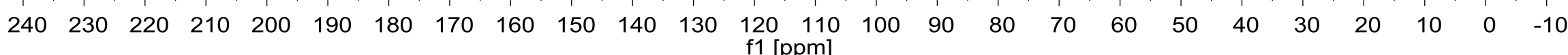


${ }^{19} \mathrm{~F}$ NMR of thianthrenium salt TT-25

DMSO- $\mathrm{d}_{6}, 25^{\circ} \mathrm{C}$
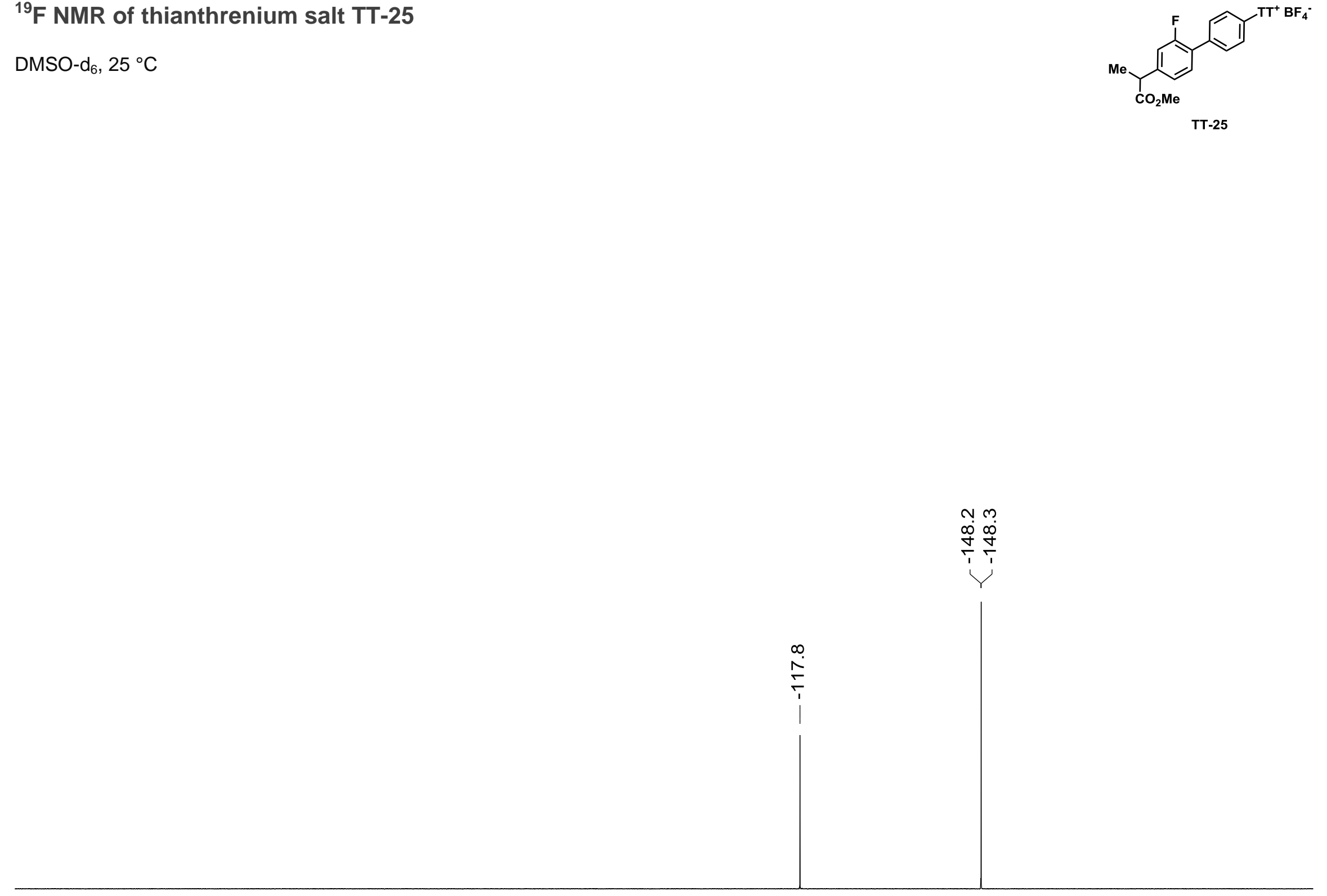

\begin{tabular}{llllllllllllllllllllllllllll}
\hline 10 & 0 & -10 & -20 & -30 & -40 & -50 & -60 & -70 & -80 & -90 & -100 & -110 & -120 & -130 & -140 & -150 & -160 & -170 & -180 & -190 & -200
\end{tabular}




\section{${ }^{1} \mathrm{H}$ NMR of aniline 25a}

$\mathrm{CDCl}_{3}, 25^{\circ} \mathrm{C}$
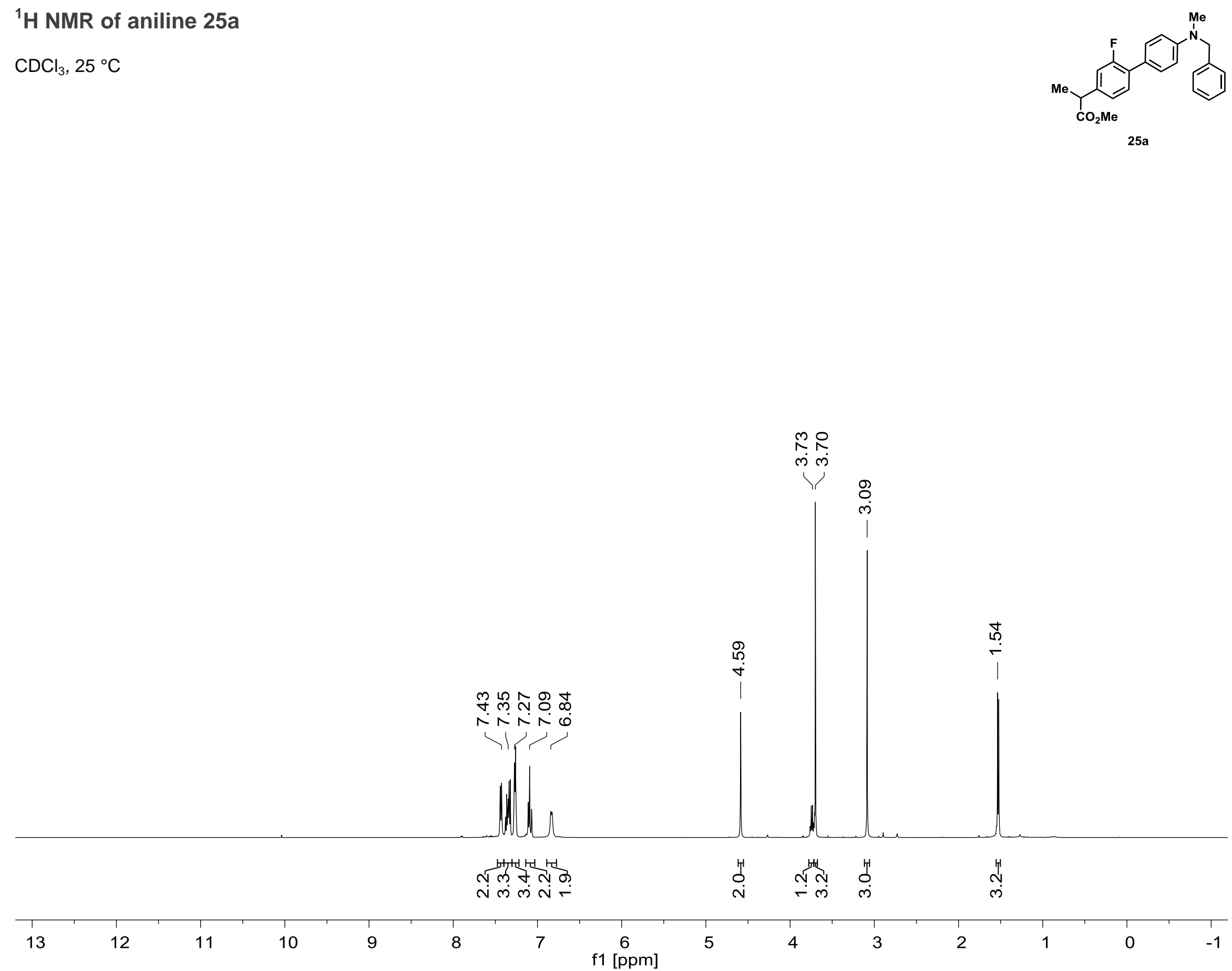


\section{${ }^{13} \mathrm{C}$ NMR of aniline $25 \mathrm{a}$}

$\mathrm{CDCl}_{3}, 25^{\circ} \mathrm{C}$

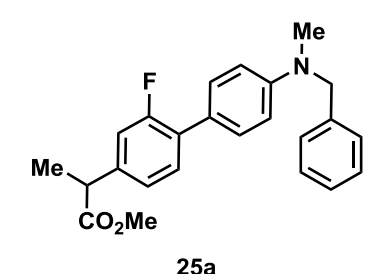

m m $, \infty, \sigma \tau \sigma$,

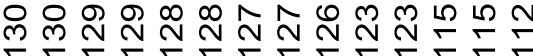

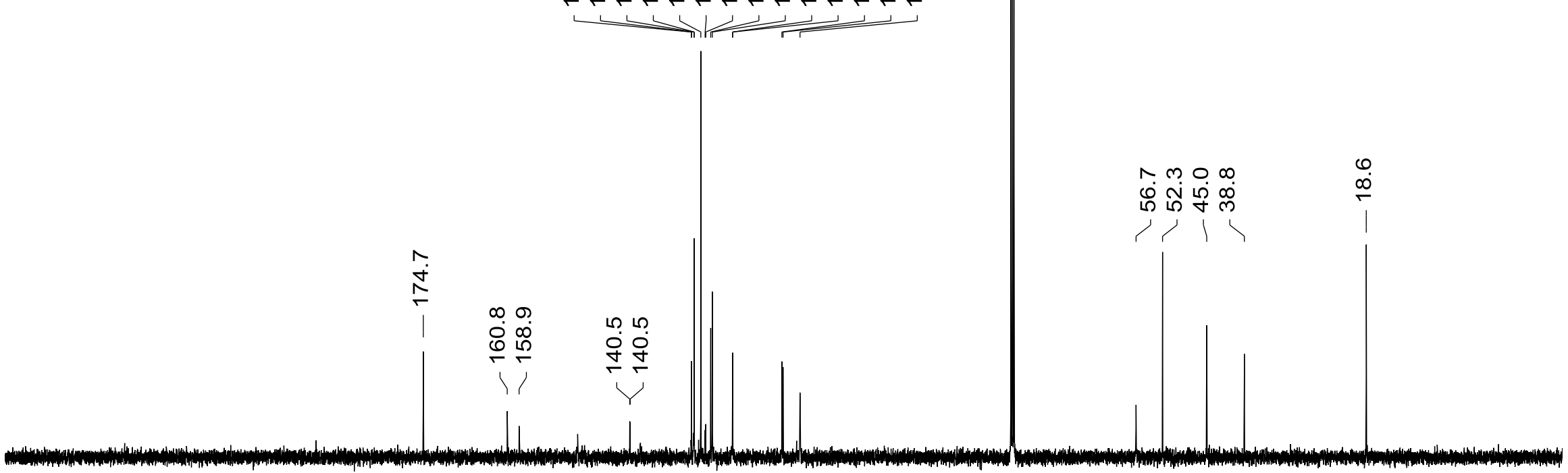


${ }^{19}$ F NMR of aniline $25 a$

$\mathrm{CDCl}_{3}, 25^{\circ} \mathrm{C}$

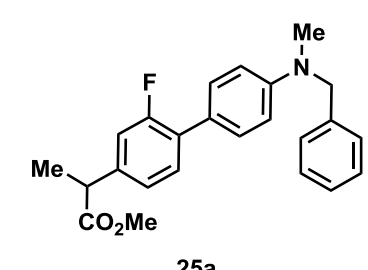

\begin{tabular}{lllllllllllllllllllllll}
\hline 10 & 0 & -10 & -20 & -30 & -40 & -50 & -60 & -70 & -80 & -90 & -100 & -110 & -120 & -130 & -140 & -150 & -160 & -170 & -180 & -190 & -200
\end{tabular}




\section{${ }^{1} \mathrm{H}$ NMR of aminoisoxazole 25b}

DMSO- $\mathrm{d}_{6}, 25^{\circ} \mathrm{C}$
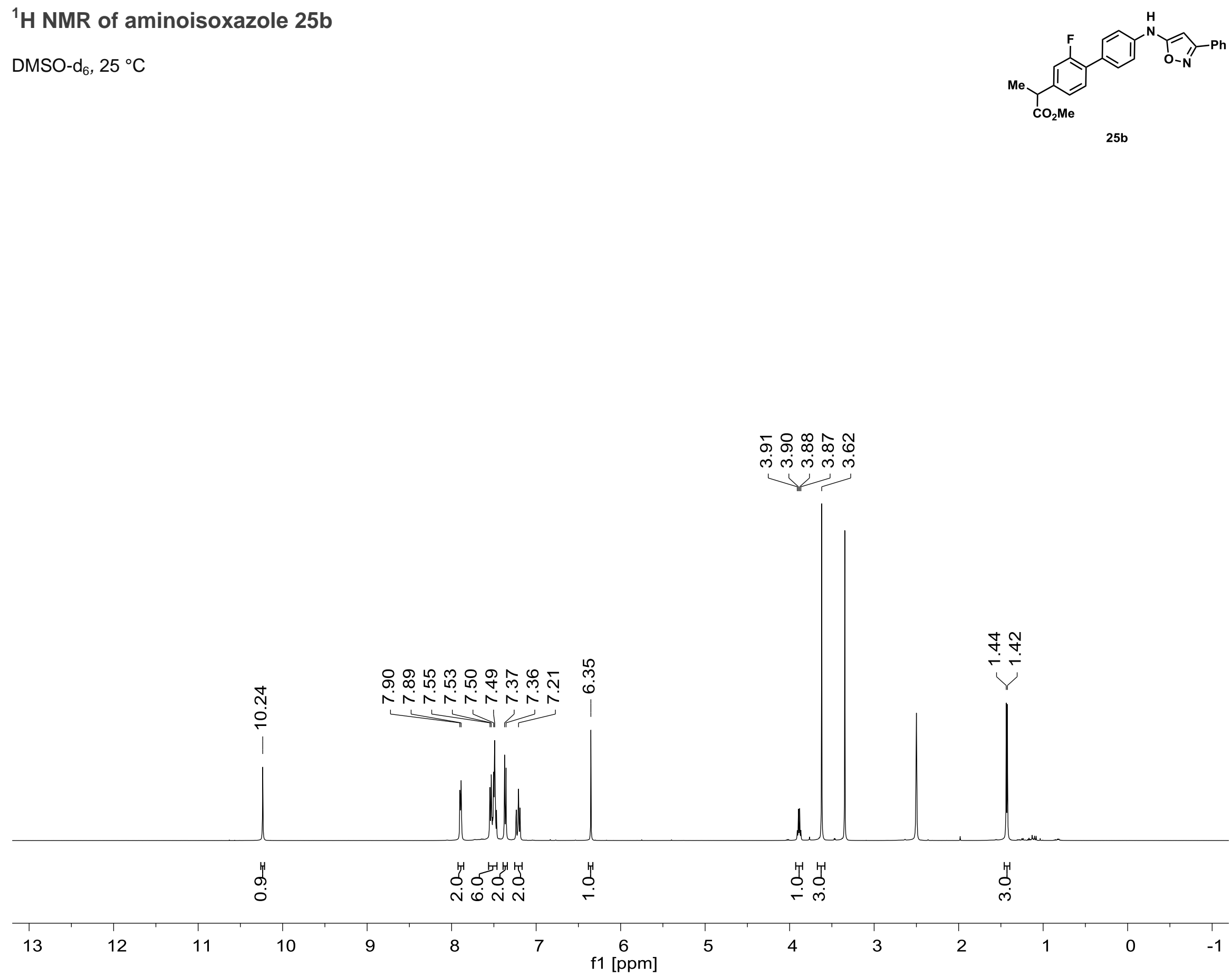


\section{${ }^{13} \mathrm{C}$ NMR of aminoisoxazole $25 \mathrm{~b}$}

DMSO- $\mathrm{d}_{6}, 25^{\circ} \mathrm{C}$

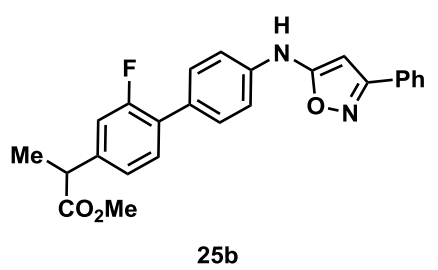

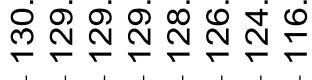

$\infty 0 \infty 00$

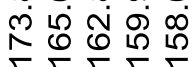

可应
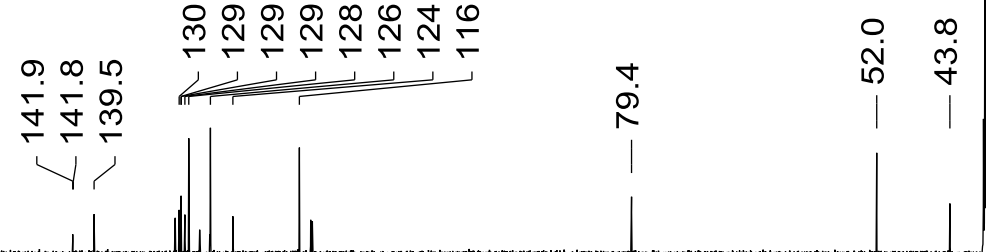

$\stackrel{\infty}{\infty}$ 
${ }^{19}$ F NMR of aminoisoxazole $25 b$

DMSO- $\mathrm{d}_{6}, 25^{\circ} \mathrm{C}$
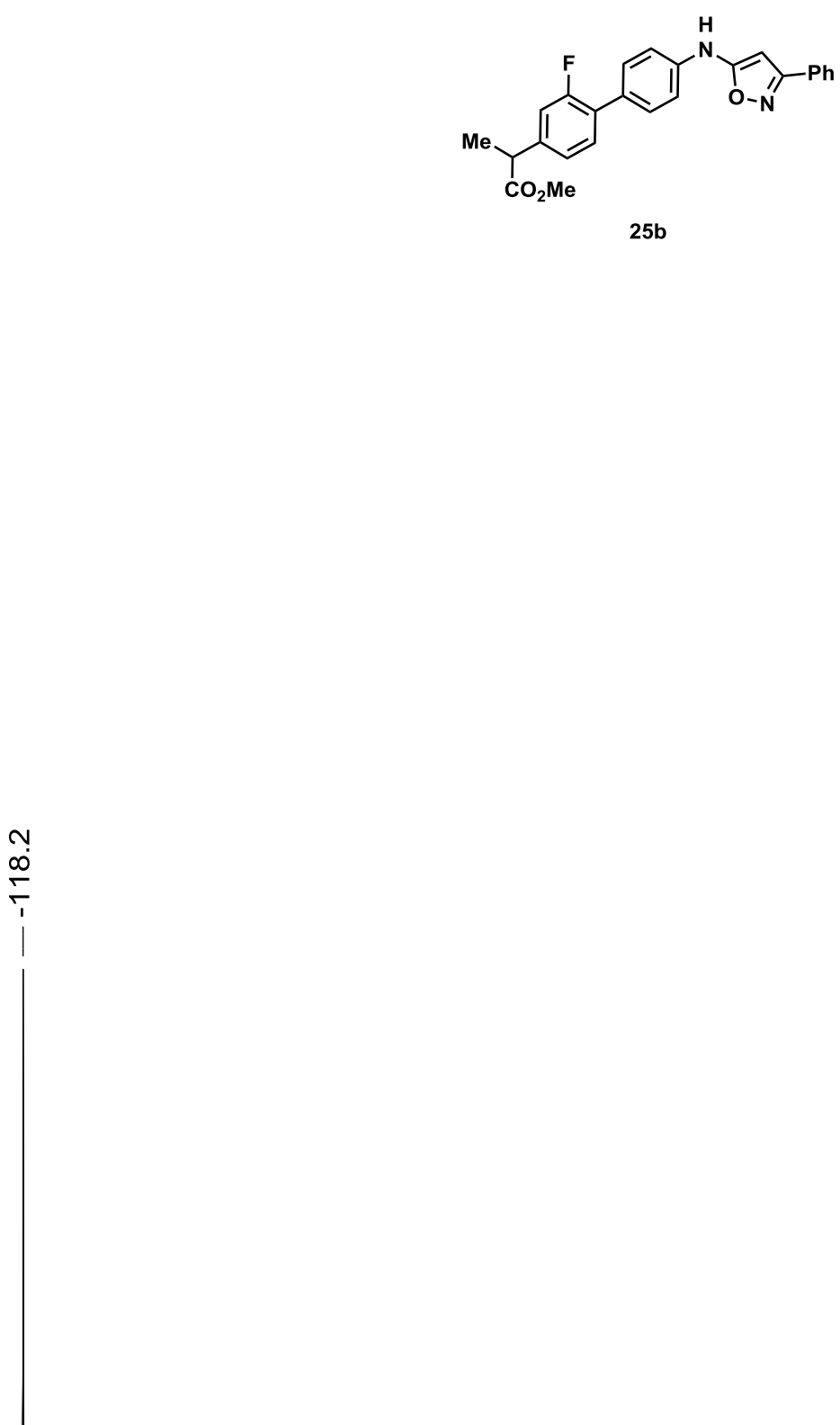

\begin{tabular}{llllllllllllllllllllll}
\hline 10 & 0 & -10 & -20 & -30 & -40 & -50 & -60 & -70 & -80 & -90 & -100 & -110 & -120 & -130 & -140 & -150 & -160 & -170 & -180 & -190 & -200
\end{tabular}




\section{${ }^{1} \mathrm{H}$ NMR of triazole 25c}

$\mathrm{CDCl}_{3}, 25^{\circ} \mathrm{C}$

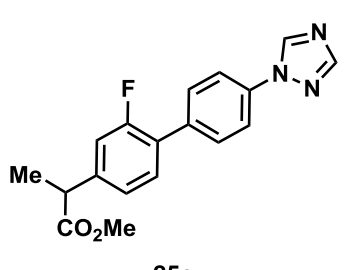

25c

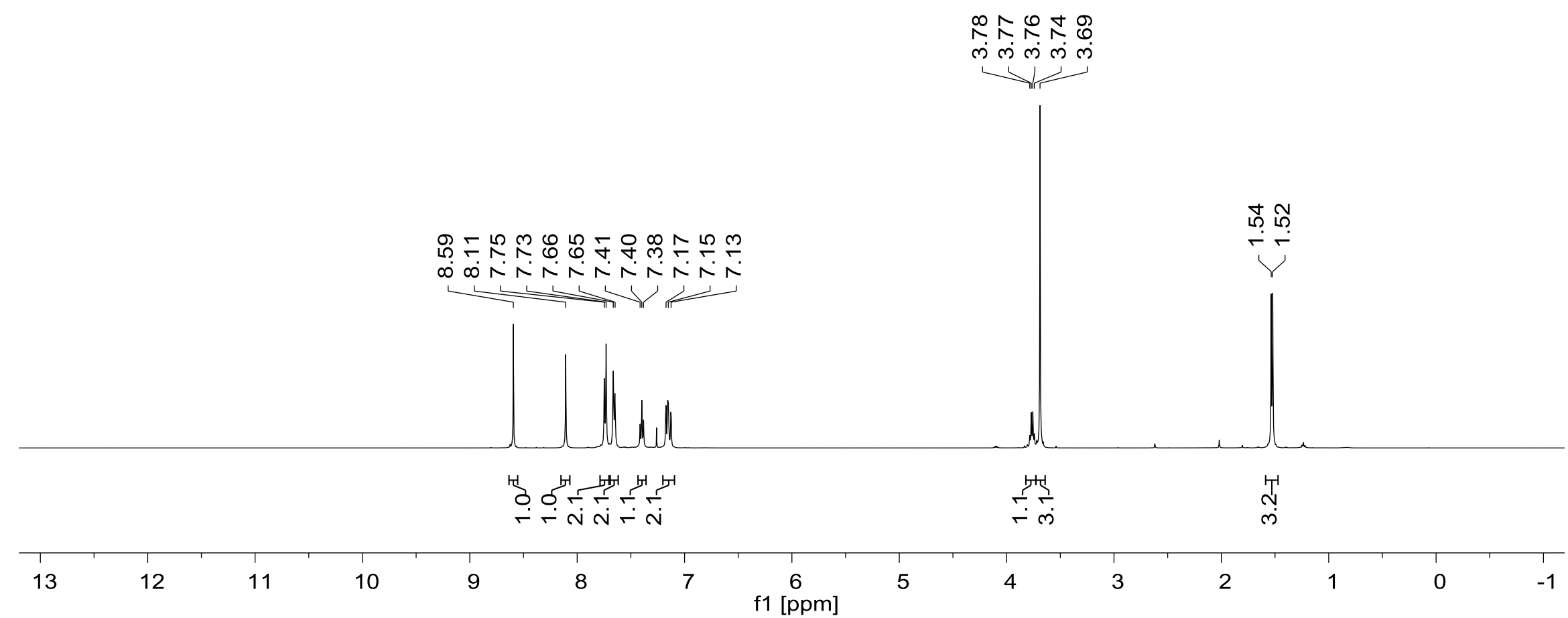




\section{${ }^{13} \mathrm{C}$ NMR of triazole $25 \mathrm{c}$}

$\mathrm{CDCl}_{3}, 25^{\circ} \mathrm{C}$

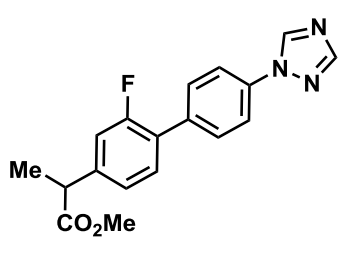

$25 \mathrm{c}$

A

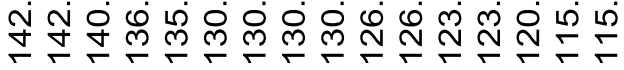

r

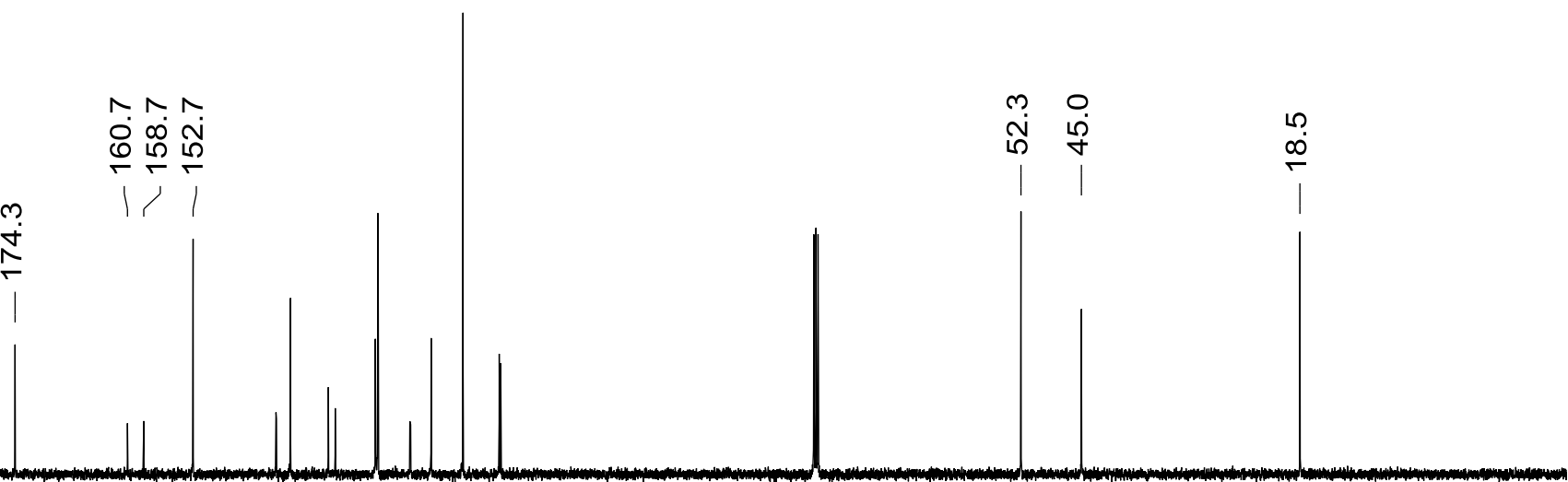

$\begin{array}{lllllllllllllllllllllllllllll}240 & 230 & 220 & 210 & 200 & 190 & 180 & 170 & 160 & 150 & 140 & 130 & 120 & 110 & 100 & 90 & 80 & 70 & 60 & 50 & 40 & 30 & 20 & 10 & 0 & -10\end{array}$ 
${ }^{19} \mathrm{~F}$ NMR of triazole $25 \mathrm{c}$

$\mathrm{CDCl}_{3}, 25^{\circ} \mathrm{C}$

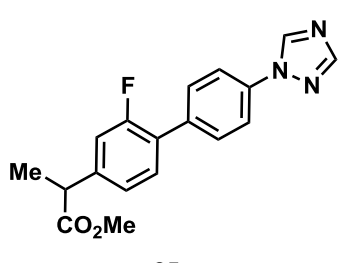

$25 \mathrm{c}$

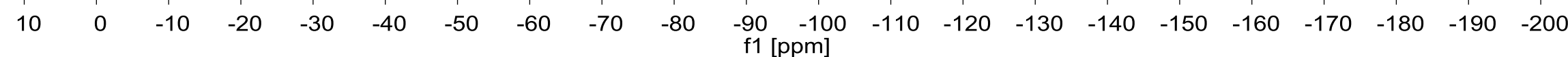




\section{${ }^{1} \mathrm{H}$ NMR of aniline $25 \mathrm{~d}$}

$\mathrm{CDCl}_{3}, 25^{\circ} \mathrm{C}$

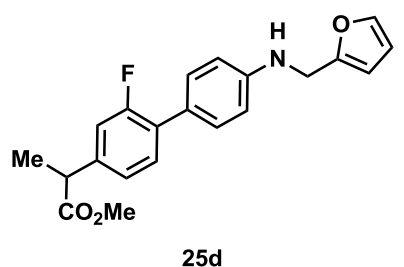

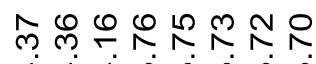

नं अंलंलाल

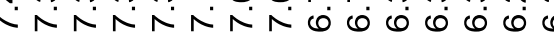

舟

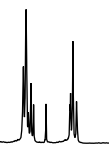

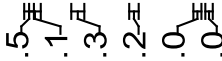

लं $\mathrm{N}$ -

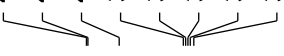




\section{${ }^{13} \mathrm{C}$ NMR of aniline $25 \mathrm{~d}$}

$\mathrm{CDCl}_{3}, 25^{\circ} \mathrm{C}$
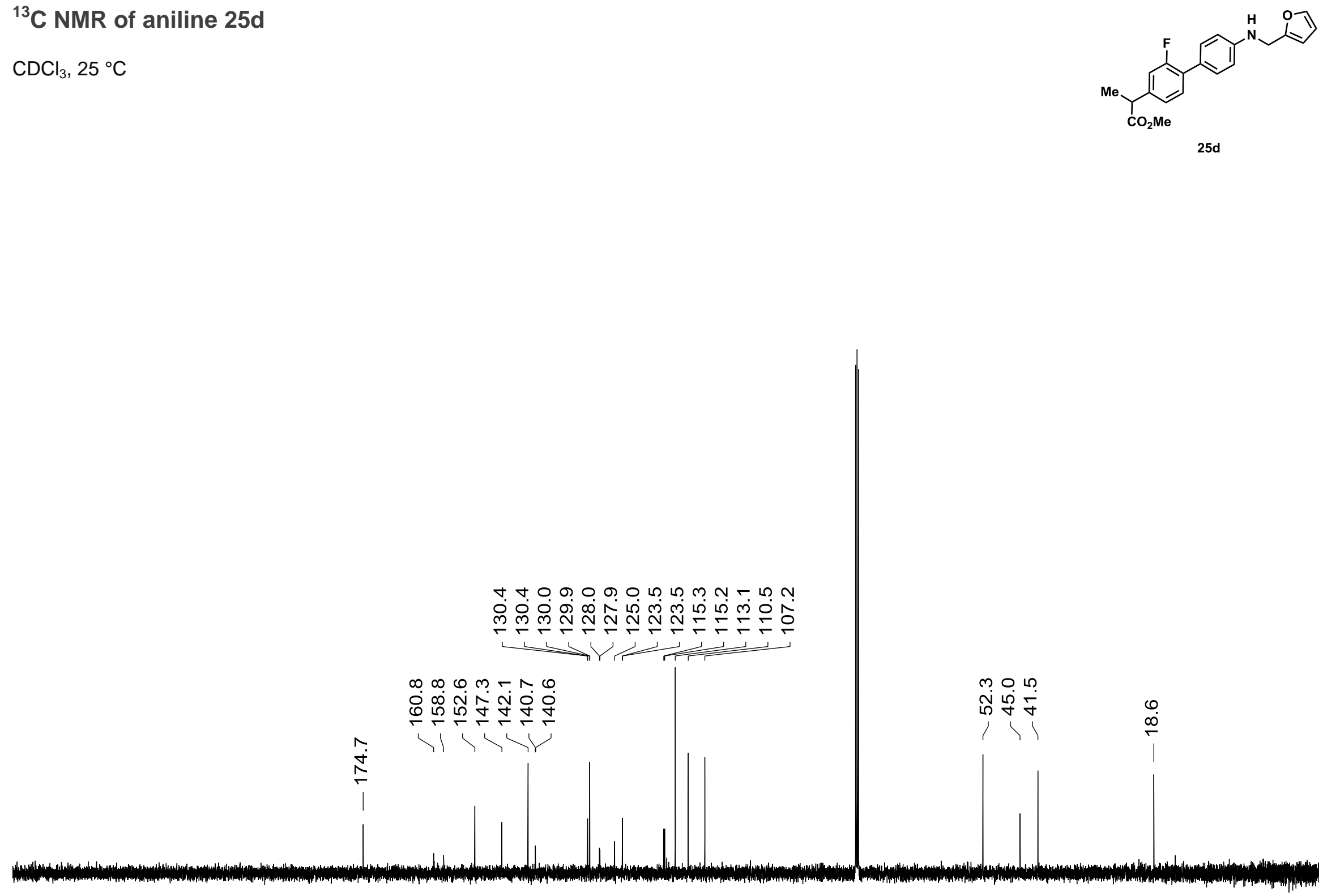

$\begin{array}{lllllllllllllllllllllllllllllll}240 & 230 & 220 & 210 & 200 & 190 & 180 & 170 & 160 & 150 & 140 & 130 & 120 & 110 & 100 & 90 & 80 & 70 & 60 & 50 & 40 & 30 & 20 & 10 & 0 & -10\end{array}$ 
${ }^{19} \mathrm{~F}$ NMR of aniline $25 \mathrm{~d}$

$\mathrm{CDCl}_{3}, 25^{\circ} \mathrm{C}$
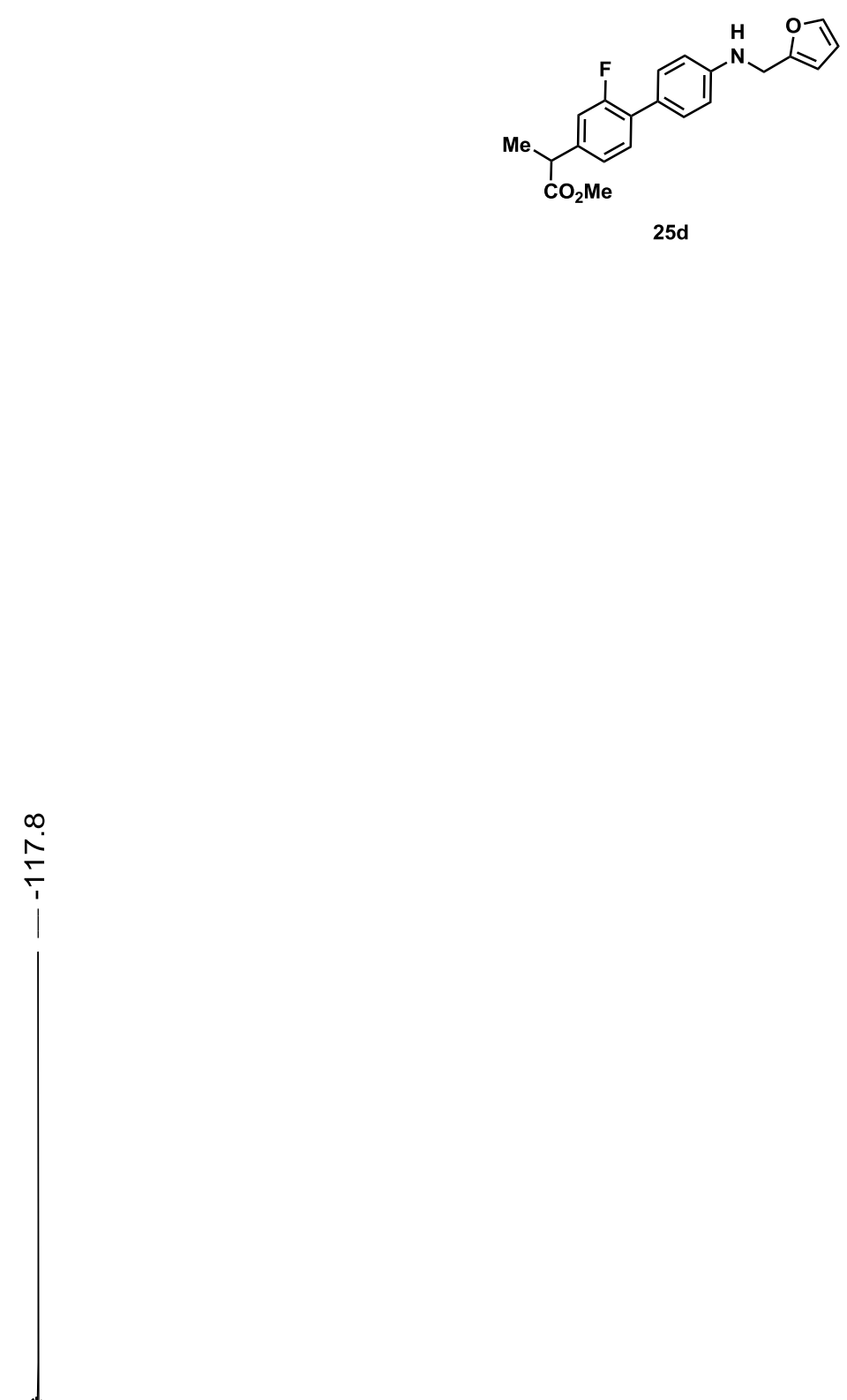

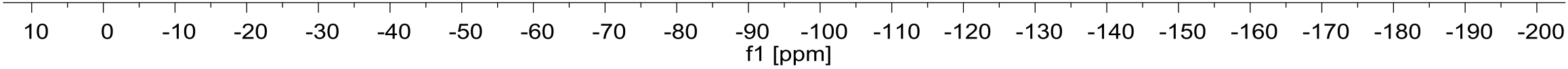

